\title{
Progress in the Development of Compressible, Multiphase Flow Modeling Capability for Nuclear Reactor Flow Applications
}

R. A. Berry

R. Saurel

F. Petitpas

E. Daniel

O. Le Métayer

S. Gavrilyuk

N. Dovetta

R. C. Martineau

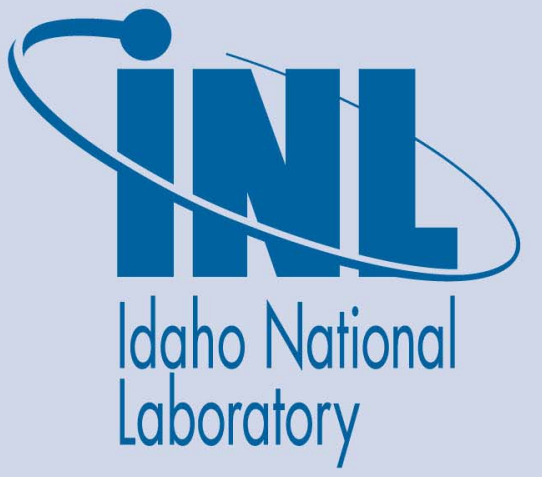

October 2008

The INL is a U.S. Department of Energy National Laboratory operated by Battelle Energy Alliance 
INL/EXT-08-15002

\section{Progress in the Development of Compressible, Multiphase Flow Modeling Capability for Nuclear Reactor Flow Applications}

${ }^{1}$ RS2N SARL

Marseille, France

(Contract 00073362)

\author{
R. A. Berry \\ R. Saurel ${ }^{1}$ \\ F. Petitpas ${ }^{1}$ \\ E. Daniel ${ }^{1}$ \\ O. Le Métayer ${ }^{1}$ \\ S. Gavrilyuk ${ }^{1}$ \\ N. Dovetta ${ }^{1}$ \\ R. C. Martineau
}

October 2008

\section{Idaho National Laboratory Idaho Falls, Idaho 83415 http://www.inl.gov}

Prepared for the U.S. Department of Energy Office of Nuclear Energy Under DOE Idaho Operations Office

Contract DE-AC07-05ID14517 


\section{DISCLAIMER}

This information was prepared as an account of work sponsored by an agency of the U.S. Government. Neither the U.S. Government nor any agency thereof, nor any of their employees, makes any warranty, expressed or implied, or assumes any legal responsibility for the accuracy, completeness, or usefulness, of any information, apparatus, product, or process disclosed, or represents that its use would not infringe privately owned rights. References herein to any specific commercial product, process, or service by trade name, trade mark, manufacturer, or otherwise, does not necessarily constitute or imply its endorsement, recommendation, or favoring by the U.S. Government or any agency thereof. The views and opinions of authors expressed herein do not necessarily state or reflect those of the U.S. Government or any agency thereof. 



\section{Contents}

Chapter 1: Introduction and Direction

Chapter 2: $\quad$ Well-Posed, Averaged Conservation Equations for Compressible Multiphase, Multi-Component, and Multi-Material Flows

Chapter 3: Simple and Efficient Relaxation Methods for Interfaces Separating Compressible Fluids and Cavitating Flows

Chapter 4: $\quad$ Toward a Low-Speed Flow Solver for Diffuse Interface Models

Chapter 5: Mathematical Models for DNS of Compressible Fluids In the Presence of Capillary Effects and Phase Transitions

Chapter 6: DEM Model for Flows in Ducts of Variable Cross-Section

Chapter 7: $\quad$ Modeling Phase Transitions in Non-Equilibrium Multiphase Compressible Flow Models 


\section{Chapter 1 \\ Introduction and Direction}




\section{Motivation}

In nuclear reactor safety and optimization there are key issues that rely on in-depth understanding of basic two-phase flow phenomena with heat and mass transfer. Within the context of multiphase flows, two bubble-dynamic phenomena - boiling (heterogeneous) and flashing or cavitation (homogeneous boiling), with bubble collapse, are technologically very important to nuclear reactor systems. The main difference between boiling and flashing is that bubble growth (and collapse) in boiling is inhibited by limitations on the heat transfer at the interface, whereas bubble growth (and collapse) in flashing is limited primarily by inertial effects in the surrounding liquid. The flashing process tends to be far more explosive (and implosive), and is more violent and damaging (at least in the near term) than the bubble dynamics of boiling. However, other problematic phenomena, such as crud deposition, appear to be intimately connecting with the boiling process. In reality, these two processes share many details.

Flashing occurs in flowing liquid systems when the pressure falls sufficiently low in some region of the flow, reaching a metastable state where the temperature is higher than the saturated one at the reduced pressure of this expanded state. Then the superheated liquid releases its metastable energy (stored as internal energy) very quickly, even explosively, producing either pure vapor (bubble) or liquid-vapor mixture at high velocity, [2]. Expansion effects in nuclear reactor systems are often due to geometrical effects, as for example in nozzles where flashing appears at locations where the pressure is relatively low and the liquid superheated. In the case of twophase blowdown (from the superheated liquid state), bubble collapse is usually not important, but the flashing of superheated liquid strongly influences critical flow rates. In other cases, besides the performance limitations which this cavitation may cause in flow systems, subsequent bubble collapse may be responsible for damage to nearby solid surfaces.

Many nuclear reactor applications rely on convective nucleate boiling to efficiently remove high heat fluxes from heated surfaces. Nucleate boiling is a very effective heat transfer mechanism, however it is well known that there exists a critical value of the heat flux at which nucleate boiling transitions to film boiling (departure from nucleate boiling (DNB) and boiling crisis), a very poor heat transfer mechanism. In most practical applications it is imperative to maintain the operating heat flux below such critical value, which is called the Critical Heat Flux (CHF). In this case, the presence of a nearby solid surface is necessary for the rapid supply of the latent heat inherent in the phase change. The presence of these surfaces is known to modify the flow patterns and other characteristics of these multiphase flows, and therefore must be interactively coupled with analyses of these phenomena. And again, as mentioned above, DNB is believed to play an integral role in performance degradation as well as the crud deposition problem. Despite several decades of intense study a consensus explanation of the physical mechanism causing CHF is yet to be found, even for the simple situation of pool boiling on a flat plate, let alone flow boiling in a rod-bundle geometry, which is the situation of interest in nuclear reactors. Many theories have been formulated, all of which rely on simple CHF models based on an idealized geometry of the vapor/liquid interface. For example, the classic hydrodynamic instability theory of $\mathrm{CHF}$ postulates an array of cylindrical vapor jets rising from the heater surface, the macrolayer dryout theory assumes a smooth liquid layer underneath a mushroom-shaped bubble, and the bubble interaction theories typically assume that nucleating bubbles are spherical. However, a very different picture of the physical situation at CHF has been revealed by recent 
studies (Theofanus et al. 2002, Nishio and Tanaka 2004) using sophisticated imaging diagnostics. Briefly, at high heat fluxes there exist numerous dry areas on the surface (with length scales of 2-3 $\mathrm{mm}$ in the $\mathrm{x}$ - and $\mathrm{y}$-directions), dispersed within an interconnected network of liquid menisci (with length scales $<50 \mu \mathrm{m}$ in the z-direction). The geometry of the liquid/vapor interface is highly irregular and its nature is dynamic, i.e., the liquid menisci advance into and retreat from the dry areas as a function of time, due to various effects, e.g., liquid inertia (sloshing), capillary forces (surface tension) and recoil forces (evaporation). Our inability to accurately predict heat transfer and heat transfer regime transitions (flow topologies) in the real nuclear reactor two-phase flow conditions and fuel bundle geometry results in increased safety margins and impedes the development of new fuel designs.

With the advent of increased availabilitly of computational power and of a new generation of codes it is believed that significant progress can be made by using computational fluid dynamics (CFD) for nuclear fuel design thus leading to a drastic reduction in development costs. As recently observed by the principal investigator at the European workshop on "Two-phase Convective Flow Boiling Flow Modelling”, our ideas for a broad spectrum of multiphase flow simulation improvements, as will be discussed subsequently, are being echoed by others. In France, CEA, EDF, AREVA and IRSN have launched the NEPTUNE project, aimed at providing tools describing two-phase flow and heat transfer that are validated in the parameter range of the industrial applications, plus others covering the entire spectrum of space and time scales. In the U.S. no such unified effort exists, but it seems necessary to promote collaborative research on this subject at the widest scale.

For example, the two-phase flow phenomena occurring inside a Boiling Water Reactor (BWR) fuel bundle includes coolant phase changes and multiple flow regimes which directly influence the coolant interaction with the fuel assembly and, ultimately, the reactor performance. The resolution of traditional sub-channel analysis codes is too coarse for analyzing the detailed intraassembly flow patterns, such as flow around a spacer element and it is now generally recognized that their basic modeling approach and computational methods no longer represent state-of-theart in the field of numerical simulation. New codes need to be developed for the fine-mesh, detailed simulation of BWR fuel assembly two-phase flow phenomena which take advantage of recent progress in Computational Fluid Dynamics (CFD) and the rapidly increasing computational power of massively parallel computers. Similar arguments can be made for the need for high resolution modeling of localized subcooled boiling and bubble collapse in Pressurized Water Reactors (PWR) to predict performance degradation, especially with regard to the crud deposition problem.

Because modern, high-resolution numerical methods/CFD codes divide the flow space into much finer computational cells it is imperative that we not:

- Utilize traditional multiphase models which are mathematically ill-posed,

- Rely on traditional "flow regime maps" used in subchannel thermal-hydraulics codes to evaluate the interface topology.

Traditional 6-equation, single pressure two-phase mixture models have a domain in which the square sound speed is negative which produces ill-posedness, non-hyperbolicity, wrong wave dynamics, and inappropriate transient solutions. Such equations have been used in the past 
because first order numerics were employed on course grids which produced large enough artificial viscosity to render a solution. However, grid convergence with such a scheme is not possible - an untenable situation. Using such equation systems negates the possibility of utilizing modern high-resolution methods (second order or higher), for example, finite volume methods based on Godunov methods with approximate Riemann solvers.

Inter-phase interactions in multiphase fluids depend on both the area and the topology of the interface. Traditional "flow-regime maps" are used in sub-channel thermal-hydraulics codes to evaluate the interface topology from cross-section-averaged flow parameters. Because CFD codes divide the flow space into much finer computational cells, they need not rely on the traditional sub-channel flow regimes. Instead, they must evaluate the local interface surface topology. The advantage realized with this approach is that the ensemble of many computational cells, with relatively simple interface surface topologies, can provide complex global topologies that include all the traditional sub-channel flow regimes. With these simplified flow regime topological maps, which will necessarily depend upon computational cell-size, only a few parameters need be used to determine the interface surface topology in each cell. These parameters will be based on experimental data and direct numerical simulation (DNS) of highly resolved phenomena on a smaller scale.

\section{Direction}

With the above motivation in mind, the appropriate research direction was established, aimed at developing well-designed physical/mathematical models along with high-resolution numerical solution methods for general multiphase flows. Specifically, research for this project is directed towards a unified physical/mathematical and numerical model development of compressible, allspeed multiphase flows spanning the following areas:

(1) General mixture level (true multiphase),

(2) Resolved interface level (DNS-like), and

(3) Multi-scale methods to resolve both (1) and (2) automatically, depending upon specified mesh resolution.

Conceptually, a well-designed 2-pressure, 2-velocity, seven-equation two-phase mixture model (as in 1) could be systematically reduced analytically to produce a 1-pressure, 1-velocity, fiveequation model (as for 2) capable of a DNS-like (Direct Numerical Simlation) resolved interface solution. If such a systematic reduction was accomplished numerically on a local spatial level, then a general algorithm (such as 1) could be made to reduce locally where appropriate to the interface resolved model (2), effectively giving an automatic multi-scale treatment (as 3).

Research is needed to lay the foundations necessary to acquire the capability to simultaneously solve fluid dynamic interface problems as well as multiphase mixtures arising from boiling, flashing or cavitation of superheated liquid, and bubble collapse, etc. in light water reactor systems. The effective nonlinear system of first order partial differential equations, along with their closure conditions, must be:

- hyperbolic and well-posed,

- in conservative form, if phase interactions and dissipative terms are neglected, and

- exhibit correct wave dynamics. 
Such two-phase flow phenomena occurring inside light water nuclear reactors includes, especially with departure from nucleate boiling (DNB) and film boiling instability (boiling crisis), coolant phase changes and multiple flow regimes which directly influence the coolant interaction with the fuel elements/assemblies and, ultimately, the reactor performance. Because of the inherent coupling, an understanding of these phemonena, along with subcooled boiling and bubble collapse, is also key to gaining an understanding of crud depostion in these systems. The goal of this research should be to provide models giving highly resolved details where necessary, simultaneously with large scale vessel/component simulation by providing a wellposed, multi-scale model that will

- Resolve interfaces for larger bubbles (direct numerical simulation, DNS-like) with single velocity, single pressure treatment, and

- Average (or homogenize) the two-phase flow field for small bubbles with two velocities, two pressures.

The principal investigator was recently asked to present an overview of this approach at the 2008 SIAM Annual Meeting (Berry and Kadioglu 2008).

The purpose of this three year LDRD project is, therefore, to lay the foundations prerequisite to developing the capability needed, as mentioned above, to simultaneously solve fluid dynamic interface problems as well as multiphase mixtures arising from boiling, cavitation, bubble collapse, etc. in light water reactor systems. This project is a collaborative team effort that, in addition to the Multiphysics Methods and High Performance Computing Groups at INL, included Prof. Richard Saurel and his SMASH team at Polytechnique University in Marseille. Professor Saurel and his group has been working for along time in multiphase flows and interface modeling in extreme thermodynamic conditions such as those related to shocks, explosions, and detonation physics. The key point here is that in these domains wave propagation is of fundamental importance and mathematical formulations as well as numerical methods must be perfectly clean. To solve compressible multiphase flows, even for weak wave problems, well-posedness is essential and correct wave dynamics (correct eigenvectors, eigenvectors, dispersion relations, etc.) is imperative. Our collaboration with Prof. Saurel is particularly ideal because of their complementary capabilities with researchers at INL. It is anticipated that other partners will contribute support in subsequent years. This informal report documents our research efforts for the nine-month authorized activity period of FY-2008 (approximately mid-December through September of 2008).

The complexity of multiphase, multi-component, and/or multi-material flow dictates that they need to be examined in an averaged sense. Traditionally, one would begin with known (or at least postulated) microscopic flow relations that hold on the "small" scale. These include continuum level conservation of mass, balance of species mass and momentum, conservation of energy, and a statement of the second law of thermodynamics often in the form of an entropy inequality (such as the Clausius-Duhem inequality). The averaged or macroscopic conservation equations and entropy inequalities are then obtained from the microscopic equations through suitable averaging procedures. At this stage a stronger form of the second law may also be postulated for the mixture of phases or materials. To render the evolutionary material flow balance system unique, constitutive equations and phase or material interaction relations are introduced from experimental observation, or by postulation, through strict enforcement of the 
constraints or restrictions resulting from the averaged entropy inequalities. These averaged equations form the governing equation system for the dynamic evolution of these mixture flows.

Most commonly, the averaging technique utilized is either volume or time averaging or a combination of the two. The flow restrictions required for volume and time averaging to be valid can be severe, and violations of these restrictions are often found. A more general, less restrictive (and far less commonly used) type of averaging known as ensemble averaging can also be used to produce the governing equation systems. In fact volume and time averaging can be viewed as special cases of ensemble averaging. In Chapter 2 an introduction to the ensemble averaging methodology is given to show how ensemble averaged balance equations and entropy inequality can be obtained from the microscopic balances. It then motivates the general need for inclusion of a separate pressure for each phase along with an addition phasic volume fraction evolution equation of at least first or second order in time. Finally, some seven-equation, twopressure, two-velocity hyperbolic, well-posed models for compressible two-phase flows are given and motivation is introduced for an alternative approach to solving the multiphase mixture flows, the discrete equation method, or DEM, to be subsequently discussed in more detail.

Though our research is ultimately aimed at developing unified, well-designed physical/mathematical models along with high-resolution numerical solution methods for general multi-scale, multiphase flow, in the interim, for numerical efficiency reasons it is expedient to build quality, analytically reduced models of the general mixture equations that can be used to produce DNS-like, interface-resolving solutions for multiple compressible fluid phases. Because of the inherent weaknesses of sharp interface methods for DNS-like simulations, most notably their inability to dynamically create interfaces and to solve interfaces separating pure media and mixtures, we focused on a diffuse interface method (DIM) which does not exhibit these weaknesses. Chapter 3 details our works in this area. The DIM considers interfaces as numerically diffused zones corresponding to artificial mixtures created by numerical diffusion. The determination of thermodynamic flow variables in these zones is achieved on the basis of multiphase flow theory. The challenge is to derive physically, mathematically, and numerically consistent thermodynamic relations for the artificial mixture. The same algorithm is implemented globally in both pure fluids and in mixture zones. For this research, a single velocity, non-conservative hyperbolic model was developed, with two energy equations involving relaxation terms, which fulfills the equation of state and energy conservation on both sides of interfaces and guarantees correct transmission of pressure waves across them. This formulation considerably simplifies numerical resolution within the context of diffuse interfaces. Codes were constructed to successfully demonstrate this methodology. Our Ph.D. student's work on this topic is of sufficient note that the world renowned scientist/mathematician S. Godunov (Russian Academy of Science), whose pioneering developments form the underpinning of modern CFD, will be traveling from Russia for his jury in December. He will be joined also by R. Abgrall, a renowned multiphase flow method developer.

Multiphase formulations have been developed to give the ability to solve problems involving both heterogeneous mixtures of materials and interfacial flows involving compressibility and phase transition. In particular, for the direct numerical simulation (DNS) of interfacial flows, of prime importance for the nuclear industry for reactor safety and optimization, the aim is to compute the critical heat flux conditions that involve a competition between bubble growth, 
surface tension, contact angle effects, heat and mass transfers at interfaces. Contrary to the approach developed by other researchers, our approach embraced a general model that accounts for complete thermodynamics in both phases. Chapter 4 details our efforts with the INL PCICE partially implicit method. In this research we verified that the partially time-implicit, all-speed flow methods developed at INL (Appendix A of Chapter 4) for single-phase can be adapted to the finite volume framework (believed to be more suitable for the numerical approximation of hyperbolic models of diffuse interfaces with complex physics). This extension was performed based on a mix of Godunov type and PCICE type concepts, with excellent results - both low speed and high speed flows were computed accurately. Work was begun also on a more sophisticated hyperbolic model of DIM type (see previous paragraph), also with the combined Godunov-PCICE method. A new variation of the PCICE predictor stage was implemented in 1$\mathrm{D}$ to correct for a lack of frame invariance in the original scheme. The multiphase novelty compared to the single-phase version is the incorporation of a pressure relaxation substep. Our other graduate student (Masters) constructed a poster of this work this summer which recently won in a competition at Ecole Normale Supérieure (ENS) in France.

This LDRD also sponsored/directed research in a related area which will, in the future, become an integral part of the other research conducted and just described. A rational derivation of the conservative part of the multiphase model is elaborated in Chapter 5 using Hamilton's principle of stationary action followed by introduction of dissipation terms which are compatible with the entropy inequality to generalize the capillary models with the introduction of heat and mass transfer relaxation terms to describe phase transition.

Typically, multiphase modeling begins with an averaged (or homogenized) system of partial differential equations (traditionally ill-posed) then discretizes this system to form a numerical scheme. This presents problems for the numerical approximation of non-conservative terms at discontinuities (interfaces, shocks) as well as unwieldy treatment of fluxes with seven waves. To solve interface problems without conservation errors and to avoid this questionable determination of average variables and the numerical approximation of the non-conservative terms in conjunction with 2 velocity mixture flows we employ a new homogenization method known as the Discrete Equations Method (DEM). Contrary to conventional methods, the averaged equations for the mixture are not used, and this method directly obtains a (well-posed) discrete equation system from the single-phase system to produce a numerical scheme which accurately computes fluxes for arbitrary numbers of phases and solves non-conservative products. The method effectively uses a sequence of single phase Riemann equation solves. Phase interactions are accounted for by Riemann solvers at each interface. Flow topology can change with changing expressions for the fluxes. Non-conservative terms are correctly approximated. Some of the closure relations missing from the traditional approach are automatically obtained. Lastly, we can sometimes identify the continuous system induced by the discrete equation. This can be very useful from a theoretical standpoint. In Chapter 6 we construct a DEM model for 1-D two compressible phases in ducts of spatially variable crosssection to test this approach. For the first time, we demonstrated on a converging-diverging twophase nozzle that this well-posed 2 pressure, 2 velocity model could be integrated to a meaningful steady-state with both phases treated as compressible. Chapter 7 gives a review of the general DEM method and investigates the modeling of mass transfer between compressible 
multiphase flows within the context of DEM through the development of a Reactive Riemann solver (RDEM) for phase change.

With the exception of the PCICE method, all of the demonstrated methods above, and documented in this report, utilized explicit time integration. To relieve time step restrictions due to stiffness and to achieve tighter coupling of equations, all methods need to evolve with implicit time integration. Toward that objective, a significant effort has been expended toward development of a method of lines (MOL) approach to the integration of the equation system. With the MOL we discretize separately the space and time domains, effectively converting to a large system of ordinary differential equations (ODE) that can be integrated with previously developed, highly refined special purpose software. A MOL framework was developed around several ODE integration packages. Though not documented in this report, we are currently incorporating the 1-D variable area, two-phase DEM algorithm described above into this framework. We will test and report on the fully implicit integration of this system at a later date. Ultimately, our plan is to be able to perform numerical integration of the stiff relaxation terms to produce a truly multi-scale method which can handle two-phase mixtures at the large, or under resolved scale, and DNS two-phase modeling on the small, or highly resolved, scale.

Simple, efficient and robust algorithms are needed to solve the well-posed models. The various ingredients employed in methods to be developed should be general enough to consider future extensions to problems involving complex multiphysics. Using the fractional step methods typically applied, and miss-applied, to calculate these types of initial value problems, that consist of different kinds of physics with multiple time-scales, some of which should be treated implicitly, requires some precautionary measures to avoid splitting and conditioning errors. We believe that a fully implicit treatment may not only be advantageous, but indeed necessary. An appropriate fully implicit approach will allow integration over the fast time scales for slow speed flows. I anticipate that using a Jacobian-Free Newton-Krylov method with physics based preconditioning will allow tightly coupled solutions of the multiphysics phenomena inherent in nuclear reactor core applications.

\section{References}

Berry R.A. and S. Kadioglu, "High-Resolution, Two-phase Flow Modeling Challenges for Light Water Nuclear Reactors," 2008 SIAM Annual Meeting, San Diego, CA, July 2008.

Nishio S. and H. Tanaka, "Visualization of Boiling Structures in High-Heat Flux Pool-Boiling," Int. J. Heat Mass Transfer, 47, 4559-4568 (2004).

Theofanus T.G. et al., "The Boiling Crisis Phenomena. Part II: Dryout Dynamics and Burnout," Experimental Thermal and Fluid Science, 26, 793-810 (2002).

"Two-phase Convective Flow Boiling Flow Modelling", organized by the Societe Hydrotechnique de France (SHF) and the International Association of Hydraulic Engineering and Research (IAHR) under sponsorship of Societe Francaise des Thermiciens (SFT), CEA, AREVA, EDF, and AFM. Workshop was held in Grenoble, France, Sept. 8-9, 2008. 


\section{Chapter 2 \\ Well-Posed, Averaged Conservation Equations for Compressible Multiphase, Multi-Component, and Multi-Material Flows}




\section{Introduction}

Many important "fluid" flows involve a combination of two or more materials having different properties. The multiple phases or components often exhibit relative motion among the phases or material classes. The microscopic motions of the individual constituents are complex and the solution to the micro-level evolutionary equations is difficult. Characteristic of such flows of multi-component materials is an uncertainty in the exact locations of the particular constituents at any particular time. For most practical purposes, it is not possible to exactly predict or measure the evolution of the details of such systems, nor is it even necessary or desirable. Instead, we are usually interested in more gross features of the motion, or the "average" behavior of the system. Here we present descriptive equations that will predict the evolution of this averaged behavior. Due to the complexities of interfaces and resultant discontinuities in fluid properties, as well as from physical scaling issues, it is essential to work with averaged quantities and parameters. We begin by tightening up, or more rigorously defining, our concept of an average. There are several types of averaging. The published literature predominantly contains two types of averaging: volume averaging [Whitaker 1999, Dobran 1991] and time averaging [Ishii 1975]. Occasionally combinations of the two are used. However, we utilize a more general approach by adopting what is known as ensemble averaging.

When the physical system has a large amount of variability, a natural interpretation of the meaning of predictions is in terms of expected values and variances. If there are many different events, or realizations, possible, then the expected value is naturally an "average" over all of these events, or the ensemble of realizations. The ensemble then is the set of all experiments with the same boundary- and initial-conditions, with some properties that we would like to associate with the mean and distribution of the components and their velocities. A realization of the flow is a possible motion that could have happened. Implicit in this concept is the intuitive idea of a "more likely" and a "less likely" realization in the ensemble. Therefore, as we shall see shortly each ensemble of realizations, corresponding to a given physical situation, has a probability measure on subsets of realizations. The ensemble average is the generalization of the elementary idea of adding the values of the variable for each realization, and dividing by the number of observations. The ensemble average then allows the interpretation of phenomena in terms of repeatability of multi-component flows.

One of the nice features of ensemble averaging, as opposed to volume averaging, is that ensemble averaging does not require that a control volume contain a large quantity of a particular component in any given realization. Consider the following example, taken directly from Drew and Lahey (1993), where the average of a particle-fluid mixture is of interest. Gas turbines are eroded by particulate matter suspended in the gas stream passing through the inlet and impacting on the various parts of the machine, e.g. the turbine blades. The trajectories of individual particles moving through the gas turbine are very complicated, depending on where and when the particles enter the inlet of the device. Such predictions are, fortunately, seldom required. A prediction, however, that is of interest to the designer is the average, or expected values, of the particle flux (or the concentration and velocities of particles) near parts in the device that are susceptible to erosion. Since the local concentration of particles is proportional to the probability that particles will be at the various points in the device at various times, and the particle velocity field will be the mean velocity that the particles will have if they are at that 
position in the device, the design engineer will be able to use this information to assess the places where erosion due to particle impact may occur. Notice it may be that there are no times for which there will be many particles in some representative control volume (or representative elementary volume, REV). So, volume averaging, which depends on the concept of having many representative particles in the averaging volume at any instant, will fail. The appropriateness of ensemble averaging is obvious. Here the ensemble is the set of motions of a single particle through the device, given that it started at a random point at the inlet at a random time during the transient flow through the device. Clearly the solution for the average concentration and average velocity gives little information about the behavior of a single particle in the device; however, the information is very appropriate for assessing the probability of damage to the device. Similar examples could be given where time averaging will fail, but where ensemble averaging is again appropriate.

The ensemble average is the more fundamentally based than either time or volume averaging. In fact, both time and volume averaging can be viewed as approximations to the ensemble average, which can be justified, respectively, for steady or homogeneous flow [Drew and Passman 1999].

\section{Ensemble Averaging}

A general method is presented here, based on the ensemble averaging concept [Kashiwa \& Rauenzahn 1994, Lhuillier 1996, Brackbill et.al. 1997, Drew \& Passman 1999], for developing averaged conservation equations for multiple materials, any one of which may be at point $\vec{x}$, at a given instant $t$. With this procedure, the most likely state at a point (the expected value) will be determined simultaneously with which material is most likely to be found at that point. Imagine running an experiment many times and collecting data about the state of the flow at each point $\vec{x}$ and time $t$. This information could include which material or phase is present, material density, velocity, pressure, temperature, concentration, etc. From this information, one can compute the ensemble average. The ensemble average of a generic property $Q_{0}$ of a fluid or material in a process is an average over the realizations

$$
\left\langle Q_{0}\right\rangle(\vec{x}, t)=\frac{1}{N_{R}} \sum_{r=1}^{N_{R}} Q_{0, r}(\vec{x}, t),
$$

where $N_{R}$ is the number of times the process or experiment is repeated, and is a large number. Now imagine that many of the realizations are near duplicates, i.e. they are essentially the same state, with $N$ occurrences. We can then rewrite the sum over the realizations as a sum over the number of states $N_{\Gamma}$ 


$$
\begin{aligned}
\left\langle Q_{0}\right\rangle(\vec{x}, t) & =\frac{1}{N_{R}} \sum_{\Gamma=1}^{N_{\Gamma}} N(\vec{x}, t, \Gamma) Q_{0}(\Gamma) \\
& =\sum_{\Gamma=1}^{N_{\Gamma}} \frac{N(\vec{x}, t, \Gamma)}{N_{R}} Q_{0}(\Gamma) \\
& =\int_{\text {all } \Gamma} Q_{0}(\Gamma) f(\vec{x}, t, \Gamma) d \Gamma,
\end{aligned}
$$

where $f(\vec{x}, t, \Gamma)=\frac{N(\vec{x}, t, \Gamma)}{N_{R}}$ is the probability of the state $\Gamma$ in the ensemble. Note that in the limit of an infinite number of repetitions of the experiment, with a sum over all of the states, we have replaced the summation with an integral form in the definition of the ensemble average. More correctly, because $\int_{\text {all } \Gamma} f(\vec{x}, t, \Gamma) d \Gamma=1.0$, we refer to $f(\vec{x}, t, \Gamma)$ as the probability density.

The state is the full thermodynamic description of the matter at a point $\vec{x}$ and time t. For example,

$$
\Gamma=\left\{\begin{array}{l}
\rho_{0}, \vec{u}_{0}, h_{0}, p_{0}, \tau_{0}, \\
\rho_{0}^{1}, \vec{u}_{0}^{1}, h_{0}^{1}, \rho_{0}^{2}, \vec{u}_{0}^{2}, h_{0}^{2}, \cdots, \\
X_{1}, X_{2}, \cdots
\end{array}\right\}
$$

where:

$X_{k}(\vec{x}, t)$ phase or material indicator function $:=1$ if material $k$ is present

$$
=0 \text { otherwise }
$$

$\rho_{0} \quad$ phase or material density

$\vec{u}_{0} \quad$ phase or material velocity

$h_{0} \quad$ phase or material specific enthalpy

$p_{0} \quad$ pressure

$\tau_{\sim} \quad$ deviatoric stress

$\rho_{0}^{s} \quad$ species partial density

$\vec{u}_{0}^{s} \quad$ species velocity

$h_{0}^{s} \quad$ species partial enthalpy,

with 


$$
\begin{aligned}
& \rho_{0}=\sum_{\text {species }} \rho_{0}^{s} \\
& \rho_{0} \vec{u}_{0}=\sum_{\text {species }} \rho_{0}^{s} \vec{u}_{0}^{s} \\
& \rho_{0} h_{0}=\sum_{\text {species }} \rho_{0}^{s} h_{0}^{s} .
\end{aligned}
$$

Other properties may also appear in the above thermodynamic state such as the phase or material temperature $\theta_{0}$, the phase or material specific internal energy $e_{0}$, and the phase or material specific entropy $s_{0}$.

In a typical multiphase flow, the ensemble averages of interest may include

$$
\begin{aligned}
& \text { Material } k \text { volume fraction : } \quad \alpha_{k} \equiv\left\langle X_{k}\right\rangle \\
& \text { Material } k \text { bulk average density : } \quad \hat{\rho}_{k} \equiv\left\langle X_{k} \rho_{0}\right\rangle \\
& \text { Material k intrinsic average density : } \quad \rho_{k} \equiv \frac{\left\langle X_{k} \rho_{0}\right\rangle}{\alpha_{k}} \\
& \text { Species } s \text { in material } k \text { bulk average density : } \quad \hat{\rho}_{k}^{s} \equiv\left\langle X_{k} \rho_{0}^{s}\right\rangle \\
& \text { Species } s \text { in material } k \text { intrinsic average density : } \quad \rho_{k}^{s} \equiv \frac{\left\langle X_{k} \rho_{0}^{s}\right\rangle}{\alpha_{k}} \\
& \text { Material } k \text { velocity : } \quad \vec{u}_{k} \equiv \frac{\left\langle X_{k} \rho_{0} \vec{u}_{0}\right\rangle}{\hat{\rho}_{k}}=\frac{\left\langle X_{k} \rho_{0} \vec{u}_{0}\right\rangle}{\alpha_{k} \rho_{k}} \\
& \text { Material } k \text { total energy : } \quad E_{k} \equiv \frac{\left\langle X_{k} \rho_{0} E_{0}\right\rangle}{\hat{\rho}_{k}}=\frac{\left\langle X_{k} \rho_{0} E_{0}\right\rangle}{\alpha_{k} \rho_{k}} \\
& \text { Material } k \text { entropy : } \\
& s_{k} \equiv \frac{\left\langle X_{k} \rho_{0} s_{0}\right\rangle}{\hat{\rho}_{k}}=\frac{\left\langle X_{k} \rho_{0} s_{0}\right\rangle}{\alpha_{k} \rho_{k}} \\
& \text { Mean mixture stress : } \quad \underset{\sim}{T} \equiv\left\langle\underset{\sim}{T_{0}}\right\rangle \\
& \text { Mean } k \text {-material stress : } \quad T_{\sim k} \equiv \frac{\left\langle X_{k} T_{\sim 0}\right\rangle}{\alpha_{k}} \\
& \text { Pressure (single pressure model) : } \quad p \equiv\left\langle p_{0}\right\rangle \\
& \text { Pressure in } k \text {-material : } \quad p_{k} \equiv \frac{\left\langle X_{k} p_{0}\right\rangle}{\alpha_{k}} \text {. }
\end{aligned}
$$

From a physical viewpoint, the bulk average density of a phase represents a summation of all of the density values that occurred for that phase, divided by the total number of experiments run. The bulk average density corresponds intuitively to the idea of the mass of phase $k$ per unit volume of mixture, or the observed material density. On the other hand, the intrinsic average density physically corresponds to a summation of all of the density values that occurred for that phase, dividing by the number of times in which that phase occurred in the experiments. The 
intrinsic average density corresponds intuitively to the idea of the mass of phase $k$ per unit volume of phase $k$, or the true material density. Some researchers prefer to work with bulk average densities [e.g. Kashiwa and Rauenzahn 1994] while others prefer working with intrinsic densities [e.g. Drew and Passman 1999]. This is mostly an issue of convenience, since one can easily be converted to the other. Here we will use intrinsic averages. Henceforth, when we say average, we shall mean intrinsic average unless indicated otherwise.

For a reasonably broad range of conditions (with common substances), the exact balance equations, valid inside each material, are

$$
\begin{array}{cr}
\dot{\rho}_{0}=-\rho_{0} \nabla \cdot \vec{u}_{0} & \text { Material mass conservation } \\
\dot{\rho}_{0}^{s}=-\rho_{0}^{s} \nabla \cdot \vec{u}_{0}-\nabla \cdot \rho_{0}^{s}\left(\vec{u}_{0}^{s}-\vec{u}_{0}\right)+\dot{r}_{0}^{s} & \text { Species mass conservation } \\
\rho_{0} \dot{\vec{u}}_{0}=\nabla \cdot T_{\sim 0}+\rho_{0} \vec{g} & \text { Material momentum balance } \\
\rho_{0} \dot{E}_{0}=\nabla \cdot\left(T_{\sim 0} \cdot \vec{u}_{0}\right)+\nabla \cdot \vec{q}_{0}+\rho_{0} \vec{g} \cdot \vec{u}_{0}+\rho_{0} \varepsilon_{0} & \text { Material energy conservation } \\
\rho_{0} \dot{s}_{0} \geq \frac{\rho_{0} \varepsilon_{0}}{\theta_{0}}-\nabla \cdot\left(\frac{\vec{q}_{0}}{\theta_{0}}\right) & \text { Material entropy inequality, }
\end{array}
$$

For these microscopic balance laws the material derivative has been used, which is defined as

$$
\dot{Q}_{0} \equiv \frac{\partial Q_{0}}{\partial t}+\vec{u}_{0} \cdot \nabla Q_{0} \quad \text { Material derivative } .
$$

Let us assume that the total variation of $f$ in the phase space $(\vec{x}, t, \Gamma)$ is [Kashiwa and Rauenzahn 1994]

$$
\frac{\partial f}{\partial t}+\vec{u}_{0} \cdot \nabla f+\dot{\Gamma} \cdot \frac{\partial f}{\partial \Gamma}=\frac{d f}{d t}=0
$$

where we are assuming that as we follow a material point through phase space its probability of occurrence remains constant. Various moments of this equation can be formed, first by multiplying equation (10) by $Q_{0}$, and then averaging this result. It can be shown [Kashiwa and Rauenzahn 1994, here corrected] that the resulting equation is

$$
\frac{\partial}{\partial t}\left\langle Q_{0}\right\rangle+\nabla \cdot\left\langle Q_{0} \vec{u}_{0}\right\rangle=\left\langle\dot{Q}_{0}+Q_{0} \nabla \cdot \vec{u}_{0}\right\rangle
$$

This result is called the moment evolution equation and the details of its derivation are given in the Appendix. The averaged conservation equations are obtained by letting our generic $Q_{0}$ be 
replaced by various "meaningful" functions and then by performing judicious manipulations on the equations to bring about physically useful forms of the equation.

\section{Mass Conservation}

By letting $Q_{0}=X_{k} \rho_{0}$ in equation (11) we get

$$
\begin{aligned}
\frac{\partial\left\langle X_{k} \rho_{0}\right\rangle}{\partial t}+\nabla \cdot\left\langle X_{k} \rho_{0} \vec{u}_{0}\right\rangle & =\left\langle\dot{X}_{k} \rho_{0}+X_{k} \dot{\rho}_{0}+X_{k} \rho_{0} \nabla \cdot \vec{u}_{0}\right\rangle \\
& =\left\langle\dot{X}_{k} \rho_{0}+X_{k}\left(\dot{\rho}_{0}+\rho_{0} \nabla \cdot \vec{u}_{0}\right)\right\rangle
\end{aligned}
$$

Introducing the pure material (microscopic) mass conservation equation and the definition of average results in

$$
\frac{\partial \alpha_{k} \rho_{k}}{\partial t}+\nabla \cdot \alpha_{k} \rho_{k} \vec{u}_{k}=\left\langle\dot{X}_{k} \rho_{0}\right\rangle
$$

Since we are taking time- and spatial-derivatives of functions that are not smooth, this averaged mass conservation equation is to be interpreted in the sense of distributions, or generalized functions [Gelfand and Shilov 1964]. Let us examine the right hand side of this equation in more detail. From the definition of a material derivative we know that

$$
\dot{X}_{k}=\frac{\partial X_{k}}{\partial t}+\vec{u}_{0} \cdot \nabla X_{k}
$$

in a generalized function sense. On the other hand, letting $\vec{u}_{i n t}$ denote the velocity of an interface of phase or material $\mathrm{k}$, the material derivative of $X_{k}$ following the interface velocity vanishes

$$
\frac{\partial X_{k}}{\partial t}+\vec{u}_{i n t} \cdot \nabla X_{k}=0
$$

This result can be easily seen by first considering points not on the interface where either $X_{k}=0$ or $X_{k}=1$ and the partial derivatives both vanish, and thus the left side of this equation vanishes identically. For points on the interface, which also move with the interface velocity, the function $X_{k}$ is a jump that remains constant so their material derivatives following the interface vanish. Therefore we can write 


$$
\begin{aligned}
\left\langle\dot{X}_{k} \rho_{0}\right\rangle & =\left\langle\rho_{0}[\left(\frac{\partial X_{k}}{\partial t}+\vec{u}_{0} \cdot \nabla X_{k}\right)-\underbrace{\left(\frac{\partial X_{k}}{\partial t}+\vec{u}_{i n t} \cdot \nabla X_{k}\right)}_{=0}]\right\rangle \\
& =\left\langle\rho_{0}\left(\vec{u}_{0}-\vec{u}_{i n t}\right) \cdot \nabla X_{k}\right\rangle,
\end{aligned}
$$

and the averaged mass conservation equation becomes

$$
\begin{aligned}
\frac{\partial \alpha_{k} \rho_{k}}{\partial t}+\nabla \cdot \alpha_{k} \rho_{k} \vec{u}_{k} & =\left\langle\rho_{0}\left(\vec{u}_{0}-\vec{u}_{i n t}\right) \cdot \nabla X_{k}\right\rangle \\
& \equiv \Omega_{k}^{\text {mass }} .
\end{aligned}
$$

We note that $\nabla X_{k}$ has the sifting property of the Dirac delta function(al). Thus the only contributors are the material interfaces. $\nabla X_{k}$ is aligned with the surface unit normal vector pointing to phase $k$ [Drew 1983, Kataoka and Serizawa 1988]

$$
\nabla X_{k}=\vec{n}_{k} \delta\left(\vec{x}-\vec{x}_{i n t}, t\right)
$$

Thus the $\Omega_{k}^{\text {mass }}$ represents the flux of mass to phase $k$ from the other phases via the interface, usually just referred to as phase change. With no storage of mass at an interface the mass conservation further requires

$$
\sum_{k=1}^{\text {no. of phases }} \Omega_{k}^{\text {mass }}=0
$$

At this point, it is convenient to introduce for later use, the concept of interfacial area density of component $k$. Defined as

$$
A_{k}=-\left\langle\hat{n}_{k} \cdot \nabla X_{k}\right\rangle
$$

where $\hat{n}_{k}$ is the unit external normal to component $k$, it is the expected value of the ratio of the interfacial area (in a small volume) to the (small) volume, in the limit as that volume approaches zero. 


\section{Generic Conservation Equation}

To more expeditiously derive the other conservation equations, let us first derive the averaged balance equation resulting from a generic, microscopic balance equation. Consider the generic, microscopic balance equation

$$
\frac{\partial \rho_{0} \psi_{0}}{\partial t}+\nabla \cdot \rho_{0} \psi_{0} \vec{u}_{0}=\nabla \cdot J_{0}+\rho_{0} g_{0}
$$

or

$$
\frac{d\left(\rho_{0} \psi_{0}\right)}{d t}+\left(\rho_{0} \psi_{0}\right) \nabla \cdot \vec{u}_{0}=\rho_{0} \dot{\psi}_{0}=\nabla \cdot J_{0}+\rho_{0} g_{0}
$$

Equation (16) and (17) hold at each point where sufficient smoothness occurs for the derivatives to be taken, as does its generic jump condition

$$
\llbracket \rho_{0} \psi_{0}\left(\vec{u}_{0}-\vec{u}_{i n t}\right)+J_{0} \rrbracket \cdot \hat{n}=m
$$

where $\psi_{0}$ is the conserved quantity, $J_{0}$ is a molecular or diffusive flux, $g_{0}$ is a source density, and $m$ is the interfacial source of $\psi_{0}$. The symbol $\llbracket \cdot \rrbracket$ here denotes the jump in the enclosed quantity across an interface. Obviously, these quantities must be added to our state space, e.g.

$$
\Gamma=\left\{\begin{array}{l}
\rho_{0}, \vec{u}_{0}, \psi_{0}, J_{0}, \cdots \\
X_{1}, X_{2}, \cdots
\end{array}\right\} .
$$

Let us also define averages of these quantities as

$$
\begin{gathered}
\psi_{k} \equiv \frac{\left\langle X_{k} \rho_{0} \psi_{0}\right\rangle}{\alpha_{k} \rho_{k}} \\
J_{k} \equiv \frac{\left\langle X_{k} J_{0}\right\rangle}{\alpha_{k}} \\
g_{k} \equiv \frac{\left\langle X_{k} \rho_{0} g_{0}\right\rangle}{\alpha_{k} \rho_{k}} .
\end{gathered}
$$

By letting $Q_{0}=X_{k} \rho_{0} \psi_{0}$ in equation (11) we get 


$$
\begin{aligned}
\frac{\partial\left\langle X_{k} \rho_{0} \psi_{0}\right\rangle}{\partial t}+\nabla \cdot\left\langle X_{k} \rho_{0} \psi_{0} \vec{u}_{0}\right\rangle & =\left\langle\frac{d\left(X_{k} \rho_{0} \psi_{0}\right)}{d t}+X_{k} \rho_{0} \psi_{0} \nabla \cdot \vec{u}_{0}\right\rangle \\
& =\left\langle\dot{X}_{k} \rho_{0} \psi_{0}+X_{k} \frac{d\left(\rho_{0} \psi_{0}\right)}{d t}+X_{k} \rho_{0} \psi_{0} \nabla \cdot \vec{u}_{0}\right\rangle \\
& =\left\langle\dot{X}_{k} \rho_{0} \psi_{0}\right\rangle+\left\langle X_{k}\left(\frac{d\left(\rho_{0} \psi_{0}\right)}{d t}+\rho_{0} \psi_{0} \nabla \cdot \vec{u}_{0}\right)\right\rangle \\
& =\left\langle\dot{X}_{k} \rho_{0} \psi_{0}+X_{k} \nabla \cdot J_{0}+X_{k} \rho_{0} g_{0}\right\rangle \\
& =\left\langle\nabla \cdot X_{k} J_{0}\right\rangle-\left\langle J_{0} \cdot \nabla X_{k}\right\rangle+\left\langle\dot{X}_{k} \rho_{0} \psi_{0}\right\rangle+\left\langle X_{k} \rho_{0} g_{0}\right\rangle \\
& =\left\langle\nabla \cdot X_{k} J_{0}\right\rangle-\left\langle J_{0} \cdot \nabla X_{k}\right\rangle+\left\langle\rho_{0} \psi_{0}\left(\vec{u}_{0}-\vec{u}_{i n t}\right) \cdot \nabla X_{k}\right\rangle+\left\langle X_{k} \rho_{0} g_{0}\right\rangle \\
& =\nabla \cdot\left\langle X_{k} J_{0}\right\rangle+\left\langle X_{k} \rho_{0} g_{0}\right\rangle+\left\langle\left[\rho_{0} \psi_{0}\left(\vec{u}_{0}-\vec{u}_{i n t}\right)-J_{0}\right] \cdot \nabla X_{k}\right\rangle .
\end{aligned}
$$

Introducing the fluctuating velocity

$$
\vec{u}_{k}^{\prime}=\vec{u}_{0}-\vec{u}_{k}
$$

into this expression finally results in

$$
\begin{aligned}
\frac{\partial \alpha_{k} \rho_{k} \psi_{k}}{\partial t}+\nabla \cdot \alpha_{k} \rho_{k} \psi_{k} \vec{u}_{k} & =\nabla \cdot \alpha_{k} J_{k}-\nabla \cdot\left\langle X_{k} \rho_{0} \psi_{0} \vec{u}_{k}^{\prime}\right\rangle+\alpha_{k} \rho_{k} g_{k}+\left\langle\left[\rho_{0} \psi_{0}\left(\vec{u}_{0}-\vec{u}_{i n t}\right)-J_{0}\right] \cdot \nabla X_{k}\right\rangle \\
& =\nabla \cdot \alpha_{k} J_{k}-\nabla \cdot\left\langle X_{k} \rho_{0} \psi_{0} \vec{u}_{k}^{\prime}\right\rangle+\alpha_{k} \rho_{k} g_{k}+\left\langle\rho_{0} \psi_{0}\left(\vec{u}_{0}-\vec{u}_{i n t}\right) \cdot \nabla X_{k}\right\rangle-\left\langle J_{0} \cdot \nabla X_{k}\right\rangle \\
& =\nabla \cdot \alpha_{k} J_{k}+\nabla \cdot \alpha_{k} J_{k}^{\text {Fluct }}+\alpha_{k} \rho_{k} g_{k}+\Omega_{k}^{\text {mass }} \psi_{k}^{\text {int }}+\Omega_{k}^{\psi},
\end{aligned}
$$

where $J_{k}^{\text {Fluct }}=-\frac{\left\langle X_{k} \rho_{0} \psi_{0} \vec{u}_{k}^{\prime}\right\rangle}{\alpha_{k}}$ is the flux of $\psi$ due to fluctuations in the phase $k$ velocity, $\psi_{k}^{\text {int }}$ is the effective value of $\psi$ that is transferred to phase $k$ from the other phases due to mass transfer, or phase change, and $\Omega_{k}^{\psi}$ is a flux of $\psi$ to phase $k$ not due to bulk mass transfer from the other phases. This is our generic, averaged balance equation. To obtain balance at the interface, our generic jump balance equation requires the constraint

$$
\sum_{k=1}^{\text {no. of phases }} \Omega_{k}^{\text {mass }} \psi_{k}^{\text {int }}+\Omega_{k}^{\psi}=M
$$

where $M=\langle m\rangle$ is the expected net effect of all the interfacial $\psi$-source terms. 


\section{Species Mass Conservation}

The microscopic species mass balance equation can be written as

$$
\frac{\partial \rho_{0}^{s}}{\partial t}+\nabla \cdot \rho_{0}^{s} \vec{u}_{0}^{s}=\dot{r}^{s}
$$

where $\rho_{0}^{s}$ is the species partial density, $\vec{u}_{0}^{s}$ is the species bulk velocity, and $\dot{r}^{s}$ is the generation or source of the species due to chemical reactions. The species mass balance equation is not usually written this way because we usually don't know much about individual species velocities. Instead, it is usually cast as

$$
\frac{\partial \rho_{0}^{s}}{\partial t}+\nabla \cdot \rho_{0}^{s} \vec{u}_{0}=\nabla \cdot \rho_{0}^{s}\left(\vec{u}_{0}-\vec{u}_{0}^{s}\right)+\dot{r}^{s}
$$

because we have (to a certain extent) acquired empirical knowledge of the behavior of the first term on the right hand side of this equation, as we shall see shortly. Let us now recast this equation as

$$
\frac{\partial}{\partial t}\left(\rho_{0} \frac{\rho_{0}^{s}}{\rho_{0}}\right)+\nabla \cdot\left(\rho_{0} \frac{\rho_{0}^{s}}{\rho_{0}} \vec{u}_{0}\right)=\nabla \cdot\left[\rho_{0} \frac{\rho_{0}^{s}}{\rho_{0}}\left(\vec{u}_{0}-\vec{u}_{0}^{s}\right)\right]+\rho_{0} \frac{\dot{r}^{s}}{\rho_{0}}
$$

which is in the form of our generic, averaged balance equation (20) with the assignments of

$$
\psi_{0}=\frac{\rho_{0}^{s}}{\rho_{0}} \quad J_{0}=\rho_{0} \frac{\rho_{0}^{s}}{\rho_{0}}\left(\vec{u}_{0}-\vec{u}_{0}^{s}\right) \quad g_{0}=\frac{\dot{r}^{s}}{\rho_{0}} .
$$

Thus the averaged species mass balance equation is

$$
\frac{\partial}{\partial t}\left\langle X_{k} \rho_{0}^{s}\right\rangle+\nabla \cdot\left\langle X_{k} \rho_{0}^{s} \vec{u}_{0}\right\rangle=\nabla \cdot\left\langle X_{k} \rho_{0}^{s}\left(\vec{u}_{0}-\vec{u}_{0}^{s}\right)\right\rangle+\left\langle X_{k} \dot{r}^{s}\right\rangle+\left\langle\left[\rho_{0}^{s}\left(\vec{u}_{0}-\vec{u}_{i n t}\right)-\rho_{0}^{s}\left(\vec{u}_{0}-\vec{u}_{0}^{s}\right)\right] \cdot \nabla X_{k}\right\rangle .
$$

Again introducing the fluctuating velocity and the definitions of averaged quantities, our final form of the averaged species mass balance equation is

$$
\begin{aligned}
\frac{\partial \alpha_{k} \rho_{k}^{s}}{\partial t}+\nabla \cdot \alpha_{k} \rho_{k}^{s} \vec{u}_{k} & =\nabla \cdot\left\langle X_{k} \rho_{0}^{s}\left(\vec{u}_{0}-\vec{u}_{0}^{s}\right)\right\rangle & & \text { relative species flux } \\
& -\nabla \cdot\left\langle X_{k} \rho_{0}^{s} \vec{u}_{k}^{\prime}\right\rangle & & \text { fluctuational diffusion } \\
& +\left\langle\rho_{0}^{s}\left(\vec{u}_{0}-\vec{u}_{i n t}\right) \cdot \nabla X_{k}\right\rangle & & \text { phase change } \\
& -\left\langle\rho_{0}^{s}\left(\vec{u}_{0}-\vec{u}_{0}^{s}\right) \cdot \nabla X_{k}\right\rangle & & \text { mass exchange } \\
& +\dot{R}_{k}^{s} & & \text { chemical reactions }
\end{aligned}
$$


where we have defined the average generation rate in phase $k$ due to chemical reactions as $\dot{R}_{k}^{s} \equiv \frac{\left\langle X_{k} \dot{r}^{s}\right\rangle}{\alpha_{k}}$.

\section{Momentum Balance}

The averaged momentum balance equation results from the generic, averaged balance equation (20) with the assignment of

$$
\psi_{0}=\vec{u}_{0} \quad J_{0}={\underset{\sim}{T}}_{0} \quad g_{0}=\vec{g}_{0}
$$

to give

$$
\frac{\partial \alpha_{k} \rho_{k} \vec{u}_{k}}{\partial t}+\nabla \cdot \alpha_{k} \rho_{k} \vec{u}_{k} \vec{u}_{k}=\nabla \cdot \alpha_{k}\left(\underset{\sim k}{T_{k}}+T_{\sim k}^{\text {Fluct }}\right)+\alpha_{k} \rho_{k} \vec{g}_{k}+\Omega_{k}^{\text {mom }}+\vec{u}_{k}^{\text {int }} \Omega_{k}^{\text {mass }}
$$

where the fluctuating stress $\underset{\sim}{T_{k}^{\text {Fluct }}}$ and the interfacial momentum source $\Omega_{k}^{\text {mom }}$ are given by

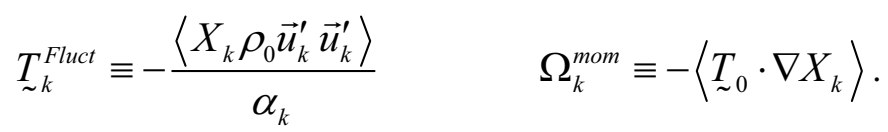

The averaged interfacial momentum balance constraint (jump condition) is

$$
\sum_{k=1}^{\text {no. of phases }} \Omega_{k}^{\text {mom }}+\vec{u}_{k}^{\text {int }} \Omega_{k}^{\text {mass }}=\vec{M}_{\text {surfacetension }},
$$

where $\vec{M}_{\text {surface tension }}$ is the interfacial momentum source, i.e. surface tension source.

\section{Energy Conservation}

The assignment of

$$
\psi_{0}=E_{0}=e_{0}+\frac{1}{2} \vec{u}_{0} \cdot \vec{u}_{0} \quad J_{0}=T_{\sim} \cdot \vec{u}_{0}+\vec{q}_{0} \quad g_{0}=\vec{g}_{0} \cdot \vec{u}_{0}+\varepsilon_{0}
$$

to the variables of the generic, averaged balance equation (20) gives the averaged energy conservation equation 


$$
\begin{aligned}
\frac{\partial}{\partial t} \alpha_{k} \rho_{k}\left(e_{k}+\frac{1}{2} \vec{u}_{k} \cdot \vec{u}_{k}+e_{k}^{F l u c t}\right)+\nabla \cdot \alpha_{k} \rho_{k} \vec{u}_{k}\left(e_{k}+\frac{1}{2} \vec{u}_{k} \cdot \vec{u}_{k}+e_{k}^{\text {Fluct }}\right) & =\nabla \cdot\left[\alpha_{k}\left(T_{\sim}+T_{\sim k}^{\text {Fluct }}\right) \cdot \vec{u}_{k}\right] \\
& -\nabla \cdot \alpha_{k}\left(\vec{q}_{k}+\vec{q}_{k}^{\text {Fluct }}\right)+\alpha_{k} \rho_{k}\left(\varepsilon_{k}+\vec{g}_{k} \cdot \vec{u}_{k}\right) \\
& +\Omega_{k}^{\text {energy }}+\Omega_{k}^{\text {mom }} \cdot \vec{u}_{k}^{\text {int }} \\
& +\Omega_{k}^{\text {mass }}\left(e_{k}^{\text {int }}+\frac{1}{2} \vec{u}_{k}^{\text {int }} \cdot \vec{u}_{k}^{\text {int }}\right)
\end{aligned}
$$

where

$$
\begin{aligned}
& e_{k}^{\text {Fluct }} \equiv \frac{1}{2} \frac{\left\langle X_{k} \rho_{0} \vec{u}_{k}^{\prime} \cdot \vec{u}_{k}^{\prime}\right\rangle}{\alpha_{k} \rho_{k}} \quad \text { fluctuation kinetic energy } \\
& \vec{q}_{k}^{\text {Fluct }} \equiv \underbrace{\frac{\left\langle X_{k} \rho_{0} \vec{u}_{k}^{\prime} e_{k}^{\prime}\right\rangle}{\alpha_{k}}}_{\text {fluctution internal energy flux }}+\underbrace{\frac{\left\langle X_{k} T_{\sim 0} \cdot \vec{u}_{k}^{\prime}\right\rangle}{\alpha_{k}}}_{\text {fluctuation shear working }}+\underbrace{\frac{1}{2} \frac{\left\langle X_{k} \rho_{0} \vec{u}_{k}^{\prime}\left(\vec{u}_{k}^{\prime} \cdot \vec{u}_{k}^{\prime}\right)\right\rangle}{\alpha_{k}}}_{\text {fluctuation kinetic energy flux }} \text { fluctuation energy flux } \\
& \varepsilon_{k} \equiv \frac{\left\langle X_{k} \rho_{0} \varepsilon_{0}\right\rangle}{\alpha_{k} \rho_{k}} \quad \text { energy source } \\
& \Omega_{k}^{\text {energy }} \equiv\left\langle\vec{q}_{0} \cdot \nabla X_{k}\right\rangle \quad \text { interfacial heat source } \\
& \Omega_{k}^{m o m} \cdot \vec{u}_{k}^{i n t} \equiv-\left\langle\underset{\sim 0}{T} \cdot \vec{u}_{0} \cdot \nabla X_{k}\right\rangle \quad \text { interfacial work } .
\end{aligned}
$$

The averaged interfacial energy balance constraint (jump condition) is

$$
\sum_{k=1}^{\text {no. of phases }} \Omega_{k}^{\text {energy }}+\Omega_{k}^{\text {mom }} \cdot \vec{u}_{k}^{\text {int }}+\Omega_{k}^{\text {mass }}\left(e_{k}^{\text {int }}+\frac{1}{2} \vec{u}_{k}^{\text {int }} \cdot \vec{u}_{k}^{\text {int }}\right)=\xi
$$

where $\xi$ is the interfacial energy source.

The kinetic energy associated with the velocity fluctuations, $e_{k}^{\text {Fluct }}$, is a type of "turbulent" kinetic energy. Sometimes the sum $e_{k}+e_{k}^{\text {Fluct }}$ is interpreted as the effective internal energy per unit mass of phase $k$.

It is sometimes useful to have an expression for the balance of fluctuation kinetic energy, $e_{k}^{\text {Fluct }}$. Its evolutionary description is derived by introducing the partition $\vec{u}_{k}^{\prime}=\vec{u}_{0}-\vec{u}_{k}$ into the microscopic pure phase momentum balance, taking the dot product of this equation with $X_{k} \vec{u}_{k}^{\prime}$, 
then performing the statistical average over configurations (keeping in mind that $\left\langle X_{k} \rho_{0} \vec{u}^{\prime}\right\rangle$ vanishes) to obtain [details are left to the reader, see e.g. Nigmatulin 1990]

$$
\begin{aligned}
\alpha_{k} \rho_{k} \frac{\partial e_{k}^{\text {Fluct }}}{\partial t}+\alpha_{k} \rho_{k} \vec{u}_{k} \cdot \nabla e_{k}^{\text {Fluct }} & =\alpha_{k} T_{\sim k}^{\text {Fluct }}: \nabla \vec{u}_{k} \\
& -\nabla \cdot\left\langle\frac{X_{k} \rho_{0}\left(\vec{u}_{k}^{\prime} \cdot \vec{u}_{k}^{\prime}\right) \vec{u}_{k}^{\prime}}{2}\right\rangle+\left\langle X_{k} \vec{u}_{k}^{\prime} \cdot\left(\nabla \cdot T_{\sim}+\rho_{0} \vec{g}_{0}\right)\right\rangle
\end{aligned}
$$

This equation bares some similarity to the equation of evolution of the fluctuational kinetic energy in a single-phase turbulent fluid [Wilcox 1998]. The first term on the right side describes the influence of the gradient of $\vec{u}_{k}$ on the development of $e_{k}^{\text {Fluct }}$, the second term is expected to diffuse $e_{k}^{\text {Fluct }}$, and the last term represents the power developed by the stresses and external forces [Lhuillier 1996].

For most multiphase flows, including some very (conceptually) simple flows such as gas flow through a packed bed or through a pebble-bed nuclear reactor, the nature of $e_{k}^{\text {Fluct }}$ is somewhat different than that of a turbulent single-phase flow. Contrary to a one-phase fluid in which the fluctuations disappear for slow flows, these fluctuations for a multiphase flow exist however slow the flow. For this reason, $e_{k}^{\text {Fluct }}$ that is produced by hydrodynamic interactions between the phases is sometimes called pseudo-turbulence [Lhuillier 1996].

\section{Entropy Inequality}

The local form of the entropy inequality (8), sometimes called the "Second Law of Thermodynamics," is used to place restrictions on the constitutive relations used to give unique phase or material behaviors. With the assignment of

$$
\psi_{0}=s_{0} \quad J_{0}=-\frac{\vec{q}_{0}}{\theta_{0}} \quad g_{0}=\frac{\varepsilon_{0}}{\theta_{0}}
$$

to the variables of the generic, averaged balance relationship (20) the averaged entropy inequality results,

$$
\frac{\partial \alpha_{k} \rho_{k} s_{k}}{\partial t}+\nabla \cdot \alpha_{k} \rho_{k} s_{k} \vec{u}_{k} \geq \nabla \cdot \alpha_{k}\left(\Phi_{k}+\Phi_{k}^{\text {Fluct }}\right)+\alpha_{k} \rho_{k} S_{k}+\Omega_{k}^{\text {entropy }}+\Omega_{k}^{\text {mass }} s_{k}^{\text {int }}
$$

where 


$$
\begin{gathered}
\vec{\Phi}_{k} \equiv-\frac{\left\langle X_{k} \frac{\vec{q}_{0}}{\theta_{0}}\right\rangle}{\alpha_{k}} \quad \text { entropy flux } \\
\vec{\Phi}_{k}^{\text {Fluct }} \equiv-\frac{\left\langle X_{k} \rho_{0} s_{k}^{\prime} \vec{u}_{k}^{\prime}\right\rangle}{\alpha_{k}} \quad \text { fluctuation entropy flux } \\
S_{k} \equiv \frac{\left\langle X_{k} \frac{\rho_{0} \varepsilon_{0}}{\theta_{0}}\right\rangle}{\alpha_{k} \rho_{k}} \quad \text { entropy source } \\
\Omega_{k}^{\text {entropy }} \equiv\left\langle\frac{\vec{q}_{0}}{\theta_{0}} \cdot \nabla X_{k}\right\rangle \quad \text { interfacial entropy source. }
\end{gathered}
$$

This entropy inequality corresponds to what Drew and Passman (1999) call the microscopic entropy inequality. A macroscopic entropy inequality can be obtained by summing inequalities (30) over all of the phases or materials present in the mixture [Truesdell 1984 and the other authors contained therein]. The macroscopic entropy inequality is useful for placing restrictions on the phasic or material interaction constitutive relations. The averaged interfacial entropy inequality (jump condition) is

$$
\sum_{k=1}^{\text {no. of phases }} \Omega_{k}^{\text {entropy }}+\Omega_{k}^{\text {mass }} s_{k}^{\text {int }} \geq 0 .
$$

\section{Volume Fraction Propagation Equations}

There remains one very important relationship to derive, a dynamic relationship that effectively reflects boundary conditions at the microscale. It accounts for the fact that the constituent volume fractions can change without affecting the gross motion and, in a sense, models the microstructural force systems operating in the multiphase mixture. Beginning with the previous Lagrangian interface material derivative relationship for $X_{k}$,

$$
\frac{\partial X_{k}}{\partial t}+\vec{u}_{i n t} \cdot \nabla X_{k}=0
$$

this equation is averaged to give 


$$
\begin{aligned}
\left\langle\frac{\partial X_{k}}{\partial t}+\vec{u}_{\mathrm{int}} \cdot \nabla X_{k}\right\rangle & =\left\langle\frac{\partial X_{k}}{\partial t}\right\rangle+\left\langle\vec{u}_{\mathrm{int}} \cdot \nabla X_{k}\right\rangle \\
& =\frac{\partial}{\partial t}\left\langle X_{k}\right\rangle+\left\langle\vec{u}_{\mathrm{int}} \cdot \nabla X_{k}\right\rangle \\
& =\frac{\partial \alpha_{k}}{\partial t}+\left\langle\vec{u}_{\mathrm{int}} \cdot \nabla X_{k}\right\rangle=0 .
\end{aligned}
$$

Introducing the fluctuating interface velocity

$$
\vec{u}_{I}^{\prime}=\vec{u}_{i n t}-\vec{u}_{I}
$$

where $\vec{u}_{I}$ is the average interface velocity, into this equation yields

$$
\begin{aligned}
\frac{\partial \alpha_{k}}{\partial t}+\left\langle\vec{u}_{\mathrm{int}} \cdot \nabla X_{k}\right\rangle & =\frac{\partial \alpha_{k}}{\partial t}+\left\langle\left(\vec{u}_{I}+\vec{u}_{I}^{\prime}\right) \cdot \nabla X_{k}\right\rangle \\
& =\frac{\partial \alpha_{k}}{\partial t}+\left\langle\vec{u}_{I} \cdot \nabla X_{k}\right\rangle+\left\langle\vec{u}_{I}^{\prime} \cdot \nabla X_{k}\right\rangle \\
& =\frac{\partial \alpha_{k}}{\partial t}+\vec{u}_{I} \cdot\left\langle\nabla X_{k}\right\rangle+\left\langle\vec{u}_{I}^{\prime} \cdot \nabla X_{k}\right\rangle \\
& =\frac{\partial \alpha_{k}}{\partial t}+\vec{u}_{I} \cdot \nabla \alpha_{k}+\left\langle\vec{u}_{I}^{\prime} \cdot \nabla X_{k}\right\rangle \\
& =\frac{\partial \alpha_{k}}{\partial t}+\vec{u}_{I} \cdot \nabla \alpha_{k}-\Omega_{k}^{v o l}=0
\end{aligned}
$$

where $\Omega_{k}^{\text {vol }}$ (for which a constituitive description is needed) is the driving function for the change of volume fraction $\alpha_{k}$. In summary, this equation is written as

$$
\frac{\partial \alpha_{k}}{\partial t}+\vec{u}_{I} \cdot \nabla \alpha_{k}=\Omega_{k}^{v o l}
$$

Because of the central role that this volume fraction propagation equation plays in modern, wellposed two-phase flow models with correct wave dynamics, we dedicate the next section to its examination.

\section{Seven Equation, Two-Pressure, Two-Velocity Hyperbolic, Well-Posed Models for Two-Phase Flows}

Prior to 1981 there had been attempts to remove the ill-posed nature from the full, two-phase flow equations, e.g. [Ransom and Scofield, 1976], [Stuhmiller, 1977], [Rousseau and Ferch, 1979], [Banerjee and Chan, 1980], [Hancox et.al., 1980], as well as others. All of these 
researchers were trying to gain closure of this equation system (the classical 6-equations system) through algebraic means - and were meeting some limited success. But they all seemed to be missing some key ingredient.

In 1981 [Nguyen, 1981] presented a paper identifying the missing ingredient in compressible two-phase flow. In this paper Nguyen utilized the entropy production for each phase to perform an Onsager-type analysis wherein a bilinear form in the thermodynamic fluxes and their conjugate forces was obtained. From this he arrived at the so-called phenomenological laws, one of which, in first approximation is:

$$
p_{k}-p_{i}=L_{\alpha k}\left(\frac{\partial \alpha_{k}}{\partial t}+w_{k} \frac{\partial \alpha_{k}}{\partial z}\right)
$$

where the notation is standard, with $w_{k}$ denoting the $z$-component of phase $k$ velocity and $L_{\alpha k}$ denoting a phenomenological coefficient, possibly to be "obtained from the flow structure." To complete the set of closure equations, Nguyen proposed to define the interface pressure $p_{i}$ as

$$
p_{i}=\frac{1}{2} \sum_{k=1}^{2}\left[p_{k}-\alpha_{k} \rho_{k} \xi_{k}\left(w_{1}, w_{2}\right)\right]
$$

where $\xi_{k}$ was to be defined consistently with the physical situation of interest. Nguyen furthermore deemed it reasonable to assume that the phenomenological coefficients were equal or

$$
L_{\alpha 1}=L_{\alpha 2}=L_{\alpha}
$$

With these assumptions Nguyen then obtained a 7-equation model with real characteristics which was hyperbolic and which could be formulated as a well-posed initial-value problem.

However, what Nguyen did not do (at least in that paper) was the following useful manipulation. Adding his equations (49) and (50) to eliminate $p_{i}$ gives

$$
\frac{\partial \alpha}{\partial t}+\frac{w_{1}+w_{2}}{2} \frac{\partial \alpha}{\partial z}=\frac{p_{1}-p_{2}}{2 L_{\alpha}}
$$

While we are enhancing the equations of Nguyen's paper, let us also do the following: Let us assume that the phenomenological coefficients are not equal. It is easily obtained that

$$
\frac{\partial \alpha}{\partial t}+\left(\frac{L_{1} w_{1}+L_{2} w_{2}}{L_{1}+L_{2}}\right) \frac{\partial \alpha}{\partial z}=\frac{p_{1}-p_{2}}{L_{1}+L_{2}} .
$$

Now, if $L_{2} \gg L_{1}$ and $w_{1}$ is of the same order as $w_{2}$ then the above relationship is approximately 


$$
\frac{\partial \alpha}{\partial t}+w_{2} \frac{\partial \alpha}{\partial z} \approx \frac{p_{1}-p_{2}}{L_{2}}
$$

These relationships are appealing because now the volume fraction change is governed by a dynamical relationship in which the pressure difference between the two phases drove the phase change. If the phases had the same pressure there would be no change in volume fraction. The denominators on the right hand side, i.e. combinations of the phenomenological coefficients for the two phases would determine how "fast" pressure equilibrium was attained. The phasic advection equation, or volume fraction propagation equation, also exhibited an advection velocity that was a weighted combination of phasic velocities (weighted with the phenomenological coefficients).

In March 1983, Passman and Nunziato at SNL and Walsh at U. of Florida published a report [Passman, Nunziato, and Walsh 1983], later to become Appendix 5C of Truesdell's classical work on rational thermodynamics [Truesdell, 1984]. In their elegant work, in addition to the traditional axioms of balance, an additional balance axiom was postulated which describes changes in volume fraction. They utilized the idea of workless constraints to describe a method of accounting, in the entropy inequality for the mixture, for the constraint requiring that the mixture be saturated. This resulted in a volume fraction propagation equation, which they called balance of equilibrated force, which accounts for the fact that the constituent volume fractions can change without affecting the gross motion. As they point out, this equation, in a sense, models the microstructural force systems operative in multiphase mixtures.

\{Note: This approach has not seen much acceptance in the two-phase fluid flow community, probably because the equation derivation was postulational [Truesdell, 1984] (as opposed to using some type of averaging) and because of the lack of physical familiarity with the terminology. However, some years later, Dobran at New York University published a monograph [Dobran, 1991] in which rigorous volume averaging is utilized, along with a basic material deformation postulate, to derive additional transport equations for multiphase mixtures that are very similar.\}

In December 1983 Baer and Nunziato of SNL released a two-phase mixture theory describing the deflagration-to-detonation transition (DDT) in reactive granular materials [Baer and Nuziato, 1983]. This research was published later in the open literature [Baer and Nunziato, 1986]. However, perhaps because of the application context, in addition to its postulational derivation, this method received little attention from the two-phase fluid dynamics community. In this work the entropy inequality for the two-phase mixture was utilized to directly establish a constitutive volume fraction propagation equation, which (in the context of their application) they called the compaction equation (with phase change)

$$
\frac{\partial \alpha_{s}}{\partial t}+v_{s} \frac{\partial \alpha_{s}}{\partial z}=\frac{\alpha_{s} \alpha_{g}}{\mu_{c}}\left[p_{s}-\left(p_{g}+\beta_{s}\right)\right]+\frac{c_{s}^{+}}{\gamma_{s}}
$$


where $\beta_{s}$ is a configuration pressure which resists changes in the packing of the bed or compaction and the coefficient $\mu_{c}$ is a compaction viscosity (again in their application context) which controls the rate at which pressure equilibrium is reached. Though derived in a different manner, this equation can also be viewed as a simpler, special case of Passman et.al.'s balance of equilibrated force equation, mentioned above. This 7-equation compressible two-phase model of Baer and Nunziato was hyperbolic and well-posed. They also constructed apparently successful numerical solution algorithms for this equation set. While the motivating applications for this model are far different from those of the two-phase fluid dynamics community, the foundational principles are very similar. Notice the similarities between their volume fraction propagation equation and that of Nguyen over two years earlier.

Baer and Nunziato's volume fraction evolutionary equation can be more generally stated as

$$
\frac{\partial \alpha_{a}}{\partial t}+v_{a} \frac{\partial \alpha_{a}}{\partial z}=\sum_{j} \frac{\alpha_{a} \alpha_{j}}{\mu_{j, a}}\left[p_{a}-\beta_{a}-\left(p_{j}-\beta_{j}\right)\right]+\frac{c_{a}^{+}}{\gamma_{a}}
$$

where $\beta_{a}$ are configuration pressures and exchange coefficients $\mu_{j, a}$ reflect interactions between the phases. These rate equations, which provide closure of the mixture model, are force balances involving the configurational pressures, phase pressures, and a viscous-like stress associated with rate-dependent volume fraction changes. The relaxation coefficients, $\mu_{j, a}$, have the units of viscosity. Moreover, like Nguyen's (but unlike Passman et.al.'s), these equations are constitutive equations, not field equations. As pointed out in [Baer, 1997], they reflect boundary conditions at the microscale. [Saurel et.al. 1994] recast the volume fraction evolution equation as a microscopic mass density evolution equation \{see notes\}. [Kashiwa and Gaffney, 2003] derive a mass density evolution equation having a somewhat different character.

To more easily see the need for a dynamic volume fraction evolution equation let us consider a cell mixture-intuitive physics model for two-phase flow. Consider a fixed volume $V$ with two immiscible constituents present (e.g. the two constituents may have been advected into a fixed cell volume). They have masses $m_{1}$ and $m_{2}$ occupying volumes $V_{1}$ and $V_{2}$, respectively, such that

$$
V_{1}+V_{2}=V
$$

Each constituent phase has material density $\rho_{1}$ and $\rho_{2}$, so

$$
\begin{aligned}
V & =V_{1}+V_{2} \\
& =\frac{m_{1}}{\rho_{1}}+\frac{m_{2}}{\rho_{2}}
\end{aligned}
$$

or 


$$
\begin{aligned}
1 & =\frac{V_{1}}{V}+\frac{V_{2}}{V} \\
& =\alpha_{1}+\alpha_{2} \\
& =\frac{m_{1}}{V \rho_{1}}+\frac{m_{2}}{V \rho_{2}}
\end{aligned}
$$

where $\alpha_{1}=\frac{V_{1}}{V}$ and $\alpha_{2}=\frac{V_{2}}{V}$ are volume fractions of each phase. For each constituent

$$
\rho_{1}=\frac{m_{1}}{V_{1}} \quad \text { and } \quad \rho_{2}=\frac{m_{2}}{V_{2}}
$$

and

$$
\begin{aligned}
p_{1} & =f_{1}\left(\rho_{1}, I_{1}\right) \\
& =f_{1}\left(\frac{m_{1}}{V_{1}}, I_{1}\right) \\
p_{2} & =f_{2}\left(\rho_{2}, I_{2}\right) \\
& =f_{2}\left(\frac{m_{2}}{V_{2}}, I_{2}\right) .
\end{aligned}
$$

Now if $V_{1}$ and $V_{2}$ are adjusted (subject to the $V_{1}+V_{2}=V$ constraint) until the phase pressures are equal to

$$
p=f_{1}\left(\frac{m_{1}}{V_{1}}, I_{1}\right)=f_{2}\left(\frac{m_{2}}{V_{2}}, I_{2}\right)
$$

with the equilibrium (or equilibration) pressure, $p$. At this equilibrium pressure the corresponding phase volumes yield the equilibrium volume fractions

$$
\begin{gathered}
\alpha_{1}^{e}=\frac{V_{1}}{V} \\
\alpha_{2}^{e}=\frac{V_{2}}{V} .
\end{gathered}
$$

This can be accomplished more generally in a dynamical fashion as follows. First, note that 


$$
\begin{aligned}
p_{1} & =f_{1}\left(\rho_{1}, I_{1}\right) \\
& =f_{1}\left(\frac{m_{1}}{\alpha_{1} V}, I_{1}\right) \\
p_{2} & =f_{2}\left(\rho_{2}, I_{2}\right) \\
& =f_{2}\left(\frac{m_{2}}{\alpha_{2} V}, I_{2}\right)
\end{aligned}
$$

and also that

$$
\begin{aligned}
\frac{d \alpha_{1}}{d t} & =-\frac{d \alpha_{2}}{d t} \\
\frac{d^{2} \alpha_{1}}{d t^{2}} & =-\frac{d^{2} \alpha_{2}}{d t^{2}} .
\end{aligned}
$$

Intuitively, we now consider the dynamical equation

$$
\frac{d \alpha_{1}}{d t}=\frac{p_{1}-p_{2}}{\tau}
$$

If $\alpha_{1}$ is compressed too much $\left(p_{1}>p_{2}\right)$ then $\alpha_{1}$ will increase with time (relax) letting $p_{1}$ reduce while $\alpha_{2}$ decreases letting $p_{2}$ increase. This process ends when $p_{1}=p_{2}=p$ and thus $\frac{d \alpha_{1}}{d t}=0$. The relaxation rate, $\tau$, controls the rate at which the phases (pressures) equilibrate.

More generally yet, we could even write

$$
\begin{aligned}
(\text { microinertia }) \cdot \frac{d^{2} \alpha_{1}}{d t^{2}}+(\text { compaction viscosity }) \cdot \frac{d \alpha_{1}}{d t} & =(\text { microstructural forces }) \\
& =F
\end{aligned}
$$

The microstructural force $F$ is a relaxation term that is intended to model the driving force or "resistance" exhibited by the mixture to changes in its configuration. For example, if we were compacting a gas-solid particle bed,

$$
F=\left\{\begin{array}{lll}
\alpha_{s} \alpha_{g}\left(p_{s}-p_{g}-\beta_{s}\right) & \text { for } & p_{s}-\beta_{s}>0 \\
-\alpha_{s} \alpha_{g} p_{g} & \text { for } & p_{s}-\beta_{s} \leq 0
\end{array}\right.
$$


in accordance with the view of compaction as an irreversible process. $\beta_{s}$ is the "configuration pressure" of the bed.

If we set the "microinertial" and the "configuration pressure" to zero we are left with

$$
\mu \frac{d \alpha_{1}}{d t}=\alpha_{1} \alpha_{2}\left(p_{1}-p_{2}\right)
$$

or

$$
\frac{d \alpha_{1}}{d t}=\frac{\alpha_{1} \alpha_{2}\left(p_{1}-p_{2}\right)}{\mu}
$$

Note the multiplicative coefficient $\alpha_{1} \alpha_{2}$ in the driving force $F$. This term is included for a couple of reasons: (1) $\alpha_{1} \alpha_{2}$ is roughly proportional to the interfacial area per unit volume, $\frac{A_{i}}{V}$. and (2) better behavior results in the limit of single phase occurring due to disappearance of the other phase, i.e. $\alpha_{1} \rightarrow 0\left(\alpha_{2} \rightarrow 1\right)$ or $\alpha_{2} \rightarrow 0\left(\alpha_{1} \rightarrow 1\right)$.

In 1998, Saurel and Abgrall [Saurel and Abgrall, 1999], who had used Baer and Nunziato's model with some slight reformulation for similar applications, and who had begun to generalize it and apply it to other multiphase mixtures of interest in a fluid dynamics context, published their 7-equation compressible multifluid/multiphase flow model. Neglecting microinertia, surface tension, and covariance terms (and assuming no mass transfer between phases) their variant is state as,

$$
\begin{gathered}
\frac{\partial \bar{\rho}_{k}}{\partial t}+\nabla \cdot \bar{\rho}_{k} \vec{u}_{k}=0 \\
\frac{\partial \bar{\rho}_{k} \vec{u}_{k}}{\partial t}+\nabla \cdot\left(\bar{\rho}_{k} \vec{u}_{k} \otimes \vec{u}_{k}\right)+\nabla \bar{p}_{k}=p_{I} \nabla \alpha_{k}+\lambda\left(\vec{u}_{m}-\vec{u}_{k}\right) \\
\frac{\partial \bar{\rho}_{k} E_{k}}{\partial t}+\nabla \cdot\left(\bar{\rho}_{k} E_{k} \vec{u}_{k}+\bar{p}_{k} \vec{u}_{k}\right)=p_{I} u_{I} \cdot \nabla \alpha_{k}+\lambda\left(\vec{u}_{m}-\vec{u}_{k}\right) u_{I}+\mu\left(p_{k}-p_{m}\right) p_{I} \\
\frac{\partial \alpha_{k}}{\partial t}+u_{I} \cdot \nabla \alpha_{k}=\mu\left(p_{k}-p_{m}\right)
\end{gathered}
$$

where $\alpha_{k}, \bar{\rho}_{k}=\alpha_{k} \rho_{k}, \vec{u}_{k}, \bar{p}_{k}=\alpha_{k} p_{k}$, and $E_{k}$ represent the fluid phase $k$ "volume fraction", mass density, velocity, pressure, and total energy, respectively. The non-equilibrium two-phase flow model derived in [Saurel et. al., 2003], a variant of the original [Baer and Nunziato, 1986] model, is preferred because of its symmetric formulation. In these models each phase is assumed compressible with its own thermodynamics. The system involves 7 partial differential equations (volume fraction and mass, momentum, and energy balance for each phase) and is hyperbolic. These equations, which represent the balance of mass, momentum, and total energy, and volume 
fraction evolution, respectively, with specific interphase transfer terms placed on the right-hand sides. In these equations, (for a two-phase flow) $k=1,2$ and correspond, respectively, with $m=2,1 ; p_{I}$ and $u_{I}$ represent the interfacial pressure and velocity. In the Baer-Nunziato model, these variables are chosen as $p_{I}=p_{2}$ and $u_{I}=\vec{u}_{1}$, while the Saurel model utilized the following interfacial values:

$$
u_{I}=\frac{\sum_{k=1,2} \alpha_{k} \rho_{k} u_{k}}{\sum_{k=1,2} \alpha_{k} \rho_{k}} \quad \text { and } \quad p_{I}=\sum_{k=1,2} \alpha_{k} p_{k}
$$

This model contains relaxation parameters $\lambda$ and $\mu$ that determine the rates at which the velocities and pressures of the two phases reach equilibrium. These equations are closed by two equations of state, the saturation constraint for the volume fractions and the stiffened gas equation of state (which holds approximately for a broad range of gases and liquids)

$$
\begin{gathered}
\alpha_{1}+\alpha_{2}=1 \\
p_{k}=\left(\gamma_{k}-1\right) \rho_{k}\left(e_{k}-q_{k}\right)-\gamma_{k} \pi_{k}
\end{gathered}
$$

where $e_{k}$ is the internal energy and $\gamma_{k}, q_{k}$, and $\pi_{k}$ are constants, specific for each phase $k$. Their model is unconditionally hyperbolic and well-posed, and is to be able to solve physical situations for which other models fail. In the original Baer and Nunziato model, which has become more popular in the literature and even has become known as the BN-model, $u_{i}$ is taken equal to the velocity of the less compressible phase and $p_{i}$, the interphase pressure, is taken equal to the pressure of the most compressible phase. In Saurel and Abgrall, $p_{i}$ is taken equal to the mixture pressure and $u_{i}$ to the velocity of the center of mass. In [Lallemand and Saurel, 2000] new and enhanced pressure relaxation procedures are presented for this method.

New variants of this theory have appeared in the literature such as the novel Discrete Equation Method (DEM) [Abgrall and Saurel, 2003]. In the traditional approach which has been presented above, notice that we first averaged the microscopic level, single phase, partial differential equations (PDE's) over an assumed phase topology distribution to obtain macroscopic level multiphase equations. Then appropriate simplifying assumptions were made for the macroscopic level PDE's. Finally, though not discussed yet, the macroscopic multiphase flow equations are numerically discretized and the resulting equations solved, using finite difference, finite volume, or finite element methods, to obtain "standard" numerical solutions. With the DEM approach one first assumes a generic phase distribution topology. Then a discretized solution is developed within the computational cell using, for example, Riemann and approximate Riemann relations. Finally, this partial solution is averaged over the cell volume and time to obtain a meaningful solution. The DEM method carries a pressure and velocity for each phase, and because it effective only solves Euler equations locally, the method hyperbolic and well-posed, and it gives correct wave dynamic solutions. But it offers an additional bonus; 
for example, [Chinnayya, Daniel, and Saurel, 2004] use this new homogenization method (DEM) to obtain, not only the seven equation model above, but also explicit closure formulas for $p_{i}$ and $u_{i}$ that are symmetric, compatible with the second law of thermodynamics, and responsible for the fulfillment of interface conditions when dealing with contact/interface problems; they also provide a general explicit formula for $\mu$. Furthermore, in the acoustic, continuous limit this method leads to the equation system (for simplicity with no mass transfer)

$$
\begin{aligned}
& \frac{\partial \alpha_{1}}{\partial t}+\nabla \alpha_{1} \cdot \vec{u}_{I}=\mu\left(p_{1}-p_{2}\right) \\
& \frac{\partial(\alpha \rho)_{1}}{\partial t}+\nabla \cdot(\alpha \rho \vec{u})_{1}=0 \\
& \frac{\partial(\alpha \rho \vec{u})_{1}}{\partial t}+\nabla \cdot(\alpha \rho \vec{u} \otimes \vec{u}+\alpha p I)_{1}=p_{I} \nabla \alpha_{1}+\lambda\left(\vec{u}_{2}-\vec{u}_{1}\right) \\
& \frac{\partial(\alpha \rho E)_{1}}{\partial t}+\nabla \cdot[\alpha(\rho E+p) \vec{u}]_{1}=p_{I} \vec{u}_{I} \cdot \nabla \alpha_{1}+\lambda \vec{u}_{I}^{\prime} \cdot\left(\vec{u}_{2}-\vec{u}_{1}\right)-\mu p_{I}^{\prime}\left(p_{1}-p_{2}\right) \\
& \frac{\partial(\alpha \rho)_{2}}{\partial t}+\nabla \cdot(\alpha \rho \vec{u})_{2}=0 \\
& \frac{\partial(\alpha \rho \vec{u})_{2}}{\partial t}+\nabla \cdot(\alpha \rho \vec{u} \otimes \vec{u}+\alpha p I)_{2}=p_{I} \nabla \alpha_{2}-\lambda\left(\vec{u}_{2}-\vec{u}_{1}\right) \\
& \frac{\partial(\alpha \rho E)_{2}}{\partial t}+\nabla \cdot[\alpha(\rho E+p) \vec{u}]_{2}=p_{I} \vec{u}_{I} \cdot \nabla \alpha_{2}-\lambda \vec{u}_{I}^{\prime} \cdot\left(\vec{u}_{2}-\vec{u}_{1}\right)+\mu p_{I}^{\prime}\left(p_{1}-p_{2}\right),
\end{aligned}
$$

where $\alpha, \rho, \vec{u}, p, E\left(E=e+\frac{1}{2} \vec{u} \cdot \vec{u}\right)$, and $e$ represent the volume fraction, density, velocity, pressure, total energy, and internal energy, respectively.

The interfacial variables have been determined in [Saurel et. al., 2003] to be

$$
\begin{aligned}
& \vec{u}_{I}=\frac{Z_{1} \vec{u}_{1}+Z_{2} \vec{u}_{2}}{Z_{1}+Z_{2}}+\frac{\nabla \alpha_{1}}{\left|\nabla \alpha_{1}\right|} \frac{p_{2}-p_{1}}{Z_{1}+Z_{2}} \\
& p_{I}=\frac{Z_{2} p_{1}+Z_{1} p_{2}}{Z_{1}+Z_{2}}+\frac{Z_{1} Z_{2}}{Z_{1}+Z_{2}} \frac{\nabla \alpha_{1}}{\left|\nabla \alpha_{1}\right|} \cdot\left(\vec{u}_{2}-\vec{u}_{1}\right) .
\end{aligned}
$$

These variables correspond to the interface velocity of, and pressure exerted on, the surface of a two phase control volume, i.e. at locations where volume fraction gradients are present. The average interfacial velocity and pressure acting inside the two-phase control volume are given by $\begin{aligned} \vec{u}_{I}^{\prime} & =\frac{Z_{1} \vec{u}_{1}+Z_{2} \vec{u}_{2}}{Z_{1}+Z_{2}} \\ p_{I}^{\prime} & =\frac{Z_{2} p_{1}+Z_{1} p_{2}}{Z_{1}+Z_{2}} .\end{aligned}$

Mechanical non-equilibrium is represented with a relaxation process whose rate is controlled by the following parameters: $\lambda=\frac{1}{2} \mu Z_{1} Z_{2} \quad$ (velocity relaxation rate) 
$\mu=\frac{A_{I}}{Z_{1}+Z_{2}} \quad$ (pressure relaxation rate),

where $A_{I}$ represents the specific interfacial area and $Z_{k}$ the acoustic impedance of phase $k$, i.e. $Z_{k}=\rho_{k} c_{k}$.

In Chapter 6 the DEM method will be applied to investigate one-dimensional, variable crosssection, two-phase flows which are both fully compressible. In Chapter 7 advanced DEM methods, which utilize the Reactive Riemann Problem to derive the cell-level solutions, will be explored. I also gives a good review of the DEM method in general.

\section{Reduced or Relaxed Equation Forms}

The single velocity pressure equilibrium model corresponds to the one of [Kapila et al., 2001]. It has been obtained as the asymptotic limit of the [Baer and Nunziato, 1986) model in the limit of both stiff velocity and pressure relaxation. In involves 5 partial differential equations, one of them being non-conservative. Its resulting speed of sound corresponds to that of [Wood, 1930] which exhibits a non-monotonic variation with volume fraction. These two difficulties (nonconservativity and non-monotonicity) present serious computational challenges. To circumvent them, a pressure non-equilibrium 6-equation model is constructed (first reduced model in Kapila et al., 2001), also non-conservative, but easier to solve with a relaxation method. This model is presented in Chapter 3.

The [Kapila et al., 2001] model is the zero-order approximation of the Baer and Nunziato model with stiff mechanical relaxation. In one dimension (with no mass transfer), with consideration of only two fluids, the model is given as:

$$
\begin{aligned}
& \frac{\partial \alpha_{1}}{\partial \mathrm{t}}+\mathrm{u} \frac{\partial \alpha_{1}}{\partial \mathrm{x}}=\frac{\rho_{2} \mathrm{c}_{2}{ }^{2}-\rho_{1} \mathrm{c}_{1}{ }^{2}}{\frac{\rho_{1} \mathrm{c}_{1}{ }^{2}}{\alpha_{1}}+\frac{\rho_{2} \mathrm{c}_{2}{ }^{2}}{\alpha_{2}}} \frac{\partial \mathrm{u}}{\partial \mathrm{x}} \\
& \frac{\partial(\alpha \rho)_{1}}{\partial \mathrm{t}}+\frac{\partial(\alpha \rho)_{1} \mathrm{u}}{\partial \mathrm{x}}=0 \\
& \frac{\partial(\alpha \rho)_{2}}{\partial \mathrm{t}}+\frac{\partial(\alpha \rho)_{2} \mathrm{u}}{\partial \mathrm{x}}=0 \\
& \frac{\partial \rho \mathrm{u}}{\partial \mathrm{t}}+\frac{\partial \rho \mathrm{u}^{2}+\mathrm{p}}{\partial \mathrm{x}}=0 \\
& \frac{\partial \rho \mathrm{E}}{\partial \mathrm{t}}+\frac{\partial(\rho \mathrm{E}+\mathrm{p}) \mathrm{u}}{\partial \mathrm{x}}=0
\end{aligned}
$$

where $\alpha, \rho, u, p, E\left(E=e+\frac{1}{2} u^{2}\right)$, and e represent respectively the volume fraction, the mixture density, the velocity, the mixture pressure, the mixture total energy and the mixture internal energy.

The mixture internal energy is defined as

$$
\mathrm{e}=\mathrm{Y}_{1} \mathrm{e}_{1}\left(\rho_{1}, \mathrm{p},\right)+\mathrm{Y}_{2} \mathrm{e}_{2}\left(\rho_{2}, \mathrm{p}\right)
$$


and the mass fraction is given by: $\mathrm{Y}_{\mathrm{k}}=\frac{(\alpha \rho)_{\mathrm{k}}}{\rho}$.

The mixture density is defined by $\rho=(\alpha \rho)_{1}+(\alpha \rho)_{2}$.

Each fluid is governed by its own convex equation of state (EOS),

$\mathrm{e}_{\mathrm{k}}=\mathrm{e}_{\mathrm{k}}\left(\rho_{\mathrm{k}}, \mathrm{p}\right)$,

which allows the determination of the phases' sound speed,

$\mathrm{c}_{\mathrm{k}}=\mathrm{c}_{\mathrm{k}}\left(\rho_{\mathrm{k}}, \mathrm{p}\right)$.

The mixture pressure $\mathrm{p}$ is determined by solving equation (II.2). In the particular case of fluids governed by the stiffened gas EOS,

$\mathrm{p}_{\mathrm{k}}=\left(\gamma_{\mathrm{k}}-1\right) \rho_{\mathrm{k}} \mathrm{e}_{\mathrm{k}}-\gamma_{\mathrm{k}} \mathrm{p}_{\infty \mathrm{k}}$,

the resulting mixture EOS reads,

$\mathrm{p}\left(\rho, \mathrm{e}, \alpha_{1}, \alpha_{2}\right)=\frac{\rho \mathrm{e}-\left(\frac{\alpha_{1} \gamma_{1} \mathrm{p}_{\infty 1}}{\gamma_{1}-1}+\frac{\alpha_{2} \gamma_{2} \mathrm{p}_{\infty 2}}{\gamma_{2}-1}\right)}{\frac{\alpha_{1}}{\gamma_{1}-1}+\frac{\alpha_{2}}{\gamma_{2}-1}}$

It is straightforward to obtain the entropy equations:

$\frac{\mathrm{ds}_{\mathrm{k}}}{\mathrm{dt}}=0, \mathrm{k}=1,2$.

Consequently, this model needs specific relations for its closure in the presence of shocks. In the limit of weak shocks, appropriate shock relations have been determined in [Saurel et al., 2007]:

$\mathrm{Y}_{\mathrm{k}}=\mathrm{Y}_{\mathrm{k}}^{0}$,

$\rho(\mathrm{u}-\sigma)=\rho^{0}\left(\mathrm{u}^{0}-\sigma\right)=\mathrm{m}$,

$\mathrm{p}-\mathrm{p}^{0}+\mathrm{m}^{2}\left(\mathrm{v}-\mathrm{v}^{0}\right)=0$,

$\mathrm{e}_{\mathrm{k}}-\mathrm{e}_{\mathrm{k}}^{0}+\frac{\mathrm{p}+\mathrm{p}^{0}}{2}\left(\mathrm{v}_{\mathrm{k}}^{*}-\mathrm{v}_{\mathrm{k}}^{0}\right)=0$,

where $\sigma$ denotes the shock speed and the upperscript ' 0 ' represents the unshocked state.

These relations have been intensively validated against a large experimental data base for weak and strong shocks in the same reference.

Also of interest, [Guillard and Murrone, 2005] use asymptotic analysis, in the limit of zero relaxation time, to reduce the 7-equation two-phase equation model (which contains relaxation terms that drive the system toward pressure and velocity equilibrium) to a five equation reduced hyperbolic system. In Chapter 3 we present a new equation system, relaxed (reduced) from the seven equation model, and numerical method to efficiently perform DNS-like simulations with the diffuse interface approach (DIM).

This whole approach is gaining momentum. It is important to get the correct wave behavior during transients and mathematical formulations as well as numerical methods must be clean. The methods discussed above provide this behavior and insure well-posedness, for both averaged multiphase flow models and DNS-like models. 


\section{References}

Abgrall, R. and R. Saurel, "Discrete Equations for Physical and Numerical Compressible Multiphase Mixtures," Journal of Computational Physics, 186, 361-396, 2003.

Baer, M.R., "Continuum Mixture Modeling of Reactive Porous Media," Chapter 3 of "HighPressure Shock Compression of Solids IV: Response of Highly Porous Solids to Shock Loading," edited by L. Davison, Y. Horie, and M. Shahinpoor, Springer, New York, 1997.

Baer, M.R. and J.W. Nunziato, "A Theory of Deflagration-to-Detonation Transition (DDT) in Granular Explosives," Sandia National Laboratory report SAND82-0293, Albuquerque, NM, December 1983.

Baer, M.R. and J.W. Nunziato, "A Two-Phase Mixture Theory for the Deflagration-toDetonation Transition (DDT) in Reactive Granular Materials," Int. J. Multiphase Flow, V. 12, p.861-889, 1986.

Banerjee, S. and A.M.C. Chan, "Separated Flow Models I, Analysis of the Averaged and Local Instantaneous Formulations," Int. J. Multiphase Flow, V. 6, p. 1-24, 1980.

Brackbill, J.U., N.L. Johnson, B.A. Kashiwa, and W.B. Vanderheyden, "Multi-phase Flows and Particle Methods," The Fifth Annual Conference of the Computational Fluid Dynamics Society of Canada, Victoria, British Columbia, May 1997.

Chinnayya, Ashwin, Eric Daniel, and Richard Saurel, "Modelling Detonation Waves in Heterogeneous Energetic Materials," Journal of Computational Physics, 196, 490-538, 2004.

Dobran, Flavio, Theory of Structured Multiphase Mixtures, Springer-Verlag, Berlin, 1991.

Drew, D.A., "Mathematical Modeling of Two-Phase Flow," Ann. Rev. Fluid Mech., V. 15, 1983.

Drew, D.A. and Lahey, R.T., "Analytical Modeling of Multiphase Flow," in Particulate TwoPhase Flow, ed. M.C. Roco, Butterworth-Heinemann, Boston, 1993.

Drew, D.A. and Passman, S.L., Theory of Multicomponent Fluids, Springer-Verlag, New York, 1999.

Gelfand, I.M. and Shilov, G.E., Generalized Functions, Vol. I, Academic, New York, 1964.

Guillard, Herve and Angelo Murrone, "A Five Equation Reduced Model for Compressible Two Phase Flow Problems,” Journal of Computational Physics, 202, 664-698, 2005.

Hancox, W.T., R.L. Ferch, W.S. Liu, and R.E. Niemann, "One-Dimensional Models for Transient Gas-Liquid Flows in Ducts," Int. J. Multiphase Flow, V. 6, p. 25-40, 1980.

Ishii, M., Thermo-Fluid Dynamic Theory of Two-Phase Flow, Eyrolles, Paris, 1975. 
Kashiwa, B.A. and Rauenzahn, R.M., "A Multimaterial Formalism," Numerical Methods in Multiphase Flows, ASME FED-Vol. 185, p.149-157, 1994.

Kashiwa, Bryan A. and Edward S. Gaffney, Design Basis for CFDLib, Los Alamos National Laboratory report No. LA-UR-03-1295, 2003.

Kataoka, I. and Serizawa, A., "Interfacial Area Concentration and Its Roles in Local Instant Formulation of Two-Phase Flow," in Transient Phenomena in Multiphase Flow, ed. N.H. Afgan, Hemisphere, New York, 1988.

Lallemand, Marie-Helene and Richard Saurel, "Pressure Relaxation Procedures for Multiphase Compressible Flows,” INRIA report No. 4038, France, October 2000.

Lhuillier, D., "The Macroscopic Modelling of Multi-Phase Mixtures," in Flow of Particles in Suspensions, ed. U. Schaflinger, Springer-Verlag Wien, New York, 1996.

Nguyen, H. , "One-Dimensional Models for Transient Two-Phase Separated Flow," Third CSNI Specialist Meeting on Transient Two-Phase Flow, California Institute of Technology, Pasadena, California, March 23-25, 1981.

Nigmatulin, R.I., Dynamics of Multiphase Media, Hemisphere, New York, 1990.

Passman, S.L., J.W. Nunziato, and E.K. Walsh, "A Theory of Multiphase Mixtures," Sandia National Laboratory report SAND82-2261, Albuquerque, NM, March 1983.

Ransom, V.H., and M.P. Scofield, "Two-Pressure Hydrodynamic Model for Two-Phase Separated Flow," Idaho National Engineering Laboratory Report SRD-50-76, January 1976 (Revised, February 1976).

Rousseau, J.C. and R.L. Ferch, "A Note on Two-Phase Separated Flow Models," Int. J. Multiphase Flow, V. 5, p. 489-494, 1979.

Saurel, R., A. Forestier, D. Veyret, and J.-C. Loraud, “A Finite Volume Scheme for Two-Phase Compressible Flows," Int. J. Numerical Methods in Fluids, 18, 803-819 (1994).

Saurel, Richard and Remi Abrgall, "A Multiphase Godunov Method for Compressible Multifluid and Multiphase Flows," Journal of Computational Physics 150, 425-467, 1999.

Stuhmiller, J.H., "The Influence of Interfacial Pressure Forces on the Character of Two-Phase Flow Model Equations," Int. J. Multiphase Flow, V. 3, p. 551-560, 1977.

Saurel, R., O. LeMetayer, J. Massoni, and S. Gavrilyuk, "Shock Jump Relations for Multiphase Mixtures with Stiff Mechanical Relaxation,” Shock Waves, 16, 209-232 (2007).

Truesdell, C., Rational Thermodynamics, $2^{\text {nd }}$ edition, Springer-Verlag, New York, 1984. 
Whitaker, S., The Method of Volume Averaging, Kluwer Academic, Dordrecht, 1999.

Wilcox, D.C., Turbulence Modeling for CFD, $2^{\text {nd }}$ edition, DCW Industries, La Canada, CA, 1998.

\section{Appendix: Moment Evolution Equation}

It is critical that special attention be given to functional dependencies in deriving the moment evolution equation. Let us begin first by defining the ensemble average of some property $Q_{0}$ as

$$
\left\langle Q_{0}\right\rangle(\vec{x}, t) \equiv \int Q_{0}\left(\Gamma_{0}\right) f\left(\vec{x}, t, \Gamma_{0}\right) d \Gamma_{0},
$$

where, for example, $\Gamma_{0}=\left\{\rho_{0}, \vec{u}_{0}, e_{0}, X_{1}, X_{2}, \cdots\right\}$. Note that, while $Q_{0}$ depends explicitly upon the state $\Gamma_{0}$, its average value $\left\langle Q_{0}\right\rangle$ depends upon position $\vec{x}$ and time $t$, which it acquired from the probability function $f\left(\vec{x}, t, \Gamma_{0}\right)$. In fact, $\left\langle Q_{0}\right\rangle$ loses its explicit dependence upon $\Gamma_{0}$ by its very definition as an integral over all possible states $\Gamma_{0}$.

The variation of the probability is

$$
d f=\frac{\partial f}{\partial t} d t+\frac{\partial f}{\partial \vec{x}} \cdot d \vec{x}+\frac{\partial f}{\partial \Gamma_{0}} \cdot d \Gamma_{0},
$$

so the time variation of the probability can be written

$$
\frac{d f}{d t}=\frac{\partial f}{\partial t}+\vec{u}_{0} \cdot \nabla f+\dot{\Gamma}_{0} \cdot \frac{\partial f}{\partial \Gamma_{0}} .
$$

If we now multiply this equation by $Q_{0}\left(\Gamma_{0}\right)$ and integrate over $\Gamma_{0}$-space we get

$$
\int Q_{0} \frac{d f}{d t} d \Gamma_{0}=\int Q_{0} \frac{\partial f}{\partial t} d \Gamma_{0}+\int Q_{0} \vec{u}_{0} \cdot \nabla f d \Gamma_{0}+\int Q_{0} \Gamma_{0} \cdot \frac{\partial f}{\partial \Gamma_{0}} d \Gamma_{0}
$$

Let us now further examine each term of this expression. Since $Q_{0}$ does not depend explicitly on time, $t$, 


$$
\begin{aligned}
\int Q_{0}\left(\Gamma_{0}\right) \frac{\partial f}{\partial t} d \Gamma_{0} & =\int \frac{\partial\left(Q_{0} f\right)}{\partial t} d \Gamma_{0} \\
& =\frac{\partial}{\partial t} \int Q_{0} f d \Gamma_{0} \\
& =\frac{\partial}{\partial t}\left\langle Q_{0}\right\rangle(\vec{x}, t) .
\end{aligned}
$$

Similarly, since $Q_{0}$ is independent of $\vec{x}$, we have

$$
\begin{aligned}
\int Q_{0} \vec{u}_{0} \cdot \nabla f d \Gamma_{0} & =\int \nabla\left(Q_{0} f\right) \cdot \vec{u}_{0} d \Gamma_{0} \\
& =\int \nabla \cdot\left(Q_{0} \vec{u}_{0} f\right) d \Gamma_{0}-\int Q_{0} f\left(\nabla \cdot \vec{u}_{0}\right) d \Gamma_{0} \\
& =\nabla \cdot \int Q_{0} \vec{u}_{0} f d \Gamma_{0}-\int Q_{0}\left(\nabla \cdot \vec{u}_{0}\right) f d \Gamma_{0} \\
& =\nabla \cdot\left\langle Q_{0} \vec{u}_{0}\right\rangle(\vec{x}, t)-\left\langle Q_{0} \nabla \cdot \vec{u}_{0}\right\rangle(\vec{x}, t) .
\end{aligned}
$$

We also know that the $\dot{\Gamma}_{0}$ are independent of $\Gamma_{0}$, so

$$
\begin{aligned}
\int Q_{0} \dot{\Gamma}_{0} \cdot \frac{\partial f}{\partial \Gamma_{0}} d \Gamma_{0} & =\dot{\Gamma}_{0} \cdot \int Q_{0} \frac{\partial f}{\partial \Gamma_{0}} d \Gamma_{0} \\
& =\dot{\Gamma}_{0} \cdot\left[\int \frac{\partial\left(Q_{0} f\right)}{\partial \Gamma_{0}} d \Gamma_{0}-\int f \frac{\partial Q_{0}}{\partial \Gamma_{0}} d \Gamma_{0}\right] \\
& =\left.\dot{\Gamma}_{0} \cdot Q_{0} f\right|_{\text {limit of } \Gamma_{0}}-\int \dot{\Gamma}_{0} \cdot \frac{\partial Q_{0}}{\partial \Gamma_{0}} f d \Gamma_{0} \\
& =0-\left\langle\dot{\Gamma}_{0} \cdot \frac{\partial Q_{0}}{\partial \Gamma_{0}}\right\rangle \\
& =-\left\langle\frac{\partial Q_{0}}{\partial \Gamma_{0}} \cdot \frac{d \Gamma_{0}}{d t}\right\rangle \\
& =-\left\langle\frac{d Q_{0}}{d t}\right\rangle=-\left\langle\dot{Q}_{0}\right\rangle(\vec{x}, t) .
\end{aligned}
$$

As long as the $Q_{0}$ are physically conserved quantities we also have

$$
\int Q_{0} \frac{d f}{d t} d \Gamma_{0}=0
$$

Putting all these expressions back into the integral equation above gives the moment evolution equation (11) 


$$
\frac{\partial\left\langle Q_{0}\right\rangle}{\partial t}+\nabla \cdot\left\langle Q_{0} \vec{u}_{0}\right\rangle=\left\langle\dot{Q}_{0}+Q_{0} \nabla \cdot \vec{u}_{0}\right\rangle .
$$

【[ Notice: By comparison with Equation (1.1) of [Kashiwa and Rauenzahn 1994],

$$
\frac{\partial\left\langle Q_{0}\right\rangle}{\partial t}+\nabla \cdot\left\langle Q_{0} \vec{u}_{0}\right\rangle=\left\langle\dot{\Gamma}_{0} \cdot \frac{\partial Q_{0}}{\partial \Gamma_{0}}\right\rangle+\int Q_{0} \frac{D f}{D t} d \Gamma_{0}
$$

theirs should read

$$
\begin{aligned}
\frac{\partial\left\langle Q_{0}\right\rangle}{\partial t}+\nabla \cdot\left\langle Q_{0} \vec{u}_{0}\right\rangle & =\left\langle\dot{\Gamma}_{0} \cdot \frac{\partial Q_{0}}{\partial \Gamma_{0}}\right\rangle+\left\langle Q_{0} \nabla \cdot \vec{u}_{0}\right\rangle+\int Q_{0} \frac{D f}{D t} d \Gamma_{0} \\
& =\left\langle\dot{Q}_{0}+Q_{0} \nabla \cdot \vec{u}_{0}\right\rangle+\int Q_{0} \frac{D f}{D t} d \Gamma_{0}
\end{aligned}
$$

to be correct. 》 


\section{Chapter 3 \\ Simple and Efficient Relaxation Methods for Interfaces Separating Compressible Fluids and Cavitating Flows}

[A preprint of an article accepted (November 2008) for publication in Journal of Computational Physics under slightly different title: "Simple and efficient relaxation methods for interfaces separating compressible fluids, cavitating flows and shocks in multiphase mixtures," Richard Saurel, Fabien Petitpas, and Ray A. Berry.] 


\title{
Simple and efficient relaxation methods for interfaces separating compressible fluids and cavitating flows
}

\author{
Richard Saurel ${ }^{1,2}$, Fabien Petitpas ${ }^{1}$ and Ray A. Berry ${ }^{3}$ \\ ${ }^{1}$ Polytech'Marseille, Aix-Marseille University and SMASH Project UMR CNRS 6595 - \\ IUSTI-INRIA , 5 rue E. Fermi, 13453 Marseille Cedex 13, France \\ ${ }^{2}$ University Institute of France, same address \\ ${ }^{3}$ Multiphysics Methods Group, Advanced Nuclear Energy Systems Department, \\ Idaho National Laboratory, P.O. Box 1625, Idaho Falls, ID 83415-3885
}

\begin{abstract}
Numerical approximation of the five-equation two-phase flow of Kapila et al. (2001) is examined. This model has shown excellent capabilities for the numerical resolution of interfaces separating compressible fluids as well as wave propagation in compressible mixtures (Murrone and Guillard, 2005, Abgrall and Perrier, 2006, Petitpas et al., 2007). However, its numerical approximation poses some serious difficulties. Among them, the nonmonotonic behavior of the sound speed causes multiple sonic transitions in the numerical diffusion zones at interfaces. Moreover, volume fraction variation across acoustic waves results in difficulties for the Riemann problem resolution, and in particular for the derivation of approximate solvers. Volume fraction positivity in the presence of shocks or strong expansion waves is another issue resulting in lack of robustness. To circumvent these difficulties, the pressure equilibrium assumption is relaxed and a pressure non-equilibrium model is developed. It results in a single velocity, non-conservative hyperbolic model with two energy equations involving relaxation terms. It fulfills the equation of state and energy conservation on both sides of interfaces and guarantees correct transmission of shocks across them. This formulation considerably simplifies numerical resolution. Following a strategy developed previously for another flow model (Saurel and Abgrall, 1999), the hyperbolic part is first solved without relaxation terms with a simple, fast and robust algorithm, valid for unstructured meshes. Second, stiff relaxation terms are solved with a Newton method that also guarantees positivity and robustness. The algorithm and model are compared to exact solutions of the Euler equations as well as solutions of the five-equation model under extreme flow conditions, for interface computation and cavitating flows involving dynamics appearance of interfaces. In order to deal with correct dynamic of shock waves propagating through multiphase mixtures, the artificial heat exchange method of Petitpas et al. (2007) is adapted to the present formulation.
\end{abstract}

Key words: hyperbolic systems, multifluid, multiphase, real gases, cavitation, multiphysics, Godunov 


\section{Introduction}

Compressible multi-material flows and multiphase mixtures arise in many natural and industrial situations including bubble dynamics, shock wave interaction with material discontinuities, detonation of high energetic materials, hypervelocity impacts, cavitating flows, combustion systems to name only a few. The motivation of the present work is the accurate and computationally efficient resolution of interface problems in extreme flow conditions (high pressure ratios $\approx 10^{7}$, high density ratios $\approx 10^{3}$ ), as well as the computation of dynamic appearance of interfaces, that occur in cavitating flows and spallation phenomena. These interfaces are often separating pure media but also mixtures of materials in which wave dynamics is also important. Such situations appear frequently in astrophysics, physics of explosives, nuclear physics, powder engineering and many other applications. The aim of the present paper is to develop a general formulation and algorithm to solve interface problems separating compressible media or mixtures in extreme situations.

Godunov-type schemes and variants have now reached a level of maturity to solve single phase flows in the presence of discontinuities. However, the presence of large discontinuities of thermodynamic variables and equations of state at material interfaces result in numerical instabilities, oscillations and computational failure (Karni, 1994, Abgrall, 1996). To circumvent these difficulties, two classes of methods have been developed:

- Methods that consider the interface as a sharp discontinuity (Sharp Interface Methods - SIM).

- Methods that consider the interface as a diffuse zone, like contact discontinuities in gas dynamics (Diffuse Interface Methods - DIM).

The Lagrangian class of SIM is the most natural (see for example Hirt et al., 1974, Farhat and Roux, 1991). In this context, the computational mesh moves and distorts with the material interface. However, when dealing with fluid flows, deformations are unbounded and resulting mesh distortions can make the Lagrangian approach unpractical (Scheffer and Zukas, 2000). Eulerian methods use a fixed mesh with an additional equation for tracking or reconstructing the material interface. In the volume of fluid (VOF) approach (Hirt and Nichols, 1981), each computational cell is assumed to possibly contain a mixture of both fluids and the volume occupied by each fluid is represented by the volume fraction, transported with the flow. This method is widely used for incompressible flows as there is no special thermodynamics to compute in mixture cells (Gueyffier et al., 1999). For compressible flows, extra energy equations are used as well as pressure relaxation procedures (Benson, 1992, Miller and Puckett, 1996). These methods seem efficient as a result of subtle management at the discrete level of the various equations. The literature doesn't provide a clear link of this discrete management to a given system of continuous partial differential equations. In the present paper, an attempt to clarify, improve and generalize these methods will be developed.

Another class of popular Eulerian methods is based on the level-set equation (Dervieux and Thomasset, 1980, Mulder et al., 1992, Osher and Fedkiw, 2001, Sethian, 2001) to locate the interface. Again, for compressible flows, special management of the interface is needed to guarantee interface conditions. Relevant work in this direction was done by Fedkiw et al. (1999) with the Ghost Fluid Method, Abgrall and Karni (2001) with a simplified version of this method 
and Khoo et al. (2005). This method is attractive for its apparent simplicity and versatility versus various problems of physics. However, its use in arbitrary conditions, with large pressure and density ratios doesn't seem obvious. Moreover, it is non-conservative regarding mixture variables (momentum and energy). The last class of SIM corresponds to Front Tracking methods, where the interface is explicitly tracked over a fixed Eulerian mesh. Considerable efforts have been done to develop computational codes employing this approach (Glimm et al., 1998, LeVeque and Shyue, 1996). It is worth mentioning that none of these methods is able to dynamically create interfaces, and to solve interfaces separating pure media and mixtures.

The second type of methods (DIM) considers interfaces as numerically diffused zones, like contact discontinuities in gas dynamics. Diffuse interfaces correspond to artificial mixtures created by numerical diffusion. A pioneering work in this direction was performed by Abgrall (1996). Determination of thermodynamic flow variables in these zones is achieved on the basis of multiphase flow theory (Saurel and Abgrall, 1999, Abgrall and Saurel, 2003, Saurel et al., 2003, Murrone and Guillard, 2005, Abgrall and Perrier, 2006, Saurel et al., 2007a, Petitpas et al., 2007). The challenge is to derive physically, mathematically, and numerically consistent thermodynamic laws for the artificial mixture. The key issue is to fulfill interface conditions within this artificial mixture. This second category possesses several advantages:

- The same algorithm is implemented globally in both pure fluids and in mixture zones. An extended hyperbolic system is used to solve every location of the flow.

- These models and methods are able to dynamically create interfaces that are not present initially, e.g. in cavitating flows where gas pockets dynamically appear in a liquid (Saurel and Le Metayer, 2001, Le Metayer et al., 2005, Saurel et al., 2008).

- These methods are also able to deal with interfaces separating pure fluids and fluid mixtures, e.g. in the computation of detonation waves in condensed explosives where chemical decomposition produces multiphase mixtures of materials (Saurel and Le Metayer, 2001, Chinnayya et al., 2004).

Methods in this second category are based on hyperbolic multiphase flow models, consisting of two main classes:

- Models for mixtures in total non-equilibrium: Baer and Nunziato (1986) model and its variants, and

- Models for mixtures in mechanical equilibrium (Stewart and Wendroff, 1984, Kapila et al., 2001).

This paper deals with the building of a simple, robust, fast and accurate formulation for single velocity and single pressure multiphase flows. The Kapila et al. (2001) model is of particular interest for the computation of interfaces separating compressible fluids, as well as barotropic and non-barotropic cavitating flows. Specific numerical schemes have been derived recently in Murrone and Guillard (2005), Abgrall and Perrier (2006), Saurel et al. (2007a), Petitpas et al. (2007).

This model is apparently simple. In the context of two fluids it is composed of two mass equations, a mixture momentum equation and a mixture energy equation. These equations express in conservative formulation. The closure is achieved by the pressure equilibrium condition that results in a differential transport equation for the volume fraction containing a non 
conservative term, involving the velocity divergence and phasic bulk moduli. However this last equation poses serious computational challenges which include:

- Shock computations within the context of a non-conservative model.

- Volume fraction positivity, when dealing with shocks and strong expansion waves. The term involving a velocity divergence in the volume fraction evolution equation is particularly difficult to approximate (Petitpas et al., 2007). This is particularly important for the dynamic appearance of interfaces in cavitating flows.

- Non-monotonic behavior of the sound speed (Wood, 1930) which produces multiple sonic points in the numerical diffusion zones at interfaces. As it is very difficult (and expensive) to solve exactly the Riemann problem (Petitpas et al., 2007), approximate Riemann solvers are used resulting in sampling errors (with multiple sonic points) in the flux computations as well as in the non-conservative equation evolution. These difficulties in the Riemann problem solution are due to volume fraction variations across shocks and expansion waves.

Moreover, in order to consider future extensions with additional physics to reach multiphysics modeling of continuous media with a multiphase approach, the computational efficiency of existing algorithms must be improved. The multiphysics challenge we consider deals with:

- Sophisticated equations of state (EOS): Mie-Gruneisen for condensed materials, JWL for explosive products (Lee et al., 1968).

- Granular materials that involve extra EOS expressing contact granular energy and contact pressure (Bdzil et al., 1999).

- Capillary effects modeling (Perigaud et al., 2005) with eventually phase transition (Saurel et al., 2008).

- Interfaces separating compressible fluids and elastic solids in extreme deformations (Miller and Colella, 2001, Titarev et al., 2007, Gavrilyuk et al., 2008, Favrie et al., 2008). This instance is particularly difficult as the EOS for solids depends on the deformation tensor.

The present paper doesn't deal with all these extensions, but it is clear that such a goal needs simple and robust multiphase formulations. The present paper addresses this issue in the context of the simplest version of the Kapila et al. (2001) model.

The main difficulty with this model comes from the pressure equilibrium condition, which results in the non-conservative equation for the volume fraction. A conservative formulation can be obtained with the help of the entropy equations. However, this conservative formulation is untenable in the presence of shocks. To circumvent these difficulties, pressure non-equilibrium effects are restored in the Kapila et al. (2001) model. This results in a 6-equation model with a single velocity but with two pressures and associated relaxation terms. This extended model was already presented as a first reduction of the Baer and Nunziato (1986) model in Kapila et al. (2001), but never considered for the description of diffuse interfaces. A seventh equation is added describing the mixture total energy in order to guarantee a correct treatment of shocks in the single phase limit. This apparent complexity with an extended model actually leads to considerable simplifications regarding numerical resolution. Indeed, this model remains hyperbolic with only three characteristic wave propagation speeds, volume fraction positivity is easily preserved and multiple sonic points no longer appear during hyperbolic evolution. The 
building of a simple and efficient method for the numerical approximation of this flow model in the context of diffuse interfaces is the aim of the present paper.

When relaxation terms are omitted the volume fraction remains constant across acoustic waves and the Riemann problem is easily solved with approximate Riemann solvers (acoustic and HLLC-type solvers, Toro, 1997). With this non-equilibrium pressure model, the sound speed has a monotonic behavior versus volume fraction and multiple sonic points no longer appear inside the numerical diffusion zone at interfaces. This feature provides robustness when considering cavitating flows (Koren et al., 2002, Sinibaldi, 2006, Petitpas et al., 2007). This hyperbolic step is then followed by a relaxation step that consists in the determination of the asymptotic solution of a differential problem in the limit of infinitely fast relaxation. This asymptotic solution results in resolution of an algebraic system. The combination of these two steps (hyperbolic + relaxation) guarantees convergence of the method to solutions of the Kapila et al. (2001) reduced model.

This paper is organized as follows. In Section II the Kapila et al. (2001) model is recalled and the non-equilibrium 6-equation model is presented. This 6-equation model tends to the 5-equation model of Kapila et al. (2001) in the limit of stiff pressure relaxation. Basic properties of these models are presented: Entropy inequality and hyperbolicity. In Section III the numerical method is built. Approximate Riemann solvers are presented for the hyperbolic part and a Godunov type scheme is built. The pressure relaxation algorithm is also presented in this Section. Special attention is given to the role of the seventh equation used to correct the computation of nonconservative energies in the single phase limit, on both sides of an interface. Various test cases are presented in Section IV, together with validations against exact solutions of the Euler equations and of the 5-equation model of Kapila et al. (2001). Some examples consider interfaces initially present in the flow, while others involve the dynamic appearance of interfaces. Section V presents the extension of the method to shock propagation in physical multiphase mixtures. This extension is not important for interfaces separating pure (or nearly pure) fluids. But it has importance when the interface separates pure fluids and mixtures of materials. In Section VI, comparisons of the 6-equation model are given with existing barotropic cavitating flow models (van Brummelen and Koren, 2003). These models are recovered as limiting cases of the present 6-equation model. Moreover, a simple algorithm is proposed to solve cavitating barotropic flows. Finally, conclusions and future investigations are discussed in Section VII.

\section{Pressure equilibrium and non-equilibrium single velocity multiphase flow models}

The single velocity pressure equilibrium model corresponds to the one of Kapila et al. (2001). It has been obtained as the asymptotic limit of the Baer and Nunziato (1986) model in the limit of both stiff velocity and pressure relaxation. In involves 5 partial differential equations, one of them being non-conservative. Its resulting speed of sound corresponds to that of Wood (1930) which exhibits a non-monotonic variation with volume fraction. These two difficulties (nonconservativity and non-monotonicity) present serious computational challenges. To circumvent them, a pressure non-equilibrium 6-equation model is constructed (first reduced model in Kapila et al., 2001), also non-conservative, but easier to solve with a relaxation method. Both models are presented hereafter. 


\section{II.1 Five-equation model}

The Kapila et al. (2001) is the zero-order approximation of the Baer and Nunziato (1986) with stiff mechanical relaxation. It reads in the context of two fluids:

$\frac{\partial \alpha_{1}}{\partial \mathrm{t}}+\mathrm{u} \frac{\partial \alpha_{1}}{\partial \mathrm{x}}=\frac{\rho_{2} \mathrm{c}_{2}{ }^{2}-\rho_{1} \mathrm{c}_{1}{ }^{2}}{\frac{\rho_{1} \mathrm{c}_{1}{ }^{2}}{\alpha_{1}}+\frac{\rho_{2} \mathrm{c}_{2}{ }^{2}}{\alpha_{2}}} \frac{\partial \mathrm{u}}{\partial \mathrm{x}}$

$\frac{\partial(\alpha \rho)_{1}}{\partial \mathrm{t}}+\frac{\partial(\alpha \rho)_{1} \mathrm{u}}{\partial \mathrm{x}}=0$

$\frac{\partial(\alpha \rho)_{2}}{\partial \mathrm{t}}+\frac{\partial(\alpha \rho)_{2} \mathrm{u}}{\partial \mathrm{x}}=0$

$\frac{\partial \rho u}{\partial t}+\frac{\partial \rho u^{2}+p}{\partial x}=0$

$\frac{\partial \rho \mathrm{E}}{\partial \mathrm{t}}+\frac{\partial(\rho \mathrm{E}+\mathrm{p}) \mathrm{u}}{\partial \mathrm{x}}=0$

where $\alpha, \rho, u, p, E\left(E=e+\frac{1}{2} u^{2}\right)$, and e represent respectively the volume fraction, the mixture density, the velocity, the mixture pressure, the mixture total energy and the mixture internal energy.

The mixture internal energy is defined as

$\mathrm{e}=\mathrm{Y}_{1} \mathrm{e}_{1}\left(\rho_{1}, \mathrm{p},\right)+\mathrm{Y}_{2} \mathrm{e}_{2}\left(\rho_{2}, \mathrm{p}\right)$

and the mass fraction is given by: $Y_{k}=\frac{(\alpha \rho)_{k}}{\rho}$. The mixture density is defined by $\rho=(\alpha \rho)_{1}+(\alpha \rho)_{2}$

Each fluid is governed by its own convex equation of state (EOS),

$\mathrm{e}_{\mathrm{k}}=\mathrm{e}_{\mathrm{k}}\left(\rho_{\mathrm{k}}, \mathrm{p}\right)$,

which allows the determination of the phases' sound speed,

$\mathrm{c}_{\mathrm{k}}=\mathrm{c}_{\mathrm{k}}\left(\rho_{\mathrm{k}}, \mathrm{p}\right)$.

The mixture pressure $\mathrm{p}$ is determined by solving equation (II.2). In the particular case of fluids governed by the stiffened gas EOS,

$\mathrm{p}_{\mathrm{k}}=\left(\gamma_{\mathrm{k}}-1\right) \rho_{\mathrm{k}} \mathrm{e}_{\mathrm{k}}-\gamma_{\mathrm{k}} \mathrm{p}_{\infty \mathrm{k}}$,

the resulting mixture EOS reads,

$$
\mathrm{p}\left(\rho, \mathrm{e}, \alpha_{1}, \alpha_{2}\right)=\frac{\rho \mathrm{e}-\left(\frac{\alpha_{1} \gamma_{1} \mathrm{p}_{\infty 1}}{\gamma_{1}-1}+\frac{\alpha_{2} \gamma_{2} \mathrm{p}_{\infty 2}}{\gamma_{2}-1}\right)}{\frac{\alpha_{1}}{\gamma_{1}-1}+\frac{\alpha_{2}}{\gamma_{2}-1}}
$$

It is straightforward to obtain the entropy equations: 
$\frac{\mathrm{ds}_{\mathrm{k}}}{\mathrm{dt}}=0, \mathrm{k}=1,2$.

Consequently, this model needs specific relations for its closure in the presence of shocks. In the limit of weak shocks, appropriate shock relations have been determined in Saurel et al. (2007b):

$\mathrm{Y}_{\mathrm{k}}=\mathrm{Y}_{\mathrm{k}}^{0}$,

$\rho(u-\sigma)=\rho^{0}\left(u^{0}-\sigma\right)=m$,

$\mathrm{p}-\mathrm{p}^{0}+\mathrm{m}^{2}\left(\mathrm{v}-\mathrm{v}^{0}\right)=0$,

$\mathrm{e}_{\mathrm{k}}-\mathrm{e}_{\mathrm{k}}^{0}+\frac{\mathrm{p}+\mathrm{p}^{0}}{2}\left(\mathrm{v}_{\mathrm{k}}^{*}-\mathrm{v}_{\mathrm{k}}^{0}\right)=0$,

where $\sigma$ denotes the shock speed and the upperscript ' 0 ' represents the unshocked state. These relations have been intensively validated against a large experimental data base for weak and strong shocks in the same reference.

Even equipped with these relations, this apparently simple model involves many difficulties:

- With the help of relations (II.5), it is possible to solve exactly or approximately the Riemann problem (Petitpas et al., 2007). Even when this solution is exact, it is shown in the same reference that convergence of a numerical scheme to the exact solution is extremely difficult as the system is non-conservative: The cell average of nonconservative variables has no physical sense. Cell averages were replaced by a relaxation procedure in Saurel et al. (2007a) and Petitpas et al. (2007). To reach convergence for shock propagating in multiphase mixtures, artificial heat exchanges were needed in the shock layer (Petitpas et al., 2007).

- Another issue is related to the volume fraction positivity in the presence of shocks and even in the presence of strong rarefaction waves. Indeed, when dealing with liquid-gas mixtures for example, the liquid compressibility is so weak that the pressure tends to become negative, resulting in computational failure in the gas sound speed computation. Such situation occurs frequently in cavitation test problems.

- An extra difficulty is related to the mixture sound speed that obeys the Wood (1930) formula $\frac{1}{\rho \mathrm{c}_{\mathrm{eq}}^{2}}=\frac{\alpha_{1}}{\rho_{1} \mathrm{c}_{1}^{2}}+\frac{\alpha_{2}}{\rho_{2} \mathrm{c}_{2}^{2}}$. The mixture sound speed has a non-monotonic variation with volume fraction, as shown in Figure 1. Here $c_{\text {eq }}$ represents the mechanical equilibrium mixture sound speed. 


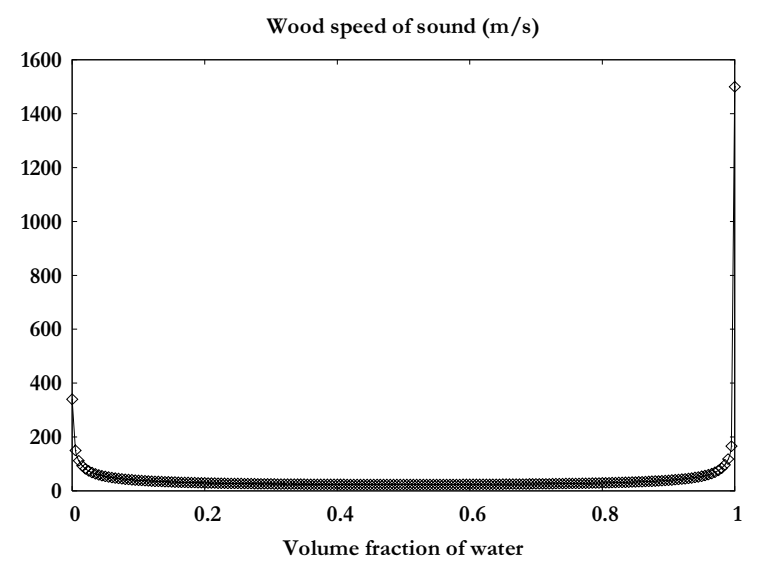

Figure 1: Representation of the mixture speed of sound $\left(\frac{1}{\rho \mathrm{c}_{\mathrm{eq}}^{2}}=\frac{\alpha_{1}}{\rho_{1} \mathrm{c}_{1}^{2}}+\frac{\alpha_{2}}{\rho_{2} \mathrm{c}_{2}^{2}}\right)$ of the 5-equation model for the liquid water - air mixture under atmospheric conditions.

This non-monotonic behavior has serious consequences for the computation of interfaces separating compressible fluids. Consider for example the advection of a liquid-gas interface, corresponding in this model to a volume fraction discontinuity. During numerical resolution, this discontinuity will become a mixture zone. In this zone, the sound speed has a non-monotonic behavior which may result in the presence of two sonic points even if the flow is subsonic in the two pure fluids. Capturing sonic points for this model poses difficulties as Riemann invariants cannot be integrated explicitly (Petitpas et al., 2007). Using an approximate Riemann solver where the left and right facing waves are treated as discontinuities is possible. When embedded in an Eulerian method a sampling error in the solution for the flux computations occurs. This error occurs twice (for each sonic point) in the interface mixture zone at each time step. As a result, computational difficulties and even failures occur.

To illustrate the difficulties related to the non-monotonic sound speed in this model, numerical results obtained with the method of Petitpas et al. (2007) are recalled. This method solves interfaces as diffuse numerical zones with the help of a Lagrange-relaxation algorithm. A onemeter long shock tube containing two chambers separated by an interface at the location $\mathrm{x}=0.8 \mathrm{~m}$ is considered. Each chamber contains a mixture of water and air. The initial density of the water is $\rho_{\text {water }}=1000 \mathrm{~kg} \cdot \mathrm{m}^{-3}$ and the stiffened gas EOS parameters are $\gamma_{\text {water }}=4.4$ and $\mathrm{p}_{\infty \text {, water }}=6.10^{8} \mathrm{~Pa}$. The initial density of air is $\rho_{\text {air }}=10 \mathrm{~kg} \cdot \mathrm{m}^{-3}$ and EOS parameters are $\gamma_{\text {air }}=1.4$ and $\mathrm{p}_{\infty, \text { air }}=0 \mathrm{~Pa}$. The left chamber contains a very small volume fraction of air $\alpha_{\text {air }}=10^{-6}$ and the pressure is equal to $10^{9} \mathrm{~Pa}$. The right chamber contains the same fluids but the volume fractions are reversed. Its pressure is equal to $10^{5} \mathrm{~Pa}$. In both chambers the initial velocity is zero. The exact solution of the single phase Euler equations and the multiphase flow model with 5 equations are compared in Figure 2 at time $\mathrm{t}=220 \mu \mathrm{s}$. 

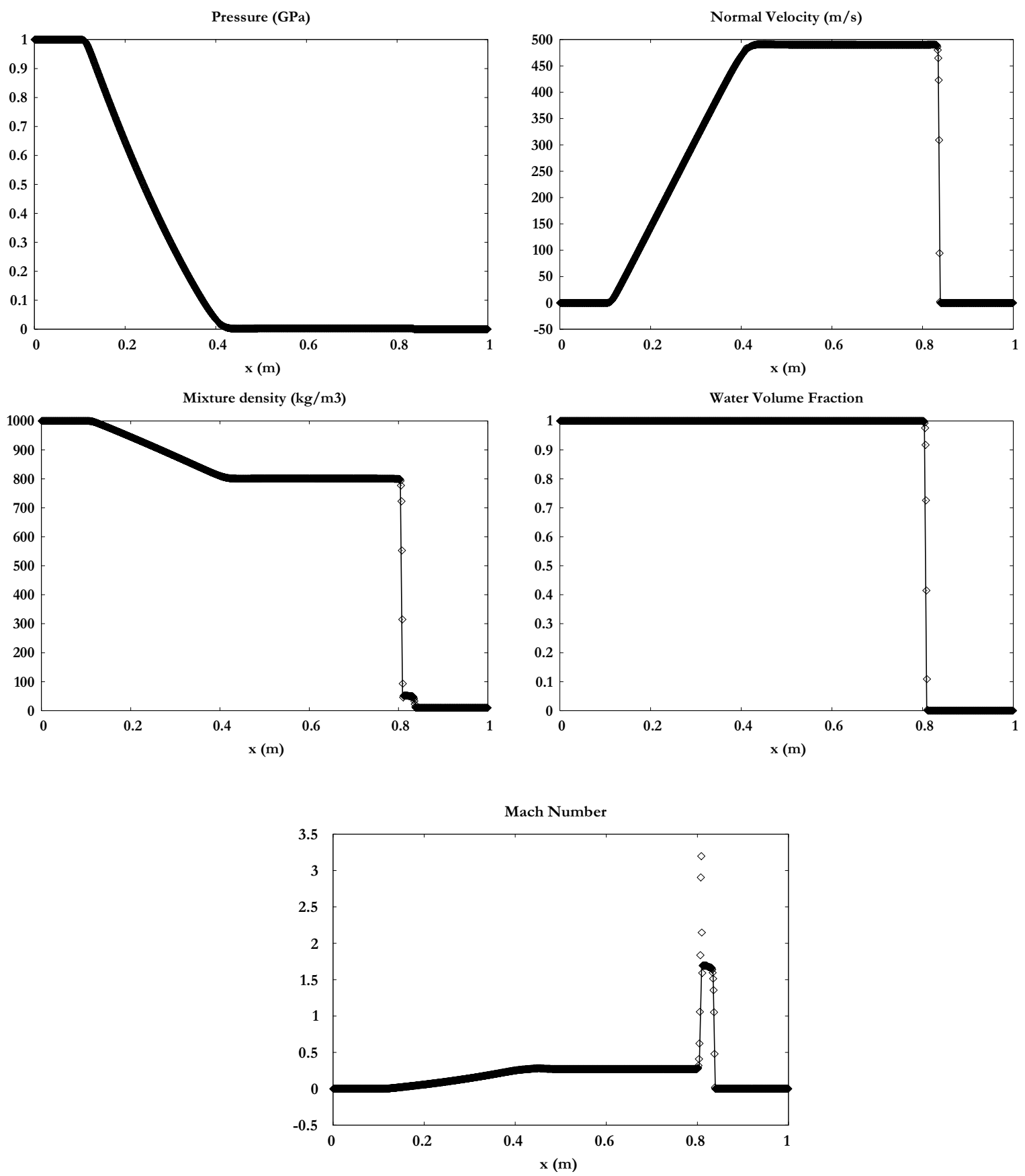

Figure 2: Liquid/Gas shock tube. The Lagrange-relaxation method (symbols) of Petitpas et al. (2007) is compared to the exact solution (solid). A 1000 cells mesh is used. The density ratio is 100 and the pressure ratio is 10000 at the initial discontinuity. A Mach oscillation appears in the numerical diffusion zone at the interface and is due to the non-monotonic behavior of the speed of sound of this model.

The method developed in Petitpas et al. (2007) is able to deal with the non-monotonic speed of sound and sonic transitions at interfaces because it uses a Lagrange-relaxation algorithm. With this method, there is no need to consider sonic points. This method is very efficient on Cartesian grids but difficult to extend to unstructured grids. This is one of the reasons why a new 
formulation is developed in the present paper. This difficulty is reported in the literature for barotropic flows with the same non-monotonic sound speed, used for example in cavitating flows (Koren et al., 2002, Coutier-Delgosha et al., 2005, Sinibaldi, 2006). These references report this problem in the simpler context of a conservative cavitation model that will be examined in Section V.

To circumvent these difficulties and develop a simple algorithm able to deal with multiphysics extensions, a pressure non-equilibrium model is considered.

\section{II.2 Six-equation model}

The 6-equation model is also derived from the 7-equation model of Baer and Nunziato (1986) in the asymptotic limit of stiff velocity relaxation only (first reduced model in Kapila et al., 2001). Pressure non-equilibrium effects are maintained. The 6-equation model should not be considered as a physical model, but more as a step-model to solve the 5-equations model (second reduced model of Kapila et al., 2001). Indeed, the model with 6-equations has better properties for numerical approximations than the mechanical equilibrium one:

- Positivity of the volume fraction is easily preserved.

- The mixture sound speed has a monotonic behavior.

These two properties are key points for the building of a simple, robust and accurate hyperbolic solver. Moreover, with proper treatment of relaxation terms, solutions of the 5-equation model will be recovered.

\section{Flow model}

The 6-equation model reads:

$$
\begin{aligned}
& \frac{\partial \alpha_{1}}{\partial \mathrm{t}}+\mathrm{u} \frac{\partial \alpha_{1}}{\partial \mathrm{x}}=\mu\left(\mathrm{p}_{1}-\mathrm{p}_{2}\right) \\
& \frac{\partial \alpha_{1} \rho_{1}}{\partial \mathrm{t}}+\frac{\partial \alpha_{1} \rho_{1} \mathrm{u}}{\partial \mathrm{x}}=0 \\
& \frac{\partial \alpha_{2} \rho_{2}}{\partial \mathrm{t}}+\frac{\partial \alpha_{2} \rho_{2} \mathrm{u}}{\partial \mathrm{x}}=0 \\
& \frac{\partial \rho \mathrm{u}}{\partial \mathrm{t}}+\frac{\partial \rho \mathrm{u}^{2}+\left(\alpha_{1} \mathrm{p}_{1}+\alpha_{2} \mathrm{p}_{2}\right)}{\partial \mathrm{x}}=0 \\
& \frac{\partial \alpha_{1} \rho_{1} \mathrm{e}_{1}}{\partial \mathrm{t}}+\frac{\partial \alpha_{1} \rho_{1} \mathrm{e}_{1} \mathrm{u}}{\partial \mathrm{t}}+\alpha_{1} \mathrm{p}_{1} \frac{\partial \mathrm{u}}{\partial \mathrm{x}}=-\mathrm{p}_{\mathrm{I}} \mu\left(\mathrm{p}_{1}-\mathrm{p}_{2}\right) \\
& \frac{\partial \alpha_{2} \rho_{2} \mathrm{e}_{2}}{\partial \mathrm{t}}+\frac{\partial \alpha_{2} \rho_{2} \mathrm{e}_{2} \mathrm{u}}{\partial \mathrm{t}}+\alpha_{2} \mathrm{p}_{2} \frac{\partial \mathrm{u}}{\partial \mathrm{x}}=\mathrm{p}_{\mathrm{I}} \mu\left(\mathrm{p}_{1}-\mathrm{p}_{2}\right)
\end{aligned}
$$

The interfacial pressure $\mathrm{p}_{\mathrm{I}}$ is obtained as the asymptotic limit of the interfacial pressure of the symmetric non-equilibrium model with 7-equations of Saurel et al. (2003). This estimate in the limit of equal velocities reads:

$\mathrm{p}_{\mathrm{I}}=\frac{\mathrm{Z}_{2} \mathrm{p}_{1}+\mathrm{Z}_{1} \mathrm{p}_{2}}{\mathrm{Z}_{1}+\mathrm{Z}_{2}}$

where $Z_{k}=\rho_{k} c_{k}$ represents the acoustic impedance of phase $\mathrm{k}$. 
The combination of the two internal energy equations with mass and momentum equations results in the additional mixture energy equation:

$$
\frac{\partial \rho\left(\mathrm{Y}_{1} \mathrm{e}_{1}+\mathrm{Y}_{2} \mathrm{e}_{2}+\frac{1}{2} \mathrm{u}^{2}\right)}{\partial \mathrm{t}}+\frac{\partial \mathrm{u}\left(\rho\left(\mathrm{Y}_{1} \mathrm{e}_{1}+\mathrm{Y}_{2} \mathrm{e}_{2}+\frac{1}{2} \mathrm{u}^{2}\right)+\left(\alpha_{1} \mathrm{p}_{1}+\alpha_{2} \mathrm{p}_{2}\right)\right)}{\partial \mathrm{x}}=0
$$

This extra equation will be important during numerical resolution, in order to correct inaccuracies due to the numerical approximation of the two non-conservative internal energy equations in the presence of shocks.

There is no difficulty to check that the second law of thermodynamics is fulfilled by this model. The phasic entropy equations are readily obtained,

$$
\begin{aligned}
& \alpha_{1} \rho_{1} \mathrm{~T}_{1} \frac{\mathrm{ds}_{1}}{\mathrm{dt}}=\mu\left(\mathrm{p}_{1}-\mathrm{p}_{2}\right)^{2} \frac{\mathrm{Z}_{1}}{\mathrm{Z}_{1}+\mathrm{Z}_{2}}, \\
& \alpha_{2} \rho_{2} \mathrm{~T}_{2} \frac{\mathrm{ds}_{2}}{\mathrm{dt}}=\mu\left(\mathrm{p}_{1}-\mathrm{p}_{2}\right)^{2} \frac{\mathrm{Z}_{2}}{\mathrm{Z}_{1}+\mathrm{Z}_{2}},
\end{aligned}
$$

insuring that the mixture entropy $\left(\mathrm{s}=\mathrm{Y}_{1} \mathrm{~s}_{1}+\mathrm{Y}_{2} \mathrm{~s}_{2}\right)$ always evolve with positive or null variations.

This model exhibits a nice feature with respect to the mixture sound speed. The mixture sound speed,

$$
\mathrm{c}_{\mathrm{f}}^{2}=\mathrm{Y}_{1} \mathrm{c}_{1}^{2}+\mathrm{Y}_{2} \mathrm{c}_{2}^{2} \text {, }
$$

has a monotonic behavior versus volume and mass fractions and represents the frozen mixture sound speed.

The model is thus strictly hyperbolic with waves speeds: $u+c_{f}, u-c_{f}, u$. A more detailed analysis of hyperbolicity and sound speed will be carried out in Section III with the approximate acoustic Riemann solver.

\section{About shock relations}

As with the previous 5-equation model, the new model is also non-conservative, and shock relations have to be prescribed. However, the preceding remarks about shock relations for the five equations model and numerical approximation of shocks with non-conservative systems yield the following conclusion:

Even when shock relations are known or accepted for a non-conservative system, it is very difficult to make the numerical solution converge naturally to the end shock state solution.

'Natural convergence' means without artifact such as artificial heat exchanges (Petitpas et al., 2007). There is thus no need to determine precise shock relations for the 6-equation model, in particular since it is intended only to approximate the 5-equation model for which shock relations are known.

However, some admissibility conditions have to be respected by a given Hugoniot approximate model. Jump conditions must at least respect (Saurel et al., 2007b):

- Energy conservation of the mixture, 
- Tangency of the mixture Hugoniot curve and mixture isentrope,

- Single phase limit for which jump conditions are unambiguously known,

- Symmetry,

- Entropy production.

Jump conditions for the mass equations are

$\alpha_{1} \rho_{1}(\mathrm{u}-\sigma)=\alpha_{1}^{0} \rho_{1}^{0}\left(\mathrm{u}^{0}-\sigma\right)=\mathrm{m}_{1}$

$\alpha_{2} \rho_{2}(u-\sigma)=\alpha_{2}^{0} \rho_{2}^{0}\left(u^{0}-\sigma\right)=m_{2}$.

Let us denote the mixture pressure by $p=\alpha_{1} p_{1}+\alpha_{2} p_{2}$ and the shock mass flow rate by $\mathrm{m}=\mathrm{m}_{1}+\mathrm{m}_{2}$. With these notations, the momentum jump condition can be written

$\mathrm{p}-\mathrm{p}^{0}+\mathrm{m}^{2}\left(\mathrm{v}-\mathrm{v}^{0}\right)=0$.

The mixture energy jump condition is:

$\mathrm{e}-\mathrm{e}^{0}+\frac{\mathrm{p}+\mathrm{p}^{0}}{2}\left(\mathrm{v}-\mathrm{v}^{0}\right)=0$,

with $\mathrm{e}=\mathrm{Y}_{1} \mathrm{e}_{1}+\mathrm{Y}_{2} \mathrm{e}_{2}$ and $\mathrm{v}=\mathrm{Y}_{1} \mathrm{v}_{1}+\mathrm{Y}_{2} \mathrm{v}_{2}\left(\mathrm{v}_{\mathrm{k}}=\frac{1}{\rho_{\mathrm{k}}}\right)$.

In the absence of relaxation effects the volume fraction jump is simply:

$\alpha_{1}=\alpha_{1}^{0}$.

The non-conservative internal energy equations are not adapted to the determination of jump conditions. Following the preceding admissibility conditions the following jump conditions are proposed:

$\mathrm{e}_{\mathrm{k}}-\mathrm{e}_{\mathrm{k}}^{0}+\frac{\mathrm{p}_{\mathrm{k}}+\mathrm{p}_{\mathrm{k}}^{0}}{2}\left(\mathrm{v}_{\mathrm{k}}-\mathrm{v}_{\mathrm{k}}^{0}\right)=0$.

The conditions that must be satisfied include:

- Energy conservation

The sum of the internal energy jump equations yields:

$\mathrm{Y}_{1}\left(\mathrm{e}_{1}-\mathrm{e}_{1}^{0}\right)+\frac{\mathrm{p}_{1}+\mathrm{p}_{1}^{0}}{2}\left(\mathrm{Y}_{1} \mathrm{v}_{1}-\mathrm{Y}_{1} \mathrm{v}_{1}^{0}\right)+\mathrm{Y}_{2}\left(\mathrm{e}_{2}-\mathrm{e}_{2}^{0}\right)+\frac{\mathrm{p}_{2}+\mathrm{p}_{2}^{0}}{2}\left(\mathrm{Y}_{2} \mathrm{v}_{2}-\mathrm{Y}_{2} \mathrm{v}_{2}^{0}\right)=0$

As $Y_{k}=\frac{\alpha_{k} v}{v_{k}}$, we have:

$\mathrm{e}-\mathrm{e}^{0}+\frac{\mathrm{p}_{1}+\mathrm{p}_{1}^{0}}{2}\left(\alpha_{1} \mathrm{v}-\alpha_{1}^{0} \mathrm{v}^{0}\right)+\frac{\mathrm{p}_{2}+\mathrm{p}_{2}^{0}}{2}\left(\alpha_{2} \mathrm{v}-\alpha_{2}^{0} \mathrm{v}^{0}\right)=0$

With the volume fraction jump relation, this equation becomes

$\mathrm{e}-\mathrm{e}^{0}+\frac{\alpha_{1} \mathrm{p}_{1}+\alpha_{2} \mathrm{p}_{2}+\alpha_{1} \mathrm{p}_{1}^{0}+\alpha_{2} \mathrm{p}_{2}^{0}}{2}\left(\mathrm{v}-\mathrm{v}^{0}\right)=0$,

or simply

$\mathrm{e}-\mathrm{e}^{0}+\frac{\mathrm{p}+\mathrm{p}^{0}}{2}\left(\mathrm{v}-\mathrm{v}^{0}\right)=0$.

This result guarantees that the phasic energy jump conditions are compatible with the mixture energy conservation. 
- Tangency of the mixture Hugoniot curve and isentrope

This is a mandatory property for the Riemann problem solution. As the volume fraction is constant across shocks and rarefaction waves (in absence of relaxation effects) and the phasic Hugoniots are tangent to phasic isentropes, the mixture Hugoniot is necessarily tangent to the mixture isentrope.

- Single phase limit

When one of the phases disappears the energy jump condition of the remaining fluid is in agreement with the single phase energy jump.

- Symmetry

Symmetry in the formulation allows an easy extension to an arbitrary number of fluids.

- Entropy production

As each phase evolves along its own Hugoniot (II.8) there is no doubt that the mixture entropy evolves positively.

Through application of these relations, the Riemann problem can now be solved. Numerical issues pertaining to the Riemann solution are addressed in the next section. Let us insist on the fact that jump conditions are not the key to shock computation in multiphase mixtures. It has been shown that even when shock relations are known, the convergence of a numerical scheme to the exact solution is very difficult. This is due to the lack of definition for cell averages of non-conservative variables (Petitpas et al., 2007).

\section{Asymptotic limit}

As the method will solve the 6-equation model with stiff relaxation terms, it is important to check that in the limit of infinitely fast pressure relaxation the 5-equation model is recovered. This proof is given in Appendix A.

\section{Numerical method}

Numerical resolution of the 6-equation model in the limit of stiff pressure relaxation is addressed in the present section. In regular zones, this model is self consistent. But in the presence of shocks the internal energy equations are inappropriate. To correct the thermodynamic state predicted by these equations in the presence of shocks, the total mixture energy equation will be used. This correction will be valid on both sides of an interface, when the flow tends to the single phase limits. The details of this correction will be examined further. For now, the 6-equation system is augmented by a redundant equation regarding the total mixture energy. The system to consider during numerical resolution thus involves 7 equations:

$$
\begin{aligned}
& \frac{\partial \alpha_{1}}{\partial \mathrm{t}}+\mathrm{u} \frac{\partial \alpha_{1}}{\partial \mathrm{x}}=\mu\left(\mathrm{p}_{1}-\mathrm{p}_{2}\right) \\
& \frac{\partial \alpha_{1} \rho_{1} \mathrm{e}_{1}}{\partial \mathrm{t}}+\frac{\partial \alpha_{1} \rho_{1} \mathrm{e}_{1} \mathrm{u}}{\partial \mathrm{x}}+\alpha_{1} \mathrm{p}_{1} \frac{\partial \mathrm{u}}{\partial \mathrm{x}}=-\mathrm{p}_{\mathrm{I}} \mu\left(\mathrm{p}_{1}-\mathrm{p}_{2}\right) \\
& \frac{\partial \alpha_{2} \rho_{2} \mathrm{e}_{2}}{\partial \mathrm{t}}+\frac{\partial \alpha_{2} \rho_{2} \mathrm{e}_{2} \mathrm{u}}{\partial \mathrm{x}}+\alpha_{2} \mathrm{p}_{2} \frac{\partial \mathrm{u}}{\partial \mathrm{x}}=\mathrm{p}_{\mathrm{I}} \mu\left(\mathrm{p}_{1}-\mathrm{p}_{2}\right)
\end{aligned}
$$




$$
\begin{aligned}
& \frac{\partial \alpha_{1} \rho_{1}}{\partial \mathrm{t}}+\frac{\partial \alpha_{1} \rho_{1} \mathrm{u}}{\partial \mathrm{x}}=0 \\
& \frac{\partial \alpha_{2} \rho_{2}}{\partial \mathrm{t}}+\frac{\partial \alpha_{2} \rho_{2} \mathrm{u}}{\partial \mathrm{x}}=0 \\
& \frac{\partial \rho \mathrm{u}}{\partial \mathrm{t}}+\frac{\partial \rho \mathrm{u}^{2}+\left(\alpha_{1} \mathrm{p}_{1}+\alpha_{2} \mathrm{p}_{2}\right)}{\partial \mathrm{x}}=0 \\
& \frac{\partial \rho\left(\mathrm{Y}_{1} \mathrm{e}_{1}+\mathrm{Y}_{2} \mathrm{e}_{2}+\frac{1}{2} \mathrm{u}^{2}\right)}{\partial \mathrm{t}}+\frac{\partial \mathrm{u}\left(\rho\left(\mathrm{Y}_{1} \mathrm{e}_{1}+\mathrm{Y}_{2} \mathrm{e}_{2}+\frac{1}{2} \mathrm{u}^{2}\right)+\left(\alpha_{1} \mathrm{p}_{1}+\alpha_{2} \mathrm{p}_{2}\right)\right)}{\partial \mathrm{x}}=0
\end{aligned}
$$

with $\mathrm{p}_{\mathrm{I}}=\frac{\mathrm{Z}_{2} \mathrm{p}_{1}+\mathrm{Z}_{1} \mathrm{p}_{2}}{\mathrm{Z}_{1}+\mathrm{Z}_{2}}$ and appropriate equations of state $\mathrm{e}_{\mathrm{k}}=\mathrm{e}_{\mathrm{k}}\left(\rho_{\mathrm{k}}, \mathrm{p}_{\mathrm{k}}\right)$.

This system is equipped with the approximate shock relations of the preceding section, in particular relation (II.8).

\section{III.1 Approximate Riemann solvers}

Two types of approximate Riemann solvers will be considered:

- acoustic linearized Riemann solver,

- HLLC Riemann solver.

These two solvers are detailed in the context of the Euler equations in Toro (1997).

\section{Acoustic solver}

This approximate solver assumes that shocks are absent or sufficiently weak. The last equation of system (III.1) can thus be suppressed. Indeed, this last equation is only used to correct some deficiencies of the numerical resolution of phase's internal energy equations in the presence of shocks. The 6-equation system free of relaxation terms can thus be written with the following variables:

$\frac{\partial \mathrm{W}}{\partial \mathrm{t}}+\mathrm{A}(\mathrm{W}) \frac{\partial \mathrm{W}}{\partial \mathrm{x}}=0$,

with $\mathrm{W}=\left(\alpha_{1}, \mathrm{~s}_{1}, \mathrm{~s}_{2}, \mathrm{u}, \mathrm{p}_{1}, \mathrm{p}_{2}\right)^{\mathrm{T}}$ and,

$$
\mathrm{A}(\mathrm{W})=\left(\begin{array}{cccccc}
\mathrm{u} & 0 & 0 & 0 & 0 & 0 \\
0 & \mathrm{u} & 0 & 0 & 0 & 0 \\
0 & 0 & \mathrm{u} & 0 & 0 & 0 \\
\frac{\mathrm{p}_{1}-\mathrm{p}_{2}}{\rho} & 0 & 0 & \mathrm{u} & \frac{\alpha_{1}}{\rho} & \frac{\alpha_{2}}{\rho} \\
0 & 0 & 0 & \rho_{1} \mathrm{c}_{1}^{2} & \mathrm{u} & 0 \\
0 & 0 & 0 & \rho_{2} \mathrm{c}_{2}^{2} & 0 & \mathrm{u}
\end{array}\right)
$$

Eigenvalues of the propagation matrix are:

$\lambda_{0}=\mathrm{u}, 4$ times fold, $\lambda_{1}=\mathrm{u}-\mathrm{c}, \lambda_{2}=\mathrm{u}+\mathrm{c}$, with,

$\mathrm{c}^{2}=\mathrm{Y}_{1} \mathrm{c}_{1}^{2}+\mathrm{Y}_{2} \mathrm{c}_{2}^{2}$

The monotonic frozen sound speed introduced in Section II is now established. 
The acoustic solver is based on characteristic equations that are readily obtained:

- Along trajectories defined by $\frac{\mathrm{dx}}{\mathrm{dt}}=\mathrm{u}$

$$
\frac{\mathrm{d}_{1}}{\mathrm{dt}}=0, \frac{\mathrm{ds}_{1}}{\mathrm{dt}}=0, \frac{\mathrm{ds}_{2}}{\mathrm{dt}}=0
$$

- Along trajectories defined by $\frac{\mathrm{dx}}{\mathrm{dt}}=\mathrm{u}-\mathrm{c}$

$$
\left.\left.\left.\left.-\frac{\mathrm{p}_{1}-\mathrm{p}_{2}}{\rho \mathrm{c}} \frac{\mathrm{d} \alpha_{1}}{\mathrm{dt}}\right)_{\mathrm{u}-\mathrm{c}}+\frac{\mathrm{du}}{\mathrm{dt}}\right)_{\mathrm{u}-\mathrm{c}}-\frac{\alpha_{1}}{\rho \mathrm{c}} \frac{\mathrm{dp}_{1}}{\mathrm{dt}}\right)_{\mathrm{u}-\mathrm{c}}-\frac{\alpha_{2}}{\rho \mathrm{c}} \frac{\mathrm{dp}_{2}}{\mathrm{dt}}\right)_{\mathrm{u}-\mathrm{c}}=0
$$

- Along trajectories defined by $\frac{\mathrm{dx}}{\mathrm{dt}}=\mathrm{u}+\mathrm{c}$

$$
\left.\left.\left.\left.\frac{p_{1}-p_{2}}{\rho c} \frac{d \alpha_{1}}{d t}\right)_{u+c}+\frac{d u}{d t}\right)_{u+c}+\frac{\alpha_{1}}{\rho c} \frac{d p_{1}}{d t}\right)_{u+c}+\frac{\alpha_{2}}{\rho c} \frac{d p_{2}}{d t}\right)_{u+c}=0
$$

These relations are used to solve the linearized Riemann problem. By assuming weak variations across left- and right-facing waves, the acoustic impedance $Z=\rho c$ (with $\mathrm{c}$ defined by III.2 and $\rho$ the mixture density) are assumed constant. The corresponding jump relations are:

- Across a right-facing wave,

$$
\begin{gathered}
\alpha_{1 \mathrm{R}}^{*}=\alpha_{1 \mathrm{R}}, \mathrm{s}_{1 \mathrm{R}}^{*}=\mathrm{s}_{1 \mathrm{R}}, \mathrm{s}_{2 \mathrm{R}}^{*}=\mathrm{s}_{2 \mathrm{R}}, \\
\left(\alpha_{1} \mathrm{p}_{1}+\alpha_{2} \mathrm{p}_{2}\right)_{\mathrm{R}}^{*}-\mathrm{Z}_{\mathrm{R}} \mathrm{u}_{\mathrm{R}}^{*}=\left(\alpha_{1} \mathrm{p}_{1}+\alpha_{2} \mathrm{p}_{2}\right)_{\mathrm{R}}-\mathrm{Z}_{\mathrm{R}} \mathrm{u}_{\mathrm{R}} \text { with } \mathrm{Z}_{\mathrm{R}}=\rho_{\mathrm{R}} \mathrm{c}_{\mathrm{R}} .
\end{gathered}
$$

- Across a left-facing wave,

$$
\begin{gathered}
\alpha_{1 \mathrm{~L}}^{*}=\alpha_{1 \mathrm{~L}}, \mathrm{~s}_{1 \mathrm{~L}}^{*}=\mathrm{s}_{1 \mathrm{~L}}, \mathrm{~s}_{2 \mathrm{~L}}^{*}=\mathrm{s}_{2 \mathrm{~L}}, \\
\left(\alpha_{1} \mathrm{p}_{1}+\alpha_{2} \mathrm{p}_{2}\right)_{\mathrm{L}}^{*}+\mathrm{Z}_{\mathrm{L}} \mathrm{u}_{\mathrm{L}}^{*}=\left(\alpha_{1} \mathrm{p}_{1}+\alpha_{2} \mathrm{p}_{2}\right)_{\mathrm{L}}+\mathrm{Z}_{\mathrm{L}} \mathrm{u}_{\mathrm{L}} \text { with } \mathrm{Z}_{\mathrm{L}}=\rho_{\mathrm{L}} \mathrm{c}_{\mathrm{L}} .
\end{gathered}
$$

The upperscript ${ }^{*} *$ ' stands for the perturbated state.

The velocity and pressure solution of the Riemann problem are thus easily obtained with the help of the interface conditions:

$$
\begin{aligned}
& \left(\alpha_{1} p_{1}+\alpha_{2} p_{2}\right)_{L}^{*}=\left(\alpha_{1} p_{1}+\alpha_{2} p_{2}\right)_{R}^{*}=\left(\alpha_{1} p_{1}+\alpha_{2} p_{2}\right)^{*}=p^{*} \\
& u_{L}^{*}=u_{R}^{*}=u^{*}
\end{aligned}
$$

Thus, the velocity and pressure solution of the Riemann problem read:

$$
\begin{aligned}
& u^{*}=\frac{Z_{L} u_{L}+Z_{R} u_{R}+p_{L}-p_{R}}{Z_{L}+Z_{R}} \\
& p^{*}=\frac{Z_{L} p_{R}+Z_{R} p_{L}+Z_{R} Z_{L}\left(u_{L}-u_{R}\right)}{Z_{L}+Z_{R}}
\end{aligned}
$$

with:

$p=\alpha_{1} p_{1}+\alpha_{2} p_{2}, Z=\rho c, \rho=\alpha_{1} \rho_{1}+\alpha_{2} \rho_{2}, c^{2}=Y_{1} c_{1}^{2}+Y_{2} c_{2}^{2}$.

Relations (III.3) are the same for the 6-equation model and for the Euler equations. The differences appear through the definitions of the mixture pressure, mixture sound speed and mixture density.

Once the pressure is determined in the star region the phase's densities are determined with the help of the entropy jumps. 
This solver is simple and efficient for subsonic flows or flows in absence of strong shocks. Characteristic relations are also useful for boundary conditions treatment. But we prefer a solver able to deal with arbitrary shocks, genuinely positive (and consequently robust), able to deal with arbitrary convex EOS. The HLLC solver of Toro et al. (1994) fulfils these requirements.

\section{HLLC type solver}

Consider a cell boundary separating a left state (L) and a right state (R). The left- and rightfacing waves speeds are readily obtained, following Davis (1988) estimates:

$\mathrm{S}_{\mathrm{R}}=\max \left(\mathrm{u}_{\mathrm{L}}+\mathrm{c}_{\mathrm{L}}, \mathrm{u}_{\mathrm{R}}+\mathrm{c}_{\mathrm{R}}\right), \mathrm{S}_{\mathrm{L}}=\min \left(\mathrm{u}_{\mathrm{L}}-\mathrm{c}_{\mathrm{L}}, \mathrm{u}_{\mathrm{R}}-\mathrm{c}_{\mathrm{R}}\right)$,

where the sound speed still obeys to Relation (III.2).

The speed of the intermediate wave (or contact discontinuity) is estimated using the HLL approximation

$S_{M}=\frac{\left(\rho u^{2}+p\right)_{L}-\left(\rho u^{2}+p\right)_{R}-S_{L}(\rho u)_{L}+S_{R}(\rho u)_{R}}{(\rho u)_{L}-(\rho u)_{R}-S_{L} \rho_{L}+S_{R} \rho_{R}}$,

with the mixture density and mixture pressure defined previously.

From these wave speeds, the following variable states are determined

$$
\begin{aligned}
& \left(\alpha_{k} \rho_{k}\right)_{R}^{*}=\left(\alpha_{k} \rho_{k}\right)_{R} \frac{S_{R}-u_{R}}{S_{R}-S_{M}}, \\
& \left(\alpha_{k} \rho_{k}\right)_{L}^{*}=\left(\alpha_{k} \rho_{k}\right)_{L} \frac{S_{L}-u_{L}}{S_{L}-S_{M}}, \\
& p^{*}=p_{R}+\rho_{R} u_{R}\left(u_{R}-S_{R}\right)-\rho_{R}^{*} S_{M}\left(S_{M}-S_{R}\right), \text { with } \rho_{R}^{*}=\sum_{k}\left(\alpha_{k} \rho_{k}\right)_{R}^{*}, \\
& E_{R}^{*}=\frac{\rho_{R} E_{R}\left(u_{R}-S_{R}\right)+p_{R} u_{R}-p^{*} S_{M}}{\rho_{R}^{*}\left(S_{M}-S_{R}\right)}, \\
& E_{L}^{*}=\frac{\rho_{L} E_{L}\left(u_{L}-S_{L}\right)+p_{L} u_{L}-p^{*} S_{M}}{\rho_{L}^{*}\left(S_{M}-S_{L}\right)}, \text { with } E=Y_{1} e_{1}+Y_{2} e_{2}+\frac{1}{2} u^{2} .
\end{aligned}
$$

The volume fraction jump is readily obtained as, in the absence of relaxation effects, the volume fraction is constant along fluid trajectories

$$
\alpha_{\mathrm{kR}}^{*}=\alpha_{\mathrm{kR}}, \quad \alpha_{\mathrm{kL}}^{*}=\alpha_{\mathrm{kL}} \text {. }
$$

As the volume fraction is constant across left- and right-facing waves, the fluid density is determined from the preceding relations to be

$$
\rho_{\mathrm{kR}}^{*}=\rho_{\mathrm{kR}}^{0} \frac{\mathrm{u}_{\mathrm{R}}-\mathrm{S}_{\mathrm{R}}}{\mathrm{S}_{\mathrm{M}}-\mathrm{S}_{\mathrm{R}}}
$$

Internal energy jumps are determined with the help of the Hugoniot relation (II.8). Let us consider the example of fluids governed by the stiffened gas EOS (II.3). With the help of the EOS, the phasic pressures are constrained along their Hugoniot curves to be functions only of the corresponding phase density: 
$\mathrm{p}_{\mathrm{k}}^{*}\left(\rho_{\mathrm{k}}^{*}\right)=\left(\mathrm{p}_{\mathrm{k}}^{0}+\mathrm{p}_{\infty \mathrm{k}}\right) \frac{\left(\gamma_{\mathrm{k}}-1\right) \rho_{\mathrm{k}}^{0}-\left(\gamma_{\mathrm{k}}+1\right) \rho_{\mathrm{k}}^{*}}{\left(\gamma_{\mathrm{k}}-1\right) \rho_{\mathrm{k}}^{*}-\left(\gamma_{\mathrm{k}}+1\right) \rho_{\mathrm{k}}^{0}}-\mathrm{p}_{\propto \mathrm{k}}$

The phase's internal energies are then determined from the EOS : $\mathrm{e}_{\mathrm{kR}}^{*}=\mathrm{e}_{\mathrm{kR}}^{*}\left(\mathrm{p}_{\mathrm{k}}^{*}, \rho_{\mathrm{k}}^{*}\right)$.

Equipped with these approximate Riemann solvers, the next step is to develop a Godunov type scheme.

\section{III.2 Godunov type method}

For the sake of simplicity, the method is presented at first-order. The extension to second-order is detailed in Appendix B.

\section{First-order method}

In the absence of relaxation terms, the conservative part of System (III.1) is updated with the conventional Godunov scheme:

$$
\mathrm{U}_{\mathrm{i}}^{\mathrm{n}+1}=\mathrm{U}_{\mathrm{i}}^{\mathrm{n}}-\frac{\Delta \mathrm{t}}{\Delta \mathrm{x}}\left(\mathrm{F}^{*}\left(\mathrm{U}_{\mathrm{i}}^{\mathrm{n}}, \mathrm{U}_{\mathrm{i}+1}^{\mathrm{n}}\right)-\mathrm{F}^{*}\left(\mathrm{U}_{\mathrm{i}-1}^{\mathrm{n}}, \mathrm{U}_{\mathrm{i}}^{\mathrm{n}}\right)\right)
$$

where $\mathrm{U}=\left((\alpha \rho)_{1}, \quad(\alpha \rho)_{2}, \quad \rho \mathrm{u}, \quad \rho \mathrm{E}\right)^{\mathrm{T}}$ and $\mathrm{F}=\left((\alpha \rho)_{1} \mathrm{u}, \quad(\alpha \rho)_{1} \mathrm{u}, \quad \rho \mathrm{u}^{2}+\mathrm{p}, \quad(\rho \mathrm{E}+\mathrm{p}) \mathrm{u}\right)^{\mathrm{T}}$,

$$
\mathrm{E}=\mathrm{Y}_{1} \mathrm{e}_{1}+\mathrm{Y}_{2} \mathrm{e}_{2}+\frac{1}{2} \mathrm{u}^{2} \text { and } \mathrm{p}=\alpha_{1} \mathrm{p}_{1}+\alpha_{2} \mathrm{p}_{2} \text {. }
$$

The volume fraction equation is also updated using the Godunov method for advection equations:

$$
\alpha_{1 i}^{n+1}=\alpha_{1 i}^{n}-\frac{\Delta t}{\Delta x}\left(\left(u \alpha_{1}\right)_{i+1 / 2}^{*}-\left(u \alpha_{1}\right)_{i-1 / 2}^{*}-\alpha_{1 i}^{n}\left(u_{i+1 / 2}^{*}-u_{i-1 / 2}^{*}\right)\right)
$$

This scheme guarantees volume fraction positivity during the hyperbolic step. Other options are possible, like for example, VOF type methods (Miller and Puckett, 1996). Using a reconstruction algorithm may have nice features when dealing with interfaces only, these interfaces having to be present at the initial time. As we also deal with dynamic appearance of interfaces, a capturing method is preferred. This is not the only difference between the Miller and Puckett (1996) method and the present one. The mixture pressure and sound speed used in the present formulation are very different from the single phase estimates used by these authors.

Regarding the non-conservative energy equations, there is no hope to determine accurate approximation in the presence of shocks (Hou and Le Floch, 1990). Therefore, we use the simplest approximation of the corresponding equations by assuming the product $(\alpha p)_{k i}{ }^{n}$ constant during the time step:

$(\alpha \rho \mathrm{e})_{\mathrm{k}_{\mathrm{i}}}^{\mathrm{n}+1}=(\alpha \rho \mathrm{e})_{\mathrm{k}_{\mathrm{i}}}^{\mathrm{n}}-\frac{\Delta \mathrm{t}}{\Delta \mathrm{x}}\left((\alpha \rho \mathrm{eu})_{\mathrm{k}_{\mathrm{i}+1 / 2}}^{*}-(\alpha \rho \mathrm{eu})_{\mathrm{k}_{\mathrm{i}-1 / 2}}^{*}+(\alpha \mathrm{p})_{\mathrm{ki}}{ }^{\mathrm{n}}\left(\mathrm{u}_{\mathrm{i}+1 / 2}^{*}-\mathrm{u}_{\mathrm{i}-1 / 2}^{*}\right)\right)$.

The lack of accuracy in the internal energy computation resulting from the present scheme is not so crucial. The internal energies will be used only to estimate the phase's pressure at the end of the hyperbolic step, before the relaxation one. The relaxation step will give a first correction to 
the internal energies, in agreement with the second law of thermodynamics. A second correction will be made with the help of the total mixture energy. The details of these two steps are described in the next two subsections. Before giving these details, let us examine a basic situation of fundamental importance when dealing with interface problems; namely uniform flow conditions.

\section{Uniform flow test}

The main difficulty in solving interface problems as diffused numerical zones lies in the building of a flow model and a numerical scheme that preserve interface conditions. This test problem was examined by Abgrall (1996) in the context of the Euler equations. Let us consider a onedimensional flow in mechanical equilibrium. A volume fraction discontinuity propagates at constant velocity $u$ in a constant pressure flow field $p_{1}=p_{2}=p$. This flow system is initially in mechanical equilibrium and therefore must remain in mechanical equilibrium during its time evolution.

Let us examine the behavior of the present Godunov method for the conservative part of this model in the particular case of uniform pressure and velocity fields. The Godunov method for the mass equations is:

$$
(\alpha \rho)_{\mathrm{ki}}^{\mathrm{n}+1}=(\alpha \rho)_{\mathrm{ki}}^{\mathrm{n}}-\frac{\Delta \mathrm{t}}{\Delta \mathrm{x}}\left((\alpha \rho u)_{\mathrm{k} i+1 / 2}^{*}-(\alpha \rho \mathrm{u})_{\mathrm{ki}-1 / 2}^{*}\right), \quad \mathrm{k}=1,2 .
$$

Because the velocity is uniform we have:

$$
(\alpha \rho)_{\mathrm{ki}_{\mathrm{i}}}^{\mathrm{n}+1}=(\alpha \rho)_{\mathrm{k}_{\mathrm{i}}}^{\mathrm{n}}-\frac{\Delta \mathrm{t}}{\Delta \mathrm{x}} \mathrm{u}\left((\alpha \rho)_{\mathrm{k}_{\mathrm{i}+1 / 2}^{*}}^{*}-(\alpha \rho)_{\mathrm{ki}-1 / 2}^{*}\right) \text {. }
$$

The mixture density thus obeys to the discrete formula:

$$
\rho_{\mathrm{i}}^{\mathrm{n}+1}=\rho_{\mathrm{i}}^{\mathrm{n}}-\frac{\Delta \mathrm{t}}{\Delta \mathrm{x}} \mathrm{u}\left(\rho_{\mathrm{i}+1 / 2}^{*}-\rho_{\mathrm{i}-1 / 2}^{*}\right)
$$

The discrete momentum equation under the same uniform flow conditions becomes:

$$
(\rho u)_{i}^{n+1}=(\rho u)_{i}^{n}-\frac{\Delta t}{\Delta x} u^{2}\left(\rho_{i+1 / 2}^{*}-\rho_{i-1 / 2}^{*}\right),
$$

that is

$$
(\rho u)_{i}^{n+1}=u(\rho)_{i}^{n+1}
$$

Thus the flow will necessarily retain its uniform velocity at the next time step: $u_{i}^{n+1}=u$.

The adopted numerical scheme for the internal energies becomes in the present situation $(\alpha \rho \mathrm{e})_{\mathrm{ki}}^{\mathrm{n}+1}=(\alpha \rho \mathrm{e})_{\mathrm{ki}}^{\mathrm{n}}-\frac{\Delta \mathrm{t}}{\Delta \mathrm{x}} \mathrm{u}\left((\alpha \rho \mathrm{e})_{\mathrm{ki}+1 / 2}^{*}-(\alpha \rho)_{\mathrm{ki}-1 / 2}^{*}\right)$

Consider, for example, the stiffened gas (SG) EOS (II.3): $\rho_{\mathrm{k}} \mathrm{e}_{\mathrm{k}}=\frac{\mathrm{p}_{\mathrm{k}}+\gamma_{\mathrm{k}} \mathrm{p}_{\infty \mathrm{k}}}{\gamma_{\mathrm{k}}-1}$.

The discrete approximation of the internal energy now becomes

$$
\left(\alpha \frac{\mathrm{p}+\gamma \mathrm{p}_{\infty}}{\gamma-1}\right)_{\mathrm{ki}}^{\mathrm{n}+1}=\left(\alpha \frac{\mathrm{p}+\gamma \mathrm{p}_{\infty}}{\gamma-1}\right)_{\mathrm{ki}_{\mathrm{i}}}^{\mathrm{n}}-\frac{\Delta \mathrm{t}}{\Delta \mathrm{x}} \mathrm{u}\left(\left(\alpha \frac{\mathrm{p}+\gamma \mathrm{p}_{\infty}}{\gamma-1}\right)_{\mathrm{k} \mathrm{i}+1 / 2}^{*}-\left(\alpha \frac{\mathrm{p}+\gamma \mathrm{p}_{\infty}}{\gamma-1}\right)_{\mathrm{ki}-1 / 2}^{*}\right)
$$

As the EOS parameters are constant in each fluid, this expression simplifies to:

$$
\left(\alpha\left(p+\gamma p_{\infty}\right)\right)_{k i}^{n+1}=\left(\alpha\left(p+\gamma p_{\infty}\right)\right)_{k i}^{n}-\frac{\Delta t}{\Delta x} \mathrm{u}\left(\left(\alpha\left(p+\gamma p_{\infty}\right)\right)_{k_{i}+1 / 2}^{*}-\left(\alpha\left(p+\gamma p_{\infty}\right)\right)_{k_{i}-1 / 2}^{*}\right)
$$


which can be rewritten as

$$
(\alpha)_{\mathrm{k}_{\mathrm{i}}}^{\mathrm{n}+1}+\left(\gamma \mathrm{p}_{\infty}\right)_{\mathrm{k}}(\alpha)_{\mathrm{i}}^{\mathrm{n}+1}=\mathrm{p}\left\{(\alpha)_{\mathrm{ki}}^{\mathrm{n}}-\frac{\Delta \mathrm{t}}{\Delta \mathrm{x}} \mathrm{u}\left((\alpha)_{\mathrm{k} i+1 / 2}^{*}-(\alpha)_{\mathrm{k}^{-}-1 / 2}^{*}\right)\right\}+\left(\gamma \mathrm{p}_{\infty}\right)_{\mathrm{k}}\left\{(\alpha)_{\mathrm{ki}}^{\mathrm{n}}-\frac{\Delta \mathrm{t}}{\Delta \mathrm{x}} \mathrm{u}\left((\alpha)_{\mathrm{k} i+1 / 2}^{*}-(\alpha)_{\mathrm{k} i-1 / 2}^{*}\right)\right\}
$$

The adopted numerical scheme for the volume fraction evolution, in uniform velocity flow conditions becomes:

$\alpha_{\mathrm{ki}}^{\mathrm{n}+1}=\alpha_{\mathrm{ki}}^{\mathrm{n}}-\frac{\Delta \mathrm{t}}{\Delta \mathrm{x}} \mathrm{u}\left(\left(\alpha_{\mathrm{k}}\right)_{\mathrm{i}+1 / 2}^{*}-\left(\alpha_{\mathrm{k}}\right)_{\mathrm{i}-1 / 2}^{*}\right)$.

Using this, the internal energy equation reduces to:

$\mathrm{p}_{\mathrm{ki}}^{\mathrm{n}+1}=\mathrm{p}$

The adopted numerical approximation thus preserves interface conditions in mechanical equilibrium flows.

When the EOS are more sophisticated than the SG one, i.e. Mie Gruneisen EOS for example that can be written under the form,

$$
\rho_{\mathrm{k}} \mathrm{e}_{\mathrm{k}}=\frac{\mathrm{p}_{\mathrm{k}}+\gamma_{\mathrm{k}} \mathrm{p}_{\infty \mathrm{k}}\left(\rho_{\mathrm{k}}\right)}{\gamma_{\mathrm{k}}-1},
$$

the same properties of interface preserving are observed experimentally. The reason is that Godunov type methods used for mass and volume fraction equations result in prolonged density field through the interface. Locally, these more sophisticated EOS thus reduce to the SG one.

\section{III.3 Relaxation step}

This step is of major importance to fulfill interface conditions in non-uniform velocity and pressure flows. It also forces the solution of the 6-equation model to converge to that of the 5equation model.

In the relaxation step we must solve

$$
\begin{aligned}
& \frac{\partial \alpha_{1}}{\partial \mathrm{t}}=\mu\left(\mathrm{p}_{1}-\mathrm{p}_{2}\right) \\
& \frac{\partial \alpha_{1} \rho_{1} \mathrm{e}_{1}}{\partial \mathrm{t}}=-\mathrm{p}_{\mathrm{I}} \mu\left(\mathrm{p}_{1}-\mathrm{p}_{2}\right) \\
& \frac{\partial \alpha_{2} \rho_{2} \mathrm{e}_{2}}{\partial \mathrm{t}}=\mathrm{p}_{1} \mu\left(\mathrm{p}_{1}-\mathrm{p}_{2}\right) \\
& \frac{\partial \alpha_{1} \rho_{1}}{\partial \mathrm{t}}=0 \\
& \frac{\partial \alpha_{2} \rho_{2}}{\partial \mathrm{t}}=0 \\
& \frac{\partial \rho \mathrm{u}}{\partial \mathrm{t}}=0 \\
& \frac{\partial \rho \mathrm{E}}{\partial \mathrm{t}}=0 \\
& \text { with } \mathrm{p}_{\mathrm{I}}=\frac{Z_{2} \mathrm{p}_{1}+Z_{1} \mathrm{p}_{2}}{Z_{1}+Z_{2}} \text { and in the limit } \mu \rightarrow+\infty .
\end{aligned}
$$


After some manipulations the internal energy equations become:

$$
\begin{aligned}
& \frac{\partial \mathrm{e}_{1}}{\partial \mathrm{t}}+\mathrm{p}_{\mathrm{I}} \frac{\partial \mathrm{v}_{1}}{\partial \mathrm{t}}=0, \\
& \frac{\partial \mathrm{e}_{2}}{\partial \mathrm{t}}+\mathrm{p}_{\mathrm{I}} \frac{\partial \mathrm{v}_{2}}{\partial \mathrm{t}}=0 .
\end{aligned}
$$

This system can be written as an integral formulation

$\mathrm{e}_{\mathrm{k}}-\mathrm{e}_{\mathrm{k}}^{0}+\hat{\mathrm{p}}_{\mathrm{Ik}}\left(\mathrm{v}_{\mathrm{k}}-\mathrm{v}_{\mathrm{k}}^{0}\right)=0$

where $\hat{\mathrm{p}}_{\mathrm{Ik}}=\frac{1}{\mathrm{v}_{\mathrm{k}}-\mathrm{v}_{\mathrm{k}}^{0}} \int_{0}^{\Delta \mathrm{t}} \mathrm{p}_{\mathrm{I}} \frac{\partial \mathrm{v}_{\mathrm{k}}}{\partial \mathrm{t}} \mathrm{dt}$.

Determination of pressure averages $\hat{\mathrm{p}}_{\mathrm{Ik}}$ has to be done in agreement with thermodynamic considerations. By summing the internal energy equations we have:

$\mathrm{Y}_{1} \mathrm{e}_{1}-\mathrm{Y}_{1} \mathrm{e}_{1}^{0}+\mathrm{Y}_{2} \mathrm{e}_{2}-\mathrm{Y}_{2} \mathrm{e}_{2}^{0}+\hat{\mathrm{p}}_{11}\left(\mathrm{Y}_{1} \mathrm{v}_{1}-\mathrm{Y}_{1} \mathrm{v}_{1}^{0}\right)+\hat{\mathrm{p}}_{\mathrm{II}}\left(\mathrm{Y}_{2} \mathrm{v}_{2}-\mathrm{Y}_{2} \mathrm{v}_{2}^{0}\right)=0$.

The mixture mass equation can be written as:

$\left(\mathrm{Y}_{1} \mathrm{v}_{1}-\mathrm{Y}_{1} \mathrm{v}_{1}^{0}\right)+\left(\mathrm{Y}_{2} \mathrm{v}_{2}-\mathrm{Y}_{2} \mathrm{v}_{2}^{0}\right)=0$.

Using these relations the mixture energy equation becomes

$\mathrm{e}-\mathrm{e}^{0}+\left(\hat{\mathrm{p}}_{\mathrm{I} 1}-\hat{\mathrm{p}}_{\mathrm{I} 2}\right)\left(\mathrm{Y}_{1} \mathrm{v}_{1}-\mathrm{Y}_{1} \mathrm{v}_{1}^{0}\right)=0$.

In order that the mixture energy conservation be fulfilled it is necessary that: $\hat{\mathrm{p}}_{\mathrm{I} 1}=\hat{\mathrm{p}}_{\mathrm{I} 2}=\hat{\mathrm{p}}_{\mathrm{I}}$. Possible estimates are $\hat{\mathrm{p}}_{\mathrm{I}}=\mathrm{p}_{\mathrm{I}}^{0}$ or $\hat{\mathrm{p}}_{\mathrm{I}}=\mathrm{p}^{*}$, the initial and relaxed pressures respectively. These estimates are compatible with the entropy inequality (Saurel et al., 2007a). With regard to the choice of one or the other estimate, upon computation of the relaxed state the resulting difference in practical computations is negligible. This negligible influence will be illustrated in the results section. The system to solve is thus composed of equations

$\mathrm{e}_{\mathrm{k}}\left(\mathrm{p}^{*}, \mathrm{v}_{\mathrm{k}}^{*}\right)-\mathrm{e}_{\mathrm{k}}^{0}\left(\mathrm{p}_{\mathrm{k}}^{0}, \mathrm{v}_{\mathrm{k}}^{0}\right)+\hat{\mathrm{p}}_{\mathrm{I}}\left(\mathrm{v}_{\mathrm{k}}^{*}-\mathrm{v}_{\mathrm{k}}^{0}\right)=0, \mathrm{k}=1,2$

which involves 3 unknowns, $\mathrm{v}_{\mathrm{k}}^{*}(\mathrm{k}=1,2)$ and $\mathrm{p}^{*}$. Its closure is achieved using the saturation constraint

$$
\begin{aligned}
& \sum_{k} \alpha_{k}=1, \\
& \text { or } \\
& \sum_{k}(\alpha \rho)_{k} v_{k}=1 .
\end{aligned}
$$

Here the $(\alpha \rho)_{\mathrm{k}}$ are constant during the relaxation process. This system can be replaced by a single equation with a single unknown $\left(\mathrm{p}^{*}\right)$. With the help of the EOS (II.3) the energy equations become

$$
\mathrm{v}_{\mathrm{k}}^{*}\left(\mathrm{p}^{*}\right)=\mathrm{v}_{\mathrm{k}}^{0} \frac{\mathrm{p}^{0}+\gamma_{\mathrm{k}} \mathrm{p}_{\infty \mathrm{k}}+\left(\gamma_{\mathrm{k}}-1\right) \hat{\mathrm{p}}_{\mathrm{I}}}{\mathrm{p}^{*}+\gamma_{\mathrm{k}} \mathrm{p}_{\infty \mathrm{k}}+\left(\gamma_{\mathrm{k}}-1\right) \hat{\mathrm{p}}_{\mathrm{I}}},
$$

and thus the only equation to solve (for $\mathrm{p}^{*}$ ) is

$\sum_{\mathrm{k}}(\alpha \rho)_{\mathrm{k}} \mathrm{v}_{\mathrm{k}}\left(\mathrm{p}^{*}\right)=1$

Once the relaxed pressure is found, the phase's specific volumes and volume fractions are determined. 
In the Miller and Puckett (1996) method, the relaxed pressure is used to advance the solution to the next time step. However, there is no guarantee that the mixture EOS or the mixture energy be in agreement with this relaxed pressure. In order to respect total energy and correct shock dynamics on both sides of the interface, the following correction is employed.

\section{III.4 Reinitialization step}

As the volume fractions have been estimated previously by the relaxation method, the mixture pressure can be determined from the mixture EOS based on the mixture energy which is known from the solution of the total energy equation. Because the mixture total energy obeys a conservation law, its evolution is accurate in the entire flow field and in particular at shocks.

Again considering fluids governed by the stiffened gas EOS, the mixture EOS in this context relates mixture energy, density and volume fractions (II.4):

$$
\mathrm{p}\left(\rho, \mathrm{e}, \alpha_{1}, \alpha_{2}\right)=\frac{\rho \mathrm{e}-\left(\frac{\alpha_{1} \gamma_{1} \mathrm{p}_{\infty 1}}{\gamma_{1}-1}+\frac{\alpha_{2} \gamma_{2} \mathrm{p}_{\infty 2}}{\gamma_{2}-1}\right)}{\frac{\alpha_{1}}{\gamma_{1}-1}+\frac{\alpha_{2}}{\gamma_{2}-1}} .
$$

This EOS is valid in pure (or nearly pure) fluids, and in the diffuse interface zone. The volume fraction has very weak variations in nearly pure fluids, so that the computed pressure is nearly exact on both sides of the interface. This guarantees correct and conservative wave dynamics on both sides of the interface. Inside the numerical diffusion zone of the interface, numerical experiments show that the method is accurate too, as the volume fractions used in the mixture EOS (II.4) have a quite accurate prediction from the relaxation method.

Once the mixture pressure is determined from (II.4) the internal energies of the phases are reinitialized with the help of their respective EOS before going to the next time step

$\mathrm{e}_{\mathrm{k}}=\mathrm{e}_{\mathrm{k}}\left(\mathrm{p}, \alpha_{\mathrm{k}} \rho_{\mathrm{k}}, \alpha_{\mathrm{k}}\right)$

\section{III.5 Summary}

The numerical method can be summarized as follows:

- At each cell boundary solve the Riemann problem of System (III.1) with favorite solver. The HLLC solver of Section (III.1) is recommended.

- Evolve all flow variables with the Godunov type method of Section (III.2).

- Determine the relaxed pressure and especially the volume fraction by solving Equation (III. 4). The Newton method is appropriate for this task.

- Compute the mixture pressure with Equation (II.4).

- Reset the internal energies with the computed pressure with the help of their respective EOS (III.5).

- Go to the first item for the next time step. 


\section{Tests and validations}

\section{IV.1 Advection of an interface in a uniform pressure and velocity flow}

A discontinuity of volume fraction (thus a mixture density discontinuity) is moving in a uniform pressure and velocity flow at $100 \mathrm{~m} / \mathrm{s}$. Initially the discontinuity is located at $\mathrm{x}=0.5 \mathrm{~m}$ in a $1 \mathrm{~m}$
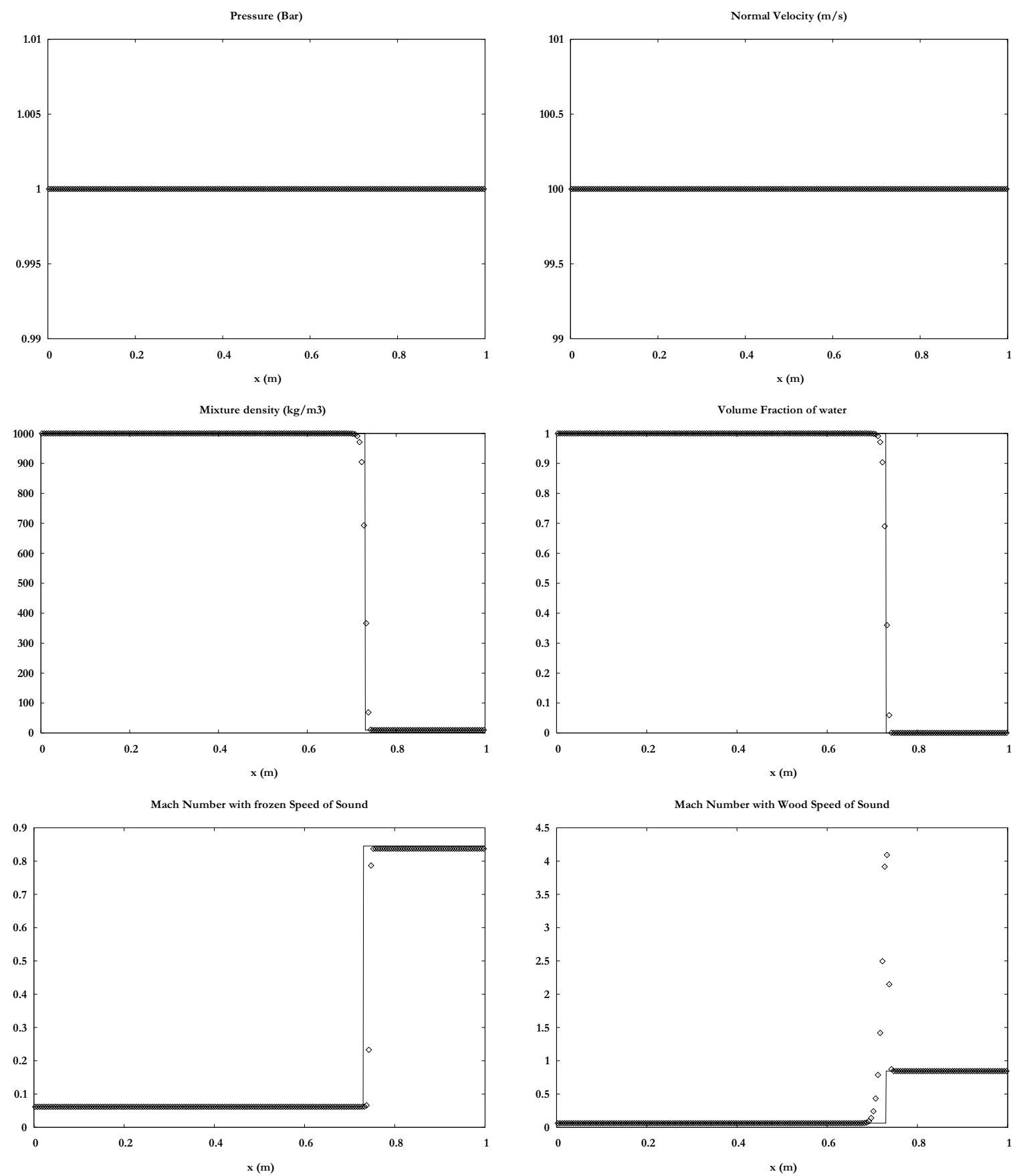

Figure 3: Advection of a volume fraction discontinuity in a uniform pressure and velocity flow. Comparison of the relaxation method with Superbee slope limiter (symbols) and the exact solution (solid). A 200 cells mesh is used. An excellent agreement is observed. 
length tube. This discontinuity separates two nearly pure fluids, liquid water on the left defined by $\rho_{\text {water }}=1000 \mathrm{~kg} \cdot \mathrm{m}^{-3}$, and the stiffened gas EOS parameters $\gamma_{\text {water }}=4.4, \mathrm{p}_{\infty \text {,water }}=6.10^{8} \mathrm{~Pa}$ and air on the right defined by $\rho_{\text {air }}=10 \mathrm{~kg} \cdot \mathrm{m}^{-3}$ with the ideal gas EOS parameters $\gamma_{\text {air }}=1.4$ and $\mathrm{p}_{\infty, \text { air }}=0 \mathrm{~Pa}$. In the left chamber, the water volume fraction is set to $\alpha_{\text {water }}=1-\varepsilon$ and in the right chamber its value is $\alpha_{\text {water }}=\varepsilon$, with $\varepsilon=10^{-8}$. The uniform pressure is set equal to $\mathrm{p}=10^{5} \mathrm{~Pa}$.

The numerical solution is plotted in Figure 3 at time $t=2.79 \mathrm{~ms}$ and is compared to the exact one. A mesh with 200 uniform cells is used with a second-order extension of the method of Section III (see Appendix B for details).

The agreement between the numerical and analytical solutions is excellent and the numerical solution is oscillation free, except for the Mach number, computed with the equilibrium sound speed $\mathrm{c}_{\mathrm{eq}}$. The present method has no difficulty resolving the sonic transition at the interface as the hyperbolic step is computed with the frozen sound speed, where this oscillation is absent.

For this test case, the flow being in mechanical equilibrium, relaxation terms present in the volume fraction and energy equations have no importance, as well as the pressure relaxation step. The respect of interface conditions is just a consequence of the clean numerical approximation with the Godunov method of conservative and non-conservative equations of Section III.2.

\section{VI.2 Shock tube with Mie Grüneisen type EOS}

In order to show the method's capabilities, in particular when dealing with more general equations of state, a test involving the Cochran-Chan (1979) EOS (CC EOS) is considered. This EOS is of Mie-Grüneisen type. The same shock tube problem presented in Saurel et al. (2007a) is considered. In this example, a single fluid is considered governed by CC EOS, with a density discontinuity in a shock tube. As there is a single fluid, the Godunov method is expected to be valid. However, it was shown in the same reference that due to the nonlinearity of $p_{\infty}(\rho)$ in the EOS, the Godunov method produced pressure and velocity oscillations. A cure to these difficulties was proposed in that same reference. Here, with the help of the multiphase flow model, these difficulties can be solved by considering the single fluid as a two-phase media with the initial discontinuity in the shock tube separating the two states.

Initially, the high pressure chamber is set to $20 \mathrm{GPa}$, while the pressure is set equal to $0.2 \mathrm{MPa}$ in the low pressure chamber. Both chambers are filled with liquid nitromethane, governed by the CC EOS in which densities are respectively set to $1134 \mathrm{~kg} / \mathrm{m}^{3}$ and $1200 \mathrm{~kg} / \mathrm{m}^{3}$. In the high pressure chamber, volume fraction of the first phase is set to $\alpha_{1}=1-\varepsilon$ and in the right chamber its value is $\alpha_{1}=\varepsilon\left(\varepsilon=10^{-8}\right)$. Thus, the model is used in the single phase limit, i.e. the same EOS is used for both fluids but with different initial densities:

$\mathrm{p}(\rho, \mathrm{e})=\rho \Gamma\left(\mathrm{e}-\mathrm{e}_{\mathrm{k}}(\rho)\right)+\mathrm{p}_{\mathrm{k}}(\rho)$, with, 


$$
\begin{aligned}
& \mathrm{e}_{\mathrm{k}}(\rho)=\frac{\mathrm{A}_{1}}{\rho_{\text {ref }}\left(\mathrm{E}_{1}-1\right)}\left(\frac{\rho}{\rho_{\text {ref }}}\right)^{\mathrm{E}_{1}-1}-\frac{A_{2}}{\rho_{\text {ref }}\left(\mathrm{E}_{2}-1\right)}\left(\frac{\rho}{\rho_{\text {ref }}}\right)^{\mathrm{E}_{2}-1}, \\
& \mathrm{p}_{\mathrm{k}}(\rho)=\mathrm{A}_{1}\left(\frac{\rho}{\rho_{\text {ref }}}\right)^{\mathrm{E}_{1}}-\mathrm{A}_{2}\left(\frac{\rho}{\rho_{\text {ref }}}\right)^{\mathrm{E}_{2}} .
\end{aligned}
$$

The data used in the present simulation are: $\Gamma=1.19, \rho_{\text {ref }}=1134 \mathrm{~kg} / \mathrm{m}^{3}, \mathrm{~A}_{1}=0.81918110^{9} \mathrm{~Pa}, \mathrm{~A}_{2}$ $=1.5083510^{9} \mathrm{~Pa}, \mathrm{E}_{1}=4.52969$ and $\mathrm{E}_{2}=1.42144$.

The solution is presented at time $\mathrm{t}=67 \mu \mathrm{s}$ in Figure 4. The present relaxation method is compared to the exact solution of the Euler equations. Results are similar to those of Saurel et al. (2007a) but the present algorithm is easier to implement. A magnified view of pressure and velocity around the contact discontinuity is given in Figure 5. It presents a solution free of oscillations.
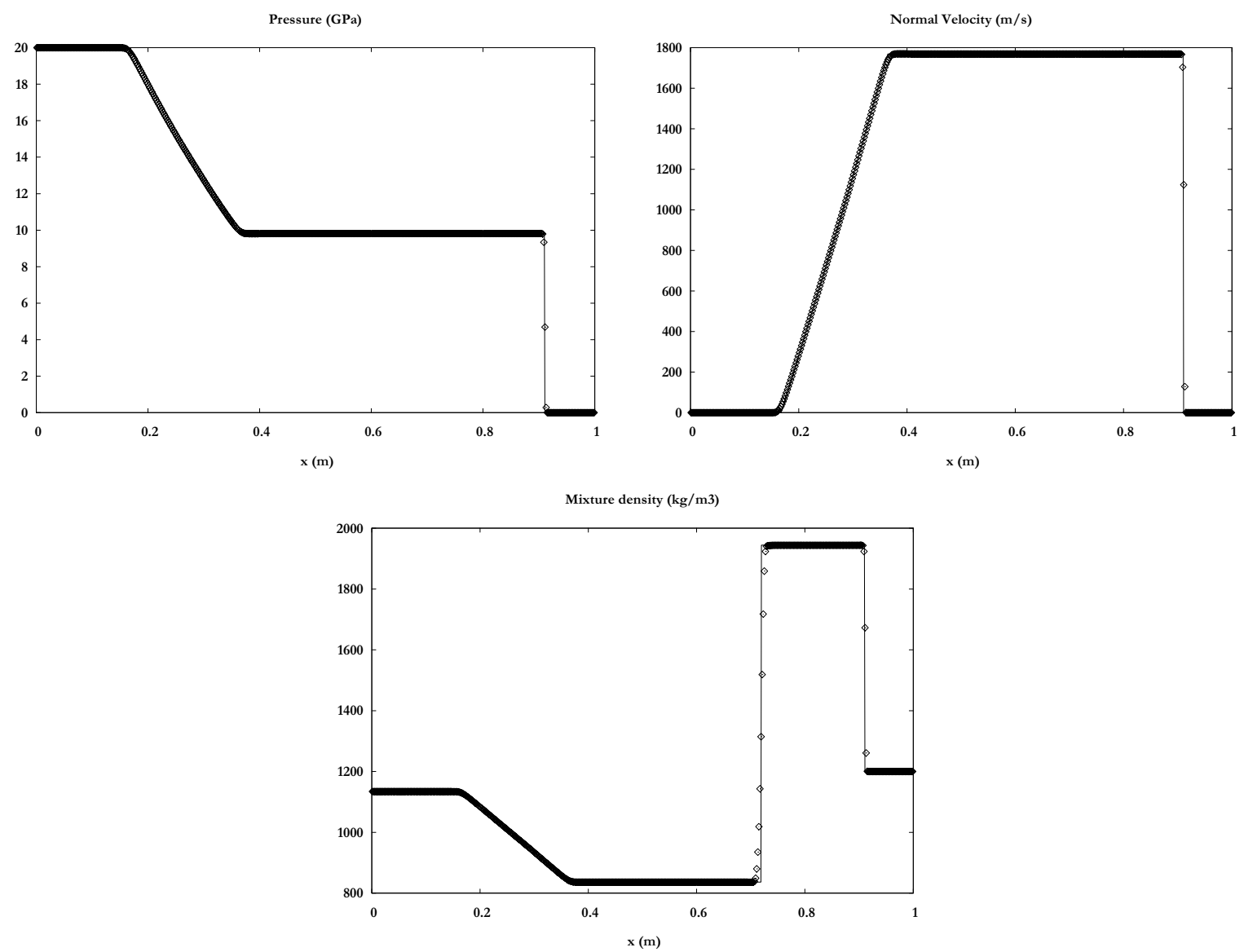

Figure 4: Shock tube with Mie-Grüneisen type EOS. The present relaxation method based on the 6-equations model (symbols) is compared to the exact solution of the Euler equations (solid). A 500 cells mesh is used. Results are in perfect agreement. 
Pressure (GPa)

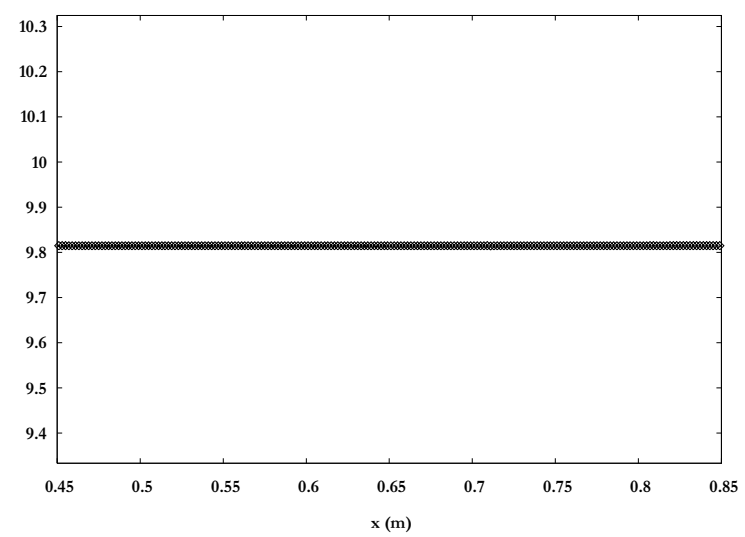

Normal Velocity $(\mathrm{m} / \mathrm{s})$

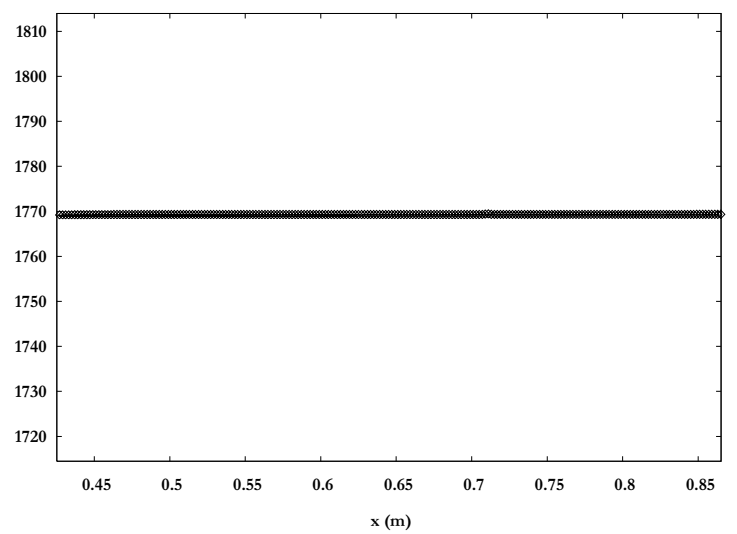

Figure 5: Shock tube with Mie-Grüneisen type EOS. Magnified view of pressure and density around the contact discontinuity. Results are in perfect agreement with the exact solution and the solution is oscillation free.

\section{IV.3 Water-Air shock tubes}

\section{Water - Air shock tube with moderate pressure ratio and high density ratio}

A $1 \mathrm{~m}$ long shock tube containing two chambers separated by an interface at the location $\mathrm{x}=0.75 \mathrm{~m}$ is considered. Each chamber contains a nearly pure fluid. The initial density of water is $\rho_{\text {water }}=1000 \mathrm{~kg} \cdot \mathrm{m}^{-3}$ and the stiffened gas EOS parameters are $\gamma_{\text {water }}=4.4$ and $\mathrm{p}_{\infty \text {, water }}=6.10^{8} \mathrm{~Pa}$. The initial density of air is $\rho_{\text {air }}=1 \mathrm{~kg} \cdot \mathrm{m}^{-3}$ and EOS parameters are $\gamma_{\text {air }}=1.4$ and $\mathrm{p}_{\infty, \text { air }}=0 \mathrm{~Pa}$. The left chamber contains a very small volume fraction of air $\alpha_{\text {air }}=10^{-6}$ and the initial pressure is set equal to $1 \mathrm{GPa}$. The right chamber contains the same fluids but the volume fractions are reversed. The initial pressure is set equal to $0.1 \mathrm{MPa}$. In both chambers the initial velocity is equal to 0 .

The numerical solution of the 6-equation model is compared to the exact solution of the Euler equations. A mesh employing 1000 uniform cells is used in Figure 6 and a mesh employing 100 cells is used in Figure 7. Comparison with the exact solution is shown in both figures at time $\mathrm{t}=240 \mu \mathrm{s}$. Again this test poses no computational difficulty. 

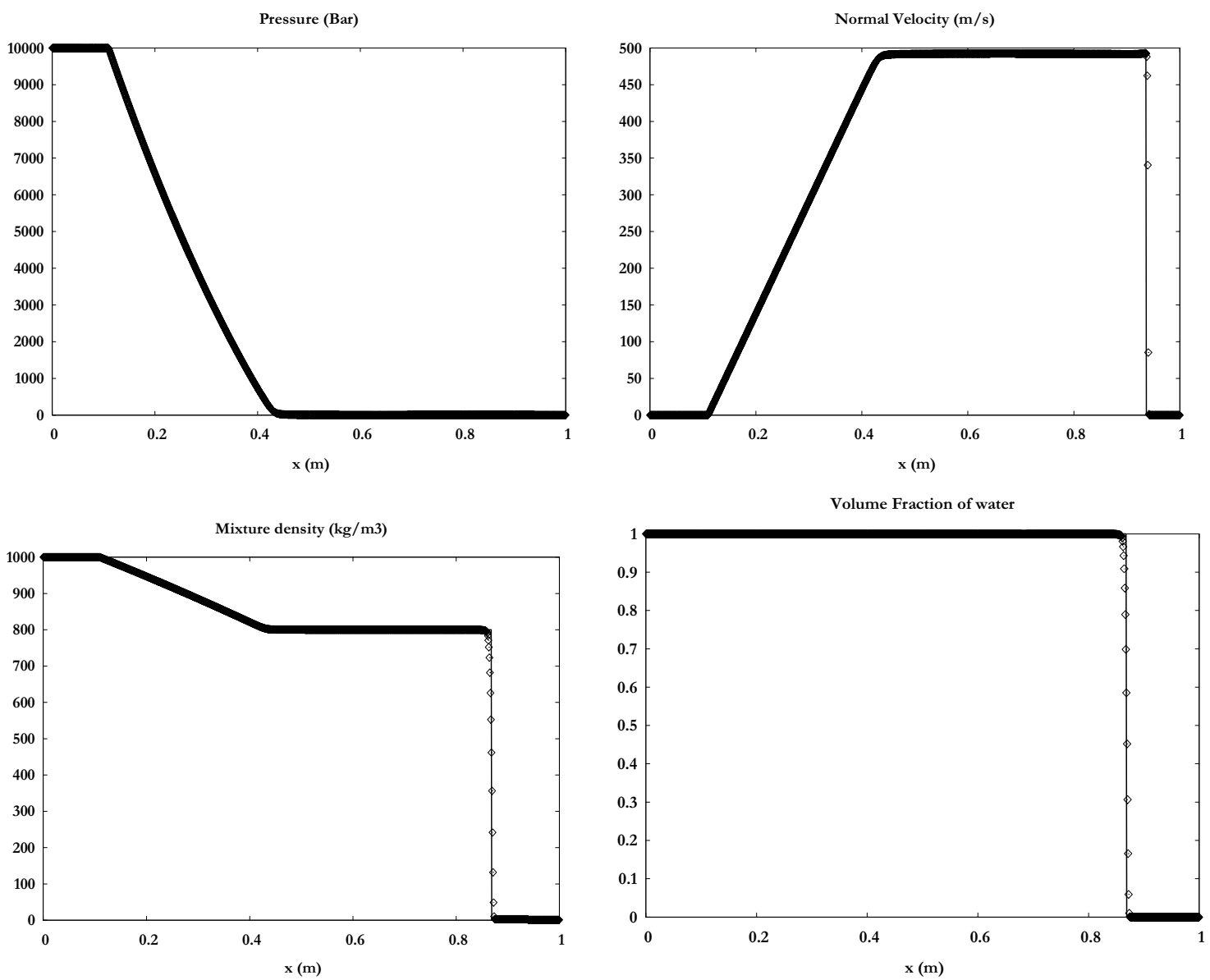

Figure 6: Liquid/Gas shock tube. The present relaxation method is used to solve the 6equation model. Numerical results are shown with symbols and compared to the exact solution (solid). A 1000 cell mesh is used. The density ratio is 1000 and the pressure ratio is 10000 at the initial discontinuity. A second order extension of the method with van Leer limiter is used.

Results are in excellent agreement. 

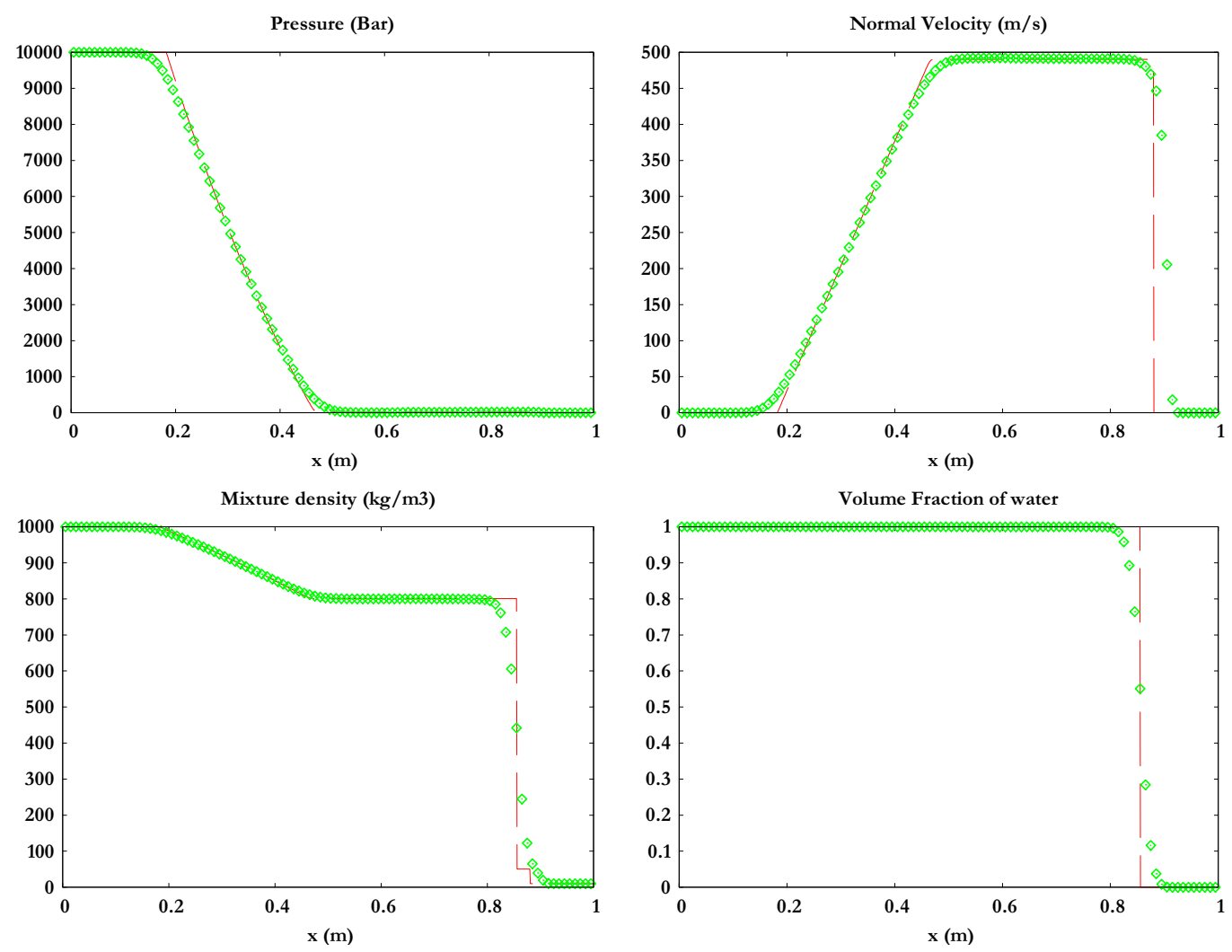

Figure 7: Same liquid/Gas shock tube as those of Figure 6 with 100 cells. Numerical results are shown with symbols and compared to the exact solution (solid). A second order extension of the method with van Leer limiter is used. Results are in good agreement.

In this test case and in all subsequent tests, strong pressure waves propagate. Relaxation terms present in the volume fraction and energy equations become important, as well as the pressure relaxation step. Robustness and convergence of the algorithm in the unsteady building of the solution are improved by pressure relaxation.

\section{Water-Air shock tube in extreme conditions}

The same shock tube problem is solved, but initially, the left chamber pressure is set to $1 \mathrm{TPa}$ (10 Mbars) and the density of air is set to $10 \mathrm{~kg} . \mathrm{m}^{-3}$. The exact solutions of the single phase Euler equations and the multiphase flow model with 6 equations are compared in Figure 8 at time $t=8.3$ $\mu \mathrm{s}$. This test illustrates the robustness and convergence of the algorithm. 

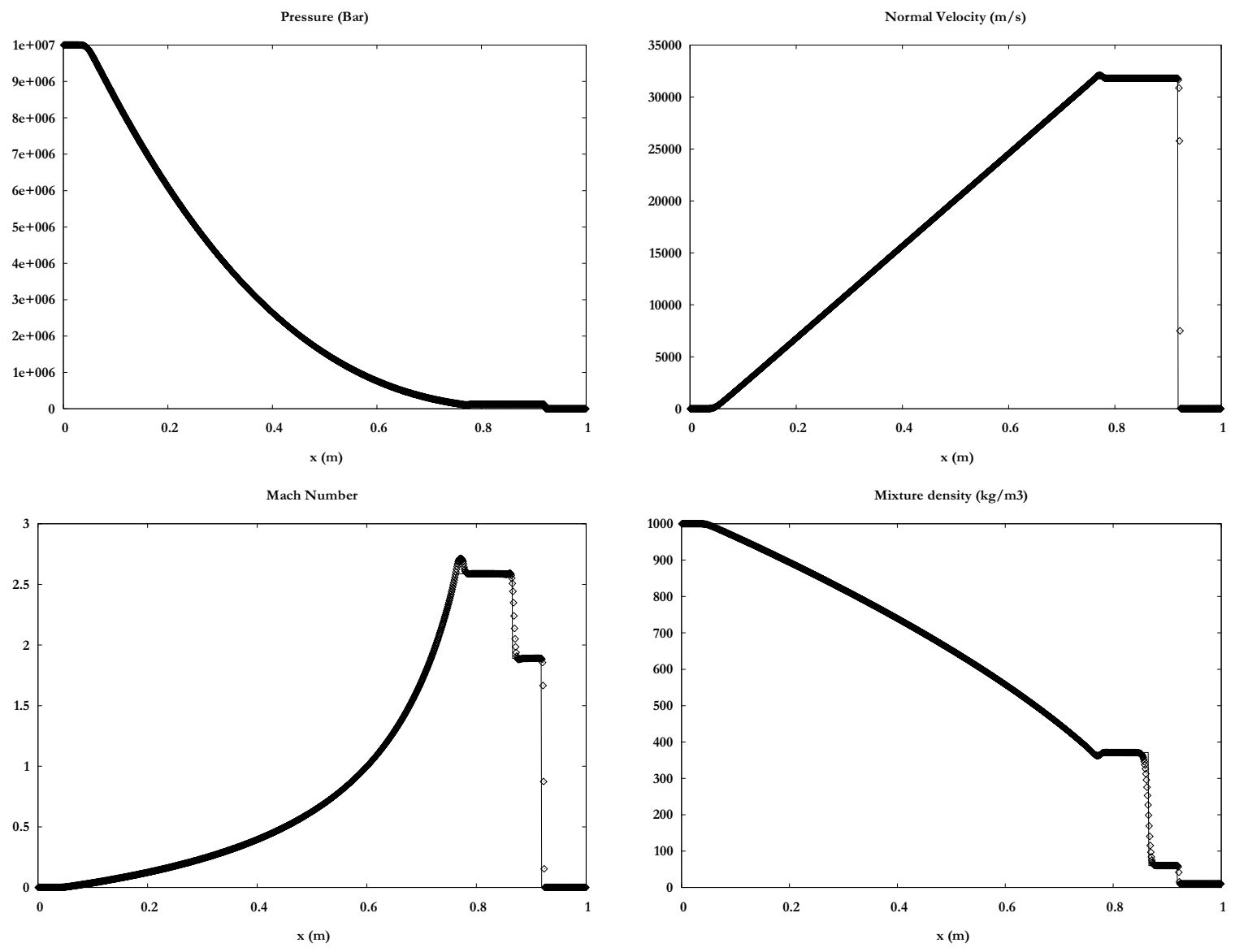

Volume Fraction of water

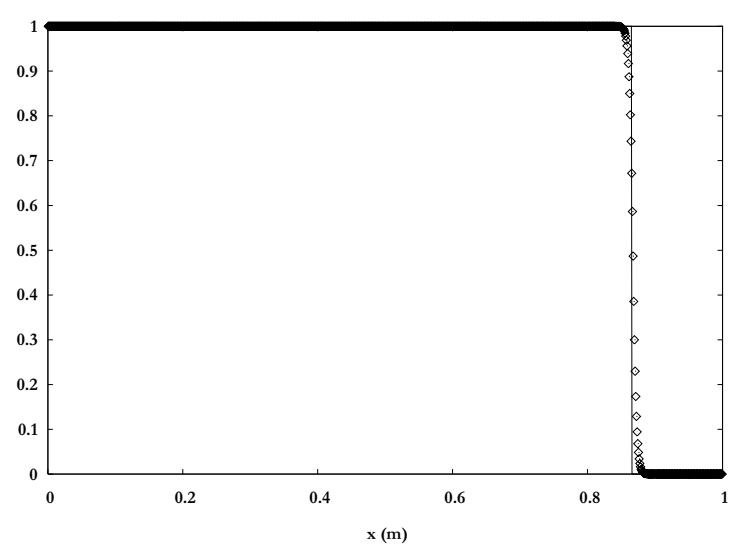

Figure 8: Liquid/Gas shock tube. The present relaxation method is used to solve the 6 equations model. Numerical results are shown with symbols and are compared to the exact solution (solid). A 1000 cell mesh is used. The initial density ratio is 100 and the initial pressure ratio is $10^{7}$. This test illustrates robustness and convergence of the algorithm.

\section{IV.4 Influence of $\hat{p}_{I}$ in the relaxation method}

During the relaxation step, we have highlighted different possible estimates for the pressure average $\hat{\mathrm{p}}_{\mathrm{I}}$. In order to demonstrate the weak influence of the estimate, the liquid/gas shock tube 
test presented in Figure 6 was run with different estimates of $\hat{\mathrm{p}}_{\mathrm{I}}$. In Figure 9, results are presented and compared with two possible estimates: $\hat{\mathrm{p}}_{\mathrm{I}}=\mathrm{p}_{\mathrm{I}}^{0}$ or $\hat{\mathrm{p}}_{\mathrm{I}}=\mathrm{p}^{*}$. No differences are visible.
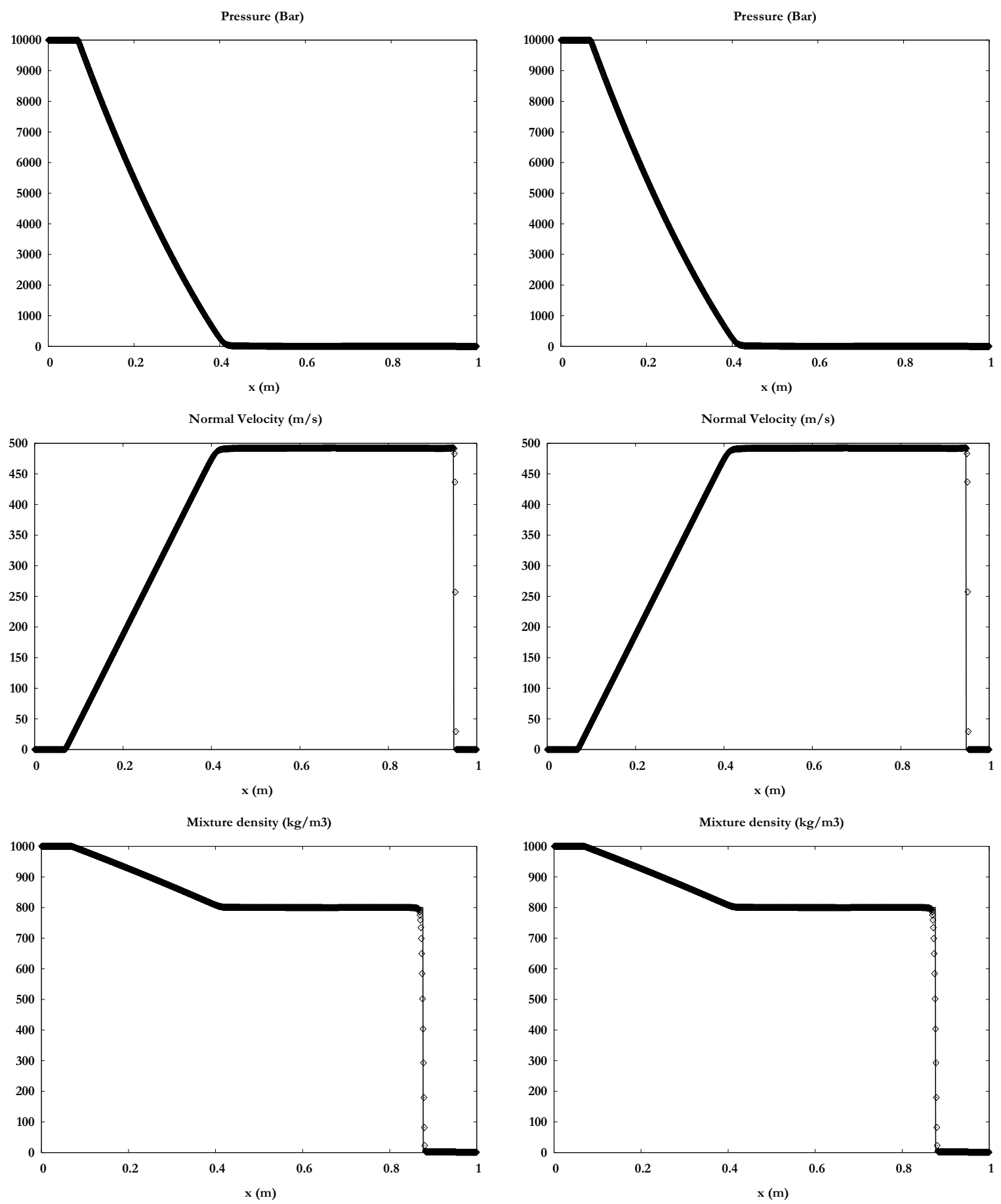

Figure 9: Comparison of two different pressure averages estimates. The test case of Figure 6 is rerun with $\hat{\mathrm{p}}_{\mathrm{I}}=\mathrm{p}_{\mathrm{I}}^{0}$ on the left the and $\hat{\mathrm{p}}_{\mathrm{I}}=\mathrm{p}^{*}$ on the right. From top to bottom, pressure, velocity and mixture density remains unchanged. 


\section{IV.5 Cavitation test}

A 1 meter length tube is filled with liquid water at atmospheric pressure and with density $\rho=$ $1000 \mathrm{~kg} / \mathrm{m}^{3}$. A small volume fraction of air $\left(\alpha_{\text {air }}=10^{-2}\right)$ is initially present everywhere. An initial velocity discontinuity is located at $\mathrm{x}=0.5 \mathrm{~m}$. On the left, the velocity is set to $\mathrm{u}=-100 \mathrm{~m} / \mathrm{s}$ and on the right, $\mathrm{u}=100 \mathrm{~m} / \mathrm{s}$. Solution is shown in Figure 10 at time $\mathrm{t}=1.85 \mathrm{~ms}$, using 1000 uniform mesh cells.

Strong rarefaction waves propagate in the tube and the liquid pressure decreases. As gas is present, the pressure cannot become negative. To maintain positive pressure, the gas volume fraction increases and creates a cavitation pocket. This results in the dynamic appearance of two interfaces that were not present initially. Excellent agreement with the exact solution of the 5equation model (Petitpas et al., 2007) is obtained. Interface creation is readily handled by the present algorithm.
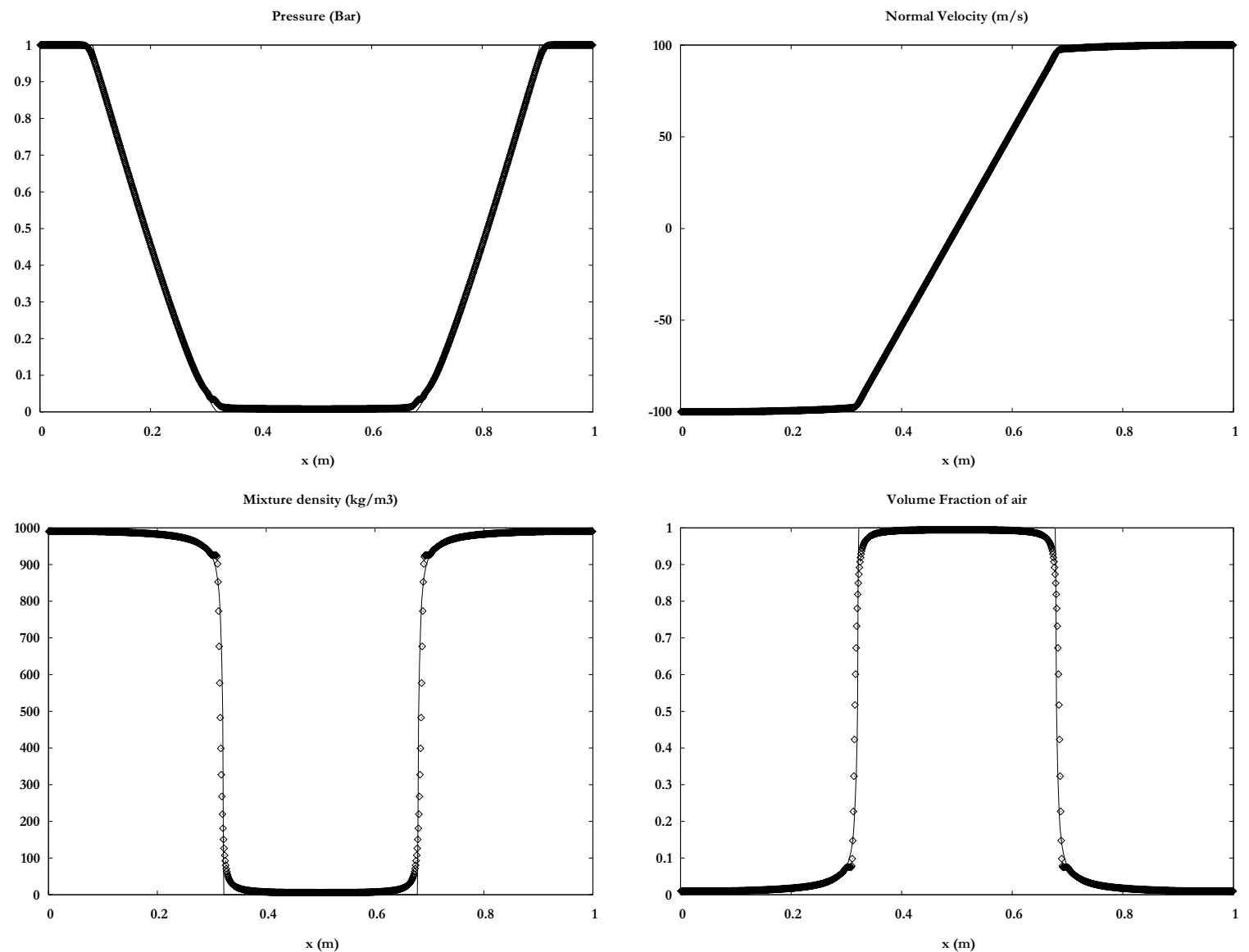

Figure 10: Expansion tube with cavitation pocket appearance. The present relaxation method is used to solve the 6-equation model. Numerical results are shown with symbols and are compared to the exact solution (solid) of the 5-equation model (Petitpas et al., 2007). A 1000 cells mesh is used. 


\section{IV.6 Multidimensional validation: Shock-Bubble interaction}

Multidimensional finite volume extension of the method is presented in Appendix C. The method is validated against shock tube experiments of shock-bubble interaction. The experiment is one of those proposed in Layes and Le Métayer (2007) where full description of the experimental setup is provided. The configuration under study consists in a shock wave

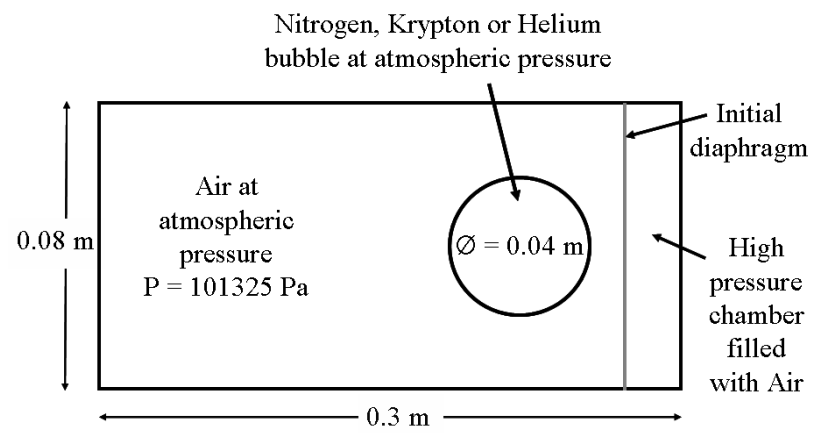

Figure 11: Initial configuration of the interaction shock - bubble.

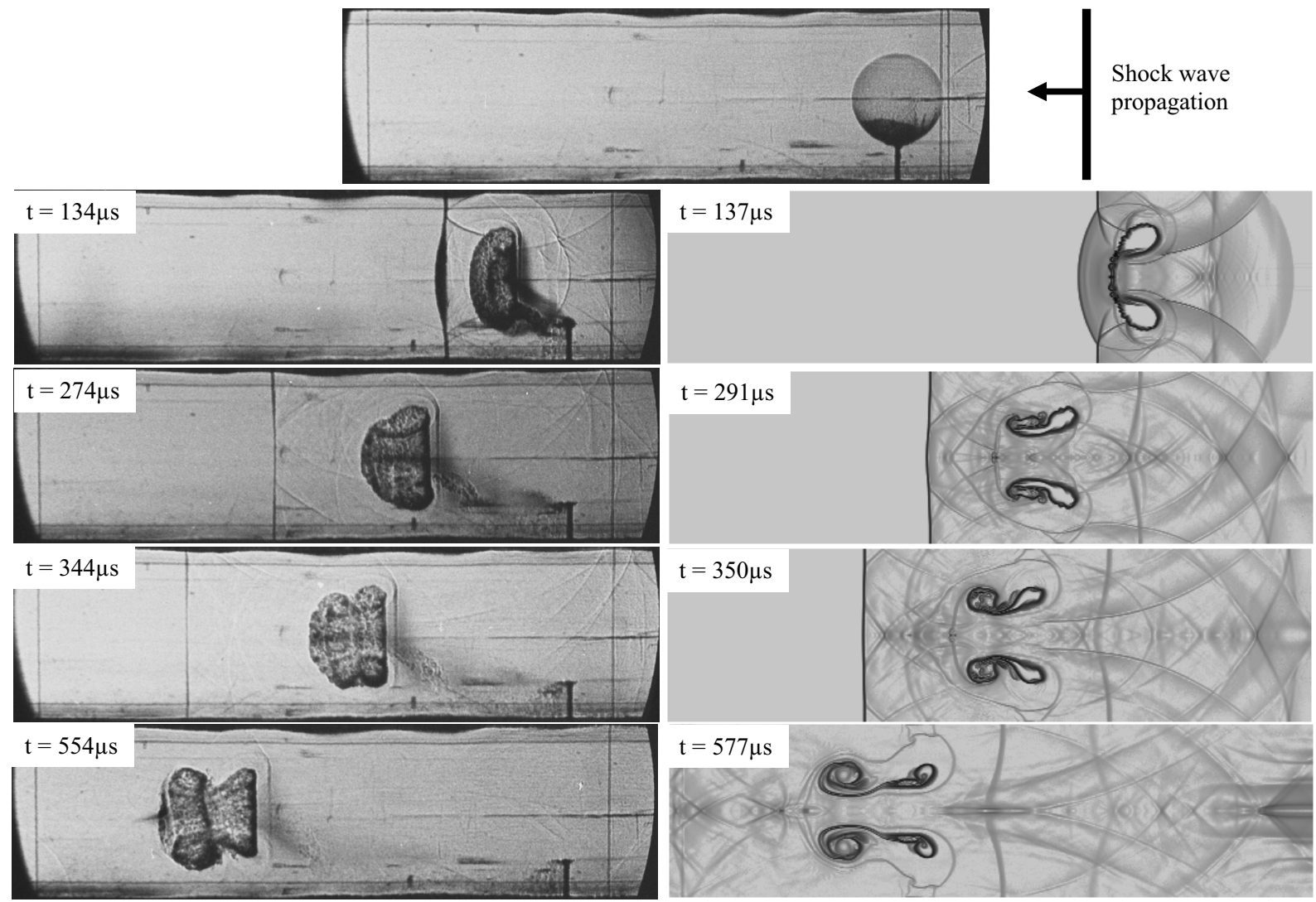

Figure 12: Shock-bubble interaction test. Experimental results (left) and computed results (right) are compared at different times. Because of the difference in gas properties, the transmitted shock wave is faster than the incident one in air. Pressure and density gradients induce vorticity generation which develops at long time scale. 
propagating at Mach number 1.5 into air at atmospheric conditions and interacting with a helium bubble. The initial density of air is $\rho_{\text {air }}=1.29 \mathrm{~kg} \cdot \mathrm{m}^{-3}$ and the initial density of helium is $\rho_{\text {helium }}=0.167 \mathrm{~kg} \cdot \mathrm{m}^{-3}$. In the simulation both fluids are considered as ideal gases with polytropic coefficients $\gamma_{\text {air }}=1.4$ and $\gamma_{\text {helium }}=1.67$. The initial configuration is represented in Figure 11. Computed results are compared with experimental ones in Figure 12.

\section{IV.7 Cavitating Richtmyer-Meshkov instability (RMI)}

To illustrate the method capabilities a 2D test involving a RMI is considered. As the liquid is not pure, new interfaces will appear during the development of the instability, due to cavitation effects. The shape of the resulting interface and the entire flow field show a non-conventional behavior, that was never computed before, as the model and method must deal with liquid gas interfaces and dynamic appearance of gas pockets in severe conditions.

The left part of the computational domain is filled with nearly pure water and the right part with nearly pure gas. They are initially separated by a curved interface. It is a portion of circle with 0.6 meter radius centered at $\mathrm{x}=1.2 \mathrm{~m}, \mathrm{y}=0.5 \mathrm{~m}$. The physical domain is $3 \mathrm{~m}$ long and $1 \mathrm{~m}$ high. The mesh contains 900 cells along x-direction and 400 cells along y-direction. Both water and gas have an initial velocity of $-200 \mathrm{~m} / \mathrm{s}$. Top, bottom and left boundaries are treated as solid walls. The initial density of water is $\rho_{\text {water }}=1000 \mathrm{~kg} \cdot \mathrm{m}^{-3}$ and the stiffened gas EOS parameters are $\gamma_{\text {water }}=4.4$ and $\mathrm{p}_{\infty \text {, water }}=6.10^{8} \mathrm{~Pa}$. The initial density of gas is $\rho_{\text {gas }}=100 \mathrm{~kg} \cdot \mathrm{m}^{-3}$ and EOS parameters are $\gamma_{\text {gas }}=1.8$ and $p_{\infty, \text { gas }}=0 \mathrm{~Pa}$. The left chamber contains a very small volume fraction of gas $\alpha_{\text {gas }}=10^{-6}$ and the right chamber contains a very small volume fraction of water $\alpha_{\text {water }}=10^{-6}$.

The initial configuration is represented in Figure 13. Results are shown in Figure 14.

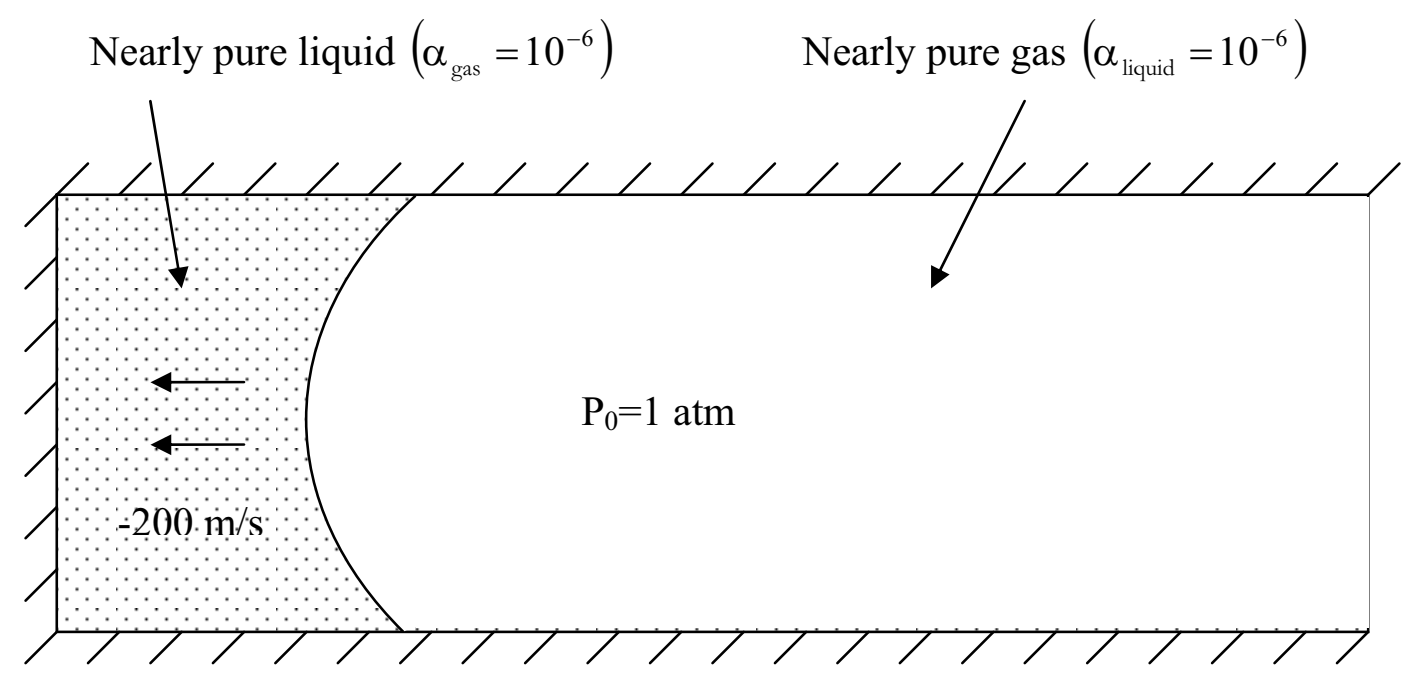

Figure 13: Initial configuration of the water-gas Richtmyer-Meshkov instability. Both liquid and gas have initial velocity of $-200 \mathrm{~m} / \mathrm{s}$. 

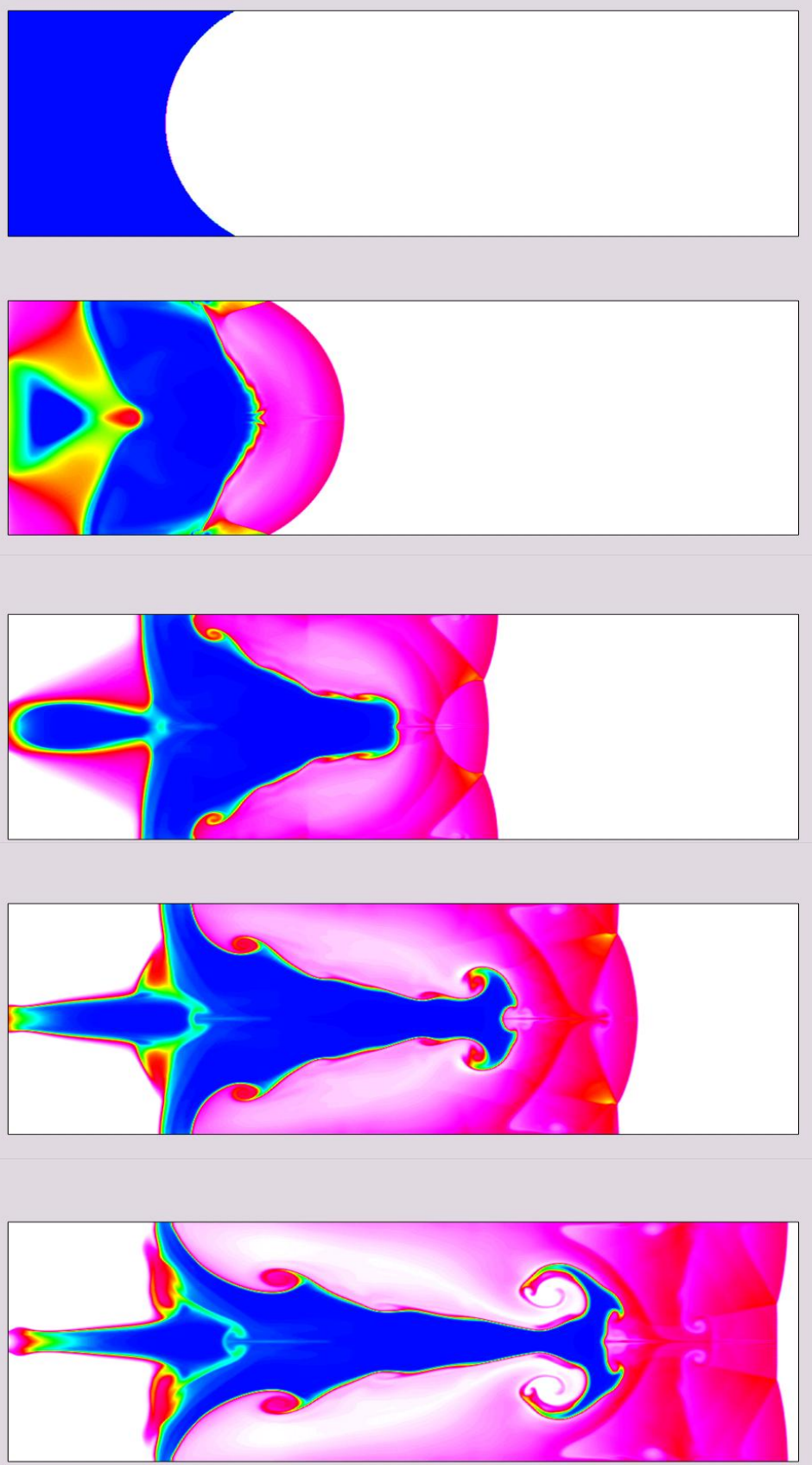

Figure 14: Water-gas Richtmyer-Meshkov instability. Mixture density contours are shown at time $\mathrm{t}_{0}=0 \mathrm{~ms}, \mathrm{t}_{1}=1.9 \mathrm{~ms}, \mathrm{t}_{2}=3.9 \mathrm{~ms}, \mathrm{t}_{3}=5.8 \mathrm{~ms}, \mathrm{t}_{4}=7.8 \mathrm{~ms}$. Blue is high density, white is low, and other colors are intermediate. New interfaces appear dynamically near the solid boundary as a result of expansion waves focusing. They result in cavitation pockets that considerably modify the jet and spike shape.

When the flow impacts the left wall, a right-facing shock propagates in the domain through the water/gas discontinuity. A conventional RMI appears first. Then expansion waves are produced 
as the jet elongates. It results in expanded zones near the solid boundary where gas inhomogeneities grow, producing dynamic appearance of gas pockets. As the pressure is very low in these zones, the jet dynamics is modified compared to conventional RMI with pure fluids. The various gas pockets near the solid boundary and in the jet core are clearly visible in Figure 15 where the gas volume fraction is shown. Relaxation terms present in the volume fraction and energy equations are responsible for the dynamic appearance of these gas pockets.
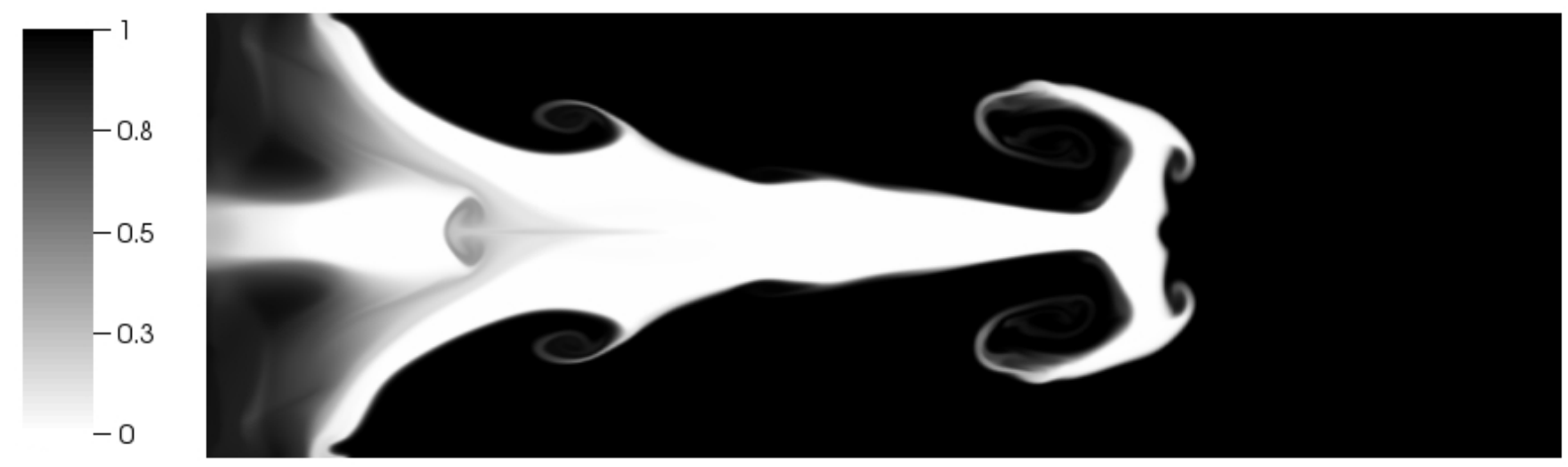

Figure 15: Water-gas Richtmyer-Meshkov instability. Volume fraction contours of gas are shown at time $t_{5}=8.6 \mathrm{~ms}$. The gas volume fraction increase into the liquid jet and near the solid wall boundary. The spike shape is also modified.

The link between the 6-equation model and conventional barotropic cavitating flows that are the most popular in cavitation modeling is detailed in Appendix D. These models are composed of one or two mass conservation equations and one momentum equation. They consist in hyperbolic systems of conservation laws. These models involve an important difficulty related to the nonmonotonic behavior of the sound speed versus volume fraction (Koren et al., 2002, CoutierDelgosha, 2005, Sinibaldi, 2006). It is thus interesting to examine how the various ingredients developed in the context of the 6-equation model can be used for these barotropic models in order to solve this difficulty.

\section{Method extension for shocks in multiphase mixtures - Artificial heat exchanges}

The present refinement of the algorithm is needed only when shock propagation in real multiphase mixtures is under study. For other situations with interfaces separating pure fluids or cavitating flows, there is no need to account for the artificial heat exchanges detailed hereafter. The artificial heat exchange is used to correct the partition of the energies in the various phases in the mixture and to propagate shocks in these mixtures at the correct speed with the correct shocked state.

Some preliminary observations of numerical schemes in the context of single phase flows are first necessary to introduce the numerical issues associated with multiphase shocks. Consider a shock wave propagating in a pure material, governed by the Euler equations. Shock capturing 
schemes produce a smearing of discontinuities and it is interesting to compare the thermodynamic path followed by the fluid in the shock layer to the theoretical Hugoniot curve. Such comparison is shown in Figure 16.

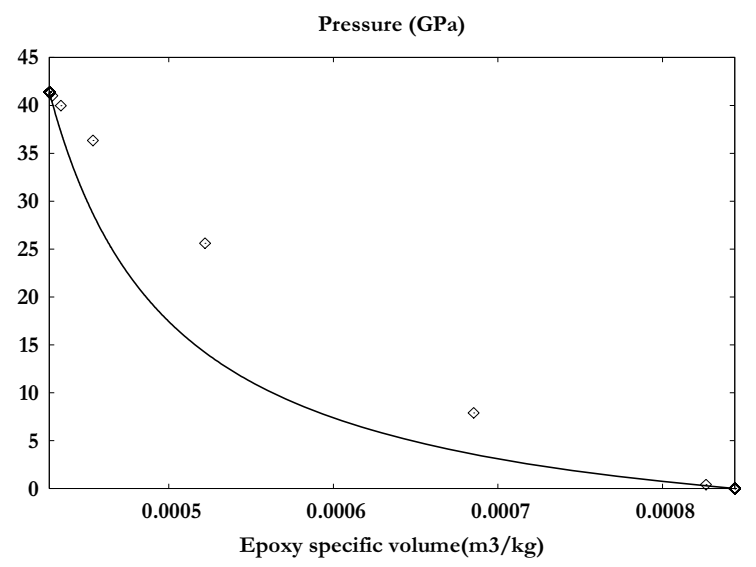

Figure 16: Comparison of the numerical Hugoniot curve (symbols) and the theoretical one (lines) in the numerical diffusion zone for single phase flows. The two thermodynamic paths are different but the end states are the same.

It appears clearly that the thermodynamic paths are very different. This is due to the succession of numerical weak shocks that propagate into the cell that do not impose the same thermodynamic transformation as a single strong shock (Courant and Friedrichs, 1948). The successive cell averages produce also transformations in disagreement with the single shock Hugoniot. However, this numerical phenomenon has no consequence on the computation of the shocked state for single phase flows. As shown in Figure 16, the end of the shock layer merges with the theoretical Hugoniot state. This is a consequence of conservation properties of the Euler equations.

When dealing with multiphase mixtures, the same deviation from the theoretical Hugoniot appears and has more serious consequences. The reason is that for each weak shock that enter the cell, the equation of state changes. Indeed, for multiphase mixtures, there is an extra degree of freedom characterized by the volume fraction. At a given point of the numerical shock, as shown in Figure 16, there is no hope that this point belongs to the theoretical mixture Hugoniot curve. It follows that the corresponding volume fraction is in error. Consequently the mixture EOS (II.4) is in error too. These errors cumulate in the shock layer and, contrary to that of single-phase flow, the end state does not belong to the mixture Hugoniot. To illustrate these difficulties, consider the following test cases.

\section{Epoxy - Spinel mixture shock tube with moderate pressure ratio}

A tube of one meter length contains two chambers separated by an interface at the location $\mathrm{x}=$ $0.6 \mathrm{~m}$. Both chambers of the tube are filled with the same mixture of epoxy and spinel. The initial density of the epoxy is $\rho_{\text {epoxy }}=1185 \mathrm{~kg} / \mathrm{m}^{3}$ and its stiffened gas EOS parameters are $\gamma_{\text {epoxy }}=2.43$ and $\mathrm{p}_{\infty, \text { epoxy }}=5.3 \times 10^{9} \mathrm{~Pa}$. The initial density of spinel is $\rho_{\text {spinel }}=3622 \mathrm{~kg} \cdot \mathrm{m}^{-3}$ and EOS parameters are $\gamma_{\text {spinel }}=1.62$ and $\mathrm{p}_{\infty, \text { spinel }}=141 \times 10^{9} \mathrm{~Pa}$. The initial volume fraction in both chambers are $\alpha_{\text {epoxy }}=0.5954\left(\alpha_{\text {spinel }}=1-\alpha_{\text {epoxy }}\right)$. The pressure at the left of the interface is equal to 
$1 \times 10^{10} \mathrm{~Pa}$, while the right chamber is at atmospheric pressure. All the materials are initially at rest. Using a 500 cell uniform mesh the solution of the multiphase flow model with 6 equations is compared with the exact solution of the 5-equation model (Petitpas et al., 2007) in Figure 17 at time $\mathrm{t}=80 \mu \mathrm{s}$. As the shock is of moderate strength, the present method converges to the exact solution without any artificial heat exchange.
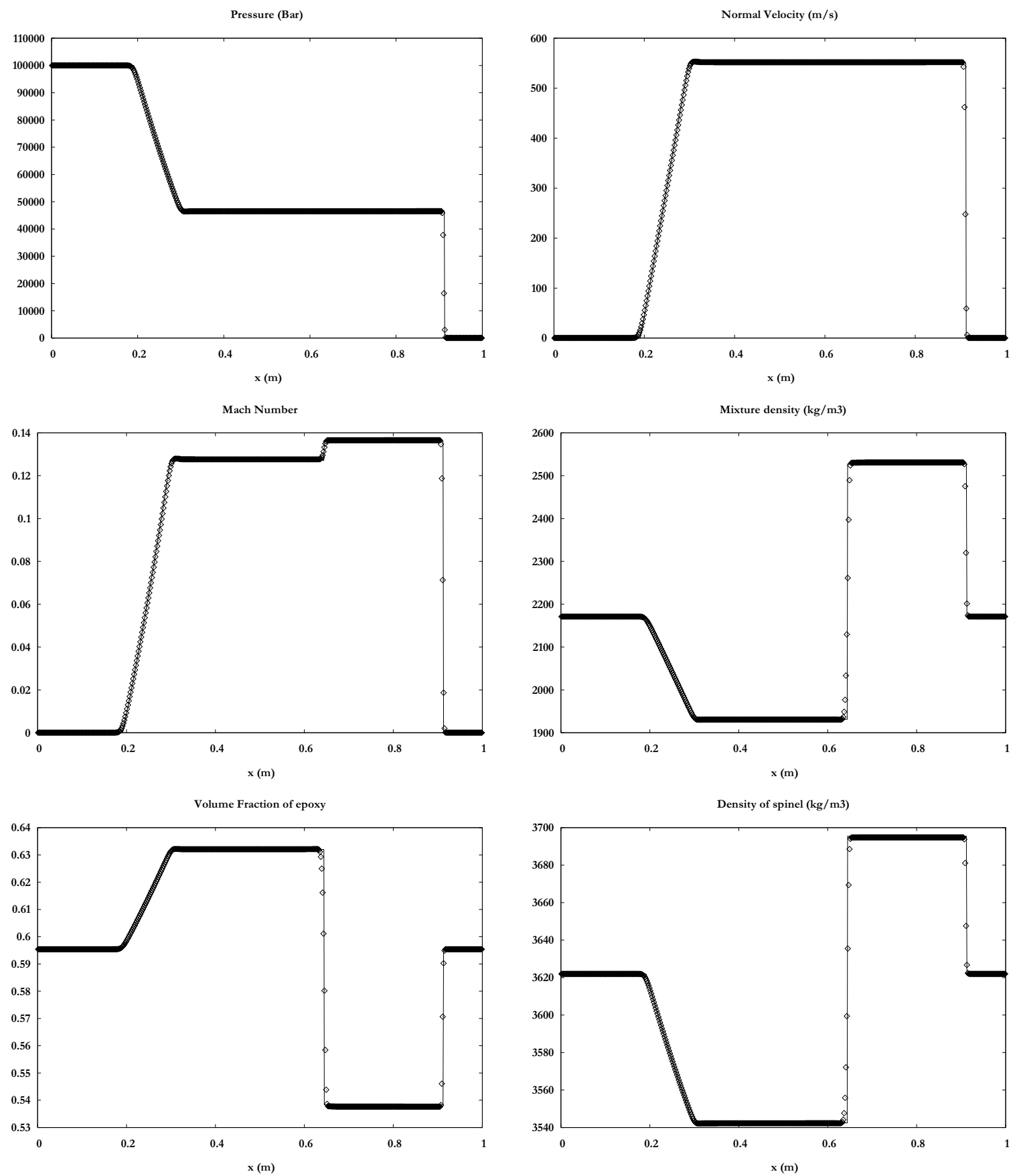

Figure 17: Epoxy-Spinel shock tube problem with moderate pressure ratio. The present relaxation method is used to solve the 6 equations model. Numerical results are shown with symbols and are compared to the exact solution of the five equations model (solid). A 500 cells mesh is used. The pressure ratio is 100000 at the initial discontinuity. 


\section{Epoxy - Spinel mixture shock tube under extreme conditions}

We consider now the same shock tube problem as previously, but the initial pressure ratio is set to $2 \cdot 10^{6}$. Results are shown in Figure 18.
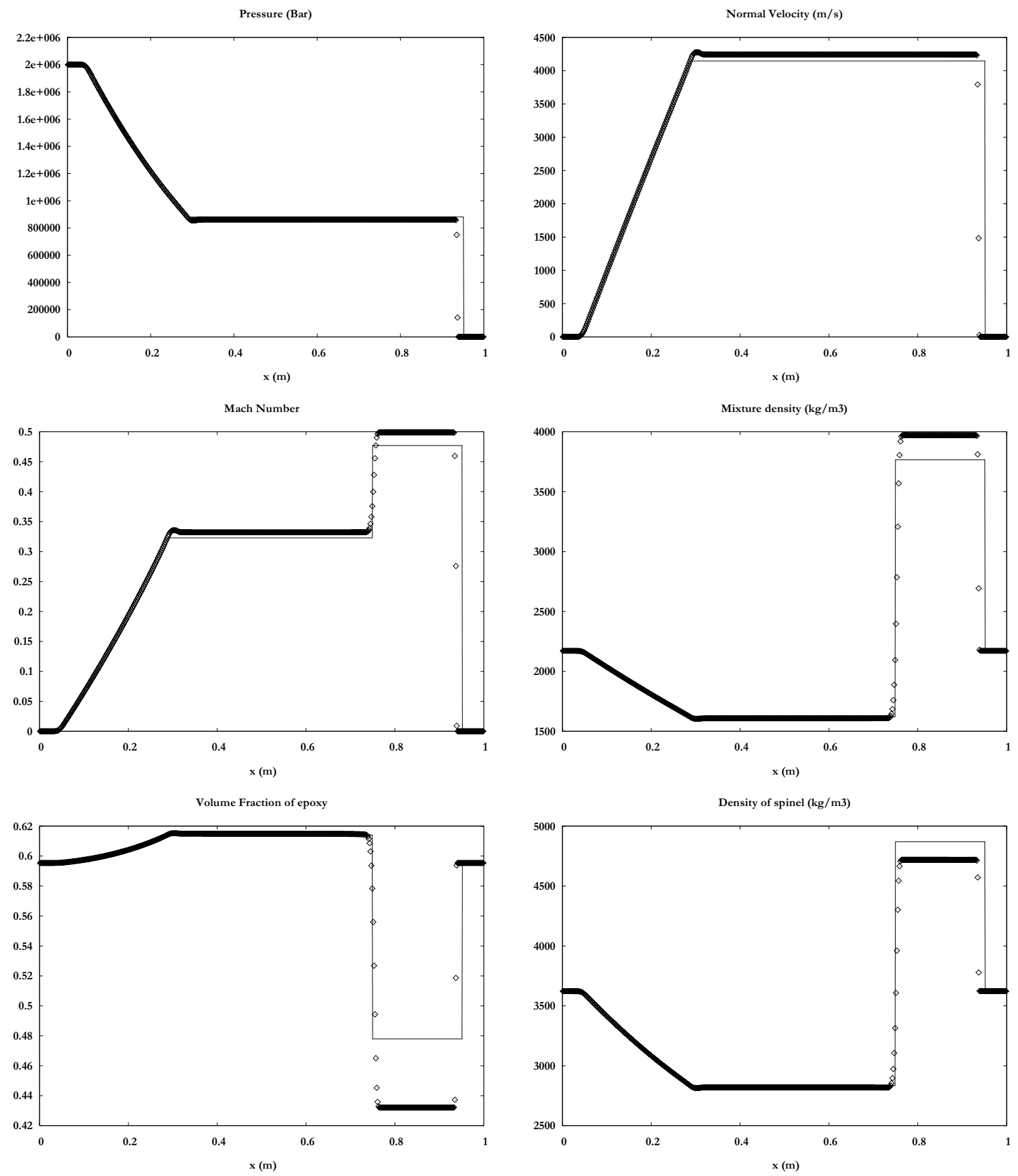

Figure 18: Epoxy-Spinel shock tube problem with extreme pressure ratio. The present relaxation method is used to solve the 6-equation model. Numerical results are shown with symbols and are compared to the exact solution of the five equation model (solid). A 500 cells mesh is used. The pressure ratio is 2000000 at the initial discontinuity.

Important differences appear between solutions as the shock is now very strong. The numerical solution does not converge to the exact solution of the 5-equation model, equipped with the 
shock relations summarized in System (II.5). This is due to the incorrect partition of internal energy in the shock layer (Petitpas et al., 2007). In order to partition the energies correctly, artificial heat exchanges are now introduced.

\section{Artificial heat exchanges in the 6-equation model}

The correct partition of the shock energy among the various phases can be achieved by shock tracking methods. Shock tracking methods have been intensively studied by Glimm et al. (1998), LeVeque and Shyue (1996), Massoni et al. (1999). Another option is to correct partition of the energies in the shock layer by introducing artificial heat transfers.

Artificial heat exchanges have been introduced in Petitpas et al. (2007) in the context of a Lagrange-relaxation method. In the present Eulerian formulation context they correspond to an extra pressure that appears in the internal energy equations:

$$
\begin{aligned}
& \frac{\partial \alpha_{1} \rho_{1} e_{1}}{\partial t}+\frac{\partial \alpha_{1} \rho_{1} e_{1} u}{\partial x}+\left(\alpha_{1} p_{1}+q\right) \frac{\partial u}{\partial x}=0 \\
& \frac{\partial \alpha_{2} \rho_{2} e_{2}}{\partial t}+\frac{\partial \alpha_{2} \rho_{2} e_{2} u}{\partial x}+\left(\alpha_{2} p_{2}-q\right) \frac{\partial u}{\partial x}=0 .
\end{aligned}
$$

The artificial heat exchange term $\pm \mathrm{q} \frac{\partial \mathrm{u}}{\partial \mathrm{x}}$ is active only in the shock layer as it is defined by $\mathrm{q}=\eta\left(\frac{\partial \mathrm{u}}{\partial \mathrm{x}}\right) v(\mathrm{v})$

where $\eta\left(\frac{\partial u}{\partial x}\right)=\left\{\begin{array}{cc}1 & \text { if } \frac{\partial u}{\partial x}<0 \\ 0 & \text { otherwise }\end{array}\right.$ and $v(v)$ is the heat exchange function.

It is more convenient and also accurate (regarding mesh independence of the results) to rewrite these equations into the form

$$
\begin{aligned}
& \frac{\partial \alpha_{1} \rho_{1} e_{1}}{\partial t}+\frac{\partial\left(\alpha_{1} \rho_{1} e_{1}+\eta\left(\frac{\partial u}{\partial x}\right) \mu(v)\right) u}{\partial x}+\alpha_{1} p_{1} \frac{\partial u}{\partial x}=0 \\
& \frac{\partial \alpha_{2} \rho_{2} e_{2}}{\partial t}+\frac{\partial\left(\alpha_{2} \rho_{2} e_{2}-\eta\left(\frac{\partial u}{\partial x}\right) \mu(v)\right) u}{\partial x}+\alpha_{2} p_{2} \frac{\partial u}{\partial x}=0 .
\end{aligned}
$$

The function $\mu(v)$ also expresses heat exchange and must be predetermined for a given twophase mixture. A method for its determination is given in Petitpas et al. (2007).

An example of the effects of function $\mu(v)$ is shown in the following example.

\section{Epoxy - Spinel mixture shock tube with artificial heat exchanges}

The test problem of Figure 18 is rerun with the artificial heat exchanges. The procedure developed in Petitpas et al. (2007) is used to determine the heat exchange function. This function depends on:

- The initial state of the mixture in which the shock propagates,

- The numerical smearing of the shock front that is inherent in a given method. 
For the present algorithm, the heat exchange function has been determined and is shown in Figure 19.

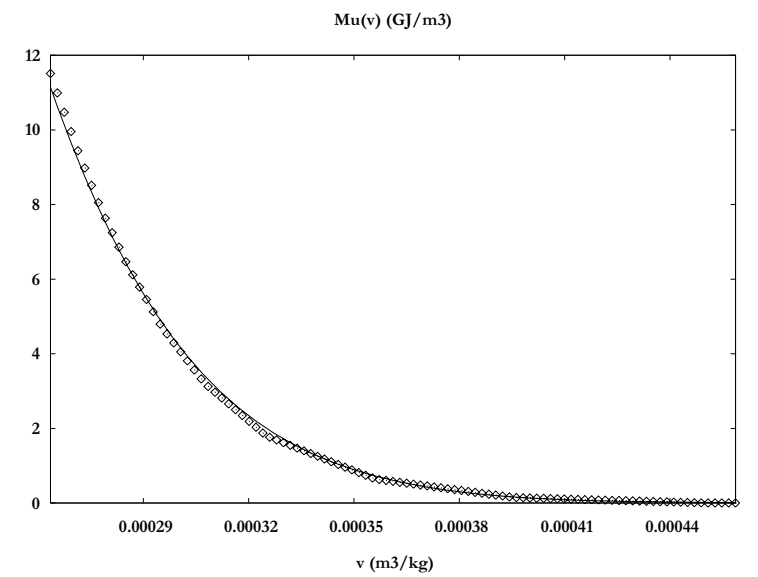

Figure 19: Values of the approximate piecewise linear function of $\mu$ (symbols) and fitting curve $\left(\bar{\mu}(\mathrm{v})=\exp \left(-5.6410^{7} \times \mathrm{v}^{2}+5.3410^{3} \times \mathrm{v}+25.6\right)\right)$ in the specific volume range $\left(2.65 \cdot 10^{-4} \sim 4.61 \cdot 10^{-4}\right.$ $\mathrm{m}^{3} / \mathrm{kg}$ ) corresponding to piston velocity range of $0 \sim 4200 \mathrm{~m} / \mathrm{s}$ and pressure range of $1 \sim 880000$ atm.

Artificial heat exchanges are used in the shock layer only. With this correction, the algorithm converges to the exact solution, as shown in Figure 20. 

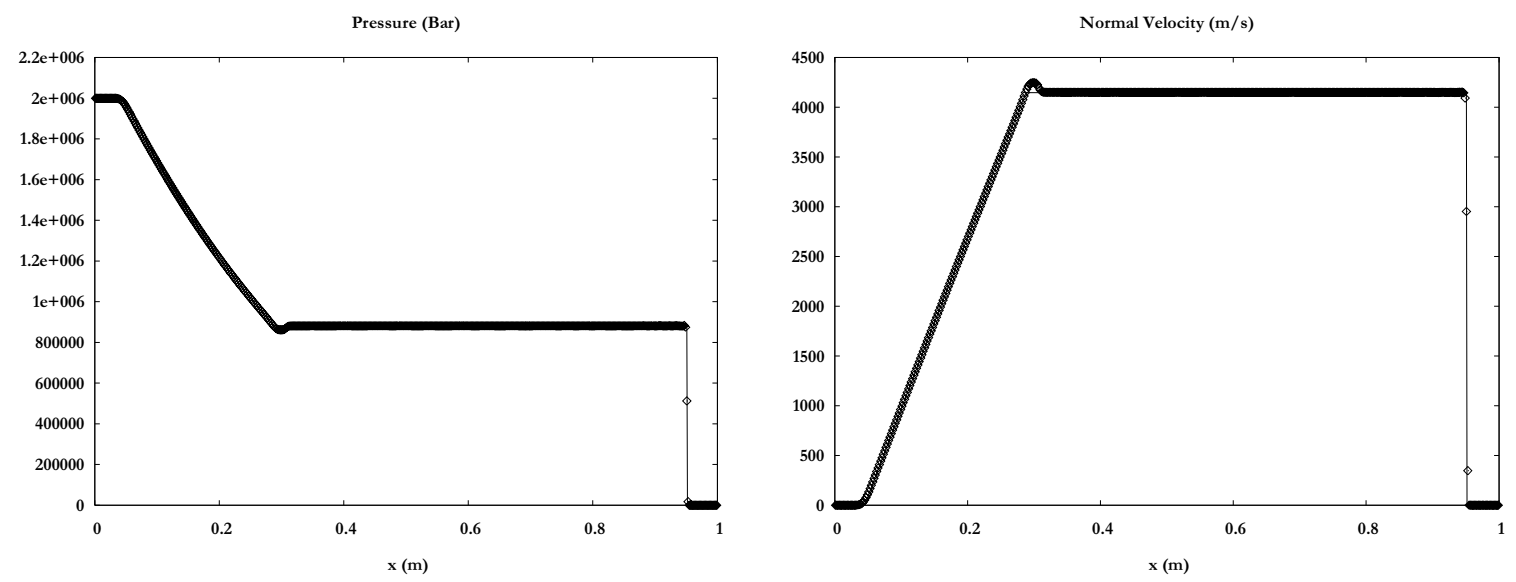

Mach Number
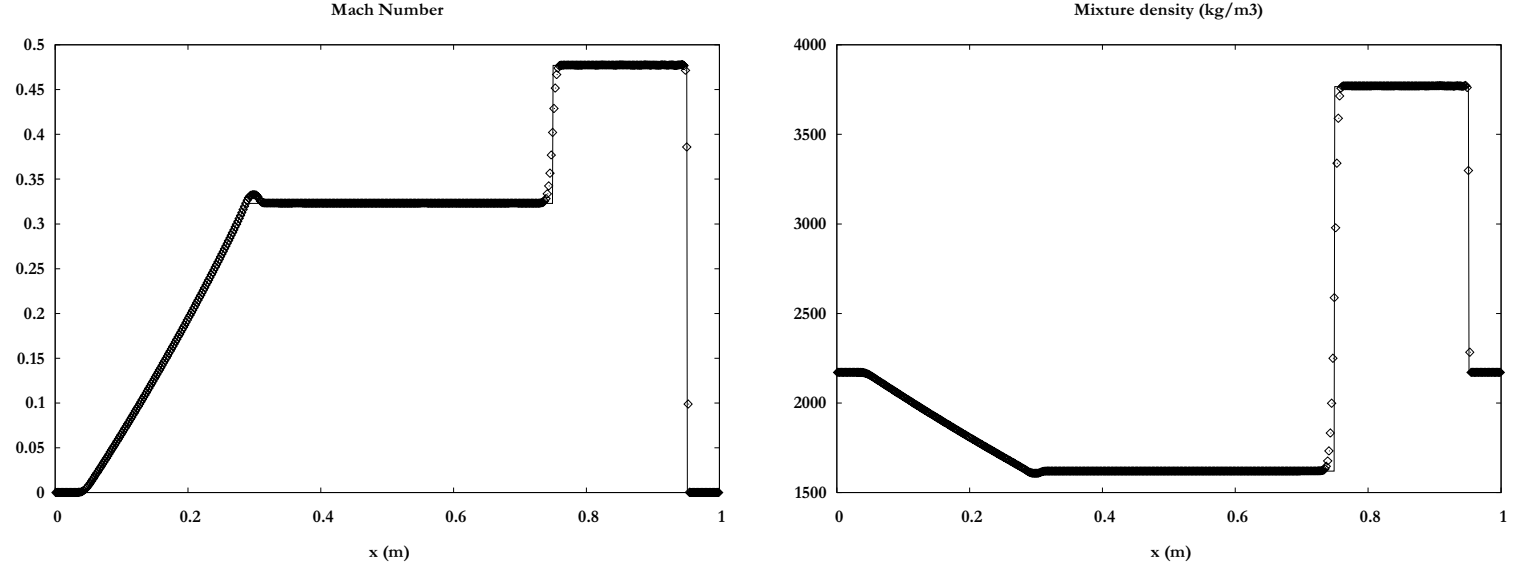

Volume Fraction of epoxy
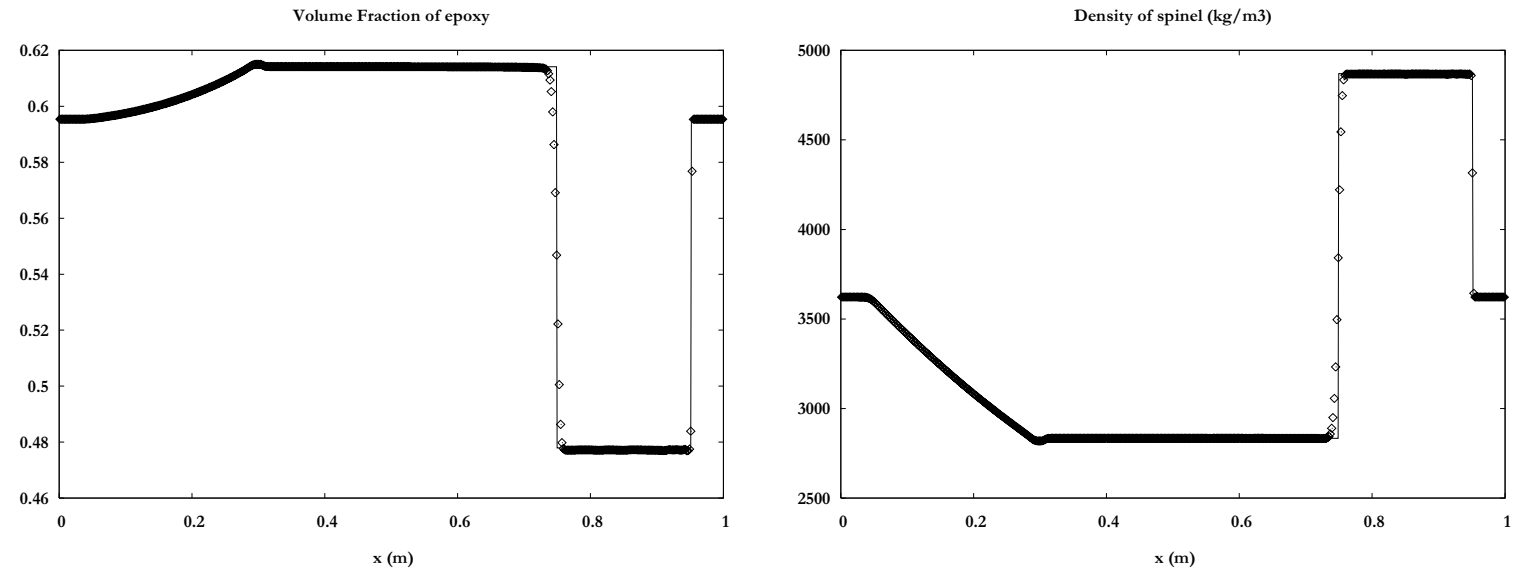

Figure 20: Epoxy-Spinel shock tube problem. The present relaxation method is used to solve the 6-equation model. Numerical results are shown with symbols and are compared to the exact solution of the 5-equation model (solid). A 500 cells mesh is used. The pressure ratio is 2 000000 at the initial discontinuity. Artificial heat exchanges are used in the shock layer only. Convergence of the results is obtained.

It is significant to note that the heat exchange function of Figure 19 provides converged results for any shock strength in the pressure range of $1 \sim 880000 \mathrm{~atm}$. Moreover, mesh independence of the solution is guaranteed. 


\section{Conclusion}

A relaxation hyperbolic model with 6 equations was built to solve interface problems, cavitating flows and shocks into mixtures. This model considerably simplifies the numerical approximation of the 5-equation model of Kapila et al. (2001). A simple, efficient and robust algorithm has been derived to solve the relaxation model. The various ingredients used by this method are general enough to consider future extensions to problems involving complex physics and large hyperbolic systems. In particular, solid-fluid coupling will be examined with the present multiphase modeling of diffuse interfaces in the context of the elastic model of Gavrilyuk et al. (2008).

\section{References}

Abgrall, R. (1996) How to prevent pressure oscillations in multicomponent flow calculations: A quasi conservative approach. Journal of Computational Physics, vol. 125, pp. 150-160

Abgrall, R. and Perrier, V. (2006) Asymptotic expansion of a multiscale numerical scheme for compressible multiphase flows. SIAM J. Multiscale and Modeling and Simulation (5), pp 84-115.

Baer, M.R. and Nunziato, J.W., (1986) A two-phase mixture theory for the deflagration-todetonation transition (DDT) in reactive granular materials. Int. J. of Multiphase Flow, vol. 12 (6), 861

Benson, D.J. (1992) Computational methods in Lagrangian and Eulerian hydrocodes. Computer Methods in Applied Mechanics and Engineering, vol. 99, pp. 235-394

Chinnayya, A., Daniel, E. and Saurel, R. (2004) Computation of detonation waves in heterogeneous energetic materials. Journal of Computational Physics, vol. 196, pp. 490-538

Cochran G. and Chan, J. (1979), Shock initiation and detonation models in one and two dimensions. CID-18024 Lawrence National Laboratory Report

Coutier-Delgosha, O., Fortes-Patella, R., Reboud, J.L., Hakimi, N. and Hirsch, C. (2005) Stability of preconditioned Navier-Stockes equations associated with a cavitation model. Computer and Fluids, 34, pp 319-349

Davis, S.F. (1988) Simplified second order Godunov type methods. SIAM J. Sci. Stat. Comput., 9: $445-473$

Dervieux, A. and Thomasset, F. (1980) A finite element method for the simulation of RayleighTaylor instability. In: Approximation methods for Navier-Stokes problems; Proceedings of the Symposium, Paderborn, West Germany, September 9-15, 1979. Springer-Verlag, Berlin, p. $145-158$.

Farhat, C. and Roux, F.X. (1991) A method for finite element tearing and interconnecting and its parallel solution algorithm. International Journal for Numerical Methods in Engineering, vol. 32,pp. 1205-1227

Favrie, N., Gavrilyuk, S. and Saurel, R. (2008) Diffuse solid-fluid interface model in cases of extreme deformations. Journal of Computational Physics, submitted

Fedkiw, R., Aslam, T., Merriman, B. and Osher, S. (1999) A Non-oscillatory Eulerian Approach to Interfaces in Multimaterial Flows (the Ghost Fluid Method). Journal of Computational Physics 152(2) , pp 457-492

Gavrilyuk, S., Favrie, N. and Saurel, R. (2008) Modelling wave dynamics of compressible elastic 
materials. Journal of Computational Physics 227(5), pp 2941-2969

Glimm, J., Grove, J.W., Li, X.L., Shyue, K.M., Zhang, Q. and Zeng, Y. (1998) Three dimensional front tracking. SIAM J. Scientific Computing, vol.19, pp. 703-727

Gueyffier, D., Li, L., Nadim, A., Scardovelli, R. and Zaleski, S. (1999) Volume-Of-Fluid Interface Tracking with Smoothed Surface Stress Methods for Three-Dimensional Flows. Journal of Computational Physics, vol. 152 , pp. 423-456

Hirt, C.W. and Nichols, B.D. (1981) Volume Of Fluid (VOF) method for the dynamics of free boundaries. Journal of Computational Physics, vol.39, pp. 201-255

Hirt, C.W., Amsden, A.A., and Cook , J.L. (1974) An Arbitrary Lagrangian Eulerian Computing Method for all flow Speeds. Journal of Computational Physics, vol.135, pp. 203-216

Kapila A.K., Menikoff R., Bdzil J.B., Son S.F. \& Stewart D.S. (2001) Two-phase modeling of deflagration-to-detonation transition in granular materials : Reduced equations. Physics of Fluids, 13 (10), pp. 3002-3024

Khoo, B.C., Liu, T.G., and Wang, C.W. The ghost fluid method for compressible gas-water simulations. Journal of Computational Physics, 204:193, 2005.

Koren, B., Lewis, M.R., van Brummelen, E.H. and van Leer, B. (2002) Riemann-problem and level-set approaches for homentropic two-fluid computations. Journal of Computational Physics 181, pp 654-674

Layes, G. and Le Métayer, O. (2007) Quantitative numerical and experimental studies of the shock accelerated heterogeneous bubbles motion. Physics of Fluids 19, 042105

Lee, E.L., Horning, H.C. and Kury, J.W. (1968) Adiabatic expansion of high explosives detonation products. Lawrence Radiation Laboratory, University of California, Livermore, TID 4500-UCRL 50422

Le Metayer, O., Massoni, J. and Saurel, R. (2005) Modeling evaporation fronts with reactive Riemann solvers. Journal of Computational Physics, 205, pp 567-610

LeVeque, R.J. and Keh-Ming Shyue (1996) Two-Dimensional Front Tracking Based on High Resolution Wave Propagation Methods. Journal of Computational Physics 123 (2), pp 354368

Massoni, J., Saurel, R., Baudin, G. and Demol, G. (1999) A mechanistic model for shock initiation of solid explosives. Physics of Fluids 11(3), pp 710-736

Miller, G.H. and Puckett, E.G. (1996) A High-Order Godunov Method for Multiple Condensed Phases. Journal of Computational Physics 128(1), pp 134-164

Miller, G.H. and Colella, P. (2001) A high-order Eulerian Godunov method for elastic-plastic flows in solids. J. Comp. Phys., 167, pp 131-176

Mulder, W., Osher, S. and Sethian, J.A. (1992) Computing interface motion : The compressible Rayleigh-Taylor and Kelvin-Helmholtz instabilities. Journal of Computational Physics, vol.100, p. 209

Murrone, A. and Guillard, H. (2005) A five equation reduced model for compressible two phase flow problems. Journal of Comp. Physics, 202 (2), pp. 664-698

Osher, S. and Fedkiw, R. (2001) Level Set Methods: An overview and some recent results. Journal of Computational Physics, vol. 169, pp. 463-502

Petitpas, F., Franquet, E., Saurel, R. and Le Metayer, O. (2007) A relaxation-projection method for compressible flows. Part II : The artificial heat exchange for multiphase shocks. Journal of Computational Physics 225 (2), pp 2214-2248

Perigaud, G. and Saurel, R. (2005) A compressible flow model with capillary effects. Journal of Computational Physics, Vol. 209, pp 139-178 
Saurel, R. and Abgrall, R. (1999) A multiphase Godunov method for multifluid and multiphase flows. Journal of Computational Physics 150, pp 425-467

Saurel, R. and Le Metayer, O. (2001) A multiphase model for interfaces, shocks, detonation waves and cavitation. Journal of Fluid Mechanics, Vol. 431, pp 239 - 271

Saurel, R., Gavrilyuk, S. and Renaud, F. (2003) A multiphase model with internal degrees of freedom: application to shock-bubble interaction. Journal of Fluid Mechanics, Vol. 495, pp 283-321

Saurel, R., Franquet, E., Daniel, E. and Le Metayer, O. (2007a) A relaxation-projection method for compressible flows. Part I : The numerical equation of state for the Euler equations. Journal of Computational Physics, vol 223(2), pp 822-845

Saurel, R. , Le Metayer, O., Massoni, J. and Gavrilyuk, S. (2007b) Shock jump relations for multiphase mixtures with stiff mechanical relaxation . Shock Waves, Vol. 16 (3), pp 209-232

Saurel, R., Petitpas, F., and Abgrall, R., (2008) Modeling phase transition in metastable liquids. Application to cavitating and flashing flows. Journal of Fluid Mechanics, 607: 313-350

Scheffer, D.R. and Zukas, J.A. (2000) Practical aspects of numerical simulation of dynamic events: material interfaces. International Journal of Impact Engineering, vol. 24, $\mathrm{n}^{\circ}$ 5-6, pp. $821-842$

Sethian, J. (2001) Evolution, Implementation and Application of Level Set and Fast Marching Methods for Advancing Fronts. Journal of Computational Physics, vol. 169, pp. 503-555

Sinibaldi, E. (2006) Implicit preconditioned numerical schemes for the simulation of threedimensional barotropic flows. PhD Thesis, Scuola Normale Superiore di Pisa, Italy

Stewart, H.B. and Wendroff, B. (1984) Two-phase flow: Models and methods. Journal of Computational Physics 56( 3), pp 363-409

Titarev, V.A., Romenski, E. and Toro, E.F. (2007) MUSTA type upwind fluxes for nonlinear elasticity. Int. J. Numerical Methods in Engineering 73(7), pp 897-926

Toro, E.F., Spruce, M. and Speares, W. (1994) Restoration of the contact surface in the HLL Riemann solver. Shock Waves, 4, pp 25-34

Toro E.F. (1997) Riemann solvers and numerical methods for fluids dynamics, Springer-Verlag, Berlin

van Brummelen, E.H. and Koren, B. (2003) A pressure-invariant conservative Godunov-type method for barotropic two-fluid flows. Journal of Computational Physics, Vol. 185, pp 289308

Wood, A.B. (1930) A textbook of sound. Bell Eds.

\section{Appendix A. Asymptotic limit of the 6-equation model in the presence of stiff pressure relaxation}

To perform the asymptotic analysis it is assumed that each flow variable $f$ obeys the following asymptotic expansion: $f=f^{\circ}+\varepsilon f^{1}$ where $f^{\circ}$ represents the equilibrium state and $f^{1}$ a small perturbation around this state. Inversely to the perturbations, pressure relaxation coefficient $\mu=\frac{\mu_{0}}{\varepsilon}$ is assumed stiff with $\varepsilon \rightarrow 0^{+}$. 
With this transformation, the equations that do not contain any relaxation parameter will be unchanged. The three equations to consider are thus the internal energy equations and the volume fraction equation. These are rewritten under following form:

$\frac{\mathrm{d} \alpha_{1}}{\mathrm{dt}}=\mu\left(\mathrm{p}_{1}-\mathrm{p}_{2}\right)$

$\alpha_{1} \rho_{1} \frac{\mathrm{de}_{1}}{\mathrm{dt}}+\alpha_{1} \mathrm{p}_{1} \frac{\partial \mathrm{u}}{\partial \mathrm{x}}=-\mathrm{p}_{\mathrm{I}} \mu\left(\mathrm{p}_{1}-\mathrm{p}_{2}\right)$,

$\alpha_{2} \rho_{2} \frac{\mathrm{de}_{2}}{\mathrm{dt}}+\alpha_{2} \mathrm{p}_{2} \frac{\partial \mathrm{u}}{\partial \mathrm{x}}=\mathrm{p}_{1} \mu\left(\mathrm{p}_{1}-\mathrm{p}_{2}\right)$,

where $\frac{\mathrm{d}}{\mathrm{dt}}=\frac{\partial}{\partial \mathrm{t}}+\mathrm{u} \frac{\partial}{\partial \mathrm{x}}$ represents the Lagrangian derivative.

Some transformations of these equations with appropriate variables are necessary before doing the asymptotic analysis. Consider the internal energy equation of phase 1 . It can be written as a pressure evolution equation as $\mathrm{e}_{1}=\mathrm{e}_{1}\left(\rho_{1}, \mathrm{p}_{1}\right)$ :

$\left.\left.\alpha_{1} \rho_{1}\left(\frac{\partial \mathrm{e}_{1}}{\partial \rho_{1}}\right)_{\mathrm{p}_{1}} \frac{\mathrm{d} \rho_{1}}{\mathrm{dt}}+\frac{\partial \mathrm{e}_{1}}{\partial \mathrm{p}_{1}}\right)_{\rho_{1}} \frac{\mathrm{dp} \mathrm{p}_{1}}{\mathrm{dt}}+\frac{\mathrm{p}_{1}}{\rho_{1}} \frac{\partial \mathrm{u}}{\partial \mathrm{x}}\right)=-\mathrm{p}_{\mathrm{I}} \mu\left(\mathrm{p}_{1}-\mathrm{p}_{2}\right)$

With the help of the phase 1 mass equation, $\frac{\mathrm{d} \alpha_{1} \rho_{1}}{\mathrm{dt}}+\alpha_{1} \rho_{1} \frac{\partial \mathrm{u}}{\partial \mathrm{x}}=0$,

that also reads, $\frac{\mathrm{d} \rho_{1}}{\mathrm{dt}}=-\frac{\rho_{1}}{\alpha_{1}} \mu\left(\mathrm{p}_{1}-\mathrm{p}_{2}\right)-\rho_{1} \frac{\partial \mathrm{u}}{\partial \mathrm{x}}$,

we get :

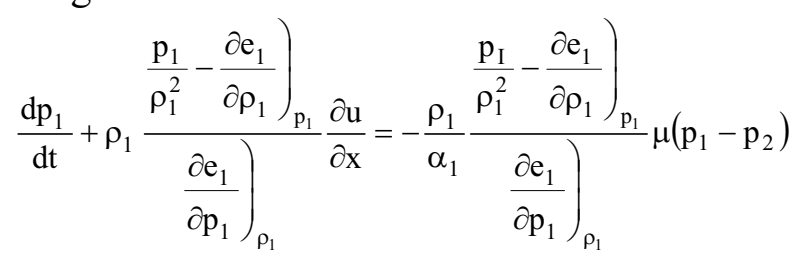

With the help of sound speed definitions,

$$
\begin{gathered}
\mathrm{c}_{1}^{2}=\frac{\left.\frac{\mathrm{p}_{1}}{\rho_{1}{ }^{2}}-\frac{\partial \mathrm{e}_{1}}{\partial \rho_{1}}\right)_{\mathrm{p}_{1}}}{\left.\frac{\partial \mathrm{e}_{1}}{\partial \mathrm{p}_{1}}\right)_{\rho_{1}}} \\
\mathrm{c}_{\mathrm{I1}}^{2}=\frac{\left.\frac{\mathrm{p}_{\mathrm{I}}}{\rho_{1}^{2}}-\frac{\partial \mathrm{e}_{1}}{\partial \rho_{1}}\right)_{\mathrm{p}_{1}}}{\left.\frac{\partial \mathrm{e}_{1}}{\partial \mathrm{p}_{1}}\right)_{\rho_{1}}}
\end{gathered}
$$

The phase 1 pressure evolution equation is obtained:

$\frac{\mathrm{dp}_{1}}{\mathrm{dt}}+\rho_{1} \mathrm{c}_{1}^{2} \frac{\partial \mathrm{u}}{\partial \mathrm{x}}=-\frac{\rho_{1} \mathrm{c}_{\mathrm{I}}^{2}}{\alpha_{1}} \mu\left(\mathrm{p}_{1}-\mathrm{p}_{2}\right)$

Regarding phase 2, a similar result is obtained: 
$\frac{\mathrm{dp}_{2}}{\mathrm{dt}}+\rho_{2} \mathrm{c}_{2}^{2} \frac{\partial \mathrm{u}}{\partial \mathrm{x}}=\frac{\rho_{2} \mathrm{c}_{\mathrm{I2}}^{2}}{\alpha_{2}} \mu\left(\mathrm{p}_{1}-\mathrm{p}_{2}\right)$

The asymptotic analysis is now carried out on the following system.

$\frac{\mathrm{d} \alpha_{1}}{\mathrm{dt}}=\mu\left(\mathrm{p}_{1}-\mathrm{p}_{2}\right)$

$\frac{\mathrm{dp}_{1}}{\mathrm{dt}}+\rho_{1} \mathrm{c}_{1}^{2} \frac{\partial \mathrm{u}}{\partial \mathrm{x}}=-\frac{\rho_{1} \mathrm{c}_{\mathrm{II}}^{2}}{\alpha_{1}} \mu\left(\mathrm{p}_{1}-\mathrm{p}_{2}\right)$

$\frac{\mathrm{dp}_{2}}{\mathrm{dt}}+\rho_{2} \mathrm{c}_{2}^{2} \frac{\partial \mathrm{u}}{\partial \mathrm{x}}=\frac{\rho_{2} \mathrm{c}_{\mathrm{I}}^{2}}{\alpha_{2}} \mu\left(\mathrm{p}_{1}-\mathrm{p}_{2}\right)$

By expanding each flow variable as $\mathrm{f}=\mathrm{f}^{\mathrm{o}}+\varepsilon \mathrm{f}^{1}$ we get:

- At order $\frac{1}{\varepsilon}$ :

$\mathrm{p}_{1}^{0}=\mathrm{p}_{2}^{0}=\mathrm{p}^{0}$

It implies on the one hand,

$\mathrm{p}_{\mathrm{I}}^{0}=\mathrm{p}^{0}$,

and on the other hand:

$\mathrm{c}_{\mathrm{I} 1}^{0^{2}}=\mathrm{c}_{1}^{0^{2}}$ and $\mathrm{c}_{\mathrm{I} 2}^{0^{2}}=\mathrm{c}_{2}^{0^{2}}$.

- At zero order the two pressure equations become:

$\frac{\mathrm{dp}^{0}}{\mathrm{dt}}+\rho_{1}^{0} \mathrm{c}_{1}^{0^{2}} \frac{\partial \mathrm{u}^{0}}{\partial \mathrm{x}}=-\frac{\rho_{1}^{0} \mathrm{c}_{1}^{0^{2}}}{\alpha_{1}^{0}}\left(\mathrm{p}_{1}^{1}-\mathrm{p}_{2}^{1}\right)$

$\frac{\mathrm{dp} \mathrm{p}^{0}}{\mathrm{dt}}+\rho_{2}^{0} \mathrm{c}_{2}^{0^{2}} \frac{\partial \mathrm{u}^{0}}{\partial \mathrm{x}}=\frac{\rho_{2}^{0} \mathrm{c}_{2}^{0^{2}}}{\alpha_{2}^{0}}\left(\mathrm{p}_{1}^{1}-\mathrm{p}_{2}^{1}\right)$

By taking the difference of these two equations, the pressure fluctuation difference is readily obtained:

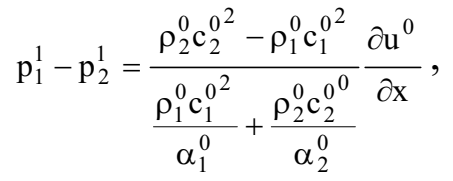

and the volume fraction equation thus becomes:

$$
\frac{\mathrm{d} \alpha_{1}^{0}}{\mathrm{dt}}=\frac{\rho_{2}^{0} \mathrm{c}_{2}^{0^{2}}-\rho_{1}^{0} \mathrm{c}_{1}^{0^{2}}}{\frac{\rho_{1}^{0} \mathrm{c}_{1}^{0^{2}}}{\alpha_{1}^{0}}+\frac{\rho_{2}^{0} \mathrm{c}_{2}^{0}}{\alpha_{2}^{0}}} \frac{\partial \mathrm{u}^{0}}{\partial \mathrm{x}} \text {. }
$$

Consequently the 5-equation model with mechanical equilibrium is recovered as the asymptotic limit of the 6-equation model in the presence of stiff pressure relaxation.

\section{Appendix B. Extension to second-order}

The first-order numerical method for the hyperbolic step presented in Section III is extended to second-order. It consists in solving the two-pressure 6-equation model (B.1) with a MUSCL type method: 


$$
\begin{aligned}
& \frac{\partial \alpha_{1}}{\partial \mathrm{t}}+\mathrm{u} \frac{\partial \alpha_{1}}{\partial \mathrm{x}}=0 \\
& \frac{\partial \alpha_{1} \rho_{1}}{\partial \mathrm{t}}+\frac{\partial \alpha_{1} \rho_{1} \mathrm{u}}{\partial \mathrm{x}}=0 \\
& \frac{\partial \alpha_{2} \rho_{2}}{\partial \mathrm{t}}+\frac{\partial \alpha_{2} \rho_{2} \mathrm{u}}{\partial \mathrm{x}}=0 \\
& \frac{\partial \rho \mathrm{u}}{\partial \mathrm{t}}+\frac{\partial \rho \mathrm{u}^{2}+\left(\alpha_{1} \mathrm{p}_{1}+\alpha_{2} \mathrm{p}_{2}\right)}{\partial \mathrm{x}}=0 \\
& \frac{\partial \alpha_{1} \rho_{1} \mathrm{e}_{1}}{\partial \mathrm{t}}+\frac{\partial \alpha_{1} \rho_{1} \mathrm{e}_{1} \mathrm{u}}{\partial \mathrm{x}}+\alpha_{1} \mathrm{p}_{1} \frac{\partial \mathrm{u}}{\partial \mathrm{x}}=0 \\
& \frac{\partial \alpha_{2} \rho_{2} \mathrm{e}_{2}}{\partial \mathrm{t}}+\frac{\partial \alpha_{2} \rho_{2} \mathrm{e}_{2} \mathrm{u}}{\partial \mathrm{x}}+\alpha_{2} \mathrm{p}_{2} \frac{\partial \mathrm{u}}{\partial \mathrm{x}}=0
\end{aligned}
$$

In the MUSCL method, the solution is assumed regular enough so that a primitive variable formulation can be used:

$$
\begin{array}{ll}
\frac{\partial \alpha_{1}}{\partial \mathrm{t}}+\mathrm{u} \frac{\partial \alpha_{1}}{\partial \mathrm{x}}=0 & \\
\frac{\partial \rho_{1}}{\partial \mathrm{t}}+\mathrm{u} \frac{\partial \rho_{1}}{\partial \mathrm{x}}+\rho_{1} \frac{\partial \mathrm{u}}{\partial \mathrm{x}}=0 & \\
\frac{\partial \rho_{2}}{\partial \mathrm{t}}+\mathrm{u} \frac{\partial \rho_{2}}{\partial \mathrm{x}}+\rho_{2} \frac{\partial \mathrm{u}}{\partial \mathrm{x}}=0 & \\
\frac{\partial \mathrm{u}}{\partial \mathrm{t}}+\mathrm{u} \frac{\partial \mathrm{u}}{\partial \mathrm{x}}+\frac{1}{\rho} \frac{\partial \mathrm{p}}{\partial \mathrm{x}}=0 & \text { or } \quad \frac{\partial \mathrm{u}}{\partial \mathrm{t}}+\mathrm{u} \frac{\partial \mathrm{u}}{\partial \mathrm{x}}+\frac{\mathrm{p}_{1}-\mathrm{p}_{2}}{\rho} \frac{\partial \alpha_{1}}{\partial \mathrm{x}}+\frac{\alpha_{1}}{\rho} \frac{\partial \mathrm{p}_{1}}{\partial \mathrm{x}}+\frac{\left(1-\alpha_{1}\right)}{\rho} \frac{\partial \mathrm{p}_{2}}{\partial \mathrm{x}}=0 \\
\frac{\partial \mathrm{p}_{1}}{\partial \mathrm{t}}+\mathrm{u} \frac{\partial \mathrm{p}_{1}}{\partial \mathrm{x}}+\rho_{1} \mathrm{c}_{1}^{2} \frac{\partial \mathrm{u}}{\partial \mathrm{x}}=0 & \\
\frac{\partial \mathrm{p}_{2}}{\partial \mathrm{t}}+\mathrm{u} \frac{\partial \mathrm{p}_{2}}{\partial \mathrm{x}}+\rho_{2} \mathrm{c}_{2}^{2} \frac{\partial \mathrm{u}}{\partial \mathrm{x}}=0 &
\end{array}
$$

Under compact form, this system reads:

$$
\frac{\partial \mathrm{W}}{\partial \mathrm{t}}+\mathrm{A}(\mathrm{W}) \frac{\partial \mathrm{W}}{\partial \mathrm{x}}=0
$$

With $W=\left(\alpha_{1}, \rho_{1}, \rho_{2}, \mathrm{u}, \mathrm{p}_{1}, \mathrm{p}_{2}\right)^{\mathrm{t}}$ and $\mathrm{A}(\mathrm{W})=\left(\begin{array}{cccccc}\mathrm{u} & 0 & 0 & 0 & 0 & 0 \\ 0 & \mathrm{u} & 0 & \rho_{1} & 0 & 0 \\ 0 & 0 & \mathrm{u} & \rho_{2} & 0 & 0 \\ \frac{\mathrm{p}_{1}-\mathrm{p}_{2}}{\rho} & 0 & 0 & \mathrm{u} & \frac{\alpha_{1}}{\rho} & \frac{1-\alpha_{1}}{\rho} \\ 0 & 0 & 0 & \rho_{1} \mathrm{c}_{1}^{2} & \mathrm{u} & 0 \\ 0 & 0 & 0 & \rho_{2} \mathrm{c}_{2}^{2} & 0 & \mathrm{u}\end{array}\right)$

Second-order extension consists in applying the following sequence of operations.

\section{Gradients limitation}

In a cell $\mathrm{i}$, at instant $\mathrm{t}^{\mathrm{n}}$, primitive variables $\mathrm{W}_{\mathrm{i}}^{\mathrm{n}}$ are known. Let us denote by $\Delta_{\mathrm{i}}^{-}$and $\Delta_{\mathrm{i}}^{+}$the gradients vector respectively on the left and right boundaries of cell $\mathrm{i}$. They are defined by: 
$\Delta_{\mathrm{i}}^{-}=\frac{\mathrm{W}_{\mathrm{i}}^{\mathrm{n}}-\mathrm{W}_{\mathrm{i}-1}^{\mathrm{n}}}{\Delta \mathrm{x}}$ and $\Delta_{\mathrm{i}}^{+}=\frac{\mathrm{W}_{\mathrm{i}+1}^{\mathrm{n}}-\mathrm{W}_{\mathrm{i}}^{\mathrm{n}}}{\Delta \mathrm{x}}$

A slope limiter function $\xi$ is used to prevent local extrema. Minmod, van Leer or Superbee limiters can be used. The limited slope is now $\Delta_{\mathrm{i}}=\xi\left(\Delta_{\mathrm{i}}^{-}, \Delta_{\mathrm{i}}^{+}\right)$.

\section{Variables extrapolation}

Within a given cell extrapolated primitive variable vectors $W_{i, L}$ and $W_{i, R}$ corresponding to the left and right boundary of cell i respectively are computed.

$$
\mathrm{W}_{\mathrm{i}, \mathrm{L}}^{\mathrm{n}}=\mathrm{W}_{\mathrm{i}}^{\mathrm{n}}-\frac{\Delta \mathrm{x}}{2} \Delta_{\mathrm{i}} \text { and } \mathrm{W}_{\mathrm{i}, \mathrm{R}}^{\mathrm{n}}=\mathrm{W}_{\mathrm{i}}^{\mathrm{n}}+\frac{\Delta \mathrm{x}}{2} \Delta_{\mathrm{i}}
$$

These variables are evolved during a half time step by:

$$
\mathrm{W}_{\mathrm{i}, \mathrm{L}, \mathrm{R}}^{\mathrm{n}+1 / 2}=\mathrm{W}_{\mathrm{i}, \mathrm{L}, \mathrm{R}}^{\mathrm{n}}+\frac{1}{2} \frac{\Delta \mathrm{t}}{\Delta \mathrm{x}} \mathrm{A}\left(\mathrm{W}_{\mathrm{i}}^{\mathrm{n}}\right)\left[\mathrm{W}_{\mathrm{i}, \mathrm{L}}^{\mathrm{n}}-\mathrm{W}_{\mathrm{i}, \mathrm{R}}^{\mathrm{n}}\right]
$$

\section{Riemann problem resolutions}

The Riemann problem is now computed at each cell boundary $i \pm 1 / 2$ allowing flux vectors $\mathrm{F}_{\mathrm{i} \pm 1 / 2}^{*}$ computation for conservative variables:

$F_{i-1 / 2}^{*}=F_{i-1 / 2}^{*}\left(W_{i-1, R}^{n+1 / 2} ; W_{i, L}^{n+1 / 2}\right)$ and $F_{i+1 / 2}^{*}=F_{i+1 / 2}^{*}\left(W_{i, R}^{n+1 / 2} ; W_{i+1, L}^{n+1 / 2}\right)$

It also provides the cell boundaries non-conservative variables:

$\alpha_{k, i \pm 1 / 2}^{*}\left(W_{i-1, R}^{n+1 / 2} ; W_{i, L}^{n+1 / 2}\right), u_{i \pm 1 / 2}^{*}\left(W_{i-1, R}^{n+1 / 2} ; W_{i, L}^{n+1 / 2}\right)$ and $(\alpha \rho e)_{k, i \pm 1 / 2}^{*}\left(W_{i-1, R}^{n+1 / 2} ; W_{i, L}^{n+1 / 2}\right)$

\section{Evolution step}

Once the inter-cells fluxes and non conservative variables are determined, the solution is evolved on the entire time step:

$$
\begin{aligned}
& \mathrm{U}_{\mathrm{i}}^{\mathrm{n}+1}=\mathrm{U}_{\mathrm{i}}^{\mathrm{n}}-\frac{\Delta \mathrm{t}}{\Delta \mathrm{x}}\left(\mathrm{F}_{\mathrm{i}+1 / 2}^{*}-\mathrm{F}_{\mathrm{i}-1 / 2}^{*}\right) \\
& \alpha_{1_{\mathrm{i}}}^{\mathrm{n}+1}=\alpha_{1_{\mathrm{i}}}^{\mathrm{n}}-\frac{\Delta \mathrm{t}}{\Delta \mathrm{x}}\left(\left(\mathrm{u} \alpha_{1}\right)_{\mathrm{i}+1 / 2}^{*}-\left(\mathrm{u} \alpha_{1}\right)_{\mathrm{i}-1 / 2}^{*}-\alpha_{1_{\mathrm{i}}}{ }^{\mathrm{n}}\left(\mathrm{u}_{\mathrm{i}+1 / 2}^{*}-\mathrm{u}_{\mathrm{i}-1 / 2}^{*}\right)\right) \\
& (\alpha \rho \mathrm{e})_{\mathrm{ki}}^{\mathrm{n}+1}=(\alpha \rho \mathrm{e})_{\mathrm{ki}}^{\mathrm{n}}-\frac{\Delta \mathrm{t}}{\Delta \mathrm{x}}\left((\alpha \rho e u)_{\mathrm{k}+1 / 2}^{*}-(\alpha \rho e \mathrm{u})_{\mathrm{ki}-1 / 2}^{*}+(\alpha \mathrm{p})_{\mathrm{ki}_{\mathrm{i}}}{ }^{\mathrm{n}}\left(\mathrm{u}_{\mathrm{i}+1 / 2}^{*}-\mathrm{u}_{\mathrm{i}-1 / 2}^{*}\right)\right),
\end{aligned}
$$

where the "*" variables are given by (A.3) and (A.4).

\section{Appendix C. Extension to multi-dimensions}

The method is extended to multi-dimensions by a finite volume method able to deal with structured and unstructured meshes. Thus, let us consider a control volume $\mathrm{V}_{\mathrm{i}}$ enclosed by a boundary surface A of normal unit vector $\vec{n}$. The conservative part of system (III.1) under integral form reads:

$\frac{\partial}{\partial \mathrm{t}} \int_{\mathrm{V}_{\mathrm{i}}} \mathrm{U}+\int_{\mathrm{A}} \mathrm{H} \bullet \overrightarrow{\mathrm{n}} \mathrm{dA}=0$ 
with $\mathrm{U}=\left((\alpha \rho)_{1},(\alpha \rho)_{2}, \rho \mathrm{u}, \rho \mathrm{v}, \rho \mathrm{E}\right)^{\mathrm{T}}$ the conservative variable vector, and $\mathrm{H}=(\mathrm{F}, \mathrm{G})$ the tensor of fluxes where:

$\mathrm{F}=\left((\alpha \rho)_{1} \mathrm{u},(\alpha \rho)_{1} \mathrm{u}, \rho \mathrm{u}^{2}+\mathrm{p}, \rho \mathrm{uv},(\rho \mathrm{E}+\mathrm{p}) \mathrm{u}\right)^{\mathrm{T}}$,

$\mathrm{G}=\left((\alpha \rho)_{1} \mathrm{v},(\alpha \rho)_{1} \mathrm{v}, \rho \mathrm{uv}, \rho \mathrm{v}^{2}+\mathrm{p},(\rho \mathrm{E}+\mathrm{p}) \mathrm{v}\right)^{\mathrm{T}}$,

with $\mathrm{E}=\mathrm{Y}_{1} \mathrm{e}_{1}+\mathrm{Y}_{2} \mathrm{e}_{2}+\frac{1}{2} \mathrm{u}^{2}+\frac{1}{2} \mathrm{v}^{2}$ and $\mathrm{p}=\alpha_{1} \mathrm{p}_{1}+\alpha_{2} \mathrm{p}_{2}$.

Boundary $A$ of $V_{i}$ is the union of $N$ straight segments $\left[A_{s} A_{s+1}\right]$, where $A_{N+1}=A_{1}$.

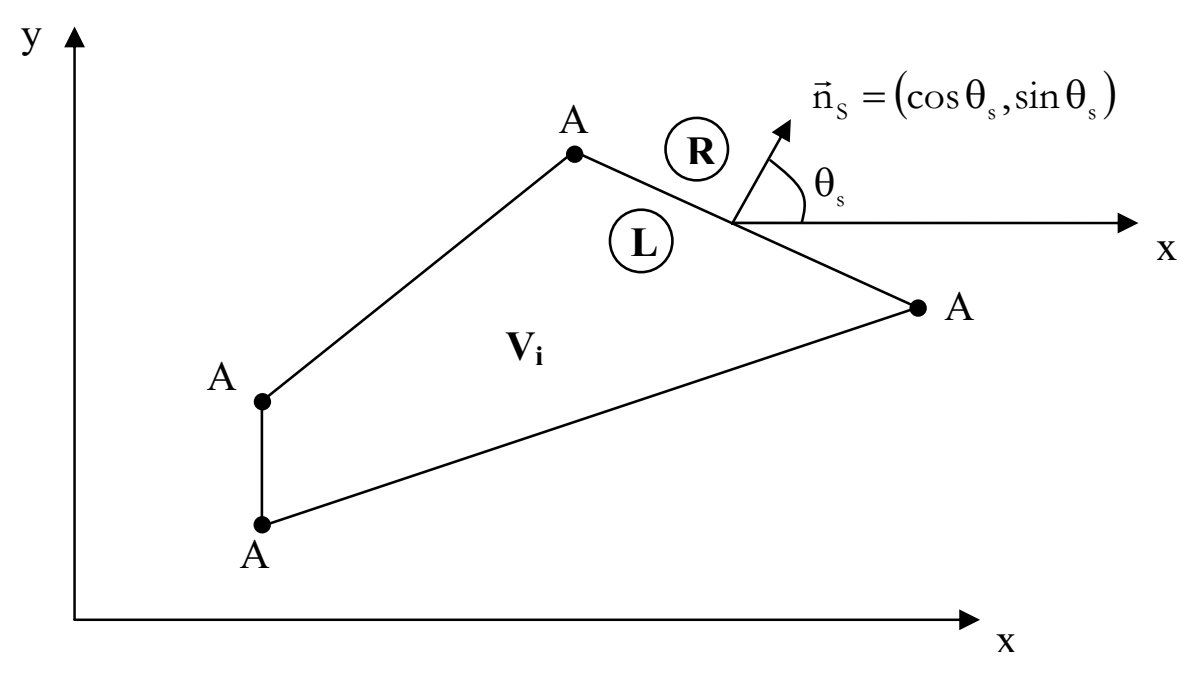

The first term of equation (C.1) is simply interpreted as time-rate of change of the average of the conservative vector $\overline{\mathrm{U}}$ inside volume $\mathrm{V}$ :

$\frac{\partial}{\partial \mathrm{t}} \int_{\mathrm{V}_{\mathrm{i}}} \mathrm{U}=\mathrm{V}_{\mathrm{i}} \frac{\partial \overline{\mathrm{U}}}{\partial \mathrm{t}}$

As the normal unit vector is expressed by $\vec{n}_{S}=\left(\cos \theta_{s}, \sin \theta_{s}\right)$, the second term of (C.1) becomes:

$\int_{\mathrm{A}} \mathrm{H} \bullet \overrightarrow{\mathrm{n}} \mathrm{dA}=\sum_{\mathrm{s}=1}^{\mathrm{N}} \int_{A_{\mathrm{s}}}^{\mathrm{A}_{\mathrm{s}+1}} \mathrm{H} \bullet \overrightarrow{\mathrm{n}}_{\mathrm{S}} \mathrm{dA}=\sum_{\mathrm{s}=1}^{\mathrm{N}} \int_{A_{\mathrm{s}}}^{\mathrm{A}_{\mathrm{s}+1}}\left(\mathrm{~F} \cdot \cos \theta_{\mathrm{s}}+\mathrm{G} \cdot \sin \theta_{\mathrm{s}}\right) \mathrm{dA}$.

Assuming that the fluxes are constant along each segment, it becomes:

$\int_{\mathrm{A}} \mathrm{H} \bullet \overrightarrow{\mathrm{n}} \mathrm{dA}=\sum_{\mathrm{s}=1}^{\mathrm{N}} \mathrm{L}_{\mathrm{s}}\left(\mathrm{F}_{\mathrm{s}} \cdot \cos \theta_{\mathrm{s}}+\mathrm{G}_{\mathrm{s}} \cdot \sin \theta_{\mathrm{s}}\right)$

where $L_{s}$ is the length of segment $\left[A_{s} A_{s+1}\right]$.

After time integration, the evolution of the conservative part of system (III.1) is given for cell $i$ by the scheme: 


$$
\mathrm{U}_{\mathrm{i}}^{\mathrm{n}+1}=\mathrm{U}_{\mathrm{i}}^{\mathrm{n}}-\frac{\Delta \mathrm{t}}{\mathrm{V}_{\mathrm{i}}} \sum_{\mathrm{s}=1}^{\mathrm{N}} \mathrm{L}_{\mathrm{s}}\left(\mathrm{F}_{\mathrm{s}}^{*} \cdot \cos \theta_{\mathrm{s}}+\mathrm{G}_{\mathrm{s}}^{*} \cdot \sin \theta_{\mathrm{s}}\right)
$$

Where $F_{s}^{*}$ and $G_{s}^{*}$ represent the fluxes solution of the Riemann problem between states $L$ and $R$ separated by the segment $\left[A_{s} A_{s+1}\right]$ with respect to normal $\vec{n}$.

The scheme for the non-conservative volume fraction equation becomes, $\alpha_{\mathrm{k}, \mathrm{i}}^{\mathrm{n}+1}=\alpha_{\mathrm{k}, \mathrm{i}}^{\mathrm{n}}-\frac{\Delta \mathrm{t}}{\mathrm{V}_{\mathrm{i}}} \sum_{\mathrm{s}=1}^{\mathrm{N}} \mathrm{L}_{\mathrm{s}}\left[\left(\mathrm{u} \alpha_{\mathrm{k}}\right)_{\mathrm{s}}^{*} \cos \theta_{\mathrm{s}}+\left(\mathrm{v} \alpha_{\mathrm{k}}\right)_{\mathrm{s}}^{*} \sin \theta_{\mathrm{s}}-\alpha_{\mathrm{k}, \mathrm{i}}^{\mathrm{n}}\left(\mathrm{u}_{\mathrm{s}}^{*} \cos \theta_{\mathrm{s}}+\mathrm{v}_{\mathrm{s}}^{*} \sin \theta_{\mathrm{s}}\right) \cdot\right]$,

and for the non-conservative energy equations it is:

$$
(\alpha \rho e)_{\mathrm{k}, \mathrm{i}}^{\mathrm{n}+1}=(\alpha \rho \mathrm{e})_{\mathrm{k}, \mathrm{i}}^{\mathrm{n}}-\frac{\Delta \mathrm{t}}{\mathrm{V}_{\mathrm{i}}} \sum_{\mathrm{s}=1}^{\mathrm{N}} \mathrm{L}_{\mathrm{s}}\left[\left((\alpha \rho \mathrm{e})_{\mathrm{k}} \mathrm{u}\right)_{\mathrm{s}}^{*} \cos \theta_{\mathrm{s}}+\left((\alpha \rho \mathrm{e})_{\mathrm{k}} \mathrm{v}\right)_{\mathrm{s}}^{*} \sin \theta_{\mathrm{s}}+(\alpha \mathrm{p})_{\mathrm{k}, \mathrm{i}}^{\mathrm{n}}\left(\mathrm{u}_{\mathrm{s}}^{*} \cos \theta_{\mathrm{s}}+\mathrm{v}_{\mathrm{s}}^{*} \sin \theta_{\mathrm{s}}\right)\right] .
$$

\section{Appendix D. The link between the 6-equation model and conventional barotropic cavitating flows}

Barotropic flow models are very popular in cavitation modeling. They are composed of one or two mass conservation equations and one momentum equation. They consist in hyperbolic systems of conservation laws. These models involve an important difficulty related to the nonmonotonic behavior of the sound speed versus volume fraction (Koren et al., 2002, CoutierDelgosha, 2005, Sinibaldi, 2006). It is thus interesting to examine how the various ingredients developed in the context of the 6-equation model can be used for these barotropic models in order to solve this difficulty.

A well posed barotropic flow model for cavitating flows can be obtained by simplifying the 5equation model of Kapila et al. (2001). In cavitating flows, shocks are assumed absent or weak, even if there is no evidence regarding this assumption. A first simplification consists in replacing the volume fraction and energy equations by entropy equations:

$$
\begin{aligned}
& \frac{\partial s_{1}}{\partial t}+u \frac{\partial s_{1}}{\partial x}=0 \\
& \frac{\partial s_{2}}{\partial t}+u \frac{\partial s_{2}}{\partial x}=0 \\
& \frac{\partial(\alpha \rho)_{1}}{\partial t}+\frac{\partial(\alpha \rho)_{1} u}{\partial x}=0 \\
& \frac{\partial(\alpha \rho)_{2}}{\partial t}+\frac{\partial(\alpha \rho)_{2} u}{\partial x}=0 \\
& \frac{\partial \rho u}{\partial t}+\frac{\partial \rho u^{2}+p}{\partial x}=0
\end{aligned}
$$

This system is closed by the pressure equilibrium condition:

$\mathrm{p}_{1}\left(\rho_{1}, \mathrm{~s}_{1}\right)=\mathrm{p}_{2}\left(\rho_{2}, \mathrm{~s}_{2}\right)=\mathrm{p}$.

Solution of this equation gives the volume fraction, and consequently the pressure $\mathrm{p}$. 
An extra assumption is used in conventional barotropic cavitating flow models. The entropies are assumed constant in the entire domain and not only along fluid's trajectories. The two entropy equations thus reduce to

$\mathrm{s}_{\mathrm{k}}=\mathrm{s}_{\mathrm{k}}^{0}, \mathrm{k}=1,2$.

The barotropic flow model thus reduces to three conservation equations:

$$
\begin{aligned}
& \frac{\partial(\alpha \rho)_{1}}{\partial \mathrm{t}}+\frac{\partial(\alpha \rho)_{1} \mathrm{u}}{\partial \mathrm{x}}=0 \\
& \frac{\partial(\alpha \rho)_{2}}{\partial \mathrm{t}}+\frac{\partial(\alpha \rho)_{2} \mathrm{u}}{\partial \mathrm{x}}=0 \\
& \frac{\partial \rho \mathrm{u}}{\partial \mathrm{t}}+\frac{\partial \rho \mathrm{u}^{2}+\mathrm{p}}{\partial \mathrm{x}}=0 .
\end{aligned}
$$

To illustrate the thermodynamic closure of this model, let us assume that each phase obeys the stiffened gas EOS (II.3). The isentropes become,

$\frac{p_{k}+p_{\infty \mathrm{k}}}{\rho_{\mathrm{k}}^{\gamma_{\mathrm{k}}}}=\frac{\mathrm{p}_{0}+\mathrm{p}_{\infty \mathrm{k}}}{\rho_{0 \mathrm{k}}^{\gamma_{\mathrm{k}}}}$,

and correspond to the Tait EOS.

The isentropic stiffened gas EOS (or Tait EOS), can be derived for any pure liquid and any ideal gas. It is a function of the phase density only

$\mathrm{p}_{\mathrm{k}}=\mathrm{p}_{\mathrm{k}}^{\mathrm{S}}\left(\rho_{\mathrm{k}}\right)=\left(\frac{\rho_{\mathrm{k}}}{\rho_{0 \mathrm{k}}}\right)^{\gamma_{\mathrm{k}}}\left(\mathrm{p}_{0}+\mathrm{p}_{\infty \mathrm{k}}\right)-\mathrm{p}_{\infty \mathrm{k}}$.

System (D.3) is thus closed by the relation

$\mathrm{p}_{1}^{\mathrm{S}}\left(\rho_{1}\right)=\mathrm{p}_{2}^{\mathrm{S}}\left(\rho_{2}\right)$.

In other words, the mixture evolves in mechanical equilibrium with isentropic evolutions for each phase. This assumption is valid provided that boundary layers, heat and mass transfer, and shock waves have negligible influence.

With the use of the isentropic stiffened gas EOS the equilibrium condition (D.5) reduces to a function the volume fraction only:

$$
f\left(\alpha_{1}\right)=\left(\frac{(\alpha \rho)_{1}}{\alpha_{1} \rho_{01}}\right)^{\gamma_{1}}\left(p_{0}+p_{\infty 1}\right)-p_{\infty 1}-\left(\frac{(\alpha \rho)_{2}}{\left(1-\alpha_{1}\right) \rho_{02}}\right)^{\gamma_{2}}\left(p_{0}+p_{\infty 2}\right)+p_{\infty 2}=0 \text {. }
$$

Its resolution gives $\alpha_{1}$, then $\rho_{1}$ as well as the pressure with the help of one of the EOS (D.4).

This model assumes that cavitation does not result from mass transfer. Cavitation pockets appear as the volume fraction increases for a small amount of gas present initially. Cavitation is thus modeled as a mechanical relaxation process, occurring at infinite rate, and not as a mass transfer process. This corresponds to a simplified limit situation compared to reality. It also presents a deficiency when pure liquid is present. Heat and mass transfers have been introduced in the 5equation model (Saurel et al., 2008) in order to deal with more realistic cavitating situations. Furthermore, the barotropic flow model, in reduced form (D.3), involves the same numerical difficulties as the 5-equation model. The sound speed for this model still obeys Wood's formulas, whose non-monotonic behavior was shown in Figure 1. 
To circumvent these difficulties, especially due to the non-monotonic behavior of the sound speed, we adapt the strategy developed in the context of the 6-equation model to this simplified situation.

\section{A relaxation model for the barotropic cavitating flow model}

The non-monotonic behavior of the sound speed that causes computational difficulties comes from the equilibrium condition (D.5). Following the analysis of Section II, a relaxation model can be built:

$$
\begin{aligned}
& \frac{\partial \alpha_{1}}{\partial \mathrm{t}}+\mathrm{u} \frac{\partial \alpha_{1}}{\partial \mathrm{x}}=\mu\left(\mathrm{p}_{1}^{\mathrm{S}}-\mathrm{p}_{2}^{\mathrm{s}}\right) \\
& \frac{\partial(\alpha \rho)_{1}}{\partial \mathrm{t}}+\frac{\partial(\alpha \rho)_{1} \mathrm{u}}{\partial \mathrm{x}}=0 \\
& \frac{\partial(\alpha \rho)_{2}}{\partial \mathrm{t}}+\frac{\partial(\alpha \rho)_{2} \mathrm{u}}{\partial \mathrm{x}}=0 \\
& \frac{\partial \rho \mathrm{u}}{\partial \mathrm{t}}+\frac{\partial \rho \mathrm{u}^{2}+\alpha_{1} \mathrm{p}_{1}+\alpha_{2} \mathrm{p}_{2}}{\partial \mathrm{x}}=0
\end{aligned}
$$

Because the model includes pressure non-equilibrium effects, the momentum equation involves pressures from both phases. This model is the isentropic analogue of the 6-equation model. Unlike the preceding models, the present one has a monotonic sound speed given by:

$c_{\mathrm{f}}^{2}=\mathrm{Y}_{1} \mathrm{c}_{1}^{2}+\mathrm{Y}_{2} \mathrm{c}_{2}^{2}$.

It is not difficult to show that in the asymptotic limit $\mu \rightarrow+\infty$ this model corresponds to system (D.3) with thermodynamic closure (D.5).

The numerical method to solve System (D.7) is a simplification of the method developed in Section III. It can be summarized as follows:

- At each cell boundary solve the Riemann problem of System (D.7) without relaxation terms with favorite solver. The HLLC solver of Section III.1 is recommended.

- Evolve all flow variables $\mathrm{W}=\left(\alpha_{1},(\alpha \rho)_{1},(\alpha \rho)_{2}, \rho \mathrm{u}\right)$ with the Godunov type method of Section (III.2).

- Determine the relaxed pressure and especially the volume fraction by solving Equation (D. 6). The Newton method is appropriate for this task.

- Go to the first item for the next time step. 


\section{Chapter 4 \\ Toward a Low-Speed Flow Solver for Diffuse Interface Models}




\section{Introduction}

The multiphase formulations developed in the RS2N Company have the ability to solve problems involving both heterogeneous mixtures of materials and interfacial flows involving shock waves, explosions, cavitation, phase transition, combustion etc. These formulations, and in particular the ones used for the direct numerical simulation (DNS) of interfacial flows present interests in the frame of nuclear reactor safety and optimization. The aim is to compute the critical heat flux conditions that involve a competition between bubble growth, surface tension, contact angle effects, heat and mass transfers at interfaces. Contrarily to the approach developed by other authors, a general model is expected, that accounts in particular complete thermodynamics in both phases.

This topic is of prime importance for the nuclear industry and it is the reason why collaboration between RSN2 and INL started a few months ago. INL has a large experience of low speed flows and also in Finite Element Methods (FEM), and its knowledge in multiphase flow is very complementary with RS2N knowledge.

In this context, the aim is to couple both competences for the building of a simple and accurate numerical scheme able to solve DNS models of interfacial flows at low speed. It poses the difficult question of the numerical approximation of compressible flow models in the incompressible limit.

In this report we present the first results of the collaboration between both parts (INL and RS2N): the main idea is to verify that the low speed flow methods developed at INL [1] can be adapted to Finite Volume framework. This last family of methods is more suitable for the numerical approximation of hyperbolic models of diffuse interfaces with complex physics.

In a first step, a single-phase flow study is conducted to verify the possible extension of low speed flow model in FV methods. The extension is done on the basis of mixed Godunov type and PCICE [1] ingredients. The obtained results are quite excellent: both low speed and high speed flows can be computed accurately.

The second part deals with a more sophisticated hyperbolic model of diffuse interface, also examined with the combined Godunov-PCICE method.

\section{Basis of Finite Volume methods}

\subsection{Introduction}


The PCICE method described in Ref. [1] has very appealing characteristics, the most important being its ability to solve low speed flows with fully compressible models. Regarding diffuse interface formulation of two-phase flows, a Finite Volume formulation is preferred to the original Finite Element one in which the PCICE method was developed. Indeed, with FV method based on Riemann solvers, a simpler control of numerical diffusion can be done. This control is particularly important with diffuse interface formulations, when each fluid is governed by a specific equation of state.

\subsection{The basis of Finite Volume method}

We suppose a general system of equations - for example the Euler equations - that can be written in a conservative form:

$\frac{\partial U}{\partial t}+\frac{\partial F(U)}{\partial x}=0$

The solution is computed over a domain $\Omega$ in the x-direction, in the range $[0, L]$. The domain $\Omega$ is divided in control volumes; the length of each volume being equal to $\Delta x$. $U$ is the vector of unknowns depending on the space coordinates $x$ and the time $t, \mathrm{~F}$ is the flux vector.

The solution is sought as the result of the integral over $\Omega$ and over the time range $T=\left[t_{1}: t_{2}\right]$. For any $\left(x_{1}, x_{2}\right)$ in $\Omega$ :

$$
\int_{x_{1}}^{x_{2}} \int_{t_{1}}^{t_{2}} \frac{\partial U}{\partial t}+\frac{\partial F(U)}{\partial x} d x d t=0
$$

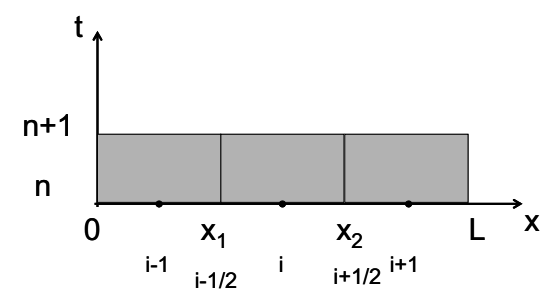

Figure 1 : Mesh and timestep used in Finite Volume methods

This integral can be calculated as follow:

$$
\left.\left.\int_{x_{1}}^{x_{2}} U\left(x, t_{2}\right)\right) d x-\int_{x_{1}}^{x_{2}} U\left(x, t_{1}\right)\right) d x+\int_{t_{1}}^{t_{2}} F\left(x, t_{2}\right) d t-\int_{t_{1}}^{t_{2}} F\left(x, t_{1}\right) d t=0
$$

Let us pose that $\Delta x=\left|x_{2}-x_{1}\right|$ and we assume that in this volume the quantity $U$ is constant, we can then define:

$$
\left.U_{i}^{n}=\frac{1}{\Delta x} \int_{x_{1}}^{x_{2}} U\left(x, t_{1}\right)\right) d x
$$

Then, the integral (3) is: 
$U_{i}^{n+1}=U_{i}^{n}-\frac{1}{\Delta x}\left(\int_{t_{1}}^{t_{2}} F\left(x_{2}, t\right) d t-\int_{t_{1}}^{t_{2}} F\left(x_{1}, t\right) d t\right)$

By the same way as for the conservative variables, let us define an average flux:

$F_{i-1 / 2}^{n}=\frac{1}{\Delta t} \int_{t_{1}}^{t_{2}} F\left(x_{1}, t\right) d t$

We obtain the explicit finite volume scheme:

$U_{i}^{n+1}=U_{i}^{n}-\frac{\Delta t}{\Delta x}\left(F_{i+1 / 2}^{n}-F_{i-1 / 2}^{n}\right)$

$F_{i+1 / 2}^{n}$ is the numerical flux at the intercell located at the index $i+1 / 2$. The value of this quantity is obtained by the means of exact or approximate Riemman solvers, which depends on the system of equations.

It is noticeable that a restriction condition exists for the value of the timestep $\Delta t$. We admit that the system of equations (1) is hyperbolic, it means that we can find as many different and real eigenvalues as the number of unknowns. Each eigenvalue is named $\lambda_{k}$ and corresponds to the velocity of waves. For example, it is well-known that the Euler equations admit 3 eigenvalues which are $\mathrm{u}, \mathrm{u}+\mathrm{c}$ and $\mathrm{u}-\mathrm{c}$ with $\mathrm{u}$ the fluid velocity and $\mathrm{c}$ is the sound speed. The conservative variables may vary only across theses waves.

Let us consider a numerical cell at the index $i$, delimited by the intercell $i-1 / 2$ and $i+1 / 2$. The quantity inside the cell is $U_{i}^{n}$ which is a constant. Consider the wave evolving with the highest and the lowest speed, $\lambda_{\max }$ and $\lambda_{\text {min }}$ respectively from a cell boundary as depicted in figure 2 . These both waves define a triangle of influence underneath the state is not perturbated.

It can be demonstrated a stability condition for a hyperbolic system, meaning that the wave from i$1 / 2$ must not go further than the opposite cell boundary at $i+1 / 2$. This yields a timestep condition:

$\Delta t \leq \frac{\Delta x}{\left|\lambda_{\max }\right|}$

In the case of the Euler equations, it is the well-known CFL condition:

$\Delta t \leq \frac{\Delta x}{|u+c|}$ 


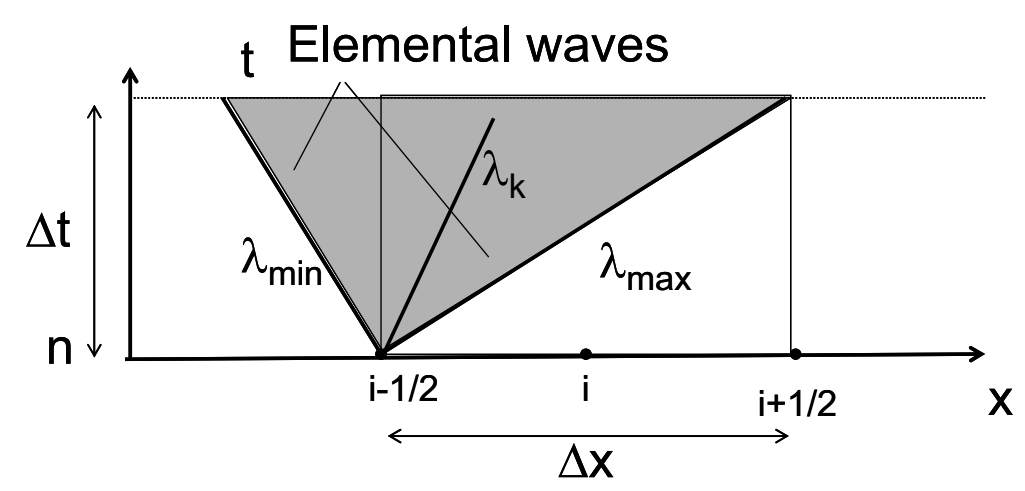

Figure 2 : General wave pattern for an hyperbolic system

Because of the definition of the average quantities, it also implies for the value of the flux calculated at time $t^{*}$ remains constant as soon as the time $t^{*}$ is in the range $[t, t+\Delta t]$.

These considerations about the timestep are of prime importance. Indeed, when compressible flows are consider the timestep must obey the CFL condition (7). The problem for low speed compressible flows is that the order of magnitude of the material velocity $u$ can be much lower than the sound velocity. The numerical solution is then driven by the acoustic wave $\Delta t \leq \frac{\Delta x}{|u+c|} \approx \frac{\Delta x}{|c|}$ although the physical problem is driven by the material velocity. Such scheme is often not accurate enough.

The main advantage of the PCICE method is that the time step is restricted by a convective Courant condition:

$$
\Delta \mathrm{t} \leq \frac{\Delta \mathrm{x}}{|\mathrm{u}|}
$$

The aim of this study is to translate the PCICE formulation in a Finite Volume formulation, to benefit advantages of both methods.

\section{The PCICE method ant its extensions for Euler equations}

\section{$\underline{3.1 \text { Introduction }}$}

The PCICE method for solving the Euler equations is based on 3 stages. The Euler equations are:

$$
\begin{aligned}
& \frac{\partial \rho}{\partial t}+\vec{\nabla} \cdot \rho \vec{u}=0 \\
& \frac{\partial \rho \vec{u}}{\partial t}+\vec{\nabla} \cdot\left(\rho \overrightarrow{u u}+p \overline{\bar{I}}_{d}\right)=0
\end{aligned}
$$


$\frac{\partial \rho E}{\partial t}+\vec{\nabla} \cdot \rho \vec{u}\left(E+\frac{p}{\rho}\right)=0$

where $\mathrm{E}$ is the total energy : $\mathrm{E}=\varepsilon+\frac{1}{2} u^{2}$ with $\varepsilon=\varepsilon(p, \rho)$.

In the original PCICE method the first stage consists in solving a modified system of equations:

$$
\begin{aligned}
& \frac{\partial \rho}{\partial t}+\vec{\nabla} \cdot \rho \vec{u}=0 \\
& \frac{\partial \rho \vec{u}}{\partial t}+\vec{\nabla} \cdot \rho \vec{u} \vec{u}=0 \\
& \frac{\partial \rho E}{\partial t}+\vec{\nabla} \cdot \rho \vec{u}\left(E+\frac{p}{\rho}\right)=0
\end{aligned}
$$

From the discrete formulation of this system combined with the EOS one can obtain a Poisson equation for the pressure: The knowledge of the pressure at the final stage leads to the correction of the quantities that have been predicted in the predictor stage.

The main goal of this study is to use a Finite Volume scheme to solve the predictor stage, with the help of Godunov type ingredients. The corresponding method will be called PCICE-VF.

\subsection{The PCICE-VF method}

The target system is the following:

$$
\begin{aligned}
& \rho^{n+1}=\rho^{n}-\frac{\Delta t}{2} \vec{\nabla} \cdot\left(\rho \vec{u}^{n}+\rho \vec{u}^{n+1}\right) \\
& \rho \vec{u}^{n+1}=\rho \vec{u}^{n}-\Delta t \vec{\nabla} \cdot \rho \vec{u} u^{n}-\Delta t \vec{\nabla} p^{n+1} \\
& \rho E^{n+1}=\rho E^{n}-\vec{\nabla} \cdot \rho \vec{u} E^{n}-\Delta t \vec{\nabla}(p u)^{n+1}
\end{aligned}
$$

\section{The Predictor stage - (Transport Stage)}

The first major change is the predictor stage: in the target system there is no pressure term and the system to solve only concern the transport of conservative variables.

$$
\begin{aligned}
& \frac{\partial \rho}{\partial t}+\vec{\nabla} \cdot \rho \vec{u}=0 \\
& \frac{\partial \rho \vec{u}}{\partial t}+\vec{\nabla} \cdot \rho \vec{u} \vec{u}=0 \\
& \frac{\partial \rho E}{\partial t}+\vec{\nabla} \cdot \rho \vec{u} E=0
\end{aligned}
$$


This system of equations is hyperbolic but not strictly: it has three times the same eigenvalue $\vec{u}$. This system is frame invariant which is not the case for the system (9). The semi-discrete solution for (11) is:

$$
\begin{gathered}
\rho^{*}=\rho^{n}-\vec{\nabla} \cdot \rho \vec{u} \\
\rho \vec{u}^{*}=\rho \vec{u}-\vec{\nabla} \cdot \rho \vec{u} \vec{u}^{n} \\
\rho E^{*}=\rho E^{n}-\vec{\nabla} \cdot \rho \vec{u} E^{n}
\end{gathered}
$$

This system is solved by the means of a Finite Volume scheme. The advantage of this new system is that the timestep is only restricted by a convective criterion: $\Delta t \leq \frac{\Delta x}{|u|}$ because $u$ is the only eigenvalue. The FV volume scheme is $U_{i}^{n+1}=U_{i}^{n}-\frac{\Delta t}{\Delta x}\left(F_{i+1 / 2}^{n}-F_{i-1 / 2}^{n}\right)$ as presented in the previous paragragh.

The Rusanov solver is used to compute the flux vector:

$\mathrm{F}_{\mathrm{i}+1 / 2}=\frac{1}{2}\left(\mathrm{~F}_{\mathrm{i}}+\mathrm{F}_{\mathrm{i}+1}\right)-\frac{1}{2} \mathrm{~S}^{+}\left(\mathrm{U}_{\mathrm{i}}+\mathrm{U}_{\mathrm{i}+1}\right)$.

With $\mathrm{U}=\left(\begin{array}{c}\rho \\ \rho \mathrm{u} \\ \rho \mathrm{E}\end{array}\right)$ and $\mathrm{F}=\left(\begin{array}{c}\rho \mathrm{u} \\ \rho \mathrm{u}^{2} \\ \rho \mathrm{Eu}\end{array}\right)$ and the wave speed is evaluated according to:

$\mathrm{S}_{\mathrm{i}+1 / 2}^{+}=\max \left(\left|\mathrm{u}_{\mathrm{i}}\right|,\left|\mathrm{u}_{\mathrm{i}+1}\right|\right)+\varepsilon$.

\section{The correction stage}

We develop now this stage because we can deduce the Poisson equation. Actually, because the mass conservation equation is a transport equation, the target equation can be used directly:

$\rho^{n+1}=\rho^{n}-\frac{\Delta t}{2} \vec{\nabla} \cdot\left(\rho \vec{u}^{n}+\rho \vec{u}^{n+1}\right)$

In this equation we use the predicted value $\rho^{*}$ to obtain:

$\rho^{\mathrm{n}+1}=\rho^{*}-\frac{\Delta \mathrm{t}}{2} \vec{\nabla} \cdot\left(\rho \mathrm{u}^{\mathrm{n}+1}-\rho \mathrm{u}^{\mathrm{n}}\right)$

In this last equation, the momentum at time $n+1$ should be removed. We use the corrected equation for the momentum. To obtain this equation, we note that in the predictor stage, only the pressure terms are not present so we naturally pose:

$\overrightarrow{\rho u}^{n+1}=\rho \vec{u}^{*}-\Delta t \vec{\nabla} \cdot p^{n+1}$

The same way for the energy equation leads to:

$\rho E^{n+1}=\rho E^{*}-\Delta t \vec{\nabla} \cdot(p \vec{u})^{n+1}$

By combining (14) and (15) we get: 
$\rho^{n+1}=\rho^{*}-\frac{\Delta t}{2} \vec{\nabla} \cdot\left(\rho \vec{u}^{*}-\rho \vec{u}^{n}\right)+\frac{\Delta t^{2}}{2} \Delta p^{n+1}$

At this time, another difference between the original PCICE method and the new version (PCICEVF) is introduced:

$\vec{\nabla} \cdot \rho \vec{u}^{*}=\vec{\nabla} \cdot \rho \vec{u}$

This assumption is justified by the fact that $\vec{\nabla} \cdot \overrightarrow{p u}^{*}$ in the time range $[t, t+\Delta t]$ is constant, under the convective time step. Indeed the fluxes computed at each cell boundary are constant by definition during the time step according to the property given in paragraph 2.

Then, assuming the pressure at $\mathrm{n}+1$ is known the correction stage reads:

$\rho^{n+1}=\rho^{*}+\frac{\Delta t^{2}}{2} \Delta p^{n+1}$
$\rho \vec{u}^{n+1}=\rho \vec{u}^{*}-\Delta t \vec{\nabla} \cdot p^{n+1}$
$\rho E^{n+1}=\rho E^{*}-\Delta t \vec{\nabla} \cdot(\overrightarrow{p u})^{n+1}$

The correction step needs the pressure determination at time $\mathrm{t}^{\mathrm{n}+1}$.

\section{Poisson equation for the pressure}

The solution for the pressure must be established. The equation of state is here used in a general differential form. In equation system (8), the closure relation is given by $\varepsilon=\varepsilon(\mathrm{p}, \rho)$ which we can rewrite $\mathrm{p}=\mathrm{p}(\varepsilon, \rho)$. Differentiation of this equation reads:

$\left.\left.\mathrm{dp}=\frac{\partial \mathrm{p}}{\partial \rho}\right)_{\varepsilon} \mathrm{d} \rho+\frac{\partial \mathrm{p}}{\partial \varepsilon}\right)_{\rho} \mathrm{d} \varepsilon$

This differential form is approximated in time variation between time $\mathrm{t}^{*}$ and the time $\mathrm{t}^{\mathrm{n}+1}$ :

$\mathrm{df}=\mathrm{f}^{\mathrm{n}+1}-\mathrm{f} *$

so

$\left.\left.\mathrm{p}^{\mathrm{n}+1}-\mathrm{p}^{*}=\frac{\partial \mathrm{p}}{\partial \rho}\right)_{\varepsilon}\left(\rho^{\mathrm{n}+1}-\rho^{*}\right)+\frac{\partial \mathrm{p}}{\partial \varepsilon}\right)_{\rho}\left(\varepsilon^{\mathrm{n}+1}-\varepsilon^{*}\right)$.

The coefficients of this equation depend on the equation of state. We have to evaluate the terms $\left(\rho^{\mathrm{n}+1}-\rho^{*}\right)$ and $\left(\varepsilon^{\mathrm{n}+1}-\varepsilon^{*}\right)$. To do this we use the associated correction equation (19):

$\rho^{\mathrm{n}+1}=\rho^{*}+\frac{\Delta \mathrm{t}^{2}}{2} \Delta \mathrm{p}^{\mathrm{n}+1}$

Thus, (23) now reads :

$\left.\left.\mathrm{p}^{\mathrm{n}+1}-\mathrm{p}^{*}=\frac{\partial \mathrm{p}}{\partial \rho}\right)_{\varepsilon} \frac{\Delta \mathrm{t}^{2}}{2} \Delta \mathrm{p}^{\mathrm{n}+1}+\frac{\partial \mathrm{p}}{\partial \varepsilon}\right)_{\rho}\left(\varepsilon^{\mathrm{n}+1}-\varepsilon^{*}\right)$ 
There is no exact expression for the internal energy at time $n+1$. The internal energy obeys the following partial differential equation:

$\rho \frac{\mathrm{d} \varepsilon}{\mathrm{dt}}+\mathrm{p} \vec{\nabla} \cdot \overrightarrow{\mathrm{u}}=0$

During the predictor step, all the transport terms have been taken into account, so in this equation the convective term of the time derivative have already been considered in the predictor step. The remaining part reads:

$\rho \frac{\partial \varepsilon}{\partial \mathrm{t}}+\mathrm{p} \vec{\nabla} \cdot \overrightarrow{\mathrm{u}}=0$

A new improvement of the method is now done thanks to this equation. Its approximation between predicted state and new time reads:

$\widetilde{\varepsilon}^{n+1} \equiv \widetilde{\varepsilon}^{n+1}=\varepsilon^{*}-\frac{\Delta t}{\tilde{\rho}} p^{n} \nabla u^{n}$

The pressure equation now reads:

$\left.\left.\mathrm{p}^{\mathrm{n}+1}-\mathrm{p}^{*}=\frac{\partial \mathrm{p}}{\partial \rho}\right)_{\varepsilon} \frac{\Delta \mathrm{t}^{2}}{2} \Delta \mathrm{p}^{\mathrm{n}+1}-\frac{\partial \mathrm{p}}{\partial \varepsilon}\right)_{\rho} \frac{\Delta \mathrm{t}}{\widetilde{\rho}} \mathrm{p}^{\mathrm{n}} \nabla \mathrm{u}^{\mathrm{n}}$

The tilde quantities are evaluated as the arithmetic average between time ${ }^{*}$ and $n$. The use of the ideal gas equation of state $\mathrm{p}=(\gamma-1) \rho \varepsilon$ leads to the coefficients:

$\left.\frac{\partial \mathrm{p}}{\partial \rho}\right)_{\varepsilon}=(\gamma-1) \varepsilon$ and $\left.\frac{\partial \mathrm{p}}{\partial \varepsilon}\right)_{\rho}=(\gamma-1) \rho$

The Poisson equation for the pressure is:

$p^{n+1}-p^{*}=(\gamma-1) \widetilde{\varepsilon} \frac{\Delta t^{2}}{2} \Delta p^{n+1}-(\gamma-1) \Delta t p^{n} \nabla u^{n}$

Or:

$\mathrm{p}^{\mathrm{n}+1}-(\gamma-1) \widetilde{\varepsilon} \frac{\Delta \mathrm{t}^{2}}{2} \Delta \mathrm{p}^{\mathrm{n}+1}=\mathrm{p}^{*}-(\gamma-1) \Delta \mathrm{t}^{\mathrm{n}} \nabla \mathrm{u}^{\mathrm{n}}$

\section{Summary of the method}

Predictor step

$$
\begin{aligned}
& \rho^{*}=\rho^{n}-\vec{\nabla} \cdot \rho \vec{u} \\
& \rho \vec{u}^{*}=\rho \vec{u}-\vec{\nabla} \cdot \rho \vec{u} \vec{u}^{n} \\
& \rho E^{*}=\rho E^{n}-\vec{\nabla} \cdot \rho \vec{u} E^{n}
\end{aligned}
$$

\section{Poisson equation}

$$
p^{n+1}-(\gamma-1) \widetilde{\varepsilon} \frac{\Delta t^{2}}{2} \Delta p^{n+1}=p^{*}-(\gamma-1) \Delta t p^{n} \nabla u^{n}
$$




\section{Corrector step}

$$
\begin{aligned}
& \rho^{n+1}=\rho^{*}+\frac{\Delta t^{2}}{2} \Delta p^{n+1} \\
& \rho \vec{u}^{n+1}=\rho \vec{u}^{*}-\Delta t \vec{\nabla} \cdot p^{n+1} \\
& \rho E^{n+1}=\rho E^{*}-\Delta t \vec{\nabla} \cdot(\vec{u})^{n+1}
\end{aligned}
$$

\section{Single-Phase results}

\subsection{Introduction}

The results presented in this section are obtained with the new method at first order in time and space. No change was done for the various test cases meaning that there is no tuning coefficient. The first test case is about the propagation of an interface moving with a constant velocity, in a constant pressure field. This test case is very important to asses the ability of the code to treat interfacial problems, which is very important for multiphase flows. The pressure and the velocity must remain constant while a density profile is propagating.

The two other test cases consider shock tube problems. All compressible effects are involved in these test cases: material interfaces, acoustics, expansion and shock waves. The numerical results are compared with exact solutions. The first test deals with strong shock: it indicates robustness of the method. The second is a weak shock tube problem, which is a simple way to obtain a low speed flow.

The fluid is supposed to obey the ideal gas law with a polytropic coefficient $\gamma=1.4$ and a specific heat at constant volume $\mathrm{Cv}=700 \mathrm{~J} / \mathrm{kg} / \mathrm{K}$.

\section{$\underline{4.2 \text { Interface problem }}$}

Let us consider a 1D domain, $1 \mathrm{~m}$ length. This domain contains a gas moving at constant velocity $\mathrm{u}=10 \mathrm{~ms}^{-1}$ and at atmospheric pressure $101325 \mathrm{~Pa}$. A density discontinuity is initially located at the abscissa $\mathrm{x}=0.5 \mathrm{~m}$, this discontinuity is the interface between a gas at higher density $\rho_{\mathrm{L}}$ (at the right) and the same gas at low density at the right of this initial interface $\rho_{R}$. 


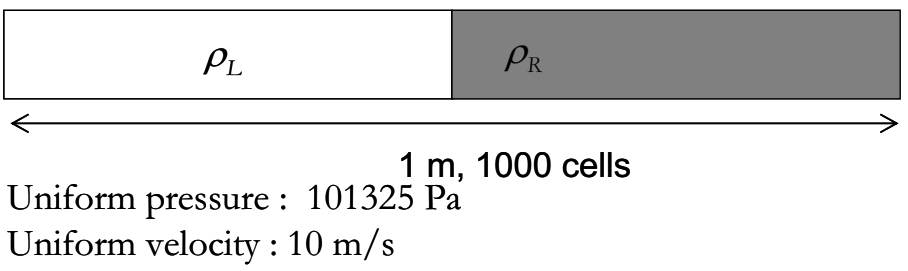

Figure 3 : Presentation of the test case for the advection

The mesh involves 1000 cells. In the first computation we consider a density jump equal to 2:

$\rho_{\mathrm{L}}=1 \mathrm{~kg} / \mathrm{m}^{3}$ and $\rho_{\mathrm{R}}=0.5 \mathrm{~kg} / \mathrm{m}^{3}$. The time step is equal $\Delta t=16 \frac{\Delta x}{u+c}$ and that is the largest we can use for the given conditions.

In Figure 4 the density profile at time $5.44 \mathrm{~ms}$ that corresponds to a 300 timesteps calculation is shown. The plateau values are perfectly respected and the velocity of the interface is also perfectly recovered. The front is smeared over a large number of cells, the main reason is the low order in space and time of the method.

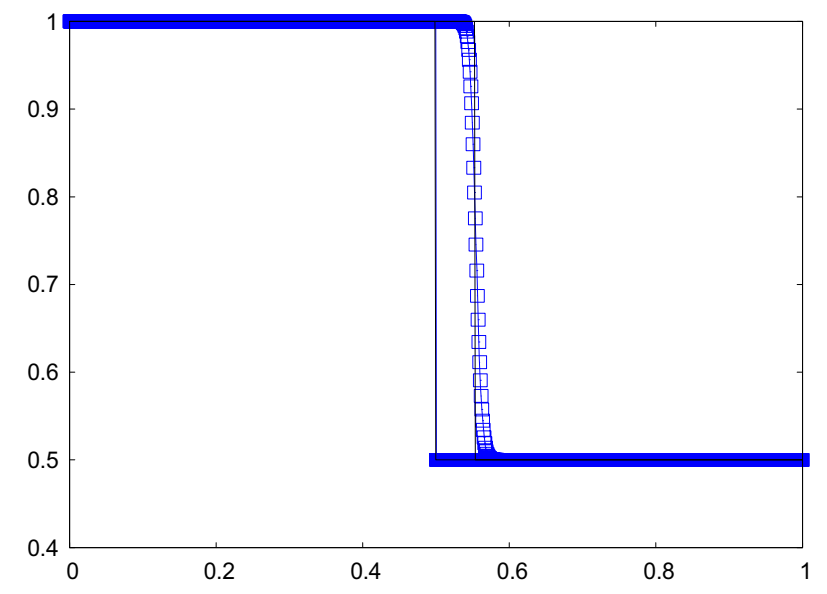

Figure 4: Advection test case : Jump density equal to 2, $\Delta \mathrm{t}=16$ CFL: Comparison of the analytical (straight line) and the numerical (symbols) solution for the density.

In the following figure 5, we plot the difference between the final pressure inside the tube and the initial pressure which was set at $101325 \mathrm{~Pa}$. The order of magnitude of the error is equal to $10^{-9} \mathrm{~Pa}$ which is very close to the numerical zero. The same plot concerning the difference between the final and the initial velocity show errors of the order of magnitude equal to $10^{-11} \mathrm{~m} / \mathrm{s}$. 


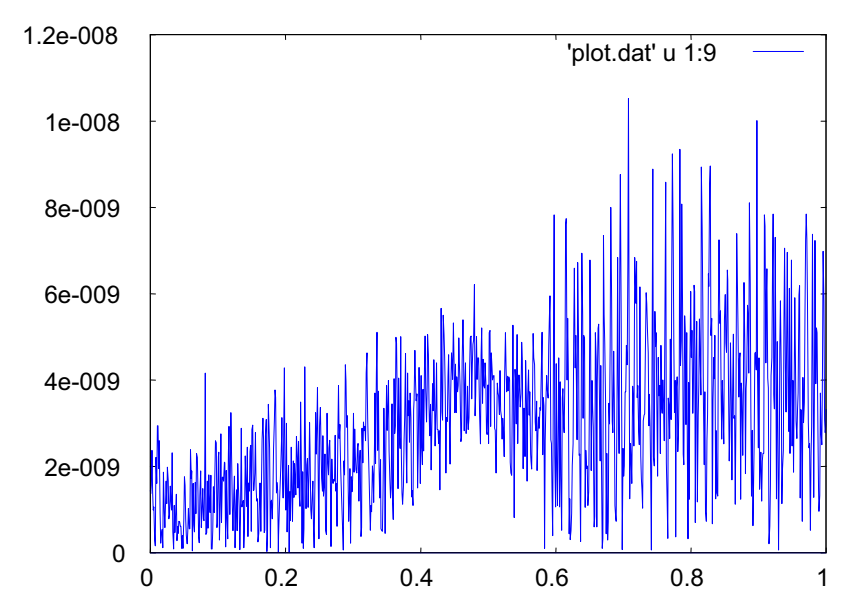

Figure 5: Advection test case : Jump density equal to 2, $\Delta \mathrm{t}=16$ CFL: Profiles of the difference between the initial pressure and the final time step pressure.

The results are quite excellent. It is noticeable that the time step, which is equal to 16 CFL, corresponds to the upper limit of the scheme as proposed in Ref. [2] that is to say $\Delta t=16 \frac{\Delta x}{u+c} \approx 0.3 \frac{\Delta x}{u}$.

Increasing the jump of the density at the interface leads to good results but the timestep is reduced. In Table 1, the largest timestep is reported according to the ratio of left and right density:

\begin{tabular}{|c|c|c|c|c|}
\hline$\frac{\rho_{L}}{\rho_{R}}$ & 2 & 10 & 100 & 1000 \\
\hline $\mathrm{CFL}$ & 16 & 12 & 8 & 3.85 \\
\hline
\end{tabular}

Table1: Evolution of the largest timestep versus the jump of density

For large density ratios the CFL number decreases but is always larger than the time step one can use with explicit methods. This problem is possibly due to the treatment of boundary conditions that is quite simplified in our code.

\subsection{Strong shock tube problem}

The strong shock tube problem consists in solving the flow created by the jump of pressure between a left and a right state initially set in a tube. This test case is interesting because every kind of waves (expansion waves, interface, shock wave) are present in the flow: the ability of a solver for compressible flows is evaluated through only one test case.

The initial pressure jump is set to $10^{6}$ : The low pressure chamber $\mathrm{p}_{\mathrm{R}}=101325 \mathrm{~Pa}$ and the high pressure is equal to $p_{L}=10^{6} p_{R}$ (Fig. 6). 


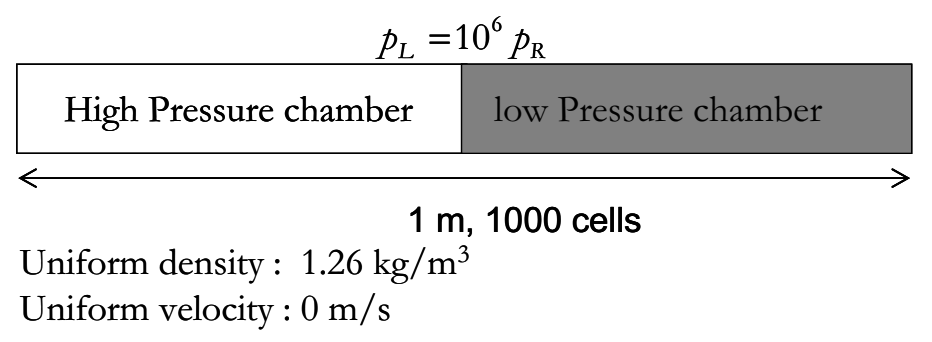

Figure 6: Presentation of the shock tune test case

The final time of the simulation is equal to $0.93 \mathrm{~ms}$. The timestep is $\Delta t=1.5 C F L$ which seems to be low compared to the time step in the previous test case. Actually, it is not so low and this time step is larger that the one proposed in Ref. [2] for the PCICE method: $\Delta t=0.5 \frac{\Delta x}{u}$. In Figure 7 the pressure, density and velocity profiles are plotted and compared to the exact solutions.

The flow solver reproduces very well the expected solution. In this case the CFL number is limited to 1.5. With these initial conditions, the acoustic wave and the convective wave are the same order of magnitude (around $180000 \mathrm{~m} / \mathrm{s}$ ): so the acoustic phenomena have at least the same importance as the transport phenomena. This test case clearly shows the robustness of the method.

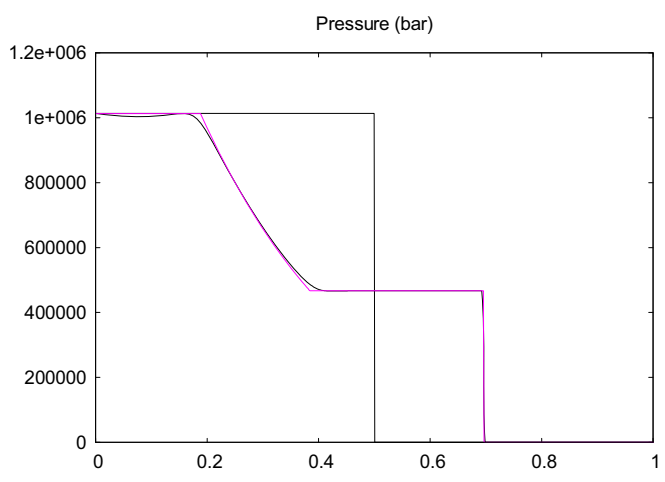



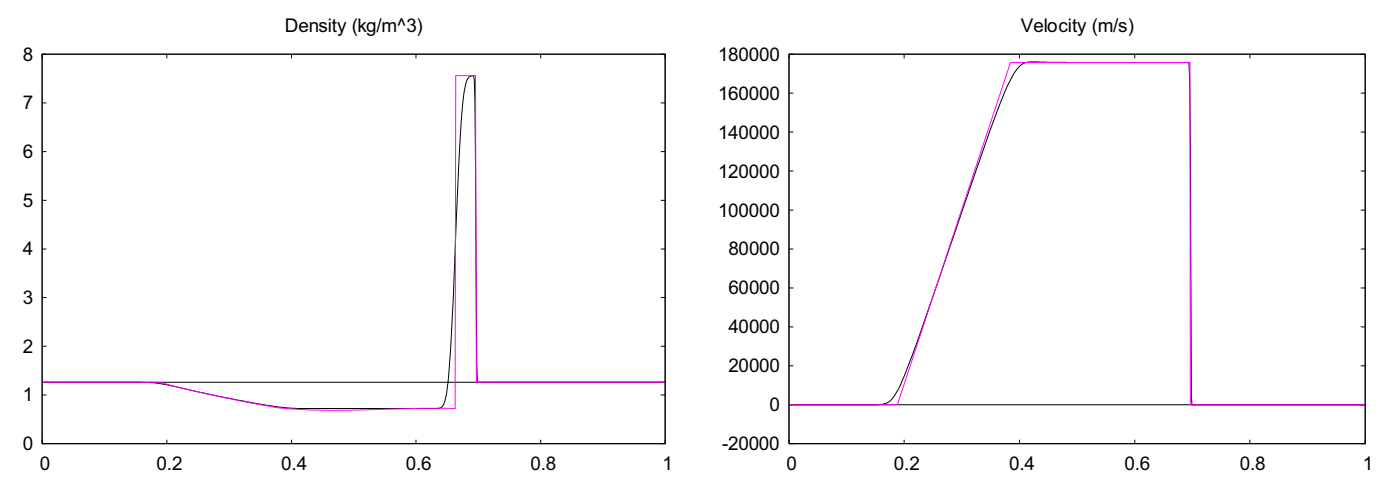

Figure 7: Comparison between analytical and numerical results for strong shock tube $p_{L}=10^{6} p_{R}$

\subsection{Weak shock tube problem}

The same shock tube problem as the one presented in the previous paragraph is computed, but we consider here a very low pressure jump. The low pressure chamber is set to $p_{R}=101325 \mathrm{~Pa}$ and the high pressure is equal to $\mathrm{p}_{\mathrm{L}}=1.05 \mathrm{p}_{\mathrm{R}}$. In the figure 8 , the numerical solution is compared to the analytical one at time $1 \mathrm{~ms}$.
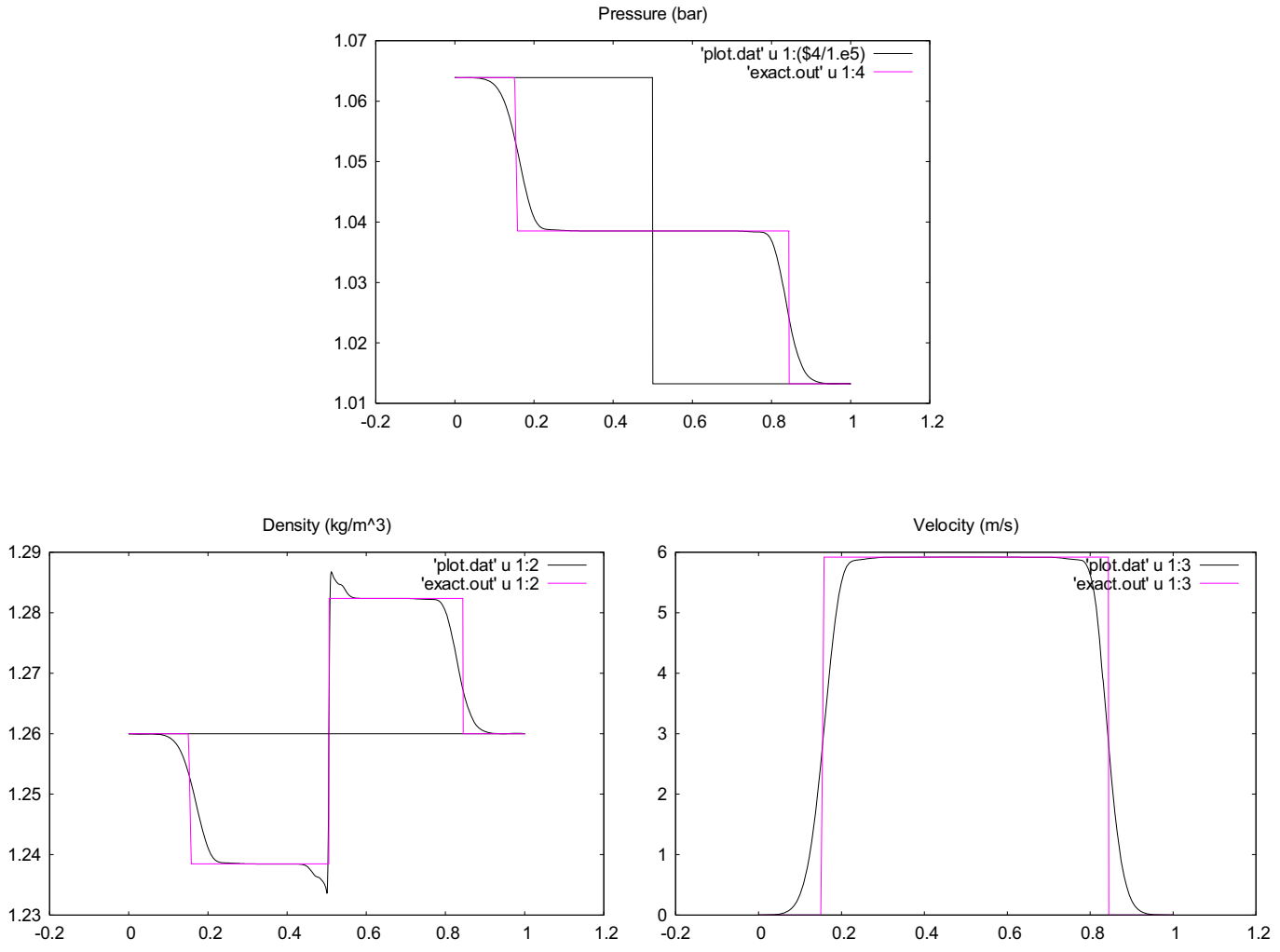
Figure 8: Comparison between analytical and numerical results for strong shock tube $p_{L}=1.05 p_{R} \cdot \Delta t=10 C F L$, number of timestep computed is 40 for a final time $1 \mathrm{~ms}$.

The final velocity is quite low, about $5 \mathrm{~m} / \mathrm{s}$. The time step used in this simulation is $\Delta t=10 C F L$. The final time is obtained after 40 time steps. The solution for the pressure and the velocity is in very good agreement with the analytical solution. The numerical solution for the density is also very good except at the interface where some spurious oscillations are present.

In figure 9, the solution obtained with CFL equal to 1 is plotted and is almost perfect for all the variables but we can already observe a small undershoot of the density at the interface.
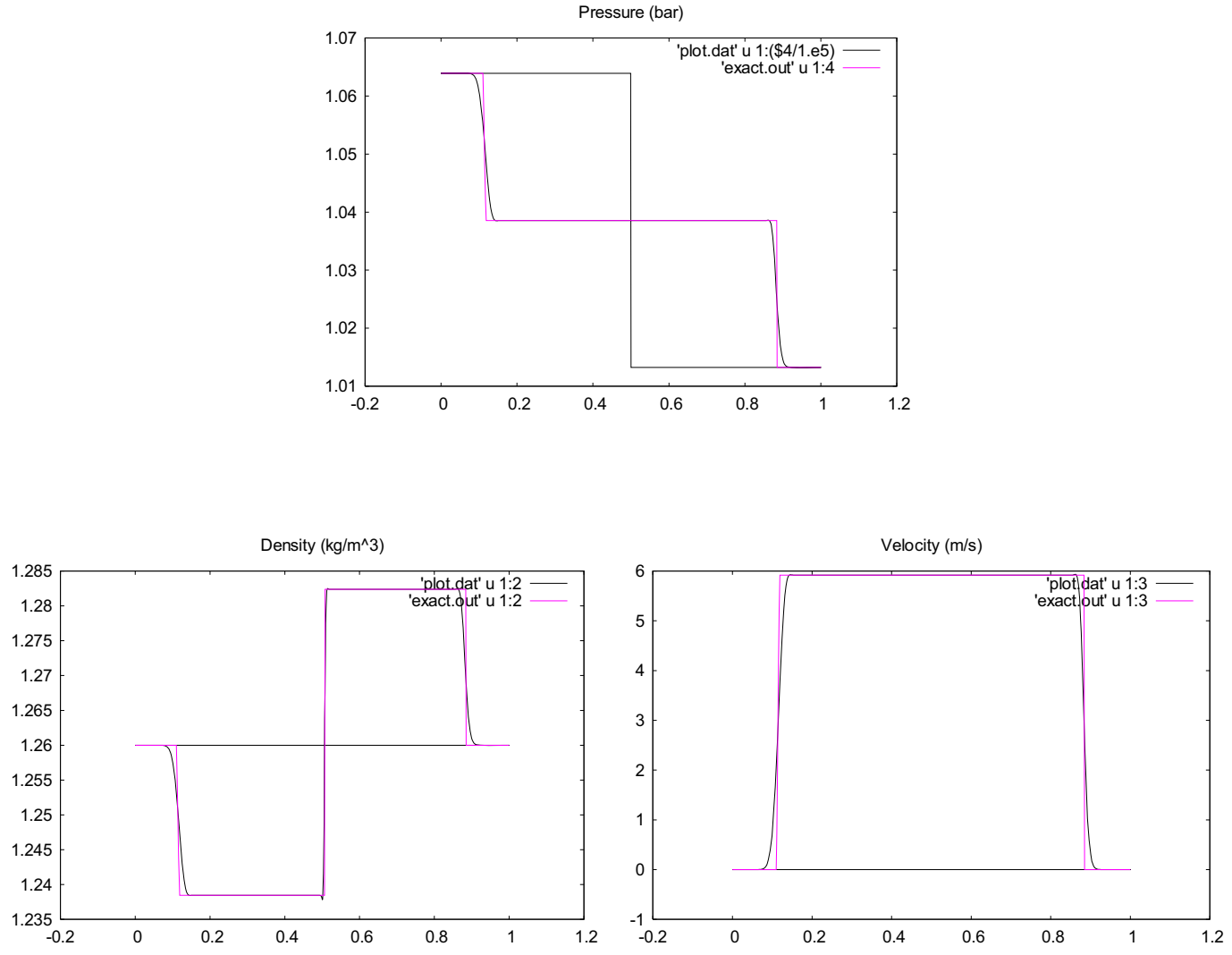

Figure 9: Comparison between analytical and numerical results for strong shock tube $p_{L}=1.05 p_{\mathrm{R}} . \Delta t=1 C F L$, number of timestep computed is 400 for a final time $1 \mathrm{~ms}$.

\section{$\underline{4.5 \text { Concluding remarks for the single-phase solver }}$}

Based on the PCICE method we have developed a new solver for single-phase flows. The objectives have been reached: a low speed flow solver is built, allowing larger timestep than conventional methods for compressible flow. 
The main possible improvements for solver consist in higher-order extension and different equations of state. The results are encouraging and the first attempt to two-phase flow solutions are presented in the next section.

\section{Diffuse-interface flow solver}

\section{$\underline{5.1 \text { Introduction }}$}

Our goal is to solve the single velocity pressure equilibrium model of Kapila et al. (2001). It has been obtained as the asymptotic limit of the Baer and Nunziato (1986) model in the limit of stiff velocity and pressure relaxation. In involves 5 partial differential equations, one of them being nonconservative. Its resulting speed of sound corresponds to that of Wood (1930) which exhibits a nonmonotonic variation with volume fraction. These two difficulties (non-conservation and nonmonotonicity) present serious computational challenges. To circumvent them, a pressure nonequilibrium 7-equation model is constructed, also non-conservative, but easier to solve with a relaxation method (Saurel, Petitpas and Berry, 2008). Both models are presented hereafter. The aim of the relaxation model is to obtain at the end of each time step a unique pressure for each phase: considering a numerical timestep starting at the mechanical equilibrium there is a time during each phase obeys its own equation of state leading to a non equilibrium state, then a relaxation occurs to obtain a new mechanical equilibrium.

The diffuse interface model are first presented. Then we briefly remind the algorithm based on the PCICE-VF method we use. In this work in progress, we have only done the needed changes in the method to take into account for the multiphase features of the flow model. The basic elements of the algorithm just developed in the context of the Euler equations are here again employed.

\subsection{Diffuse interface models}

\subsubsection{Five-equation model}

The Kapila et al. (2001) model is the zero-order approximation of the Baer and Nunziato (1986) with stiff mechanical relaxation. In the context of two fluids it reads

$$
\begin{aligned}
& \frac{\partial \alpha_{1}}{\partial t}+\nabla \alpha_{1} \cdot \vec{u}=\frac{\rho_{2} c_{2}^{2}-\rho_{1} c_{1}^{2}}{\frac{\rho_{1} c_{1}^{2}}{\alpha_{1}}+\frac{\rho_{2} c_{2}^{2}}{\alpha_{2}}} \nabla \cdot \vec{u} \\
& \frac{\partial \alpha_{1} \rho_{1}}{\partial t}+\nabla \cdot\left(\alpha_{1} \rho_{1} \vec{u}\right)=0 \\
& \frac{\partial \alpha_{2} \rho_{2}}{\partial t}+\nabla \cdot\left(\alpha_{2} \rho_{2} \vec{u}\right)=0 \\
& \frac{\partial \rho \vec{u}}{\partial t}+\nabla \cdot[(\rho \vec{u} \otimes \vec{u})+p I]=0 \\
& \frac{\partial \rho E}{\partial t}+\nabla \cdot(\rho E+p) \vec{u}=0
\end{aligned}
$$


where $\alpha, \rho, \vec{u}, p, E\left(E=\varepsilon+\frac{1}{2} u^{2}\right)$, and $\varepsilon$ represent respectively the volume fraction, the mixture density, the velocity, the mixture pressure, the mixture total energy and the mixture internal energy.

The mixture internal energy is defined as

$\varepsilon=Y_{1} \varepsilon_{1}\left(p_{1}, \rho_{1}\right)+Y_{2} \varepsilon_{2}\left(p_{2}, \rho_{2}\right)$

and the mass fractions are given by $Y_{k}=\frac{(\alpha \rho)_{k}}{\rho}$. The mixture density is defined by $\rho=\alpha_{1} \rho_{1}+\alpha_{2} \rho_{2}$. Each fluid is governed by its own convex equation of state (EOS), $e_{k}=e_{k}\left(\rho_{k}, p\right)$, which allows the determination of the phases' sound speed, $c_{k}=c_{k}\left(\rho_{k}, p\right)$. In the particular case of fluids governed by the stiffened gas EOS:

$p_{k}=\left(\gamma_{k}-1\right) \rho_{k} \varepsilon_{k}-\gamma_{k} p_{\infty k}$

the resulting mixture EOS reads

$$
p\left(\rho, \varepsilon, \alpha_{1}, \alpha_{2}, Y_{1}, Y_{2}\right)=\frac{\rho \varepsilon-\left(\frac{\alpha_{1} \gamma_{1} p_{\infty 1}}{\gamma_{1}-1}+\frac{\alpha_{2} \gamma_{2} p_{\infty 2}}{\gamma_{2}-1}\right)}{\frac{\alpha_{1}}{\gamma_{1}-1}+\frac{\alpha_{2}}{\gamma_{2}-1}}
$$

Thus, while this model maintains mechanical equilibrium, it provides for thermal non-equilibrium, which is what physically occurs through a phase interface.

\subsubsection{Seven-equation model}

The 7-equation model we intend to solve is as follows:

$$
\begin{aligned}
& \frac{\partial \alpha_{1}}{\partial t}+\nabla \alpha_{1} \cdot \vec{u}=\mu\left(p_{1}-p_{2}\right) \\
& \frac{\partial \alpha_{1} \rho_{1}}{\partial t}+\nabla \cdot\left(\alpha_{1} \rho_{1} \vec{u}\right)=0 \\
& \frac{\partial \alpha_{2} \rho_{2}}{\partial t}+\nabla \cdot\left(\alpha_{2} \rho_{2} \vec{u}\right)=0 \\
& \frac{\partial \rho \vec{u}}{\partial t}+\nabla \cdot\left[(\rho \vec{u} \otimes \vec{u})+\left(\alpha_{1} p_{1}+\alpha_{2} p_{2}\right) I\right]=0 \\
& \frac{\partial \alpha_{1} \rho_{1} \varepsilon_{1}}{\partial t}+\vec{\nabla} \cdot\left(\alpha_{1} \rho_{1} \varepsilon_{1} \vec{u}\right)+\alpha_{1} p_{1} \vec{\nabla} \cdot \vec{u}=-p_{I} \mu\left(p_{1}-p_{2}\right) \\
& \frac{\partial \alpha_{2} \rho_{2} \varepsilon_{2}}{\partial t}+\vec{\nabla} \cdot\left(\alpha_{2} \rho_{2} \varepsilon_{2} \vec{u}\right)+\alpha_{2} p_{2} \vec{\nabla} \cdot \vec{u}=p_{I} \mu\left(p_{1}-p_{2}\right) \\
& \frac{\partial \rho\left(Y_{1} \varepsilon_{1}+Y_{2} \varepsilon_{2}+\frac{1}{2} \vec{u}^{2}\right)}{\partial t}+\vec{\nabla} \cdot\left(\rho\left(Y_{1} \varepsilon_{1}+Y_{2} \varepsilon_{2}+\frac{1}{2} \vec{u}^{2}\right)+\alpha_{1} p_{1}+\alpha_{2} p_{2}\right) \vec{u}=0
\end{aligned}
$$


with

$p_{I}=\frac{Z_{2} p_{1}+Z_{1} p_{2}}{Z_{1}+Z_{2}}$

If this 7 -equation model is pressure-relaxed $(\mu \rightarrow \infty)$ the phasic pressures become equal $\left(p_{1}=p_{2}=p\right)$ and the system of equations tends asymptotically to the 5-equation system above. Our task herein is to design a PCICE-VF numerical scheme to solve this 7-equation model, as it presents better properties for the design of numerical schemes (Saurel et al., 2008) and because in the limit of stiff pressure relaxation, the 5-equation model solutions are recovered.

\subsection{PCICE-VF multiphase solver}

Predictor stage

The novelty compared to the one-phase flow solver is a new second sub-step. The system considered here is (32) in absence of pressure relaxation term, considered latter. In order to clarify the presentation by comparison with one-phase flow solver, the solved equations are written as:

$$
\begin{aligned}
& \alpha_{1 i}^{*}=\alpha_{1 i}^{n}-\frac{\Delta t}{\Delta x}\left(\left(u \alpha_{1}\right)_{i+1 / 2}^{n}-\left(u \alpha_{1}\right)_{i-1 / 2}^{n}-\alpha_{1 i}^{n}\left(u_{i+1 / 2}^{n}-u_{i-1 / 2}^{n}\right)\right) \\
& Y_{k i}^{*}=Y_{k i}^{n}-\frac{\Delta t}{\Delta x}\left(\left(u Y_{k}\right)_{i+1 / 2}^{n}-\left(u Y_{k}\right)_{i-1 / 2}^{n}-Y_{k i}^{n}\left(u_{i+1 / 2}^{n}-u_{i-1 / 2}^{n}\right)\right) \\
& \left.\rho_{i}^{*}=\rho_{i}^{n}-\frac{\Delta t}{\Delta x}\left((\rho u)_{i+1 / 2}^{n}-(\rho u)_{i-1 / 2}^{n}\right)\right) \\
& \left.(\rho u)_{i}^{*}=(\rho u)_{i}^{n}-\frac{\Delta t}{\Delta x}\left(\left(\rho u^{2}\right)_{i+1 / 2}^{n}-\left(\rho u^{2}\right)_{i-1 / 2}^{n}\right)\right) \\
& (\alpha \rho e)_{k i}^{*}=(\alpha \rho e)_{k i}^{n}-\frac{\Delta t}{\Delta x}\left((\alpha \rho e u)_{k i+1 / 2}^{n}-(\alpha \rho e u)_{k i-1 / 2}^{n}+(\alpha p)_{k i}^{n}\left(u_{i+1 / 2}^{n}-u_{i-1 / 2}^{n}\right)\right) \\
& (\rho E)^{*}=(\rho E)^{n}-\frac{\Delta t}{\Delta x}\left((\rho E u)_{i+1 / 2}^{n}-(\rho E u)_{k i-1 / 2}^{n}\right)
\end{aligned}
$$

The mass equations of each phase have been replaced by evolution equations for the mass fractions. The interest of this formulation will appear during the building of the Poisson equation.

\section{Relaxation stage}

The relaxation substep will give a correction to the internal energies, in agreement with the second law of thermodynamics. A second correction will be made with the help of the total mixture energy. Up to this point, the following system has been solved:

$$
\frac{\partial \alpha_{1}}{\partial t}+\nabla \alpha_{1} \cdot \vec{u}=0
$$


$\frac{\partial \alpha_{1} \rho_{1}}{\partial t}+\nabla \cdot\left(\alpha_{1} \rho_{1} \vec{u}\right)=0$

$\frac{\partial \alpha_{2} \rho_{2}}{\partial t}+\nabla \cdot\left(\alpha_{2} \rho_{2} \vec{u}\right)=0$

$\frac{\partial \rho \vec{u}}{\partial t}+\nabla \cdot(\rho \vec{u} \otimes \vec{u})=0$

$\frac{\partial \alpha_{1} \rho_{1} \varepsilon_{1}}{\partial t}+\vec{\nabla} \cdot\left(\alpha_{1} \rho_{1} \varepsilon_{1} \vec{u}\right)+\alpha_{1} p_{1} \vec{\nabla} \cdot \vec{u}=0$

$\frac{\partial \alpha_{2} \rho_{2} \varepsilon_{2}}{\partial \mathrm{t}}+\vec{\nabla} \cdot\left(\alpha_{2} \rho_{2} \varepsilon_{2} \overrightarrow{\mathrm{u}}\right)+\alpha_{2} \mathrm{p}_{2} \vec{\nabla} \cdot \overrightarrow{\mathrm{u}}=0$

$\frac{\partial \rho \mathrm{E}}{\partial \mathrm{t}}+\operatorname{div}(\rho \mathrm{Eu})=0$

Due to the velocity divergence in the mass and energy equations, pressure disequilibrium occurs. The pressure relaxation is thus necessary, which will determine the relaxed or equilibrium pressure with the corresponding phasic volume fractions and densities. It is this pressure relaxation substep to which we now turn.

This step is of major importance to fulfil interface conditions in non-uniform velocity and pressure flows. It also forces the solution of the 6-equation model to converge to that of the 5-equation model.

In the relaxation step, we must solve:

$\frac{\partial \alpha_{1}}{\partial t}=\mu\left(p_{1}-p_{2}\right)$

$\frac{\partial \alpha_{1} \rho_{1} e_{1}}{\partial t}=-p_{I} \mu\left(p_{1}-p_{2}\right)$

$\frac{\partial \alpha_{2} \rho_{2} e_{2}}{\partial t}=p_{I} \mu\left(p_{1}-p_{2}\right)$

$\frac{\partial \alpha_{1} \rho_{1}}{\partial t}=0$

$\frac{\partial \alpha_{2} \rho_{2}}{\partial t}=0$

$\frac{\partial \rho \vec{u}}{\partial t}=0$

$\frac{\partial \rho E}{\partial t}=0$

with $p_{I}=\frac{Z_{2} p_{1}+Z_{1} p_{2}}{Z_{1}+Z_{2}}$ and in the limit $\mu \rightarrow+\infty$. 
After some manipulations the internal energy equations become

$$
\begin{aligned}
& \frac{\partial e_{1}}{\partial t}+p_{I} \frac{\partial v_{1}}{\partial t}=0 \\
& \frac{\partial e_{2}}{\partial t}+p_{I} \frac{\partial v_{2}}{\partial t}=0 .
\end{aligned}
$$

where $v_{k}$ are the phasic specific volumes. This system can be written as an integral formulation

$$
e_{k}-e_{k}^{0}+\hat{p}_{I k}\left(v_{k}-v_{k}^{0}\right)=0
$$

where $\hat{p}_{I k}=\frac{1}{v_{k}-v_{k}^{0}} \int_{0}^{\Delta t} p_{I} \frac{\partial v_{k}}{\partial t} d t$.

To determine the pressure averages $\hat{\mathrm{p}}_{\mathrm{Ik}}$ begin by summing the internal energy equations

$$
Y_{1} e_{1}-Y_{1} e_{1}^{0}+Y_{2} e_{2}-Y_{2} e_{2}^{0}+\hat{p}_{I 1}\left(Y_{1} v_{1}-Y_{1} v_{1}^{0}\right)+\hat{p}_{I 2}\left(Y_{2} v_{2}-Y_{2} v_{2}^{0}\right)=0
$$

The mixture mass equation can be written as

$\left(Y_{1} v_{1}-Y_{1} v_{1}^{0}\right)+\left(Y_{2} v_{2}-Y_{2} v_{2}^{0}\right)=0$.

Using these relations the mixture energy equation becomes

$e-e^{0}+\left(\hat{p}_{I 1}-\hat{p}_{I 2}\right)\left(Y_{1} v_{1}-Y_{1} v_{1}^{0}\right)=0$.

In order that the mixture energy conservation be fulfilled it is necessary that: $\hat{p}_{I 1}=\hat{p}_{I 2}=\hat{p}_{I}$. Possible estimates are $\hat{\mathrm{p}}_{\mathrm{I}}=\hat{\mathrm{p}}_{\mathrm{I}}^{*}$ or $\hat{p}_{I}=p^{R}$, the initial and relaxed pressures respectively. These estimates are compatible with the entropy inequality. With regard to the choice of one or the other estimate, upon computation of the relaxed state the resulting difference in practical computations is negligible. The system to solve is thus composed of equations

$e_{k}\left(p^{R}, v_{k}^{R}\right)-e_{k}^{0}\left(p_{k}^{0}, v_{k}^{0}\right)+\hat{p}_{I}\left(v_{k}^{R}-v_{k}^{0}\right)=0, \quad \mathrm{k}=1,2$

which involves 3 unknowns, $v_{k}^{R}(\mathrm{k}=1,2)$ and $p^{R}$. Its closure is achieved using the saturation constraint

$$
\sum_{k} \alpha_{k}=1
$$

$\sum_{k}^{\text {or }}(\alpha \rho)_{k} v_{k}=1$.

Here the $(\alpha \rho)_{k}$ are constant during the relaxation process. This system can be replaced by a single equation with a single unknown $\left(p^{R}\right)$. With the help of the EOS the energy equations become

$$
v_{k}^{R}\left(p^{R}\right)=v_{k}^{0} \frac{p_{k}^{0}+\gamma_{k} p_{\infty k}+\left(\gamma_{k}-1\right) \hat{p}_{I}}{p^{R}+\gamma_{k} p_{\infty k}+\left(\gamma_{k}-1\right) \hat{p}_{I}},
$$

and thus the only equation to solve (for $p^{R}$ ) is

$$
\sum_{k}(\alpha \rho)_{k} v_{k}^{R}\left(p^{R}\right)=1 \text {. }
$$


Once the relaxed pressure is found, the phase's specific volumes $v_{k}^{R}\left(p^{R}\right)$ and volume fractions $\alpha_{k}^{R}=(\alpha \rho)_{k} v_{k}^{R}\left(p^{R}\right)$ corresponding to this pressure relaxed state are determined.

At the end of the relaxation step, we have solved

$$
\begin{aligned}
& \frac{\partial \alpha_{1}}{\partial t}+\nabla \alpha_{1} \cdot \vec{u}=\frac{\rho_{2} c_{2}^{2}-}{\frac{\rho_{1} c_{1}^{2}}{\alpha_{1}}+} \\
& \frac{\partial \alpha_{1} \rho_{1}}{\partial t}+\nabla \cdot\left(\alpha_{1} \rho_{1} \vec{u}\right)=0 \\
& \frac{\partial \alpha_{2} \rho_{2}}{\partial t}+\nabla \cdot\left(\alpha_{2} \rho_{2} \vec{u}\right)=0 \\
& \frac{\partial \rho \vec{u}}{\partial t}+\nabla \cdot(\rho \vec{u} \otimes \vec{u})=0 \\
& \frac{\partial \rho \mathrm{E}}{\partial \mathrm{t}}+\operatorname{div}(\rho \mathrm{Eu})=0
\end{aligned}
$$

And now $p_{1}=p_{2}=p$.

\section{Poisson equation}

At this point, we have solved a mixture model characterised by a unique pressure. The Poisson equation can be obtained by the same way as for the Euler equations. The mixture equation of state has changed and we now have $p\left(\rho, \varepsilon, \alpha_{1}, \alpha_{2}, Y_{1}, Y_{2}\right)$. Then the differential form of the pressure is:

$$
\left.\left.\left.\left.\left.\left.d p=\frac{\partial p}{\partial \rho}\right) d \rho+\frac{\partial p}{\partial \varepsilon}\right) d \varepsilon+\frac{\partial p}{\partial \alpha_{1}}\right) d \alpha_{1}+\frac{\partial p}{\partial \alpha_{2}}\right) d \alpha_{2}+\frac{\partial p}{\partial Y_{1}}\right) d Y_{1}+\frac{\partial p}{\partial Y_{2}}\right) d Y_{2} .
$$

We assume that the mass fraction $Y_{k}$ will remain constant during the acoustic relaxation (the mass fraction is constant through any elemental wave). We also assume that during the first stage, the volume fraction have been transported and require no correction. This assumes that the right hand side of the volume fraction is neglected. The corresponding sub-model is also able to solve interface problems (Massoni et al., 2002).

So we have during this stage:

$$
\left.\left.d p=\frac{\partial p}{\partial \rho}\right) d \rho+\frac{\partial p}{\partial \varepsilon}\right) d \varepsilon
$$

The Poisson equation is then formally the same as in the single-phase flow solver.

\section{Correction stage}

Some quantities are corrected thanks to the mixture pressure at time $\mathrm{n}+1$ according:

$$
\mathrm{Y}_{\mathrm{k}}^{\mathrm{n}+1}=\mathrm{Y}_{\mathrm{k}}^{\mathrm{p}}
$$




$$
\begin{aligned}
& \rho^{n+1}=\rho^{*}+\frac{\Delta t^{2}}{2} \Delta\left(p^{n+1}\right) \\
& \rho u^{n+1}=\rho u^{*}-\Delta t \vec{\nabla}\left(p^{n+1}\right)
\end{aligned}
$$

then

$$
\rho E^{n+1}=\rho E^{*}-\Delta t \vec{\nabla}\left(p u^{n+1}\right)
$$

We also correct the volume fraction according:

$$
\alpha_{k}^{n+1}=\alpha_{k}^{R}+\frac{\Delta t}{\Delta x} K^{\text {new }}\left(u_{i+1 / 2}^{n+1}-u_{i-1 / 2}^{n+1}\right)
$$

Each phase quantity can be deduced from these relations (41)-(45) with the help of the equation of state.

\subsection{Summary of the two-phase PCIC-VF algorithm}

\section{Predictor stage}

$$
\begin{aligned}
& \alpha_{1 i}^{*}=\alpha_{1 i}^{n}-\frac{\Delta t}{\Delta x}\left(\left(u \alpha_{1}\right)_{i+1 / 2}^{n}-\left(u \alpha_{1}\right)_{i-1 / 2}^{n}-\alpha_{1 i}^{n}\left(u_{i+1 / 2}^{n}-u_{i-1 / 2}^{n}\right)\right) \\
& Y_{k i}^{*}=Y_{k i}^{n}-\frac{\Delta t}{\Delta x}\left(\left(u Y_{k}\right)_{i+1 / 2}^{n}-\left(u Y_{k}\right)_{i-1 / 2}^{n}-Y_{k i}^{n}\left(u_{i+1 / 2}^{n}-u_{i-1 / 2}^{n}\right)\right) \\
& \left.\rho_{i}^{*}=\rho_{i}^{n}-\frac{\Delta t}{\Delta x}\left((\rho u)_{i+1 / 2}^{n}-(\rho u)_{i-1 / 2}^{n}\right)\right) \\
& \left.(\rho u)_{i}^{*}=(\rho u)_{i}^{n}-\frac{\Delta t}{\Delta x}\left(\left(\rho u^{2}\right)_{i+1 / 2}^{n}-\left(\rho u^{2}\right)_{i-1 / 2}^{n}\right)\right) \\
& (\alpha \rho e)_{k i}^{*}=(\alpha \rho e)_{k i}^{n}-\frac{\Delta t}{\Delta x}\left((\alpha \rho e u)_{k i+1 / 2}^{n}-(\alpha \rho e u)_{k i-1 / 2}^{n}+(\alpha p)_{k i}^{n}\left(u_{i+1 / 2}^{n}-u_{i-1 / 2}^{n}\right)\right) \\
& (\rho E)^{*}=(\rho E)^{n}-\frac{\Delta t}{\Delta x}\left((\rho E u)_{i+1 / 2}^{n}-(\rho E u)_{k i-1 / 2}^{n}\right)
\end{aligned}
$$

\section{Relaxation procedure}

To obtain $\mathrm{p}_{1}=\mathrm{p}_{2}=\mathrm{p}$.

\section{Poisson equation}

\section{Correction stage}

\section{$\underline{5.2 \text { Results }}$}

In this work-in-progress we had time to only obtain results for the propagation of a two-phase interface in a uniform flow at constant velocity and constant pressure. The solutions presented in this section are obtained with no correction for the volume fraction, meaning that the term $\mathrm{K}^{\text {new }}$ in (45) is set equal to zero. The time step used in this calculation is equal to 1 CFL. The problem simulated is shown in Figure 10: 


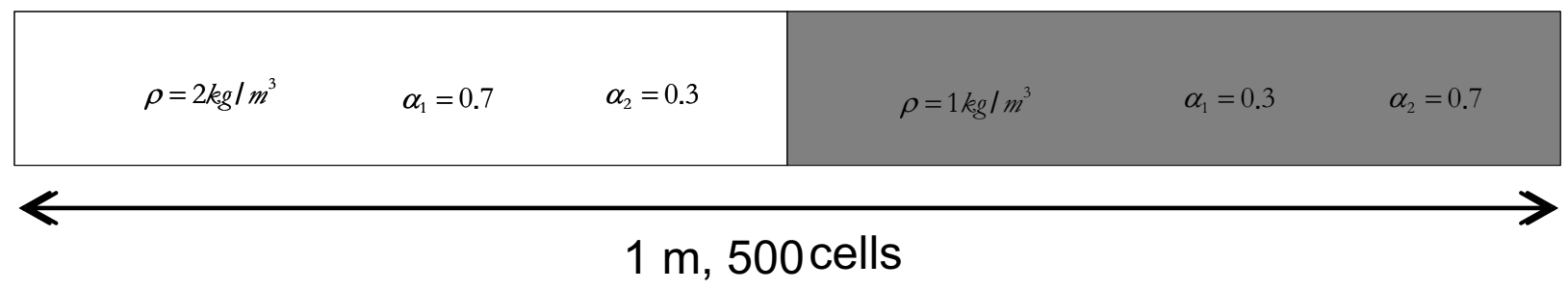

\section{Uniform pressure : $101325 \mathrm{~Pa}$ \\ Uniform velocity : $10 \mathrm{~m} / \mathrm{s}$}

Figure 10 : Propagation of a material interface in a multiphase flow

It is noticeable that the interface concerns the mixture density as well as the volume fraction. The solutions are plotted at time $1 \mathrm{~ms}$ for the mixture density (Figure 11) and the volume fraction of phase 1 (Figure 12). The solution is in very good agreement with the exact one.

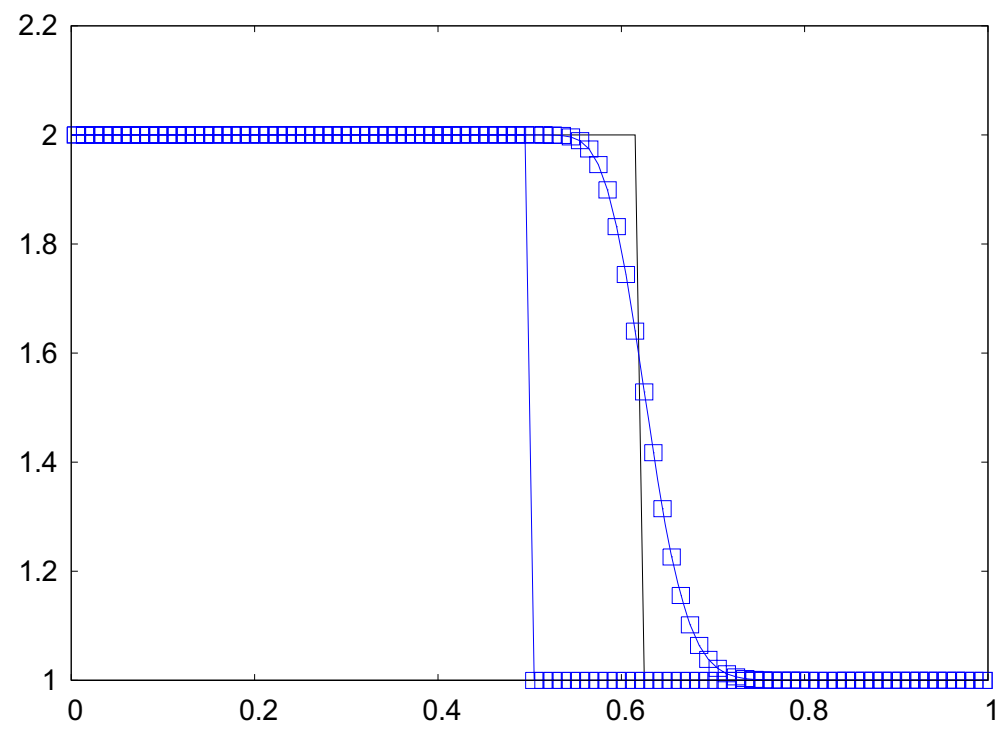

Figure 11 : The density of the mixture in a $10 \mathrm{~m} / \mathrm{s}$ moving flow. In symbol the numerical results compared to the analytical solution 


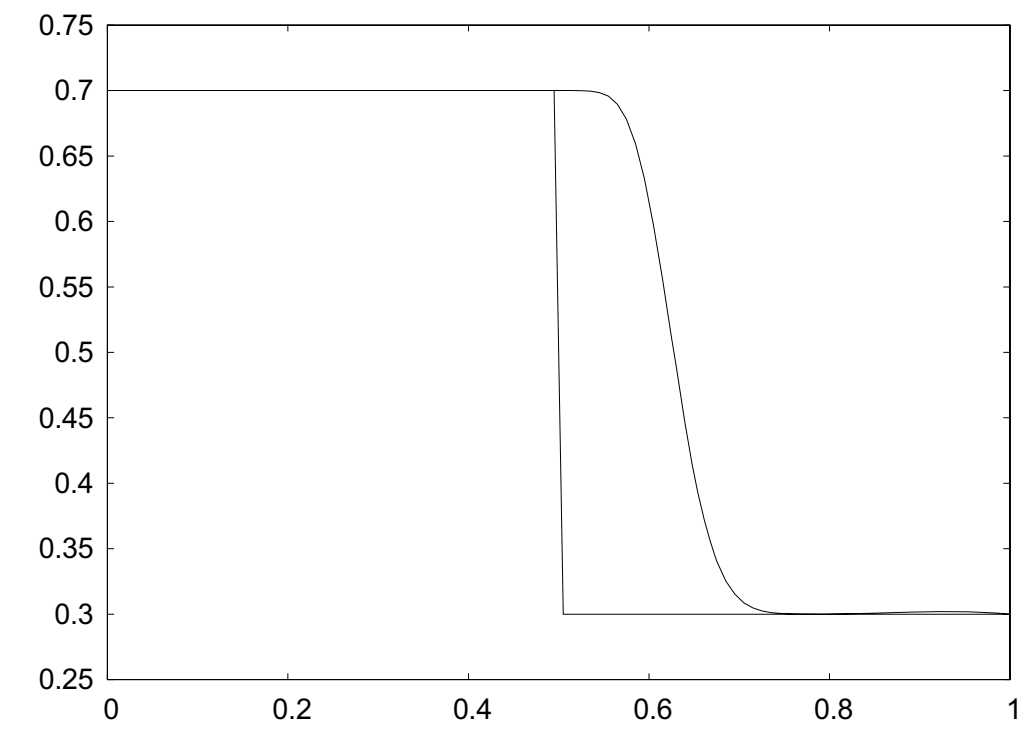

Figure 12 : Evolution of the volume fraction of phase 1 in the advection test case.

\section{Concluding remarks}

We have developed a Finite Volume version of the PCICE method. Some changes have been done compared to the original version to obtain a pressure and velocity oscillation free method. Extra work is needed to extend the method at higher order in space and time. Also, very large density ratios at interfaces induce time step restrictions that have to be understood in order to improve computational efficiency in these conditions.

Some first results are presented concerning the two-phase model of diffuse interface. Extra efforts are needed to succeed with shock tube test cases with interfaces.

\section{References}

[1] Berry R.A and Martineau R.C. "Examination of the PCICE method in the nearly incompressible as well as strictly incompressible limits", J.Power and Energy Systems, 2, 295, 2008. Included with this Chapter as Appendix A.

[2] Martineau R.C. and Berry R.A " An efficient, semi-implicit pressure based scheme employing a high-resolution FEM for simulating transient Acoustic and steady, inviscid and viscous, compressible flows on unstructured grids", INL report, 2004.

[3] Kapila A.K., Menikoff R., Bdzil J.B., Son S.F. \& Stewart D.S. (2001) Two-phase modeling of deflagration-to-detonation transition in granular materials : Reduced equations. Physics of Fluids, 13 (10), pp. 3002-3024.

[4] Baer, M.R. and Nunziato, J.W., (1986) A two-phase mixture theory for the deflagration-todetonation transition (DDT) in reactive granular materials. Int. J. of Multiphase Flow, 12 (6), 861 
[5] Saurel, R., Petitpas, F., and Berry, R., (2008) Simple and efficient relaxation methods for interfaces separating compressible fluids, cavitating flows and shocks in multiphase mixtures. Journal of Computational Physics, in revision.

[6] Massoni, J., Saurel, R., Nkonga, R. and Abgrall, R. (2002) Proposition de méthodes et modèles eulériens pour les problèmes à interfaces entre fluides compressibles en présence de transfert de chaleur. International Journal of Heat and Mass Transfer, Vol 45, N 6, pp 1287-1307. 


\section{Appendix 4A \\ Examination of the PCICE Method in the Nearly Incompressible, as well as Strictly Incompressible, Limits}

Reproduction of article published in Journal of Power and Energy Systems, 2 (2), pp. 598-610 (2008). 


\title{
Examination of the PCICE Method in the Nearly Incompressible, as well as Strictly Incompressible, Limits *
}

\author{
Ray A. Berry** and Richard C. Martineau*** \\ ** Idaho National Laboratory \\ Multiphysics Methods Group, P.O. Box 1625, Idaho Falls, Idaho 83415-3840 USA \\ E-mail:Ray.Berry@inl.gov \\ *** Idaho National Laboratory \\ Multiphysics Methods Group, P.O. Box 1625, Idaho Falls, Idaho 83415-3840 USA
}

\begin{abstract}
The conservative-form, pressure-based PCICE numerical method (Martineau and Berry, 2004) (Berry, 2006), recently developed for computing transient fluid flows of all speeds from very low to very high (with strong shocks), is simplified and generalized. Though the method automatically treats a continuous transition of compressibility, three distinct, limiting compressibility regimes are formally defined for purposes of discussion and comparison with traditional methods - the strictly incompressible limit, the nearly incompressible limit, and the fully compressible limit. The PCICE method's behavior is examined in each limiting regime. In the strictly incompressible limit the PCICE algorithm reduces to the traditional MACtype method with velocity divergence driving the pressure Poisson equation. In the nearly incompressible limit the PCICE algorithm is found to reduce to a generalization of traditional incompressible methods, i.e. to one in which not only the velocity divergence effect, but also the density gradient effect is included as a driving function in the pressure Poisson equation. This nearly incompressible regime has received little attention, and it appears that in the past, strictly incompressible methods may have been conveniently applied to flows in this regime at the expense of ignoring a potentially important coupling mechanism. This could be significant in many important flows; for example, in natural convection flows resulting from high heat flux. In the fully compressible limit or regime, the algorithm is found to reduce to an expression equivalent to density-based methods for high-speed flow.
\end{abstract}

Key words: compressible, incompressible, strictly incompressible, nearly incompressible, fully compressible

\section{Introduction}

Single phase flows of practical import for current and future nuclear reactor systems cover a wide range of flow speeds and compressibilities. Seldom do they belong exclusively to the restrictive class of strictly incompressible flow. Most reactor flows are of a mixed nature meaning that they possess regions dominated by, for example, nearly incompressible flow as well as regions dominated by strongly compressible effects. Or, for transients, during part of 
the time compressible effects will dominate while at different time the flow will be nearly incompressible. To be able to handle such flows, the pressure-corrected variant of the implicit continuous-fluid Eulerian (ICE) (Harlow and Amsden, 1971), or PCICE numerical method has been developed and presented (Martineau and Berry, 2004) as a finite element method, PCICE-FEM, for computing fluid flows of all speeds from low subsonic or nearly incompressible to high supersonic compressible. PCICE is a predictor-corrector method for approximating the solution of the conservative form of the Euler/Navier-Stokes equations:

$$
\begin{aligned}
& \frac{\partial \rho}{\partial t}+\nabla \cdot \rho \vec{u}=0 \\
& \frac{\partial \rho \vec{u}}{\partial t}+\nabla \cdot(\rho \vec{u} \otimes \vec{u})=-\nabla p+\nabla \cdot \tau \\
& \frac{\partial \rho e_{t}}{\partial t}+\nabla \cdot \rho \vec{u} h_{t}=\nabla \cdot(\tau \cdot \vec{u})+\nabla \cdot k \nabla T+i(T)
\end{aligned}
$$

where $\rho, \vec{u}, p$, and $T$ represent the fluid mass density, velocity, pressure, and temperature, respectively. In these equations, $\tau$ is the shear stress, $e_{t}=e+\frac{\vec{u} \cdot \vec{u}}{2}$ is the total energy density (where $e$ is the internal energy density), $h_{t}=\frac{\rho e_{t}+p}{\rho}$ is the specific total enthalpy, and $i(T)$ is a temperature-dependent energy source term. Fourier's law for thermal conduction has been assumed with $k$ denoting the thermal conductivity. These equations represent the balance of mass, momentum, and total energy, respectively. Because the PCICE method is not restricted to any specific equation of state, the general functional form

$$
p=f(\rho, e)
$$

will be utilized throughout this development.

The objectives of this short note are two-fold. First, as in (Berry, 2006), it presents a simplified, yet generalized, description of the PCICE method, independent of specific spatial discretizations and equations of state. Second, the behavior of the PCICE method is examined in the strictly incompressible limit, the nearly incompressible limit ( $c \Delta t>>l$, where $c$ is the acoustic wave speed, $\Delta t$ represents the time resolution of interest, and $l$ represents the characteristic length of interest), as well as in the fully compressible limit $(c \Delta t<<l)$.

\subsection{PCICE ALGORITHM}

The pressure-corrected implicit continuous-fluid Eulerian, or PCICE algorithm (Martineau and Berry, 2004)(Berry, 2006), is an ideal basis with which to construct a fully coupled unified physics computer analysis code. This scheme, developed for all-speed compressible and nearly incompressible flows, improves upon previous pressure-based methods in terms of accuracy and numerical efficiency and gives a wider range of applicability. Because of the need to simulate flows with shocks it is essential that both the governing equations and their discretized approximations be in conservative form (Lax and Wendroff, 1960)(Leveque, 1990). Unlike other ICE variants that have been proposed in the past, most of which are entirely or partially in primitive form, the PCICE algorithm solves the conservative form of the governing equations.

Other researchers have coupled, to varying extent, energy effects into ICE-type algorithms (Casulli and Greenspan, 1984)(Liles and Reed, 1978)(Westbrook, 1978) (Xiao, et al., 1997), but most have utilized, entirely or partially, nonconservative forms which led to algorithms which are restricted to smooth transient solutions (no discontinuity waves) or to steady solutions with sonic- and lower speeds. Patnaik, et. al. (Patnaik, et al., 1987) 
developed a "barely implicit" ICE-type algorithm in conservative form which primarily couples the momentum and energy equations, similar to that of Cassuli and Greenspan (Casulli and Greenspan, 1984). The PCICE algorithm efficiently incorporates an even higher degree of implicitness into a very general conservative framework which can be utilized with either finite difference, finite volume, or finite element spatial representations. In the PCICE algorithm, the total energy equation is sufficiently coupled to the pressure Poisson equation to avoid iteration between the pressure Poisson equation and the pressure-correction equations. The pressure Poisson equation then has the time-advanced internal energy information it requires to yield an accurate implicit pressure. At the end of a time step, the conserved values of mass, momentum, and total energy are all pressure-corrected. As a result, the iterative process usually associated with pressure-based schemes is not required. This aspect has been found advantageous when computing transient compressible flows, including flows with significant energy deposition, chemical reactions, or phase change.

The pressure-based PCICE solution algorithm is composed of two fractional steps. The first fractional step is composed of an explicit predictor-corrector Heun's method applied without the pressure gradient term present in the momentum equation (projection-type approach). In the second fractional step an implicit pressure correction is employed - an elliptic Poisson equation is solved for new-time pressures followed by an explicit correction with the new pressures. The pressure, momentum, and density in the governing hydrodynamic equations are treated in an implicit fashion. The so-called mass-momentum coupling is obtained by substituting the momentum balance equations into the mass conservation equation to eliminate time-advanced momentum-density (or mass flux) as an unknown. The time rate of density change in the mass conservation equation is then expressed in terms of pressure and internal energy change by employing the equation of state. These substitutions result in a single second-order elliptic differential equation in terms of pressure (pressure Poisson equation). This semi-implicit treatment has two advantages over explicit schemes. First, the acoustic component from the explicit time step size stability criteria is removed, thus eliminating the time integration stiffness that results from slow flows. Second, the pressure obtained with this semi-implicit treatment corrects the momentum to satisfy mass conservation requirements. This allows nearly incompressible flows to be simulated with compressible flow equations, which can be used to simulate flows from very low speeds to supersonic, including mixed flows with all flow speeds present.

Though our original description of the PCICE algorithm was in the context of a finite-element based method, PCICE-FEM (Martineau and Berry, 2004), with an ideal gas equation of state, it can be generally implemented within the context of other spatial discretization methods (finite-difference, finite-volume, grid-free, etc.) (Berry, 2006). Therefore, the description of the PCICE algorithm given here will be kept free of specific spatial discretizations and equations of state.

\subsection{TEMPORAL DISCRETIZATION}

The PCICE algorithm is a predictor-corrector method for solving the following time discretization of balance Eqns. (1), (2), and (3) for mass, momentum, and energy, respectively (for illustration, here simplified to the Euler equations):

$$
\begin{aligned}
& \rho^{n+1}=\rho^{n}-\frac{\Delta t}{2} \nabla \cdot\left[(\rho \vec{u})^{n+1}+(\rho \vec{u})^{n}\right] \\
& (\rho \vec{u})^{n+1}=(\rho \vec{u})^{n}-\Delta t \vec{\nabla} \cdot(\rho \vec{u} \otimes \vec{u})^{n+\frac{1}{2}}-\frac{\Delta t}{2} \vec{\nabla}\left(p^{n+1}+p^{n}\right) \\
& \left(\rho e_{t}\right)^{n+1}=\left(\rho e_{t}\right)^{n}-\frac{\Delta t}{2} \vec{\nabla} \cdot\left[(\rho \vec{u})^{n+1} h_{t}^{n+1}+(\rho \vec{u})^{n} h_{t}^{n}\right] .
\end{aligned}
$$


The divergence term in the momentum equation is at a partially time-advanced level obtained by utilizing an explicit two-step, modified Heun's method which will be described subsequently. In the original PCICE-FEM method (Martineau and Berry, 2004), an efficient Taylor-Galerkin method was used, however the modified Heun's method exhibits lower phase errors near discontinuities. These equations are approximated with the following fractional two-step process.

\subsection{Fractional Step 1}

The first fractional step solves a portion of Eqns. (5)-(7), with a predictor step:

$$
\begin{aligned}
& \rho^{*}=\rho^{n}-\Delta t \nabla \cdot(\rho \vec{u})^{n} \\
& (\rho \vec{u})^{*}=(\rho \vec{u})^{n}-\Delta t \vec{\nabla} \cdot(\rho \vec{u} \otimes \vec{u})^{n} \\
& \left(\rho e_{t}\right)^{*}=\left(\rho e_{t}\right)^{n}-\Delta t \vec{\nabla} \cdot(\rho \vec{u})^{n} h_{t}^{n} \\
& \vec{u}^{*}=\frac{(\rho \vec{u})^{*}}{\rho^{*}} \quad e_{t}^{*}=\frac{\left(\rho e_{t}\right)^{*}}{\rho^{*}} \quad e^{*}=e_{t}^{*}-\frac{\vec{u}^{*} \cdot \vec{u}^{*}}{2} \\
& p^{*}=f\left(\rho^{*}, e^{*}\right) \quad h_{t}^{*}=\frac{\left(\rho e_{t}\right)^{*}+p^{*}}{\rho^{*}}
\end{aligned}
$$

followed by a corrector step:

$$
\begin{aligned}
\rho^{* * *} & =\rho^{n}-\frac{\Delta t}{2} \nabla \cdot\left[(\rho \vec{u})^{*}+(\rho \vec{u})^{n}\right] \\
& =\rho^{*}-\frac{\Delta t}{2} \nabla \cdot\left[(\rho \vec{u})^{*}-(\rho \vec{u})^{n}\right] \\
(\rho \vec{u})^{* *} & =(\rho \vec{u})^{n}-\frac{\Delta t}{2} \vec{\nabla} \cdot\left[(\rho \vec{u} \otimes \vec{u})^{*}+(\rho \vec{u} \otimes \vec{u})^{n}\right] \\
& =(\rho \vec{u})^{*}-\frac{\Delta t}{2} \vec{\nabla} \cdot\left[(\rho \vec{u} \otimes \vec{u})^{*}-(\rho \vec{u} \otimes \vec{u})^{n}\right] \\
\left(\rho e_{t}\right)^{* *} & =\left(\rho e_{t}\right)^{n}-\frac{\Delta t}{2} \vec{\nabla} \cdot\left[(\rho \vec{u})^{*} h_{t}^{*}+(\rho \vec{u})^{n} h_{t}^{n}\right] \\
& =\left(\rho e_{t}\right)^{*}-\frac{\Delta t}{2} \vec{\nabla} \cdot\left[(\rho \vec{u})^{*} h_{t}^{*}-(\rho \vec{u})^{n} h_{t}^{n}\right] \\
\vec{u}^{* *}= & \frac{(\rho \vec{u})^{* *}}{\rho^{* *}} \quad e_{t}^{* *}=\frac{\left(\rho e_{t}\right)^{* *}}{\rho * *}-\frac{\vec{u}^{* *} \cdot \vec{u}^{* *}}{2} \\
p^{* *}= & f\left(\rho^{* *}, e^{* *}\right) \quad h_{t}^{* *}=\frac{\left(\rho e_{t}\right)^{* *}+p^{* *}}{\rho^{* *}}
\end{aligned}
$$

It is important that the quantities $\rho,(\rho \vec{u})$, and $\left(\rho e_{t}\right)$, for both the predictor $\left(^{*}\right)$ and corrector $(* *)$ steps, be advanced with high-order monotonic algorithms such as FCT, TVD, ENO, etc. or that they be smoothed with another appropriate smoother such as the variable diffusion method of Swanson and Turkel (Swanson and Turkel, 
1992) used in the finite element version PCICE-FEM (Martineau and Berry, 2004).

As with projection-type schemes, the pressure gradient is not included in the partial momentum balance Eqns. (9) and (13). Instead, it will be included implicitly in the next fractional step. Therefore the time step stability restriction for this fractional step is the satisfaction of the material Courant condition, or the Courant condition based on flow speed. If the shear stress, heat transfer, or energy source terms are included and treated explicitly, a stable time step based on these terms may be too restrictive. To obtain additional stability with larger time steps, such terms can be treated implicitly, either here in this fractional step, or in an additional (subsequent or previous) fractional step.

\subsection{Fractional Step 2}

This fractional step seeks to solve the following portion of the original discretized Eqns. (5)-(7) to obtain new time pressure.

Momentum

$$
\begin{aligned}
(\rho \vec{u})^{n+1} & =(\rho \vec{u})^{* *}-\frac{\Delta t}{2} \nabla\left(p^{n+1}+p^{n}\right) \\
& =(\rho \vec{u})^{* *}-\frac{\Delta t}{2} \nabla\left(p^{n+1}-p^{n}\right)-\Delta t \nabla p^{n}
\end{aligned}
$$

Mass

$$
\rho^{n+1}=\rho^{* *}-\frac{\Delta t}{2} \nabla \cdot\left[(\rho \vec{u})^{n+1}-(\rho \vec{u})^{*}\right]
$$

Total Energy

$$
\left(\rho e_{t}\right)^{n+1}=\left(\rho e_{t}\right)^{* *}-\frac{\Delta t}{2} \nabla \cdot\left[(\rho \vec{u})^{n+1} h_{t}^{n+1}-(\rho \vec{u})^{*} h_{t}^{*}\right] .
$$

Eqn. (16) is required to achieve the target discretization, and is obtained by subtracting Eqn. (13) from Eqn. (6) using

$$
(\rho \vec{u} \otimes \vec{u})^{n+\frac{1}{2}}=\frac{1}{2}\left[(\rho \vec{u} \otimes \vec{u})^{*}+(\rho \vec{u} \otimes \vec{u})^{n}\right] .
$$

Similarly, Eqn. (17) is obtained by subtracting Eqn. (12) from (5) and Eqn. (18) results from subtracting Eqn. (14) from (7).

The solution to Eqns. (16) - (18) is accomplished in a couple of steps, first by constructing a pressure Poisson equation which is solved to obtain pressures at the new-time level, then correcting the dependent variables with these new-time pressures. 
The pressure Poisson equation is constructed by first substituting Eqn. (16) into Eqn. (5)

$$
\rho^{n+1}=\rho^{n}-\frac{\Delta t}{2} \nabla \cdot\left[(\rho \vec{u})^{* *}-\frac{\Delta t}{2} \nabla \cdot\left(p^{n+1}-p^{n}\right)-\Delta t \nabla p^{n}+(\rho \vec{u})^{n}\right]
$$

or with rearrangement

$$
\begin{gathered}
\rho^{n+1}-\rho^{n}=\frac{\Delta t^{2}}{4} \nabla \cdot \nabla\left(p^{n+1}-p^{n}\right)+\frac{\Delta t^{2}}{2} \nabla \cdot \nabla p^{n} \\
-\frac{\Delta t}{2} \nabla \cdot\left[(\rho \vec{u})^{* *}+(\rho \vec{u})^{n}\right]
\end{gathered} .
$$

From the equation of state (EOS)

$$
p=f(\rho, e)
$$

one can obtain

$$
\delta \rho=\frac{1}{\frac{\partial f}{\partial \rho}} \delta p-\frac{\frac{\partial f}{\partial e}}{\frac{\partial f}{\partial \rho}} \delta e
$$

where $\delta$ just indicates a perturbation or change in a quantity. This leads to the simple approximation

$$
\rho^{n+1}-\rho^{n} \approx \frac{1}{\left(\frac{\partial f}{\partial \rho}\right)^{* *}}\left(p^{n+1}-p^{n}\right)-\frac{\left(\frac{\partial f}{\partial e}\right)^{* *}}{\left(\frac{\partial f}{\partial \rho}\right)^{* *}}\left(e^{* *}-e^{n}\right) .
$$

The pressure Poisson equation, in terms of $\delta p=p^{n+1}-p^{n}$, is finally obtained by substituting Eqn. (20) into Eqn. (19) giving

$$
\begin{aligned}
\frac{1}{\left(\frac{\partial f}{\partial \rho}\right)^{* *}}\left(p^{n+1}-p^{n}\right)-\frac{\Delta t^{2}}{4} \nabla \cdot \nabla\left(p^{n+1}-p^{n}\right) & =\frac{\left(\frac{\partial f}{\partial e}\right)^{* *}}{\left(\frac{\partial f}{\partial \rho}\right)^{* *}}\left(e^{* *}-e^{n}\right) \\
& -\frac{\Delta t}{2} \nabla \cdot\left[(\rho \vec{u})^{* *}+(\rho \vec{u})^{n}\right] \\
& +\frac{\Delta t^{2}}{2} \nabla \cdot \nabla p^{n} .
\end{aligned}
$$

Numerical solution of this equation by an efficient, elliptic partial differential equation solver yields the new pressure distribution, $p^{n+1}$.

The other dependent variables are then updated, or corrected, with the new-time pressures as follows, in order: 
Momentum

$(\rho \vec{u})^{n+1}=(\rho \vec{u})^{* *}-\frac{\Delta t}{2} \nabla\left[p^{n+1}+p^{n}\right]$

Mass

$\rho^{n+1}=\rho^{* *}-\frac{\Delta t}{2} \nabla \cdot\left[(\rho \vec{u})^{n+1}-(\rho \vec{u})^{*}\right]$

Total Energy

$h_{t}^{n+1}=\frac{\left(\rho e_{t}\right)^{* *}+p^{n+1}}{\rho^{n+1}}$

$\left(\rho e_{t}\right)^{n+1}=\left(\rho e_{t}\right)^{* *}-\frac{\Delta t}{2} \nabla \cdot\left[(\rho \vec{u})^{n+1} h_{t}^{n+1}-(\rho \vec{u})^{*} h_{t}^{*}\right]$

\subsection{COMPRESSIBLE AND INCOMPRESSIBLE LIMITS}

The objective here is to identify and examine various compressible limiting forms of the PCICE algorithm. Dividing the pressure Poisson Eqn. (21) by $\Delta t$, noting that the isentropic sound speed $c$ is given by $c^{2}=\frac{p}{\rho^{2}}\left(\frac{\partial f}{\partial e}\right)+\left(\frac{\partial f}{\partial \rho}\right)$,

and introducing

$\frac{1}{\left(\frac{\partial f}{\partial \rho}\right)}=\frac{A}{c^{2}} \quad$ where $A=\left[1+\frac{p\left(\frac{\partial f}{\partial e}\right)}{\rho^{2}\left(\frac{\partial f}{\partial \rho}\right)}\right]$,

transforms pressure Poisson equation into

$$
\begin{aligned}
\frac{A^{* *}}{\left(c^{* *}\right)^{2}} \frac{p^{n+1}-p^{n}}{\Delta t} & =\frac{\Delta t}{4} \nabla^{2} p^{n+1}+\frac{A^{* *}\left(\frac{\partial f}{\partial e}\right)^{* *}}{\left(c^{* *}\right)^{2}} \frac{e^{* *}-e^{n}}{\Delta t} \\
& -\frac{1}{2} \nabla \cdot\left[(\rho \vec{u})^{* *}+(\rho \vec{u})^{n}\right] \\
& +\frac{\Delta t}{4} \nabla^{2} p^{n} .
\end{aligned}
$$

If Eqn. (26) is multiplied by a characteristic length, $l$, and a characteristic time,

$$
\begin{aligned}
\tau_{c} \frac{p^{n+1}-p^{n}}{\Delta t} & =\frac{l c^{* *} \Delta t}{4 A^{* *}} \nabla^{2} p^{n+1}+\tau_{c}\left(\frac{\partial f}{\partial e}\right)^{* *} \frac{e^{* *}-e^{n}}{\Delta t} \\
& -\frac{l c^{* *}}{2 A^{* *}} \nabla \cdot\left[(\rho \vec{u})^{* *}+(\rho \vec{u})^{n}\right] \\
& +\frac{l c^{* *} \Delta t}{4 A^{* *}} \nabla^{2} p^{n} .
\end{aligned}
$$

The characteristic time $\tau_{c}$ approximates the time it takes an acoustic wave (traveling with velocity $c^{* *}$ to propagate the distance $(l)$ characterizing the portion of our solution domain of interest and effecting the solution 
change. While incompressible fluids don't physically exist, such a mathematical model can be conceived by supposing the time resolution of interest corresponds to $\Delta t$. Thus if the case is desired in which $\tau_{c}<<t$, then necessarily the resolution of the physical acoustic waves which produce the solution change is not of interest.

Furthermore, a characteristic time $\tau_{u}=\frac{l}{|\vec{u}|}$ can be identified which approximates the time it takes to advect the solution a distance $l$.

\subsection{Nearly Incompressible Limit}

In the nearly incompressible limit $\tau_{c}<<\Delta t<\tau_{u}$, or in other words $c^{*} \Delta t>>l$, which implies that $\frac{\tau_{c}}{\Delta t} \rightarrow 0$ and the pressure Poisson Eqn. (27) effectively reduces to

$$
\nabla^{2} p^{n+1}=\frac{2}{\Delta t} \nabla \cdot\left[(\rho \vec{u})^{* *}+(\rho \vec{u})^{n}\right]-\nabla^{2} p^{n} .
$$

Obviously the nearly incompressible limit also implies that $\frac{|\vec{u}|}{c^{*}}<<\frac{|\vec{u}| \Delta t}{l}<1$, or the Mach Number $<<1$.

\subsection{Strictly Incompressible Limit}

Substituting the gradient of the equation of state

$\nabla \rho=\frac{1}{\left(\frac{\partial f}{\partial \rho}\right)} \nabla p-\frac{\left(\frac{\partial f}{\partial e}\right)}{\left(\frac{\partial f}{\partial \rho}\right)} \nabla e=\frac{A}{c^{2}} \nabla p-\frac{A\left(\frac{\partial f}{\partial e}\right)}{c^{2}} \nabla e$

into the expanded third term on the right side of Eqn. (27) gives

$$
\begin{aligned}
-\frac{l c^{* *}}{2 A^{* *}} \nabla \cdot\left[(\rho \vec{u})^{* *}+(\rho \vec{u})^{n}\right] & =-\frac{l c^{* *}}{2 A^{* *}}\left[\vec{u}^{* *} \cdot \nabla \rho^{* *}+\rho^{* *}\left(\nabla \cdot \vec{u}^{* *}\right) \vec{u}^{n} \cdot \nabla \rho^{n}+\rho^{n}\left(\nabla \cdot \vec{u}^{n}\right)\right] \\
& =-\frac{\tau_{c}}{2} \vec{u}^{* *} \cdot\left[\nabla p^{* *}-\left(\frac{\partial f}{\partial e}\right)^{* *} \nabla e^{* *}\right]-\frac{l c^{* *}}{2 A^{* *}} \rho^{* *}\left(\nabla \cdot \vec{u}^{* *}\right) \\
& -\frac{\tau_{c}^{n}}{2}\left(\frac{A^{n}}{A^{* *}}\right)\left(\frac{c^{* *}}{c^{n}}\right) \vec{u}^{n} \cdot\left[\nabla p^{n}-\left(\frac{\partial f}{\partial e}\right)^{n} \nabla e^{n}\right] \\
& -\frac{l c^{* *}}{2 A^{* *}} \rho^{n}\left(\nabla \cdot \vec{u}^{n}\right)
\end{aligned}
$$

In the strictly incompressible limit, $\tau_{c} \rightarrow 0, \tau_{c}^{n} \rightarrow 0$ (because sound speeds are infinite), so Eqn. (30) becomes

$$
\begin{aligned}
-\lim _{\tau_{c} \rightarrow 0} \lim _{\tau_{c} \rightarrow 0} \frac{l c^{* *}}{A^{* *}} \nabla \cdot\left[(\rho \vec{u})^{* *}+(\rho \vec{u})^{n}\right]= \\
-\frac{l c^{* *}}{2 A^{* *}}\left[\rho^{* *}\left(\nabla \cdot \vec{u}^{* *}\right)+\rho^{n}\left(\nabla \cdot \vec{u}^{n}\right)\right]
\end{aligned}
$$

and the pressure Poisson Eqn. (27) reduces to

$$
\nabla^{2} p^{n+1}=\frac{2}{\Delta t}\left[\rho^{* *}\left(\nabla \cdot \vec{u}^{* *}\right)+\rho^{n}(\nabla \cdot \vec{u})\right]-\nabla^{2} p^{n}
$$

Note that $\tau_{c} \rightarrow 0$ and $\tau_{c}^{n} \rightarrow 0$ imply, physically, that the characteristic length scale of interest $l$ is very small relative to the sound speed, as opposed to considering incompressible flow to possess an infinite sound speed, as some researchers choose. If $\nabla \cdot \vec{u}^{n}=0$, Eqn. (32) can be compared directly with the corresponding, traditional 
MAC-type pressure Poisson equation (Harlow and Welch, 1965), without convective terms,

$$
\nabla^{2} p^{n+1}=\frac{\rho^{n} \nabla \cdot \vec{u}^{* *}}{\Delta t} \text {. }
$$

In these two equations, the computed pressure field will be consistent with the requirement for incompressible flow fields that the velocity fields be divergence-free. It is, however, an advantage to have density included at a partially updated time as in Eqn. (32) instead of being evaluated at old time as in the MAC-type Eqn. (33). Moreover, in the nearly incompressible limit Eqn. (28) additionally allows for potentially important spatial gradients in the density field to drive the pressure Poisson equation, and thus to be coupled with the pressure and velocity fields. This could be important, for example, in natural convection flows. It is apparent that if a primitive variable strictly incompressible approach is adopted along with a Boussinesque approximation, the only way bouyant forces could enter the pressure Poisson equation is via the velocity divergence term in Eqn. (32), which in turn comes from an explicit velocity (momentum) predictor equation. Unlike the nearly incompressible limit Poisson Eqn. (28), the density gradient effects would then be neglected.

\subsection{Fully Compressible Limit}

On the other end of the spectrum, for fully compressible flows $\tau_{u}>\tau_{c} \gg \Delta t$, or $c^{* *} \Delta t<<l$, and the pressure Poisson Eqn. (27) can be rewritten as

$$
\frac{1}{\left(\frac{\partial f}{\partial \rho}\right)^{* *}} \frac{p^{n+1}-p^{n}}{\Delta t}-\frac{\left(\frac{\partial f}{\partial e}\right)^{* *}}{\left(\frac{\partial f}{\partial \rho}\right)^{* *}} \frac{e^{* *}-e^{n}}{\Delta t}=-\nabla \cdot(\rho \vec{u})^{* *}
$$

(as seen from Eqn. (17)) which is effectively a density-based compressible flow algorithm since it is easily recognized that the left side of this equation is an approximation for the term $\frac{\partial \rho}{\partial t}$. In fact, the combination of fractional step 1 above with pressure Eqn. (34) and the correction Eqns. (22)-(25), to which the PCICE methods reduces in this limit, constitutes an explicit, predictor-corrector algorithm for fully compressible flows.

\subsection{EXAMPLES}

We give here three example simulations computed with PCICE-FEM in two spatial dimensions, one for each of the three limiting classes discussed above: strictly incompressible, nearly incompressible, and fully compressible. For the strictly incompressible regime we utilize the thermally driven flow in a square cavity. A von Karman vortex street behind a cylinder will be used as an example illustrating the nearly incompressible regime. For the fully compressible regime we simulate an intense spherical explosion, sometimes known as the Sedov blast wave problem. 


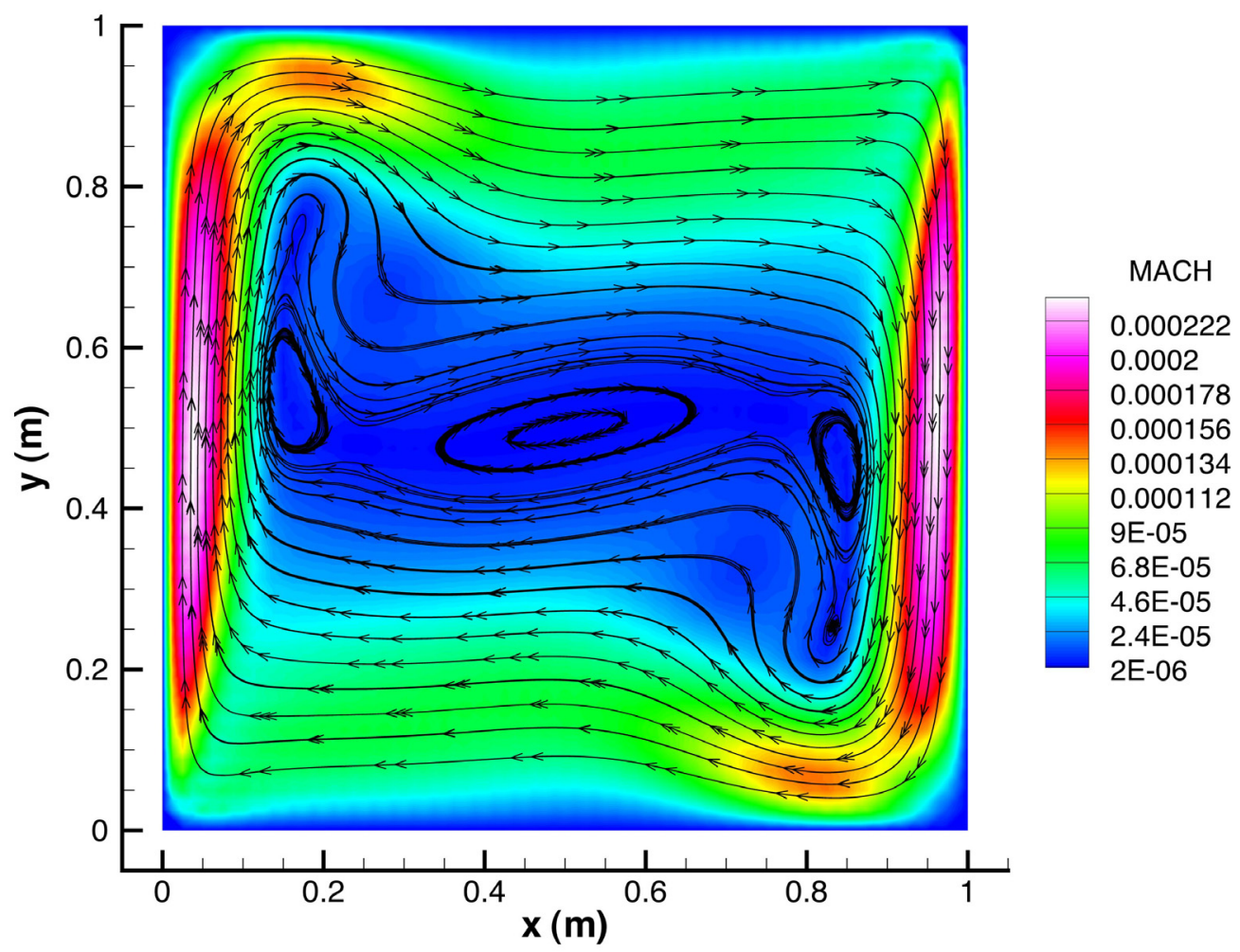

Figure 1. THERMALLY DRIVEN CAVITY, $\mathrm{Ra}=10^{6}$, Wall Temperature Difference $=12 \mathrm{~K}$.

\subsection{Strictly Incompressible Example}

We consider the thermally driven, circulatory flow of air in an upright square domain (Heuveline, 2003). The two horizontal walls are defined as no-slip, adiabatic solid walls and the two vertical walls are defined as no-slip isothermal walls, $606 \mathrm{~K}$ on the left wall and $594 \mathrm{~K}$ on the right. Fig. 1 shows the Mach number isolines (color shading) along with the streamline contours for a Raleigh number of $10^{6}$. This agrees very closely with the results obtained by (Heuveline, 2003) as well as those obtained assuming strictly incompressible flow with the Boussinesq approximation. Note, however, that if we changed the temperatures, e.g. to $960 \mathrm{~K}$ and $240 \mathrm{~K}$ keeping the Raleigh number the same (thus, viscosity must be raised), the heat flux would be much higher and density gradients would play a much more dominant role. Then the flow would be within the nearly incompressible regime, and the results would not agree with those obtained assuming incompressible flow with the Boussinesq approximation. This case is also shown in (Heuveline, 2003).

\subsection{Nearly Incompressible Example}

A common benchmark problem for transient algorithms employing the incompressible Navier-Stokes equations is the low Reynolds number flow around a cylinder in a cross flow. Beyond a Reynolds number of 40, the solution becomes unsteady and a periodic shedding of vortices occurs, known as the von Karman vortex street. These vortices are laminar for Reynolds numbers up to approximately 5000. Here we use our compressible, all-speed PCICE-FEM formulation with a free stream velocity equivalent to a Mach number of $M=0.05$. For a free stream temperature of $300 \mathrm{~K}$ and a free stream pressure of $101325 \mathrm{~Pa}$, the free stream velocity is $17.36 \mathrm{~m} / \mathrm{s}$. The top and bottom walls are set to free-slip. Fig. 2 shows an instantaneous computed Mach number distribution. At this same time, the corresponding instantaneous computed temperature distribution is shown in Fig. 3. Note the small Mach number and the small temperature differences, due to the slight compressibility effects, are resolved on the vortex street, even though the flows are very slow. An incompressible solution would not be able produce this result. 


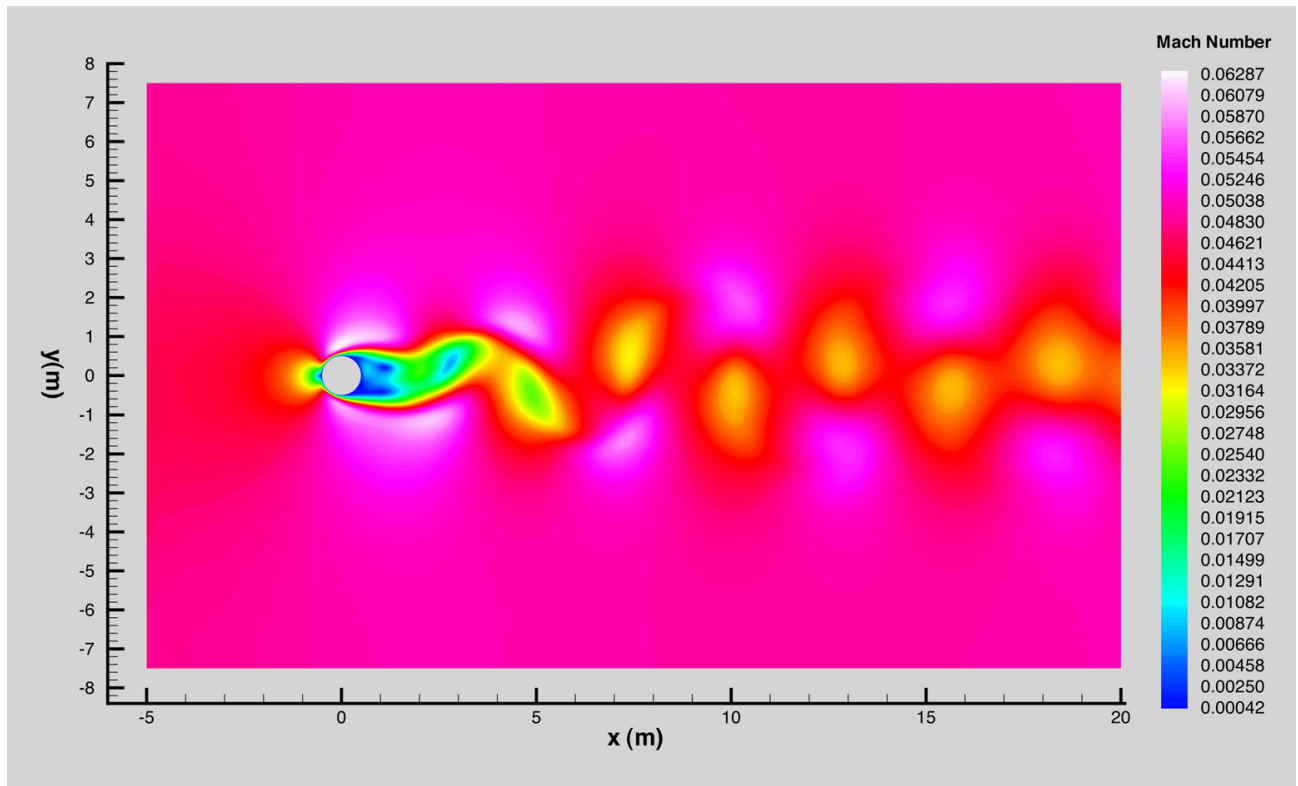

Figure 2. VON KARMAN VORTEX STREET MACH NUMBER SOLUTION, time=4.0 s.

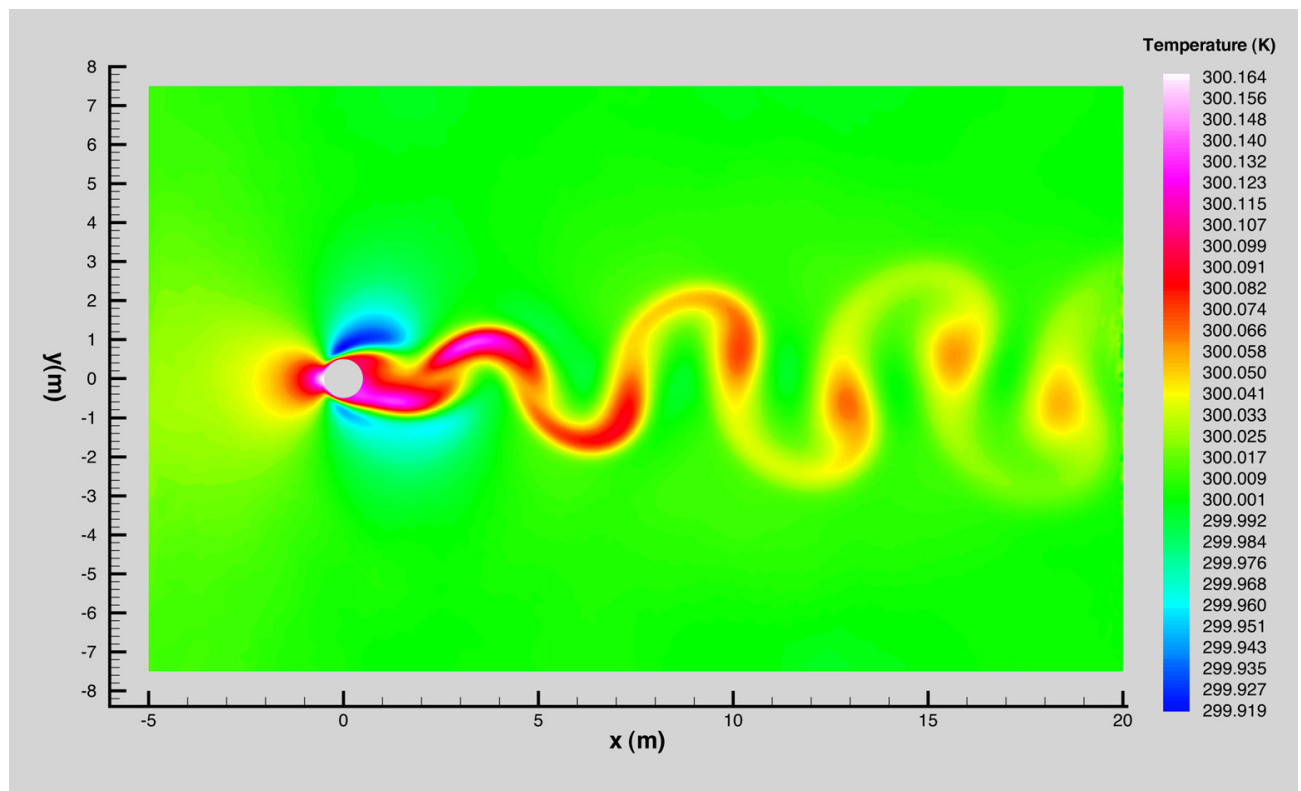

Figure 3. VON KARMAN VORTEX STREET TEMPERATURE SOLUTION, time $=4.0 \mathrm{~s}$.

\subsection{Fully Compressible Example}

The Sedov blast wave Sedov, 1959) problem is characterized as an intense point explosion in a uniform ideal gas, initially at rest, with negligible initial pressure and finite initial density. This axisymmetrical PCICE-FEM (Martineau, 2007) simulation incorporates the initial conditions of the 1945 Trinity fission bomb in New Mexico and is compared to Sedov's analytical solution. A $100 \mathrm{~m}$ radius hemispherical domain is represented by one quadrant of a circular region integrated about the axis of symmetry (x-axis). The estimated explosion energy of the Trinity test is $7.19 \times 10^{13} \mathrm{~J}$. To approximate this explosion, $3.595 \times 101^{3} \mathrm{~J}$ (one-half the explosion energy for one-half of the sphere) is instantly deposited in the finite elements with an elemental distance less than $4.5 \mathrm{~m}$ from the origin. The balance of the domain is at atmospheric conditions, where pressure is initially set to $P=9.41831 \times 10^{10} \mathrm{~Pa}$ and temperature is initially set to $T=2.62497 \times 10^{8} \mathrm{~K}$. This constitutes a 941,831 to 1 drop in pressure for the first time step. The Sedov analytical solution for this explosion energy instantly deposited at the origin with an initial density of $\rho_{0}=1.25 \mathrm{~kg} / \mathrm{m}^{3}$ 
results in a shock wave location of $84.57 \mathrm{~m}$ from the origin at $8.0 \times 10^{-3} \mathrm{~s}$ after the explosion is initiated. Fig. 4 shows computed density distribution $\left(\rho / \rho_{0}\right)$ at this time and Fig. 5 shows a comparison of this density and the pressure solutions with Sedov's analytical solutions along the coordinate axis. Both compare very favorably with Sedov's solution.

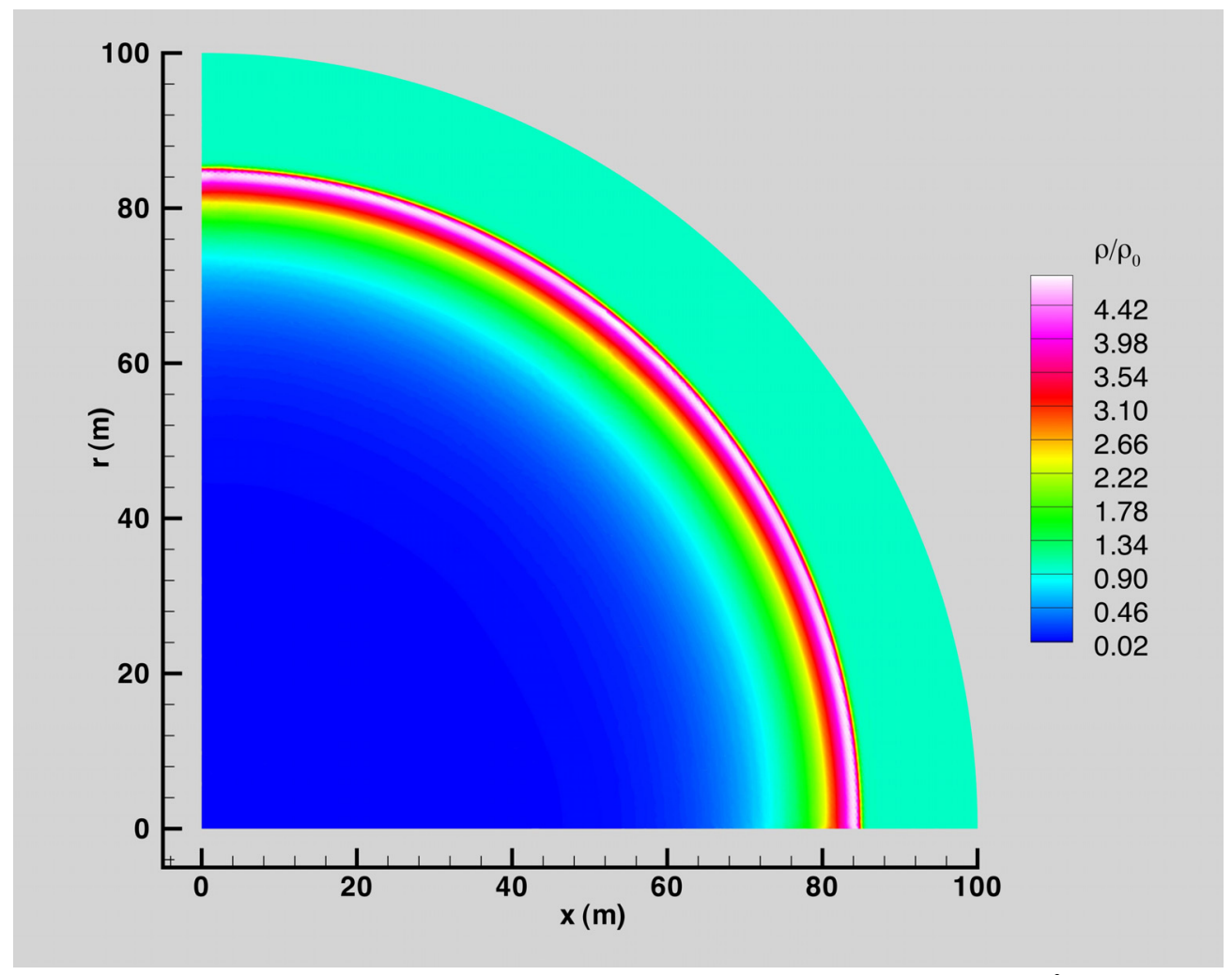

Figure 4. SEDOV BLAST WAVE MASS DENSITY SOLUTION, time $=8.0 \times 10^{-3} \mathrm{~s}$.
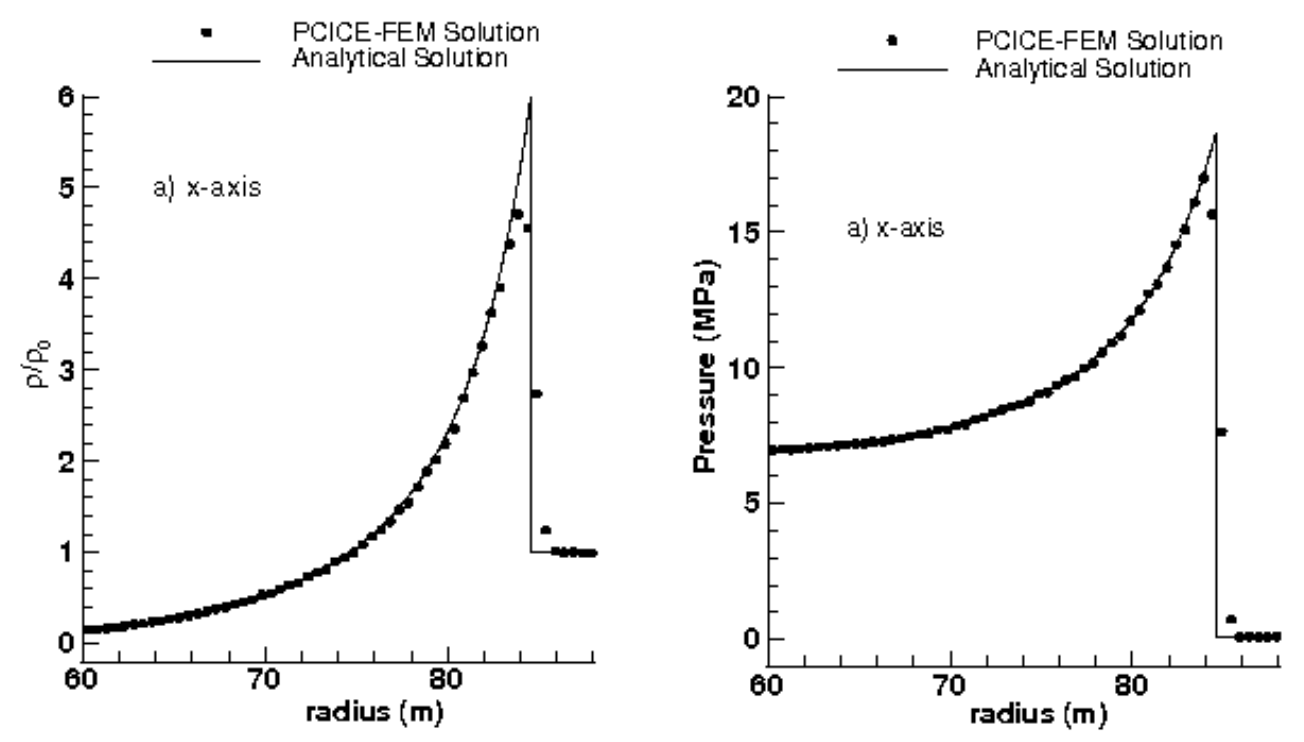

Figure 5. SEDOV BLAST WAVE COMPUTED DENSITY AND PRESSURE COMPARISON TO ANALYTICAL, time $=8.0 \times 10^{-3} \mathrm{~s}$. 


\subsection{CONCLUSIONS}

The PCICE method has been shown to reduce to a variant of the traditional MAC-type method for strictly incompressible flows. However, most single phase flows of practical interest in nuclear energy systems do not fall within this narrowly defined restrictive class. Rather, they can be classed as nearly incompressible or fully compressible. For nearly incompressible flows (e.g. in natural convection flows where heat flux is significant the strictly incompressible flow assumption coupled with a Boussinesq approximation is physically unrealistic) the PCICE method has been shown to reduce to an appropriate generalization in which density gradients directly serve as a driving mechanism in the flow field. Unlike most other pressure-based methods which claim to allow for compressibility effects, the PCICE method is formulated in conservative form so that supersonic flows and flows containing strong shock waves can be easily considered. The PCICE method has been shown to approximate a conservative-form density-based method for such fully compressible flows. Since the method automatically treats a continuous transition or continuum of compressibility, mixed flows with low speed, even incompressible, in one region and high speed or shock waves in another can be treated.

\section{ACKNOWLEDGMENT}

The author acknowledges the US Department of Energy, Office of Nuclear Energy for funding the preparation of this manuscript at the Idaho National Laboratory under DOE Idaho Operations Office Contract DE-AC0705 ID14517.

\section{REFERENCES}

Martineau, R.C. and Berry, R.A., The pressure-corrected ICE finite element method (PCICE-FEM) for compressible flows on unstructured meshes, J. Comput. Phys. 198 (2004) 659.

Berry, R.A., Notes on the PCICE method: simplification, generalization, and compressibility properties, J. Comput. Phys. 215 (2006) 6.

Harlow, F.H. and Amsden, A.A., A numerical fluid dynamics calculation for all flow speeds, J. Comput. Phys. 8 (1971) 197.

Lax, P. and Wendroff, B., Systems of conservation laws, Comm. on Pure and Applied Math. 13 (1960) 217.

Leveque, R.J., Numerical Methods for Conservation Laws, Birkhauser Verlag, Basel, 1990.

Casulli, V. and Greenspan, D., Pressure method for the numerical solution of transient, compressible fluid flow, Int. J. for Num. Meth. in Fluids 4 (1984) 1001.

Liles, D.R. and Reed, W.H., Semi-implicit method for two-phase fluid dynamics, J. Comput. Phys. 26 (1978) 390.

Westbrook, C.K., A generalized ICE method for chemically reactive flows in combustion systems, J. Comput. Phys. 28 (1978) 67.

Xiao, F., et al., An algorithm for simulating solid objects suspended in stratified flow, Computer Phys. Comm. 102 (1997) 147.

Patnaik, G., et al., A barely implicit correction for flux-corrected transport, J. Comp. Phys. 71 (1987) 1.

Swanson, R.C. and Turkel, E., On central-difference and upwind schemes, J. Comput. Phys. 101 (1992) 297.

Harlow, F.H. and Welch, J.E., Numerical calculation of time-dependent viscous incompressible flowof fluids with free surface, Phys. Fluids 8 (1965) 2182.

Heuveline, V., On higher-order mixed FEM for low Mach number flows: application to a natural convection benchmark problem, Int. J. Numer. Meth. Fluids 41 (2003) 1339.

Sedov, L.I., Similarity and Dimensional Methods in Mechanics, Academic Press, New York, 1959.

Martineau, R.C., The PCICE-FEM scheme for highly compressible axisymmetric flows, Int. J. Comput. Fluids, accepted for publication. 


\section{Chapter 5 \\ Mathematical Models for DNS of Compressible Fluids in the Presence of Capillary Effects and Phase Transitions}




\section{Introduction}

The aim of this report is the derivation of a diffuse interface model for the direct numerical simulation of liquid-vapor interfaces appearing, for example, in the vapor bubble growth due to the phase transition. The model accounts for the phase compressibility, in particular that of the gas phase, surface tension effects and phase transitions.

First, a rational derivation of the conservative part of the multiphase model is elaborated by using Hamilton's principle of stationary action. Then, dissipation terms which are compatible with the entropy inequality, are introduced (see examples of such a derivation in Gavrilyuk \& Gouin (1999), and Gavrilyuk \& Saurel (2002)). The model generalizes the capillary model of Perigaud \& Saurel (2005) with the introduction of heat and mass transfer relaxation terms to describe phase transition. The introduction of relaxation terms follows Saurel, Petitpas \& Abgrall (2008).

Two main classes of methods are commonly used for description of interfaces separating fluids. One of them considers the interfaces as sharp discontinuities : Lagrangian approaches (Hirt et al, 1974, Farhat \& Roux, 1991), level-set methods (Osher \& Fedkiw, 2001, Sethian, 2001), tracking methods (Glimm et $a l, 1998$ ) and boundary integral methods ( Hou et al, 2001).

In the second approach the interfaces are diffused. The study of diffused interfaces in compressible fluids is usually based on the second gradient theory (also called in the literature as the theory of Korteweg-type fluids). This theory works quite well in the vicinity of the thermodynamic critical point where there no difference between a liquid and its vapor. The fluid density is considered as the order parameter, and the fluid internal energy is then a function of the density and the density gradient. There has been considerable efforts in this direction (see a survey by D. M. Anderson, G. B. McFadden \& A. A. Wheeler, 1998). Our aim is to study the interfaces far from the thermodynamic critical

point, hence the density can not be considered as the order parameter. A new approach is needed.

\section{Variational principle}

Consider the following transport equation for a sharp interface given by the equation $\phi(t, \mathbf{x})=0$ and having the interface velocity $\mathbf{u}_{i}$ :

$$
\phi_{t}+\mathbf{u}_{i} \cdot \nabla \phi=0
$$

In general, $\phi(t, \mathbf{x})$ is not discontinuous (Heaviside step function), but represents a rather smooth transition zone. In this case, the following modified equation should be resolved (Brackbill, Kothe \& Zemach (1992), Sethian (1999), J. A. Sethian \& P. Smereka (2003)):

$$
\frac{D \phi}{D t}=\phi_{t}+\mathbf{u} \cdot \nabla \phi=0
$$


Here $\mathbf{u}$ is the extension of the interface velocity $\mathbf{u}_{i}$ off the interface. Now we have to couple the equation (2) with the governing equations of non-equilibrium compressible mixtures. First, we will construct a dissipation-free equilibrium model by using Hamilton's principle of stationary action (Gavrilyuk \& Gouin, 1999, Gavrilyuk \& Saurel, 2002). Consider the Lagrangian which is the difference between the kinetic and the potential energy of the mixture :

$$
L=\rho\left(\frac{|\mathbf{u}|^{2}}{2}-\varepsilon\right)-\frac{\lambda}{m}|\nabla \phi|^{m}
$$

Here $\mathbf{u}$ is the velocity (we will use a one-velocity description of the interfaces), $\rho$ is the mixture density, $\varepsilon$ is the mixture specific internal energy :

$$
\rho=\alpha_{1} \rho_{1}+\alpha_{2} \rho_{2}, \quad \varepsilon=y_{1} \varepsilon_{1}+y_{2} \varepsilon_{2}
$$

The volume fractions $\alpha_{i}$ and the mass fractions $y_{i}$ are defined by

$$
\alpha_{1}+\alpha_{2}=1, \quad y_{1}+y_{2}=1, \quad y_{i}=\frac{\alpha_{i} \rho_{i}}{\rho}=\alpha_{i} \rho_{i} v, v=\frac{1}{\rho} .
$$

The Gibbs identity is verified for each phase

$$
\theta_{i} d \eta_{i}=d \varepsilon_{i}+p_{i} d\left(\frac{1}{\rho_{i}}\right)
$$

where $\rho_{i}, p_{i}, \varepsilon_{i}, \theta_{i}, \eta_{i}$ are the densities, pressures, specific energies, temperatures, and specific entropies of each component, respectively. The capillarity parameter $\lambda$ characterizes the diffuse interface. The interface is sharp if $m=1$, and thick if $1<m<\infty$. In other words, if $m=1$, and $\phi$ is the Heaviside step function, the integral of $|\nabla \phi|$ over the volume occupied by the mixture is the total area of the interface separating the phases, and the constant $\lambda$ is the surface tension. In the case $1<m<\infty$ the separate interpretation of the integral of $|\nabla \phi|^{m}$ and the parameter $\lambda$ is less obvious. Only the combination $\frac{\lambda}{m}|\nabla \phi|^{m}$ has a sense, it is a volume capillary energy.

Consider the Hamilton action

$$
a=\int_{t_{1}}^{t_{2}} \int_{D} L d t d D
$$

The Hamilton principle expresses that the particle trajectories are stationary curves of (4) submitted to the following constraints

$$
\rho_{t}+\operatorname{div}(\rho \mathbf{u})=0, \quad \eta_{i t}+\mathbf{u} \cdot \nabla \eta_{i}=0, \quad y_{t}+\mathbf{u} \cdot \nabla y=0, \quad \phi_{t}+\mathbf{u} \cdot \phi=0 .
$$

We will obtain now the governing equations by calculating the first variation of Hamilton's action submitted constraints (5). The variations of unknown variables in Eulerian coordinates in terms of virtual displacements $\delta \mathbf{x}$ are given by (Gavrilyuk \& Gouin, 1999) :

$\delta \rho=-\operatorname{div}(\rho \delta \mathbf{x}), \quad \delta \eta_{i}=-\nabla \eta_{i} \cdot \delta \mathbf{x}, \quad \delta y_{2}=-\nabla y_{2} \cdot \delta \mathbf{x}, \quad \delta \mathbf{u}=\frac{D \delta \mathbf{x}}{D t}-\frac{\partial \mathbf{u}}{\partial \mathbf{x}} \delta \mathbf{x}, \quad \delta \phi=-\nabla \phi \cdot \delta \mathbf{x}$ 
In particular, the definition of $\delta \phi$ implies

$$
\begin{gathered}
\delta(|\nabla \phi|)=\frac{\nabla \phi \cdot \nabla(\delta \phi)}{|\nabla \phi|}, \\
\delta\left(\frac{|\nabla \phi|^{m}}{m}\right)=|\nabla \phi|^{m-2} \nabla \phi \cdot \nabla(\delta \phi)= \\
=\operatorname{div}\left(\delta \phi|\nabla \phi|^{m-2} \nabla \phi\right)-\operatorname{div}\left(|\nabla \phi|^{m-2} \nabla \phi\right) \delta \phi= \\
=\operatorname{div}\left(\delta \phi|\nabla \phi|^{m-2} \nabla \phi\right)+\operatorname{div}\left(|\nabla \phi|^{m-2} \nabla \phi\right) \nabla \phi \cdot \delta \mathbf{x}
\end{gathered}
$$

But

$$
\begin{gathered}
\operatorname{div}\left(|\nabla \phi|^{m-2} \nabla \phi\right) \nabla \phi=\operatorname{div}\left(|\nabla \phi|^{m-2} \nabla \phi \otimes \nabla \phi\right)-|\nabla \phi|^{m-2} \phi^{\prime \prime} \nabla \phi= \\
=\operatorname{div}\left(|\nabla \phi|^{m-2} \nabla \phi \otimes \nabla \phi-\frac{|\nabla \phi|^{m}}{m} I\right)
\end{gathered}
$$

Hence,

$$
\delta\left(\frac{|\nabla \phi|^{m}}{m}\right)=\operatorname{div}\left(\delta \phi|\nabla \phi|^{m-2} \nabla \phi\right)+\operatorname{div}\left(|\nabla \phi|^{m-2} \nabla \phi \otimes \nabla \phi-\frac{|\nabla \phi|^{m}}{m} I\right) \cdot \delta \mathbf{x}
$$

By using (6) we can present the variation of (4) as a linear functional of the virtual displacement $\delta \mathbf{x}$ :

$$
\begin{gathered}
\delta L=\delta \rho \frac{|\mathbf{u}|^{2}}{2}+\rho \mathbf{u} \cdot \delta \mathbf{u}-\delta E= \\
=-\operatorname{div}(\rho \delta \mathbf{x}) \frac{|\mathbf{u}|^{2}}{2}+\rho \mathbf{u} \cdot\left(\frac{d \delta \mathbf{x}}{d t}-\frac{\partial \mathbf{u}}{\partial \mathbf{x}} \delta \mathbf{x}\right)-\delta E
\end{gathered}
$$

This implies

$\delta L=\frac{\partial}{\partial t}(\rho \mathbf{u} \cdot \delta \mathbf{x})+\operatorname{div}\left(\left(\rho \mathbf{u} \otimes \mathbf{u}-\rho \frac{|\mathbf{u}|^{2}}{2} I\right) \delta \mathbf{x}\right)-\frac{\partial \rho \mathbf{u}}{\partial t} \cdot \delta \mathbf{x}-\operatorname{div}(\rho \mathbf{u} \otimes \mathbf{u}) \cdot \delta \mathbf{x}-\delta E$

The variation of the Lagrangian (8) contains also the the variation of the volume energy $\delta E$ which is not yet estimated.

$$
E=\rho \varepsilon+\frac{\lambda}{m}|\nabla \phi|^{m}
$$

The thermodynamic volume energy verifies the identity

$$
d(\rho \varepsilon)=h d \rho+\rho\left(h_{2}-h_{1}\right) d y_{2}-\left(p_{2}-p_{1}\right) d \alpha_{2}+\rho \sum_{i=1}^{2} y_{i} \theta_{i} d \eta_{i},
$$

where

$$
h=\varepsilon+\frac{p}{\rho}, \quad p=\alpha_{1} p_{1}+\alpha_{2} p_{2}, \quad h_{i}=\varepsilon_{i}+\frac{p_{i}}{\rho_{i}} .
$$


This identity can also be written in the form

$$
\rho d h=d p+\rho\left(h_{2}-h_{1}\right) d y_{2}-\left(p_{2}-p_{1}\right) d \alpha_{2}+\rho \sum_{i=1}^{2} y_{i} \theta_{i} d \eta_{i}
$$

Finally, the variation of the energy $E$ is given by

$$
\delta E=h \delta \rho+\rho\left(h_{2}-h_{1}\right) \delta y_{2}-\left(p_{2}-p_{1}\right) \delta \alpha_{2}+\rho \sum_{i=1}^{2} y_{i} \theta_{i} \delta \eta_{i}+\lambda \delta\left(\frac{|\nabla \phi|^{m}}{m}\right)
$$

Since $\delta \alpha_{2}$ varies independently (it is not related with the virtual displacement $\delta \mathbf{x})$, one has the equilibrium condition

$$
p_{2}-p_{1}=0
$$

Then the variation of the the total internal energy simplifies :

$$
\begin{aligned}
& \delta E=-h \operatorname{div}(\rho \delta \mathbf{x})-\rho\left(h_{2}-h_{1}\right) \nabla y_{2} \cdot \delta \mathbf{x}-\rho \sum_{i=1}^{2} y_{i} \theta_{i} \nabla \eta_{i} \cdot \delta \mathbf{x}+\lambda \delta\left(\frac{|\nabla \phi|^{m}}{m}\right)= \\
& =-\operatorname{div}(h \rho \delta \mathbf{x})+\rho \nabla h \cdot \delta \mathbf{x}-\rho\left(h_{2}-h_{1}\right) \nabla y_{2} \cdot \delta \mathbf{x}-\rho \sum_{i=1}^{2} y_{i} \theta_{i} \nabla \eta_{i} \cdot \delta \mathbf{x}+\lambda \delta\left(\frac{|\nabla \phi|^{m}}{m}\right)
\end{aligned}
$$

Using (9) we get

$$
\begin{gathered}
\delta E=-\operatorname{div}(h \rho \delta \mathbf{x})+\nabla p \cdot \delta \mathbf{x}+\lambda \delta\left(\frac{|\nabla \phi|^{m}}{m}\right)= \\
=-\operatorname{div}\left(h \rho \delta \mathbf{x}+\lambda|\nabla \phi|^{m-2} \nabla \phi(\nabla \phi \cdot \delta \mathbf{x})\right)+\nabla p \cdot \delta \mathbf{x}+\lambda \operatorname{div}\left(|\nabla \phi|^{m-2} \nabla \phi \otimes \nabla \phi-\frac{|\nabla \phi|^{m}}{m} I\right) \cdot \delta \mathbf{x} .
\end{gathered}
$$

Finally, taking into account (7) the variation of the Lagrangian will be

$$
\begin{aligned}
& \delta L=-\left(\frac{\partial \rho \mathbf{u}}{\partial t}+\operatorname{div}\left(\rho \mathbf{u} \otimes \mathbf{u}+p I+\lambda\left(|\nabla \phi|^{m-2} \nabla \phi \otimes \nabla \phi-\frac{|\nabla \phi|^{m}}{m} I\right)\right)\right) \cdot \delta \mathbf{x}+ \\
& +\frac{\partial}{\partial t}(\rho \mathbf{u} \cdot \delta \mathbf{x})+\operatorname{div}\left(\left(\rho \mathbf{u} \otimes \mathbf{u}-\rho \frac{|\mathbf{u}|^{2}}{2} I\right) \delta \mathbf{x}+h \rho \delta \mathbf{x}+\lambda|\nabla \phi|^{m-2} \nabla \phi(\nabla \phi \cdot \delta \mathbf{x})\right),
\end{aligned}
$$

The terms

$$
\frac{\partial}{\partial t}(\rho \mathbf{u} \cdot \delta \mathbf{x})
$$

and

$$
\operatorname{div}\left(\left(\rho \mathbf{u} \otimes \mathbf{u}-\rho \frac{|\mathbf{u}|^{2}}{2} I\right) \delta \mathbf{x}+h \rho \delta \mathbf{x}+\lambda|\nabla \phi|^{m-2} \nabla \phi(\nabla \phi \cdot \delta \mathbf{x})\right)
$$

are linear with respect to $\delta \mathbf{x}$, and hence they vanish after using the GaussOstrogradsky theorem or integration with respect to time, because $\left.\delta \mathbf{x}\right|_{\partial D}=0$ 
and $\left.\delta \mathbf{x}\right|_{t=t_{1}}=\left.\delta \mathbf{x}\right|_{t=t_{2}}=0$.To satisfy the condition $\delta L=0$ for any $\delta \mathbf{x}$, the momentum equation should be verified :

$$
\frac{\partial \rho \mathbf{u}}{\partial t}+\operatorname{div}(\rho \mathbf{u} \otimes \mathbf{u}-T)=0
$$

with the following symmetric stress tensor :

$$
T=-p I-\lambda\left(|\nabla \phi|^{m-2} \nabla \phi \otimes \nabla \phi-\frac{|\nabla \phi|^{m}}{m} I\right), p=\alpha_{1} p_{1}+\alpha_{2} p_{2}=p_{1}=p_{2} .
$$

$T$ can be given into the following equivalent form :

$$
T=-\left(p+\lambda \frac{m-1}{m}|\mathbf{w}|^{m}\right) I+\lambda|\nabla \phi|^{m} \Pi=-P I+\lambda|\nabla \phi|^{m} \Pi .
$$

Here we introduced the total pressure

$$
P=p+\lambda \frac{m-1}{m}|\mathbf{w}|^{m} .
$$

and the projector operator on the surface $\phi=$ const in the normal direction :

$$
\Pi=I-\frac{\nabla \phi}{|\nabla \phi|} \otimes \frac{\nabla \phi}{|\nabla \phi|}, \quad \Pi=\Pi^{2} .
$$

In the case of sharp interfaces $(m=1)$ the stress tensor is

$$
T=-p I+\lambda|\nabla \phi| \Pi, \quad \Pi=I-\frac{\nabla \phi}{|\nabla \phi|} \otimes \frac{\nabla \phi}{|\nabla \phi|} .
$$

A non-conservative form of the momentum equation can be written by using the identity

$$
\nabla(|\nabla \phi|)=\frac{\phi^{\prime \prime} \nabla \phi}{|\nabla \phi|}=\frac{\partial \mathbf{w}}{\partial \mathbf{x}} \frac{\mathbf{w}}{|\mathbf{w}|}=\left(\frac{\partial \mathbf{w}}{\partial \mathbf{x}}\right)^{T} \frac{\mathbf{w}}{|\mathbf{w}|}
$$

because

$$
\frac{\partial \mathbf{w}}{\partial \mathbf{x}}-\left(\frac{\partial \mathbf{w}}{\partial \mathbf{x}}\right)^{T}=0
$$

Then

$$
\begin{gathered}
\operatorname{div} T=-\nabla P+\lambda \operatorname{div}\left(|\nabla \phi|^{m} \Pi\right)= \\
=-\nabla P+\lambda \operatorname{div}\left(|\nabla \phi|^{m} I-|\nabla \phi|^{m-1} \frac{\nabla \phi}{|\nabla \phi|} \otimes \nabla \phi\right)= \\
=-\nabla P+\lambda(m-1)|\nabla \phi|^{m-2} \phi^{\prime \prime} \nabla \phi-\lambda \operatorname{div}\left(|\nabla \phi|^{m-1} \frac{\nabla \phi}{|\nabla \phi|}\right) \nabla \phi= \\
=-\nabla P+\lambda(m-1)|\nabla \phi|^{m-2} \phi^{\prime \prime} \nabla \phi-\lambda|\nabla \phi|^{m-1} \operatorname{div}\left(\frac{\nabla \phi}{|\nabla \phi|}\right) \nabla \phi-\lambda(m-1)|\nabla \phi|^{m-2} \frac{(\nabla(|\nabla \phi|) \cdot \nabla \phi)}{|\nabla \phi|} \nabla \phi=
\end{gathered}
$$




$$
\begin{gathered}
=-\nabla P-\lambda|\nabla \phi|^{m-1} \operatorname{div}\left(\frac{\nabla \phi}{|\nabla \phi|}\right) \nabla \phi+\lambda(m-1)|\nabla \phi|^{m-2}\left(\phi^{\prime \prime} \nabla \phi-\frac{(\nabla(|\nabla \phi|) \cdot \nabla \phi)}{|\nabla \phi|} \nabla \phi\right)= \\
=-\nabla P-\lambda|\nabla \phi|^{m-1} \operatorname{div}\left(\frac{\nabla \phi}{|\nabla \phi|}\right) \nabla \phi+\lambda(m-1)|\nabla \phi|^{m-2}\left(\phi^{\prime \prime} \nabla \phi-\frac{\left(\left(\phi^{\prime \prime} \nabla \phi\right) \cdot \nabla \phi\right)}{|\nabla \phi|^{2}} \nabla \phi\right)= \\
=-\nabla P-\lambda|\nabla \phi|^{m-1} \operatorname{div}\left(\frac{\nabla \phi}{|\nabla \phi|}\right) \nabla \phi+\lambda(m-1)|\nabla \phi|^{m-2}\left(I-\frac{\nabla \phi}{|\nabla \phi|} \otimes \frac{\nabla \phi}{|\nabla \phi|}\right) \phi^{\prime \prime} \nabla \phi= \\
=-\nabla P-\lambda|\nabla \phi|^{m-1} \operatorname{div}\left(\frac{\nabla \phi}{|\nabla \phi|}\right) \nabla \phi+\lambda(m-1)|\nabla \phi|^{m-2} \Pi \phi^{\prime \prime} \nabla \phi .
\end{gathered}
$$

Consequently, another useful form of the momentum equation is :

$\frac{\partial \rho \mathbf{u}}{\partial t}+\operatorname{div}(\rho \mathbf{u} \otimes \mathbf{u}+P I)=-\lambda|\mathbf{w}|^{m-1} \operatorname{div}\left(\frac{\mathbf{w}}{|\mathbf{w}|}\right) \mathbf{w}+\lambda(m-1)|\mathbf{w}|^{m-2} \Pi \frac{\partial \mathbf{w}}{\partial \mathbf{x}} \mathbf{w} \equiv \lambda \operatorname{div}\left(|\nabla \phi|^{m} \Pi\right)$.

The conservative form of the equation of energy following from the entropy equations is :

$$
\begin{gathered}
\left(\frac{\rho|\mathbf{u}|^{2}}{2}+E\right)_{t}+\operatorname{div}\left(\mathbf{u}\left(\frac{\rho|\mathbf{u}|^{2}}{2}+E\right)-T \mathbf{u}\right)=0, \\
E=\rho \varepsilon+\frac{\lambda}{m}|\nabla \phi|^{m} .
\end{gathered}
$$

Another useful form of the energy equation is :

$$
\left(\frac{\rho|\mathbf{u}|^{2}}{2}+E\right)_{t}+\operatorname{div}\left(\mathbf{u}\left(\frac{\rho|\mathbf{u}|^{2}}{2}+E\right)+P \mathbf{u}\right)=\operatorname{div}\left(\lambda|\nabla \phi|^{m} \Pi\right) \cdot \mathbf{u}+\lambda|\nabla \phi|^{m} \operatorname{tr}\left(\Pi \frac{\partial \mathbf{u}}{\partial \mathbf{x}}\right) .
$$

Taking into account the isentropic character of motion, the equilibrium condition (10) can also be rewritten in the form :

$$
\frac{D \alpha_{2}}{D t}+K \operatorname{div} \mathbf{u}=\mathbf{0}, \quad K=\frac{\rho_{2} c_{2}^{2}-\rho_{1} c_{1}^{2}}{\frac{\rho_{1} c_{1}^{2}}{\alpha_{1}}+\frac{\rho_{2} c_{2}^{2}}{\alpha 2}} .
$$

Here $c_{i}^{2}$ are sound velocities of each component :

$$
c_{i}^{2}=\left.\frac{\partial p_{i}\left(\rho_{i}, \eta_{i}\right)}{\partial \rho_{i}}\right|_{\eta_{i}=\text { const }}
$$

The system of equations governing compressible fluids with capillary effects is :

$$
\begin{gathered}
\rho_{t}+\operatorname{div}(\rho \mathbf{u})=0, \\
y_{2 t}+\mathbf{u} \cdot \nabla y_{2}=0, \\
\phi_{t}+\mathbf{u} \cdot \nabla \phi=0,
\end{gathered}
$$




$$
\begin{gathered}
\frac{\partial \rho \mathbf{u}}{\partial t}+\operatorname{div}(\rho \mathbf{u} \otimes \mathbf{u}-T)=0, T=-P I+\lambda|\nabla \phi|^{m}\left(I-\frac{\nabla \phi}{|\nabla \phi|} \otimes \frac{\nabla \phi}{|\nabla \phi|}\right), \\
\alpha_{2 t}+\mathbf{u} \cdot \nabla \alpha_{2}+K \operatorname{div} \mathbf{u}=\mathbf{0}, \quad K=\frac{\rho_{2} c_{2}^{2}-\rho_{1} c_{1}^{2}}{\frac{\rho_{1} c_{1}^{2}}{\alpha_{1}}+\frac{\rho_{2} c_{2}^{2}}{\alpha_{2}}} \\
\eta_{i t}+\mathbf{u} \cdot \nabla \eta_{i}=0,
\end{gathered}
$$

with the energy equation :

$$
\left(\frac{\rho|\mathbf{u}|^{2}}{2}+E\right)_{t}+\operatorname{div}\left(\mathbf{u}\left(\frac{\rho|\mathbf{u}|^{2}}{2}+E\right)-T \mathbf{u}\right)=0, E=\rho \varepsilon+\frac{\lambda}{m}|\nabla \phi|^{m} .
$$

The initial conditions for $\nabla \phi$ can be taken the same as for $\nabla y_{2}$ : the order parameter $\phi$ can be identified with the mass fraction. If we introduce

$$
\mathbf{w}=\nabla \phi
$$

then, taking the gradient of the transport equation for $\phi$, we can write it in the form

$$
\mathbf{w}_{t}+\nabla(\mathbf{w} \cdot \mathbf{u})=0
$$

It means that the equation for $\phi$ can be eliminated and replaced by the following set of equations :

$$
\mathbf{w}_{t}+\nabla(\mathbf{w} \cdot \mathbf{u})=0, \quad \operatorname{rot}(\mathbf{w})=\mathbf{0} .
$$

An alternative formulation of the equilibrium model describing diffused interfaces is :

$$
\begin{gathered}
\rho_{t}+\operatorname{div}(\rho \mathbf{u})=0, \\
\mathbf{w}_{t}+\nabla(\mathbf{w} \cdot \mathbf{u})=0, \quad \operatorname{rot}(\mathbf{w})=\mathbf{0}, \\
\frac{\partial \rho \mathbf{u}}{\partial t}+\operatorname{div}(\rho \mathbf{u} \otimes \mathbf{u}-T)=0, T=-P I+\lambda|\mathbf{w}|^{m} \Pi, \quad \Pi=I-\frac{\mathbf{w}}{|\mathbf{w}|} \otimes \frac{\mathbf{w}}{|\mathbf{w}|}, \\
\alpha_{2 t}+\mathbf{u} \cdot \nabla \alpha_{2}+K \operatorname{div} \mathbf{u}=0, \quad K=\frac{\rho_{2} c_{2}^{2}-\rho_{1} c_{1}^{2}}{\frac{\rho_{1} c_{1}^{2}}{\alpha_{1}}+\frac{\rho_{2} c_{2}^{2}}{\alpha_{2}}} \\
y_{2 t}+\mathbf{u} \cdot \nabla y_{2}=0, \\
\eta_{i t}+\mathbf{u} \cdot \nabla \eta_{i}=0 .
\end{gathered}
$$

It admits the same energy equation :

$$
\left(\frac{\rho|\mathbf{u}|^{2}}{2}+E\right)_{t}+\operatorname{div}\left(\mathbf{u}\left(\frac{\rho|\mathbf{u}|^{2}}{2}+E\right)-T \mathbf{u}\right)=0, E=\rho \varepsilon+\frac{\lambda}{m}|\nabla \phi|^{m} .
$$

The present model generalizes the model in Perigaud \& Saurel (2005) where the volume fraction was taken as the order parameter while here the mass fraction is the order parameter. 


\section{Hyperbolicity of the diffuse interface equilib- rium model}

The aim of this Section is to study the hyperbolicity of the model (16). Without loss of generality, we consider the one-dimensional case where the constraint $\operatorname{rot}(\mathbf{w})=\mathbf{0}$ is automatically verified. In one-dimension, the model reads :

$$
\begin{gathered}
\frac{D \rho}{D t}+\rho u_{x}=0, \quad \eta_{i t}+u \eta_{i x}=0, \quad y_{2 t}+u y_{2 x}=0, \quad w_{t}+(u w)_{x}=0, \\
\frac{D \alpha_{2}}{D t}+K u_{x}=0, K=\frac{\rho_{2} c_{2}^{2}-\rho_{1} c_{1}^{2}}{\frac{\rho_{1} c_{1}^{2}}{\alpha_{1}}+\frac{\rho_{2} c_{2}^{2}}{\alpha_{2}}} \\
\rho \frac{D u}{D t}+\left(p+\frac{\lambda(m-1)}{m}|w|^{m}\right)_{x}=0,
\end{gathered}
$$

It can also be rewritten in terms of variables $\rho, u, \eta_{i}, y_{2}, w$ and $p$. Indeed, we have :

$$
\begin{gathered}
d p=d\left(\alpha_{1} p_{1}+\alpha_{2} p_{2}\right)=\left(p_{2}-p_{1}\right) d \alpha_{2}+\alpha_{2} c_{2}^{2} d\left(\frac{y_{2} \rho}{\alpha_{2}}\right)+\alpha_{1} c_{1}^{2} d\left(\frac{y_{1} \rho}{\alpha_{1}}\right)+\frac{\partial p_{1}}{\partial \eta_{1}} d \eta_{1}+\frac{\partial p_{2}}{\partial \eta_{2}} d \eta_{2}= \\
=\alpha_{2} y_{2} c_{2}^{2} d\left(\frac{\rho}{\alpha_{2}}\right)+\alpha_{1} y_{1} c_{1}^{2} d\left(\frac{\rho}{\alpha_{1}}\right)=\left(y_{2} c_{2}^{2}+y_{1} c_{1}^{2}\right) d \rho+\left(\frac{y_{1} c_{1}^{2} \rho}{\alpha_{1}}-\frac{y_{2} c_{2}^{2} \rho}{\alpha_{2}}\right) d \alpha_{2}= \\
=\left(y_{2} c_{2}^{2}+y_{1} c_{1}^{2}\right) d \rho+\left(\frac{y_{1} c_{1}^{2}}{\alpha_{1}}-\frac{y_{2} c_{2}^{2}}{\alpha_{2}}\right) \rho d \alpha_{2} .
\end{gathered}
$$

But

$$
\frac{D \alpha_{2}}{D t}-\frac{K}{\rho} \frac{D \rho}{D t}=0, \quad K=\frac{\rho_{2} c_{2}^{2}-\rho_{1} c_{1}^{2}}{\frac{\rho_{1} c_{1}^{2}}{\alpha_{1}}+\frac{\rho_{2} c_{2}^{2}}{\alpha_{2}}}
$$

Taking into account (18) we finally obtain :

$$
d p=c_{w}^{2} d \rho,
$$

where the Wood sound velocity $c_{w}$ is defined by

$$
\frac{1}{\rho c_{w}^{2}}=\frac{\alpha_{1}}{\rho_{1} c_{1}^{2}}+\frac{\alpha_{2}}{\rho_{2} c_{2}^{2}} \text {. }
$$

Consequently, the one-dimensional system reads :

$$
\begin{gathered}
p_{t}+u p_{x}+\rho c_{w}^{2} u_{x}=0, \\
u_{t}+u u_{x}+\frac{p_{x}}{\rho}+\frac{\lambda(m-1)}{\rho}|w|^{m-1} \operatorname{sgn}(w) w_{x}=0 \\
w_{t}+u w_{x}+w u_{x}=0 \\
\alpha_{2 t}+u \alpha_{2 x}+K u_{x}=0
\end{gathered}
$$




$$
\begin{gathered}
y_{2 t}+u y_{2 x}=0, \\
\eta_{i t}+u \eta_{i x}=0, \quad i=1,2
\end{gathered}
$$

We introduce the vector of unknowns

$$
\mathbf{U}^{T}=\left(p, u, w, \alpha_{2}, y_{2}, \eta_{1}, \eta_{2}\right)
$$

and present (19) in a condensed form :

$$
\mathbf{U}_{t}+A \mathbf{U}_{x}=0
$$

with

$$
A=\left(\begin{array}{ccccccc}
u & \rho c_{w}^{2} & 0 & 0 & 0 & 0 & 0 \\
\frac{1}{\rho} & u & \frac{\lambda(m-1)}{\rho}|w|^{m-1} \operatorname{sgn}(w) & 0 & 0 & 0 & 0 \\
0 & w & u & 0 & 0 & 0 & 0 \\
0 & K & 0 & u & 0 & 0 & 0 \\
0 & 0 & 0 & 0 & u & 0 & 0 \\
0 & 0 & 0 & 0 & 0 & u & 0 \\
0 & 0 & 0 & 0 & 0 & 0 & u
\end{array}\right)
$$

The eigenvalues of the matrix $A$ are :

$$
\begin{gathered}
\mu_{1,2,3,4,5}=u \\
\mu_{6,7}=u \pm \sqrt{c_{W}^{2}+\frac{\lambda(m-1)}{\rho}|w|^{m}}
\end{gathered}
$$

It can be shown that there exist exactly seven independent eigenvectors of $A$. Hence, the system is hyperbolic.

\section{Jump relations across discontinuities}

The system of conservation laws for unknowns $\left(\rho, y_{2}, \mathbf{w}=\nabla \phi, \mathbf{u}, p, \eta_{1}, \eta_{2}\right)$ can be rewritten in the form :

$$
\begin{gathered}
\rho_{t}+\operatorname{div}(\rho \mathbf{u})=0, \quad\left(\rho y_{2}\right)_{t}+\operatorname{div}\left(\rho y_{2} \mathbf{u}\right)=0, \\
\mathbf{w}_{t}+\nabla(\mathbf{w} \cdot \mathbf{u})=0, \\
\operatorname{rot} \mathbf{w}=\mathbf{0}, \\
\frac{\partial(\rho \mathbf{u})}{\partial t}+\operatorname{div}(\rho \mathbf{u} \otimes \mathbf{u}-T)=0, T=-P I+\lambda|\mathbf{w}|^{m} \Pi, \\
\left(\frac{\rho|\mathbf{u}|^{2}}{2}+E\right)_{t}+\operatorname{div}\left(\mathbf{u}\left(\frac{\rho|\mathbf{u}|^{2}}{2}+E\right)-T \mathbf{u}\right)=0, \quad E=\rho \varepsilon+\frac{\lambda}{m}|\mathbf{w}|^{m} .
\end{gathered}
$$


The volume fraction and entropies are related by

$$
p_{1}\left(\frac{\left(1-\alpha_{2}\right)\left(1-y_{2}\right)}{\rho}, \eta_{1}\right)=p_{2}\left(\frac{\alpha_{2} y_{2}}{\rho}, \eta_{2}\right)=p
$$

It follows from here that on discontinuities with the normal vector $\mathbf{n}$ and the tangent vectors $\tau$, and propagating with the normal velocity $D_{n}$ we have :

$$
\begin{gathered}
{\left[\rho\left(\mathbf{u} \cdot \mathbf{n}-D_{n}\right)\right]=0,} \\
{\left[\rho y_{2}\left(\mathbf{u} \cdot \mathbf{n}-D_{n}\right)\right]=0,} \\
{[\mathbf{w} \cdot \tau]=0, \quad\left[(\mathbf{w} \cdot \mathbf{u}) \mathbf{n}-D_{n} \mathbf{w}\right]=0,} \\
{\left[\rho \mathbf{u}\left(\mathbf{u} \cdot \mathbf{n}-D_{n}\right)-T \mathbf{n}\right]=0,} \\
{\left[\left(\mathbf{u} \cdot \mathbf{n}-D_{n}\right)\left(\frac{\rho|\mathbf{u}|^{2}}{2}+E\right)-\mathbf{n} \cdot T \mathbf{u}\right]=0 .}
\end{gathered}
$$

We distinguish two types of discontinuities : contact discontinuities (or interfaces) where $\mathbf{u} \cdot \mathbf{n}-D_{n}=0$ and shock waves where $\mathbf{u} \cdot \mathbf{n}-D_{n} \neq 0$. Moreover, in the following we will always consider a simplified case where the direction of the normal vector to the discontinuity coincides with the vector $\mathbf{w}$ :

$$
\mathbf{n}=\frac{\mathbf{w}}{|\mathbf{w}|}
$$

In particular,

$$
\mathbf{w} \cdot \tau=\mathbf{0}, \quad \mathbf{w}=(\mathbf{w} \cdot \mathbf{n}) \mathbf{n} .
$$

We call these discontinuities homogeneous in tangential direction.

\subsection{Contact discontinuities}

We consider first the interfaces $\mathbf{u} \cdot \mathbf{n}-D_{n}=0$. The first relation

$$
[\mathbf{w} \cdot \tau]=0
$$

and the second relation for $\mathbf{w}$

$$
\left[(\mathbf{w} \cdot \mathbf{u}) \mathbf{n}-D_{n} \mathbf{w}\right]=0
$$

are automatically verified. Indeed,

$$
\left[\mathbf{w} \cdot \mathbf{u}-D_{n} \mathbf{w} \cdot \mathbf{n}\right]=\left[\mathbf{w} \cdot((\mathbf{u} \cdot \mathbf{n}) \mathbf{n}+(\mathbf{u} \cdot \tau) \tau)-D_{n} \mathbf{w} \cdot \mathbf{n}\right]=[(\mathbf{w} \cdot \tau)(\mathbf{u} \cdot \tau)]=0 .
$$

On the contact interface we may have the discontinuity of $y_{2}$ and $\mathbf{w} \cdot \mathbf{n}$. The momentum equation on the contact discontinuity gives :

$$
-[T \mathbf{n}]=\left[P \mathbf{n}+\lambda\left(|\mathbf{w}|^{m-2} \mathbf{w}(\mathbf{w} \cdot \mathbf{n})-|\mathbf{w}|^{m} \mathbf{n}\right)\right]=[P] \mathbf{n}=0 .
$$


The energy equation gives a relation which is automatically fulfilled :

$$
\begin{gathered}
-[\mathbf{n} \cdot T \mathbf{u}]=-[\mathbf{u} \cdot T \mathbf{n}]=\left[\mathbf{u} \cdot\left(P \mathbf{n}+\lambda\left(|\mathbf{w}|^{m-2} \mathbf{w}(\mathbf{w} \cdot \mathbf{n})-|\mathbf{w}|^{m} \mathbf{n}\right)\right)\right]= \\
=[(\mathbf{u} \cdot \mathbf{n}) P]=0 .
\end{gathered}
$$

Finally, the relations on the contact discontinuity are :

$$
\mathbf{w} \cdot \tau=0, \quad[\mathbf{u} \cdot \mathbf{n}]=0, \quad[P]=0 .
$$

However, a priori,

$$
\left[y_{2}\right] \neq 0, \quad[\mathbf{u} \cdot \tau] \neq 0, \quad[\mathbf{w} \cdot \mathbf{n}] \neq 0 .
$$

\section{Compressive discontinuities}

Let us consider the Rankine-Hugoniot relations for shocks : $\mathbf{u} \cdot \mathbf{n}-D_{n} \neq 0$ at the condition

$$
\mathbf{w} \cdot \tau=0 .
$$

The mass conservation laws imply :

$$
\begin{gathered}
{\left[\rho\left(\mathbf{u} \cdot \mathbf{n}-D_{n}\right)\right]=0,} \\
{\left[y_{2}\right]=0 .}
\end{gathered}
$$

The same representation of $\mathbf{n}$ can be taken :

$$
\mathbf{n}=\frac{\mathbf{w}}{|\mathbf{w}|}
$$

The momentum equation is then equivalent to

$$
\left[\rho \mathbf{u}\left(\mathbf{u} \cdot \mathbf{n}-D_{n}\right)+P \mathbf{n}\right]=0
$$

It gives two relations

$$
\left[\rho\left(\mathbf{u} \cdot \mathbf{n}-D_{n}\right)^{2}+P\right]=0, \quad[\mathbf{u} \cdot \tau]=0 .
$$

Then the equation for $\mathbf{w}$

$$
\begin{gathered}
{\left[\mathbf{w} \cdot \mathbf{u}-D_{n} \mathbf{w} \cdot \mathbf{n}\right]=\left[\mathbf{w} \cdot((\mathbf{u} \cdot \mathbf{n}) \mathbf{n}+(\mathbf{u} \cdot \tau) \tau)-D_{n} \mathbf{w} \cdot \mathbf{n}\right]=} \\
=\left[(\mathbf{w} \cdot \mathbf{n})\left((\mathbf{u} \cdot \mathbf{n})-D_{n}\right)+(\mathbf{w} \cdot \tau)(\mathbf{u} \cdot \tau)\right]=\left[\frac{(\mathbf{w} \cdot \mathbf{n})}{\rho} \rho\left((\mathbf{u} \cdot \mathbf{n})-D_{n}\right)\right] .
\end{gathered}
$$

implies

$$
\left[\frac{\mathbf{w} \cdot \mathbf{n}}{\rho}\right]=0
$$


Or

$$
\left[\frac{|\mathbf{w}|}{\rho}\right]=0
$$

Finally, the energy equation

$$
\left[\left(\mathbf{u} \cdot \mathbf{n}-D_{n}\right)\left(\frac{\rho|\mathbf{u}|^{2}}{2}+E\right)+\mathbf{n} \cdot T \mathbf{u}\right]=0
$$

can be transformed to

$$
\begin{aligned}
{\left[\frac{E+p+\lambda \frac{m-1}{m}|\mathbf{w}|^{m}}{\rho}+\frac{\left(\mathbf{u} \cdot \mathbf{n}-D_{n}\right)^{2}}{2}\right] } & =\left[\frac{E}{\rho}+\frac{P}{\rho}+\frac{\left(\mathbf{u} \cdot \mathbf{n}-D_{n}\right)^{2}}{2}\right]=0, \\
E=\rho \varepsilon+\frac{\lambda}{m}|\nabla \phi|^{m}, \quad P & =p+\lambda \frac{m-1}{m}|\mathbf{w}|^{m} .
\end{aligned}
$$

To summarize, the shock relations in the case $\mathbf{w} \cdot \tau=0$ are

$$
\begin{gathered}
{\left[\rho\left(\mathbf{u} \cdot \mathbf{n}-D_{n}\right)\right]=0, \quad\left[y_{2}\right]=0,} \\
{[\mathbf{u} \cdot \tau]=0, \quad\left[\frac{\mathbf{w} \cdot \mathbf{n}}{\rho}\right]=0,} \\
{\left[\rho\left(\mathbf{u} \cdot \mathbf{n}-D_{n}\right)^{2}+P\right]=0,} \\
{\left[\frac{E}{\rho}+\frac{P}{\rho}+\frac{\left(\mathbf{u} \cdot \mathbf{n}-D_{n}\right)^{2}}{2}\right]=0 .}
\end{gathered}
$$

We will introduce the mass flux through the shock surface

$$
q=\rho\left(\mathbf{u} \cdot \mathbf{n}-D_{n}\right)
$$

Let index " 0 " means the state before the front. We obtain then the equation of the Rayleigh line (generalized)

$$
P-P_{0}+q^{2}\left(v-v_{0}\right)=0
$$

and the Hugoniot curve (generalized)

$$
\frac{E}{\rho}-\frac{E_{0}}{\rho_{0}}+\frac{1}{2}\left(P+P_{0}\right)\left(v-v_{0}\right)=0,
$$

or, equivalently,

$$
\varepsilon+\frac{\lambda v}{m}|\mathbf{w}|^{m}-\varepsilon_{0}-\frac{\lambda v_{0}}{m}\left|\mathbf{w}_{0}\right|^{m}+\frac{1}{2}\left(P+P_{0}\right)\left(v-v_{0}\right)=0 .
$$




\subsection{The case of sharp interfaces $(m=1)$}

It follows from $(13)$ that in the case of sharp interfaces $(m=1)$ the RankineHugoniot relations for the shocks are :

$$
p-p_{0}+q^{2}\left(v-v_{0}\right)=0, \quad \varepsilon-\varepsilon_{0}+\frac{1}{2}\left(p+p_{0}\right)\left(v-v_{0}\right)=0,
$$

because

$$
P=p, \quad v|\mathbf{w}|=v|\mathbf{w} \cdot \mathbf{n}|=v_{0}\left|\mathbf{w}_{0} \cdot \mathbf{n}\right|=v_{0}\left|\mathbf{w}_{0}\right|
$$

\subsection{General case $(m>1)$}

In general case, we have

$$
P-P_{0}+q^{2}\left(v-v_{0}\right)=0, \quad P=p+\lambda \frac{m-1}{m}|\mathbf{w}|^{m}=p+\lambda \frac{m-1}{m}\left(\frac{\rho}{\rho_{0}}\right)^{m}\left|\mathbf{w}_{0}\right|^{m}
$$

and the Hugoniot curve (generalized) is :

$$
\varepsilon-\varepsilon_{0}+\frac{\lambda\left|\mathbf{w}_{0}\right|^{m} v}{m}\left(\left(\frac{\rho}{\rho_{0}}\right)^{m}-\frac{\rho}{\rho_{0}}\right)+\frac{1}{2}\left(P+P_{0}\right)\left(v-v_{0}\right)=0 .
$$

In both situations, one more relation is necessary to determine the volume fraction after the shock.

\subsection{Additivity principle}

As mentioned before, one more relation is needed to determine the volume fraction jump. This problem is well known in the mechanics of the heterogeneous mixtures, and its solution is given through the "additivity principle" which is mainly due to Russian experimentalists Altschuler, and Dremin and Karpukhin (see the article by Saurel et al 2007 where this principle is discussed and its partial justification proposed). This principle says that in a multiphase shock front (which is indeed dispersed) each material follows its own Hugoniot. This principle was verified experimentally in a large scale of pressures and densities. We will generalize the additivity principle to our case :

$$
\varepsilon_{i}-\varepsilon_{i 0}+\frac{\lambda\left|\mathbf{w}_{0}\right|^{m} v_{i}}{m}\left(\left(\frac{\rho}{\rho_{0}}\right)^{m}-\frac{\rho}{\rho_{0}}\right)+\frac{1}{2}\left(P+P_{0}\right)\left(v_{i}-v_{i 0}\right)=0, \quad i=1,2 .
$$

Summing (24) multiplied by $y_{i}$ we obtain the conservation of the total energy. In the case of sharp interfaces $(m=1)$ this corresponds exactly to the classical additivity principle (see Saurel et al 2007).

If we take the internal energies of components in the form :

$$
\varepsilon_{i}=\varepsilon_{i}\left(v_{i}, p\right)=\varepsilon_{i}\left(v_{i}, P-\lambda \frac{m-1}{m}\left(\frac{\rho}{\rho_{0}}\right)^{m}\left|\mathbf{w}_{0}\right|^{m}\right)
$$


we can find implicitly from (24)

$$
v_{i}=v_{i}(P, v)
$$

Then we can determine the Hugoniot curve $v$ as a function of $P$ from the implicit equation :

$$
v=y_{1} v_{1}(P, v)+y_{2} v_{2}(P, v) .
$$

\section{Mechanical relaxation}

The mechanical equilibrium model has several disadvantages which are discussed in the paper by Saurel, Petitpas \& Berry (2008). The main disadvantage is related to the fact that the equilibrium sound speed is not a monotone function with respect to the volume fraction. Another issue is related to the non-conservative volume fraction equation that yields to positivity preserving difficulties when rarefaction or compression waves are present at interfaces. This is why a pressure non-equilibrium model is preferred. The pressure nonequilibrium models seem more complex, but it simplifies numerical resolution with operator splitting strategies. The corresponding non-equilibrium model is

$$
\begin{gathered}
\rho_{t}+\operatorname{div}(\rho \mathbf{u})=0, \quad\left(\rho y_{2}\right)_{t}+\operatorname{div}\left(\rho y_{2} \mathbf{u}\right)=0 \\
\mathbf{w}_{t}+\nabla(\mathbf{w} \cdot \mathbf{u})=0, \\
\operatorname{rot} \mathbf{w}=\mathbf{0}, \\
\frac{\partial \rho \mathbf{u}}{\partial t}+\operatorname{div}(\rho \mathbf{u} \otimes \mathbf{u}-T)=0, \\
T=-P I+\lambda|\mathbf{w}|^{m} \Pi=-\left(p+\lambda \frac{m-1}{m}|\mathbf{w}|^{m}\right) I+\lambda|\mathbf{w}|^{m}\left(I-\frac{\mathbf{w}}{|\mathbf{w}|} \otimes \frac{\mathbf{w}}{|\mathbf{w}|}\right), \\
\left(\frac{\rho|\mathbf{u}|^{2}}{2}+E\right)_{t}+\operatorname{div}\left(\mathbf{u}\left(\frac{\rho|\mathbf{u}|^{2}}{2}+E\right)-T \mathbf{u}\right)=0, \quad E=\rho+\frac{\lambda}{m}|\mathbf{w}|^{m}, \\
\frac{D \alpha_{2}}{D t}=\mu\left(p_{2}-p_{1}\right) .
\end{gathered}
$$

The initial conditions for $\mathbf{w}$ should be agreed with those of $\nabla y_{2}$ :

$$
\left.\mathbf{w}\right|_{t=0}=\left.\nabla y_{2}\right|_{t=0} .
$$

It implies that for any time

$$
\mathbf{w}=\nabla y_{2}
$$

Closure of system (25) needs an additional energy or entropy equation, that has to be determined. To this aim, let us note that the energy equation can be transformed :

$\rho \frac{D \varepsilon}{D t}+p \operatorname{div}(\mathbf{u})+\frac{\lambda}{m}\left(\left(|\mathbf{w}|^{m}\right)_{t}+\operatorname{div}\left(\mathbf{u}|\mathbf{w}|^{m}\right)\right)+\lambda \operatorname{tr}\left(\left(|\mathbf{w}|^{m-2} \mathbf{w} \otimes \mathbf{w}-\frac{|\mathbf{w}|^{m}}{m} I\right) \frac{\partial \mathbf{u}}{\partial \mathbf{x}}\right)=0$. 
But

$$
\begin{gathered}
\frac{1}{m}\left(\left(|\mathbf{w}|^{m}\right)_{t}+\operatorname{div}\left(\mathbf{u}|\mathbf{w}|^{m}\right)\right)+\operatorname{tr}\left(\left(|\mathbf{w}|^{m-2} \mathbf{w} \otimes \mathbf{w}-\frac{|\mathbf{w}|^{m}}{m} I\right) \frac{\partial \mathbf{u}}{\partial \mathbf{x}}\right)= \\
=|\mathbf{w}|^{m-2} \mathbf{w} \cdot \mathbf{w}_{t}+|\mathbf{w}|^{m-2} \mathbf{u} \cdot\left(\frac{\partial \mathbf{w}}{\partial \mathbf{x}}\right)^{T} \mathbf{w}+|\mathbf{w}|^{m-2} \mathbf{w} \cdot \frac{\partial \mathbf{u}}{\partial \mathbf{x}} \mathbf{w}= \\
=|\mathbf{w}|^{m-2}\left(\mathbf{w} \cdot \mathbf{w}_{t}+\mathbf{u} \cdot\left(\frac{\partial \mathbf{w}}{\partial \mathbf{x}}\right)^{T} \mathbf{w}+\mathbf{w} \cdot \frac{\partial \mathbf{u}}{\partial \mathbf{x}} \mathbf{w}\right)=\mathbf{0}
\end{gathered}
$$

because

$$
\left(\frac{\partial \mathbf{w}}{\partial \mathbf{x}}\right)^{T}-\left(\frac{\partial \mathbf{w}}{\partial \mathbf{x}}\right)=0
$$

Hence,

$$
\rho \frac{D \varepsilon}{D t}+p \operatorname{div}(\mathbf{u})=0 .
$$

But

$$
\begin{gathered}
d \varepsilon=y_{1} d \varepsilon_{1}+y_{2} d \varepsilon_{2}=y_{1}\left(\theta_{1} d \eta_{1}-p_{1} d v_{1}\right)+y_{2}\left(\theta_{2} d \eta_{2}-p_{2} d v_{2}\right)= \\
=y_{1} \theta_{1} d \eta_{1}+y_{2} \theta_{2} d \eta_{2}-p_{1} d\left(y_{1} v_{1}\right)-p_{2} d\left(y_{2} v_{2}\right)= \\
=y_{1} \theta_{1} d \eta_{1}+y_{2} \theta_{2} d \eta_{2}-p d v+\left(p_{1}-p_{2}\right) v d \alpha_{2} .
\end{gathered}
$$

Hence, it is equivalent to :

$$
\rho\left(y_{1} \theta_{1} \frac{D \eta_{1}}{D t}+y_{2} \theta_{2} \frac{D \eta_{2}}{D t}\right)=\left(p_{2}-p_{1}\right) \frac{D \alpha_{2}}{D t}=\mu\left(p_{2}-p_{1}\right)^{2} \geq 0 .
$$

The equations for the entropy of each component can be taken in the form (for any $\left.p_{I}\right)$ :

$$
\rho y_{1} \theta_{1} \frac{D \eta_{1}}{D t}=\left(p_{1}-p_{I}\right) \frac{D \alpha_{1}}{D t}, \quad \rho y_{2} \theta_{2} \frac{D \eta_{2}}{D t}=\left(p_{2}-p_{I}\right) \frac{D \alpha_{2}}{D t}
$$

Summing them, we obtain (26).

The following definition of $p_{I}$ is proposed in Saurel, Gavrilyuk and Renaud, 2003 :

$$
p_{I}=\frac{Z_{2} p_{1}+Z_{1} p_{2}}{Z_{1}+Z_{2}}
$$

where $Z_{i}$ are acoustical impedances. Then

$$
p_{1}-p_{I}=\frac{Z_{1}\left(p_{1}-p_{2}\right)}{Z_{1}+Z_{2}}, \quad p_{2}-p_{I}=\frac{Z_{2}\left(p_{2}-p_{1}\right)}{Z_{1}+Z_{2}}
$$

we obtain

$$
\rho y_{1} \theta_{1} \frac{D \eta_{1}}{D t}=\frac{\mu Z_{1}}{Z_{1}+Z_{2}}\left(p_{1}-p_{2}\right)^{2}, \quad \rho y_{2} \theta_{2} \frac{D \eta_{2}}{D t}=\frac{\mu Z_{2}}{Z_{1}+Z_{2}}\left(p_{2}-p_{1}\right)^{2}
$$

These two equations achieve the closure of system (25). The entropy inequality is fulfilled :

$$
\rho\left(y_{1} \frac{D \eta_{1}}{D t}+y_{2} \frac{D \eta_{2}}{D t}\right)=\frac{\mu}{Z_{1}+Z_{2}}\left(\frac{Z_{1}}{\theta_{1}}+\frac{Z_{2}}{\theta_{2}}\right)\left(p_{2}-p_{1}\right)^{2} \geq 0 .
$$




\subsection{Hyperbolicity of the non-equilibrium model}

As in the case of the pressure equilibrium model, it is sufficient to study the one-dimensional case. The equations are

$$
\begin{gathered}
\rho_{t}+(u \rho)_{x}=0 \\
u_{t}+u u_{x}+\frac{p_{x}}{\rho}+\frac{\lambda(m-1)}{\rho}|w|^{m-1} \operatorname{sgn}(w) w_{x}=0 \\
w_{t}+u w_{x}+w u_{x}=0 \\
\alpha_{2 t}+u \alpha_{2 x}=\mu\left(p_{2}-p_{1}\right) \\
y_{2 t}+u y_{2 x}=0 \\
\eta_{i t}+u \eta_{i x}=0, \quad i=1,2
\end{gathered}
$$

We introduce the vector of unknowns

$$
\mathbf{V}^{T}=\left(\rho, u, w, \alpha_{2}, y_{2}, \eta_{1}, \eta_{2}\right)
$$

Taking into account the relation

$$
p_{x}=\left(y_{1} c_{1}^{2}+y_{2} c_{2}^{2}\right) \rho_{x}+\left(p_{2}-p_{1}+\rho_{1} c_{1}^{2}-\rho_{2} c_{2}^{2}\right) \alpha_{2 x}+\rho\left(c_{2}^{2}-c_{1}^{2}\right) y_{2 x},
$$

we present (27) in the matrix form

$$
\begin{gathered}
\mathbf{V}_{t}+B \mathbf{V}_{x}=0, \\
B=\left(\begin{array}{ccccccc}
u & \rho & 0 & 0 & 0 & 0 & 0 \\
\frac{y_{1} c_{1}^{2}+y_{2} c_{2}^{2}}{\rho} & u & \frac{\lambda(m-1)}{\rho}|w|^{m-1} \operatorname{sgn}(w) & \frac{p_{2}-p_{1}+\rho_{1} c_{1}^{2}-\rho_{2} c_{2}^{2}}{\rho} & c_{2}^{2}-c_{1}^{2} & 0 & 0 \\
0 & w & u & 0 & 0 & 0 & 0 \\
0 & 0 & 0 & u & 0 & 0 & 0 \\
0 & 0 & 0 & 0 & u & 0 & 0 \\
0 & 0 & 0 & 0 & 0 & u & 0 \\
0 & 0 & 0 & 0 & 0 & 0 & u
\end{array}\right)
\end{gathered}
$$

The system is hyperbolic with eigenvalues of the matrix $B$ given by :

$$
\mu_{1,2,3,4,5}=u, \quad \mu_{6,7}=u \pm \sqrt{c_{f}^{2}+\frac{\lambda(m-1)}{\rho}|w|^{m}}
$$

where

$$
c_{f}^{2}=y_{1} c_{1}^{2}+y_{2} c_{2}^{2}
$$

is a frozen sound speed. The sound speed presents a monotonic behavior versus the mass and volume fraction. This facilitates numerical resolution (Saurel, Petitpas \& Berry, 2008). The Riemann solver can be constructed in the same way as in this reference. The corresponding shock relations take into account the conservation of the volume fraction. 


\section{Computational results}

To illustrate the method capability to treat capillary flows, two test cases are proposed. An oscillating square droplet is presented to highlight surface tension effects. An impact between two droplets is then presented to show that the method is able to deal with interface disappearance and interface creation. No phase transition is considered. In both computations, the liquid is governed by the stiffened gas equation of state with parameters $\gamma_{l i q}=2.1$ and $p_{\infty, l i q}=$ $10^{7} \mathrm{~Pa}$. The surrounding air is governed by the ideal gas equation of state with polytropic exponent $\gamma_{a i r}=1.4$.

\subsection{Oscillating droplet}

Consider a square liquid droplet placed in air. Initially, the pressure is set $1 \mathrm{~atm}$ every where in the computational domain. A 100x100 cells mesh is used. The initial shape of the droplet is given hereafter:

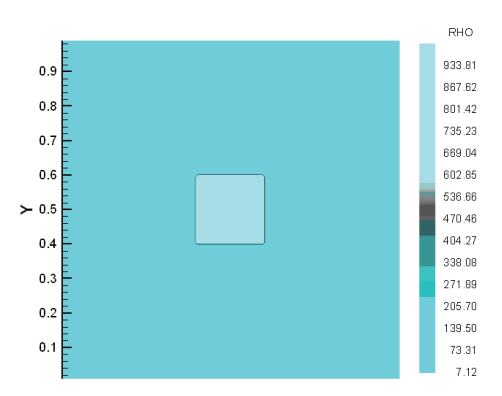

Figure 1: Initial condition for the square droplet

Due to surface tension effects $\lambda=1000 N / m$, the droplet tends to decrease its surface energy. This implies oscillations up to the equilibrium state. Results are presented at different instants.

At steady state, the droplet finds a circular shape of radius $0.11 \mathrm{~m}$ with an average pressure of $8600 \mathrm{~Pa}$. This is in agreement with the Laplace law which predicts a pressure jump of $9090 \mathrm{~Pa}$. The error in the pressure jump is lower than $6 \%$.

\subsection{Droplets impact}

A collision between two liquid droplets is presented. The two droplets move at the relative velocity $10 \mathrm{~m} / \mathrm{s}$. The surface tension coefficient $\lambda$ is taken equal to $850 \mathrm{~N} / \mathrm{m}$. A 150x150 cells mesh is used. The two droplets first merge. Then, the intensity of the impact induces an expansion phase. During this expansion, 

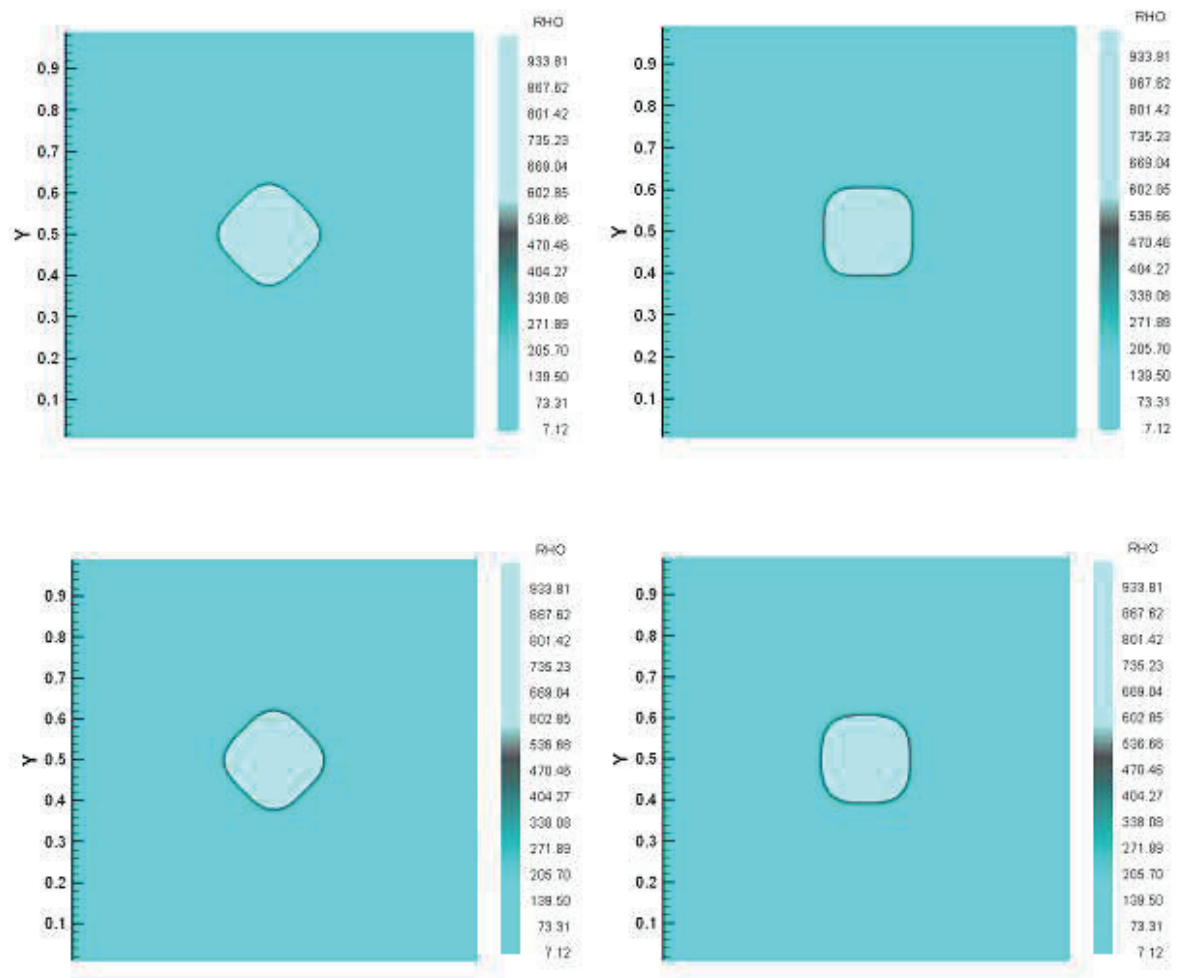

Figure 2: Oscillation of the droplet due to surface tension effects. Results are shown at instants $t_{1}=15 \mathrm{~ms}, t_{2}=34 \mathrm{~ms}, t_{3}=53 \mathrm{~ms}$ and $t_{4}=72 \mathrm{~ms}$

the droplet breaks up and two new droplets appears. Then, the droplets oscillate to restore a circular shape.

\subsection{Phase transition modelling}

The following natural splitting can be done when both phenomena - relaxation and phase transition - are present. At the first step, the relaxation takes place. When the mechanical equilibrium is attained :

$$
p_{1}\left(\rho_{1}, \eta_{1}\right)=p_{2}\left(\rho_{2}, \eta_{2}\right)
$$

then the phase transition takes place. The first step being described previously (relaxation towards to equilibrium), we need only to describe the second step. We will take the equations of mass in the form (see Saurel, Petitpas \& Abgrall, 
2008) :

$$
\frac{\partial\left(\rho y_{k}\right)}{\partial t}+\operatorname{div}\left(\rho y_{k} \mathbf{u}\right)=\rho \frac{D y_{k}}{D t}=\rho \dot{y}_{k},
$$

The gradient $\mathbf{w}$ of the order parameter $\phi$ verifies the equation :

$$
\mathbf{w}_{t}+\nabla(\mathbf{w} \cdot \mathbf{u})=0
$$

The initial condition for $\mathbf{w}$ can be agreed with those of $\nabla y_{2}$ at $t=0$ :

$$
\left.\mathbf{w}\right|_{t=0}=\left.\nabla y_{2}\right|_{t=0}
$$

However, now it does not guarantee that for any time

$$
\mathbf{w}=\nabla y_{2} .
$$

When mass transfer terms are added in equation (28), the next step is to determine the associated volume fraction transfer. They are determined at the basis of the entropy production analysis in each phase and in the mixture. To determine entropy equations, the following analysis has to be done. The energy equation

$$
\left(\frac{\rho|\mathbf{u}|^{2}}{2}+E\right)_{t}+\operatorname{div}\left(\mathbf{u}\left(\frac{\rho|\mathbf{u}|^{2}}{2}+E\right)-T \mathbf{u}\right)=0
$$

can be transformed to

$\rho \frac{D \varepsilon}{D t}+p \operatorname{div}(\mathbf{u})+\frac{\lambda}{m}\left(\left(|\mathbf{w}|^{m}\right)_{t}+\operatorname{div}\left(\mathbf{u}|\mathbf{w}|^{m}\right)\right)+\lambda \operatorname{tr}\left(\left(|\mathbf{w}|^{m-2} \mathbf{w} \otimes \mathbf{w}-\frac{|\mathbf{w}|^{m}}{m} I\right) \frac{\partial \mathbf{u}}{\partial \mathbf{x}}\right)=0$.

But

$$
\begin{gathered}
\frac{1}{m}\left(\left(|\mathbf{w}|^{m}\right)_{t}+\operatorname{div}\left(\mathbf{u}|\mathbf{w}|^{m}\right)\right)+\operatorname{tr}\left(\left(|\mathbf{w}|^{m-2} \mathbf{w} \otimes \mathbf{w}-\frac{|\mathbf{w}|^{m}}{m} I\right) \frac{\partial \mathbf{u}}{\partial \mathbf{x}}\right)= \\
=|\mathbf{w}|^{m-2} \mathbf{w} \cdot \mathbf{w}_{t}+|\mathbf{w}|^{m-2} \mathbf{u} \cdot\left(\frac{\partial \mathbf{w}}{\partial \mathbf{x}}\right)^{T} \mathbf{w}+|\mathbf{w}|^{m-2} \mathbf{w} \cdot \frac{\partial \mathbf{u}}{\partial \mathbf{x}} \mathbf{w}= \\
=|\mathbf{w}|^{m-2}\left(\mathbf{w} \cdot \mathbf{w}_{t}+\mathbf{u} \cdot\left(\frac{\partial \mathbf{w}}{\partial \mathbf{x}}\right)^{T} \mathbf{w}+\mathbf{w} \cdot \frac{\partial \mathbf{u}}{\partial \mathbf{x}} \mathbf{w}\right)= \\
=|\mathbf{w}|^{m-2}\left(\mathbf{w} \cdot \mathbf{w}_{t}+\mathbf{u} \cdot\left(\left(\frac{\partial \mathbf{w}}{\partial \mathbf{x}}\right)^{T}-\frac{\partial \mathbf{w}}{\partial \mathbf{x}}\right) \mathbf{w}+\mathbf{u} \cdot \frac{\partial \mathbf{w}}{\partial \mathbf{x}} \mathbf{w}+\mathbf{w} \cdot \frac{\partial \mathbf{u}}{\partial \mathbf{x}} \mathbf{w}\right)=\mathbf{0}
\end{gathered}
$$

Hence,

$$
\rho \frac{D \varepsilon}{D t}+p \operatorname{div}(\mathbf{u})=0
$$

Since

$$
d \varepsilon=y_{1} \theta_{1} d \eta_{1}+y_{2} \theta_{2} d \eta_{2}-p d v+\left(h_{2}-h_{1}\right) d y_{2},
$$


the energy equation is equivalent to :

$$
y_{1} \theta_{1} \frac{D \eta_{1}}{D t}+y_{2} \theta_{2} \frac{D \eta_{2}}{D t}+\left(h_{1}-h_{2}\right) \dot{y}_{1}=0 .
$$

Taking the material derivative of the pressure equilibrium condition we obtain :

$$
\frac{D p_{1}\left(\frac{\rho y_{1}}{\alpha_{1}}, \eta_{1}\right)}{D t}-\frac{D p_{2}\left(\frac{\rho y_{2}}{\alpha_{2}}, \eta_{2}\right)}{D t}=0 .
$$

It is equivalent to

$$
\begin{aligned}
& c_{1}^{2}\left(\frac{\rho}{\alpha_{1}} \frac{D y_{1}}{D t}+\frac{y_{1}}{\alpha_{1}} \frac{D \rho}{D t}-\frac{\rho y_{1}}{\alpha_{1}^{2}} \frac{D \alpha_{1}}{D t}\right)+\rho_{1} \theta_{1} \Gamma_{1} \frac{D \eta_{1}}{D t}= \\
& =c_{2}^{2}\left(\frac{\rho}{\alpha_{2}} \frac{D y_{2}}{D t}+\frac{y_{2}}{\alpha_{2}} \frac{D \rho}{D t}-\frac{\rho y_{2}}{\alpha_{2}^{2}} \frac{D \alpha_{2}}{D t}\right)+\rho_{2} \theta_{2} \Gamma_{2} \frac{D \eta_{2}}{D t}
\end{aligned}
$$

Here $\Gamma_{i}$ are the Grüneisen coefficients :

$$
\Gamma_{i}=\frac{1}{\rho_{i} \theta_{i}} \frac{\partial p_{i}}{\partial \eta_{i}}
$$

We can solve equations (29), (30) to find finally

$$
\begin{gathered}
\rho y_{1} y_{2} \theta_{1}\left(\frac{\Gamma_{1}}{\alpha_{1}}+\frac{\Gamma_{2}}{\alpha_{2}}\right) \frac{D \eta_{1}}{D t}= \\
=y_{2}\left(c_{2}^{2}\left(\frac{\rho}{\alpha_{2}} \frac{D y_{2}}{D t}+\frac{y_{2}}{\alpha_{2}} \frac{D \rho}{D t}-\frac{\rho y_{2}}{\alpha_{2}^{2}} \frac{D \alpha_{2}}{D t}\right)-c_{1}^{2}\left(\frac{\rho}{\alpha_{1}} \frac{D y_{1}}{D t}+\frac{y_{1}}{\alpha_{1}} \frac{D \rho}{D t}-\frac{\rho y_{1}}{\alpha_{1}^{2}} \frac{D \alpha_{1}}{D t}\right)\right)- \\
-\rho_{2} \Gamma_{2}\left(h_{1}-h_{2}\right) \dot{y}_{1}, \\
\rho y_{1} y_{2} \theta_{2}\left(\frac{\Gamma_{1}}{\alpha_{1}}+\frac{\Gamma_{2}}{\alpha_{2}}\right) \frac{D \eta_{2}}{D t}= \\
=-y_{1}\left(c_{2}^{2}\left(\frac{\rho}{\alpha_{2}} \frac{D y_{2}}{D t}+\frac{y_{2}}{\alpha_{2}} \frac{D \rho}{D t}-\frac{\rho y_{2}}{\alpha_{2}^{2}} \frac{D \alpha_{2}}{D t}\right)-c_{1}^{2}\left(\frac{\rho}{\alpha_{1}} \frac{D y_{1}}{D t}+\frac{y_{1}}{\alpha_{1}} \frac{D \rho}{D t}-\frac{\rho y_{1}}{\alpha_{1}^{2}} \frac{D \alpha_{1}}{D t}\right)\right)- \\
-\rho_{1} \Gamma_{1}\left(h_{1}-h_{2}\right) \dot{y}_{1} .
\end{gathered}
$$

Taking the volume fraction equation in the form :

$$
\begin{gathered}
\frac{D \alpha_{1}}{D t}=K \operatorname{div}(\mathbf{u})+\frac{\left(\frac{\Gamma_{1}}{\alpha_{1}}+\frac{\Gamma_{2}}{\alpha_{2}}\right)}{\frac{\rho_{1} c_{1}^{2}}{\alpha_{1}}+\frac{\rho_{2} c_{2}^{2}}{\alpha_{2}}} Q_{1}+\frac{\rho}{\rho_{I}} \dot{y}_{1}, \\
K=\frac{\rho_{2} c_{2}^{2}-\rho_{1} c_{1}^{2}}{\frac{\rho_{1} c_{1}^{2}}{\alpha_{1}}+\frac{\rho_{2} c_{2}^{2}}{\alpha_{2}}}, \quad Q_{1}=H\left(\theta_{2}-\theta_{1}\right), \quad H=\text { const }, \quad \rho_{I}=\frac{\frac{\rho_{1} c_{1}^{2}}{\alpha_{1}}+\frac{\rho_{2} c_{2}^{2}}{\alpha_{2}}}{\frac{c_{1}^{2}}{\alpha_{1}}+\frac{c_{2}^{2}}{\alpha_{2}}},
\end{gathered}
$$




$$
\dot{y}_{1}=\nu\left(\bar{g}_{2}-\bar{g}_{1}\right), \quad \bar{g}_{i}=h_{i}-\bar{\theta} \eta_{i}, \quad \bar{\theta}=\frac{\theta_{1} \theta_{2}}{\theta_{I}}, \quad \theta_{I}=\frac{\frac{\Gamma_{1} \theta_{1}}{\alpha_{1}}+\frac{\Gamma_{2} \theta_{2}}{\alpha_{2}}}{\frac{\Gamma_{1}}{\alpha_{1}}+\frac{\Gamma_{2}}{\alpha_{2}}}
$$

we obtain the governing equations in the form presented in Saurel, Petitpas and Abgrall (2008). After each step of calculation, we have to re-initialize the initial conditions for $\mathbf{w}$ by taking

$$
\mathbf{w}=\nabla y_{2}
$$

For completeness, we present the full system of governing equations describing the equilibrium phase transitions :

$$
\begin{aligned}
& \frac{\partial\left(\rho y_{k}\right)}{\partial t}+\operatorname{div}\left(\rho y_{k} \mathbf{u}\right)=\rho \dot{y}_{k}, \\
& \mathbf{w}_{t}+\nabla(\mathbf{w} \cdot \mathbf{u})=0 \\
& \frac{\partial \rho \mathbf{u}}{\partial t}+\operatorname{div}(\rho \mathbf{u} \otimes \mathbf{u}-T)=0, \\
& T=-P I+\lambda|\mathbf{w}|^{m} \Pi=-\left(p+\lambda \frac{m-1}{m}|\mathbf{w}|^{m}\right) I+\lambda|\mathbf{w}|^{m}\left(I-\frac{\mathbf{w}}{|\mathbf{w}|} \otimes \frac{\mathbf{w}}{|\mathbf{w}|}\right), \\
& \left(\frac{\rho|\mathbf{u}|^{2}}{2}+E\right)_{t}+\operatorname{div}\left(\mathbf{u}\left(\frac{\rho|\mathbf{u}|^{2}}{2}+E\right)-T \mathbf{u}\right)=0, \quad E=\rho \varepsilon+\frac{\lambda}{m}|\mathbf{w}|^{m}, \\
& \frac{D \alpha_{1}}{D t}=K \operatorname{div}(\mathbf{u})+\frac{\left(\frac{\Gamma_{1}}{\alpha_{1}}+\frac{\Gamma_{2}}{\alpha_{2}}\right)}{\frac{\rho_{1} c_{1}^{2}}{\alpha_{1}}+\frac{\rho_{2} c_{2}^{2}}{\alpha_{2}}} Q_{1}+\frac{\rho}{\rho_{I}} \dot{y}_{1}, \\
& K=\frac{\rho_{2} c_{2}^{2}-\rho_{1} c_{1}^{2}}{\frac{\rho_{1} c_{1}^{2}}{\alpha_{1}}+\frac{\rho_{2} c_{2}^{2}}{\alpha_{2}}}, \quad Q_{1}=H\left(\theta_{2}-\theta_{1}\right), \quad H=\text { const }, \quad \rho_{I}=\frac{\frac{\rho_{1} c_{1}^{2}}{\alpha_{1}}+\frac{\rho_{2} c_{2}^{2}}{\alpha_{2}}}{\frac{c_{1}^{2}}{\alpha_{1}}+\frac{c_{2}^{2}}{\alpha_{2}}}, \\
& \dot{y}_{1}=\nu\left(\bar{g}_{2}-\bar{g}_{1}\right), \quad \bar{g}_{i}=h_{i}-\bar{\theta} \eta_{i}, \quad \bar{\theta}=\frac{\theta_{1} \theta_{2}}{\theta_{I}}, \quad \theta_{I}=\frac{\frac{\Gamma_{1} \theta_{1}}{\alpha_{1}}+\frac{\Gamma_{2} \theta_{2}}{\alpha_{2}}}{\frac{\Gamma_{1}}{\alpha_{1}}+\frac{\Gamma_{2}}{\alpha_{2}}} .
\end{aligned}
$$

\section{Conclusion}

Two mathematical models corresponding to two steps in calculation of diffused liquid-gas interfaces have been built. The first step corresponds to the model (25) where only the pressure relaxation is calculated. The phase transition is "frozen" at this step. During the second step, where the mechanical equilibrium is achieved, the phase transition takes place. Compared with ill-posed mathematical models based on the Van-der-Waals type equations of state, our model is hyperbolic and uses the equations of state of individual components. The ability of the model to describe correctly physical phenomena is demonstrated on the non-equilibrium motion of droplets in a gas. The capability of the model to describe phase transition phenomena was previously demonstrated in Saurel, Petitpas \& Abgrall (2008). 


\section{References}

D. M. Anderson, G. B. McFadden \& A. A. Wheeler, Diffuse-Interface Methods in Fluid Mechanics, Annual Review of Fluid Mechanics, 30, 139-165 (1998)

J. U. Brackbill, D. B. Kothe \& C. Zemach, A continuum model for modeling surface tension. J. Comp. Physics. 100 335-354 (1992)

C. Farhat \& F. Roux, A method for finite element tearing and interconnecting and its parallel solution algorithm, International J. Numerical Meth. in Engineering, 32, 457-492 (1991).

S. L. Gavrilyuk \& H. Gouin, A new form of governing equations of fluids arising from Hamilton's principle. Int. J. Eng. Sci., 37(1999) 1495-1520.

S. L. Gavrilyuk \& R. Saurel, Mathematical and numerical modeling of twophase compressible flows with micro-inertia, J. Comput. Physics, 175, 326-360 (2002).

J. Glimm, J. Grove, X. Li, K. Shyue, Q. Zhang \& Y. Zeng, Three-dimensional front tracking, SIAM J. Scientific Computing, 19, 703-727 (1998).

C. Hirt, A. Amsden \& J. Cook, An arbitrary Lagrangian-Eulerian computing method for all flow speeds, J. Comput. Physics, 14, 227-253 (1974).

T. Y. Hou, J. S. Lowengrub \& M. J. Shelley, Boundray integral methods for multicomponnet fluids and multiphase materials, J. Comput. Physics, 169, 302-362 (2001).

S. Osher \& R. Fedkiw, Level set methods : an overview and some recent results, J. Comput. Physics, 169, 463-502 (2001).

G. Perigaud \& R. Saurel, A compressible flow model with capillary effects, J. of Comput. Physics. 209 139-178 (2005).

J. A. Sethian, Level Set Methods and Fast Marching Methods: Evolving Interfaces in Computational Geometry, Fluid Mechanics, Computer Vision and Material Sciences. Cambridge, MA: Cambridge University Press (1999).

J. A. Sethian \& P. Smereka, Level Set Methods for Fluid Interfaces, Annual Review of Fluid Mechanics, 35, 341-372 (2003).

R. Saurel, S. Gavrilyuk \& F. Renaud, A multiphase model with internal degrees of freedom : application to shock-bubble interaction, Journal of Fluid Mech. 495, 283-321 (2003).

R. Saurel, O. Lemetayer, J. Massoni \& S. Gavrilyuk, Shock jump relations for multiphase mixtures with stiff mechanical relaxation, Shock waves, 16, 209232 (2007).

R. Saurel, F. Petitpas \& R. Abgrall, Modelling phase transition in metastable liquids: application to cavitating and flashing flows, Journal of Fluid Mech. 607, 313-350 (2008).

R. Saurel, F. Petitpas \& R. A. Berry, Simple and efficient relaxation methods for interfaces separating compressible fluids, cavitating flows and shocks in multiphase mixture, J. Comput. Physics (in revision) (2008). 

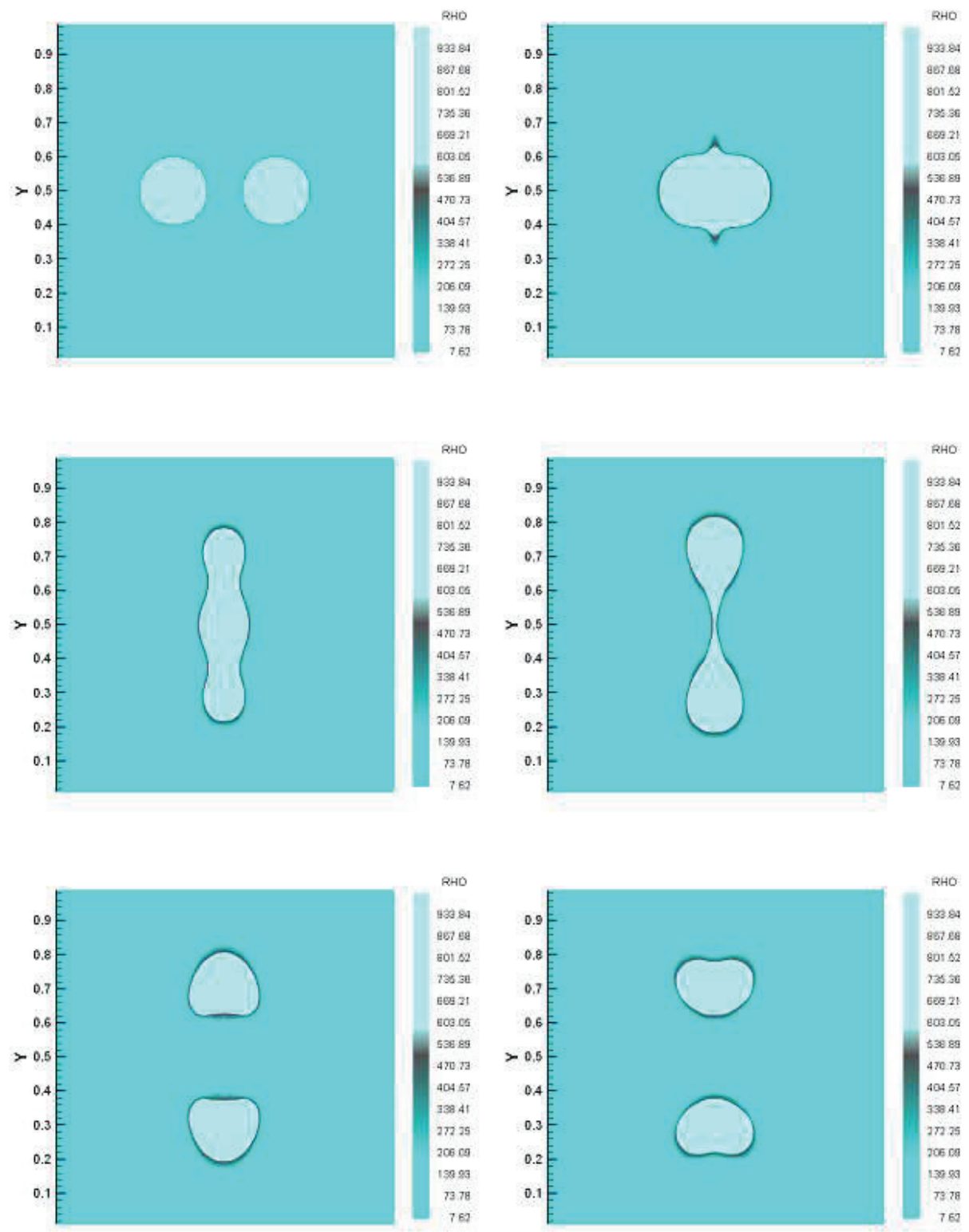

Figure 3: Impact of two droplets moving at relative velocity of $10 \mathrm{~m} / \mathrm{s}$. Results are shown at instants $t_{0}=0 \mathrm{~ms}, t_{1}=21 \mathrm{~ms}, t_{2}=52 \mathrm{~ms}, t_{3}=98 \mathrm{~ms}$, $t_{4}=120 \mathrm{~ms}$ and $t_{5}=155 \mathrm{~ms}$ 


\section{Chapter 6 \\ DEM Model for Flows in Ducts of Variable Cross-Section}




\section{DEM model for flows in ducts of variable cross sections}

Typically, multiphase modeling begins with an averaged (or homogenized) system of partial differential equations (traditionally ill-posed) then discretizes this system to form a numerical scheme. This presents problems for the numerical approximation of non-conservative terms at discontinuities (interfaces, shocks) as well as unwieldy treatment of fluxes with seven waves. To solve interface problems without conservation errors and to avoid this questionable determination of average variables and the numerical approximation of the non-conservative terms in conjunction with 2 velocity mixture flows we employ a new homogenization method known as the Discrete Equations Method (DEM). Contrary to conventional methods, the averaged equations for the mixture are not used, and this method directly obtains a (well-posed) discrete equation system from the single-phase system to produce a numerical scheme which accurately computes fluxes for arbitrary numbers of phases and solves non-conservative products. The method effectively uses a sequence of single phase Riemann equation solves. Phase interactions are accounted for by Riemann solvers at each interface. Flow topology can change with changing expressions for the fluxes. Non-conservative terms are correctly approximated. Some of the closure relations missing from the traditional approach are automatically obtained. Lastly, we can sometimes identify the continuous system induced by the discrete equation. This can be very useful from a theoretical standpoint. In this chapter we outline the construction of a DEM model for 1-D two compressible phases in ducts of spatially variable cross-section to test this approach. For the first time, we demonstrated on a convergingdiverging two-phase nozzle that this well-posed 2 pressure, 2 velocity model could be integrated to a meaningful steady-state with both phases treated as compressible. In the following chapter we give a review of the general DEM method and investigates the modeling of mass transfer between compressible multiphase flows within the context of DEM through the development of a Reactive Riemann solver (RDEM) for phase change.

Simple, efficient and robust algorithms are needed to solve the well-posed models. The various ingredients employed in methods to be developed should be general enough to consider future extensions to problems involving complex multiphysics. Using the fractional step methods typically applied, and miss-applied, to calculate these types of initial value problems, that consist of different kinds of physics with multiple time-scales, some of which should be treated implicitly, requires some precautionary measures to avoid splitting and conditioning errors. With the exception of the PCICE method, all of the methods developed so far, and documented in this report, utilized explicit time integration. To relieve time step restrictions due to stiffness and to achieve tighter coupling of equations, we believe that a fully implicit treatment may not only be advantageous, but indeed necessary. An appropriate fully implicit approach will allow integration over the fast time scales for slow speed flows. We anticipate that using a JacobianFree Newton-Krylov method with physics based preconditioning will allow tightly coupled solutions of the multiphysics phenomena inherent in nuclear reactor core applications. Toward that objective, a significant effort has been expended toward development of a method of lines (MOL) approach to the integration of the equation system. With the MOL we discretize separately the space and time domains, effectively converting to a large system of ordinary differential equations (ODE) that can be integrated with previously developed, highly refined special purpose software. Though not documented in this report, we are currently incorporating the 1-D variable area, two-phase DEM algorithm to be described below into this framework. 
Thus this explicit model, to be described, also serves as a necessary beginning for the subsequent implicit methods development. We will test and report on the fully implicit integration of this system at a later date.

We will assume here that each phase obeys the single phase Euler equations

$$
\begin{aligned}
& \frac{\partial \rho}{\partial \mathrm{t}}+\operatorname{div}(\rho \overrightarrow{\mathrm{u}})=0 \\
& \frac{\partial \rho \overrightarrow{\mathrm{u}}}{\partial \mathrm{t}}+\operatorname{div}(\rho \overrightarrow{\mathrm{u}} \otimes \overrightarrow{\mathrm{u}})+\operatorname{grad}(\mathrm{P})=0 \\
& \frac{\partial \rho \mathrm{E}}{\partial \mathrm{t}}+\operatorname{div}((\rho \mathrm{E}+\mathrm{P}) \overrightarrow{\mathrm{u}})=0
\end{aligned}
$$

with the following notation

$\rho$ density,

$\overrightarrow{\mathrm{u}}$ velocity vector,

P pressure,

E total energy,

e internal energy.

Total energy is defined by : $E=e+1 / 2 \vec{u} \cdot \vec{u}$.

Each phase is characterized by its phase function $\mathrm{X}_{\mathrm{k}}$ that obeys the following evolution equation:

$$
\frac{\partial \mathrm{X}_{\mathrm{k}}}{\partial \mathrm{t}}+\overrightarrow{\mathrm{u}}_{\mathrm{I}} \operatorname{grad}\left(\mathrm{X}_{\mathrm{k}}\right)=0
$$

where $\overrightarrow{\mathrm{u}}_{\mathrm{I}}$ represents the local interfacial velocity.

The DEM proceeds in a sequence of steps:

- fluid selection,

- integration over a control volume,

- determination of the phase contacts at cell boundaries,

- ...

The first step consists in the fluid selection. We have to multiply the Euler equations, corresponding to a system of conservation laws by the characteristic (or phase) function: 
$\mathrm{X}_{\mathrm{k}}\left(\frac{\partial \mathrm{U}}{\partial \mathrm{t}}+\operatorname{div}(\mathrm{F})\right)=0$

Where the extended system of conservative variables is :

$\mathrm{U}=(1, \rho, \rho \overrightarrow{\mathrm{u}}, \rho \mathrm{E})^{\mathrm{T}}$

and corresponding flux :

$\mathrm{U}=(0, \rho \overrightarrow{\mathrm{u}}, \rho \overrightarrow{\mathrm{u}} \otimes \overrightarrow{\mathrm{u}}+\mathrm{P} \overline{\mathrm{I}},(\rho \mathrm{E}+\mathrm{P}) \overrightarrow{\mathrm{u}})^{\mathrm{T}}$

After some calculations we get :

$\frac{\partial \mathrm{X}_{\mathrm{k}} \mathrm{U}}{\partial \mathrm{t}}+\operatorname{div}\left(\mathrm{X}_{\mathrm{k}} \mathrm{F}\right)=\left(\mathrm{F}-\overrightarrow{\mathrm{u}}_{\mathrm{I}} \mathrm{U}\right) \operatorname{grad}\left(\mathrm{X}_{\mathrm{k}}\right)$

The second step consists in the space integration of this equation:

$\int_{V}\left(\frac{\partial X_{k} U}{\partial t}+\operatorname{div}\left(X_{k} F\right)\right) d V=\int_{V}\left(F-\vec{u}_{I} U\right) \operatorname{grad}\left(X_{k}\right) d V$

The control volume being time invariant, the first term becomes :

$\int_{\mathrm{V}} \frac{\partial \mathrm{X}_{\mathrm{k}} \mathrm{U}}{\partial \mathrm{t}} \mathrm{dV}=\frac{\partial}{\partial \mathrm{t}} \int_{\mathrm{V}} \mathrm{X}_{\mathrm{k}} \mathrm{UdV}=\frac{\partial \mathrm{V}\left\langle\mathrm{X}_{\mathrm{k}} \mathrm{U}\right\rangle}{\partial \mathrm{t}}$

with the following definition of cell averages : $\left\langle X_{k} U\right\rangle=\frac{1}{V} \int_{V} X_{k} U d V$.

The second term can be transformed thanks to the Gauss theorem:

$$
\int_{V} \operatorname{div}\left(X_{k} F\right) d V=\int_{S} X_{k} F \cdot \vec{n} d S
$$

This surface integral transforms to a sum of fluid surfaces intergrals and solid surfaces integrals:

$$
\int_{S} X_{k} F \cdot \vec{n} d S=\int_{S f} X_{k} F \cdot \vec{n} d S+\int_{S s} X_{k} F \cdot \vec{n} d S
$$

The integral over fluid surfaces needs particular attention and will be achieved with the DEM. The solid surface one can be simplified by noting that the flux expresses:

$$
\int_{S s} X_{k} F \cdot \vec{n} d S=\int_{S s} X_{k}\left(\begin{array}{c}
0 \\
\rho \vec{u} \\
\rho \vec{u} \otimes \vec{u}+P \bar{I} \\
(\rho E+P) \vec{u}
\end{array}\right) \cdot \vec{n} d S
$$

By using boundary conditions on solid surfaces we have : 


$$
\int_{S s} X_{k}\left(\begin{array}{c}
0 \\
\rho \vec{u} \\
\rho \vec{u} \otimes \vec{u}+P \bar{I} \\
(\rho E+P) \vec{u}
\end{array}\right) \cdot \vec{n} d S=\left(\begin{array}{c}
0 \\
0 \\
\int_{S s} X_{k} P \vec{n} d S \\
0
\end{array}\right)
$$

Consider the following example :

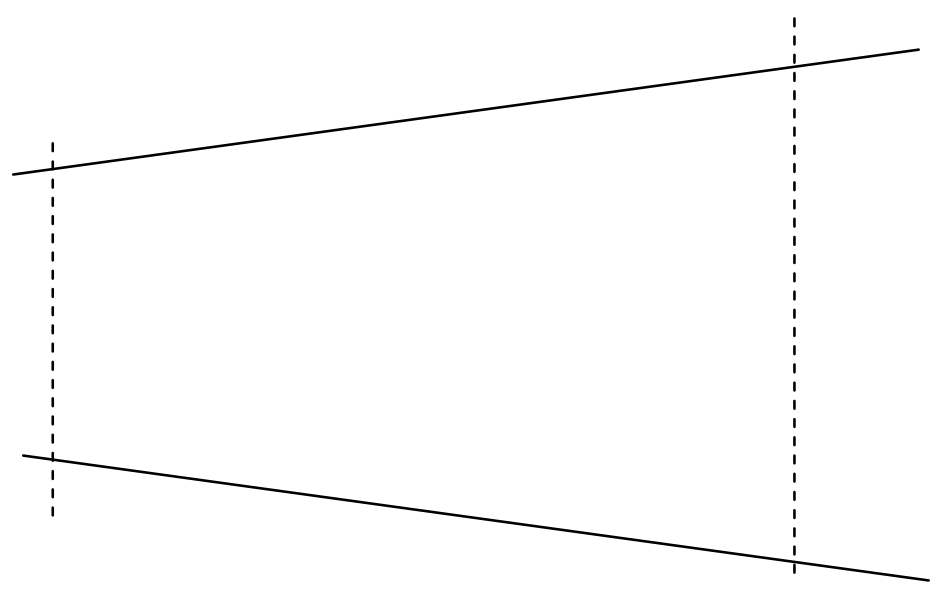

Part of the wall is occupied by phase 1 , the rest being occupied by phase 2 . We assume that phase 1 is occupying the upper wall with the same concentration as with the lower wall. On a part only of the wall the pressure of phase 1 acts. Thus we have to compute:

$$
\int_{S S} X_{k} P \vec{n} d S=\int_{S s k} P_{k} \vec{n} d S
$$

where ssk represents the wall surface occupied by phase k.

Assuming the pressure is uniform in the given phase:

$$
\int_{S s k} P_{k} \vec{n} d S=-\int_{\text {Complement of } S s k} P_{k} \vec{n} d S
$$

Thus,

$$
\int_{\text {ssk }} \mathrm{P}_{\mathrm{k}} \overrightarrow{\mathrm{n}} \mathrm{d} S=-\mathrm{P}_{\mathrm{k}}\left(\mathrm{A}_{\mathrm{k}, \text { out }}-\mathrm{A}_{\mathrm{k}, \text { in }}\right) \overrightarrow{\mathrm{i}}
$$

The two surfaces $A_{\text {kout }}$ and $A_{\text {kin }}$ are included in the two phase control volume: 


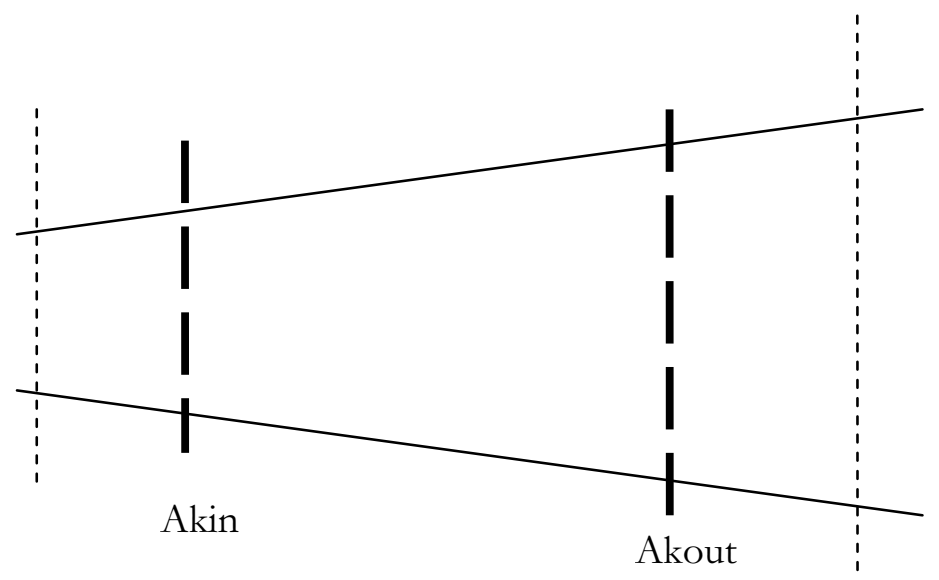

These two surfaces close the $\mathrm{k}$ phase control volume when all bubbles present at the walls are in contact. Let us denote by $\mathrm{L}$ the distance between these two cross sections. These intermediate surfaces are related to the fluid control volume surfaces by:

$$
\frac{A_{k, \text { out }}-A_{k, \text { in }}}{L}=\frac{A_{i+1 / 2}-A_{i-1 / 2}}{\Delta x}
$$

Consequently :

$A_{k, \text { out }}-A_{k, \text { in }}=\frac{L}{\Delta x}\left(A_{i+1 / 2}-A_{i-1 / 2}\right)$

Assuming the two phase mixture homogenous enough :

$$
\frac{\mathrm{L}}{\Delta \mathrm{x}} \cong \alpha_{\mathrm{k}}
$$

Consequently,

$$
\int_{\text {Ssk }} \mathrm{P}_{\mathrm{k}} \overrightarrow{\mathrm{n}} \mathrm{d} S=-\mathrm{P}_{\mathrm{k}} \alpha_{\mathrm{k}}\left(\mathrm{A}_{\mathrm{i}+1 / 2}-\mathrm{A}_{\mathrm{i}-1 / 2}\right) \overrightarrow{\mathrm{i}}
$$

The solid surface integral thus reads

$$
\int_{S s} X_{k}\left(\begin{array}{c}
0 \\
\rho \vec{u} \\
\rho \vec{u} \otimes \vec{u}+P \bar{I} \\
(\rho E+P) \vec{u}
\end{array}\right) \cdot \vec{n} d S=\left(\begin{array}{c}
0 \\
0 \\
-P_{k} \alpha_{k}\left(A_{i+1 / 2}-A_{i-1 / 2}\right) \vec{i} \\
0
\end{array}\right)
$$


The fluid surface integral has now to be considered: $\int_{S f} X_{k} F \cdot \vec{n} d S$.

Consider for example the inflow cross section $A_{i-1 / 2}$. We thus have to compute $\int_{A_{i-1 / 2}} X_{k} F \cdot \vec{n} d S$.

The inflow and outflow sections are perfectly determined, for example with relations like $A_{i-1 / 2}=\min \left(A_{i-1}, A_{i}\right)$.

Each cross section is partly occupied by a given phase. Indeed, several types of contact are present, as summarized in the next table:

\begin{tabular}{|c|l|l|c|}
\hline Type of Contact & \multicolumn{1}{|c|}{ Contact area } & Indicator function 1 & Convective flux \\
\hline $1-1$ & $\mathrm{~S}_{11}=\mathrm{A}_{\mathrm{i}-1 / 2} \operatorname{Min}\left(\alpha_{1, \mathrm{i}-1}, \alpha_{1, \mathrm{i}}\right)$ & $\mathrm{X}_{1}^{*}(1,1)=1$ & $\mathrm{~F}^{*}(1,1)$ \\
\hline $1-2$ & $\mathrm{~S}_{12}=\mathrm{A}_{\mathrm{i}-1 / 2} \operatorname{Max}\left(\alpha_{1, \mathrm{i}-1}-\alpha_{1, \mathrm{i}}, 0\right)$ & $\mathrm{X}_{1}^{*}(1,2)=\left\{\begin{array}{l}1 \text { if } \mathrm{u}^{*}(1,2)>0 \\
0 \text { otherwise }\end{array}\right.$ & $\mathrm{F}^{*}(1,2)$ \\
\hline $2-1$ & $\mathrm{~S}_{21}=\mathrm{A}_{\mathrm{i}-1 / 2} \operatorname{Max}\left(\alpha_{1, \mathrm{i}}-\alpha_{1, \mathrm{i}-1}, 0\right)$ & $\mathrm{X}_{1}^{*}(2,1)=\left\{\begin{array}{l}1 \text { if u }{ }^{*}(2,1)<0 \\
0 \text { otherwise }\end{array}\right.$ & $\mathrm{F}^{*}(2,1)$ \\
\hline $2-2$ & $\mathrm{~S}_{22}=\mathrm{A}_{\mathrm{i}-1 / 2} \operatorname{Min}\left(\alpha_{2, \mathrm{i}-1}, \alpha_{2, \mathrm{i}}\right)$ & $\mathrm{X}_{1}^{*}(2,2)=0$ & $\mathrm{~F}^{*}(2,2)$ \\
\hline
\end{tabular}

The fluid integral is thus obtained from the preceding table:

$$
\int_{A_{\mathrm{i}-1 / 2}} \mathrm{X}_{1} \mathrm{~F} \cdot \vec{n} \mathrm{~d} S=\sum_{\operatorname{lm}} \mathrm{S}_{\operatorname{lm}} \mathrm{X}_{1}^{*}(1, \mathrm{~m}) \mathrm{F}^{*}(1, \mathrm{~m})
$$

It remains to compute the last integral associated to interaction effects : $\int_{V}\left(F-\vec{u}_{I} U\right) \operatorname{grad}\left(X_{k}\right) d V$.

For the sake of simplicity we consider only this integral at cell boundaries, where volume fraction jumps are present. Inside the control volume we already know that these terms correspond to relaxation terms, that have been determined previously (see Chinnayya et al., 2004 for example).

We now note that at each point where $\operatorname{grad}\left(X_{k}\right)$ is non zero, the difference $\left(F-\vec{u}_{I} U\right)$ is necessarily localy constant (this difference correspond to the Rankine Hugoniot conditions, or interface conditions in the present context).

Therefore, the volume integral (here a surface integral, as we consider a 2D flow) transforms: 
$\int_{V}\left(F-\vec{u}_{I} U\right) \operatorname{grad}\left(X_{k}\right) d V=\int_{S}\left(F-\vec{u}_{I} U\right) \operatorname{grad}\left(X_{k}\right) d S$ (we have just changed notations, $S$ is $V$ in two dimensions).

$$
\int_{S}\left(F-\overrightarrow{\mathrm{u}}_{\mathrm{I}} \mathrm{U}\right) \operatorname{grad}\left(\mathrm{X}_{\mathrm{k}}\right) \mathrm{dS}=\int_{\mathrm{x}_{\mathrm{i}-1 / 2}-\varepsilon}^{\mathrm{x}_{\mathrm{i}-1 / 2}+\varepsilon} \int_{\mathrm{y}=-0.5 \mathrm{H}_{\mathrm{i}-1: 2}}^{0.5 \mathrm{H}_{\mathrm{i}-1 / 2}}\left(\mathrm{~F}-\overrightarrow{\mathrm{u}}_{\mathrm{I}} \mathrm{U}\right) \operatorname{grad}\left(\mathrm{X}_{\mathrm{k}}\right) \mathrm{dxdy}+\int_{\mathrm{x}_{\mathrm{i}+1 / 2}-\varepsilon}^{\mathrm{x}_{\mathrm{i}+1 / 2}+\varepsilon} \int_{\mathrm{j}=-0.5 \mathrm{H}_{\mathrm{i}+1: 2}}^{0.5 \mathrm{H}_{\mathrm{i}+1 / 2}}\left(\mathrm{~F}-\overrightarrow{\mathrm{u}}_{\mathrm{I}} \mathrm{U}\right) \operatorname{grad}\left(\mathrm{X}_{\mathrm{k}}\right) \mathrm{dxdy}
$$

Here two integrals only are present because internal bubbles are omitted. The two integrals correspond to the two boundary surfaces.

We now transform one of these surface integrals into a contour one, by using the fact that the interface conditions are local constants:

$$
\int_{S}\left(F-\vec{u}_{I} U\right) \operatorname{grad}\left(X_{k}\right) d S=\int_{x_{i-1 / 2}-\varepsilon}^{x_{i-1 / 2}+\varepsilon} \int_{y=-0.5 H_{i-1: 2}}^{0.5 H_{i-1 / 2}}\left(F-\vec{u}_{I} U\right) \operatorname{grad}\left(X_{k}\right) d x d y+\int_{x_{i+1 / 2}-\varepsilon}^{x_{i+1 / 2}+\varepsilon} \int_{y=-0.5 H_{i+1: 2}}^{0.5 H_{i+1 / 2}}\left(F-\vec{u}_{I} U\right) \operatorname{grad}\left(X_{k}\right) d x d y
$$

Where $\varepsilon \rightarrow 0^{+}$. We now focus on one of these two integrals :

$$
\begin{aligned}
& \int_{\mathrm{x}_{\mathrm{i}-1 / 2}-\varepsilon}^{\mathrm{x}_{\mathrm{i}-1 / 2}+\varepsilon} \int_{\mathrm{j}=-0.5 \mathrm{H}_{\mathrm{i}-1: 2}}^{0.5 \mathrm{H}_{\mathrm{i}-1 / 2}}\left(\mathrm{~F}-\overrightarrow{\mathrm{u}}_{\mathrm{I}} \mathrm{U}\right) \operatorname{grad}\left(\mathrm{X}_{\mathrm{k}}\right) \mathrm{dxdy} \\
& =\left(\mathrm{F}-\overrightarrow{\mathrm{u}}_{\mathrm{I}} \mathrm{U}\right)_{\mathrm{i}-1 / 2} \int_{\mathrm{x}_{\mathrm{i}-1 / 2-1 / 2}^{+\varepsilon}}^{\mathrm{x}_{\mathrm{i}}-\varepsilon} \int_{\mathrm{y}=-0.5 \mathrm{H}_{\mathrm{i}-1: 2}}^{0.5 \mathrm{H}_{\mathrm{i}-1 / 2}} \operatorname{grad}\left(\mathrm{X}_{\mathrm{k}}\right) \mathrm{dxdy} \\
& =\left(\mathrm{F}-\overrightarrow{\mathrm{u}}_{\mathrm{I}} \mathrm{U}\right)_{12, \mathrm{i}-1 / 2}\left[\mathrm{X}_{\mathrm{k}}\right]_{12} \mathrm{~S}_{12}+\left(\mathrm{F}-\overrightarrow{\mathrm{u}}_{\mathrm{I}} \mathrm{U}\right)_{21, \mathrm{i}-1 / 2}\left[\mathrm{X}_{\mathrm{k}}\right]_{21} \mathrm{~S}_{21}
\end{aligned}
$$




\begin{tabular}{|c|c|c|c|}
\hline $\begin{array}{l}\text { Type of } \\
\text { Contact }\end{array}$ & Surface of Contact & Jump of the indicator function 1 & Lagrangian flux \\
\hline $1-1$ & $S_{11}=A_{i-1 / 2} \operatorname{Min}\left(\alpha_{1, i-1}, \alpha_{1, i}\right)$ & {$\left[\mathrm{X}_{1}^{*}\right](1,1)=0$} & $\mathrm{~F}^{\mathrm{lag}, *}(1,1)$ \\
\hline $1-2$ & $S_{12}=A_{i-1 / 2} \operatorname{Max}\left(\alpha_{1, i-1}-\alpha_{1, i}, 0\right)$ & {$\left[\mathrm{X}_{1}^{*}\right](1,2)=\left\{\begin{array}{l}-1 \text { if } \mathrm{u}_{1}^{*}(1,2)>0 \\
0 \text { otherwise }\end{array}\right.$} & $\mathrm{F}^{\operatorname{lag}, *}(1,2)$ \\
\hline $2-1$ & $S_{21}=A_{i-1 / 2} \operatorname{Max}\left(\alpha_{1, i}-\alpha_{1, i-1}, 0\right)$ & {$\left[\mathrm{X}_{1}^{*}\right](2,1)= \begin{cases}1 & \text { if } \mathrm{u}_{1}^{*}(2,1)>0 \\
0 & \text { otherwise }\end{cases}$} & $\mathrm{F}^{\operatorname{lag}, *}(2,1)$ \\
\hline $2-2$ & $S_{22}=A_{i-1 / 2} \operatorname{Min}\left(\alpha_{2, i-1}, \alpha_{2, i}\right)$ & {$\left[\mathrm{X}_{1}^{*}\right](2,2)=0$} & $\mathrm{~F}^{\operatorname{lag}, *}(2,2)$ \\
\hline
\end{tabular}

\section{Summary}

$\frac{\partial \mathrm{V}\left\langle\mathrm{X}_{\mathrm{k}} \mathrm{U}\right\rangle}{\partial \mathrm{t}}+\left\{\sum_{\mathrm{lm}} \mathrm{S}_{\operatorname{lm}} \mathrm{X}_{1}^{*}(1, \mathrm{~m}) \mathrm{F}^{*}(1, \mathrm{~m})\right\}_{\mathrm{i}+1 / 2}-\left\{\sum_{\operatorname{lm}} \mathrm{S}_{\operatorname{lm}} \mathrm{X}_{1}^{*}(1, \mathrm{~m}) \mathrm{F}^{*}(1, \mathrm{~m})\right\}_{\mathrm{i}-1 / 2}=$ $\left\{\left(\mathrm{F}-\overrightarrow{\mathrm{u}}_{\mathrm{I}} \mathrm{U}\right)_{12}\left[\mathrm{X}_{\mathrm{k}}\right]_{12} \mathrm{~S}_{12}+\left(\mathrm{F}-\overrightarrow{\mathrm{u}}_{\mathrm{I}} \mathrm{U}\right)_{21}\left[\mathrm{X}_{\mathrm{k}}\right]_{21} \mathrm{~S}_{21}\right\}_{\mathrm{i}-1 / 2}+\left\{\left(\mathrm{F}-\overrightarrow{\mathrm{u}}_{\mathrm{I}} \mathrm{U}\right)_{12}\left[\mathrm{X}_{\mathrm{k}}\right]_{12} \mathrm{~S}_{12}+\left(\mathrm{F}-\overrightarrow{\mathrm{u}}_{\mathrm{I}} \mathrm{U}\right)_{21}\left[\mathrm{X}_{\mathrm{k}}\right]_{21} \mathrm{~S}_{21}\right\}_{\mathrm{i}+1 / 2}$ $+\left(\begin{array}{c}0 \\ 0 \\ P_{k} \alpha_{k}\left(A_{i+1 / 2}-A_{i-1 / 2}\right) \vec{i} \\ 0\end{array}\right)$

$\frac{\partial \mathrm{A}\left\langle\mathrm{X}_{\mathrm{k}} \mathrm{U}\right\rangle}{\partial \mathrm{t}}+\frac{\left\{\sum_{\operatorname{lm}} \mathrm{S}_{\operatorname{lm}} \mathrm{X}_{1}^{*}(1, \mathrm{~m}) \mathrm{F}^{*}(1, \mathrm{~m})\right\}_{\mathrm{i}+1 / 2}-\left\{\sum_{\operatorname{lm}} \mathrm{S}_{\operatorname{lm}} \mathrm{X}_{1}^{*}(1, \mathrm{~m}) \mathrm{F}^{*}(1, \mathrm{~m})\right\}_{\mathrm{i}-1 / 2}}{\Delta \mathrm{x}}=$ $\frac{\left\{\left(\mathrm{F}-\overrightarrow{\mathrm{u}}_{\mathrm{I}} \mathrm{U}\right)_{12}\left[\mathrm{X}_{\mathrm{k}}\right]_{12} \mathrm{~S}_{12}+\left(\mathrm{F}-\overrightarrow{\mathrm{u}}_{\mathrm{I}} \mathrm{U}\right)_{21}\left[\mathrm{X}_{\mathrm{k}}\right]_{21} \mathrm{~S}_{21}\right\}_{\mathrm{i}-1 / 2}+\left\{\left(\mathrm{F}-\overrightarrow{\mathrm{u}}_{\mathrm{I}} \mathrm{U}\right)_{12}\left[\mathrm{X}_{\mathrm{k}}\right]_{12} \mathrm{~S}_{12}+\left(\mathrm{F}-\overrightarrow{\mathrm{u}}_{\mathrm{I}} \mathrm{U}\right)_{21}\left[\mathrm{X}_{\mathrm{k}}\right]_{21} \mathrm{~S}_{21}\right\}_{\mathrm{i}+1 / 2}}{\Delta \mathrm{x}}$ $+\left(\begin{array}{c}0 \\ 0 \\ P_{k} \alpha_{k}\left(\frac{A_{i+1 / 2}-A_{i-1 / 2}}{\Delta x}\right) \vec{i} \\ 0\end{array}\right)$

$\frac{\partial \alpha_{\mathrm{k}} \mathrm{AU}}{\partial \mathrm{t}}+\frac{\Delta\left(\alpha_{\mathrm{k}} \mathrm{AF}\right)}{\Delta \mathrm{x}}=\frac{\mathrm{F}^{\operatorname{lag}} \mathrm{A} \Delta \alpha}{\Delta \mathrm{x}}+\left(\begin{array}{c}0 \\ 0 \\ \mathrm{P}_{\mathrm{k}} \alpha_{\mathrm{k}}\left(\frac{\mathrm{A}_{\mathrm{i}+1 / 2}-\mathrm{A}_{\mathrm{i}-1 / 2}}{\Delta \mathrm{x}}\right) \overrightarrow{\mathrm{i}}\end{array}\right)$

The continuous system (without relaxation terms and with no heat and mass transfer between phases) can be written from the above as : 
Volume fraction

$\frac{\partial \alpha_{k}}{\partial t}+u_{I} \frac{\partial \alpha}{\partial x}=0$

$\underline{\text { Mass }}$

$\frac{\partial \alpha_{k} \rho_{k} A}{\partial t}+\frac{\partial \alpha_{k} \rho_{k} u_{k} A}{\partial x}=0$

Momentum

$\frac{\partial \alpha_{k} \rho_{k} u_{k} A}{\partial t}+\frac{\partial \alpha_{k} A\left(\rho_{k} u_{k}^{2}+p_{k}\right)}{\partial x}=p_{I} A \frac{\partial \alpha_{k}}{\partial x}+P_{k} \alpha_{k} \frac{\partial A}{\partial x}$

$\frac{\partial \alpha_{k} \rho_{k} u_{k} A}{\partial t}+\frac{\partial \alpha_{k} A \rho_{k} u_{k}{ }^{2}}{\partial x}+A p_{k} \frac{\partial \alpha_{k}}{\partial x}+\alpha_{k} \frac{\partial A p_{k}}{\partial x}+\alpha_{k} A \frac{\partial p_{k}}{\partial x}+\alpha_{k} p_{k} \frac{\partial A}{\partial x}=p_{I} A \frac{\partial \alpha_{k}}{\partial x}+P_{k} \alpha_{k} \frac{\partial A}{\partial x}$

$\frac{\partial \alpha_{k} \rho_{k} u_{k} A}{\partial t}+\frac{\partial \alpha_{k} A \rho_{k} u_{k}^{2}}{\partial x}+\alpha_{k} A \frac{\partial p_{k}}{\partial x}=\left(p_{I}-p_{k}\right) A \frac{\partial \alpha_{k}}{\partial x}$

$\left.\frac{\mathrm{du}_{\mathrm{k}}}{\mathrm{dt}}\right)_{\mathrm{u}_{\mathrm{k}}}+\frac{1}{\rho_{\mathrm{k}}} \frac{\partial \mathrm{p}_{\mathrm{k}}}{\partial \mathrm{x}}=\frac{\left(\mathrm{p}_{\mathrm{I}}-\mathrm{p}_{\mathrm{k}}\right)}{\alpha_{\mathrm{k}} \rho_{\mathrm{k}}} \frac{\partial \alpha_{\mathrm{k}}}{\partial \mathrm{x}}$

Now if $A$ is constant:

$\frac{\partial \alpha_{k} \rho_{k} u_{k} A}{\partial t}+\frac{\partial \alpha_{k} A\left(\rho_{k} u_{k}^{2}+p_{k}\right)}{\partial x}=p_{I} A \frac{\partial \alpha_{k}}{\partial x}+P_{k} \alpha_{k} \frac{\partial A}{\partial x}$
$\frac{\partial \alpha_{k} \rho_{k} u_{k}}{\partial t}+\frac{\partial \alpha_{k}\left(\rho_{k} u_{k}{ }^{2}+p_{k}\right)}{\partial x}=p_{I} \frac{\partial \alpha_{k}}{\partial x}$

Now if $p$ is constant and alpha varies:

$\frac{\partial \alpha_{k} \rho_{k} u_{k}}{\partial t}+\frac{\partial \alpha_{k}\left(\rho_{k} u_{k}^{2}+p_{k}\right)}{\partial x}=p_{I} \frac{\partial \alpha_{k}}{\partial x}$

$\frac{\partial \alpha_{k} \rho_{k} u_{k}}{\partial t}+\frac{\partial \alpha_{k}\left(\rho_{k} u_{k}^{2}\right)}{\partial x}=0$

If $A$ varies and alpha constant:

$\frac{\partial \alpha_{k} \rho_{k} u_{k} A}{\partial t}+\frac{\partial \alpha_{k} A\left(\rho_{k} u_{k}{ }^{2}+p_{k}\right)}{\partial x}=p_{I} A \frac{\partial \alpha_{k}}{\partial x}+P_{k} \alpha_{k} \frac{\partial A}{\partial x}$

$\frac{\partial \rho_{k} u_{k} A}{\partial t}+\frac{\partial A\left(\rho_{k} u_{k}{ }^{2}+p_{k}\right)}{\partial x}=P_{k} \frac{\partial A}{\partial x}$

Energy

$\frac{\partial \alpha_{k} \rho_{k} E_{k} A}{\partial}+\frac{\partial \alpha_{k}\left(\rho_{k} E_{k}+p_{k}\right) u_{k} A}{\partial x}=p_{I} u_{k} A \frac{\partial \alpha_{k}}{\partial x}$ 


\section{RESULTS}

In the following we first present results for single phase, 1-D variable area simulation with the compressible Euler equations using a stiffened gas equation of state (liquid water). This 1-D, volume-centered, finite volume method uses the HLLC approximate Riemann solver to determine the fluxes at cell faces. The scheme uses explicit time stepping to brute for the solution to steady state. This steady state solution is used as an initial benchmark solution for the initial two phase DEM results (described next).

The DEM two phase methods is then employed with this same compressible liquid and with a compressible gas. Again stiffened gas EOS is used for each phase. First relaxation is turned off, which means that the phases do not interact with each other. That is two independent, variable area solutions should evolve, one for each phase. The volume fractions should not change. The solution for the liquid phase should be identical to the single phase result first presented.

Then two different degrees of relaxation are turned on via specification of the specific interfacial area $(\mathrm{SSV}=1$ and $\mathrm{SSV}=100)$, i.e. interfacial area per unit volume. Results are successively presented for each phase. Finally, relaxation is left on, specific interfacial area SSV=100, and crude (not physically correct, but which still add a qualitatively similar effect) mass and heat transfer models are added for a first look at their effects. Results are then displayed for each phase (plots with blue background) on the same page for comparison. 
Single Phase, 1D EULER STIFFENED GAS (1-D Finite Volume, Benchmark Solution)

data $\mathrm{kmax} / 1000000 /, \mathrm{kprint} / 10000 /$

c data $\mathrm{kmax} / 1 / \mathrm{kprint} / 1 /$

data ltube/1.d0/,dt/1.d-12/

c Indices des raccords

data idisc1/100/,idisc2/300/,idisc3/500/

c Conditions initiales data rho01/1000.d0/,rho0r/1000.d0/ data $\mathrm{u} 01 / 0 . \mathrm{d} 0 /, \mathrm{u} 0 \mathrm{r} / 0 . \mathrm{d} 0 /$ data $\mathrm{pg} 01 / 1 . \mathrm{d} 5 /, \mathrm{pg} 0 \mathrm{r} / 1 . \mathrm{d} 5 /$

c Donnees geometriques des troncons data sent $1 / 0.2 \mathrm{~d} 0 /$,ssort $1 / 0.2 \mathrm{~d} 0 /$ data sent $2 / 0.2 \mathrm{~d} 0 /$,ssort $2 / 0.15 \mathrm{~d} 0 /$ data sent $3 / 0.15 \mathrm{~d} 0 /$, ssort $3 / 0.2 \mathrm{~d} 0 /$

c Thermo data gamma/4.4d0/,pinf $/ 6 . d 8 /$

c Donnees de controle data $\mathrm{cfl} / 0.5 \mathrm{~d} 0 /$,temps $/ 0 . \mathrm{d} 0 /$

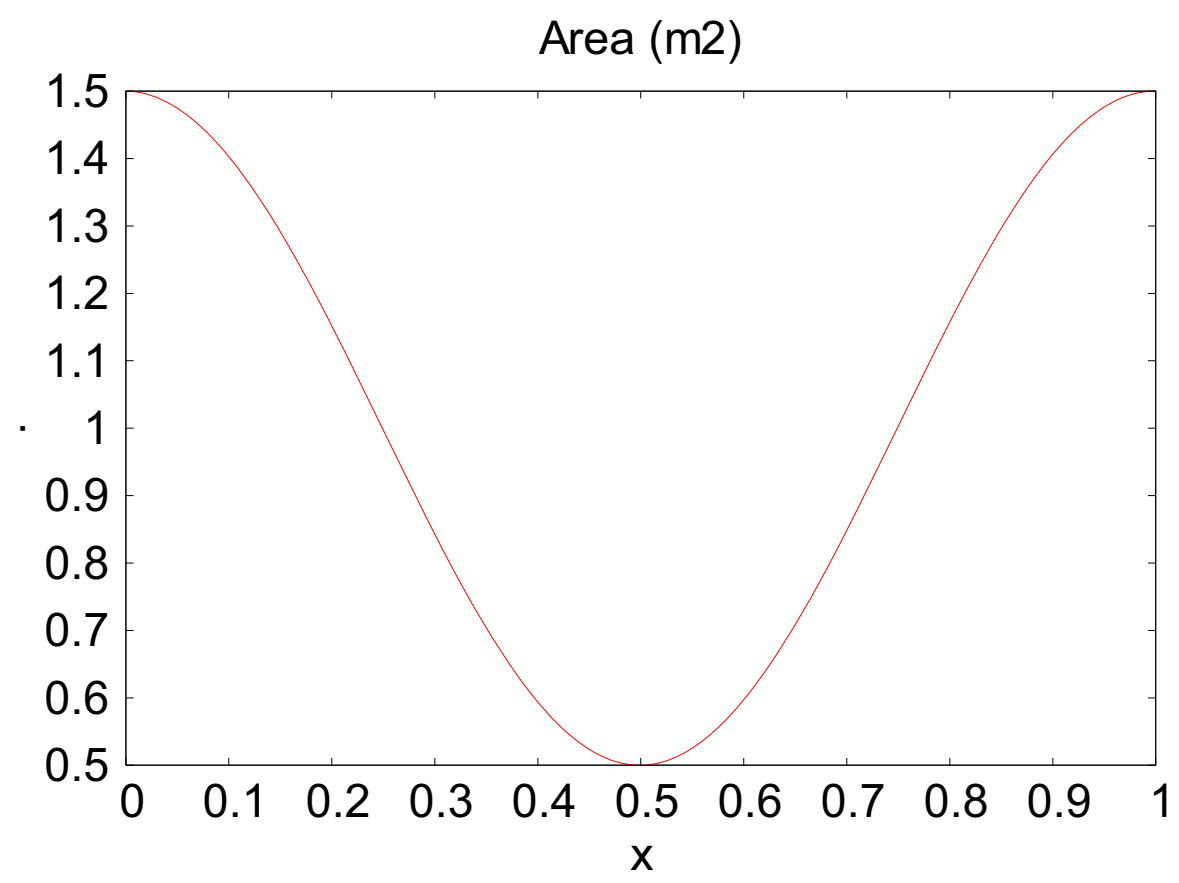



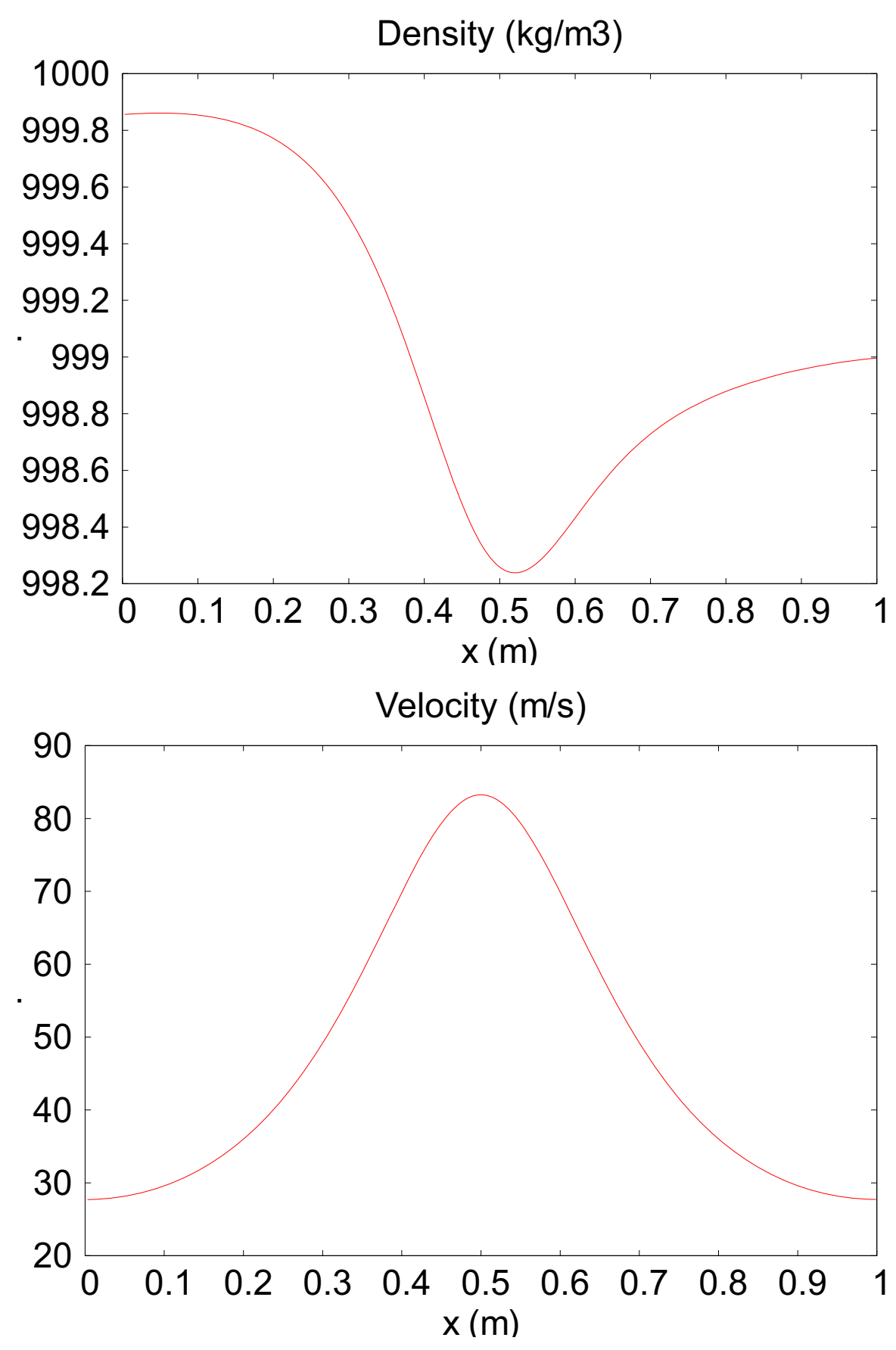

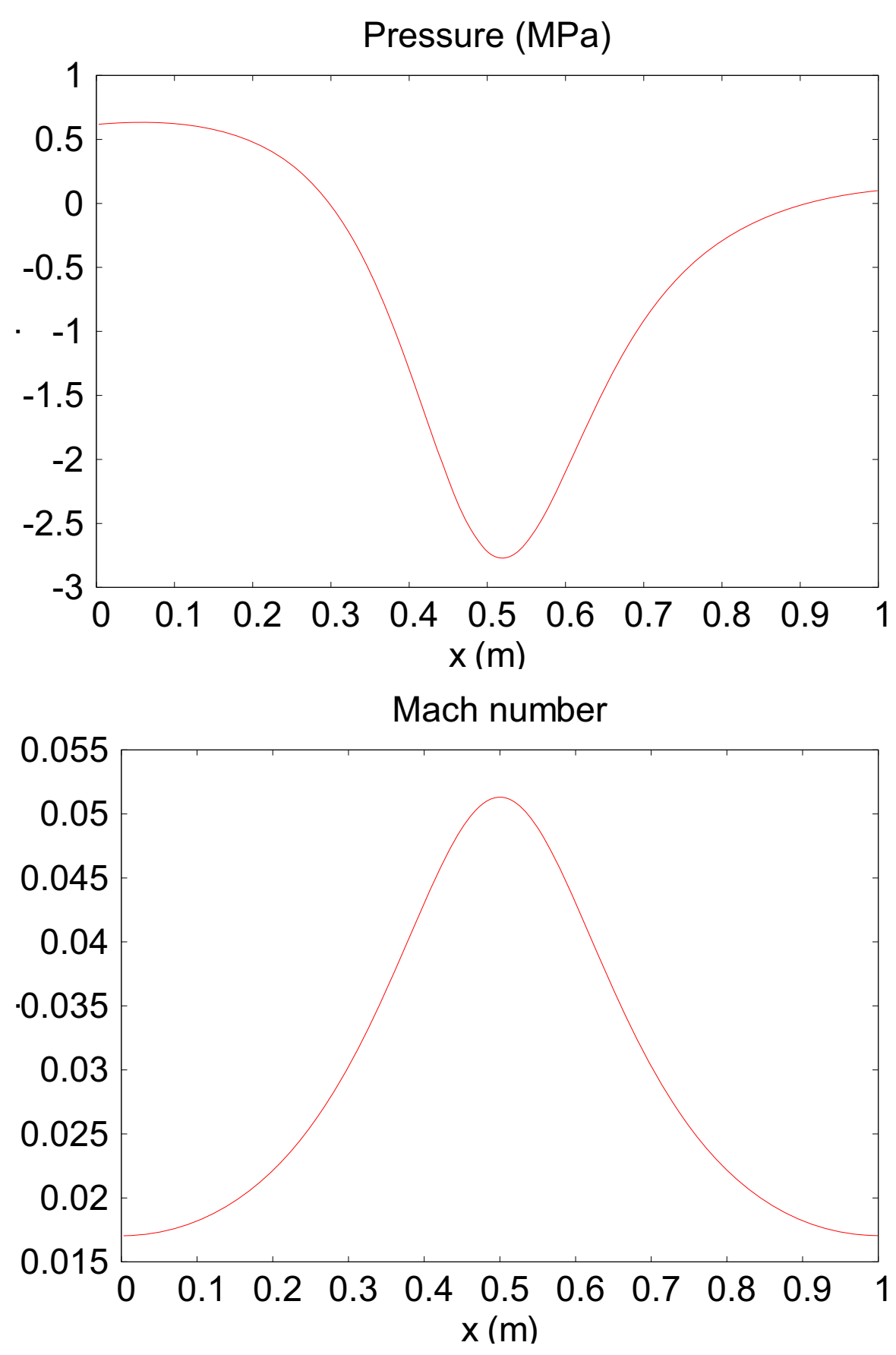


\section{TWO PHASE DEM CODE}

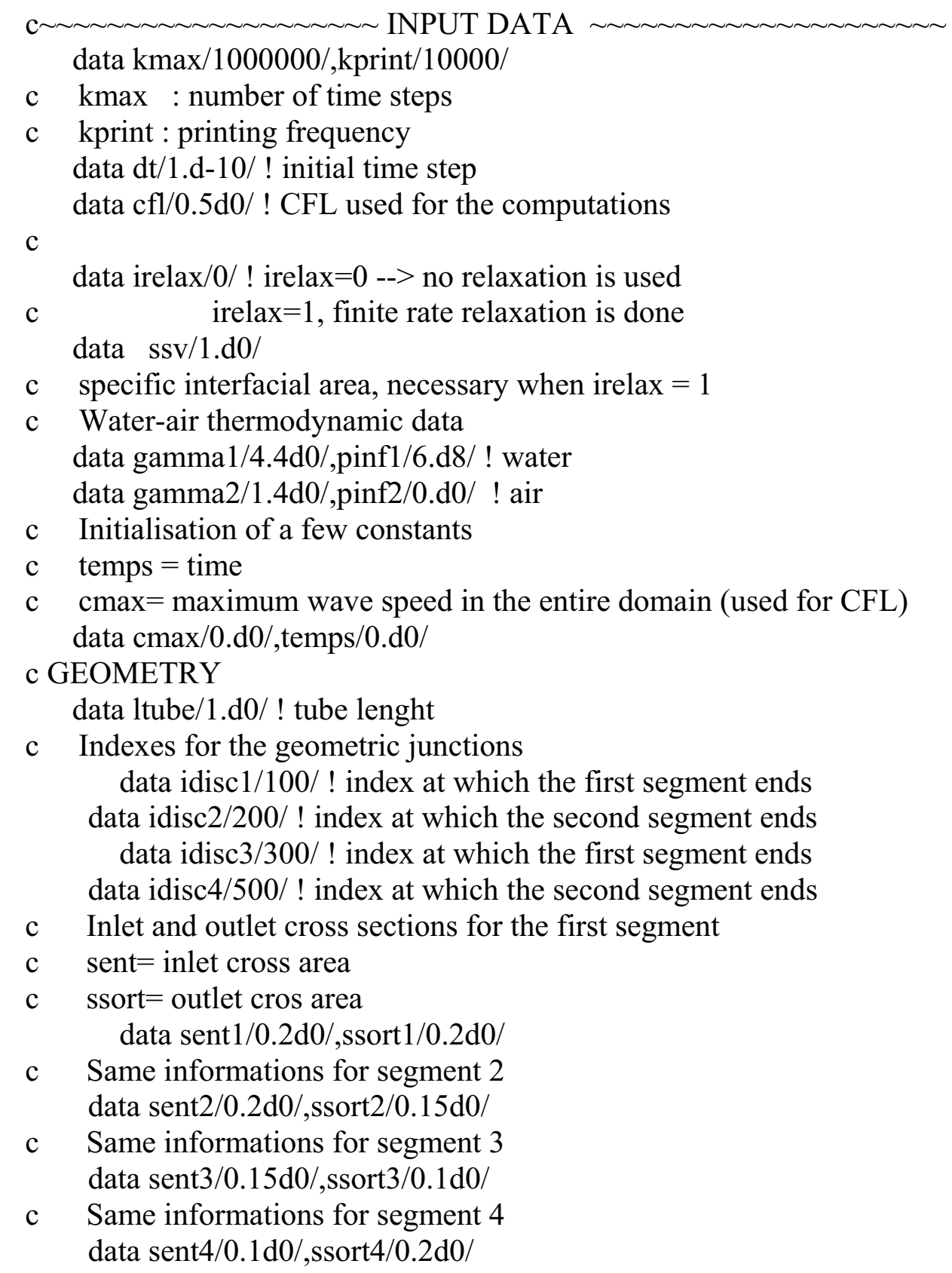




\section{LIQUID VARIABLES}
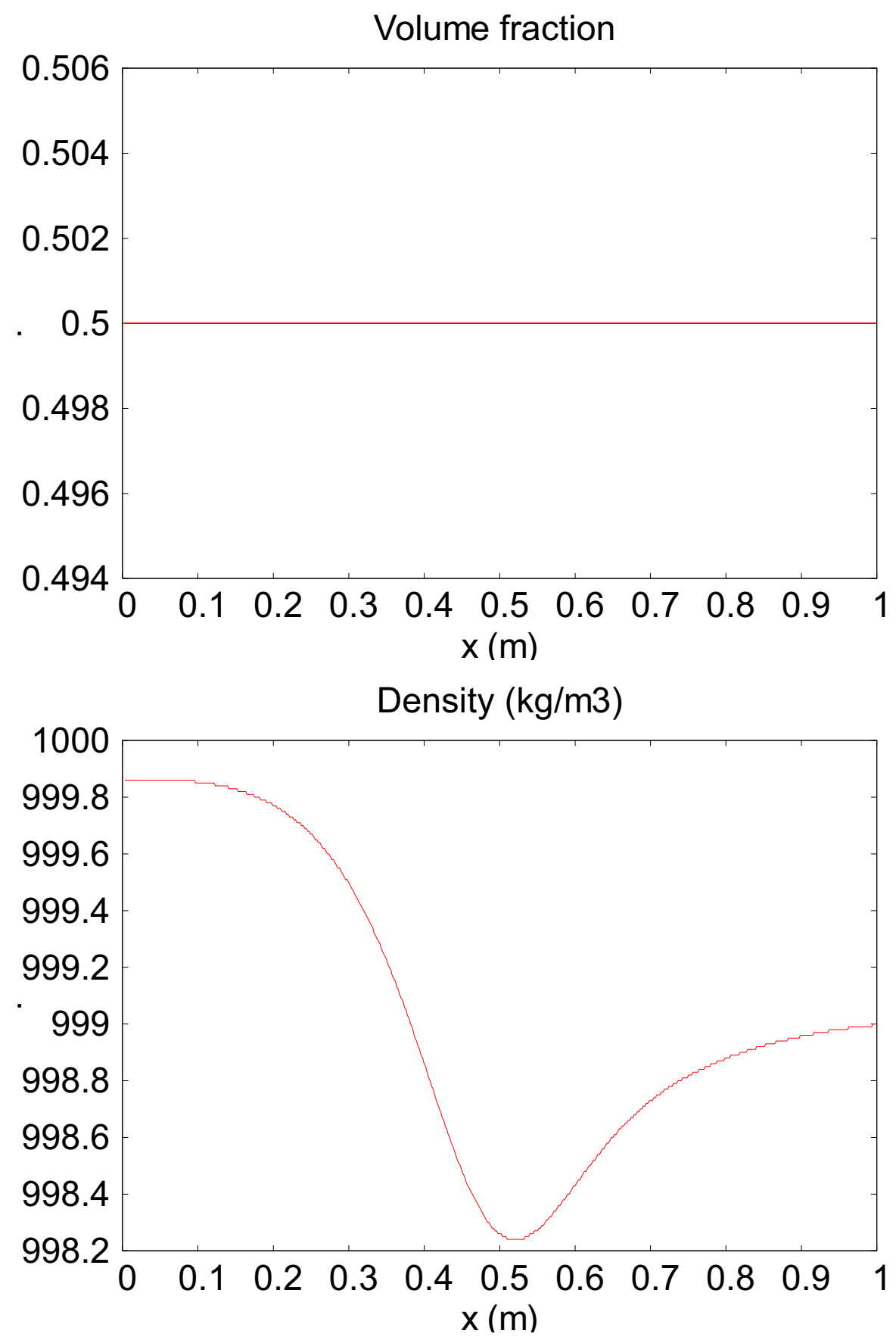

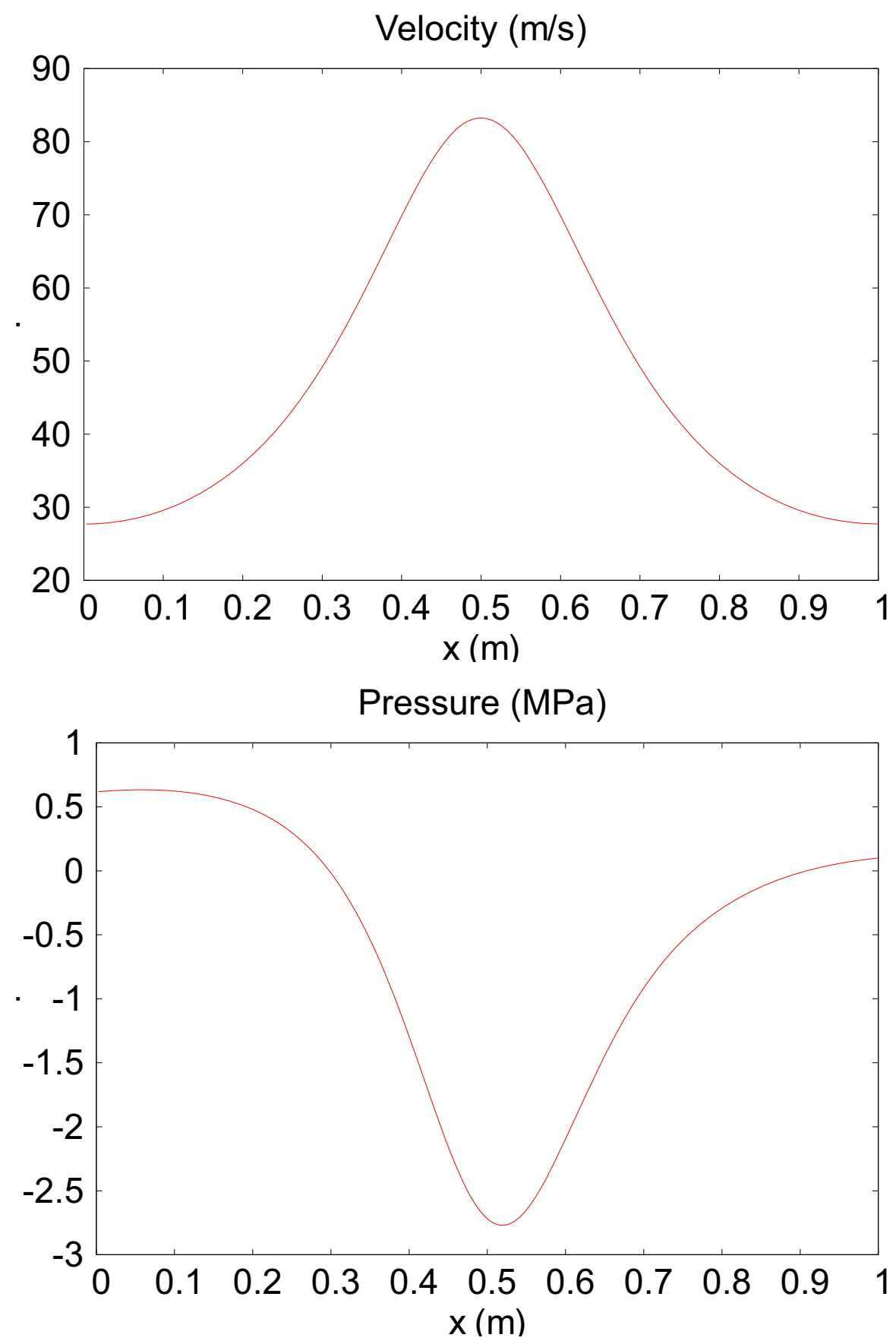


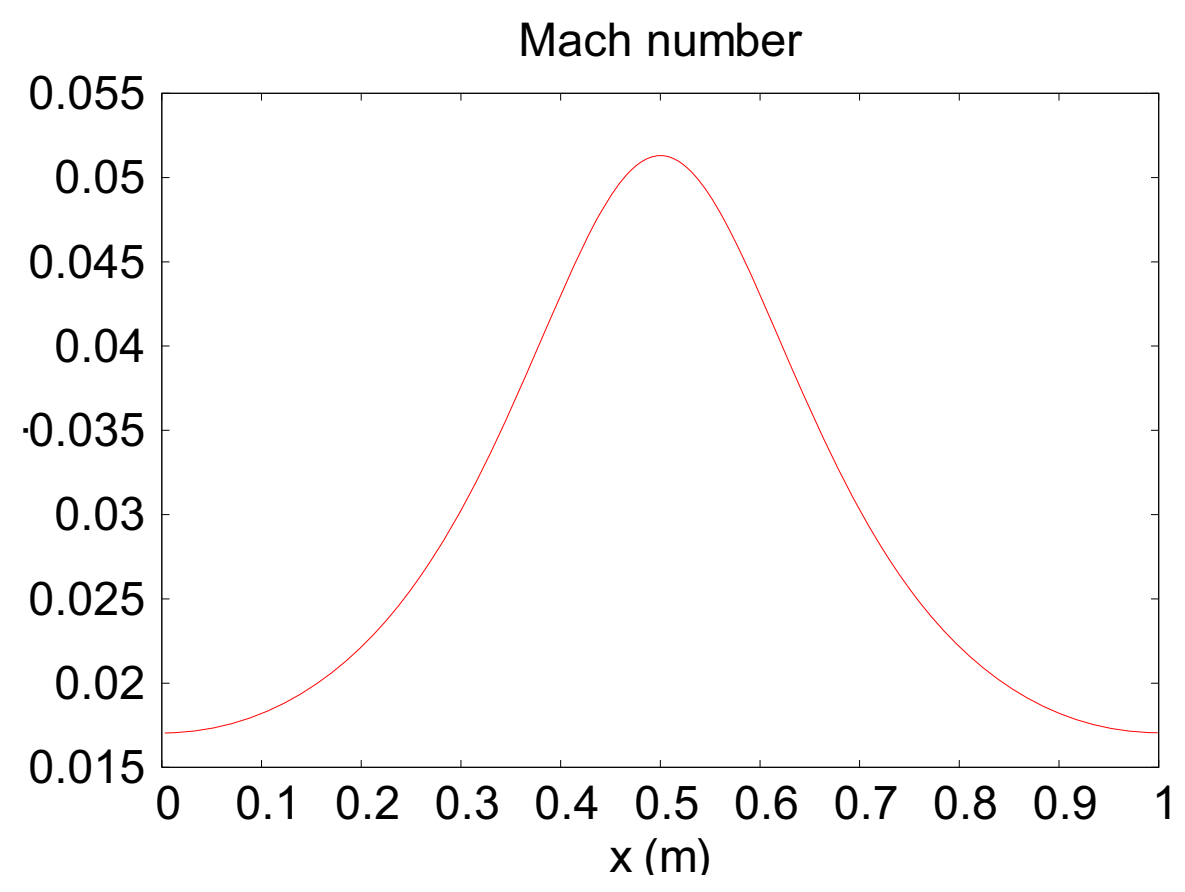

\section{GAS VARIABLES}

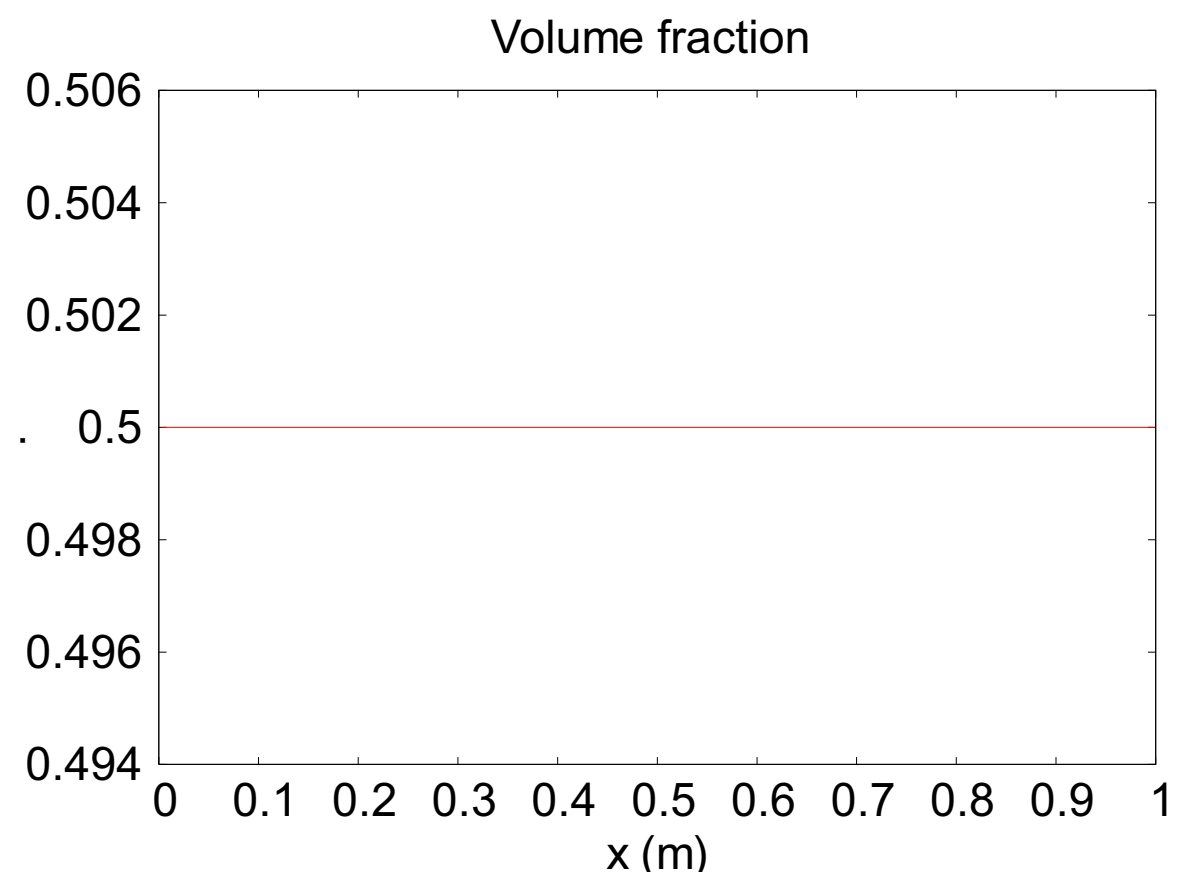



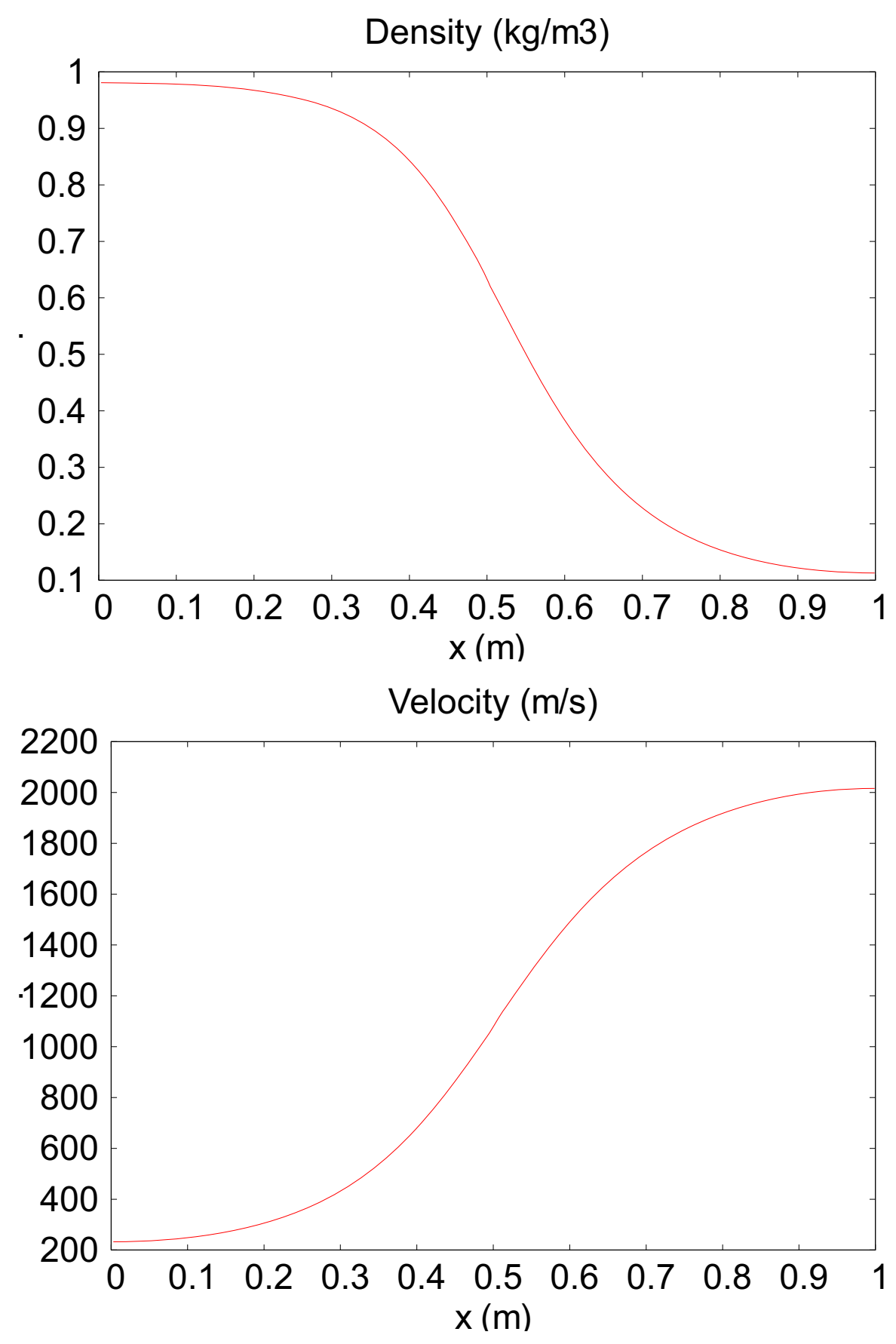

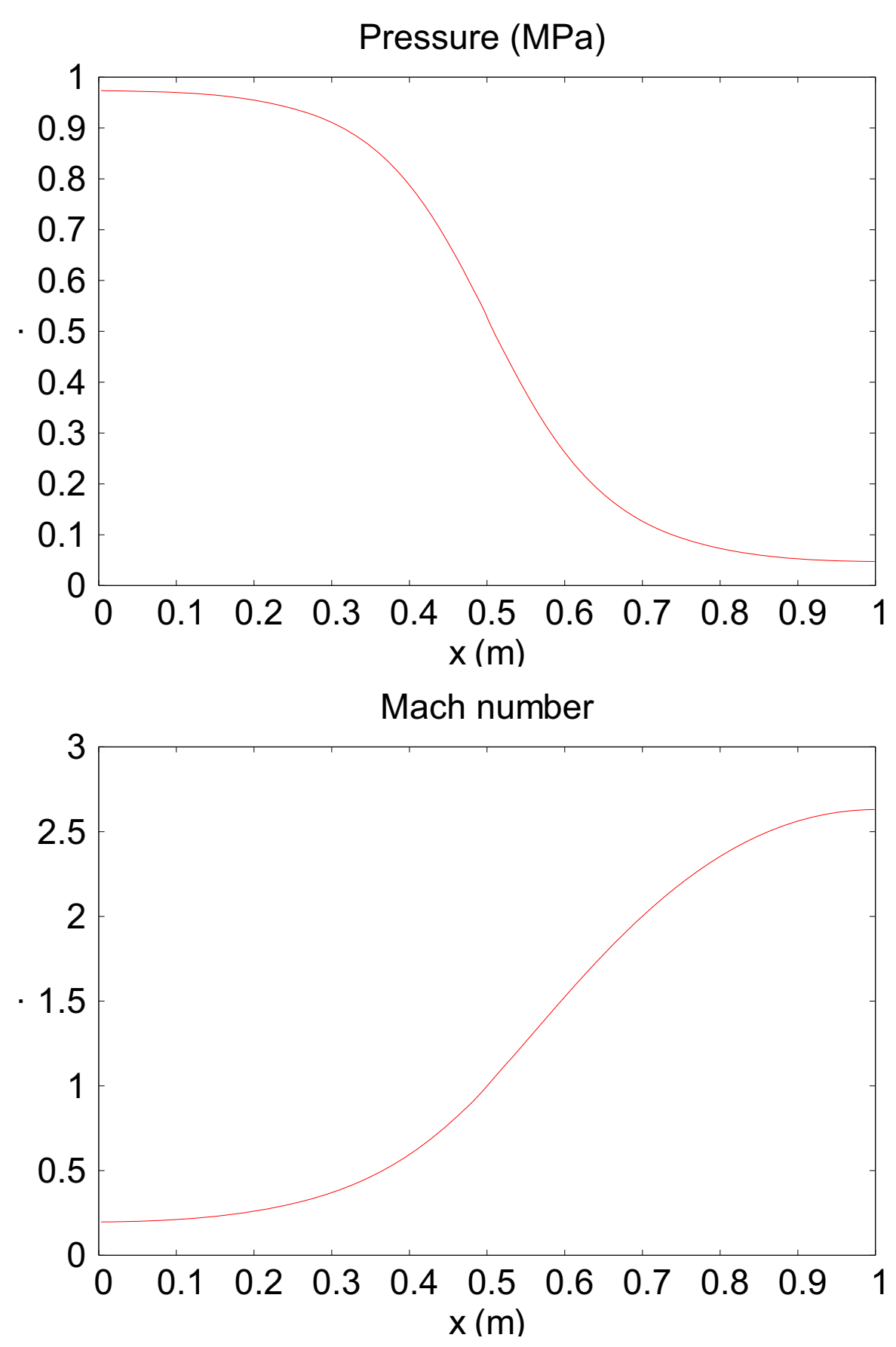


\section{COMPARISON EULER SINGLE-PHASE (Left) AND TWO-PHASE LIQUID-GAS CODE (Right). THE COMPARISON IS DONE ON LIQUID VARIABLES.}
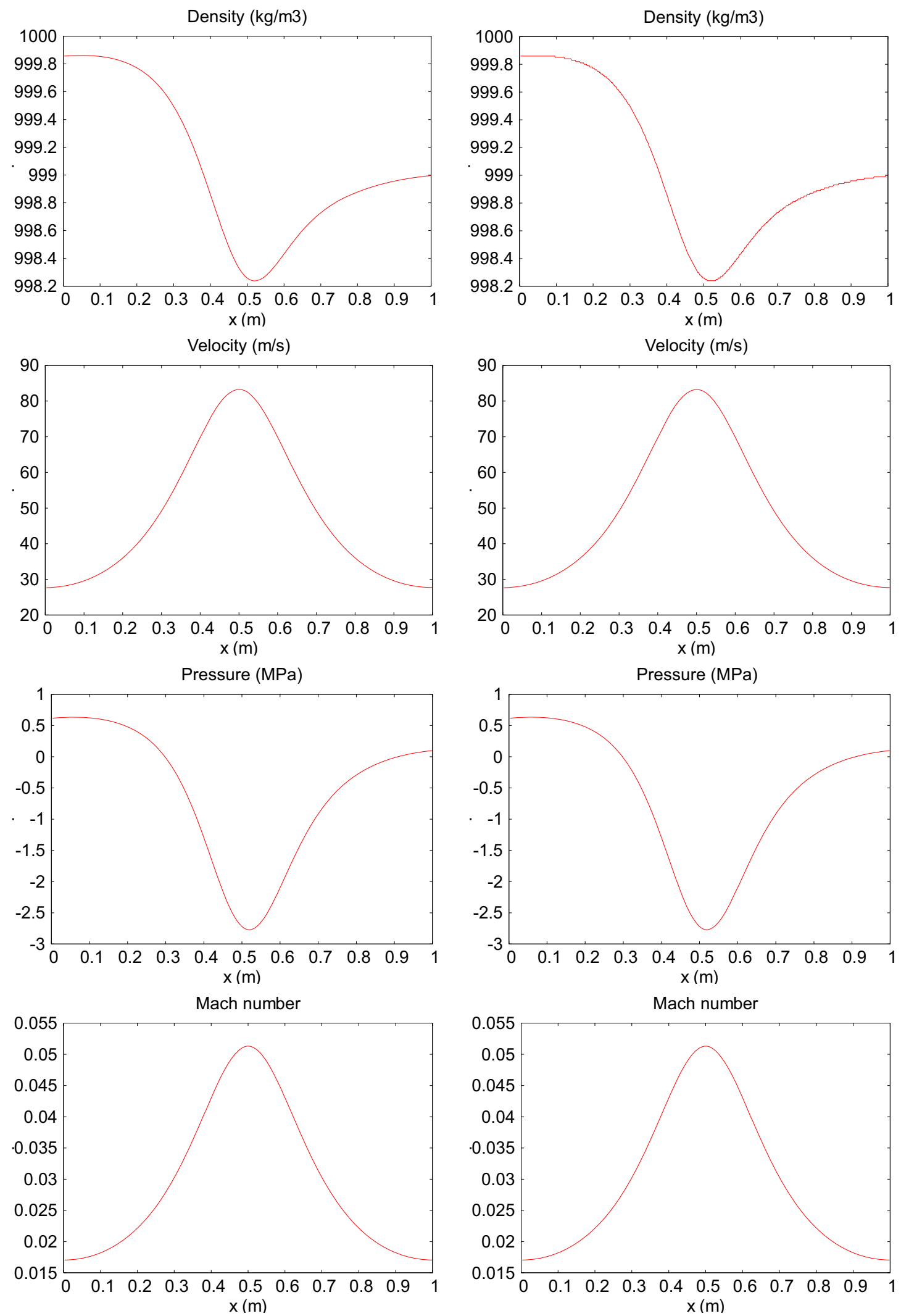
TWO "PHASE WITH RELAXATION

(BOTH PRESSURES AND VELOCITIES WITH SSV=1)

\section{LIQUID PHASE VARIABLES}
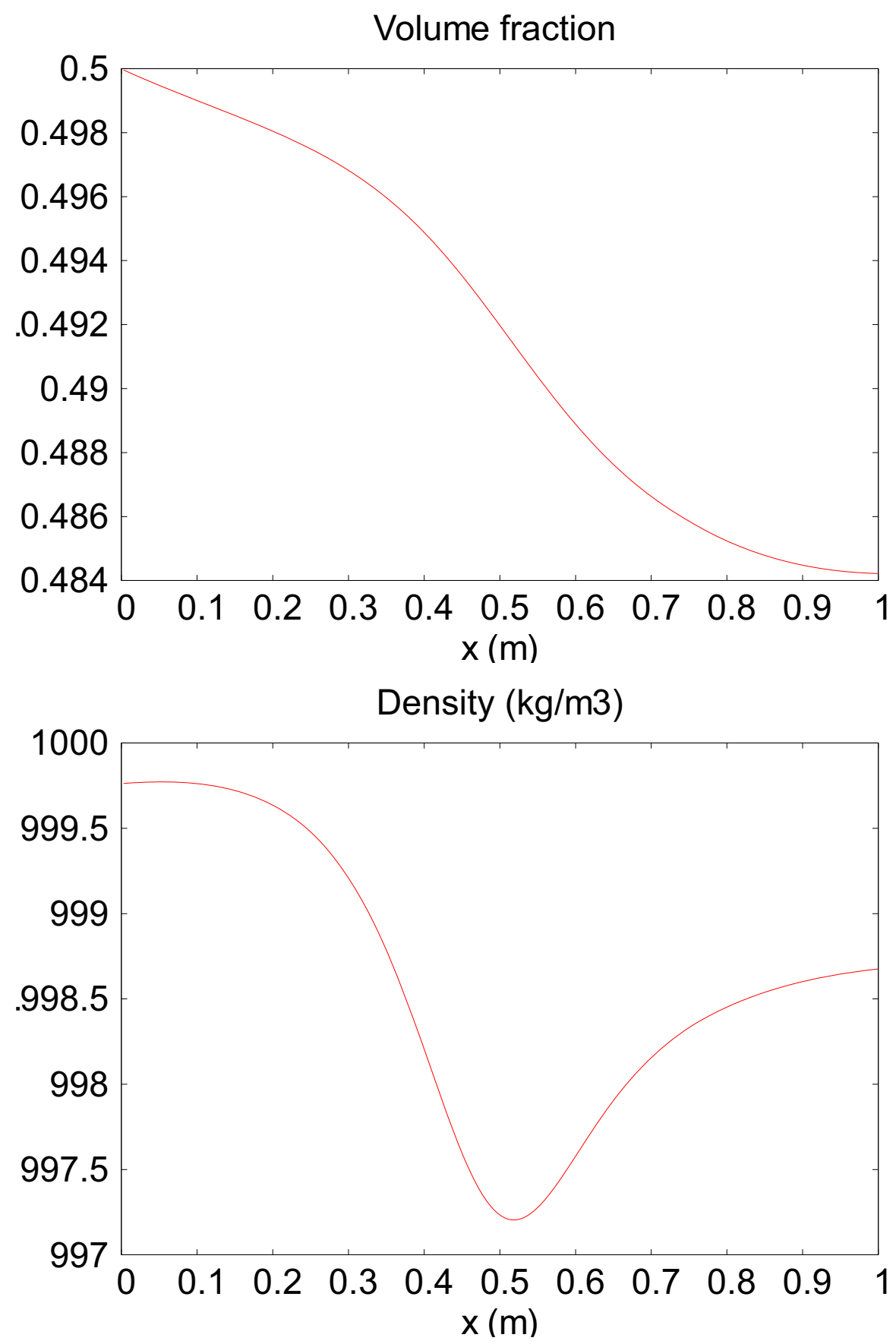

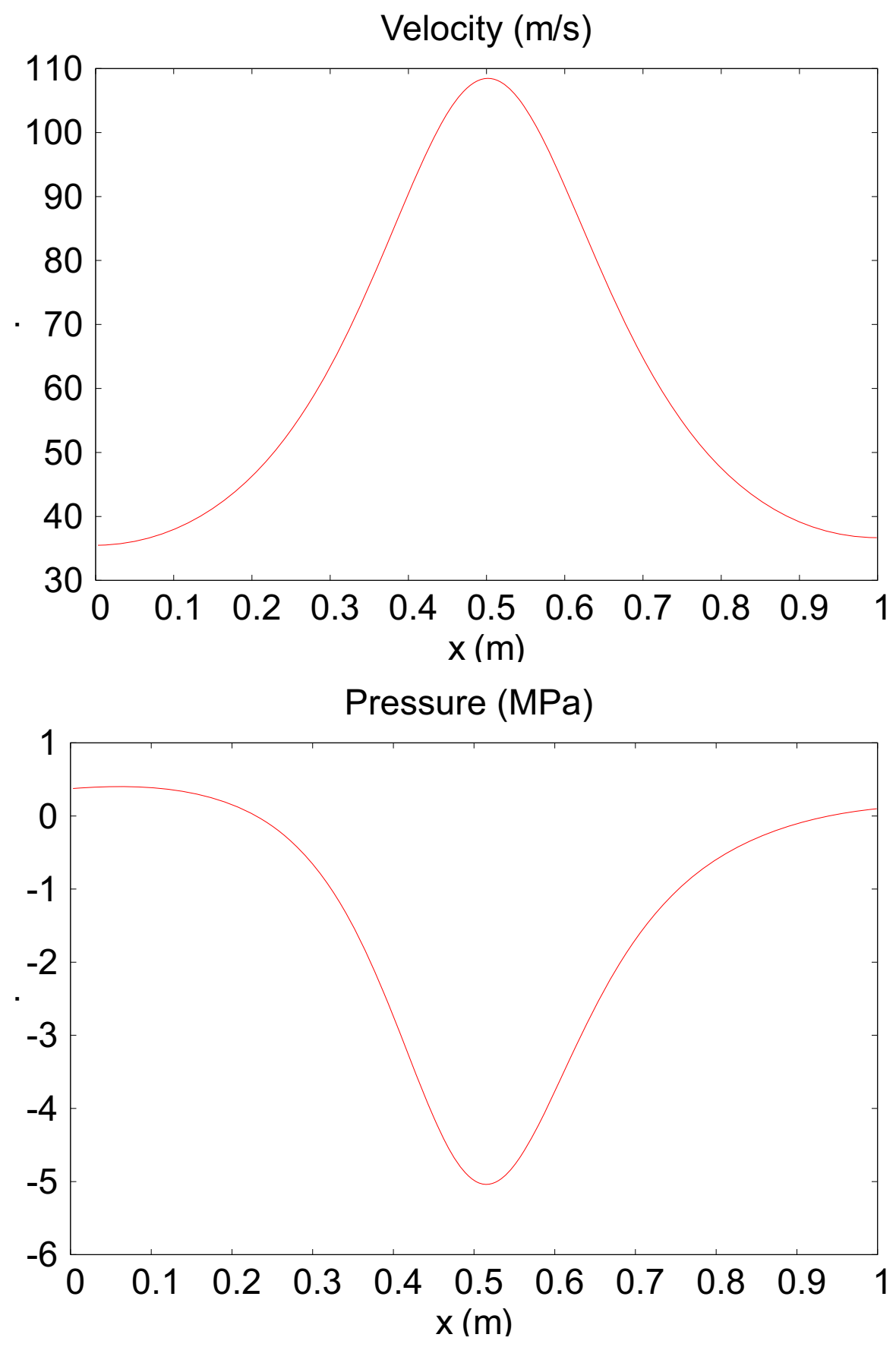


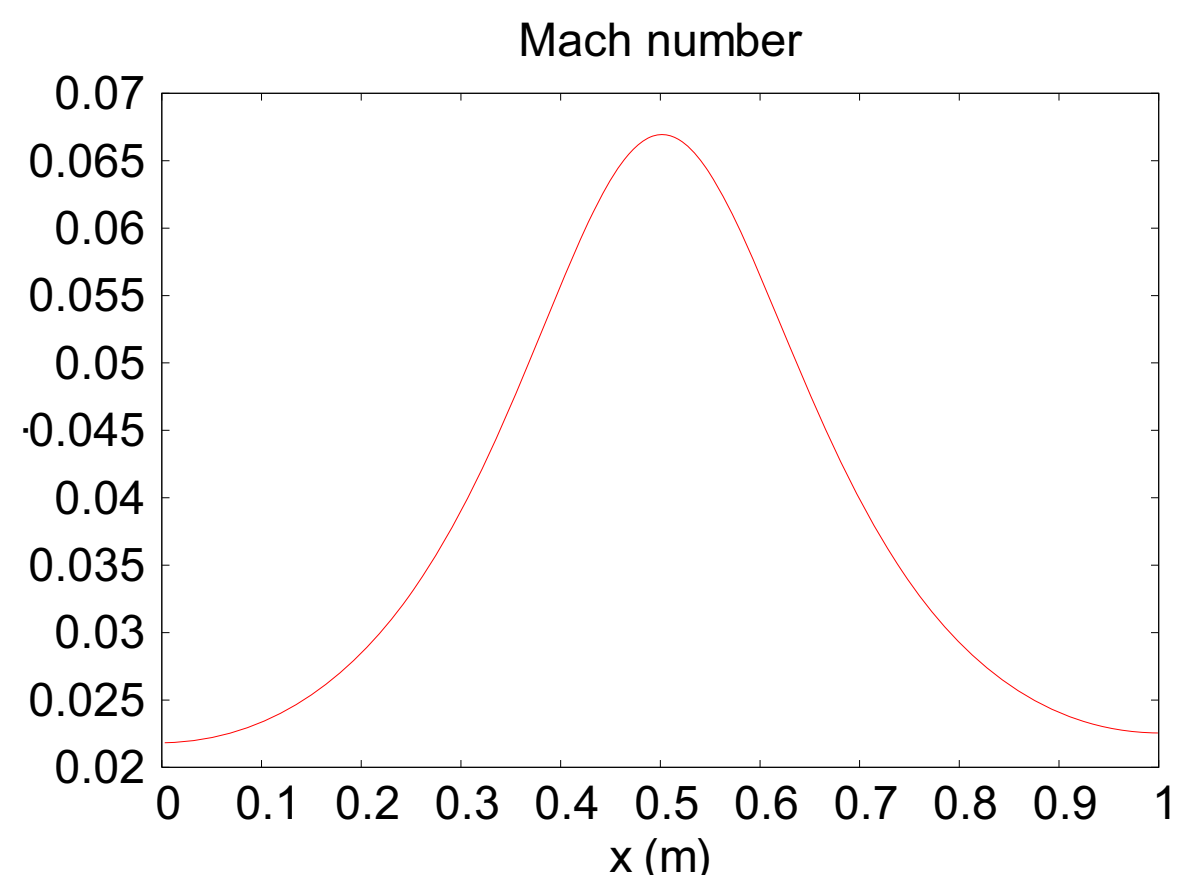

GAS PHASE VARIABLES

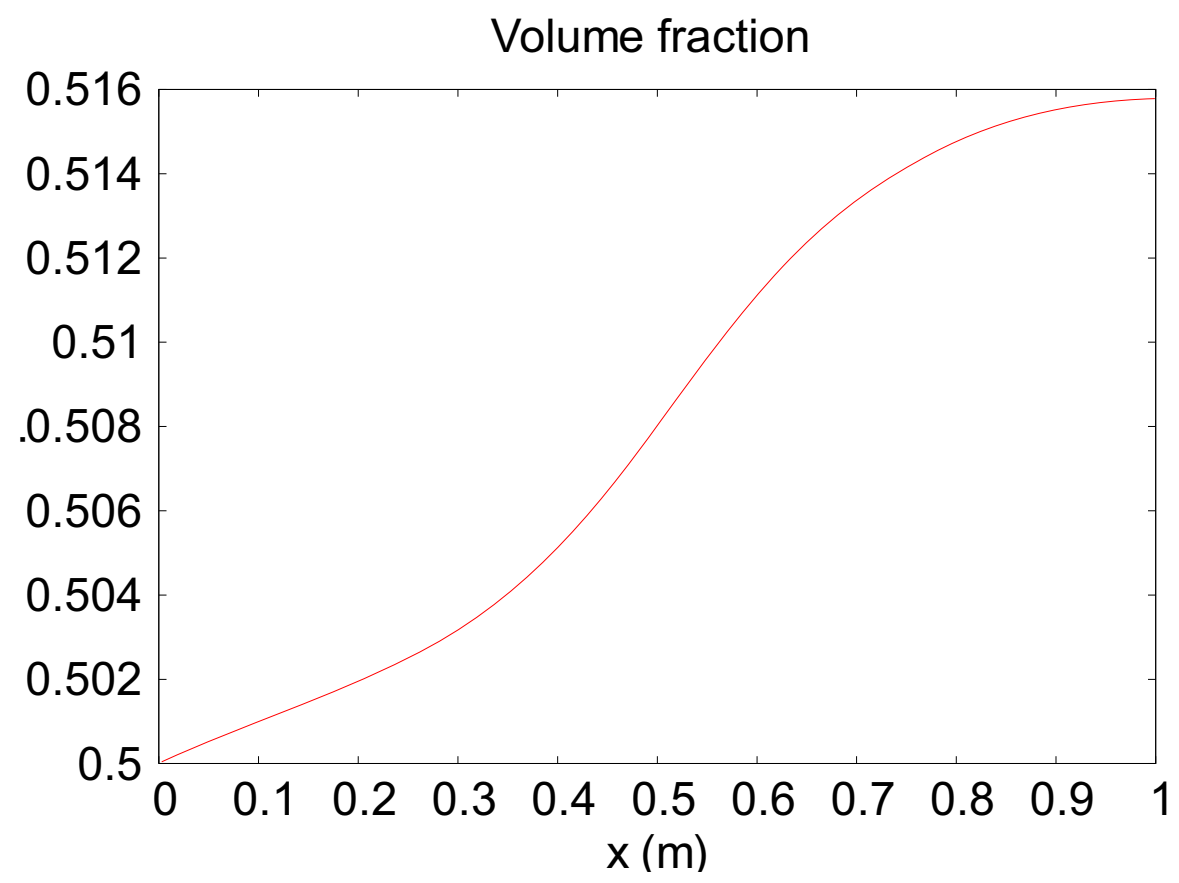



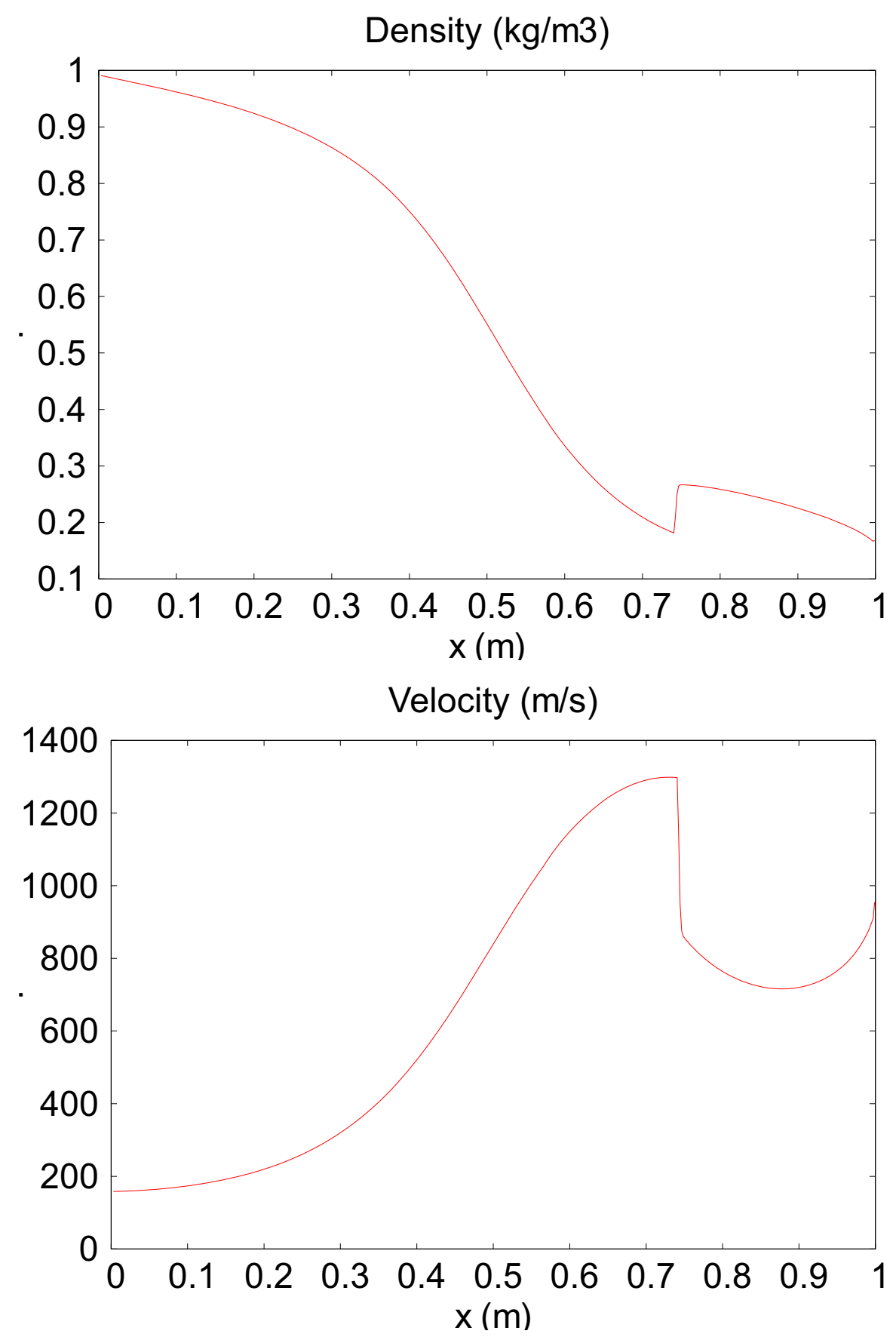

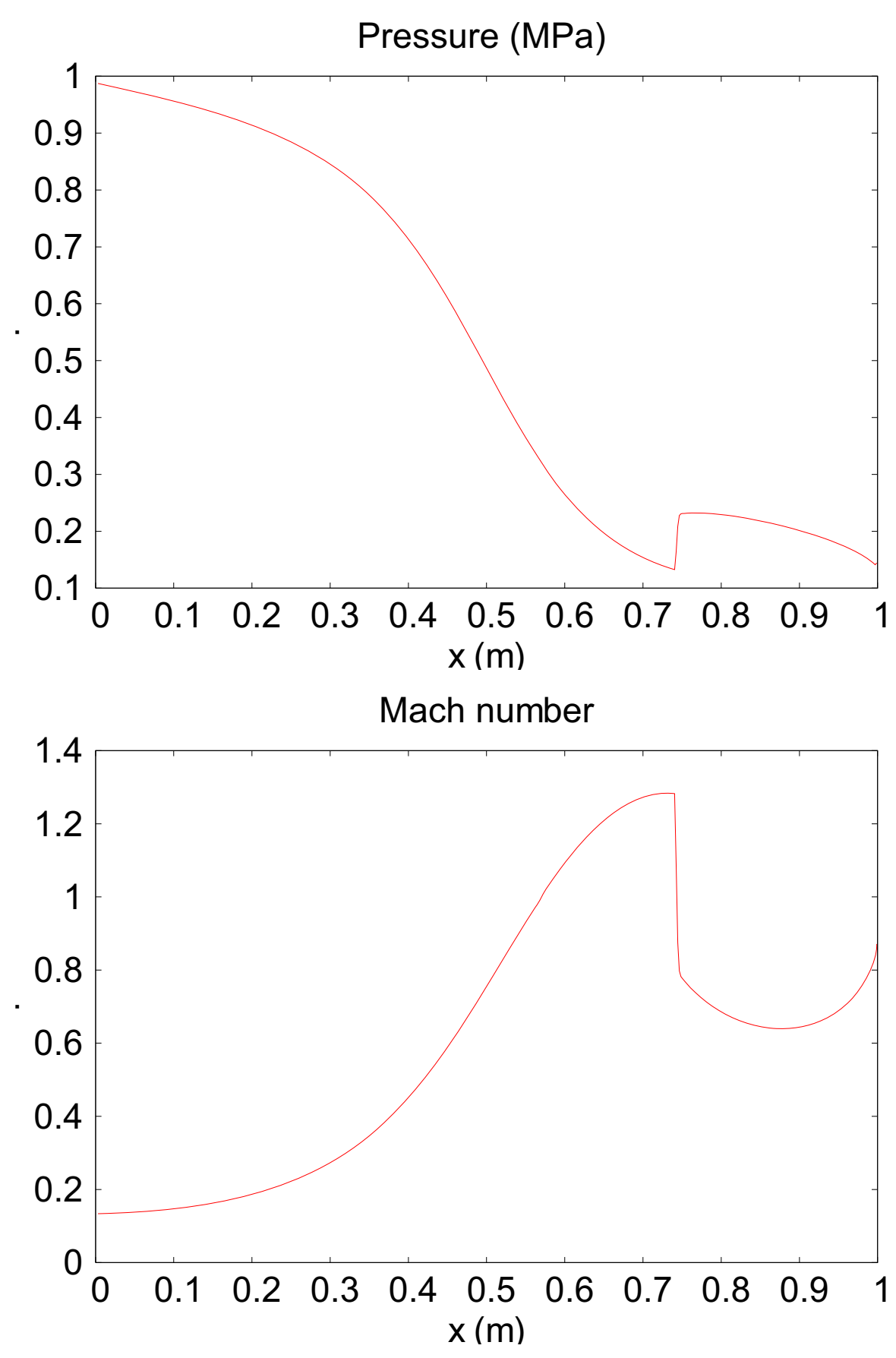
TWO "PHASE WITH RELAXATION

(BOTH PRESSURES AND VELOCITIES WITH SSV=100)

\section{LIQUID PHASE VARIABLES}

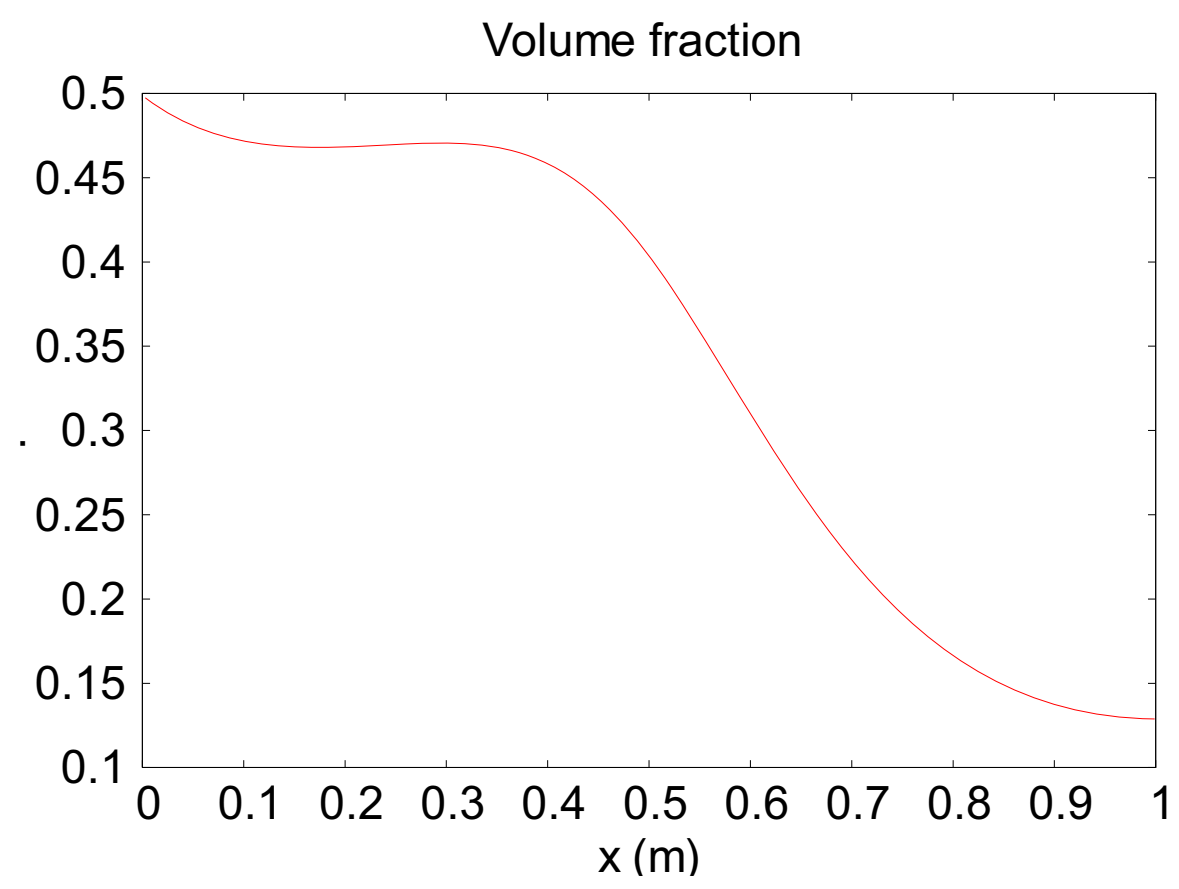

Density $(\mathrm{kg} / \mathrm{m} 3)$

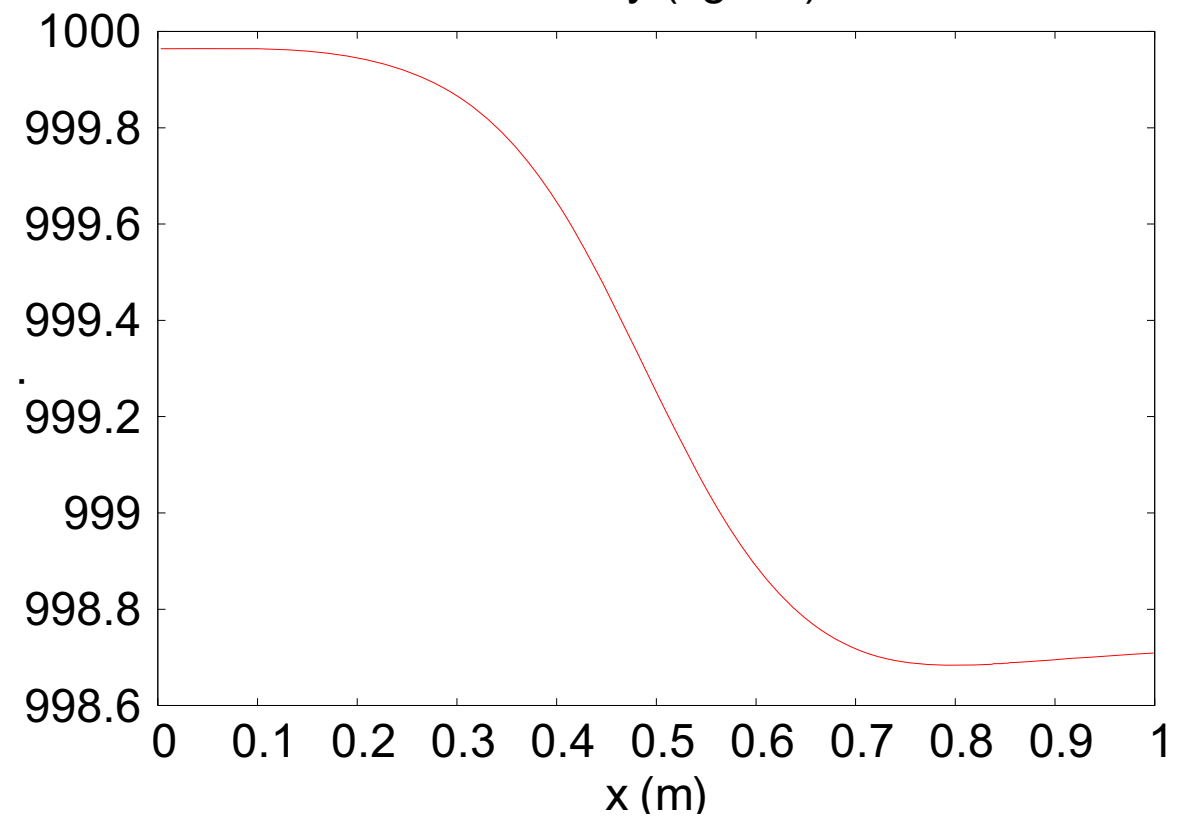



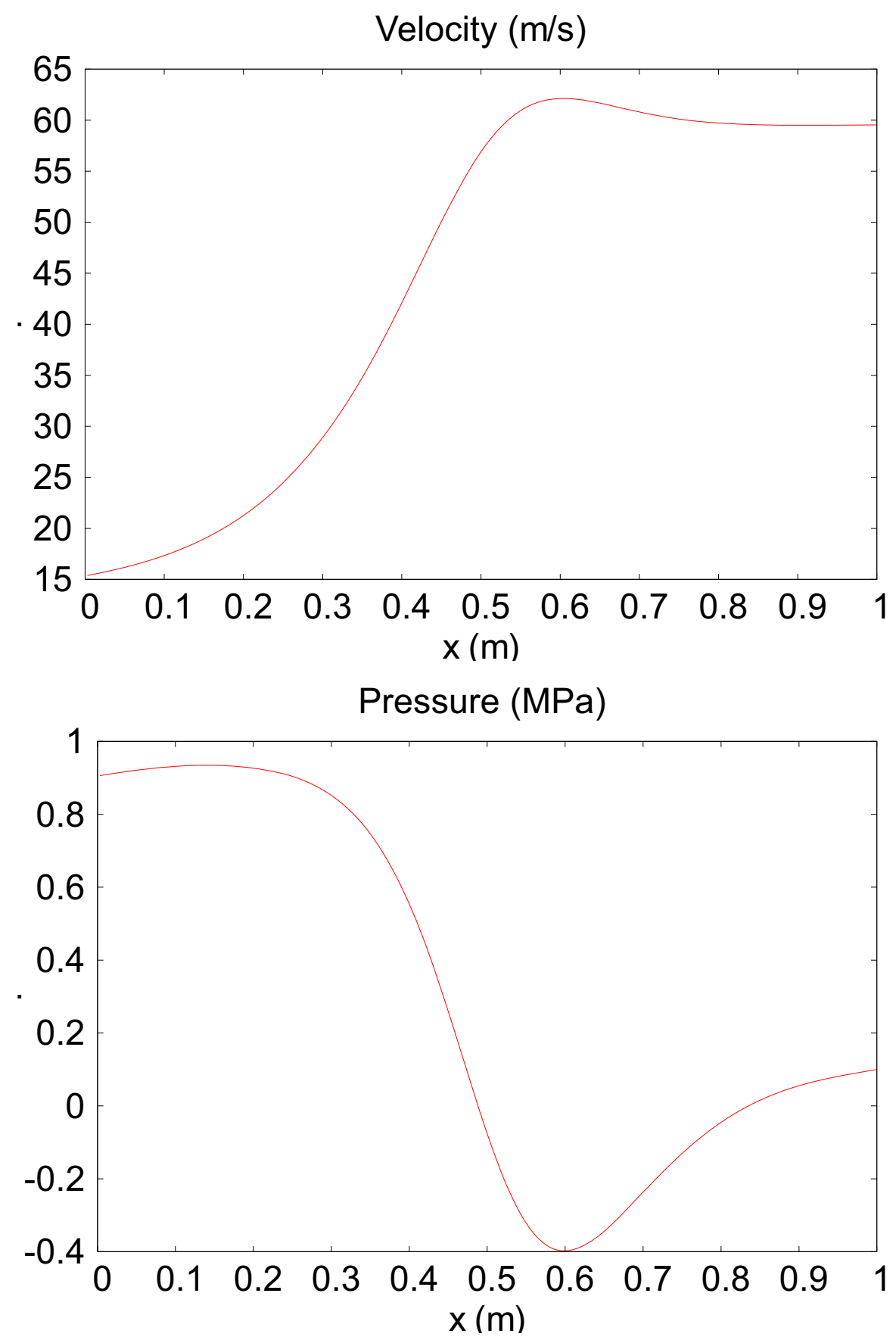


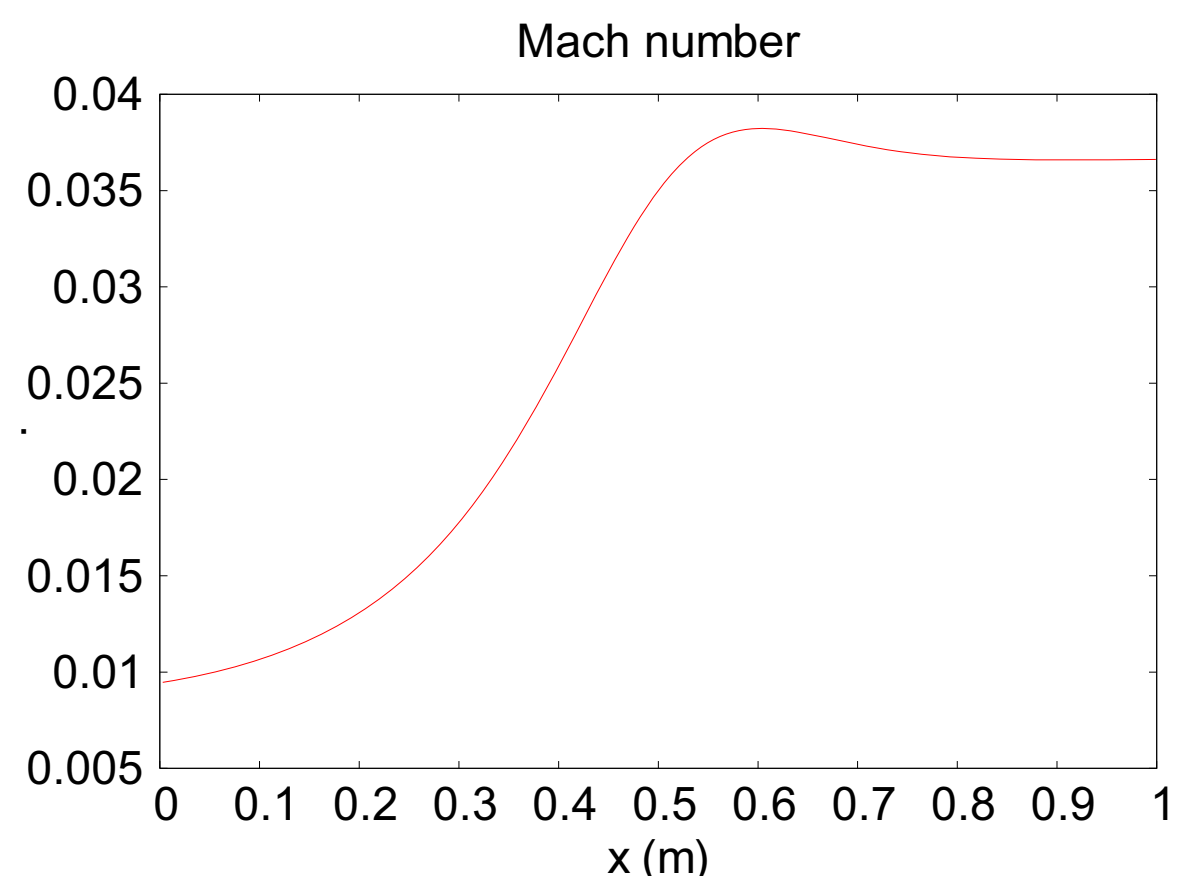

GAS PHASE VARIABLES

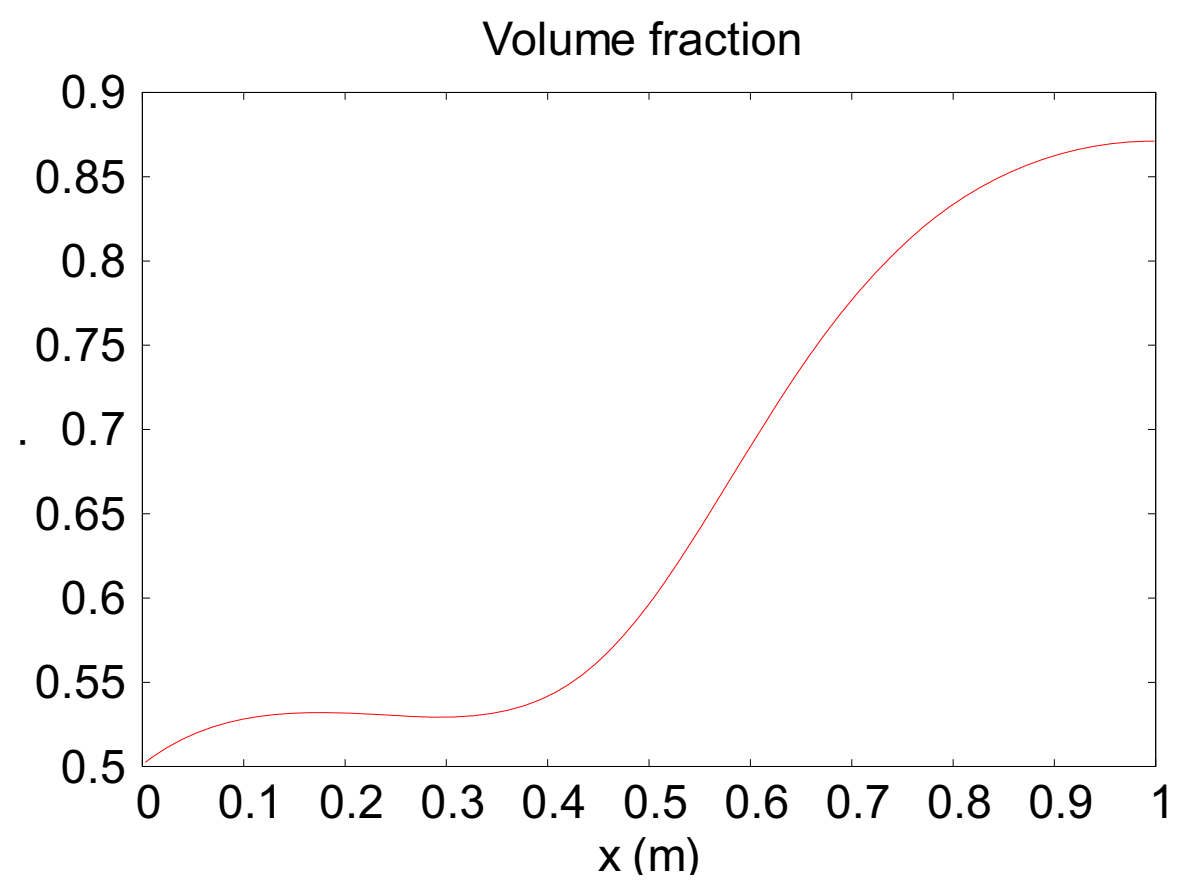



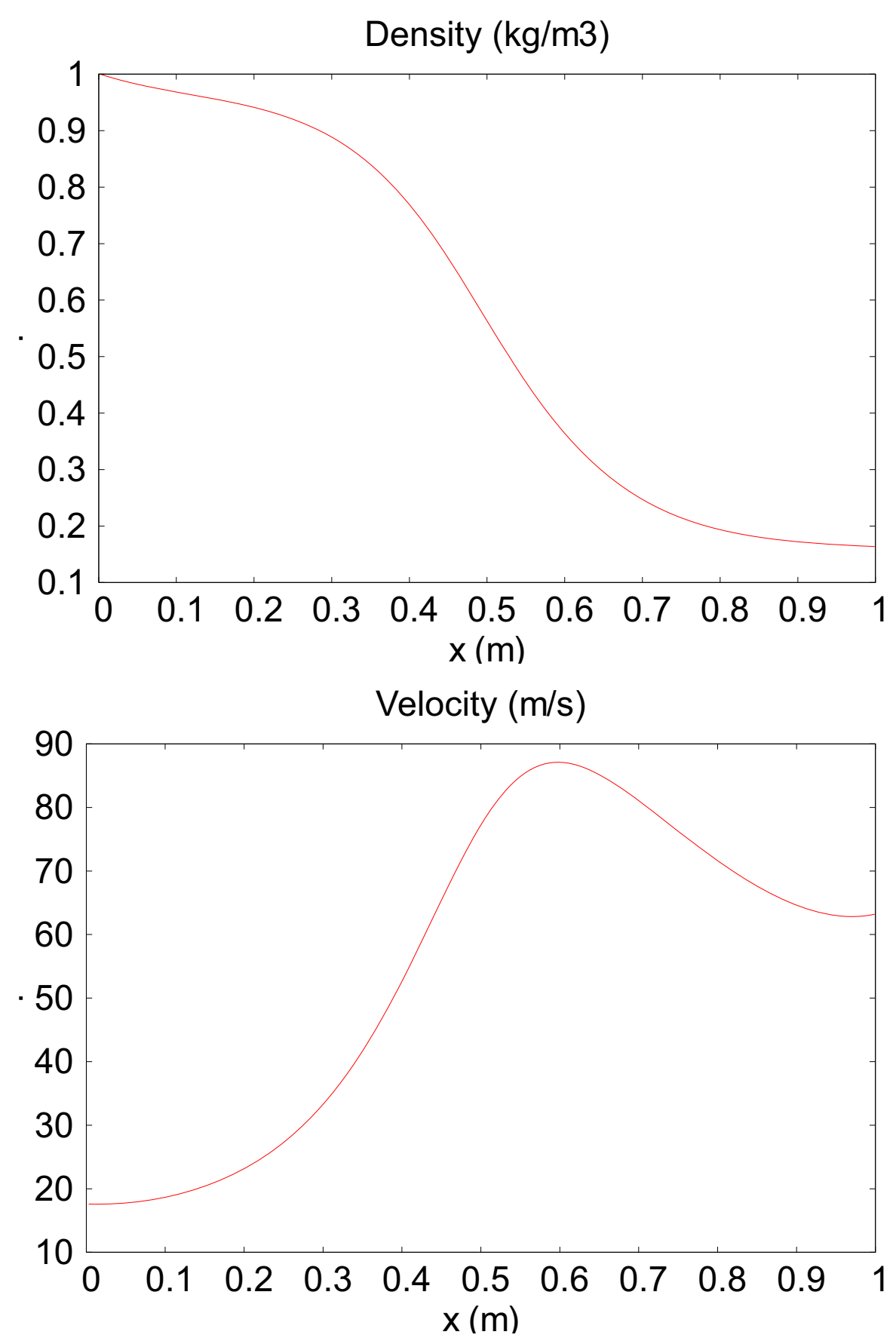

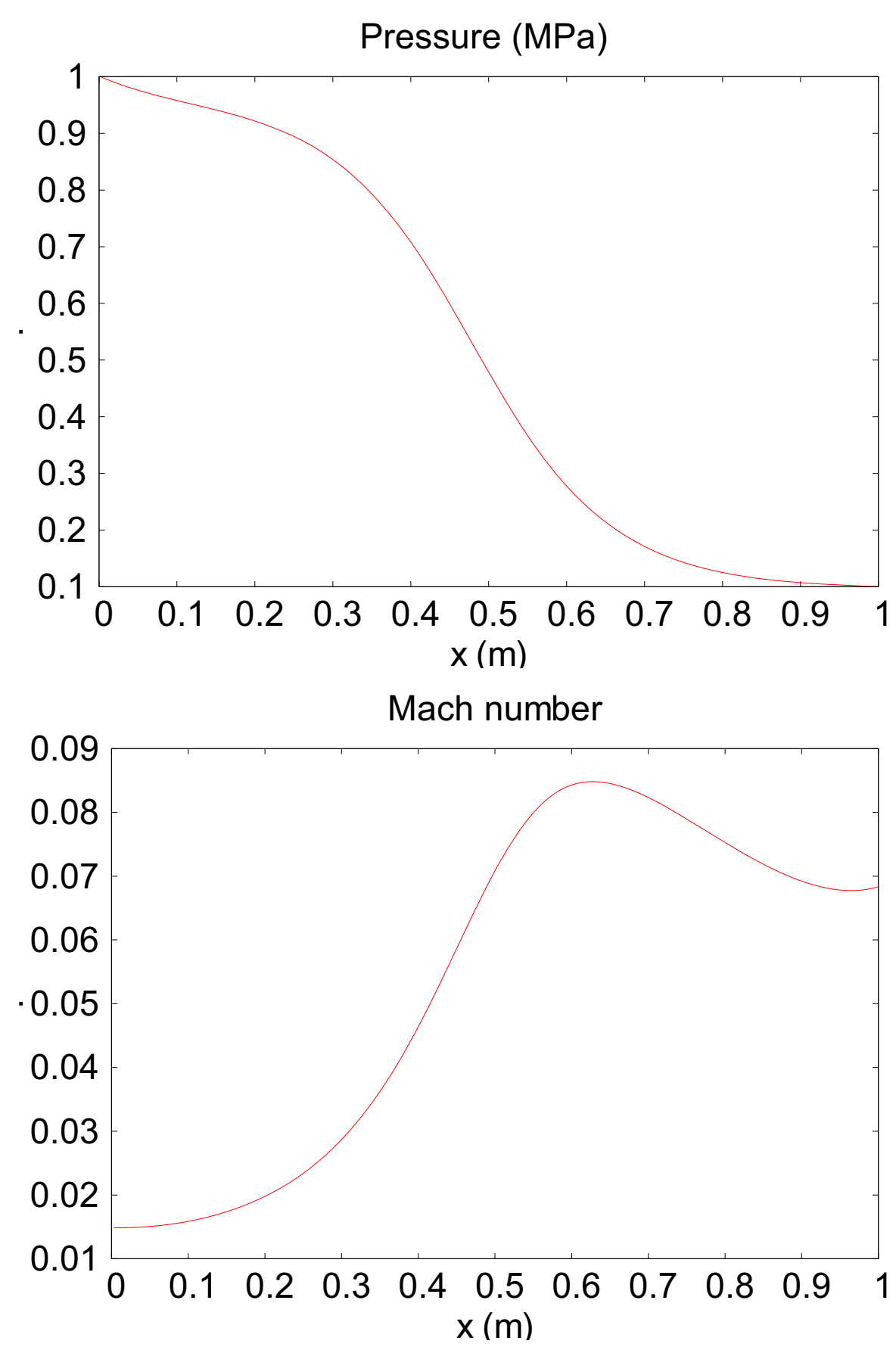


\section{HEAT AND MASS TRANSERS (SIMPLIFIED VERSION)}

heatex $=1 . \mathrm{d} 6$

lvap $=2 . \mathrm{d} 6$

qdot $=$ heatex $*(\mathrm{t} 2-\mathrm{t} 1)$

mdot $=$ qdot/lvap

c

$$
\begin{aligned}
& \text { pinter }=(\mathrm{zk}(1) * \mathrm{pk}(2)+\mathrm{zk}(2) * \mathrm{pk}(1)) /(\mathrm{zk}(1)+\mathrm{zk}(2)) \\
& \text { vinter }=(\mathrm{zk}(1) * \mathrm{uk}(1)+\mathrm{zk}(2) * \mathrm{uk}(2)) /(\mathrm{zk}(1)+\mathrm{zk}(2))
\end{aligned}
$$

c

source $(1,1)=\mathrm{mu}^{*}(\mathrm{pk}(1)-\mathrm{pk}(2))-\mathrm{mdot} / \mathrm{rhoi}$

source $(1,2)=-\operatorname{mdot}$

source $(1,3)=\operatorname{lambda} *(\mathrm{uk}(2)-\mathrm{uk}(1))-\mathrm{mdot}^{*}$ ui

source $(1,4)=-$ pinter* $\operatorname{source}(1,1)$

$\mathrm{S}$

+ vinter*lambda*(uk(2)-uk(1))

-mdot*ei+qdot

source $(2,1)=$-source $(1,1)$

source $(2,2)=$-source $(1,2)$

source $(2,3)=$-source $(1,3)$

source $(2,4)=$ pinter* $\operatorname{source}(1,1)$

S

-vinter*lambda*(uk(2)-uk(1))

$\mathrm{S}$

+ mdot*(ei-lvap) 


\section{LIQUID PHASE VARIABLES}
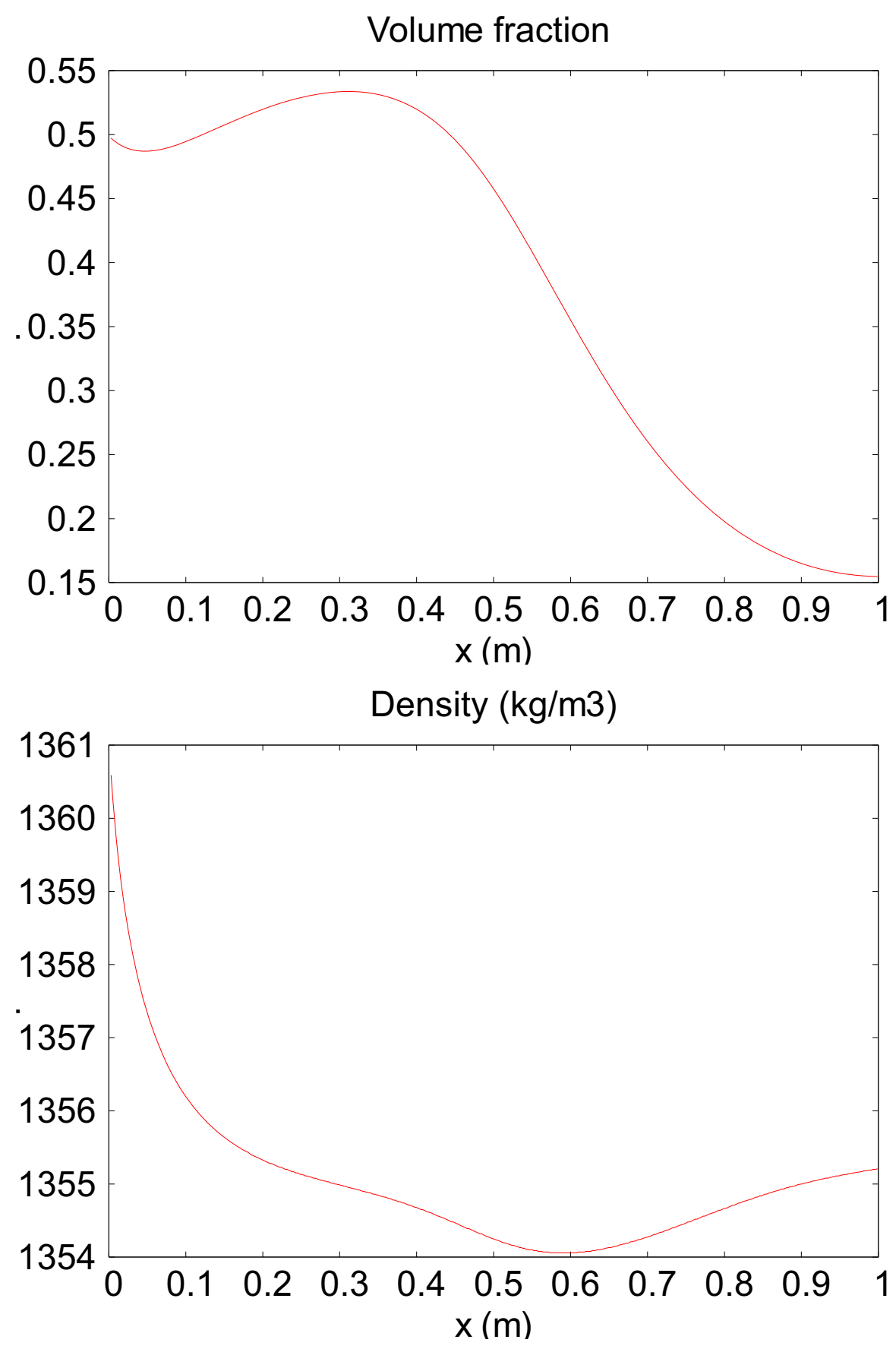

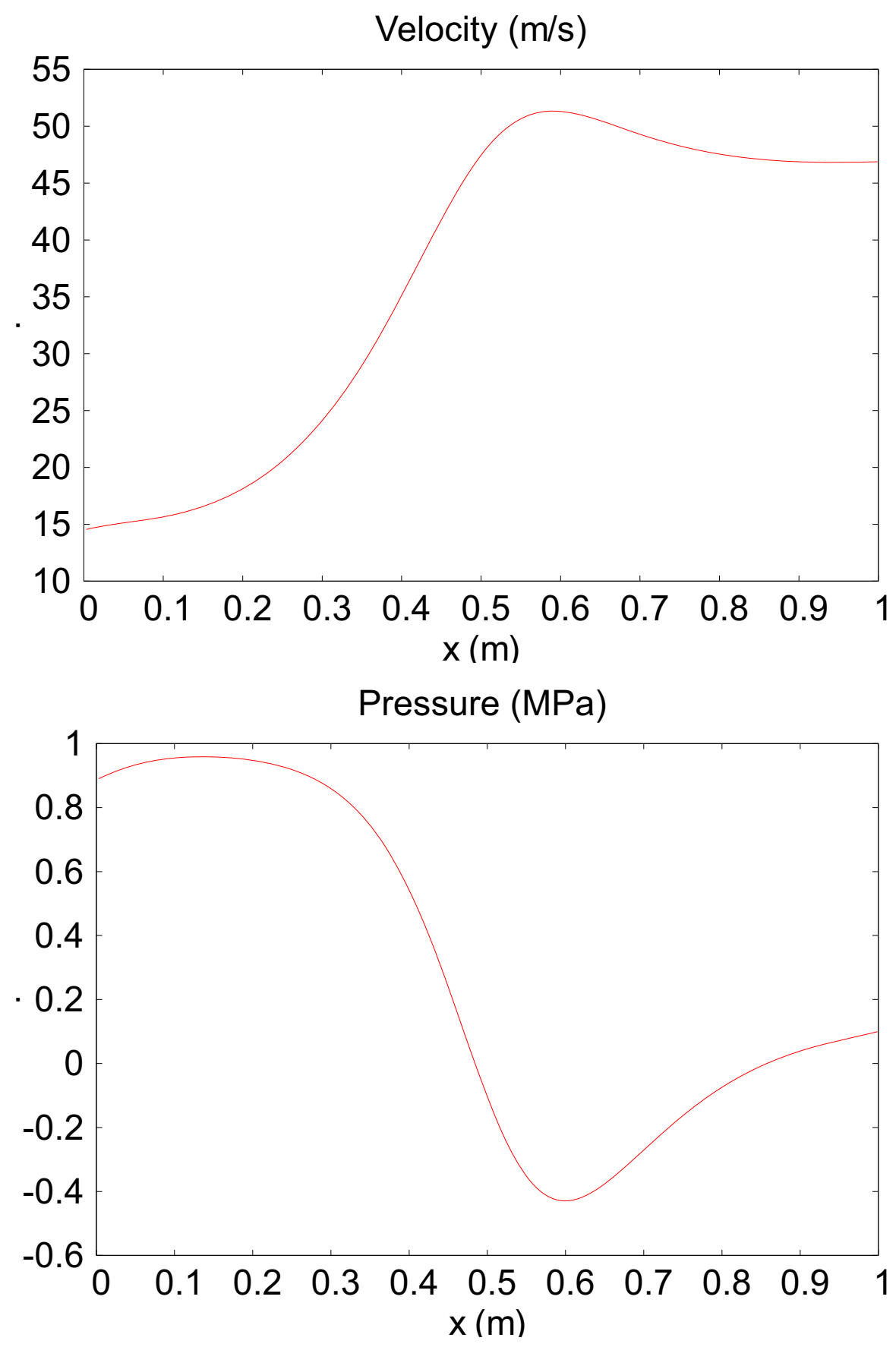


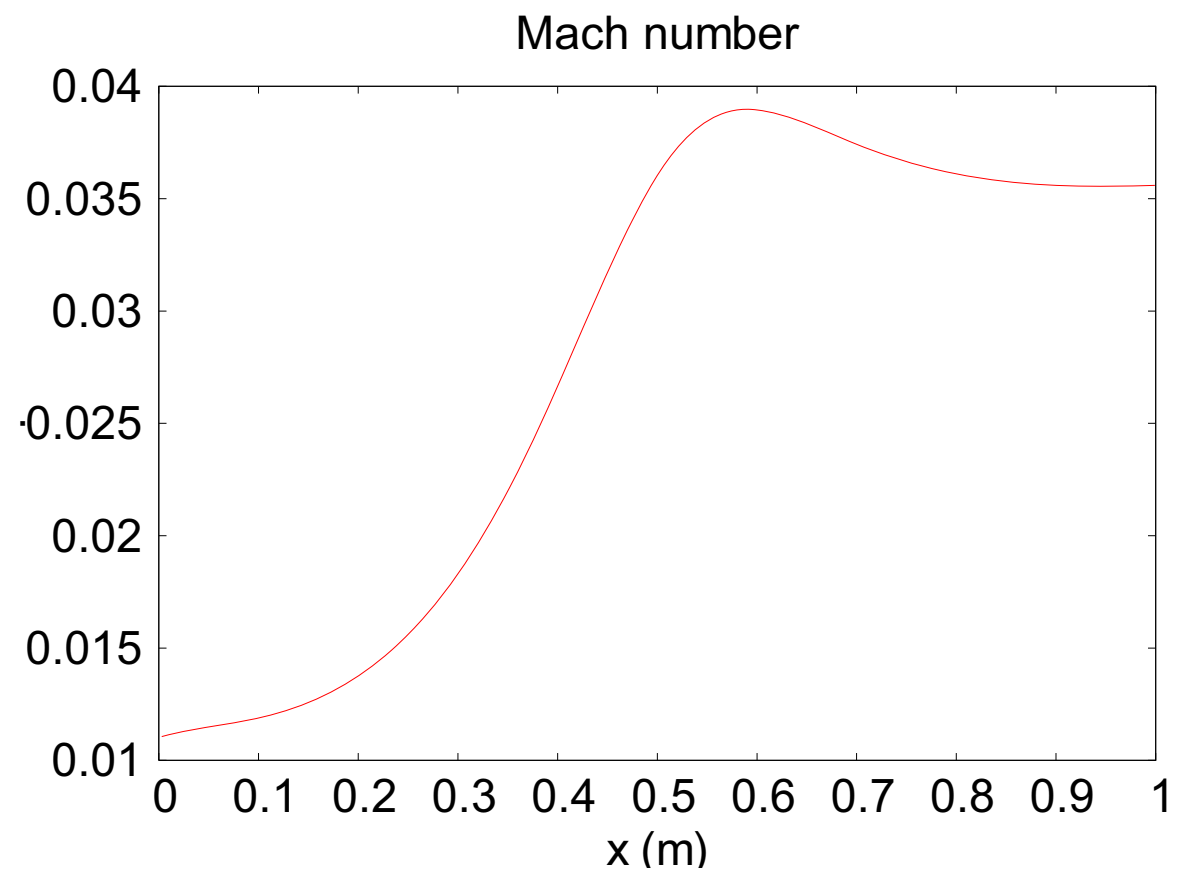

Temperature (K)

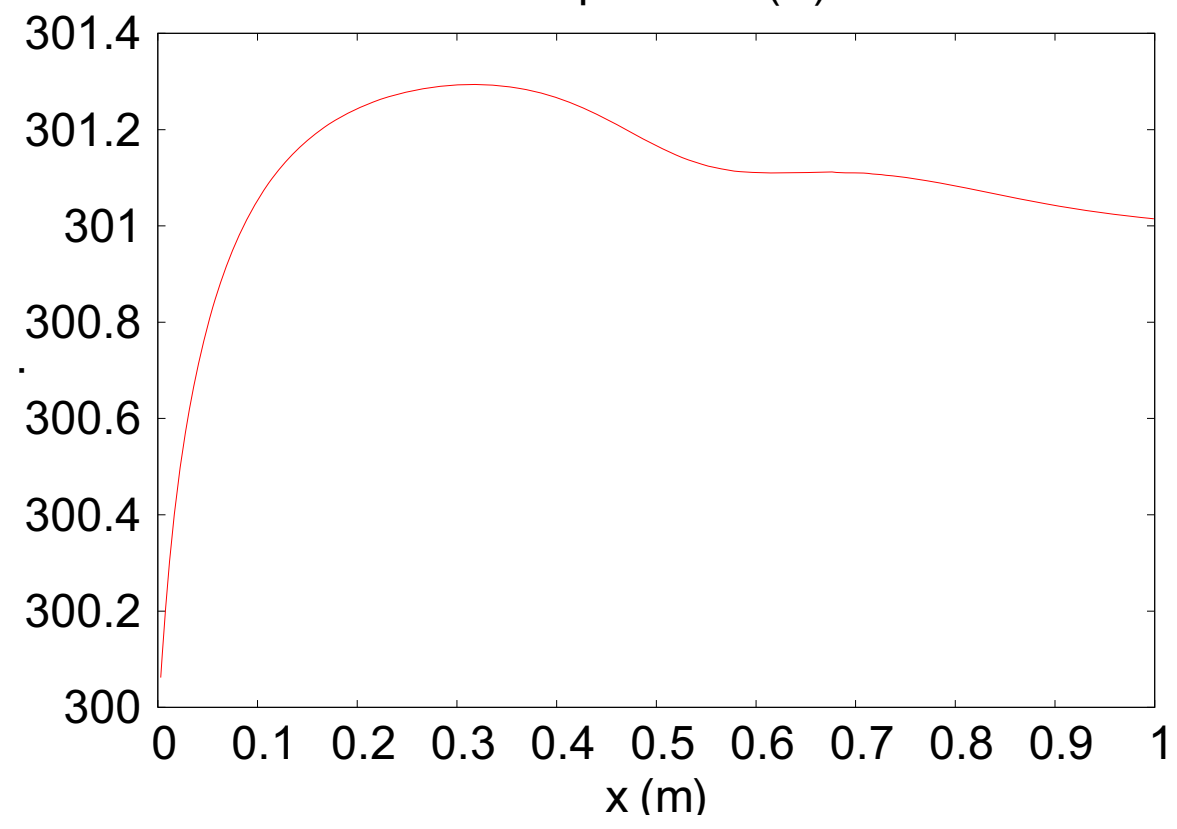




\section{GAS PHASE VARIABLES}
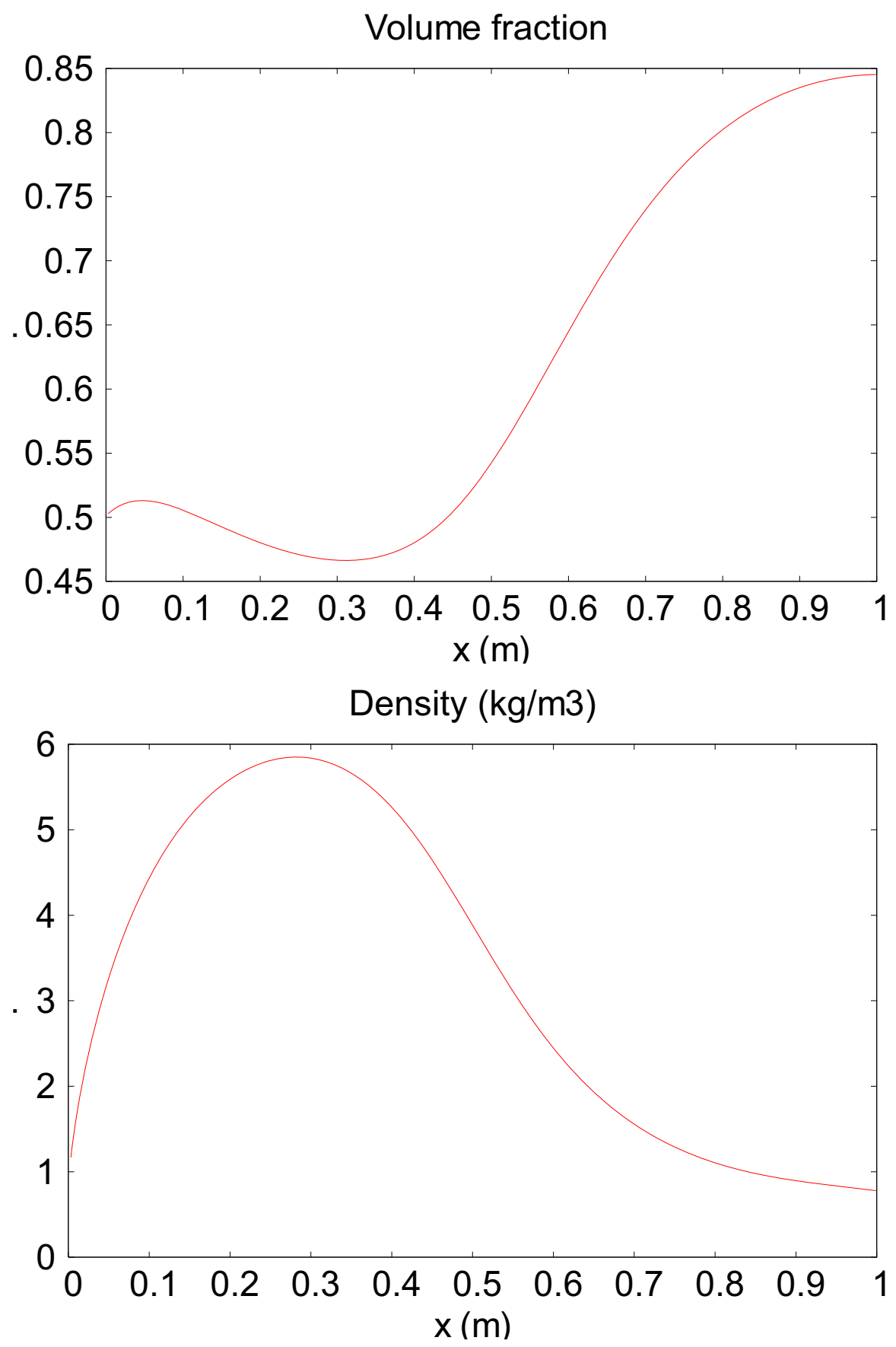

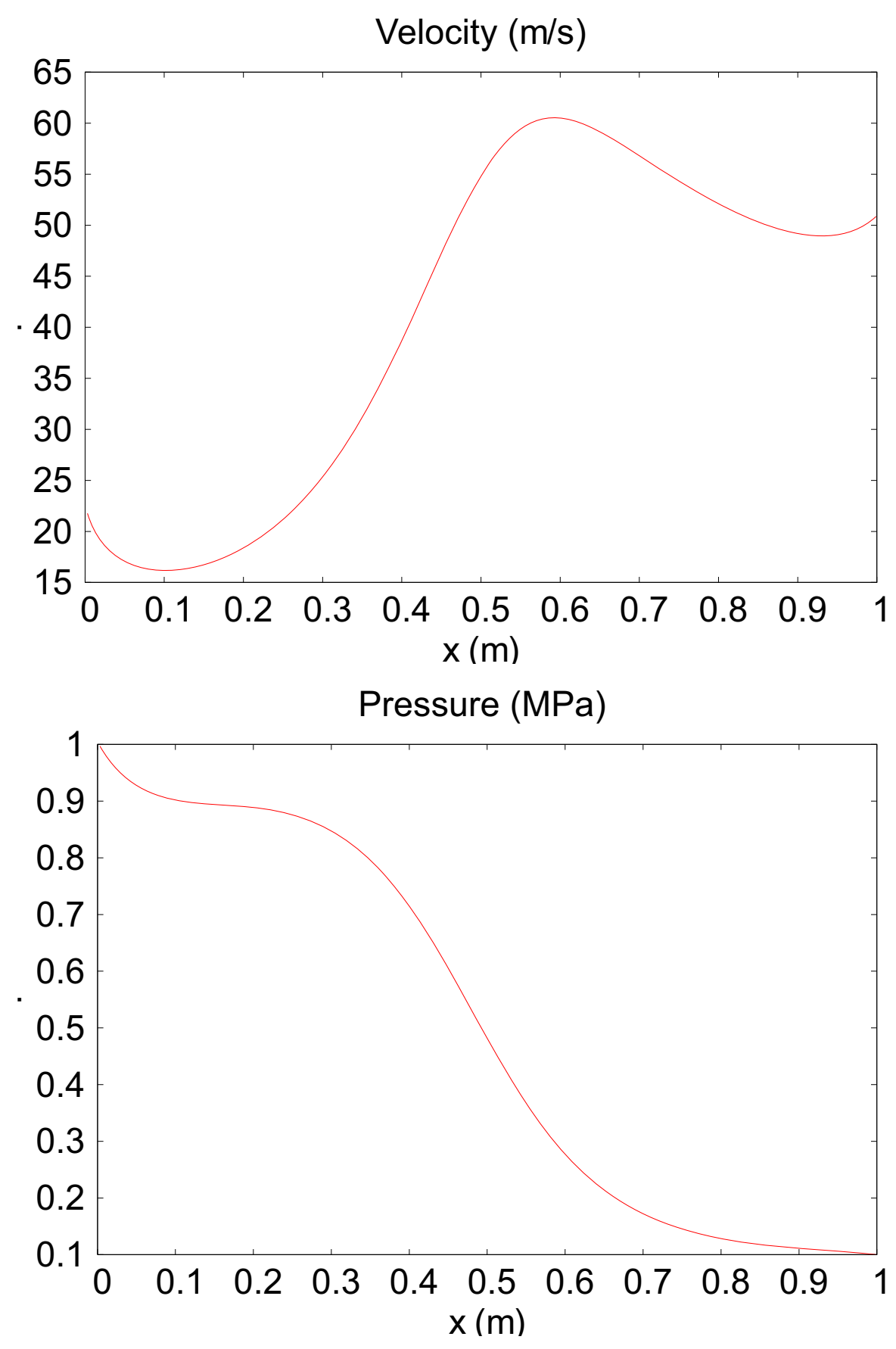

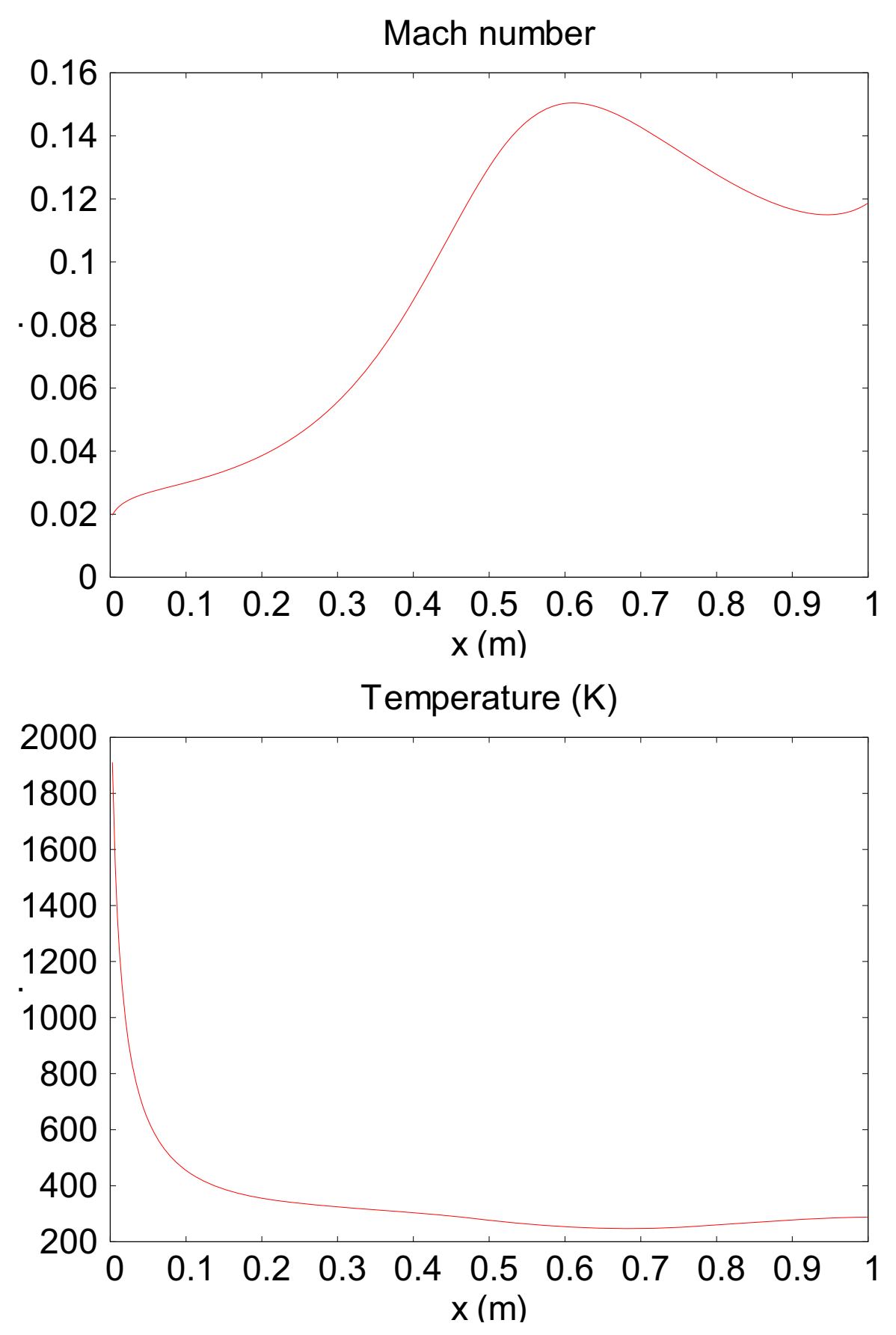

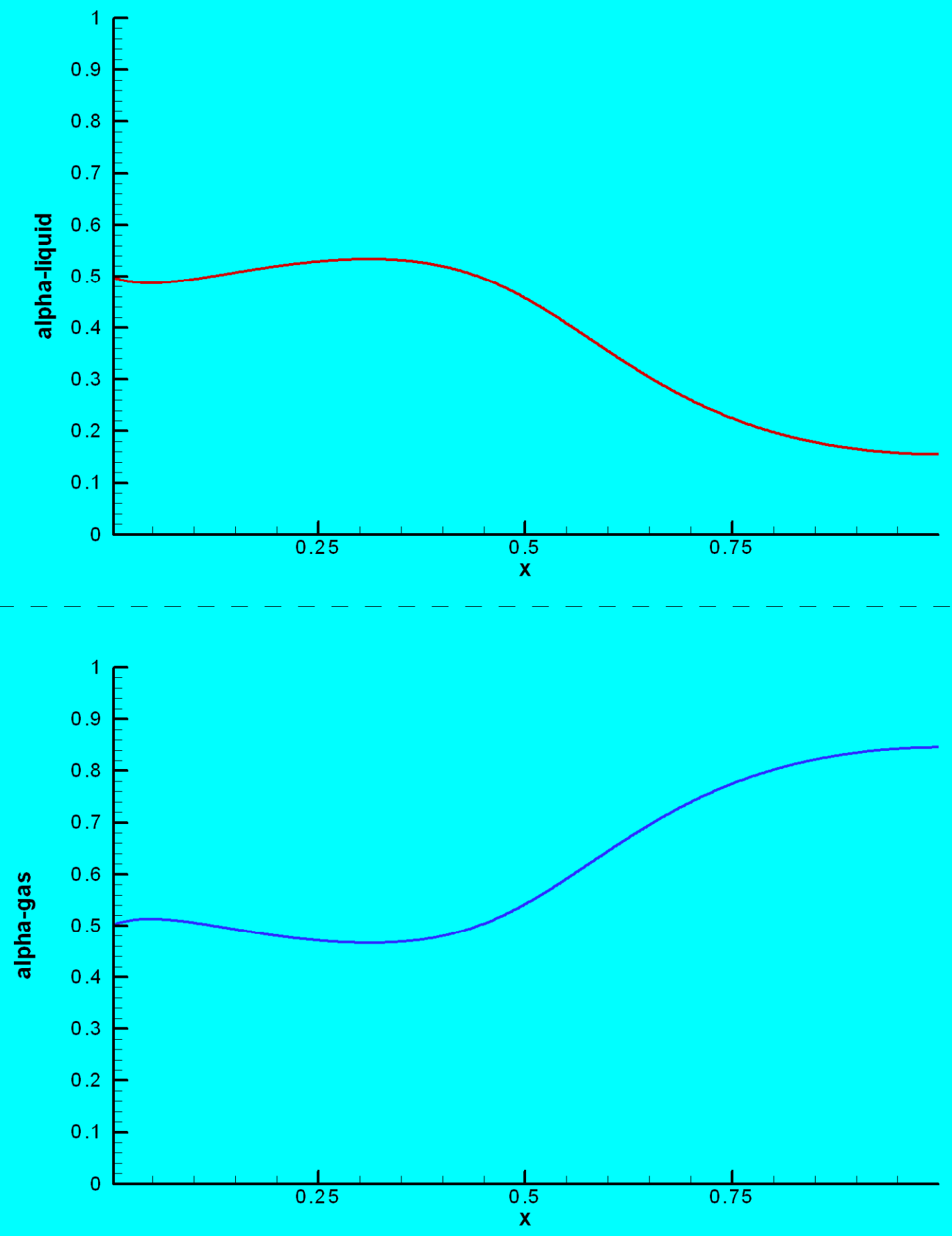

Steady State Volume Fractions. SSV=100, Simplified Heat and Mass Transfer. 


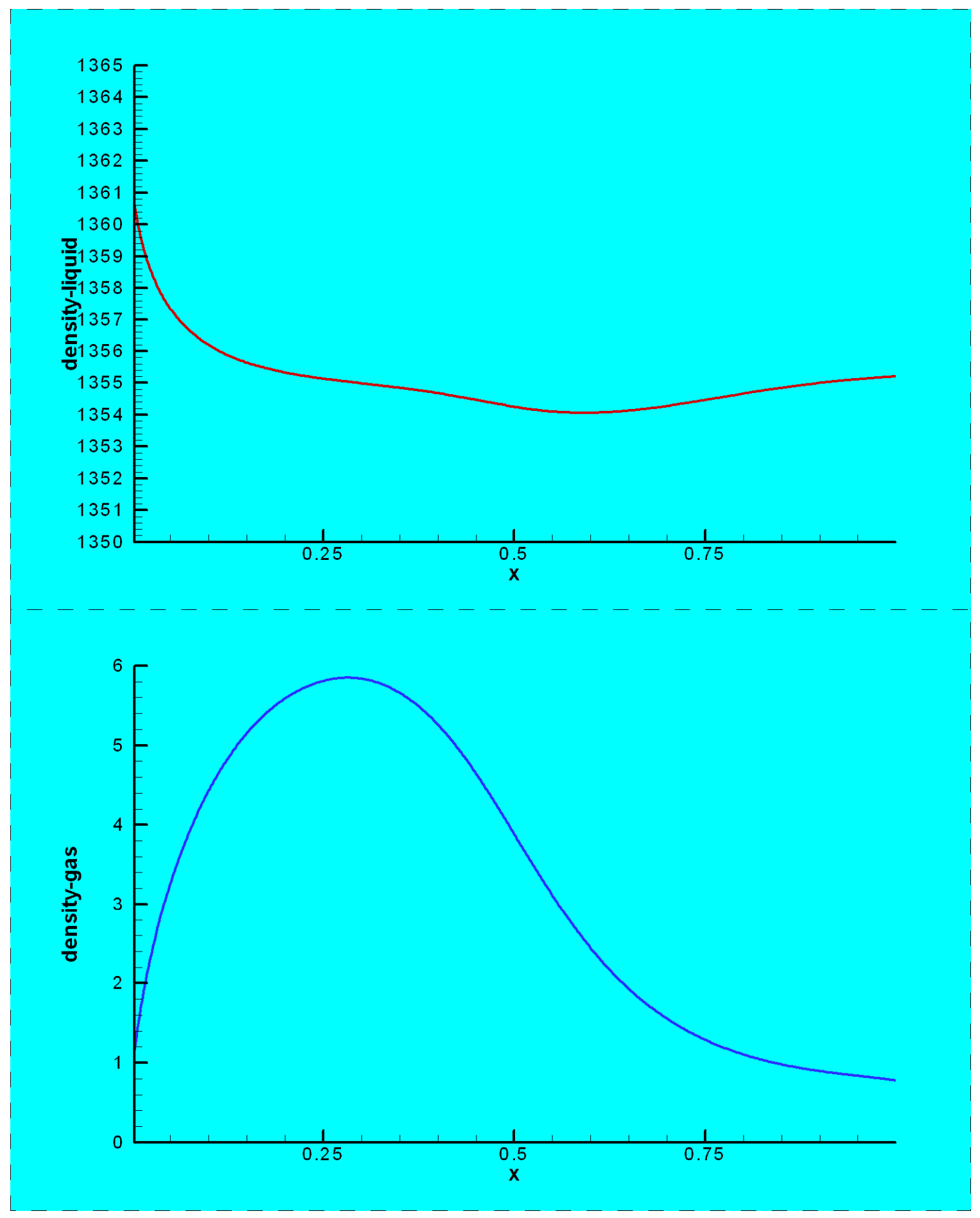

Steady State Mass Densities. SSV=100, Simplified Heat and Mass Transfer. 


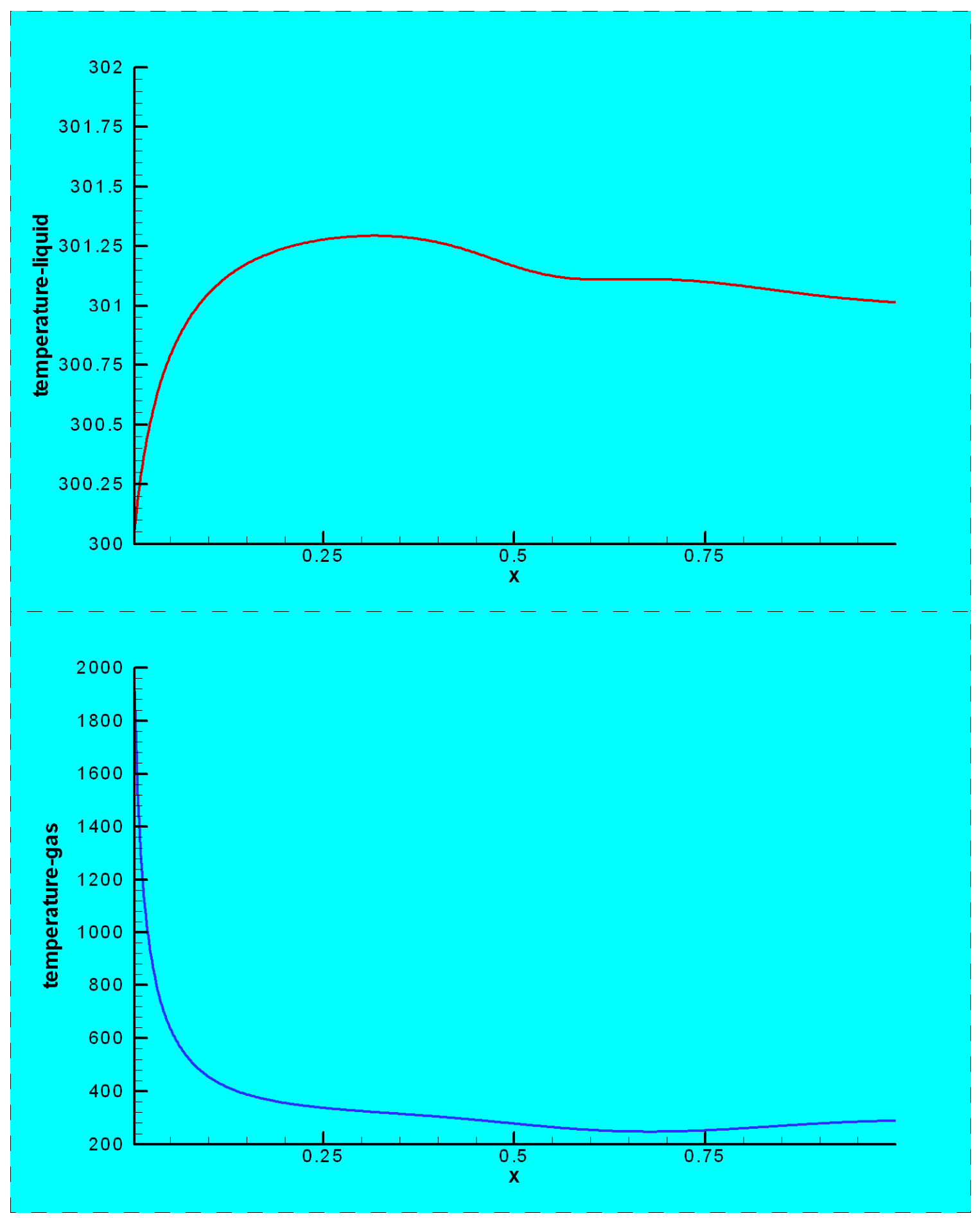

Steady State Temperatures. SSV=100, Simplified Heat and Mass Transfer. 


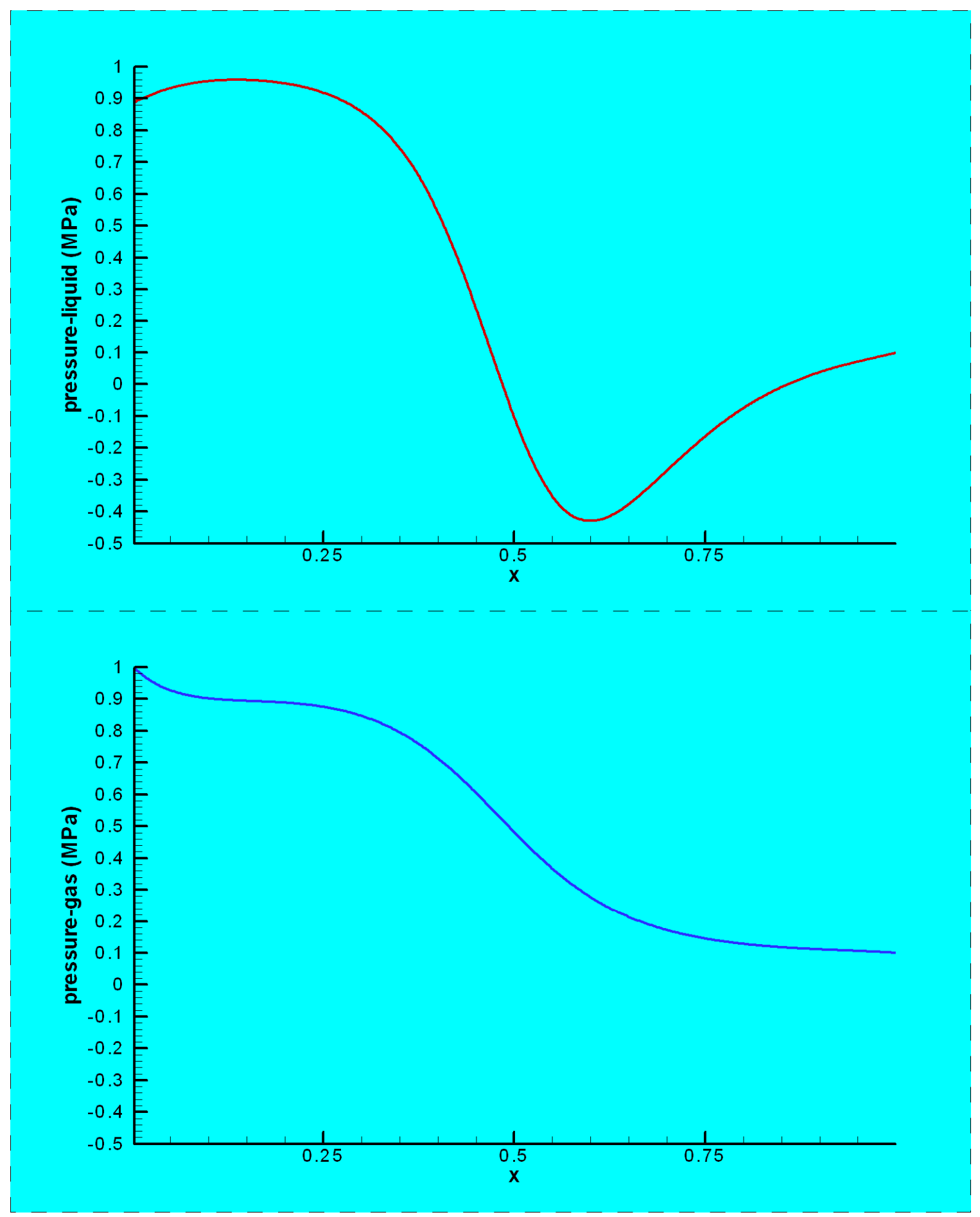

Steady State Pressures. SSV=100, Simplified Heat and Mass Transfer. 


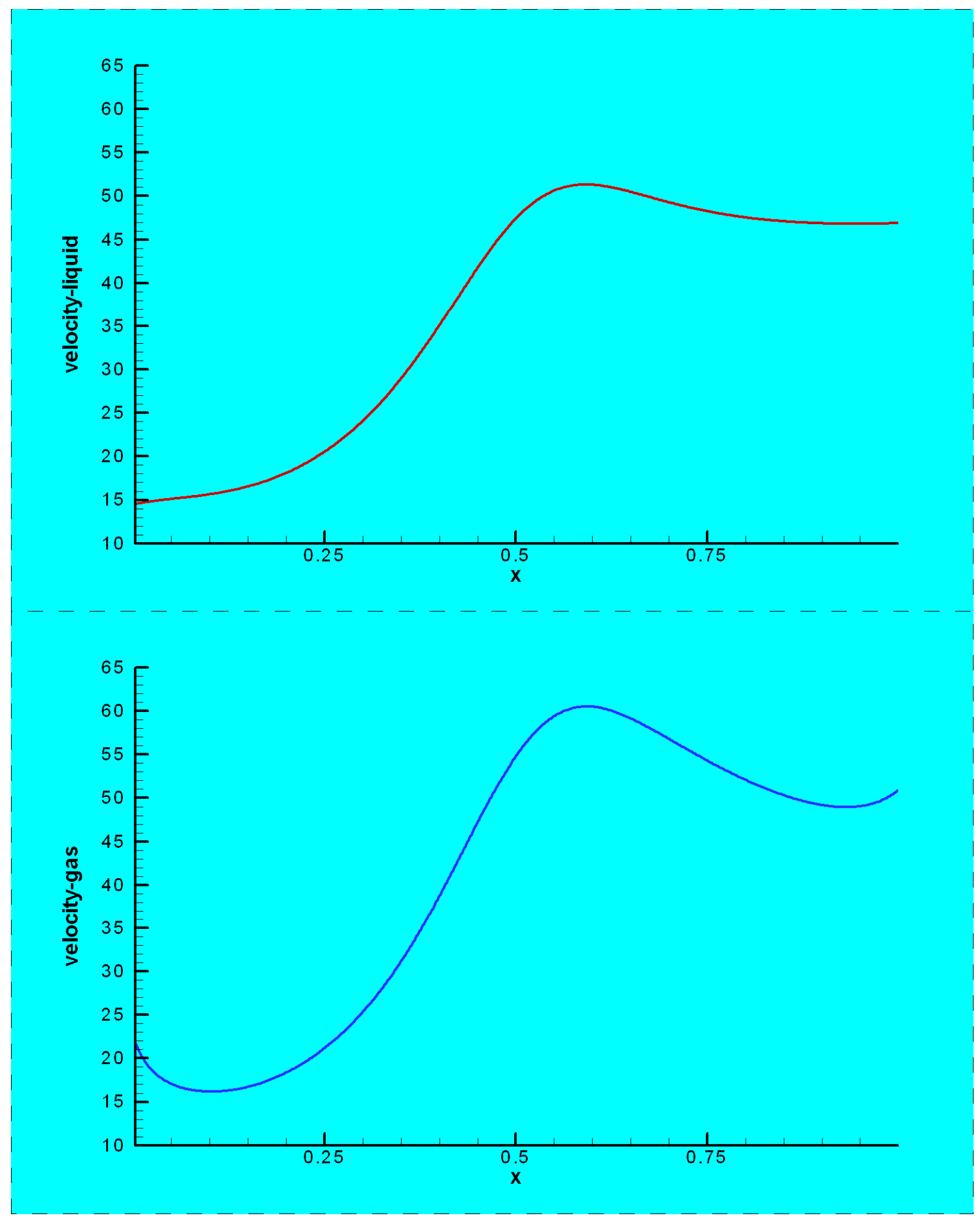

Steady State Velocities. SSV=100, Simplified Heat and Mass Transfer. 


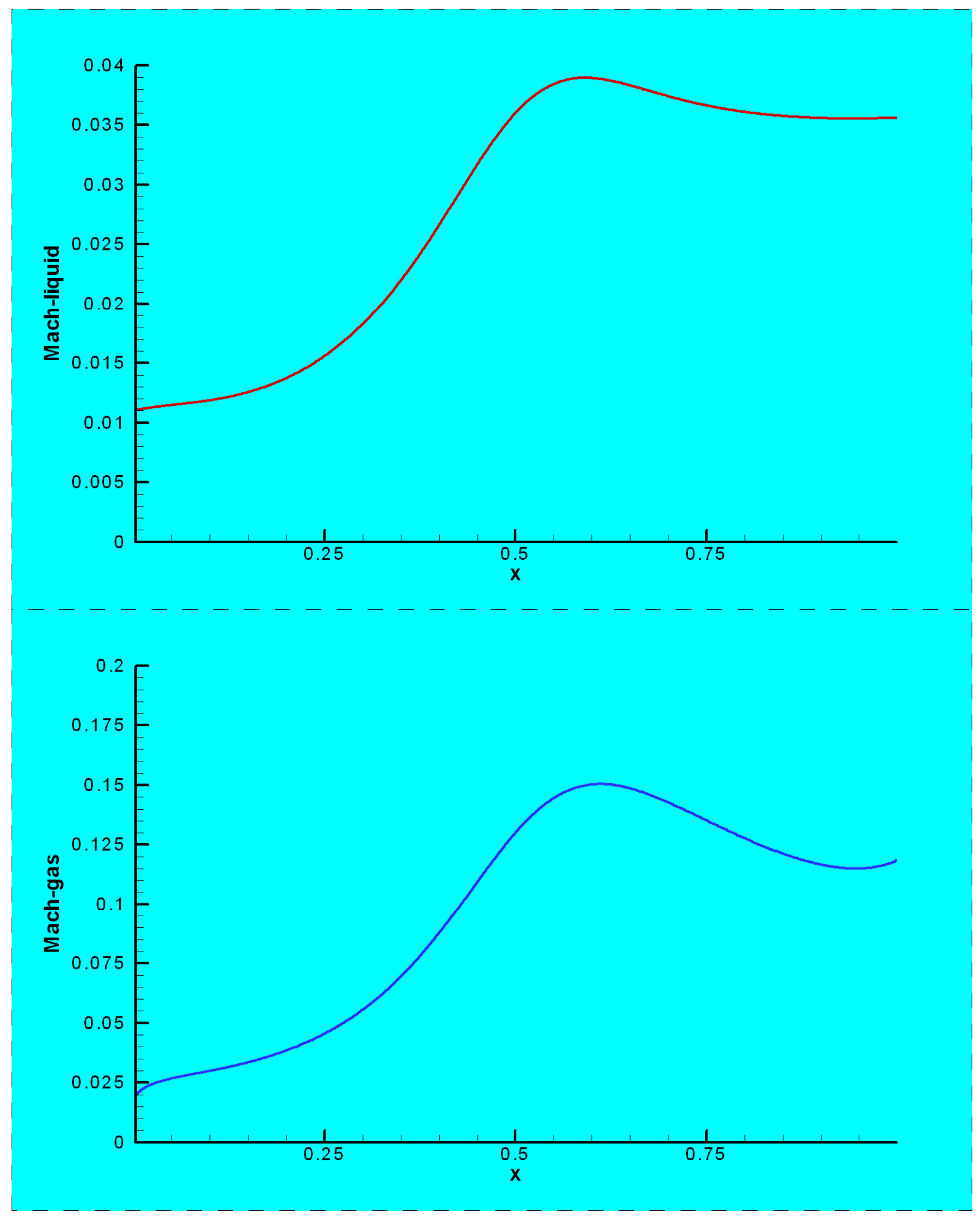

Steady State Mach Numbers. SSV=100, Simplified Heat and Mass Transfer. 


\section{Chapter 7 \\ Modeling Phase Transitions in Non-Equilibrium Multiphase Compressible Flow Models}




\section{Introduction}

This work deals with mass transfer modelling in multiphase compressible flows models $[1,2,3,13$, 16, 18]. A particular attention is given to evaporation processes appearing in boiling flows or cavitating systems. Boiling or cavitating flows appear in many industrial applications such as nuclear reactors, injection devices or hypervelocity projectiles in liquids. The relevant models able to treat these phenomena are multiphase compressible models where phases or fluids are in mechanical and/or thermodynamic disequilibria in accordance with the flow conditions. It means that each pure phase has its own set of equations (balance of mass, momentum and energy) and obeys its own equation of state. Additional equations (linked to the advection of each phase volume fraction) as well as relaxation and mass transfer terms are present and represent physical interactions between phases. The multiphase compressible flow models mentionned above express in generic form (in 1-space dimension) for phase $k$ :

$$
\left\{\begin{array}{l}
\frac{\partial \alpha_{k}}{\partial t}+u_{I} \frac{\partial \alpha_{k}}{\partial x}= \pm \mu \Delta P \pm \frac{\dot{m}}{\tilde{\rho}_{I}} \\
\frac{\partial(\alpha \rho)_{k}}{\partial t}+\frac{\partial(\alpha \rho u)_{k}}{\partial x}= \pm \dot{m} \\
\frac{\partial(\alpha \rho u)_{k}}{\partial t}+\frac{\partial\left(\alpha\left(\rho u^{2}+P\right)\right)_{k}}{\partial x}=P_{I} \frac{\partial \alpha_{k}}{\partial x} \pm \lambda \Delta u \pm \dot{m} \tilde{u}_{I} \\
\frac{\partial(\alpha \rho E)_{k}}{\partial t}+\frac{\partial(\alpha(\rho E+P) u)_{k}}{\partial x}=(P u)_{I} \frac{\partial \alpha_{k}}{\partial x} \pm \lambda \overline{u_{I}} \Delta u \mp \mu \overline{P_{I}} \Delta P \pm \dot{m} \tilde{H}_{I}
\end{array}\right.
$$

where $\alpha_{k}, \rho_{k}, u_{k}, E_{k}=e_{k}+\frac{1}{2} u_{k}^{2}$ and $P_{k}$ represent the volume fraction, the density, the material velocity, the total energy and the pressure of phase $k$ respectively. The use of an arbitrary equation of state $e_{k}=e\left(P_{k}, \rho_{k}\right)\left(e_{k}\right.$ is the internal energy of phase $k$ ) for each phase allows the thermodynamic closure of the system.

As mentionned earlier additional variables are present representing interactions between phases such as the interface velocity $u_{I}$ and pressure $P_{I}$ as well as pressure and velocity relaxation coefficients $\mu$ and $\lambda$ respectively and terms associated to mass transfer process such as $\dot{m}$ (mass flow rate), $\tilde{\rho}_{I}, \tilde{u}_{I}$ and $\tilde{H}_{I}$.

One of the main difficulties in system (1) is the modelling of these interface variables, relaxation coefficients and particularly the mass transfer terms. In [2] simple expressions of these variables and coefficients are proposed but for specific phenomena (detonations) only in limit cases. Recently, [3, 17] obtained general expressions of these terms but for situations where mass transfer is absent. In [3] mass transfer terms were considered but again for detonation problems in limit cases. Most recently, [13] developped a method for evaporation fronts. But the source terms associated to mass transfer phenomena in system (1) were not considered and were replaced by the use of a reactive Riemann problem instead.

Thus the aim of this work is to build and to obtain general explicit expressions of mass transfer terms directly suitable for numerical resolution when evaporation occurs. This study is divided in two steps. First, analytical expressions of the interfacial density $\tilde{\rho}_{I}$, velocity $\tilde{u}_{I}$ and energy $\tilde{H}_{I}$ are investigated assuming that the mass flow rate $\dot{m}$ is given and known. To overcome this difficulty, the reactive Riemann problem built in [13] including subsonic evaporation fronts is considered and is linearized. The same approach, based on Riemann problems solved at each interface, was used in $[3,17]$ to obtain the interfacial 
pressure $P_{I}$ and velocity $u_{I}$ when mass transfer is absent. The linearized reactive solver is now used to obtain the mass transfer terms of system (1) according to the same method. This method necessitates extensions that are detailed in this document.

Second, the determination of the mass flow rate $\dot{m}$ is addressed by considering local physical phenomena governing the dynamics of the evaporation process at the microscopic scale. In this context diffusive effects are considered such as heat and mass diffusion.

In Section 2, the reactive Riemann Problem for the Euler equations when evaporation occurs is briefly recalled and its associated linearization is presented. In Section 3 the way to obtain the macroscopic mass transfer terms in multiphase flows models (1) using the linearized reactive Riemann problem and the discrete equations method (DEM) is shown. Then Section 4 is devoted to the determination of the mass flow rate $\dot{m}$. The method presented briefly in this report involves new ingredients and in particular the resolution of an algebraic ' $0 D$ ' system taking into account local physical phenomena such as heat transfer and mass diffusion at the microscopic scale.

\section{The Reactive Riemann Problem for the Euler Equations}

When a liquid initially in thermodynamic equilibrium undergoes strong rarefaction waves, it may reach a metastable state where the temperature is higher than the saturated one at the final pressure of the expanded state. Then the superheated liquid releases its metastable energy (stored as internal energy) very quickly, even explosively, producing either pure vapor or liquid-vapor mixture at thermodynamic equilibrium, flowing at high velocity. This phenomenon is often called cavitation. Such situations appear frequently in nature and in many industrial applications, as liquid flows around hypervelocity projectiles, airfoils, or inside nozzles such as fuel injector systems. Cavitation in these systems always produces strong disturbances. In most applications, cavitation appears as a multidimensional process due to geometrical effects. This multidimensional character complicates both experiments and theories. By using one-dimensional expansion tubes, many authors $[7,11,15,21]$ and particularly [19] succeeded in isolating the main phenomenon we propose to summarize hereafter. These experiments consisted in connecting a vertical tube filled with a liquid in thermodynamic equilibrium at atmospheric pressure (or higher) to a very low-pressure chamber (Figure 1).

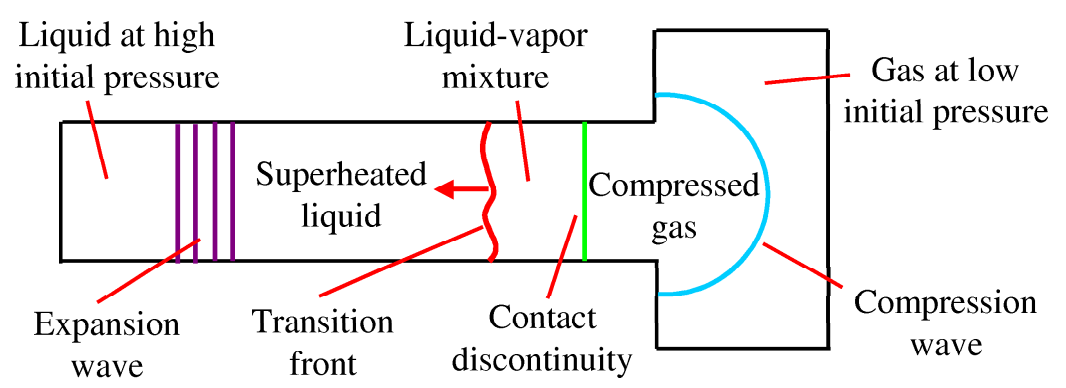

Figure 1: Simplified experimental setup of the expansion tube in [19] and associated waves paths 
As soon as the membrane between the liquid and the vacuum chamber is ruptured, rarefaction waves propagate through the liquid producing a superheated liquid (Figure 2).

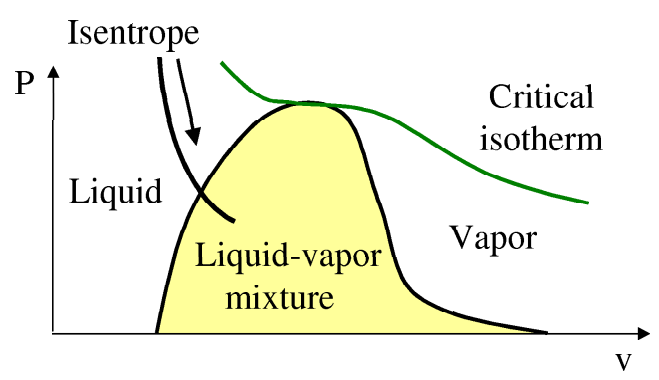

Figure 2: Liquid thermodynamic process associated to the expansion wave producing a superheated liquid

Then a subsonic phase transition front propagates through the superheated liquid producing a high velocity vapor towards the low-pressure chamber. The front velocity is approximately $1 \mathrm{~m} / \mathrm{s}$ while the ejected vapor velocity is of the order of $100 \mathrm{~m} / \mathrm{s}$.

For the applications under consideration (hypervelocity underwater projectiles, liquid flows in nozzles, boiling flows, ...), the evaporation front is considered as a discontinuity without solving its internal structure. Obviously, it poses some difficulties that are summarized hereafter and that have been solved in $[13]$.

The first difficulty deals with the thermodynamic closure. Since the front is considered as a discontinuity separating the liquid phase and a pure vapor, appropriate EOS on both sides of the front are needed to close the conservation laws. These ones correspond to the Euler equations. In this case, pure liquid and vapor EOS are needed and are strongly coupled. Indeed, at thermodynamic equilibrium, combination of both EOS in conjunction with pressure, temperature and chemical potential equilibrium must reproduce the phase diagram as detailed in [12]. This combination also results in a mixture EOS corresponding to the well known saturated vapor pressure $P=P_{\text {sat }}(T)$ characteristic of the liquid-vapor couple.

Moreover, in the experiments described below, an acoustic wave (expansion wave) is preceding the evaporation front, itself preceding a compression wave and a contact discontinuity as represented in the Figure 1. The corresponding waves pattern is shown in the $(x, t)$ diagram in the Figure 3 .

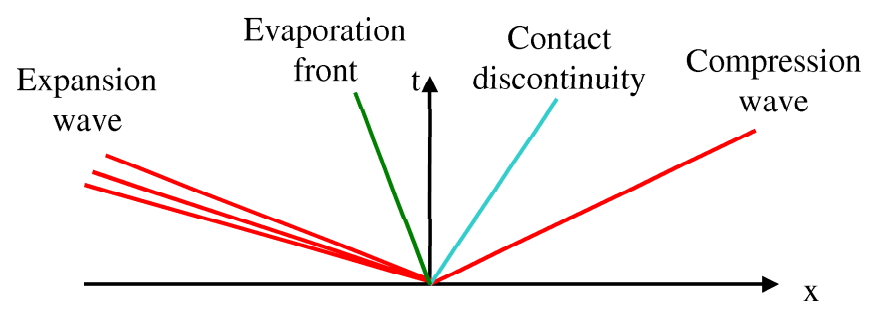

Figure 3: A typical waves pattern of the reactive Riemann problem associated to evaporation waves

Such a configuration is known in the literature as a reactive Riemann problem. This one has been solved 
by $[4,9,20]$ for detonation and deflagration problems with ideal gas EOS. For evaporation problems, it has been solved in [13]. Since the front is considered as a discontinuity, the Rankine-Hugoniot relations for the Euler equations express the mass, momentum and energy conservation principles across the front. However, this front being subsonic, a precursor acoustic wave is present. In order to remove the indeterminacy produced by the extra state resulting from the precursor wave propagation, an additional relation is required : the 'kinetic relation'. This one selects the front velocity and uniqueness of the reactive Riemann problem solution is recovered. The kinetic relation results from the resolution of the front internal structure. An approximate solution is given in Section 4.

With the help of the Riemann problem solution it is possible to build both numerical methods [1] and mathematical models of non-equilibrium multiphase flows. We consider here the second option where this method is used to obtain closure relations for $\tilde{\rho}_{I}, \tilde{u}_{I}$ and $\tilde{H}_{I}$.

Before dealing with the reactive Riemann problem, we recall the basis of a conventional Riemann solver for the Euler equations. The reactive Riemann solver will use the same ingredients, completed by additional relations associated to the evaporation front.

\subsection{Recalls on the Inert Riemann Problem}

The inert Riemann problem associated to the Euler equations is given by :

$$
\left\{\begin{array}{c}
\frac{\partial U(x, t)}{\partial t}+\frac{\partial F(U(x, t))}{\partial x}=0 \\
U(x, 0)= \begin{cases}U_{L} & \text { if } x<0 \\
U_{R} & \text { if } x>0\end{cases}
\end{array}\right.
$$

where $U(x, t)=(\rho, \rho u, \rho E)^{T}$ and $F(U(x, t))=\left(\rho u, \rho u^{2}+P,(\rho E+P) u\right)^{T}$.

$U_{L}$ and $U_{R}$ are constant initial states. $\rho, u$ and $P$ are respectively the density, the material velocity and the pressure. The total energy is defined as $E=e+1 / 2 u^{2}$ where $e$ is the internal energy. The system (2) is closed by the use of an equation of state $e=e(P, \rho)$.

A simple typical EOS is the 'Stiffened Gas' EOS [10]. Indeed, this EOS contains the main properties of the matter that are repulsive effects in gases, repulsive and attractive effects in liquids. The 'Stiffened Gas' EOS reads :

$$
e(P, \rho)=\frac{P+\gamma P_{\infty}}{(\gamma-1) \rho}+q
$$

where $e, P$ and $\rho$ are respectively the specific internal energy, the pressure and the density of the fluid. $\gamma, P_{\infty}$ and $q$ (energy of the fluid at a given reference state) are constant parameters, characteristic of the

thermodynamic behaviour of the fluid. The sound speed, for this particular EOS, reads $c^{2}=\frac{\gamma\left(P+P_{\infty}\right)}{\rho}$. More details about the determination of such EOS parameters may be found in [12].

The problem (2) consists in finding the intermediate constant states $U_{L}^{*}$ and $U_{R}^{*}$ delimited by the various waves (expansions, shocks or contact discontinuities) present in the medium.

The different jump relations available across the various waves are of three types : Riemann invariants (isentropic relations) for rarefaction waves, Rankine-Hugoniot relations for shock waves and interface conditions for the contact discontinuity $u$.

Across this one, the interface conditions are $u=c s t$ and $P=c s t$. 
Across rarefaction waves, the isentropic relations are :

$$
\begin{cases}\rho d u= \pm c d \rho & (a) \\ d P=c^{2} d \rho & (b)\end{cases}
$$

with \pm for right- and left-facing expansion waves respectively.

When using the 'Stiffened Gas' EOS (3), the isentropic relations (4) become :

$$
\left\{\begin{array}{l}
u=u_{0} \pm \frac{2 c_{0}}{\gamma-1}\left(\left(\frac{P+P_{\infty}}{P_{0}+P_{\infty}}\right)^{\frac{\gamma-1}{2 \gamma}}-1\right) \\
\rho=\rho_{0}\left(\frac{P+P_{\infty}}{P_{0}+P_{\infty}}\right)^{\frac{1}{\gamma}}
\end{array}\right.
$$

with \pm for right- and left-facing expansion waves respectively. The state with subscript 0 is the state in which rarefaction waves propagate.

The Rankine-Hugoniot relations are given by :

$$
\left\{\begin{array}{l}
u=u_{0}+m\left(\frac{1}{\rho_{0}}-\frac{1}{\rho}\right) \\
P=P_{0}+m^{2}\left(\frac{1}{\rho_{0}}-\frac{1}{\rho}\right) \\
e(P, \rho)=e_{0}\left(P_{0} \rho_{0}\right)+\frac{P+P_{0}}{2}\left(\frac{1}{\rho_{0}}-\frac{1}{\rho}\right)
\end{array}\right.
$$

where $m=\rho_{0}\left(\sigma-u_{0}\right)$ represents the mass flow rate ( $\sigma$ is the shock speed) and the state with subscript 0 is the state in which the shock propagates.

Using the 'Stiffened Gas' EOS (3), system (6) reads :

$$
\left\{\begin{array}{l}
u=u_{0} \pm \frac{P-P_{0}}{\rho_{0} c_{0} \sqrt{\frac{\gamma+1}{2 \gamma}\left(\frac{P+P_{\infty}}{P_{0}+P_{\infty}}\right)+\frac{\gamma-1}{2 \gamma}}} \\
m^{2}=\left(\rho_{0} c_{0}\right)^{2}\left(\frac{\gamma+1}{2 \gamma}\left(\frac{P+P_{\infty}}{P_{0}+P_{\infty}}\right)+\frac{\gamma-1}{2 \gamma}\right) \\
\rho=\rho_{0} \frac{(\gamma+1)\left(\frac{P+P_{\infty}}{P_{0}+P_{\infty}}\right)+\gamma-1}{(\gamma-1)\left(\frac{P+P_{\infty}}{P_{0}+P_{\infty}}\right)+\gamma+1}
\end{array}\right.
$$

with \pm for right- and left-facing shock waves respectively.

Relations (5.a) and (7.a) read under the same form :

$$
u=u_{0} \pm \phi_{0}(P)
$$

with

$$
\phi_{0}(P)= \begin{cases}\frac{P-P_{0}}{\rho_{0} c_{0} \sqrt{\frac{\gamma+1}{2 \gamma}\left(\frac{P+P_{\infty}}{P_{0}+P_{\infty}}\right)+\frac{\gamma-1}{2 \gamma}}} & \text { if } P>P_{0} \\ \frac{2 c_{0}}{\gamma-1}\left(\left(\frac{P+P_{\infty}}{P_{0}+P_{\infty}}\right)^{\frac{\gamma-1}{2 \gamma}}-1\right) & \text { otherwise }\end{cases}
$$


Now substituting subscript 0 in (8) and (9) by subscripts $L$ and $R$ corresponding to the left and right states respectively, we get :

$$
\begin{aligned}
& u^{*}=u_{L}-\phi_{L}\left(P^{*}\right) \\
& u^{*}=u_{R}+\phi_{R}\left(P^{*}\right)
\end{aligned}
$$

where the interface conditions $u_{L}^{*}=u_{R}^{*}=u^{*}$ and $P_{L}^{*}=P_{R}^{*}=P^{*}$ have been used.

Thus, the combination of (10) and (11) leads to a scalar equation :

$$
F\left(P^{*}\right)=u_{R}-u_{L}+\phi_{R}\left(P^{*}\right)+\phi_{L}\left(P^{*}\right)=0
$$

The solution of (12) is obtained by using an iterative numerical method (Newton-Raphson for example). Once the pressure $P^{*}$ is obtained, the other flow variables are calculated from the appropriate relations given previously. More details about the resolution of the inert Riemann problem are described for example in $[4,9,14,22]$.

The exact resolution of the Riemann problem is possible but the various flow variables cannot be expressed as algebraic functions of the known left $L$ and right $R$ states. With the discrete equations method, when explicit formulas are seeked for the various interfacial variables explicit Riemann problem solution is also required. Such analytical solution is available only with linearized isentropic and shock relations. This is the frame of the so called 'acoustic solver'. It means that waves of weak amplitude are only allowed in the multiphase mixture. The acoustic solver is based on linearized relations (10) and (11) around initial states.

These relations become in this case :

$$
u^{*}=u_{L}-\frac{P^{*}-P_{L}}{Z_{L}} \quad u^{*}=u_{R}+\frac{P^{*}-P_{R}}{Z_{R}}
$$

where $Z=\rho c$ represents the acoustic impedance of the fluid.

The Riemann problem solutions for $P^{*}$ and $u^{*}$ are :

$$
\begin{gathered}
u^{*}=\frac{Z_{L} u_{L}+Z_{R} u_{R}+P_{L}-P_{R}}{Z_{L}+Z_{R}} \\
P^{*}=\frac{Z_{R} P_{L}+Z_{L} P_{R}+Z_{L} Z_{R}\left(u_{L}-u_{R}\right)}{Z_{L}+Z_{R}}
\end{gathered}
$$

\subsection{The Reactive Riemann Problem}

Isentropic and shock relations described in the previous part are used again in the reactive Riemann problem but are valid only in pure fluids. The physical phenomenon described in Figure 1 leads to an additional front through which vapor appears. Like any discontinuity, this front obeys the RankineHugoniot relations (6). But, contrary to shocks, the EOS parameters are different on both sides of the front. In addition, as reported in introduction, a kinetic relation must complete the Rankine-Hugoniot relations to solve exactly this particular Riemann problem.

\subsubsection{Available Relations across Reactive Fronts}

The thermodynamic behaviour of the superheated liquid (subscript 0) obeys :

$$
e_{0}\left(P_{0}, \rho_{0}\right)=\frac{P_{0}+\gamma_{0} P_{\infty 0}}{\left(\gamma_{0}-1\right) \rho_{0}}+q_{0}
$$


While the appearing fluid (vapor) obeys :

$$
e(P, \rho)=\frac{P+\gamma P_{\infty}}{(\gamma-1) \rho}+q
$$

Combining relations (6.c), (16) and (17), we obtain :

$$
\rho=\rho_{0} \frac{\frac{\gamma+1}{\gamma-1}\left(P+P_{\infty}\right)+P_{0}+P_{\infty}}{\frac{\gamma_{0}+1}{\gamma_{0}-1}\left(P_{0}+P_{\infty 0}\right)+P+P_{\infty 0}-2 \rho_{0} \Delta q}
$$

where $\Delta q=q-q_{0}$ denotes the difference between reference energies. The relation (18) represents the Crussard curve relative to the initial state $\left(\rho_{0}, P_{0}\right)$. This curve (see Figure 4$)$ is divided in three parts.

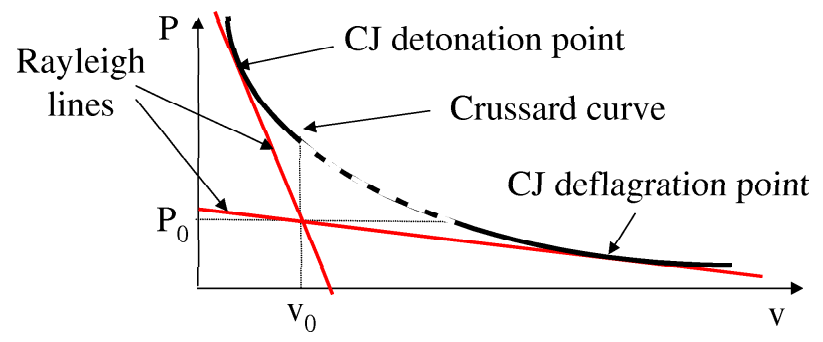

Figure 4: Crussard curve and Rayleigh line in a $(P, v)$ diagram associated to a superheated liquid state (subscript 0)

One of them (dotted lines) is a non physical area because it corresponds to positive slopes $\left(m^{2}<0\right)$ of the Rayleigh line represented by relation (6.b). Two points delimit this non-admissible area. The first one corresponds to a constant pressure deflagration $\left(P=P_{0}\right)$ whose associated density is given by :

$$
\rho_{p}=\frac{\gamma\left(P_{0}+P_{\infty}\right)}{\frac{\gamma-1}{\gamma_{0}-1} c_{0}^{2}-(\gamma-1) \Delta q}<\rho_{0}
$$

The second point corresponds to constant volume transformation $\left(\rho=\rho_{0}\right)$ whose associated pressure reads :

$$
P_{v}=\frac{\gamma-1}{\gamma_{0}-1}\left(P_{0}+\gamma_{0} P_{\infty 0}\right)-\gamma P_{\infty}-(\gamma-1) \rho_{0} \Delta q>P_{0}
$$

The upper part of the Crussard curve in the Figure 4 corresponds to the detonation branch $\left(P>P_{v}\right)$ while the lower part represents the deflagration branch $\left(P<P_{0}\right)$. The detonation branch cannot be reached because vapor appearance would be nonsense in this case. Indeed this zone corresponds to situations where compression waves are present in the medium, bringing the liquid back to a non-superheated state. Consequently, the solution locates in the deflagration area : the evaporation front is undercompressive. Now, combining relations (6.b) and (18), the mass flow rate reads :

$$
m^{2}=\frac{\rho_{0}}{2}\left((\gamma+1)\left(P+P_{\infty}\right)+(\gamma-1)\left(P_{0}+P_{\infty}\right)\right) \frac{P-P_{0}}{P-P_{v}}
$$

The combination of relations (6.a), (6.b) and (21) gives the following material velocity expression :

$$
u=u_{0} \pm s g\left(P-P_{0}\right) \sqrt{\frac{2\left(P-P_{0}\right)\left(P-P_{v}\right)}{\rho_{0}\left((\gamma+1)\left(P+P_{\infty}\right)+(\gamma-1)\left(P_{0}+P_{\infty}\right)\right)}}
$$


with \pm for a right(left)-facing front respectively.

It is important to note also the presence of two particular points in the Figure 4 . These are tangential points of the Rayleigh line and the Crussard curve. The high-pressure solution $\left(P>P_{v}\right)$ and the lowpressure solution $\left(P<P_{0}\right)$ are named respectively the Chapman-Jouguet detonation and deflagration points (CJ points).

\subsubsection{Exact solution of the Reactive Riemann Problem}

As the evaporation front is subsonic with respect to the medium in which it propagates, it is preceded by a precursor wave (shock or expansions) propagating approximately at the liquid sound speed, as represented in the Figure 1. By enumerating the various unknowns (pressure, density and flow velocity for each intermediate state and two wave speeds : shock and evaporation front) and the available relations (three Rankine-Hugoniot relations for shock and evaporation front, two relations for expansion waves and contact discontinuity), we have eleven unknowns and ten relations. The kinetic relation that controls the phase change rate achieves the Riemann problem closure.

During the resolution of the reactive Riemann problem we designate by 'composite wave' the waves group composed of the precursor and the evaporation front. The precursor could be compressive or undercompressive according to the pressure behind the front as represented in the Figure 5.
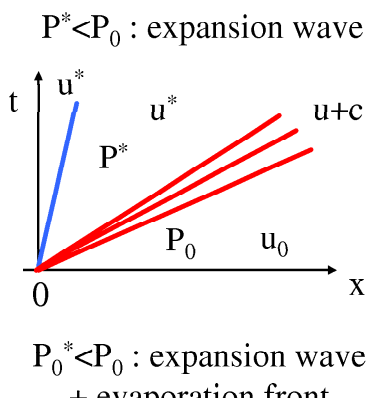
+ evaporation front

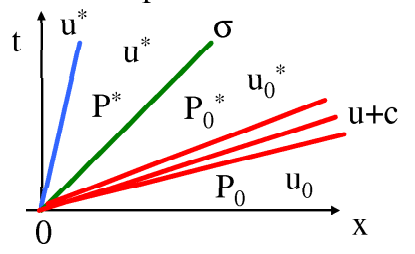

$\mathrm{P}^{*}>\mathrm{P}_{0}:$ inert shock

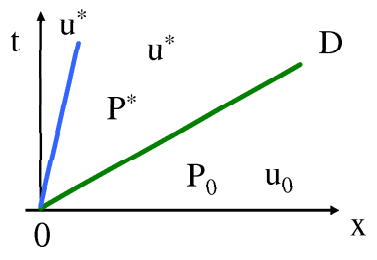

$$
\mathrm{P}_{0}^{*}>\mathrm{P}_{0}: \text { inert shock }
$$$$
+ \text { evaporation front }
$$

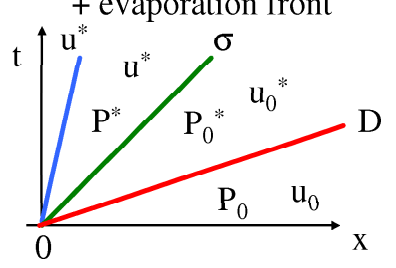

Figure 5: Waves patterns in the reactive Riemann problem associated to evaporation fronts

The reactive Riemann problem is solved using an iterative method, like in the inert case. At each iteration, the pressure $P^{*}$ determines the waves pattern (simple or composite waves) that must be considered so as to connect the state associated to $P^{*}$ to the left and right states denoted by subscripts $L$ and $R$.

Let us recall that in the inert case, two instances are possible :

- if $P^{*} \geq P_{0}$, the wave corresponds to a shock and we use the Rankine-Hugoniot relations $(7)$,

- if $P^{*} \leq P_{0}$, the wave is an expansion and we use the isentropic relations (5). 
If the fluid is a liquid, an evaporation front connects the state $P^{*}$ to an intermediate state $0^{*}$ (superheated liquid). This front is preceded by the precursor wave connecting the state $0^{*}$ and the initial state 0 of the liquid. Then two cases are possible to connect the states $0^{*}$ and 0 :

- if $P_{0}^{*}>P_{0}$, the precursor is a shock and we use the relations (7),

- if $P_{0}^{*} \leq P_{0}$, the precursor is an expansion wave and we use the relations (5).

The relations associated to the previous waves patterns can be written under a common form :

$$
u^{*}=u_{0} \pm \phi_{0}\left(P^{*}\right)
$$

with

$$
\phi_{0}\left(P^{*}\right)= \begin{cases}\frac{P^{*}-P_{0}}{\rho_{0} c_{0} \sqrt{\frac{\gamma_{0}+1}{2 \gamma_{0}}\left(\frac{P^{*}+P_{\infty 0}}{P_{0}+P_{\infty 0}}\right)+\frac{\gamma_{0}-1}{2 \gamma_{0}}}} & \text { if } P^{*}>P_{0} \text { (inert) } \\ \frac{2 c_{0}}{\gamma_{0}-1}\left(\frac{P^{*}+P_{\infty 0}}{P_{0}+P_{\infty 0}}\right)^{\left.\frac{\gamma_{0}-1}{2 \gamma_{0}}-1\right)} & \text { if } P^{*} \leq P_{0} \text { (inert) } \\ u_{0}^{*}-u_{0}+\frac{P^{*}-P_{0}^{*}}{m\left(P^{*}\right)} & \text { (reactive) }\end{cases}
$$

where $m\left(P^{*}\right)$ is the mass flow rate through the reactive front. This one is calculated considering an explicit kinetic relation so that the pressure $P^{*}$ coincides exactly to the pressure behind the evaporation front. For each value of $P^{*}$, a unique associated state $0^{*}$ is obtained by an iterative method we detail hereafter. For each value of $P_{0}^{*}$, the associated density is given by :

$$
\rho_{0}^{*}= \begin{cases}\rho_{0} \frac{\left(\gamma_{0}+1\right)\left(\frac{P_{0}^{*}+P_{\infty 0}}{P_{0}+P_{\infty 0}}\right)+\gamma_{0}-1}{\left(\gamma_{0}-1\right)\left(\frac{P_{0}^{*}+P_{\infty 0}}{P_{0}+P_{\infty 0}}\right)+\gamma_{0}+1} & \text { if } P_{0}^{*}>P_{0} \\ \rho_{0}\left(\frac{P_{0}^{*}+P_{\infty 0}}{P_{0}+P_{\infty 0}}\right) \frac{1}{\gamma_{0}} & \text { otherwise }\end{cases}
$$

The density $\rho^{*}$ (behind the front) and the mass flow rate $m$ are obtained by relations (18) and (21). Then we use the Rayleigh line equation (6.b) to update $P_{0}^{*}$ at each iteration :

$$
P_{0}^{*}=P^{*}-m^{2}\left(\frac{1}{\rho_{0}^{*}}-\frac{1}{\rho^{*}}\right)
$$

The value of $P_{0}^{*}$ is then modified until convergence is obtained. The other variables associated to the state $0^{*}$ are determined either by relations $(7)$ or by relations $(5)$ in accordance with $P_{0}^{*}$.

Now substituting subscript 0 in (23) and (24) by subscripts $L$ and $R$ corresponding to the left and right states respectively, we get :

$$
\begin{aligned}
& u^{*}=u_{L}-\phi_{L}\left(P^{*}\right) \\
& u^{*}=u_{R}+\phi_{R}\left(P^{*}\right)
\end{aligned}
$$

Combining relations (27) and (28), a scalar equation is recovered :

$$
F\left(P^{*}\right)=u_{R}-u_{L}+\phi_{R}\left(P^{*}\right)+\phi_{L}\left(P^{*}\right)=0
$$


The solution $P^{*}$ is determined again by an iterative method (Newton-Raphson for example). Once $P^{*}$ is obtained, the waves pattern and the whole solution of the reactive Riemann problem are uniquely determined.

In the next part a linearization of the reactive Riemann problem is achieved.

\subsection{The Linearized Reactive Riemann Problem for the Euler Equations}

Let us consider a configuration of the reactive Riemann problem where the liquid is present at the right and its associated vapor at the left as represented in the Figure 6.

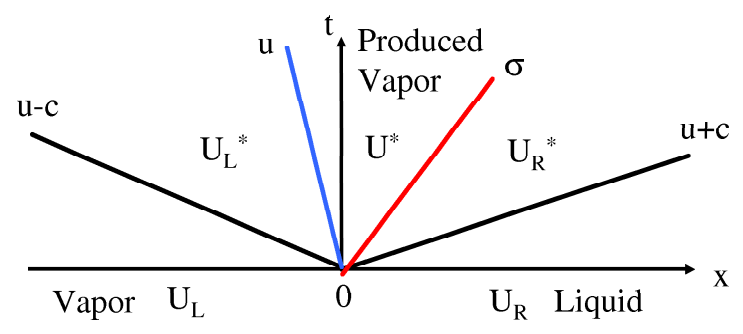

Figure 6: Configuration under study for the linearized reactive Riemann problem

The available relations valid across rarefaction or shock waves are the acoustic relations (13) :

$$
u_{L}^{*}=u_{L}-\frac{P_{L}^{*}-P_{L}}{Z_{L}} \quad u_{R}^{*}=u_{R}+\frac{P_{R}^{*}-P_{R}}{Z_{R}}
$$

By using the interface conditions $u_{L}^{*}=u^{*}$ and $P_{L}^{*}=P^{*}$ across the contact discontinuity the previous relations become :

$$
u^{*}=u_{L}-\frac{P^{*}-P_{L}}{Z_{L}} \quad u_{R}^{*}=u_{R}+\frac{P_{R}^{*}-P_{R}}{Z_{R}}
$$

Combining relations (31), we get :

$$
u^{*}-u_{R}^{*}=u_{L}-u_{R}+\frac{P_{L}}{Z_{L}}+\frac{P_{R}}{Z_{R}}-\frac{P^{*}}{Z_{L}}-\frac{P_{R}^{*}}{Z_{R}}=P_{I}\left(\frac{1}{Z_{L}}+\frac{1}{Z_{R}}\right)-\frac{P^{*}}{Z_{L}}-\frac{P_{R}^{*}}{Z_{R}}
$$

where $P_{I}$ is the pressure solution (15) of the linearized Riemann problem without evaporation front. Now the Rankine-Hugoniot relations at the reactive front level are considered :

$$
\left\{\begin{array}{l}
u^{*}=u_{R}^{*}+m_{R}\left(\frac{1}{\rho_{R}^{*}}-\frac{1}{\rho^{*}}\right) \\
P^{*}=P_{R}^{*}+m_{R}^{2}\left(\frac{1}{\rho_{R}^{*}}-\frac{1}{\rho^{*}}\right) \\
e^{*}=e_{R}^{*}+\frac{P^{*}+P_{R}^{*}}{2}\left(\frac{1}{\rho_{R}^{*}}-\frac{1}{\rho^{*}}\right)
\end{array}\right.
$$

where $m_{R}$ is the mass flow rate through the reactive front and is assumed to be known.

Combining relations (33.a), (33.b) and (32), an explicit relation linking $P^{*}$ and $P_{R}^{*}$ is obtained :

$$
P^{*}=P_{I}-\frac{Z_{L}}{Z_{R}} \frac{\left(Z_{R}-m_{R}\right)}{\left(Z_{L}+m_{R}\right)}\left(P_{I}-P_{R}^{*}\right)
$$

Another relation between these variables is needed to determine the solution of the linearized reactive Riemann problem. This relation is provided by the use of the last available equation (33.c). Combining 
relations (33.b) and (33.c), we get :

$$
2 m_{R}^{2}\left(e^{*}-e_{R}^{*}\right)=\left(P^{*}+P_{R}^{*}\right)\left(P^{*}-P_{R}^{*}\right)
$$

Another ingredients are still needed. Indeed, $e^{*}$ is obtained by the explicit use of the following vapor equation of state :

$$
e^{*}=e^{*}\left(P^{*}, \rho^{*}\right)=\frac{P^{*}}{\Gamma^{*} \rho^{*}}+q
$$

where $\Gamma=\gamma-1$ and $q$ are the Grüneisen coefficient and the energy constant of vapor phase respectively. The variable $\rho^{*}$ obeys the relation $(33 . \mathrm{b})$ :

$$
\frac{1}{\rho^{*}}=\frac{1}{\rho_{R}^{*}}-\frac{\left(P^{*}-P_{R}^{*}\right)}{m_{R}^{2}}
$$

Using the last relation in (36) leads to :

$$
e^{*}=\frac{P^{*}}{\Gamma^{*}}\left(\frac{1}{\rho_{R}^{*}}-\frac{\left(P^{*}-P_{R}^{*}\right)}{m_{R}^{2}}\right)+q
$$

The variables $\rho_{R}^{*}$ and $e_{R}^{*}$ are obtained by the following linearized relations :

$$
\begin{gathered}
\frac{1}{\rho_{R}^{*}}=\frac{1}{\rho_{R}}+\frac{\left(P_{R}-P_{R}^{*}\right)}{Z_{R}^{2}} \\
e_{R}^{*}=e_{R}+P_{R} \frac{\left(P_{R}^{*}-P_{R}\right)}{Z_{R}^{2}}
\end{gathered}
$$

Using relation (39) in (38), we have :

$$
e^{*}=\frac{P^{*}}{\Gamma^{*}}\left(\frac{1}{\rho_{R}}+\frac{\left(P_{R}-P_{R}^{*}\right)}{Z_{R}^{2}}-\frac{\left(P^{*}-P_{R}^{*}\right)}{m_{R}^{2}}\right)+q
$$

and then relation (35) expresses the second relation linking $P^{*}$ and $P_{R}^{*}$ :

$$
2 m_{R}^{2}\left[\frac{P^{*}}{\Gamma^{*}}\left(\frac{1}{\rho_{R}}+\frac{\left(P_{R}-P_{R}^{*}\right)}{Z_{R}^{2}}-\frac{\left(P^{*}-P_{R}^{*}\right)}{m_{R}^{2}}\right)+q-e_{R}-P_{R} \frac{\left(P_{R}^{*}-P_{R}\right)}{Z_{R}^{2}}\right]=\left(P^{*}+P_{R}^{*}\right)\left(P^{*}-P_{R}^{*}\right)
$$

Now the explicit relation $(34)$ of $P^{*}$ in function of $P_{R}^{*}$ is embedded into the previous one and after some calculations, a second order polynomial equation over the variable $P_{R}^{*}$ is obtained :

$$
A\left(P_{I}-P_{R}^{*}\right)^{2}+B\left(P_{I}-P_{R}^{*}\right)+C
$$

where the constants are given by :

$$
\begin{gathered}
A=Z_{L}\left[\begin{array}{c}
m_{R}\left(\Gamma^{*}+1-\frac{m_{R}}{Z_{R}}-\left(\frac{m_{R}}{Z_{R}}\right)^{2}+\left(\frac{m_{R}}{Z_{R}}\right)^{3}\right) \\
+Z_{L}\left(\frac{\Gamma^{*}}{2}\left(\frac{m_{R}}{Z_{L}}\right)^{2}+\left(\Gamma^{*}+1\right) \frac{m_{R}}{Z_{R}}-\left(\frac{\Gamma^{*}}{2}+2\right)\left(\frac{m_{R}}{Z_{R}}\right)^{2}+\left(\frac{m_{R}}{Z_{R}}\right)^{3}\right)
\end{array}\right] \\
B=Z_{L}\left(Z_{L}+m_{R}\right)\left[\begin{array}{l}
-\frac{m_{R}^{2}}{\rho_{R}}\left(1-\frac{m_{R}}{Z_{R}}\right)+P_{R}\left(\frac{m_{R}}{Z_{R}}\right)^{2}\left(\Gamma^{*}-1+\Gamma^{*} \frac{m_{R}}{Z_{L}}+\frac{m_{R}}{Z_{R}}\right) \\
-P_{I}\left(\left(\Gamma^{*}+1\right) \frac{m_{R}}{Z_{L}}+\left(\Gamma^{*}+1\right) \frac{m_{R}}{Z_{R}}-\left(2+\frac{m_{R}}{Z_{L}}\right)\left(\frac{m_{R}}{Z_{R}}\right)^{2}+\left(\frac{m_{R}}{Z_{R}}\right)^{3}\right)
\end{array}\right] \\
C=\left(Z_{L}+m_{R}\right)^{2}\left[m_{R}^{2} \Gamma^{*}\left(q-h_{R}\right)+\left(\Gamma^{*} P_{R}+P_{I}\right)\left(\frac{m_{R}^{2}}{\rho_{R}}+\left(P_{R}-P_{I}\right)\left(\frac{m_{R}}{Z_{R}}\right)^{2}\right)\right]
\end{gathered}
$$


Nevertheless the previous coefficients are quite complex and asymptotic extensions are necessary to obtain simple formulas. The assumption that is used is the subsonic character of the evaporation process. It means that $Z_{R}$ (acoustic impedance of the liquid phase) is much larger than the mass flow rate $m_{R}$. When neglecting all the powers of the term $\frac{m_{R}}{Z_{R}}$ in the previous expressions, the coefficients become :

$$
\begin{gathered}
A=\left(\Gamma^{*}+1\right) Z_{L}+\frac{\Gamma^{*}}{2} m_{R} \\
B=-\left(Z_{L}+m_{R}\right)\left(Z_{L} \frac{m_{R}}{\rho_{R}}+\left(\Gamma^{*}+1\right) P_{I}\right) \\
C=m_{R}\left(Z_{L}+m_{R}\right)^{2}\left(\frac{P_{I}}{\rho_{R}}+\Gamma^{*}\left(q-e_{R}\right)\right)
\end{gathered}
$$

Now simple explicit expressions of all variables, solutions of the linearized reactive Riemann problem, may be obtained. This work is in progress.

\section{Implementation of the Linearized Reactive Riemann Solver in the Discrete Equations Method (DEM)}

In order to treat multi-dimensional propagation of evaporation fronts, an innovative numerical method named DEM (Discrete Equations Method) proposed by $[1,17,3]$ for interface problems and multiphase mixtures has been extended to reactive fronts in [13]. This method consists in connecting several systems of equations, each one being associated to a pure fluid. It leads to discrete equations that can be used for interface problems or multiphase flows with several velocities. It may be considered as an homogenization method belonging to the class of averaging methods.

Usually, multiphase flows equations are obtained by mathematical averaging procedures [6]. Another option is based on the Hamilton principle as given in [8] for example. Both methods yield to non-conservative systems where average interfacial variables appear. In all cases, the resulting system must be solved by numerical approximations. In this kind of approach, the determination of the average variables as well as the numerical approximation of the non-conservative terms is always questionable.

These difficulties are solved by the present method. Contrary to conventional methods, the averaged equations for the mixture are not used. Interface problems between pure fluids are solved locally with the help of their own governing equations. In [1], it was done with the Euler equations for each fluid resulting in interface problem solution obtained with conventional Riemann solver. In [17], a turbulent flow model was used in each fluid with the associated Riemann problem solution. The local interface variables are determined at each interface of the two-phase flow mixture whose topology is given a priori. Then, an averaging procedure is applied over all these solutions. In that sense, it proceeds like a conventional Godunov scheme in each phase with a moving boundary. The averaged interface solutions provide a set of discrete equations that can be used directly as a numerical scheme. Their continuous limit was obtained in [17] and identified to the Baer-Nunziato model [2] with more general closure relations for averaged interface variables.

In the present paper, the continuous limit of the extended reactive discrete equations method is investigated. Important extensions are necessary since the reactive Riemann problem presented in Section 2 is 
used.

In the following, we first recall the basis of this method when mass transfer is absent. We then extend the method when evaporation fronts are considered. Additional terms (reactive fluxes) appear and modifications of the Eulerian and Lagrangian fluxes introduced in $[1,17]$ are necessary.

\subsection{Recalls on the Discrete Equations Method}

With this method, for any type of two-phase mixture (liquid drops into a gas, gas bubbles into a liquid, stratified and annular flows) as well as flows with macroscopic interfaces, each pure fluid is assumed to obey the Euler equations. They interact via their interfaces at the microscopic scale when dealing with mixtures and at macroscopic scale when dealing with interface problems. Averaging the various interactions inside a two phase control volume yield coupling terms that appear as macroscopic variables like the volume fraction and non conservative terms or Lagrangian fluxes as introduced in $[1,17,3]$. In the following we summarize this method considering a stratified flow topology only.

The interfaces under study evolving in two dimensions, each pure fluid obeys the $2 D$ Euler equations :

$$
\frac{\partial U}{\partial t}+\frac{\partial F}{\partial x}+\frac{\partial G}{\partial y}=0
$$

where $U=[1, \rho, \rho u, \rho v, \rho E]^{T}, F=\left[0, \rho u, \rho u^{2}+P, \rho u v,(\rho E+P) u\right]^{T}$ and

$G=\left[0, \rho v, \rho u v, \rho v^{2}+P,(\rho E+P) v\right]^{T}$.

The first trivial equation $\frac{\partial 1}{\partial t}+\frac{\partial 0}{\partial x}+\frac{\partial 0}{\partial y}=0$ comes from an evolution equation expressing the link between the Lagrangian and Eulerian coordinates. This trivial identity will be necessary to obtain the volume fraction numerical equation.

To obtain the local equations of each pure fluid $k$, we multiply the Euler equations (44) by the phase function $\chi_{k}$ of each fluid. This one only admits two values : $\chi_{k}(M, t)=1$ if the point $M$ is located in the phase $k$ at time $t$ and $\chi_{k}(M, t)=0$ otherwise. This function obeys the local evolution equation:

$$
\frac{\partial \chi_{k}}{\partial t}+\sigma_{x} \frac{\partial \chi_{k}}{\partial x}+\sigma_{y} \frac{\partial \chi_{k}}{\partial y}=0
$$

where $\sigma_{x}$ and $\sigma_{y}$ are the components of the local interface velocity.

After some algebraic manipulations, the local pure fluids equations are given by :

$$
\frac{\partial \chi_{k} U}{\partial t}+\frac{\partial \chi_{k} F}{\partial x}+\frac{\partial \chi_{k} G}{\partial y}=F^{l a g} \frac{\partial \chi_{k}}{\partial x}+G^{l a g} \frac{\partial \chi_{k}}{\partial y}
$$

where the Lagrangian fluxes are defined as $F^{l a g}=F-\sigma_{x} U$ and $G^{l a g}=G-\sigma_{y} U$.

For interface problems between pure inert fluids we have $\sigma_{x}=u$ and $\sigma_{y}=v$. In this case, the Lagrangian fluxes write $F^{l a g}=[-u, 0, P, 0, P u]^{T}$ and $G^{l a g}=[-v, 0,0, P, P v]^{T}$.

Next we integrate in time and space over each computational cell $\left.C_{i, j}=\right] x_{i-1 / 2}, x_{i+1 / 2}[\times] y_{j-1 / 2}, y_{j+1 / 2}[$ the equation (46) :

$$
\int_{0}^{\Delta t} \int_{C_{i, j}}\left(\frac{\partial \chi_{k} U}{\partial t}+\frac{\partial \chi_{k} F}{\partial x}+\frac{\partial \chi_{k} G}{\partial y}\right) d V d t=\int_{0}^{\Delta t} \int_{C_{i, j}}\left(F^{l a g} \frac{\partial \chi_{k}}{\partial x}+G^{l a g} \frac{\partial \chi_{k}}{\partial y}\right) d V d t
$$

Relation (47) can be written as :

$$
I_{1}+I_{2}+I_{2}^{\prime}=I_{3}+I_{3}^{\prime}
$$


with

$$
\begin{aligned}
& I_{1}=\int_{0}^{\Delta t} \int_{C_{i, j}} \frac{\partial \chi_{k} U}{\partial t} d V d t \\
& I_{2}=\int_{0}^{\Delta t} \int_{C_{i, j}} \frac{\partial \chi_{k} F}{\partial x} d V d t \quad I_{2}^{\prime}=\int_{0}^{\Delta t} \int_{C_{i, j}} \frac{\partial \chi_{k} G}{\partial y} d V d t \\
& I_{3}=\int_{0}^{\Delta t} \int_{C_{i, j}} F^{l a g} \frac{\partial \chi_{k}}{\partial x} d V d t \quad I_{3}^{\prime}=\int_{0}^{\Delta t} \int_{C_{i, j}} G^{l a g} \frac{\partial \chi_{k}}{\partial y} d V d t
\end{aligned}
$$

Now these five integrals have to be determined to obtain the discrete macroscopic equations of each fluid. As an interface is not solved as a true discontinuity but rather as a diffusion zone (because of artificial smearing), it corresponds to a series of cells with various levels (volume fractions) of fluids. In this way, at each cell boundary, we define a contact surface between fluids. For example, $S_{12, i-1 / 2}$ represents the contact surface at cell boundary $i-1 / 2$ between fluid 1 on the left (present inside the cell $C_{i-1, j}$ ) and fluid 2 on the right (present inside the cell $C_{i, j}$ ). At each cell boundary, only 4 types of contact between pure fluids are possible. Each type of contact is associated to the corresponding surface.

Let us denote by $(l, m)$ the configuration where the fluid $l$ is present on the left and the fluid $m$ is present on the right at a given cell boundary. At each contact $(l, m)$, the corresponding Riemann problem is solved. Its solution, denoted in the following by the superscript ' $*$ ', is used for the computation of the various fluxes (Eulerian and Lagrangian) that are then averaged over the cell surface.

In order to compute the integrals in (48), we define the following averaging operators :

- Volume average : $\langle f\rangle=\frac{1}{V_{i j}} \int_{V_{i j}} f d V$

- Surface averages : $\widehat{f}=\frac{1}{\Delta x} \int_{x_{i-1 / 2}}^{x_{i+1 / 2}} f d x, \bar{f}=\frac{1}{\Delta y} \int_{y_{j-1 / 2}}^{y_{j+1 / 2}} f d y$

- Time average : $\widetilde{f}=\frac{1}{\Delta t} \int_{0}^{\Delta t} f d t$

\subsubsection{Integration of the Temporal Term $I_{1}$}

The first integral $I_{1}$ reads :

$$
I_{1}=\int_{0}^{\Delta t} \int_{C_{i j}} \frac{\partial \chi_{k} U}{\partial t} d V d t=\int_{C_{i j}}\left(\chi_{k} U\right)^{n+1} d V-\int_{C_{i j}}\left(\chi_{k} U\right)^{n} d V=\left(\left\langle\chi_{k} U\right\rangle_{i j}^{n+1}-\left\langle\chi_{k} U\right\rangle_{i j}^{n}\right) V_{i j}
$$

Using the volume average of the phase function $\chi_{k}$ in the cell $C_{i j}$, we have :

$$
\left\langle\chi_{k}\right\rangle_{i j}=\frac{1}{V_{i j}} \int_{C_{i j}} \chi_{k} d V=\frac{V_{k, i j}}{V_{i j}} \stackrel{\text { def }}{=} \alpha_{k, i j}
$$

where $V_{k, i j}$ represents the volume occupied by the phase $k$ in the cell $C_{i j}$.

Then, the term $I_{1}$ writes :

$$
I_{1}=\left(\left(\alpha_{k}\left\{U_{k}\right\}\right)_{i j}^{n+1}-\left(\alpha_{k}\left\{U_{k}\right\}\right)_{i j}^{n}\right) V_{i j}
$$

where $\left\{U_{k}\right\}_{i j} \stackrel{\text { def }}{=} \frac{1}{V_{k, i j}} \int_{C_{k, i j}} U_{k} d V$ is the volume average of $U_{k}$ in $V_{k, i j}$. 


\subsubsection{Integration of the Convective Fluxes $I_{2}$ and $I_{2}^{\prime}$}

The second integral $I_{2}$ reads :

$$
I_{2}=\int_{0}^{\Delta t} \int_{C_{i j}} \frac{\partial \chi_{k} F}{\partial x} d V d t=\int_{0}^{\Delta t} \int_{y_{j-1 / 2}}^{y_{j+1 / 2}}\left(\chi_{k} F\right)_{i+1 / 2} d y d t-\int_{0}^{\Delta t} \int_{y_{j-1 / 2}}^{y_{j+1 / 2}}\left(\chi_{k} F\right)_{i-1 / 2} d y d t
$$

Using the previous averages, we obtain :

$$
\left.I_{2}=\Delta y \int_{0}^{\Delta t}\left(\overline{\chi_{k} F}\right)_{i+1 / 2} d t-\Delta y \int_{0}^{\Delta t}\left(\overline{\chi_{k} F}\right)_{i-1 / 2} d t=\Delta t \Delta y\left(\widetilde{\left(\widetilde{\chi_{k} F}\right.}\right)_{i+1 / 2}-\left(\widetilde{\overline{\chi_{k} F}}\right)_{i-1 / 2}\right)
$$

In order to determine the average Eulerian flux $\widetilde{\overline{\chi_{k} F}}$ at each cell boundary, we first compute the following surface averages :

$$
\int_{y_{j-1 / 2}}^{y_{j+1 / 2}}\left(\chi_{k} F\right)_{i \pm 1 / 2} d y=\sum_{l, m}\left(S \chi_{k}^{*} F^{*}\right)_{l m, i \pm 1 / 2}
$$

where $S_{l m}$ represents the contact surface for each pair of fluids in contact. Their expressions, reported in the Table i for cell boundary $i-1 / 2$, are obtained following simple arguments provided in [17].

$\chi_{k, l m}^{*}$ represents the phase function of fluid $k$ for the same pair. Its value is reported in the same table and is obtained from the Riemann problem solution, according to the location of fluid $k$.

When fluid $k$ is present on both sides of the interface, $\chi_{k}^{*}=1$. When the other fluid is present on both sides, $\chi_{k}^{*}=0$. These situations correspond to the first and last lines of the Table i respectively.

When fluid $k$ is present on the left, $\chi_{k}^{*}=1$ only if the fluid $k$ is entering the cell (line 2 of the Table i). When it is present on the right, $\chi_{k}^{*}=1$ only if the fluid $k$ is leaving the cell (line 3 of the Table i).

\begin{tabular}{|c|c|c|c|}
\hline Contact & Surface & Eulerian Flux & Phase function $\chi_{1}^{*}$ \\
\hline $1-1$ & $S_{11}=\operatorname{\Delta yMin}\left(\alpha_{1, i-1}, \alpha_{1, i}\right)$ & $F_{11}^{*}$ & $\chi_{1,11}^{*}=1$ \\
\hline $1-2$ & $S_{12}=\Delta y \operatorname{Max}\left(0, \alpha_{1, i-1}-\alpha_{1, i}\right)$ & $F_{12}^{*}$ & $\chi_{1,12}^{*}=\left\{\begin{array}{l}1 \text { if } u_{12}^{*}>0 \\
0 \text { otherwise }\end{array}\right.$ \\
\hline $2-1$ & $S_{21}=\Delta y \operatorname{Max}\left(0, \alpha_{1, i}-\alpha_{1, i-1}\right)$ & $F_{21}^{*}$ & $\chi_{1,21}^{*}=\left\{\begin{array}{l}1 \text { if } u_{21}^{*}<0 \\
0 \text { otherwise }\end{array}\right.$ \\
\hline $2-2$ & $S_{22}=\operatorname{\Delta yMin}\left(\alpha_{2, i-1}, \alpha_{2, i}\right)$ & $F_{22}^{*}$ & $\chi_{1,22}^{*}=0$ \\
\hline
\end{tabular}

Table i: The different configurations for Eulerian fluxes at cell boundary $i-1 / 2$ for fluid $k=1$

As the Riemann problem is solved to determine the speed of the contact discontinuity $u_{l m}^{*}$, the whole solution is sampled along the axis $x / t=0$ and allows the various fluxes computation $F_{l m}^{*}$.

The Eulerian fluxes are supposed to be constant during a time step (CFL condition), thus we have :

$$
I_{2}=\Delta t\left(\sum_{l, m}\left(S \chi_{k}^{*} F^{*}\right)_{l m, i+1 / 2}-\sum_{l, m}\left(S \chi_{k}^{*} F^{*}\right)_{l m, i-1 / 2}\right)
$$

The third integral $I_{2}^{\prime}$ is obtained as previously :

$$
I_{2}^{\prime}=\int_{0}^{\Delta t} \int_{C_{i j}} \frac{\partial \chi_{k} G}{\partial y} d V d t=\Delta t \Delta x\left(\left(\widetilde{\widetilde{\chi_{k} G}}\right)_{j+1 / 2}-\left(\widetilde{\widetilde{\chi_{k} G}}\right)_{j-1 / 2}\right)
$$


The Eulerian flux is obtained again by summing the different configurations of pairs in contact. Then, the integral $I_{2}^{\prime}$ reads :

$$
I_{2}^{\prime}=\Delta t\left(\sum_{l, m}\left(S \chi_{k}^{*} G^{*}\right)_{l m, j+1 / 2}-\sum_{l, m}\left(S \chi_{k}^{*} G^{*}\right)_{l m, j-1 / 2}\right)
$$

\subsubsection{Integration of the Non Conservative Terms $I_{3}$ and $I_{3}^{\prime}$}

The fourth integral $I_{3}$ provides the Lagrangian fluxes. It reads :

$$
I_{3}=\int_{0}^{\Delta t} \int_{C_{i j}} F^{l a g} \frac{\partial \chi_{k}}{\partial x} d V d t=\int_{0}^{\Delta t} \int_{y_{j-1 / 2}}^{y_{j+1 / 2}} \int_{x_{i-1 / 2}}^{x_{i+1 / 2}} F^{l a g} \frac{\partial \chi_{k}}{\partial x} d x d y d t
$$

The interfaces present in the flow topology are located at the cell boundaries and inside the cell. Then, $I_{3}$ writes:

$$
\begin{aligned}
I_{3}= & \int_{0}^{\Delta t} \int_{y_{j-1 / 2}}^{y_{j+1 / 2}}\left(F^{l a g}\left[\chi_{k}^{x}\right]\right)_{i-1 / 2} d y d t+\int_{0}^{\Delta t} \int_{y_{j-1 / 2}}^{y_{j+1 / 2}}\left(F^{l a g}\left[\chi_{k}^{x}\right]\right)_{i+1 / 2} d y d t \\
& +\sum_{\text {int }} \int_{0}^{\Delta t} \int_{y_{j-1 / 2}}^{y_{j+1 / 2}}\left(F^{l a g}\left[\chi_{k}^{x}\right]\right)_{i n t} d y d t
\end{aligned}
$$

where $\left[\chi_{k}^{x}\right]$ represents the jump of $\chi_{k}$ in the $x$ direction through an interface. As explained in $[1]$, the preceding integration is allowed because the Lagrangian flux $F^{l a g}$ is uniform at every location where $\frac{\partial \chi_{k}}{\partial x}$ is non zero.

By using the definitions of averages, we obtain :

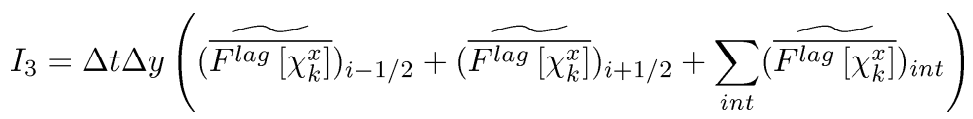

As for the calculations of the Eulerian fluxes, we use the considerations described in the Table ii (for cell boundary $i-1 / 2)$.

\begin{tabular}{|c|c|c|c|}
\hline Contact & Surface & Lagrangian Flux & Jump $\left[\chi_{1}^{x}\right]^{*}$ \\
\hline $1-1$ & $S_{11}$ & $F_{11}^{\text {lag,* }}$ & {$\left[\chi_{1}^{x}\right]_{11}^{*}=0$} \\
\hline $1-2$ & $S_{12}$ & $F_{12}^{\text {lag,* }}$ & {$\left[\chi_{1}^{x}\right]_{12}^{*}=\left\{\begin{array}{l}-1 \text { if } u_{12}^{*}>0 \\
0 \text { otherwise }\end{array}\right.$} \\
\hline $2-1$ & $S_{21}$ & $F_{21}^{\text {lag,* }}$ & {$\left[\chi_{1}^{x}\right]_{21}^{*}=\left\{\begin{array}{l}1 \text { if } u_{21}^{*}>0 \\
0 \text { otherwise }\end{array}\right.$} \\
\hline $2-2$ & $S_{22}$ & $F_{22}^{\text {lag,* }}$ & {$\left[\chi_{1}^{x}\right]_{22}^{*}=0$} \\
\hline
\end{tabular}

Table ii: The different configurations for Lagrangian fluxes at cell boundary $i-1 / 2$ for fluid $k=1$

By summing the different terms, we obtain :

$$
\int_{y_{j-1 / 2}}^{y_{j+1 / 2}}\left(F^{l a g}\left[\chi_{k}^{x}\right]\right)_{i-1 / 2} d y=\sum_{l, m}\left(S F^{l a g, *}\left[\chi_{k}^{x, *}\right]\right)_{l m, i-1 / 2}
$$

The jump of the phase function is zero when the same fluid is present on both sides of the interface (lines 1 and 4 of Table ii). When the fluid under interest lies initially on the left and is entering the cell (line 
2 of Table ii), the jump of its associated phase function is -1 . When the fluid under interest lies on the right and the other fluid is entering the cell (line 3 of Table ii), the jump of its phase function is 1 . The Lagrangian fluxes, in each type of contact, involve only the corresponding interface velocity and pressure that are provided by the Riemann solver.

For cell boundary $i+1 / 2$, we also have :

$$
\int_{y_{j-1 / 2}}^{y_{j+1 / 2}}\left(F^{l a g}\left[\chi_{k}^{x}\right]\right)_{i+1 / 2} d y=\sum_{l, m}\left(S F^{l a g, *}\left[\chi_{k}^{x, *}\right]\right)_{l m, i+1 / 2}
$$

For internal interfaces inside the cell, we have similarly :

$$
\int_{y_{j-1 / 2}}^{y_{j+1 / 2}}\left(F^{l a g}\left[\chi_{k}^{x}\right]\right)_{i n t} d y=\sum_{l, m}\left(S F^{l a g, *}\left[\chi_{k}^{x, *}\right]\right)_{l m, i n t}
$$

Assuming the Lagrangian fluxes are constant during the time step, we have :

$$
I_{3}=\Delta t\left(\sum_{l, m}\left(S F^{l a g, *}\left[\chi_{k}^{x, *}\right]\right)_{l m, i-1 / 2}+\sum_{l, m}\left(S F^{l a g, *}\left[\chi_{k}^{x, *}\right]\right)_{l m, i+1 / 2}+\sum_{i n t} \sum_{l, m}\left(S F^{l a g, *}\left[\chi_{k}^{x, *}\right]\right)_{l m, i n t}\right)
$$

The last integral $I_{3}^{\prime}$ is obtained similarly :

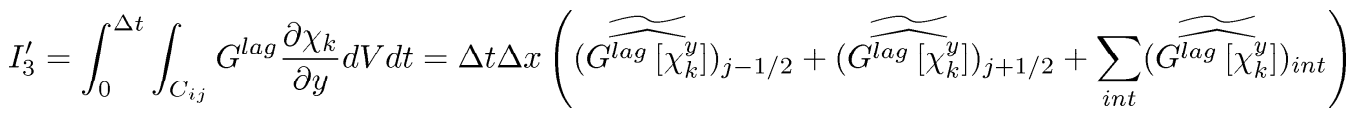

and

$$
I_{3}^{\prime}=\Delta t\left(\sum_{l, m}\left(S G^{l a g, *}\left[\chi_{k}^{y, *}\right]\right)_{l m, j-1 / 2}+\sum_{l, m}\left(S G^{l a g, *}\left[\chi_{k}^{y, *}\right]\right)_{l m, j+1 / 2}+\sum_{i n t} \sum_{l, m}\left(S G^{\text {lag, }, *}\left[\chi_{k}^{y, *}\right]\right)_{l m, \text { int }}\right)
$$

Finally, the numerical scheme (48) reads, for each fluid $k$ :

$$
\begin{aligned}
& \frac{\left(\alpha_{k}\left\{U_{k}\right\}\right)_{i j}^{n+1}-\left(\alpha_{k}\left\{U_{k}\right\}\right)_{i j}^{n}}{\Delta t}+\frac{\left.\widetilde{\left(\overline{\chi_{k} F}\right.}\right)_{i+1 / 2}-\left(\widetilde{\overline{\chi_{k} F}}\right)_{i-1 / 2}}{\Delta x}+\frac{\widetilde{\left(\widetilde{\chi_{k} G}\right)_{j+1 / 2}-\left(\widetilde{\chi_{k} G}\right)_{j-1 / 2}}}{\Delta y}
\end{aligned}
$$

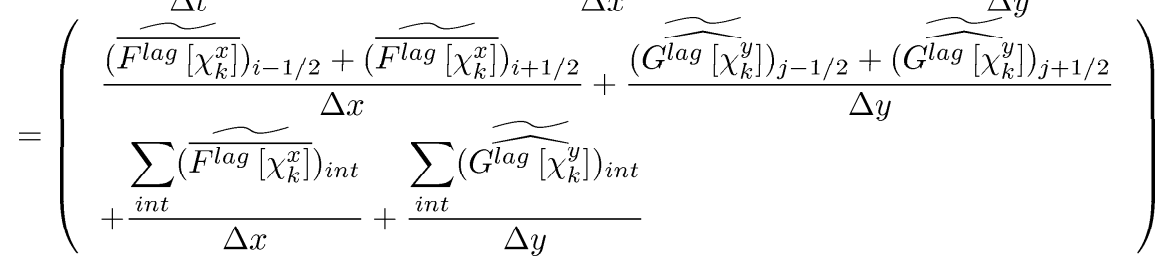

where all terms have been divided by $\Delta x \Delta y \Delta t$ and with

$$
\begin{aligned}
& \left.\left(\widetilde{\overline{\chi_{k} F}}\right)_{i \pm 1 / 2}=\frac{1}{\Delta y} \sum_{l, m}\left(S \chi_{k}^{*} F^{*}\right)_{l m, i \pm 1 / 2} \quad \widetilde{\widetilde{\left(\chi_{k} G\right.}}\right)_{j \pm 1 / 2}=\frac{1}{\Delta x} \sum_{l, m}\left(S \chi_{k}^{*} G^{*}\right)_{l m, j \pm 1 / 2} \\
& \left(\widetilde{F^{\operatorname{lag}\left[\chi_{k}^{x}\right]}}\right)_{i \pm 1 / 2}=\frac{1}{\Delta y} \sum_{l, m}\left(S F^{\text {lag }, *}\left[\chi_{k}^{x, *}\right]\right)_{l m, i \pm 1 / 2}
\end{aligned}
$$

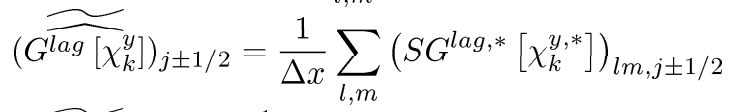

$$
\begin{aligned}
& \left.\widetilde{\left(\overline{F^{\operatorname{lag}}\left[\chi_{k}^{x}\right]}\right.}\right)_{\text {int }}=\frac{1}{\Delta y} \sum_{l, m}\left(S F^{l a g, *}\left[\chi_{k}^{x, *}\right]\right)_{l m, i n t}
\end{aligned}
$$

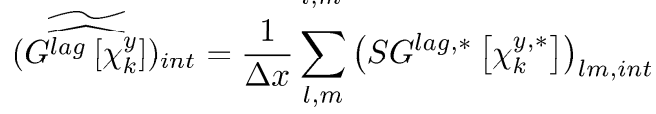




\subsubsection{Continuous Limit of the Discrete Equations Method}

The continuous limit of the discrete equations method (49) provides general closure laws : the analytical expressions of the pressure and velocity relaxation terms and the interfacial velocity and pressure variables contained in the generic system (1). This limit is obtained when $\Delta t, \Delta x$ and $\Delta y$ tends simultaneously to zero in the algebraic system (49).

This system (49) writes :

$$
T_{1}+T_{2}+T_{2}^{\prime}=T_{3}+T_{3}^{\prime}+T_{\text {int }}
$$

where $T_{i n t}$ represents the non conservative terms inside the cell $C_{i j}$.

\section{Temporal term $T_{1}$}

The temporal term $T_{1}$ writes :

$$
\lim _{\Delta x \rightarrow 0} \lim _{\Delta y \rightarrow 0} \frac{\left(\alpha_{k}\left\{U_{k}\right\}\right)_{i j}^{n+1}-\left(\alpha_{k}\left\{U_{k}\right\}\right)_{i j}^{n}}{\Delta t}=\frac{\left(\alpha_{k} U_{k}\right)^{n+1}-\left(\alpha_{k} U_{k}\right)^{n}}{\Delta t}
$$

Concerning the temporal limit, we have :

$$
\lim _{\Delta t \rightarrow 0} \frac{\left(\alpha_{k} U_{k}\right)^{n+1}-\left(\alpha_{k} U_{k}\right)^{n}}{\Delta t}=\frac{\partial \alpha_{k} U_{k}}{\partial t}
$$

Then

$$
\lim _{\Delta x \rightarrow 0} \lim _{\Delta y \rightarrow 0} \lim _{\Delta t \rightarrow 0} T_{1}=\frac{\partial \alpha_{k} U_{k}}{\partial t}
$$

Flux term $T_{2}$

$$
T_{2}=\frac{\left(\widetilde{\overline{\chi_{k} F}}\right)_{i+1 / 2}-\left(\widetilde{\overline{\chi_{k} F}}\right)_{i-1 / 2}}{\Delta x}
$$

Concerning the temporal limit we have :

$$
\lim _{\Delta t \rightarrow 0} \frac{\left(\widetilde{\overline{\chi_{k} F}}\right)_{i+1 / 2}-\left(\widetilde{\overline{\chi_{k} F}}\right)_{i-1 / 2}}{\Delta x}=\frac{\left(\overline{\chi_{k} F}\right)_{i+1 / 2}-\left(\overline{\chi_{k} F}\right)_{i-1 / 2}}{\Delta x}
$$

Using the definition, we have, at each cell boundary :

$$
\overline{\chi_{k} F}=\frac{1}{\Delta y} \int_{y_{j-1 / 2}}^{y_{j+1 / 2}} \chi_{k} F d y=\frac{S_{k}}{\Delta y} \frac{1}{S_{k}} \int_{S_{k}} F_{k} d y=\overline{\chi_{k}}\left\{F_{k}\right\}
$$

where $\left\{F_{k}\right\}$ is the fluxes average of fluid $k$ across the surface $S_{k}$.

$\overline{\chi_{k}}$ is only the surface fraction through which fluid $k$ crosses the cell from cell boundary to cell boundary.

It can be linked with the volume fraction of fluid $k$ :

$$
\lim _{\Delta y \rightarrow 0} \frac{\left(\overline{\chi_{k} F}\right)_{i+1 / 2}-\left(\overline{\chi_{k} F}\right)_{i-1 / 2}}{\Delta x}=\frac{\left(\alpha_{k} F_{k}\right)_{i+1 / 2}-\left(\alpha_{k} F_{k}\right)_{i-1 / 2}}{\Delta x}
$$

Then the following limit holds :

$$
\lim _{\Delta x \rightarrow 0} \frac{\left(\alpha_{k} F_{k}\right)_{i+1 / 2}-\left(\alpha_{k} F_{k}\right)_{i-1 / 2}}{\Delta x}=\frac{\partial \alpha_{k} F_{k}}{\partial x}
$$

Then

$$
\lim _{\Delta x \rightarrow 0} \lim _{\Delta y \rightarrow 0} \lim _{\Delta t \rightarrow 0} T_{2}=\frac{\partial \alpha_{k} F_{k}}{\partial x}
$$




\section{Flux term $T_{2}^{\prime}$}

Following the same previous ingredients, this term writes :

$$
\lim _{\Delta x \rightarrow 0} \lim _{\Delta y \rightarrow 0} \lim _{\Delta t \rightarrow 0} T_{2}^{\prime}=\frac{\partial \alpha_{k} G_{k}}{\partial y}
$$

\section{Non conservative term $T_{3}$}

$$
T_{3}=\frac{\left(\widetilde{F^{\operatorname{lag}}\left[\chi_{k}^{x}\right]}\right)_{i-1 / 2}+\left(\widetilde{F^{\operatorname{lag}\left[\chi_{k}^{x}\right]}}\right)_{i+1 / 2}}{\Delta x}
$$

The temporal limit leads to the following relation :

$$
\lim _{\Delta t \rightarrow 0} \frac{\left(\widetilde{F^{\operatorname{lag}}\left[\chi_{k}^{x}\right]}\right)_{i-1 / 2}+\left(\widetilde{F^{\operatorname{lag}}\left[\chi_{k}^{x}\right]}\right)_{i+1 / 2}}{\Delta x}=\frac{\left(\overline{F^{\operatorname{lag}}\left[\chi_{k}^{x}\right]}\right)_{i-1 / 2}+\left(\overline{F^{\operatorname{lag}}\left[\chi_{k}^{x}\right]}\right)_{i+1 / 2}}{\Delta x}
$$

At each cell boundary we have :

$$
\overline{F^{l a g}\left[\chi_{k}^{x}\right]}=\frac{1}{\Delta y} \int_{y_{j-1 / 2}}^{y_{j+1 / 2}} F^{l a g}\left[\chi_{k}^{x}\right] d y=\frac{1}{\Delta y}\left(\int_{y_{j-1 / 2}}^{y_{j+1 / 2}} F^{l a g} \chi_{k, d}^{x} d y-\int_{y_{j-1 / 2}}^{y_{j+1 / 2}} F^{l a g} \chi_{k, g}^{x} d y\right)
$$

where the relation $\left[\chi_{k}^{x}\right]=\chi_{k, d}^{x}-\chi_{k, g}^{x}$ holds representing the jump of the characteristic function between left and right states across the cell boundary. Then, denoting by $S_{k, d}$ and $S_{k, g}$ the presence surface of fluid $k$ at the right and the left of the cell boundary respectively, we get :

$$
\overline{F^{l a g}\left[\chi_{k}^{x}\right]}=\frac{1}{\Delta y}\left(\int_{S_{k, d}} F^{l a g} d y-\int_{S_{k, g}} F^{l a g} d y\right)
$$

The Lagrangian fluxes being constant through interfaces between pure fluids the following relation is deduced :

$$
\overline{F^{l a g}\left[\chi_{k}^{x}\right]}=\frac{1}{\Delta y} \int_{S_{k, d}-S_{k, g}} F^{l a g} d y=\frac{1}{\Delta y} \frac{S_{k, d}-S_{k, g}}{\left|S_{k, d}-S_{k, g}\right|} \int_{\left|S_{k, d}-S_{k, g}\right|} F^{l a g} d y
$$

where $\left|S_{k, d}-S_{k, g}\right|$ is the total contact surface through which the jump of $\chi_{k}^{x}$ is non zero. The relation becomes :

$$
\overline{F^{l a g}\left[\chi_{k}^{x}\right]}=\frac{S_{k, d}-S_{k, g}}{\Delta y} \frac{1}{\left|S_{k, d}-S_{k, g}\right|} \int_{\left|S_{k, d}-S_{k, g}\right|} F^{l a g} d y=\frac{S_{k, d}-S_{k, g}}{\Delta y}\left\{F_{k}^{l a g}\right\}
$$

where $\left\{F_{k}^{l a g}\right\}$ is the average of the Lagrangian fluxes associated to fluid $k$ in the considered cell boundary. In addition, using the definitions of the contact surfaces, we have $\frac{S_{k, d}-S_{k, g}}{\Delta y}=\alpha_{k, d}-\alpha_{k, g}$ because these surfaces are multiple of $\Delta y$.

Then, denoting by $\left[\alpha_{k}^{x}\right]$ the jump of the volume fraction of phase $k$ through the cell boundary, we get :

$$
\overline{F^{l a g}\left[\chi_{k}^{x}\right]}=\overline{\left[\chi_{k}^{x}\right]}\left\{F_{k}^{l a g}\right\}=\left[\alpha_{k}^{x}\right]\left\{F_{k}^{l a g}\right\}
$$

Now the following limit is done :

$$
\lim _{\Delta y \rightarrow 0}\left[\alpha_{k}^{x}\right]\left\{F_{k}^{l a g}\right\}=\left[\alpha_{k}^{x}\right] F_{k}^{l a g}
$$

Taking the expression of the term $T_{3}$, we have :

$$
\frac{\left(\overline{F^{\operatorname{lag}}\left[\chi_{k}^{x}\right]}\right)_{i-1 / 2}+\left(\overline{F^{\operatorname{lag}}\left[\chi_{k}^{x}\right]}\right)_{i+1 / 2}}{\Delta x}=\frac{\left(\left[\alpha_{k}^{x}\right] F_{k}^{\text {lag }}\right)_{i-1 / 2}+\left(\left[\alpha_{k}^{x}\right] F_{k}^{\text {lag }}\right)_{i+1 / 2}}{\Delta x}
$$


For each fluid $k$, the jump of $\alpha_{k}$ inside the cell is zero because $\alpha_{k}$ is assumed to be constant inside the cell. Then the preceding relation becomes :

$$
\lim _{\Delta x \rightarrow 0} \frac{\left(\left[\alpha_{k}^{x}\right] F_{k}^{l a g}\right)_{i-1 / 2}+\left(\left[\alpha_{k}^{x}\right] F_{k}^{l a g}\right)_{i+1 / 2}}{\Delta x}=\lim _{\Delta x \rightarrow 0} \frac{1}{\Delta x} \int_{x_{i-1 / 2}}^{x_{i+1 / 2}} F_{k}^{l a g} \frac{\partial \alpha_{k}}{\partial x} d x=F_{k}^{l a g} \frac{\partial \alpha_{k}}{\partial x}
$$

and

$$
\lim _{\Delta x \rightarrow 0} \lim _{\Delta y \rightarrow 0} \lim _{\Delta t \rightarrow 0} T_{3}=F_{k}^{l a g} \frac{\partial \alpha_{k}}{\partial x}
$$

Remark : the components of $F_{k}^{\text {lag }}$ in the general case may be expressed in a discontinuity of $\alpha_{k}$, for example in a cell boundary. The integration formulas of the method write in this case :

$$
F_{k}^{l a g}=\frac{\overline{F^{l a g}\left[\chi_{k}^{x}\right]}}{\overline{\left[\chi_{k}^{x}\right]}}=\frac{\sum_{l, m}\left(S F^{l a g, *}\left[\chi_{k}^{x, *}\right]\right)_{l m}}{\sum_{l, m}\left(S\left[\chi_{k}^{x, *}\right]\right)_{l m}}=\frac{\left(S F^{l a g, *}\left[\chi_{k}^{x, *}\right]\right)_{12}+\left(S F^{l a g, *}\left[\chi_{k}^{x, *}\right]\right)_{21}}{\left(S\left[\chi_{k}^{x, *}\right]\right)_{12}+\left(S\left[\chi_{k}^{x, *}\right]\right)_{21}}
$$

Using the relation $\left[\chi_{k}^{x, *}\right]_{21}=-\left[\chi_{k}^{x, *}\right]_{12}$, we get :

$$
F_{k}^{l a g}=\frac{\left(S F^{\text {lag }, *}\right)_{12}-\left(S F^{\text {lag }, *}\right)_{21}}{S_{12}-S_{21}}=\frac{1}{S_{12}-S_{21}}\left(\begin{array}{l}
-S_{12} u_{12}^{*}+S_{21} u_{21}^{*} \\
0 \\
S_{12} P_{12}^{*}-S_{21} P_{21}^{*} \\
0 \\
S_{12} P_{12}^{*} u_{12}^{*}-S_{21} P_{21}^{*} u_{21}^{*}
\end{array}\right)
$$

If $S_{12}=0$ then $S_{21} \neq 0$ necessarily using the definition of the contact surfaces between phases. In this case, we also have $\frac{\partial \alpha_{1}}{\partial x}>0$ and $\frac{\partial \alpha_{2}}{\partial x}<0$.

The preceding relation reduces to :

$$
F_{k}^{l a g}=F_{21}^{l a g, *}=\left(\begin{array}{l}
-u_{21}^{*} \\
0 \\
P_{21}^{*} \\
0 \\
P_{21}^{*} u_{21}^{*}
\end{array}\right)
$$

In a symmetric manner when $S_{12} \neq 0, S_{21}=0, \frac{\partial \alpha_{1}}{\partial x}<0$ and $\frac{\partial \alpha_{2}}{\partial x}>0$, the Lagrangian flux writes :

$$
F_{k}^{l a g}=F_{12}^{l a g, *}=\left(\begin{array}{l}
-u_{12}^{*} \\
0 \\
P_{12}^{*} \\
0 \\
P_{12}^{*} u_{12}^{*}
\end{array}\right)
$$

More generally, denoting by $f^{+}=\operatorname{Max}(0, f)$ the positive part of an arbitrary variable, the expression of the Lagrangian flux becomes :

$$
F_{k}^{l a g}=\left(s g\left(\frac{\partial \alpha_{1}}{\partial x}\right)\right)^{+} F_{21}^{l a g, *}+\left(s g\left(\frac{\partial \alpha_{2}}{\partial x}\right)\right)^{+} F_{12}^{l a g, *}
$$


or

$$
F_{k}^{l a g}=\left(\begin{array}{l}
-u_{I} \\
0 \\
P_{I, x} \\
0 \\
(P u)_{I, x}
\end{array}\right)
$$

Now the expressions of these parameters (interface velocity and pressure) are to be determined. For this, solutions from acoustic Riemann solvers are considered :

$$
\begin{aligned}
& P_{12}^{*}=\frac{Z_{1} P_{2}+Z_{2} P_{1}+Z_{1} Z_{2}\left(u_{1}-u_{2}\right)}{Z_{1}+Z_{2}} \text { and } u_{12}^{*}=\frac{Z_{1} u_{1}+Z_{2} u_{2}+P_{1}-P_{2}}{Z_{1}+Z_{2}} \\
& P_{21}^{*}=\frac{Z_{2} P_{1}+Z_{1} P_{2}+Z_{1} Z_{2}\left(u_{2}-u_{1}\right)}{Z_{1}+Z_{2}} \text { and } u_{21}^{*}=\frac{Z_{2} u_{2}+Z_{1} u_{1}+P_{2}-P_{1}}{Z_{1}+Z_{2}}
\end{aligned}
$$

where $Z=\rho c$ represents the impedance acoustic and $c$ the sound speed of the fluid. The variables $P_{1}$, $P_{2}, u_{1}$ and $u_{2}$ are also those of the considered fluid.

The general expression of the interface velocity becomes :

$$
u_{I}=\left(s g\left(\frac{\partial \alpha_{1}}{\partial x}\right)\right)^{+} u_{21}^{*}+\left(s g\left(\frac{\partial \alpha_{2}}{\partial x}\right)\right)^{+} u_{12}^{*}=\frac{Z_{1} u_{1}+Z_{2} u_{2}}{Z_{1}+Z_{2}}+s g\left(\frac{\partial \alpha_{1}}{\partial x}\right) \frac{P_{2}-P_{1}}{Z_{1}+Z_{2}}
$$

The interface pressure obeys the following relation :

$$
P_{I, x}=\left(s g\left(\frac{\partial \alpha_{1}}{\partial x}\right)\right)^{+} P_{21}^{*}+\left(s g\left(\frac{\partial \alpha_{2}}{\partial x}\right)\right)^{+} P_{12}^{*}=\frac{Z_{2} P_{1}+Z_{1} P_{2}}{Z_{1}+Z_{2}}+s g\left(\frac{\partial \alpha_{1}}{\partial x}\right) Z_{1} Z_{2} \frac{u_{2}-u_{1}}{Z_{1}+Z_{2}}
$$

Non conservative term $T_{3}^{\prime}$

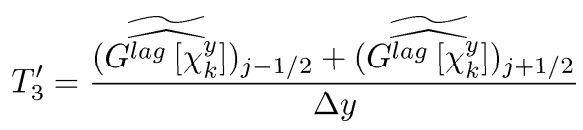

The same previous ingredients provide the relation :

$$
\lim _{\Delta y \rightarrow 0} \lim _{\Delta x \rightarrow 0} \lim _{\Delta t \rightarrow 0} T_{3}^{\prime}=G_{k}^{l a g} \frac{\partial \alpha_{k}}{\partial y}
$$

with

$$
\begin{gathered}
G_{k}^{l a g}=\left(\begin{array}{l}
-v_{I} \\
0 \\
0 \\
P_{I, y} \\
(P v)_{I, y}
\end{array}\right) \\
v_{I}=\frac{Z_{1} v_{1}+Z_{2} v_{2}}{Z_{1}+Z_{2}}+s g\left(\frac{\partial \alpha_{1}}{\partial y}\right) \frac{P_{2}-P_{1}}{Z_{1}+Z_{2}} \\
P_{I, y}=\frac{Z_{2} P_{1}+Z_{1} P_{2}}{Z_{1}+Z_{2}}+s g\left(\frac{\partial \alpha_{1}}{\partial y}\right) Z_{1} Z_{2} \frac{v_{2}-v_{1}}{Z_{1}+Z_{2}}
\end{gathered}
$$

Internal term $T_{\text {int }}$

$$
T_{i n t}=\frac{\sum_{i n l}\left(\widetilde{F^{\operatorname{lag}\left[\chi_{k}^{x}\right]}}\right)_{i n t}}{\Delta x}+\frac{\sum_{i n t}\left(\widehat{\left.G^{\operatorname{lag}\left[\chi_{k}^{y}\right.}\right]}\right)_{i n t}}{\Delta y}
$$


This term involves interactions between fluids inside the cell.

The temporal limit leads to :

$$
\lim _{\Delta t \rightarrow 0} T_{i n t}=\frac{\sum_{i n t}\left(\overline{F^{\operatorname{lag}}\left[\chi_{k}^{x}\right]}\right)_{i n t}}{\Delta x}+\frac{\sum_{i n t}\left(\widehat{\left.G^{\text {lag }\left[\chi_{k}^{y}\right.}\right]}\right)_{i n t}}{\Delta y}
$$

Using the definitions of the averages we get :

$$
\frac{\sum_{\text {int }}\left(\overline{F^{\text {lag }}\left[\chi_{k}^{x}\right]}\right)_{i n t}}{\Delta x}=\frac{\sum_{\text {int }}\left(\left(S F^{\text {lag }, *}\left[\chi_{k}^{x, *}\right]\right)_{12}+\left(S F^{\text {lag }, *}\left[\chi_{k}^{x, *}\right]\right)_{21}\right)_{i n t}}{\Delta x \Delta y}
$$

The relation $\left[\chi_{k}^{x, *}\right]_{21}=-\left[\chi_{k}^{x, *}\right]_{12}$ leads to :

$$
\frac{\sum_{\text {int }}\left(\overline{F^{l a g}\left[\chi_{k}^{x}\right]}\right)_{i n t}}{\Delta x}=\left[\chi_{k}^{x, *}\right]_{12} \frac{\sum_{\text {int }}\left(\left(S F^{\text {lag }, *}\right)_{12}-\left(S F^{\text {lag }, *}\right)_{21}\right)_{i n t}}{\Delta x \Delta y}
$$

The Lagrangian fluxes $F_{12}^{l a g, *}$ and $F_{21}^{l a g, *}$ being constant through interfaces between pure fluids inside the cell, the preceding relation writes :

$$
\frac{\sum_{\text {int }}\left(\overline{F^{l a g}\left[\chi_{k}^{x}\right]}\right)_{i n t}}{\Delta x}=\left[\chi_{k}^{x, *}\right]_{12} \frac{F_{12}^{\text {lag,* }} \sum_{\text {int }} S_{12, \text { int }}-F_{21}^{\text {lag }, *} \sum_{\text {int }} S_{21, i n t}}{\Delta x \Delta y}
$$

In this expression, $\sum_{i n t} S_{12, i n t}$ (resp. $\sum_{\text {int }} S_{21, i n t}$ ) represents the strictly internal total surface for which fluid 1 (resp. 2) at the left is in contact with fluid 2 (resp. 1) at the right.

Then the relation $\sum_{\text {int }} S_{12, \text { int }}=\sum_{\text {int }} S_{21, \text { int }}$ holds if symmetric entities are considerd inside the numerical cell (bubbles for example).

Denoting by $S_{x}=\sum_{\text {int }} S_{12, \text { int }}+\sum_{\text {int }} S_{21, \text { int }}=2 \sum_{\text {int }} S_{12, \text { int }}$ the total contact surface between fluids 1 and 2 inside the cell according to the $x$ direction and $\left[\chi_{k}^{x, *}\right]_{12}=\delta_{k 2}-\delta_{k 1}\left(\delta_{k l}\right.$ being the Kronecker symbol), we get :

$$
\frac{\sum_{\text {int }}\left(\overline{F^{l a g}\left[\chi_{k}^{x}\right]}\right)_{\text {int }}}{\Delta x}=\frac{\left(\delta_{k 2}-\delta_{k 1}\right) S_{x}}{2 \Delta x \Delta y}\left(F_{12}^{l a g, *}-F_{21}^{l a g, *}\right)=\frac{\left(\delta_{k 2}-\delta_{k 1}\right) S_{x}}{2 \Delta x \Delta y}\left(\begin{array}{l}
-u_{12}^{*}+u_{21}^{*} \\
0 \\
P_{12}^{*}-P_{21}^{*} \\
0 \\
(P u)_{12}^{*}-(P u)_{21}^{*}
\end{array}\right)
$$

Taking again the expressions of the pressure and velocity from the acoustic Riemann solver :

$$
\begin{aligned}
& P_{12}^{*}=\frac{Z_{1} P_{2}+Z_{2} P_{1}+Z_{1} Z_{2}\left(u_{1}-u_{2}\right)}{Z_{1}+Z_{2}} \text { et } u_{12}^{*}=\frac{Z_{1} u_{1}+Z_{2} u_{2}+P_{1}-P_{2}}{Z_{1}+Z_{2}} \\
& P_{21}^{*}=\frac{Z_{2} P_{1}+Z_{1} P_{2}+Z_{1} Z_{2}\left(u_{2}-u_{1}\right)}{Z_{1}+Z_{2}} \text { et } u_{21}^{*}=\frac{Z_{2} u_{2}+Z_{1} u_{1}+P_{2}-P_{1}}{Z_{1}+Z_{2}}
\end{aligned}
$$

Denoting $\Delta P=P_{2}-P_{1}$ and $\Delta u=u_{1}-u_{2}$, the following relations are easily obtained :

$$
\begin{gathered}
-u_{12}^{*}+u_{21}^{*}=\frac{2}{Z_{1}+Z_{2}} \Delta P \\
P_{12}^{*}-P_{21}^{*}=\frac{2 Z_{1} Z_{2}}{Z_{1}+Z_{2}} \Delta u
\end{gathered}
$$




$$
(P u)_{12}^{*}-(P u)_{21}^{*}=\frac{2 Z_{1} Z_{2}}{Z_{1}+Z_{2}} \frac{Z_{1} u_{1}+Z_{2} u_{2}}{Z_{1}+Z_{2}} \Delta u-\frac{2}{Z_{1}+Z_{2}} \frac{Z_{1} P_{2}+Z_{2} P_{1}}{Z_{1}+Z_{2}} \Delta P
$$

Then :

$$
\frac{\sum_{\text {int }}\left(\overline{F^{l a g}\left[\chi_{k}^{x}\right]}\right)_{i n t}}{\Delta x}=\frac{\left(\delta_{k 2}-\delta_{k 1}\right) S_{x}}{\Delta x \Delta y\left(Z_{1}+Z_{2}\right)}\left(\begin{array}{l}
\Delta P \\
0 \\
Z_{1} Z_{2} \Delta u \\
0 \\
Z_{1} Z_{2} \frac{Z_{1} u_{1}+Z_{2} u_{2}}{Z_{1}+Z_{2}} \Delta u-\frac{Z_{1} P_{2}+Z_{2} P_{1}}{Z_{1}+Z_{2}} \Delta P
\end{array}\right)
$$

Using the same hypothesis in the $y$ direction, we have :

$$
\frac{\left.\sum_{\text {int }}\left(G^{\text {lag }\left[\chi_{k}^{y}\right.}\right]\right)_{\text {int }}}{\Delta y}=\frac{\left(\delta_{k 2}-\delta_{k 1}\right) S_{y}}{\Delta x \Delta y\left(Z_{1}+Z_{2}\right)}\left(\begin{array}{l}
\Delta P \\
0 \\
0 \\
Z_{1} Z_{2} \Delta v \\
Z_{1} Z_{2} \frac{Z_{1} v_{1}+Z_{2} v_{2}}{Z_{1}+Z_{2}} \Delta v-\frac{Z_{1} P_{2}+Z_{2} P_{1}}{Z_{1}+Z_{2}} \Delta P
\end{array}\right)
$$

where $S_{y}$ represents the total contact surface between fluids insid the cell according to the $y$ direction and $\Delta v=v_{1}-v_{2}$.

Now denoting by $S_{v x}=\frac{S_{x}}{\Delta x \Delta y}$ and $S_{v y}=\frac{S_{y}}{\Delta x \Delta y}$ the contact surfaces per volume unit in the two directions, as well as $S_{v}=S_{v x}+S_{v y}$ the total contact surface per volume unit, the spatial limit leads easily to the following relation :

$$
\lim _{\Delta x \rightarrow 0} \lim _{\Delta y \rightarrow 0} \lim _{\Delta t \rightarrow 0} T_{i n t}=\left(\delta_{k 2}-\delta_{k 1}\right)\left(\begin{array}{l}
\mu \Delta P \\
0 \\
\lambda \Delta u \\
\nu \Delta v \\
\lambda \overline{u_{I}} \Delta u+\nu \overline{v_{I}} \Delta v-\mu \overline{P_{I}} \Delta P
\end{array}\right)
$$

where

$$
\begin{gathered}
\mu=\frac{S_{v}}{Z_{1}+Z_{2}} \quad \lambda=S_{v x} \frac{Z_{1} Z_{2}}{Z_{1}+Z_{2}} \quad \nu=S_{v y} \frac{Z_{1} Z_{2}}{Z_{1}+Z_{2}} \\
\overline{P_{I}}=\frac{Z_{1} P_{2}+Z_{2} P_{1}}{Z_{1}+Z_{2}} \quad \overline{u_{I}}=\frac{Z_{1} u_{1}+Z_{2} u_{2}}{Z_{1}+Z_{2}} \quad \overline{v_{I}}=\frac{Z_{1} v_{1}+Z_{2} v_{2}}{Z_{1}+Z_{2}}
\end{gathered}
$$


All the preceding developments concerning the different terms lead to a well known partial differential system (1) presented in introduction :

$$
\left\{\begin{array}{l}
\frac{\partial \alpha_{k}}{\partial t}+u_{I} \frac{\partial \alpha_{k}}{\partial x}+v_{I} \frac{\partial \alpha_{k}}{\partial y}=\left(\delta_{k 2}-\delta_{k 1}\right) \mu \Delta P \\
\frac{\partial(\alpha \rho)_{k}}{\partial t}+\frac{\partial(\alpha \rho u)_{k}}{\partial x}+\frac{\partial(\alpha \rho v)_{k}}{\partial y}=0 \\
\frac{\partial(\alpha \rho u)_{k}}{\partial t}+\frac{\partial\left(\alpha\left(\rho u^{2}+P\right)\right)_{k}}{\partial x}+\frac{\partial(\alpha \rho u v)_{k}}{\partial y}=P_{I x} \frac{\partial \alpha_{k}}{\partial x}+\left(\delta_{k 2}-\delta_{k 1}\right) \lambda \Delta u \\
\frac{\partial(\alpha \rho v)_{k}}{\partial t}+\frac{\partial(\alpha \rho u v)_{k}}{\partial x}+\frac{\partial\left(\alpha\left(\rho v^{2}+P\right)\right)_{k}}{\partial y}=P_{I y} \frac{\partial \alpha_{k}}{\partial y}+\left(\delta_{k 2}-\delta_{k 1}\right) \nu \Delta v \\
(P u)_{I x} \frac{\partial \alpha_{k}}{\partial x}+(P v)_{I y} \frac{\partial \alpha_{k}}{\partial y} \\
+\left(\delta_{k 2}-\delta_{k 1}\right)\left(\lambda \overline{u_{I}} \Delta u+\nu \overline{v_{I}} \Delta v\right) \\
-\left(\delta_{k 2}-\delta_{k 1}\right) \mu \overline{P_{I}} \Delta P
\end{array}\right)
$$

with the following expressions of the different variables :

$$
\begin{gathered}
\mu=\frac{S_{v}}{Z_{1}+Z_{2}} \quad \lambda=S_{v x} \frac{Z_{1} Z_{2}}{Z_{1}+Z_{2}} \quad \nu=S_{v y} \frac{Z_{1} Z_{2}}{Z_{1}+Z_{2}} \\
\overline{P_{I}}=\frac{Z_{1} P_{2}+Z_{2} P_{1}}{Z_{1}+Z_{2}} \quad \overline{u_{I}}=\frac{Z_{1} u_{1}+Z_{2} u_{2}}{Z_{1}+Z_{2}} \quad \overline{v_{I}}=\frac{Z_{1} v_{1}+Z_{2} v_{2}}{Z_{1}+Z_{2}} \\
u_{I}=\overline{u_{I}}+s g\left(\frac{\partial \alpha_{1}}{\partial x}\right) \frac{\Delta P}{Z_{1}+Z_{2}} \quad P_{I, x}=\overline{P_{I}}-s g\left(\frac{\partial \alpha_{1}}{\partial x}\right) Z_{1} Z_{2} \frac{\Delta u}{Z_{1}+Z_{2}} \\
v_{I}=\overline{v_{I}}+s g\left(\frac{\partial \alpha_{1}}{\partial y}\right) \frac{\Delta P}{Z_{1}+Z_{2}} \quad P_{I, y}=\overline{P_{I}}-s g\left(\frac{\partial \alpha_{1}}{\partial y}\right) Z_{1} Z_{2} \frac{\Delta v}{Z_{1}+Z_{2}}
\end{gathered}
$$

The analytical expressions of coefficients and interfacial variables present in system (50) have been obtained with the help of the acoustic Riemann solver. Following the same ingredients the aim of this work is now to use the linearized reactive Riemann solver and the reactive discrete equations method to obtain analytical expressions of mass tranfer terms between phases when evaporation occurs. This is the topic of the following section.

\subsection{Implementation of the Reactive Riemann Solver into the DEM}

In the Figure 3, we have represented an example of waves pattern in a $(x, t)$ diagram representing the solution of the reactive Riemann problem associated to an evaporation front. An additional wave appears compared to the non reactive case : the evaporation front. This one is a discontinuity separating the superheated liquid and its pure vapor.

In addition, such a front obeys the Rankine-Hugoniot relations $[F-\sigma U]=0$ ( $\sigma$ is the front velocity) where $F$ and $U$ represent respectively the flux and the conservative variables vector of pure fluid Euler equations.

Thus two fundamental informations are available :

- the evaporation front is the location where the phase functions have jumps,

- the front obeys the Rankine-Hugoniot relations $[F-\sigma U]=0$. 
Indeed, the dynamic vapor appearance behind the front creates a jump of its phase function. Then a jump of the liquid phase function is created which is opposite to the vapor one. The terms $F-\sigma U$ associated to evaporation fronts, named reactive fluxes, have similar effects and properties as Lagrangian fluxes corresponding to the particular case $\sigma=u$. These reactive fluxes are constant across phase functions discontinuities.

It is also important to note that the dynamic vapor appearance behind the front will modify the jumps of the phase functions across the contact discontinuity as represented in the Figure 7.

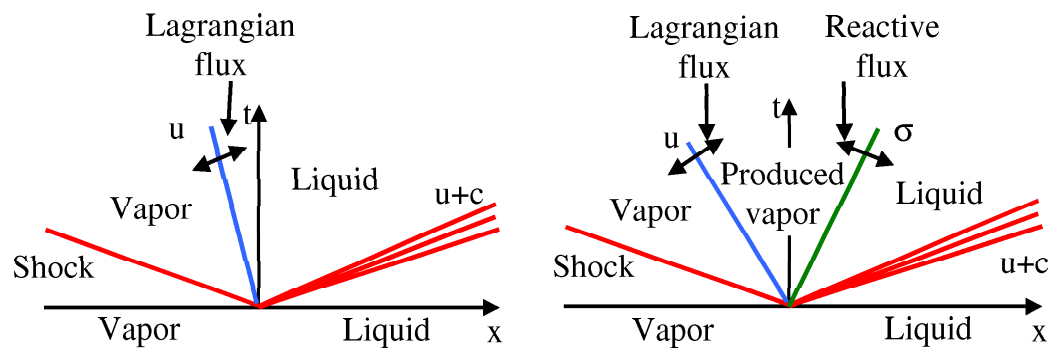

Figure 7: Resolution of the inert (left) and the reactive (right) Riemann problem

Indeed, in the inert case, the evaporation front is absent and only the fluids initially present are recovered on both sides of the contact discontinuity. In the reactive case, vapor appears at the contact discontinuity. Then the Lagrangian fluxes are modified as well as the jumps of the phase functions, as shown in the Figure 7 . The jumps are zero at the contact discontinuity for the situation depicted on this figure. The same type of remarks holds at the front level.

For the sake of clarity, we adopt some notations indicating if the resolution of a Riemann problem is inert or reactive. The Riemann problem solution corresponds to the inert one when the liquid is not superheated, i.e. if the 'reactivity' test $\left(P^{*}<P_{s a t}\left(T^{*}\right)\right)$ is not fulfilled. The different fluxes and the associated phase functions will be given in accordance with these notations, described in the Table iii.

\begin{tabular}{|c|c|}
\hline Notations & Significations \\
\hline$(R)$ & reactive Riemann problem \\
\hline$(I)$ & inert Riemann problem \\
\hline
\end{tabular}

Table iii: Notations associated to the Riemann problems resolution

In addition, in the particular Riemann problem $(1-1)$, two evaporation fronts may appear. They will be denoted by the subscripts $l$ and $r$ when they are facing respectively to the left and to the right.

Now we consider the integrals of equation (48) that must be determined in the reactive case.

The temporal term $I_{1}$ remains unchanged while the convective fluxes $I_{2}$ and $I_{2}^{\prime}$ and the non-conservative terms $I_{3}$ and $I_{3}^{\prime}$ have to be modified. 


\subsubsection{Integration of the Convective Fluxes $I_{2}$ and $I_{2}^{\prime}$}

The dynamic vapor appearance changes the values of the phase function $\chi_{k}^{*}$. For example, the solution of the reactive Riemann problem represented in the Figure 7 indicates that vapor is present at $x / t=0$ while the associated inert solution leads to the presence of liquid.

Assuming fluid 1 is a liquid and fluid 2 is its associated vapor, the values of $\chi_{1}^{*}$ in the Table i must be modified when the Riemann problems are reactive. Then, the sampling at $x / t=0$ is done with respect to $u^{*}$ (contact discontinuity velocity) and $\sigma^{*}$ (front velocity) as represented in the Table iv.

\begin{tabular}{|c|c|c|c|c|}
\hline Contact & Surface & Eulerian Flux & Type & Phase function $\chi_{1}^{*}$ \\
\hline \multirow{4}{*}{$1-1$} & \multirow{4}{*}{$S_{11}$} & \multirow{4}{*}{$F_{11}^{*}$} & $(I)$ & $\chi_{1,11}^{*}=1$ \\
\hline & & & $(R)_{l}$ & $\begin{array}{l}0 \text { if } \sigma_{11, l}^{*}<0<u_{11}^{*} \\
1 \text { otherwise }\end{array}$ \\
\hline & & & $(R)_{r}$ & $\begin{array}{l}0 \text { if } u_{11}^{*}<0<\sigma_{11, r}^{*} \\
1 \text { otherwise }\end{array}$ \\
\hline & & & $\begin{array}{l}(R)_{l} \\
(R)_{r}\end{array}$ & $\begin{array}{l}0 \text { if } \sigma_{11, l}^{*}<0<\sigma_{11, r}^{*} \\
1 \text { otherwise }\end{array}$ \\
\hline \multirow[t]{2}{*}{$1-2$} & \multirow[t]{2}{*}{$S_{12}$} & \multirow[t]{2}{*}{$F_{12}^{*}$} & $(I)$ & $\chi_{1,12}^{*}=\left\{\begin{array}{l}1 \text { if } u_{12}^{*}>0 \\
0 \text { otherwise }\end{array}\right.$ \\
\hline & & & $(R)$ & $\begin{array}{l}1 \text { if } \sigma_{12}^{*}>0 \\
0 \text { otherwise }\end{array}$ \\
\hline \multirow[t]{2}{*}{$2-1$} & \multirow[t]{2}{*}{$S_{21}$} & \multirow[t]{2}{*}{$F_{21}^{*}$} & $(I)$ & $\begin{array}{l}1 \text { if } u_{21}^{*}<0 \\
0 \text { otherwise }\end{array}$ \\
\hline & & & $(R)$ & $\chi_{1,21}^{*}=\left\{\begin{array}{l}1 \text { if } \sigma_{21}^{*}<0 \\
0 \text { otherwise }\end{array}\right.$ \\
\hline $2-2$ & $S_{22}$ & $F_{22}^{*}$ & $(I)$ & $\chi_{1,22}^{*}=0$ \\
\hline
\end{tabular}

Table iv: The different configurations for Eulerian fluxes at cell boundary $i-1 / 2$ for fluid $k=1$ in the presence of evaporation fronts

When vapor is present initially on both sides of the contact (last line of Table iv), the phase function of fluid 1 is necessary 0 .

When fluid 1 is present on one side only, two instances have to be considered. This fluid may remain liquid, the inert Riemann problem thus provides the same solution regarding $\chi_{1}^{*}$ and $F^{*}$ as in Table i (lines 5 and 7 of Table iv). When fluid 1 becomes superheated, vapor is produced behind the front.

Thus, when fluid 1 is initially on the left and the front is entering the cell, $\chi_{1}^{*}=1$ (line 6 of Table iv). Such instance occurs when the liquid velocity is positive and larger than the front velocity that faces to left. Symmetrically, when fluid 1 is initially on the right and the front is leaving the cell, $\chi_{1}^{*}=1$ (line 8 of Table iv). Such case occurs when the liquid velocity is negative and larger than the front velocity that faces to right. The first line of Table iv corresponds to the case with two inert liquids in contact already 
examined in Table i. The three next cases are more complex.

We consider first the case where only the liquid on the left is superheated (line 2 of Table iv) corresponding to a reactive front facing to left $(R)_{l}$. In this case, the contact discontinuity separates vapor produced behind the front on the left and inert liquid on the right. When the front velocity is negative and the velocity of the contact discontinuity is positive, then only vapor is present along the $x / t=0$ axis. Otherwise, fluid 1 crosses the cell boundary.

The third line of Table iv summarizes the symmetric instance (evaporation front facing to right). The fourth line of Table iv summarizes the instance of two reactive fronts facing respectively to left and right producing both pure vapor.

Taking into account all instances summarized in Table iv, the formulas of the convective fluxes remain unchanged :

$$
\begin{aligned}
& I_{2}=\Delta t \Delta y\left(\widetilde{\left(\widetilde{\chi_{k} F}\right)_{i+1 / 2}}-\left(\widetilde{\widetilde{\chi_{k} F}}\right)_{i-1 / 2}\right)=\Delta t\left(\sum_{l, m}\left(S \chi_{k}^{*} F^{*}\right)_{l m, i+1 / 2}-\sum_{l, m}\left(S \chi_{k}^{*} F^{*}\right)_{l m, i-1 / 2}\right)
\end{aligned}
$$

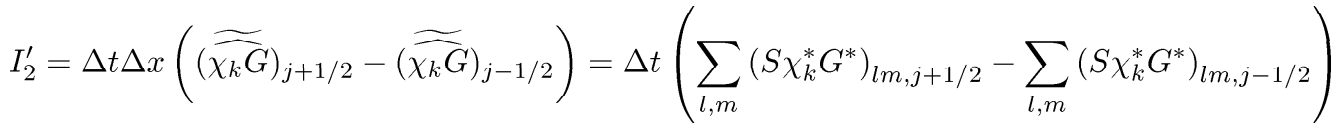

\subsubsection{Integration of the Non-Conservative Terms $I_{3}$ and $I_{3}^{\prime}$}

Contrary to convective fluxes, the integration of the terms $\int_{y_{j-1 / 2}}^{y_{j+1 / 2}}(F-\sigma U)\left[\chi_{k}^{x}\right] d y$ on each cell boundary leads to additional terms named reactive fluxes.

For each reactive Riemann problem (see the Figure 8), this integral may be splitted in two types of terms.
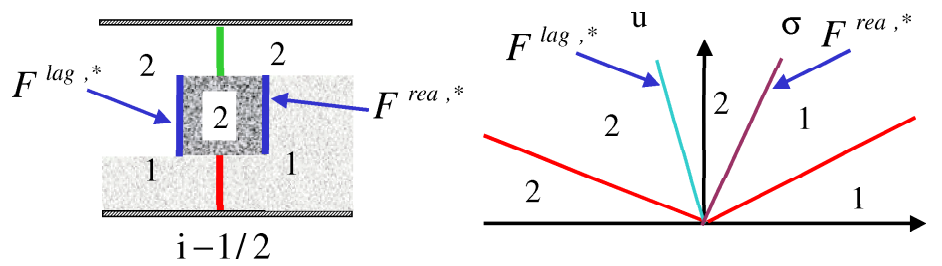

Figure 8: Lagrangian and reactive fluxes in the Riemann problem $(2-1)$

The first one comes from the contact discontinuity (Lagrangian fluxes) and the second one is associated to the presence of the reactive front (reactive fluxes) :

$$
\int_{y_{j-1 / 2}}^{y_{j+1 / 2}}(F-\sigma U)\left[\chi_{k}^{x}\right] d y=\sum_{l, m} S_{l m}\left(F^{l a g, *}\left[\chi_{k}^{x}\right]^{*}+F^{r e a, *}\left[\chi_{k}^{x}\right]^{*}\right)_{l m}
$$

Integrations are possible because the Lagrangian and the transfer fluxes are uniform across contact discontinuities and reactive fronts respectively :

$$
F^{l a g}=[-u, 0, P, 0, P u]^{T} \quad F^{r c a}=[-\sigma, m, m u+P, m u v, m E+P u]^{T}
$$

where $m=\rho(u-\sigma)$ is the mass flow rate across the reactive front.

The Lagrangian fluxes and the associated jumps of the phase function $\chi_{1}$ across the contact discontinuities 
are summarized in the Table $\mathrm{v}$ for cell boundary $i-1 / 2$.

\begin{tabular}{|c|c|c|c|c|}
\hline Contact & Surface & Lagrangian Flux & Type & $\operatorname{Jump}\left[\chi_{1}^{x}\right]^{*}$ \\
\hline \multirow{4}{*}{$1-1$} & \multirow{4}{*}{$S_{11}$} & \multirow{4}{*}{$F_{11}^{l a g, *}$} & $(I)$ & {$\left[\chi_{1}^{x}\right]_{11}^{*}=0$} \\
\hline & & & $(R)_{l}$ & {$\left[\chi_{1}^{x}\right]_{11}^{*}=\left\{\begin{array}{l}1 \text { if } u_{11}^{*}>0 \\
0 \text { otherwise }\end{array}\right.$} \\
\hline & & & $(R)_{r}$ & {$\left[\chi_{1}^{x}\right]_{11}^{*}=\left\{\begin{array}{l}-1 \text { if } u_{11}^{*}>0 \\
0 \text { otherwise }\end{array}\right.$} \\
\hline & & & $\begin{array}{l}(R)_{l} \\
(R)_{r}\end{array}$ & {$\left[\chi_{1}^{x}\right]_{11}^{*}=0$} \\
\hline \multirow[t]{2}{*}{$1-2$} & \multirow[t]{2}{*}{$S_{12}$} & \multirow[t]{2}{*}{$F_{12}^{l a g, *}$} & $(I)$ & $\begin{array}{l}-1 \text { if } u_{12}^{*}>0 \\
0 \text { otherwise }\end{array}$ \\
\hline & & & $(R)$ & {$\left[\chi_{1}^{x}\right]_{12}^{*}=0$} \\
\hline \multirow[t]{2}{*}{$2-1$} & \multirow[t]{2}{*}{$S_{21}$} & \multirow[t]{2}{*}{$F_{21}^{l a g, *}$} & $(I)$ & $=\left\{\begin{array}{l}1 \text { if } u_{21}^{*}>0 \\
0 \text { otherwise }\end{array}\right.$ \\
\hline & & & $(R)$ & {$\left[\chi_{1}^{x}\right]_{21}^{*}=0$} \\
\hline $2-2$ & $S_{22}$ & $F_{22}^{\text {lag,* }}$ & $(I)$ & {$\left[\chi_{1}^{x}\right]_{22}^{*}=0$} \\
\hline
\end{tabular}

Table v: The different configurations for Lagrangian fluxes at cell boundary $i-1 / 2$ for fluid $k=1$ in the presence of total evaporation fronts

We detail the first type of contact $(1-1)$ that is the most complicated. The first line of Table v corresponds to the absence of reactive fronts. Fluid 1 remaining present on both sides of the contact discontinuity, the jump of its phase function is necessarily zero. Similarly, when two fronts are present (line 4 of Table v) there is no jump of the phase function since vapor is present on both sides of the contact discontinuity. We now consider presence of superheated liquid only on the left (line 2 of Table v). When the velocity of the contact discontinuity, separating the produced vapor on the left and the inert liquid on the right, is negative, no jump of the phase function is present inside the cell. Otherwise, this jump is necessarily 1. Symmetrically, when the superheated liquid lies on the right only (line 3 of Table v) and the velocity of the contact discontinuity is positive, the jump is -1 since the produced vapor is on the right and the inert liquid is on the left. The other instances of Table $\mathrm{v}$ follow the same logic.

When reactive Riemann problems are considered, we can notice that the jump of the phase functions are zero in most cases, contrarily to the inert case (see the Table ii). Thus most interactions between fluids (liquid and vapor) are carried out from contact discontinuities to reactive fronts. The different configurations concerning these additional reactive fluxes are summarized in the Table vi.

We detail the situations that appear when fluid 1 is initially on both sides of the contact. When a reactive front is present and faces to left (line 1 of Table vi), the jump of the phase function is -1 when the front velocity is positive. Otherwise, the jump is zero. Symmetrically, when a front faces to right (line 2 of Table vi), the jump is 1 when the front velocity is positive. The same conclusions provide the data of 


\begin{tabular}{|c|c|c|c|c|}
\hline Contact & Surface & Type & Reactive Flux & Jump $\left[\chi_{1}^{x}\right]^{*}$ \\
\hline $1-1$ & $S_{11}$ & $(R)_{l}$ & $F_{11, l}^{\text {rea, } *}$ & {$\left[\chi_{1}^{x}\right]_{11, l}^{*}=\left\{\begin{array}{l}-1 \text { if } \sigma_{11, l}^{*}>0 \\
0 \text { otherwise }\end{array}\right.$} \\
\cline { 3 - 5 } & $(R)_{r}$ & $F_{11, r}^{\text {rea,*}}$ & {$\left[\chi_{1}^{x}\right]_{11, r}^{*}=\left\{\begin{array}{l}1 \text { if } \sigma_{11, r}^{*}>0 \\
0 \text { otherwise }\end{array}\right.$} \\
\hline $1-2$ & $S_{12}$ & $(R)$ & $F_{12}^{\text {rea,*}}$ & {$\left[\chi_{1}^{x}\right]_{12}^{*}=\left\{\begin{array}{l}-1 \text { if } \sigma_{12}^{*}>0 \\
0 \text { otherwise }\end{array}\right.$} \\
\hline $2-1$ & $S_{21}$ & $(R)$ & $F_{21}^{\text {rea,*}}$ & {$\left[\chi_{1}^{x}\right]_{21}^{*}=\left\{\begin{array}{l}1 \text { if } \sigma_{21}^{*}>0 \\
0 \text { otherwise }\end{array}\right.$} \\
\hline
\end{tabular}

Table vi: The different configurations for reactive fluxes at cell boundary $i-1 / 2$ for fluid $k=1$ in the presence of total evaporation fronts

lines 3 and 4 of Table vi.

The large number of configurations mentioned previously in the different tables shows the complexity of the coupling between the reactive Riemann problem and the discrete equations method. According to the solutions provided by the resolution of the Riemann problem, additional terms appear and modify the terms present initially in the numerical scheme (49).

Then, the numerical scheme of the reactive discrete equations method (RDEM) reads for each fluid $k$ :

$$
\begin{aligned}
& \frac{\left(\alpha_{k}\left\{U_{k}\right\}\right)_{i j}^{n+1}-\left(\alpha_{k}\left\{U_{k}\right\}\right)_{i j}^{n}}{\Delta t}+\frac{\left(\widetilde{\overline{\chi_{k} F}}\right)_{i+1 / 2}-\left(\widetilde{\overline{\chi_{k} F}}\right)_{i-1 / 2}}{\Delta x}+\frac{\left(\widetilde{\widetilde{\chi_{k} G}}\right)_{j+1 / 2}-\left(\widetilde{\widetilde{\chi_{k} G}}\right)_{j-1 / 2}}{\Delta y}
\end{aligned}
$$

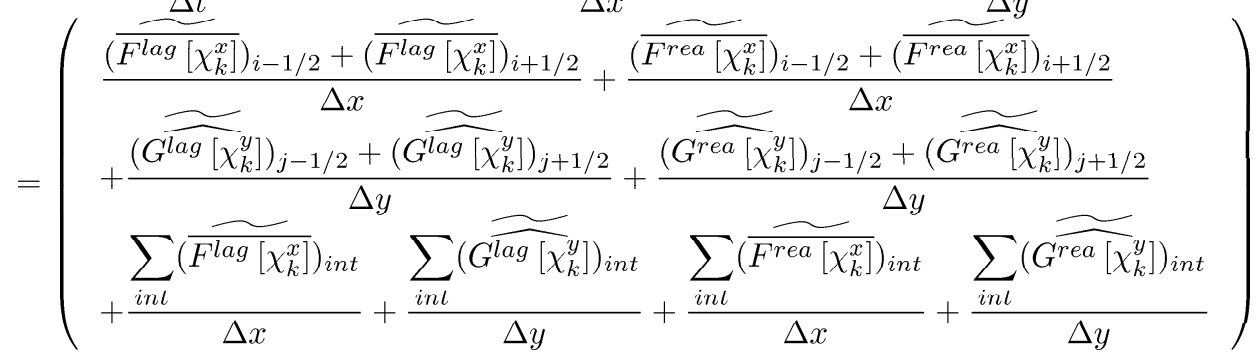

where

$$
\begin{aligned}
& \left.\left.\widetilde{\overline{\left(\chi_{k} F\right.}}\right)_{i \pm 1 / 2}=\frac{1}{\Delta y} \sum_{l, m}\left(S \chi_{k}^{*} F^{*}\right)_{l m, i \pm 1 / 2} \quad \widetilde{\widetilde{\left(\chi_{k} G\right.}}\right)_{j \pm 1 / 2}=\frac{1}{\Delta x} \sum_{l, m}\left(S \chi_{k}^{*} G^{*}\right)_{l m, j \pm 1 / 2} \\
& \widetilde{\left(\frac{F^{\text {lag }}\left[\chi_{k}^{x}\right]}{]}\right)_{i \pm 1 / 2}}=\frac{1}{\Delta y} \sum_{l, m}\left(S F^{l a g, *}\left[\chi_{k}^{x, *}\right]\right)_{l m, i \pm 1 / 2} \quad \widetilde{\left(\frac{F^{\text {rea }}\left[\chi_{k}^{x}\right]}{i \pm 1 / 2}\right.}=\frac{1}{\Delta y} \sum_{l, m}\left(S F^{\text {rea }, *}\left[\chi_{k}^{x, *}\right]\right)_{l m, i \pm 1 / 2} \\
& \left(\widetilde{\left.\left.G^{\operatorname{lag}\left[\chi_{k}^{y}\right.}\right]\right)_{j \pm 1 / 2}}=\frac{1}{\Delta x} \sum_{l, m}\left(S G^{l a g, *}\left[\chi_{k}^{y, *}\right]\right)_{l m, j \pm 1 / 2} \quad \widetilde{\widetilde{G^{\text {rea }}\left[\chi_{k}^{y}\right]}}\right)_{j \pm 1 / 2}=\frac{1}{\Delta x} \sum_{l, m}\left(S G^{r e a, *}\left[\chi_{k}^{y, *}\right]\right)_{l m, j \pm 1 / 2}
\end{aligned}
$$

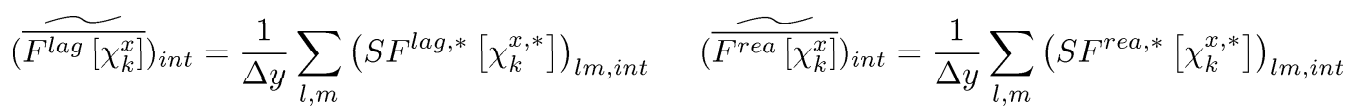

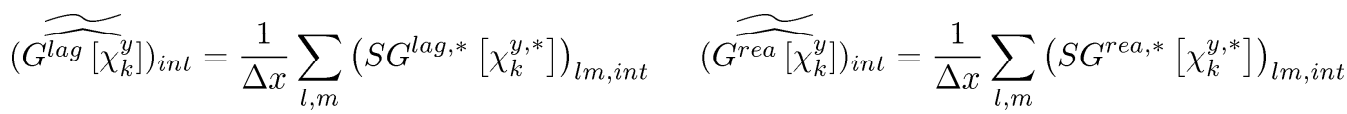




\subsubsection{Continuous Limit of the reactive Discrete Equations Method}

The continuous limit of the reactive discrete equations method (51) provides additional general closure laws : new analytical expressions of pressure and velocity relaxation terms, interfacial velocity and pressure variables as well as mass transfer terms. The determination of these terms of fundamental importance is the aim of this report. This limit is obtained when $\Delta t, \Delta x$ and $\Delta y$ tends simultaneously to zero in the algebraic system (51).

System (51) reads :

$$
T_{1}+T_{2}+T_{2}^{\prime}=T_{3}+T_{3}^{r e a}+T_{3}^{\prime}+T_{3}^{\prime r e a}+T_{i n t}+T_{i n t}^{r e a}
$$

where $T_{i n t}$ and $T_{i n t}^{r e a}$ represent the non conservative terms, inert and reactive respectively, inside the cell $C_{i j}$.

The limit of the temporal and the convective terms remains unchanged :

\section{Temporal term $T_{1}$}

$$
\lim _{\Delta x \rightarrow 0} \lim _{\Delta y \rightarrow 0} \lim _{\Delta t \rightarrow 0} T_{1}=\frac{\partial \alpha_{k} U_{k}}{\partial t}
$$

Flux term $T_{2}$

$$
\lim _{\Delta x \rightarrow 0} \lim _{\Delta y \rightarrow 0} \lim _{\Delta t \rightarrow 0} T_{2}=\frac{\partial \alpha_{k} F_{k}}{\partial x}
$$

Flux term $T_{2}^{\prime}$

$$
\lim _{\Delta x \rightarrow 0} \lim _{\Delta y \rightarrow 0} \lim _{\Delta t \rightarrow 0} T_{2}^{\prime}=\frac{\partial \alpha_{k} G_{k}}{\partial y}
$$

Non conservative term $T_{3}$

$$
T_{3}=\frac{\widetilde{\left(\overline{F^{\operatorname{lag}}\left[\chi_{k}^{x}\right]}\right)_{i-1 / 2}}+\left(\widetilde{\left.F^{\operatorname{lag}\left[\chi_{k}^{x}\right]}\right)_{i+1 / 2}}\right.}{\Delta x}
$$

Assuming that all Riemann problems involved in these terms are reactive at each cell boundary, we have $\left[\chi_{k}^{x}\right]=0$ at the contact discontinuities whatever the fluid and the configuration are. The only discontinuities of the phase functions are located at the reactive front level. These jumps will be taken into account in the term $T_{3}^{r e a}$. Consequently the term $T_{3}$ vanishes and the limit is :

$$
\lim _{\Delta x \rightarrow 0} \lim _{\Delta y \rightarrow 0} \lim _{\Delta t \rightarrow 0} T_{3}=0
$$

Non conservative term $T_{3}^{\text {rea }}$

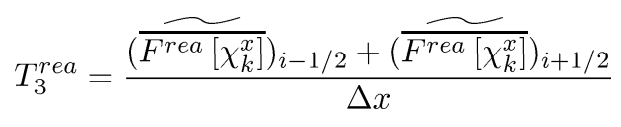

The temporal limit leads to the following relation:

$$
\lim _{\Delta t \rightarrow 0} \frac{\widetilde{\left(\overline{F^{r e a}\left[\chi_{k}^{x}\right]}\right)_{i-1 / 2}}+\left(\widetilde{F^{\text {rea }}\left[\chi_{k}^{x}\right]}\right)_{i+1 / 2}}{\Delta x}=\frac{\left(\overline{F^{r e a}\left[\chi_{k}^{x}\right]}\right)_{i-1 / 2}+\left(\overline{F^{\text {rea }}\left[\chi_{k}^{x}\right]}\right)_{i+1 / 2}}{\Delta x}
$$

At each cell boundary we have :

$$
\overline{F^{r e a}\left[\chi_{k}^{x}\right]}=\frac{1}{\Delta y} \int_{y_{j-1 / 2}}^{y_{j+1 / 2}} F^{r e a}\left[\chi_{k}^{x}\right] d y=\frac{1}{\Delta y}\left(\int_{y_{j-1 / 2}}^{y_{j+1 / 2}} F^{r e a} \chi_{k, d}^{x} d y-\int_{y_{j-1 / 2}}^{y_{j+1 / 2}} F^{r e a} \chi_{k, g}^{x} d y\right)
$$


where the relation $\left[\chi_{k}^{x}\right]=\chi_{k, d}^{x}-\chi_{k, g}^{x}$ is used representing the characteristic function jump between left and right states in a cell boundary. Then denoting $S_{k, d}$ and $S_{k, g}$ the presence surfaces of fluid $k$ at the right and the left sides of the cell boundary respectively, we get :

$$
\overline{F^{r e a}\left[\chi_{k}^{x}\right]}=\frac{1}{\Delta y}\left(\int_{S_{k, d}} F^{r e a} d y-\int_{S_{k, g}} F^{r e a} d y\right)
$$

The transfer fluxes being constant across reactive fronts the following relation holds :

$$
\overline{F^{r e a}\left[\chi_{k}^{x}\right]}=\frac{1}{\Delta y} \int_{S_{k, d}-S_{k, g}} F^{r e a} d y=\frac{1}{\Delta y} \frac{S_{k, d}-S_{k, g}}{\left|S_{k, d}-S_{k, g}\right|} \int_{\left|S_{k, d}-S_{k, g}\right|} F^{r e a} d y
$$

where $\left|S_{k, d}-S_{k, g}\right|$ is the total contact surface through which the jump of $\chi_{k}^{x}$ is non zero (reactive fronts).

Then :

$$
\overline{F^{r e a}\left[\chi_{k}^{x}\right]}=\frac{S_{k, d}-S_{k, g}}{\Delta y} \frac{1}{\left|S_{k, d}-S_{k, g}\right|} \int_{\left|S_{k, d}-S_{k, g}\right|} F^{r e a} d y=\frac{S_{k, d}-S_{k, g}}{\Delta y}\left\{F_{k}^{r e a}\right\}
$$

where $\left\{F_{k}^{r c a}\right\}$ is the average of reactive fluxes associated to fluid $k$ in the considered cell boundary.

Moreover using the definitions of contact surfaces, we have $\frac{S_{k, d}-S_{k, g}}{\Delta y}=\alpha_{k, d}-\alpha_{k, g}$ because these surfaces are multiple of $\Delta y$.

Then, denoting $\left[\alpha_{k}^{x}\right]$ the jump of volume fraction of fluid $k$ through the cell boundary, we have :

$$
\overline{F^{r e a}\left[\chi_{k}^{x}\right]}=\overline{\left[\chi_{k}^{x}\right]}\left\{F_{k}^{r e a}\right\}=\left[\alpha_{k}^{x}\right]\left\{F_{k}^{r e a}\right\}
$$

The following limit can now be adopted :

$$
\lim _{\Delta y \rightarrow 0}\left[\alpha_{k}^{x}\right]\left\{F_{k}^{r e a}\right\}=\left[\alpha_{k}^{x}\right] F_{k}^{r e a}
$$

The expression of the general term $T_{3}^{r e a}$ becomes :

$$
\frac{\left.\left(\overline{F^{r e a}\left[\chi_{k}^{x}\right]}\right)_{i-1 / 2}+\left(\overline{F^{r e a}\left[\chi_{k}^{x}\right.}\right]\right)_{i+1 / 2}}{\Delta x}=\frac{\left(\left[\alpha_{k}^{x}\right] F_{k}^{r e a}\right)_{i-1 / 2}+\left(\left[\alpha_{k}^{x}\right] F_{k}^{r e a}\right)_{i+1 / 2}}{\Delta x}
$$

Besides for each fluid $k$ the jump of $\alpha_{k}$ inside the cell is zero because $\alpha_{k}$ is assumed to be constant. Then :

$$
\lim _{\Delta x \rightarrow 0} \frac{\left(\left[\alpha_{k}^{x}\right] F_{k}^{r e a}\right)_{i-1 / 2}+\left(\left[\alpha_{k}^{x}\right] F_{k}^{r e a}\right)_{i+1 / 2}}{\Delta x}=\lim _{\Delta x \rightarrow 0} \frac{1}{\Delta x} \int_{x_{i-1 / 2}}^{x_{i+1 / 2}} F_{k}^{r e a} \frac{\partial \alpha_{k}}{\partial x} d x=F_{k}^{r e a} \frac{\partial \alpha_{k}}{\partial x}
$$

or

$$
\lim _{\Delta x \rightarrow 0} \lim _{\Delta y \rightarrow 0} \lim _{\Delta t \rightarrow 0} T_{3}^{r e a}=F_{k}^{r e a} \frac{\partial \alpha_{k}}{\partial x}
$$

Remark : the components of $F_{k}^{r e a}$ may be expressed in the genaral case at a discontinuity of $\alpha_{k}$, for example in a cell boundary. For this the integration formulas of the method are considered :

$$
F_{k}^{r e a}=\frac{\overline{F^{r e a}\left[\chi_{k}^{x}\right]}}{\overline{\left[\chi_{k}^{x}\right]}}=\frac{\sum_{l, m}\left(S F^{r e a, *}\left[\chi_{k}^{x, *}\right]\right)_{l m}}{\sum_{l, m}\left(S\left[\chi_{k}^{x, *}\right]\right)_{l m}}=\frac{\left(S F^{r e a, *}\left[\chi_{k}^{x, *}\right]\right)_{12}+\left(S F^{r e a, *}\left[\chi_{k}^{x, *}\right]\right)_{21}}{\left(S\left[\chi_{k}^{x, *}\right]\right)_{12}+\left(S\left[\chi_{k}^{x, *}\right]\right)_{21}}
$$

Following the trivial relation $\left[\chi_{k}^{x, *}\right]_{21}=-\left[\chi_{k}^{x, *}\right]_{12}$, we get :

$$
F_{k}^{r e a}=\frac{\left(S F^{r e a, *}\right)_{12}-\left(S F^{r e a, *}\right)_{21}}{S_{12}-S_{21}}=\frac{1}{S_{12}-S_{21}}\left(\begin{array}{l}
-S_{12} \sigma_{12}^{*}+S_{21} \sigma_{21}^{*} \\
S_{12} m_{12}^{*}-S_{21} m_{21}^{*} \\
S_{12}(m u+P)_{12}^{*}-S_{21}(m u+P)_{21}^{*} \\
S_{12}(m v)_{12}^{*}-S_{21}(m v)_{21}^{*} \\
S_{12}(m E+P u)_{12}^{*}-S_{21}(m E+P u)_{21}^{*}
\end{array}\right)
$$


Now if $S_{12}=0$ then $S_{21} \neq 0$ using the definition of the contact surfaces between fluids. In this case we also have the relations $\frac{\partial \alpha_{1}}{\partial x}>0$ and $\frac{\partial \alpha_{2}}{\partial x}<0$.

The preceding relation reduces to :

$$
F_{k}^{r e a}=F_{21}^{r e a, *}=\left(\begin{array}{l}
-\sigma_{21}^{*} \\
m_{21}^{*} \\
(m u+P)_{21}^{*} \\
(m v)_{21}^{*} \\
(m E+P u)_{21}^{*}
\end{array}\right)
$$

The symmetric case where $S_{12} \neq 0$ and $S_{21}=0$ also holds. In this case $\frac{\partial \alpha_{1}}{\partial x}<0, \frac{\partial \alpha_{2}}{\partial x}>0$ and the reactive flux writes :

$$
F_{k}^{r e a}=F_{12}^{r e a, *}=\left(\begin{array}{l}
-\sigma_{12}^{*} \\
m_{12}^{*} \\
(m u+P)_{12}^{*} \\
(m v)_{12}^{*} \\
(m E+P u)_{12}^{*}
\end{array}\right)
$$

More generally, denoting by $f^{+}=\operatorname{Max}(0, f)$ the positive part of an arbitrary variable $f$, the expression of the reactive flux becomes :

$$
F_{k}^{r e a}=\left(s g\left(\frac{\partial \alpha_{1}}{\partial x}\right)\right)^{+} F_{21}^{r e a, *}+\left(s g\left(\frac{\partial \alpha_{2}}{\partial x}\right)\right)^{+} F_{12}^{r e a, *}
$$

This expression may also be written as :

$$
F_{k}^{r e a}=\left(\begin{array}{l}
-\sigma_{I, x} \\
m_{I, x} \\
(m u+P)_{I, x} \\
(m v)_{I, x} \\
(m E+P u)_{I, x}
\end{array}\right)
$$

Now the expressions of the various components of the reactive flux vector can be computed with the help of the linearized reactive Riemann solver.

\section{Non conservative term $T_{3}^{\prime}$}

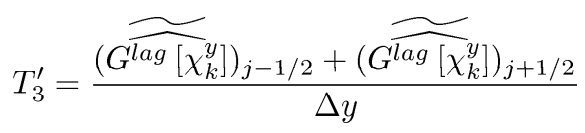

Following the same developments as the term $T_{3}$ we have $T_{3}^{\prime}=0$ and then :

$$
\lim _{\Delta y \rightarrow 0} \lim _{\Delta x \rightarrow 0} \lim _{\Delta t \rightarrow 0} T_{3}^{\prime}=0
$$

Reactive non conservative term $T_{3}^{\prime}$,rea

Again following the same developments as the term $T_{3}^{r e a}$, we have :

$$
\lim _{\Delta x \rightarrow 0} \lim _{\Delta y \rightarrow 0} \lim _{\Delta t \rightarrow 0} T_{3}^{\prime}{ }^{\text {,rea }}=G_{k}^{r e a} \frac{\partial \alpha_{k}}{\partial y}
$$


with

$$
G_{k}^{r e a}=\left(\begin{array}{l}
-\sigma_{I, y} \\
m_{I, y} \\
(m u)_{I, y} \\
(m v+P)_{I, y} \\
(m E+P v)_{I, y}
\end{array}\right)
$$

Internal term $T_{\text {int }}$

$$
T_{\text {int }}=\frac{\sum_{i n t}\left(\widetilde{F^{\operatorname{lag}}\left[\chi_{k}^{x}\right]}\right)_{i n t}}{\Delta x}+\frac{\sum_{i n t}\left(\widetilde{\left.\left.G^{\operatorname{lag}\left[\chi_{k}^{y}\right.}\right]\right)_{i n t}}\right.}{\Delta y}
$$

Again the Riemann problems involved inside the cell are assumed to be reactive ones. In this case $\left[\chi_{k}^{x, *}\right]=\left[\chi_{k}^{y, *}\right]=0$ at the contact discontinuities whatever the configuration is.

The limit reduces to :

$$
\lim _{\Delta y \rightarrow 0} \lim _{\Delta x \rightarrow 0} \lim _{\Delta t \rightarrow 0} T_{i n t}=0
$$

Reactive internal term $T_{\text {int }}^{\text {rea }}$

$$
T_{\text {int }}^{\text {rea }}=\frac{\sum_{i n t}\left(\widetilde{F^{\text {rea }}\left[\chi_{k}^{x}\right]}\right)_{\text {int }}}{\Delta x}+\frac{\sum_{\text {int }}\left(\widetilde{G^{\text {rea }}\left[\chi_{k}^{y}\right]}\right)_{\text {int }}}{\Delta y}
$$

The temporal limit is :

$$
\lim _{\Delta t \rightarrow 0} T_{i n t}^{r e a}=\frac{\sum_{i n t}\left(\overline{F^{r e a}\left[\chi_{k}^{x}\right]}\right)_{i n t}}{\Delta x}+\frac{\sum_{i n t}\left(\widehat{G^{r e a}\left[\chi_{k}^{y}\right]}\right)_{i n t}}{\Delta y}
$$

Using the average definitions we have :

$$
\frac{\sum_{i n t}\left(\overline{F^{r e a}\left[\chi_{k}^{x}\right]}\right)_{i n t}}{\Delta x}=\frac{\sum_{i n t}\left(\left(S F^{r e a, *}\left[\chi_{k}^{x, *}\right]\right)_{12}+\left(S F^{r e a, *}\left[\chi_{k}^{x, *}\right]\right)_{21}\right)_{i n t}}{\Delta x \Delta y}
$$

Now using the simple relation $\left[\chi_{k}^{x, *}\right]_{21}=-\left[\chi_{k}^{x, *}\right]_{12}$, we get :

$$
\frac{\sum_{i n t}\left(\overline{F^{r e a}\left[\chi_{k}^{x}\right]}\right)_{i n t}}{\Delta x}=\left[\chi_{k}^{x, *}\right]_{12} \frac{\sum_{i n t}\left(\left(S F^{r e a, *}\right)_{12}-\left(S F^{r e a, *}\right)_{21}\right)_{i n t}}{\Delta x \Delta y}
$$

The reactive fluxex $F_{12}^{r e a, *}$ et $F_{21}^{r e a, *}$ being constant across reactive fronts inside the cell, the previous relation writes :

$$
\frac{\sum_{i n t}\left(\overline{F^{r e a}\left[\chi_{k}^{x}\right]}\right)_{i n t}}{\Delta x}=\left[\chi_{k}^{x, *}\right]_{12} \frac{F_{12}^{r e a, *} \sum_{i n t} S_{12, \text { int }}-F_{21}^{r e a, *} \sum_{i n t} S_{21, i n t}}{\Delta x \Delta y}
$$

In this relation, $\sum_{i n t} S_{12, i n t}$ (resp. $\sum_{i n t} S_{21, i n t}$ ) represents the strictly internal total surface for which fluid 1 (resp. 2) at the left is in contact with fluid 2 (resp. 1) at the right.

Then the following relation $\sum_{i n t} S_{12, i n t}=\sum_{i n t} S_{21, i n t}$ holds. Indeed this assumption holds for strictly internal symmetric entities such as bubbles.

Besides $S_{x}=\sum_{\text {int }} S_{12, \text { int }}+\sum_{\text {int }} S_{21, \text { int }}=2 \sum_{\text {int }} S_{12, \text { int }}$ denotes the total contact surface between fluids 1 
and 2 inside the cell and according to the $x$ direction.

In this case, denoting $\left[\chi_{k}^{x, *}\right]_{12}=\delta_{k 2}-\delta_{k 1}\left(\delta_{k l}\right.$ being the Kronecker symbol), we have :

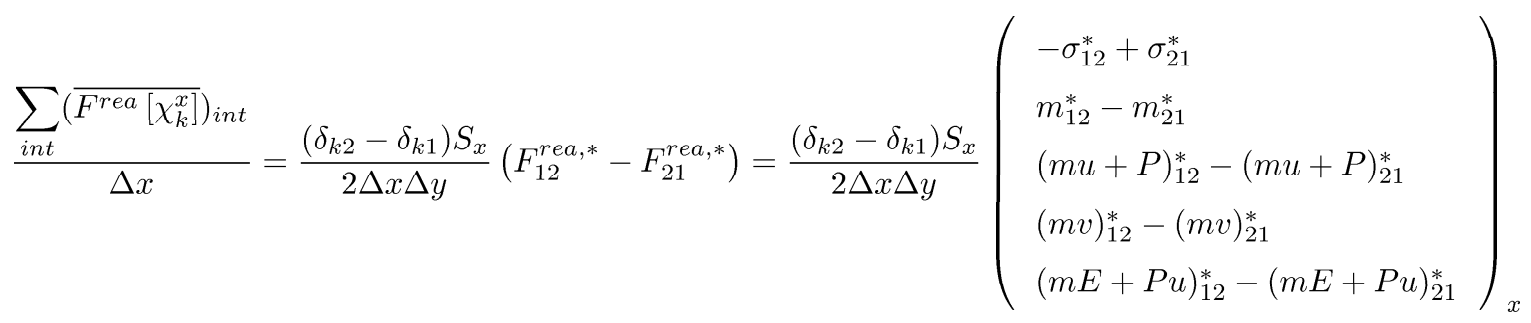

Symmetrically, using the same ingredients in the $y$ direction, we have :

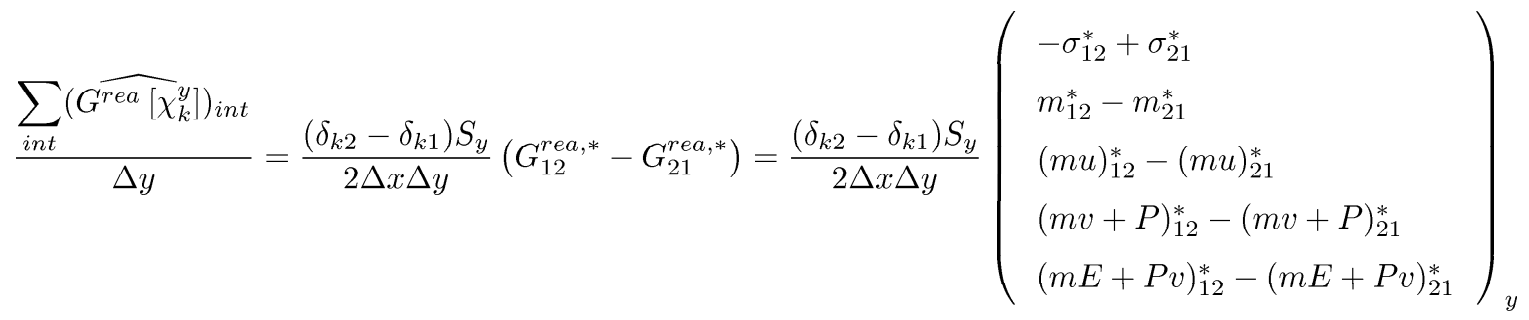

where $S_{y}$ represents the total contact surface between fluids inside the cell according to the $y$ direction.

Now the analytical expressions of variables from the linearized reactive Riemann solver must be used to express the general source terms of the discrete equations method as in the inert cases. This work is in progress.

\section{Determination of the mass flow rate}

The aim of this section is the modelling of the mass flow rate between phases. Local mechanisms of the evaporation process are considered between a liquid phase and its associated vapor in presence of temperature gradients. Indeed a liquid phase change may occur according to 2 main mechanisms. The first one is related to evaporation induced by external heating or concentration gradients in a quite constant pressure environment. The second case corresponds to 'flashing' evaporation such as cavitation induced by a strong and sudden depressurization of the liquid phase. In this process no extra energy is needed for the phase change. The necessary energy is already contained in the liquid in the form of internal energy. The mechanisms of interest that are considered in this report are the diffusive ones : heat diffusion at the interfaces. In the present approach an important development is done compared to existing models of the literature. Indeed drops are usually assumed to be instantaneously at the saturation temperature or present a uniform temperature profile. In the approach adopted in this report the characteristic time scale of heating is taken into account. For the sake of clarity thermal diffusion only at the microscopic interface between a liquid and its vapor is presented in this document.

The local equations under consideration are the mass and energy equations :

$$
\left\{\begin{array}{l}
\frac{\partial \rho_{k}}{\partial t}+\vec{\nabla} \cdot(\rho \vec{u})_{k}=0 \\
\frac{\partial(\rho E)_{k}}{\partial t}+\vec{\nabla} \cdot(\rho H \vec{u}+\vec{q})_{k}=0
\end{array}\right.
$$


where phase $k$ is either liquid $(l)$ or vapor $(g)$ phases and $\vec{q}_{k}=-\lambda_{k} \vec{\nabla} T_{k}$ is the diffusive heat flux.

The momentum equation is no longer useful since the quasi-isobaric assumption is made. This hypothesis consists in neglecting the pressure and kinetic energies variations in the total energy equation. Indeed an evaporation front propagates slowly (in the order of $1 \mathrm{~m} / \mathrm{s}$ ) compared to acoustic waves present in the medium (in the order of $1000 \mathrm{~m} / \mathrm{s}$ ). These acoustic waves allows a quasi-instantaneous pressure equilibrium through evaporation fronts. Thus the quasi-isobaric assumption commonly used for flame propagation is also fulfilled in the present case.

When dealing with interface conditions and integrating system (52) over an arbitrary control volume containing a steady interface, the following algebraic system is obtained :

$$
\left\{\begin{array}{l}
\dot{m}_{l}+\dot{m}_{g}=0 \\
\dot{m}_{l} h_{l, I}-\lambda_{l} \vec{\nabla} T_{l, I} \cdot \vec{n}_{l}+\dot{m}_{g} h_{g, I}-\lambda_{g} \vec{\nabla} T_{g, I} \cdot \vec{n}_{g}=0
\end{array}\right.
$$

where subscript $I$ denotes interface variables.

The diffusive heat fluxes may be defined as :

$$
\vec{q}_{k} \cdot \vec{n}_{k}=-\lambda_{k} \vec{\nabla} T_{k, I} \cdot \vec{n}_{k}=H_{T k}\left(T_{I}-T_{k \infty}\right)
$$

where $H_{T k}$ represents the diffusive exchange coefficient in each phase based on correlations including the Nusselt number from experiments. $T_{I}$ is the common interface temperature of both phases and $T_{k \infty}$ is the temperature of phase $k$ given by a macroscopic two-phase flow model.

Combining equations of system (53) a simple expression of the mass flow rate is obtained :

$$
\dot{m}_{g}=\frac{H_{T l}\left(T_{l \infty}-T_{I}\right)+H_{T g}\left(T_{g \infty}-T_{I}\right)}{L_{v}\left(T_{I}\right)}
$$

where the relation $h_{g I}-h_{l I}=L_{v}\left(T_{I}\right)$ has been used ( $L_{v}$ is the latent heat of vaporization). Since the temperature is determined by the saturation constraint $T_{I}=T_{\text {sat }}(P)$ and the pressure $P$ is known (from the macroscopic model) the mass flow rate is computed by relation (55).

Generalization of this method to additional diffusive effects (mass concentration gradients) and an arbitrary number of phases present in the mixture needs some further developments.

\section{Conclusion}

This work deals with the modelling of mass transfer between phases in multiphase compressible flows models $[1,2,3,13]$. The expressions of the different terms present in system (1) have been partially obtained in this document. Two complementary steps were necessary. The first one was the obtention of explicit expressions of the mass transfer terms. The linearized reactive Riemann problem has been considered and solved. The discrete equations method (DEM) has been extended to reactive subsonic evaporation fronts and mass transfer terms have been obtained when dealing with the continuous limit of the numerical system. The second step were related to the determination of the mass flow rate considering local diffusive effects at the microscopic scale. More efforts are still necessary and are in progress to achieve this work. 


\section{References}

[1] Abgrall, R. \& Saurel, R. (2003) Discrete Equations for Physical and Numerical Compressible Multiphase Mixtures. J. Comp. Physics, 186, pp 361-396

[2] Baer, M.R. \& Nunziato, J.W. (1986) A Two-Phase Mixture Theory for the Deflagration-toDetonation Transition (DDT) in Reactive Granular Materials. Int. J. of Multiphase Flows, 12, pp $861-889$

[3] Chinnayya, A., Daniel, E. \& Saurel, R. (2004) Modelling Detonation Waves in Heterogeneous Energetic Materials. J. Comp. Physics, 196, pp 490-538

[4] Chorin, A.J. (1977) Random Choice Methods with Applications to Reacting Gas Flow. J. Comp. Physics, 25, pp 253-272

[5] Clavin, P. \& Garcia, P. (1983) The Influence of the Temperature Dependence of Diffusivities on the Dynamics Flame Fronts. J. Mécanique Théorique et Appliquée, 2, pp 245-263

[6] Drew, D.A. \& Passman, S.L. (1998) Theory of Multicomponent Fluids. Springer

[7] Frost, D.L., Lee, J.H.S. \& Ciccarelli, G. (1991) The Use of Hugoniot Analysis for the Propagation of Vapor Explosion Waves. Shock Waves, 1, pp 99-110

[8] Gavrilyuk, S. \& Saurel, R. (2002) Mathematical and Numerical Modelling of Two-Phase Compressible Flows with Micro-Inertia. J. Comp. Phys., 175(1), pp 326-360

[9] Godlewski, E. \& Raviart, P.-A. (1996) Numerical Approximation of Hyperbolic Systems of Conservation Laws. Applied Mathematical Sciences, 118, Springer-Verlag

[10] Harlow, F. \& Amsden, A. (1971) Fluid dynamics. monograph LA-4700, Los Alamos National Laboratory, NM

[11] Kurschat, Th., Chaves, H. \& Meier, G.E.A. (1992) Complete Adiabatic Evaporation of Highly Superheated Liquid Jets. J. Fluid Mech., 236, pp 43-59

[12] Lemetayer, O., Massoni, J. \& Saurel, R. (2004) Elaborating Equations of State of a Liquid and its Vapor for Two-Phase Flow Models. Int. J. of Thermal Sciences, 43, pp 265-276

[13] Lemetayer, O., Massoni, J. \& Saurel, R. (2005) Modelling Evaporation Fronts with Reactive Riemann Solvers. J. Comp. Physics, 205, pp 567-610

[14] Menikoff, R. \& Plohr, B.J. (1989) The Riemann Problem for Fluid Flow of Real Materials. Rev. Mod. Physics, 61, pp 75-130

[15] Reinke, P. \& Yadigaroglu, G. (2001) Explosive Vaporization of Superheated Liquids by Boiling Fronts. Int. J. of Multiphase Flows, 27, pp 1487-1516

[16] Saurel, R. \& Abgrall, R. (1999) A Multiphase Godunov Method for Compressible Multifluid and Multiphase Flows. J. Comp. Physics, 150, pp 425-467 
[17] Saurel, R., Gavrilyuk, S. \& Renaud, F. (2003) A Multiphase Model with Internal Degrees of Freedom : Application to Shock-Bubble Interaction. J. Fluid. Mechanics, 495, pp 283-321

[18] Saurel, R. \& LeMetayer, O. (2001) A Multiphase Model for Compressible Flows with Interfaces, Shocks, Detonation Waves and Cavitation. J. Fluid. Mechanics, 431, pp 239-271

[19] Simões-Moreira, J.R. \& Shepherd, J.E. (1999) Evaporation Waves in Superheated Dodecane. J. Fluid Mech., 382, pp 63-86

[20] Teng, Z.-H., Chorin, A.J. \& LiU, T.-P. (1982) Riemann Problems for Reacting Gas, with Applications to Transition. SIAM J. Appl. Math., 42(5), pp 964-981

[21] Thompson, P.A., Chaves, H., Meier, G.E.A., Kim, Y.-G. \& Speckmann, H.-D. (1987) Wave Splitting in a Fluid of Large Heat Capacity. J. Fluid Mech., 185, pp 385-414

[22] Toro, E.F. (1997) Riemann Solvers and Numerical Methods for Fluid Dynamics. Springer 




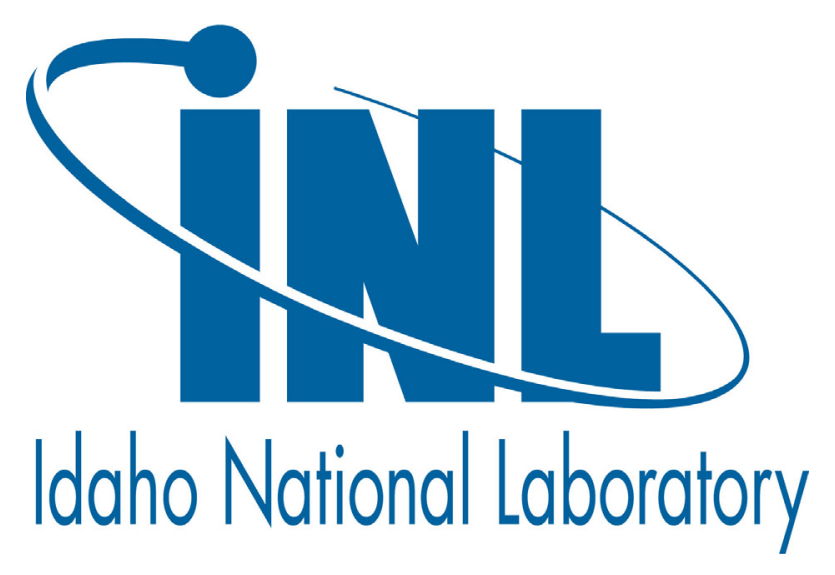

